Supplementary information for:

\title{
Ethylene-Triggered Formation of Ruthenium Alkylidene from Decomposed Catalyst
}

\author{
Wietse Smit, Marco Foscato, ${ }^{*}$ Giovanni Occhipinti,* and Vidar R. Jensen* \\ Department of Chemistry, University of Bergen, Allégaten 41, N-5007 Bergen, Norway
}

\section{Table of contents}

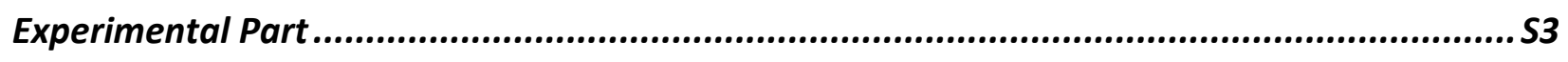

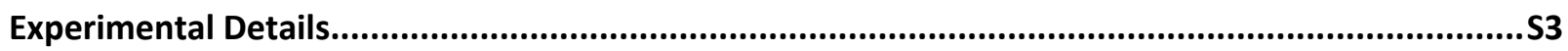

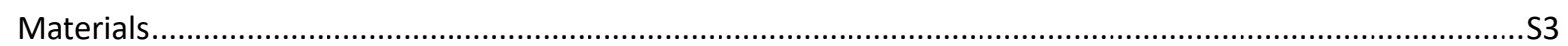

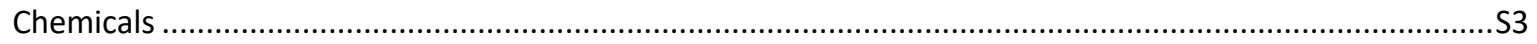

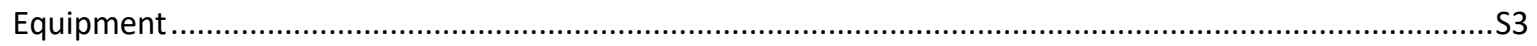

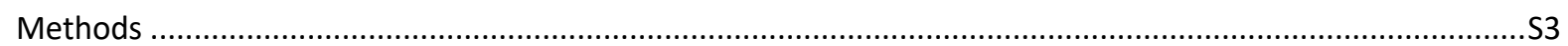

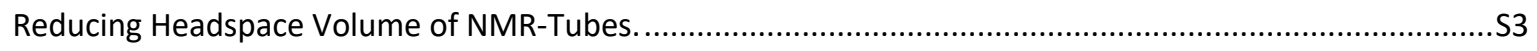

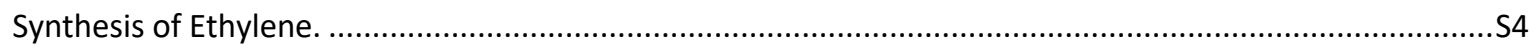

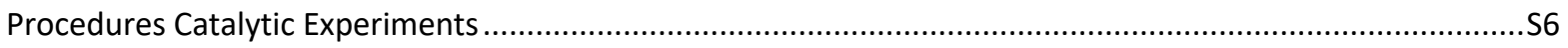

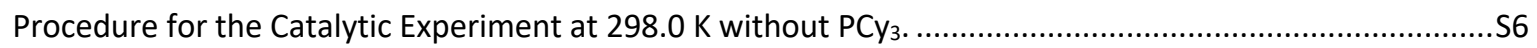

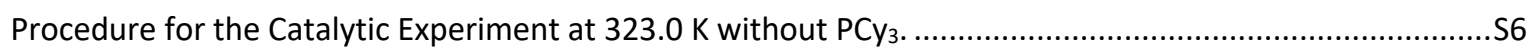

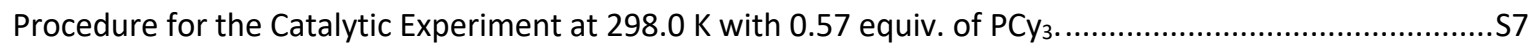

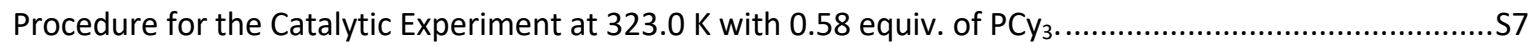

Procedure for the Catalytic Experiments with Variable Concentration of 1 ( $323.0 \mathrm{~K}$ with 0.6 equiv. of $\mathrm{PC} \mathrm{y}_{3}$ ).

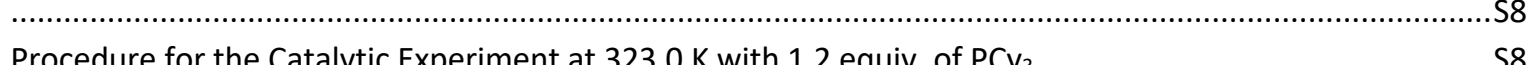

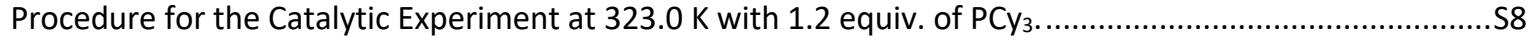

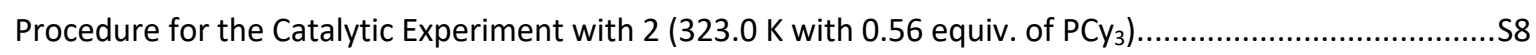

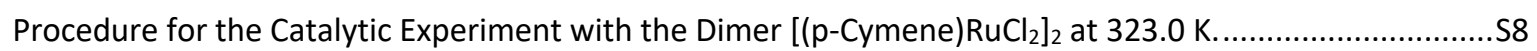

Ring Closing Metathesis (RCM) of Diethyl Diallylmalonate (DEDAM) ....................................................59

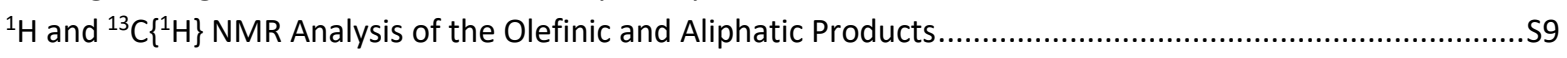

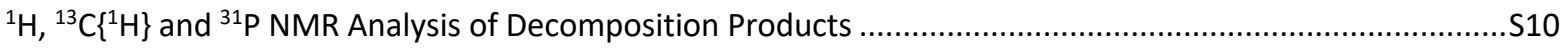

Calculation of Turnovers and Rates of Product Formation ....................................................................S10

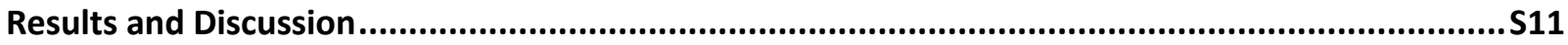

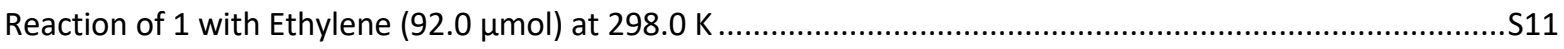

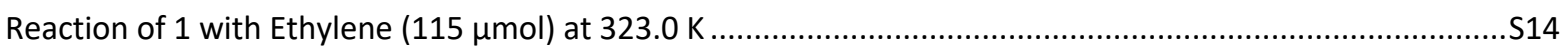

Reaction of 1 with Ethylene $(115.9 \mu \mathrm{mol})$ at $298.0 \mathrm{~K}$ in the Presence of $\mathrm{PCY}_{3}(0.57$ equiv. $)$............................S17

Reaction of $1(4.1 \mu \mathrm{mol})$ with Ethylene $(78.5 \mu \mathrm{mol})$ at $323.0 \mathrm{~K}$ in the Presence of $\mathrm{PCy}_{3}(0.58$ equiv.) .............S20

Reaction of $2(4.1 \mu \mathrm{mol})$ with Ethylene $(89.5 \mu \mathrm{mol})$ at $323.0 \mathrm{~K}$ in the Presence of $\mathrm{PCy}_{3}(0.58$ equiv.) .............S25

Reaction of $1(3.6 \mu \mathrm{mol})$ with Ethylene $(84.3 \mu \mathrm{mol})$ at $323.0 \mathrm{~K}$ in the Presence of $\mathrm{PCy}_{3}(1.2$ equiv.) ...............S27

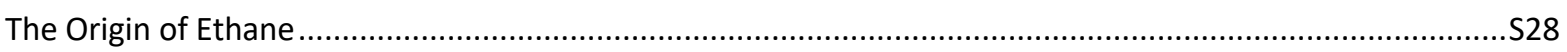

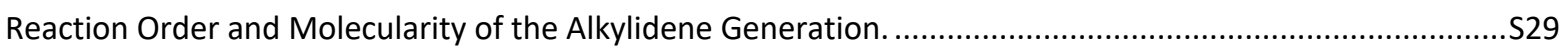

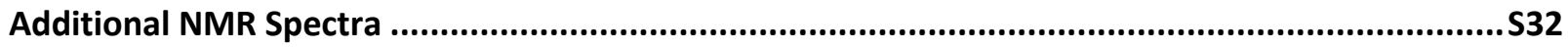

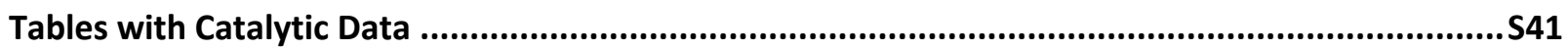




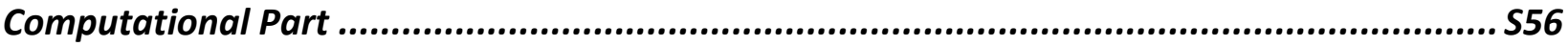

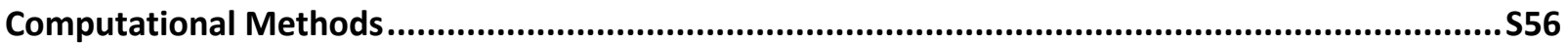

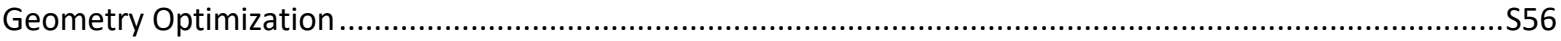

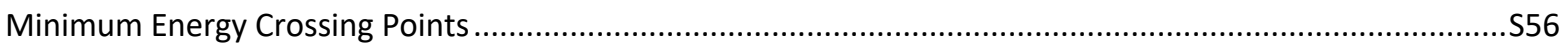

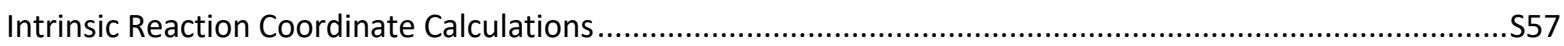

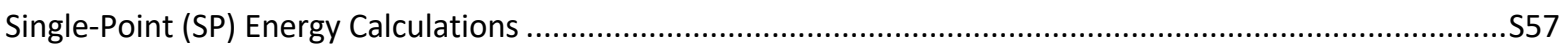

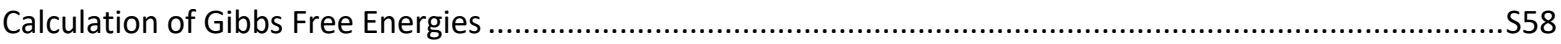

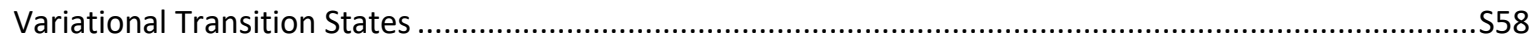

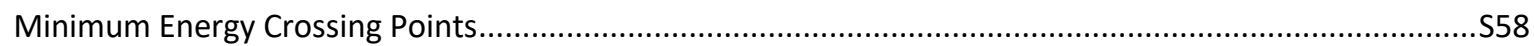

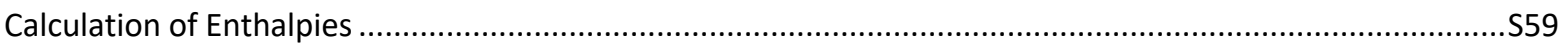

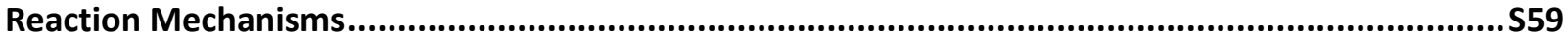

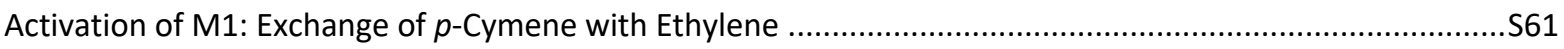

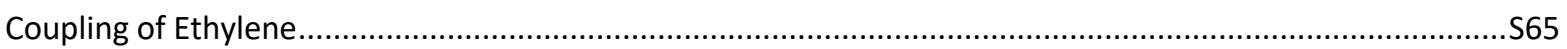

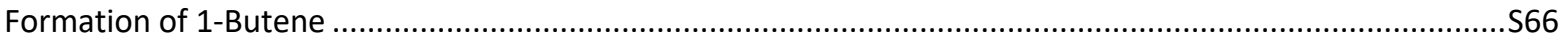

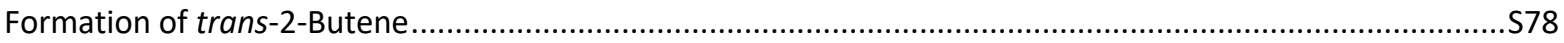

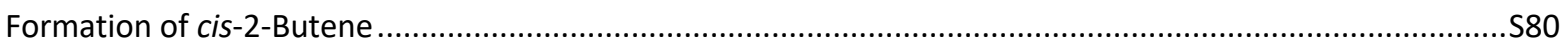

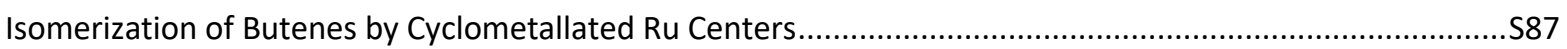

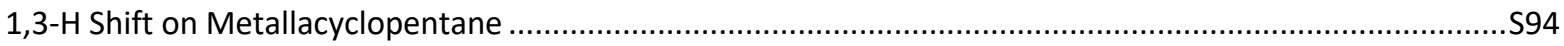

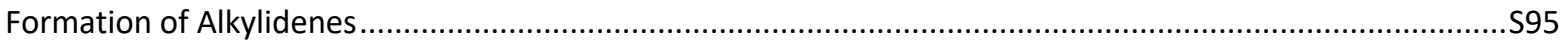

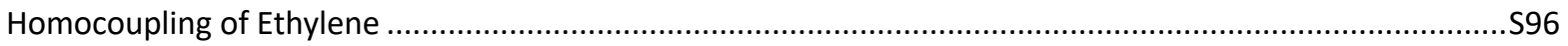

Formation of Propene from Decomposition of Unsubstituted Metallacyclobutane.......................................S97

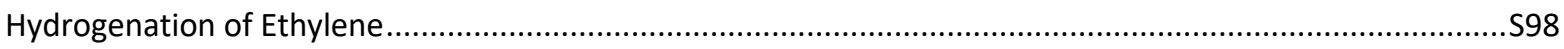

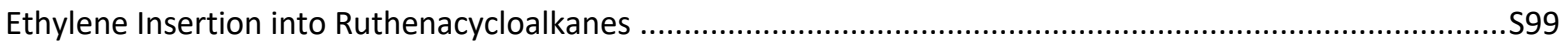

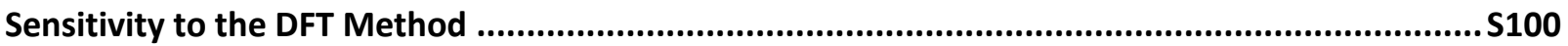

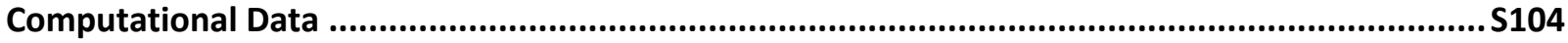

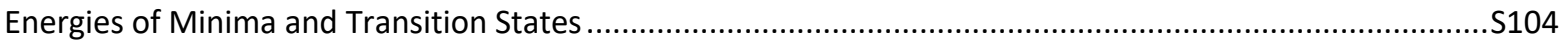

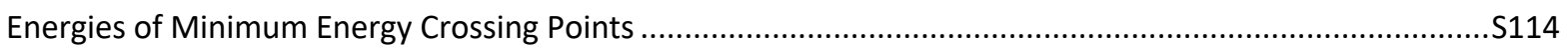

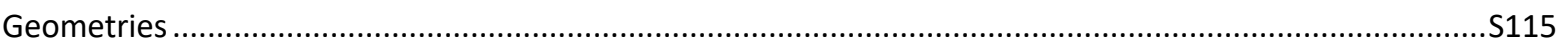

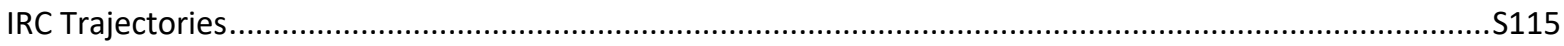

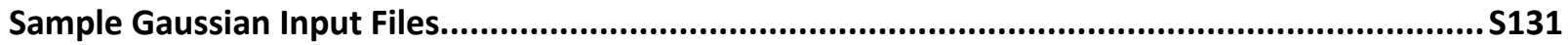

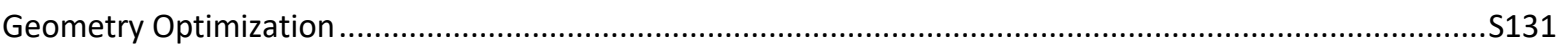

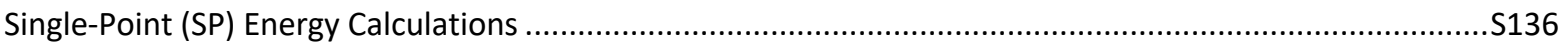

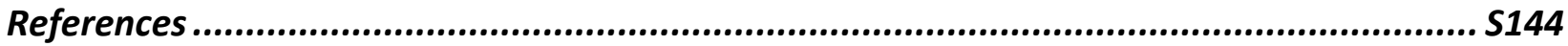




\section{Experimental Part}

\section{Experimental Details}

\section{Materials}

\section{Chemicals}

All chemicals were obtained from Sigma-Aldrich unless otherwise stated. Grubbs Catalyst ${ }^{\mathrm{TM}}$ C859 (Piers second-generation catalyst), Grubbs Catalyst ${ }^{\mathrm{TM}} \mathrm{C} 633$ (Grubbs Z-selective catalyst), Grubbs secondgeneration catalyst and tricyclohexylphosphine $\left(\mathrm{PCy}_{3}\right)$ were used as received. 2,2,3,3-Tetramethylbutane and palladium on carbon (10\% Pd, reduced and dry) were obtained from Tokyo Chemical Industries (TCI) and Strem Chemicals, respectively, and both were used as received. Benzene- $d_{6}$ was degassed, dried over $\mathrm{CaH}_{2}$ and subsequently filtered over basic alumina (Brockmann I, activated three days at $220{ }^{\circ} \mathrm{C}$ in vacuo). Styrene was obtained from Fluka and degassed before use. Diethyl diallylmalonate (Sigma Aldrich) was degassed, dried over molecular sieves $(4 \AA)$ and passed through a column of activated basic alumina. Compound $\mathbf{1}$, (SIMes) $\left(\eta^{6}-p\right.$-cymene) $\mathrm{RuCl}_{2}$, was synthesized according to literature procedure. ${ }^{1}$ Ethylene (purity $>99.99 \%$, as determined by GC) was produced according to the procedure described in the section "Synthesis of ethylene". Propene was obtained as Messer ${ }^{\circledR}$ CANGas at $99.5 \%$ purity and hydrogen from Praxair with quality 5.0 Ultra, both of which were used as received. A Restek Rt ${ }^{\mathbb{B}}$-Alumina BOND $/ \mathrm{Na}_{2} \mathrm{SO}_{4} 50 \mathrm{~m} \times 0.32 \mathrm{~mm} \times 5 \mu \mathrm{m}$ PLOT GC-column was obtained from VWR and pretreated according to the procedure indicated by the manufacturer.

\section{Equipment}

Catalytic reactions were carried out in Gas-Tight (J. Young valve), low-pressure/vacuum (LPV) Shigemi NMR-tubes with an outer diameter of $5 \mathrm{~mm}$ and a bottom length of $8 \mathrm{~mm}$ that is compatible with Bruker NMR instruments (Wilmad-LabGlass, product number: CMS-005B-LPV; ordered via: Fluorochem Ltd, Hadfield, Derbyshire, United Kingdom). Two additional PTFE holders (Wilmad-LabGlass, product number: 529-B) were ordered from Sigma-Aldrich (product nr: Z286672). Further information, describing the justification for the use of this type of NMR tubes and how they were used in experiments, is found in the section "Reducing headspace volume of NMR-tubes".

NMR spectra were recorded on Bruker BioSpin AV500, NEO600 and 850 Ascend. Chemical shifts in ${ }^{1} \mathrm{H}$ and ${ }^{13} \mathrm{C}\left\{{ }^{1} \mathrm{H}\right\}$ NMR spectra are reported relative to residual solvent peaks. ${ }^{2}$ In the ${ }^{31} \mathrm{P}$ NMR spectra chemical shifts were referenced using a solution of tricyclohexylphosphine in benzene- $d_{6}$ as external standard $(\delta=10.9 \mathrm{ppm}) .{ }^{3}$ Generally, a delay (D1) of at least five times the spin-lattice relaxation time $\left(T_{1}\right)$ is advised between consecutive Rf-pulses to obtain $>99 \% z$-magnetization recovery, and thus, to allow quantitative NMR-analysis. ${ }^{4,5}$ Therefore, a D1 interval of 60 seconds was used for both ${ }^{1} \mathrm{H}$ and ${ }^{13} \mathrm{C}\left\{{ }^{1} \mathrm{H}\right\}$ NMR spectra recorded during catalytic experiments.

Gas chromatography (GC) analysis were performed on an Agilent technologies 7890A instrument equipped with a split/splitless injection port, Restek $\mathrm{Rt}^{\mathbb{R}}$-Alumina BOND/Na $\mathrm{NO}_{4} 50 \mathrm{~m}$ x $0.32 \mathrm{~mm}$ x $5 \mu \mathrm{m}$ PLOT column and FID detector. Injections of gas mixtures were performed manually using a gas tight syringe (500R-V-GT, SGE Analytical Science). Molar ratios between the different gasses were calculated from the area percentages according to their respective effective carbon number $(\mathrm{ECN}){ }^{6}$

\section{Methods}

\section{Reducing Headspace Volume of NMR-Tubes.}

Standard low-pressure/vacuum (LPV) NMR tubes contain a relatively large headspace volume $(\approx 2 \mathrm{ml})$. Thus, when investigating dissolved gaseous compounds by NMR spectroscopy, a significant amount of these compounds may be lost from the solution to the headspace. Quantification of these gasses in the headspace is not possible with conventional NMR, lowering the apparent yield. In addition, a large headspace volume most likely hampers the reaction of gaseous compounds with the catalyst in solution. 
Shigemi NMR tubes with J. Young valves (Figure S1) offer the most appropriate way in reducing the headspace volume of an NMR-tube. With this type of tube, most of the volume is filled up by sliding a glass plunger attached to a PTFE holder into the tube on top of the to-be-measured solution. In our experiments, the remaining headspace volume above this plunger and holder was even further reduced by sliding two additional PTFE holders in on top. The headspace volume thus obtained was estimated at $\approx 0.5$ $\mathrm{ml}$, a significant reduction from the $\approx 2 \mathrm{ml}$ in standard LPV NMR tubes. Other researchers have reduced the headspace volume by simply using a larger sample volume. ${ }^{7,8}$ However, this was deemed unsuitable for our experiments since large sample volumes makes the NMR-tube too heavy, which increases the risk of breakage. Large-volume samples also reduce the shimming quality and thus the resolution of the NMR spectra.

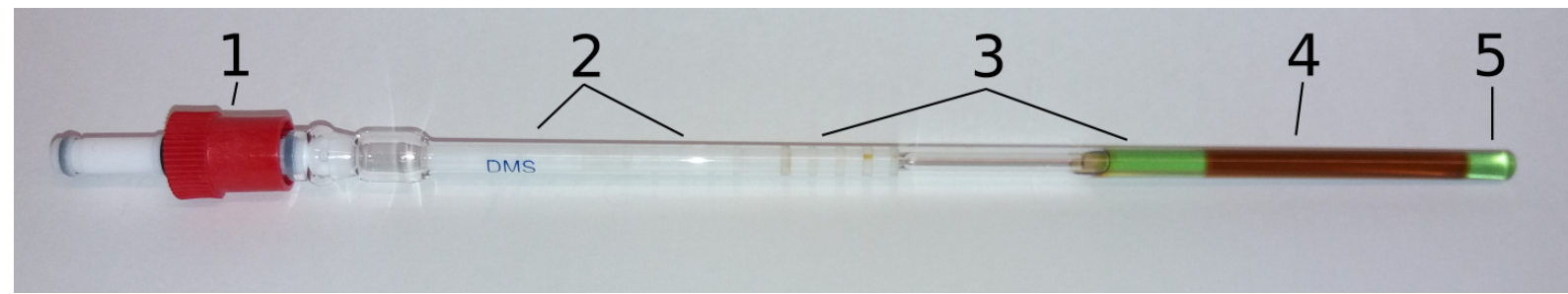

Figure S1. Experimental setup of using a LPV Shigemi tube set in combination with two additional PTFE holders to reduce headspace. 1. J. Young valve, 2. Additional PTFE holders (2x), 3. PTFE holder with connected glass plunger, 4. Reaction mixture, 5. Glass tube-bottom.

Differences in solubility between the gaseous compounds in the sample might give distorted ratios in solution, even if the headspace volume has been reduced. First, to investigate if there is any difference in the ratios of gaseous products dissolved in benzene- $d_{6}$ versus the ratios in the complete sample (headspace + solution) a catalytic sample was analyzed through ${ }^{1} \mathrm{H}$ NMR (for the gasses in solution) and gas chromatography (gasses in the complete sample). This sample was obtained with a procedure similar to the ones described below, with the exception that a standard LPV tube with J. Young valve was used here. After the ratios of the different products were established through ${ }^{1} \mathrm{H}$ NMR, the sample was completely evaporated into an evacuated $1 \mathrm{~L}$ Schlenk flask. This was achieved through connecting the J. Young valve of the NMR tube via a rubber septum and glass tube to the flask and slowly opening both the valve on the Schlenk flask and NMR tube. Some warm water was used to heat the NMR tube to aid the complete evaporation of the benzene- $d_{6}$ into the Schlenk flask. Subsequently, all valves were closed, the NMR tube was removed and the Schlenk vial was filled to standard pressure with argon. Then, through a septum, a $50 \mu \mathrm{L}$ sample of the gas mixture in the Schlenk flask was taken using a gas tight syringe and injected manually on a GC with the settings shown in Table S1. Using the effective carbon number (ECN) the molar ratios of the gasses were calculated from area percentages. ${ }^{6}$ The results of this experiment (Table S2) show a maximum relative difference (Rel\%) of $6.6 \%$. This indicates that there is not much difference in molar ratios between the different gaseous compounds in solution and those determined including also the portions present in the headspace.

\section{Synthesis of Ethylene.}

Due to the sensitivity of $\mathbf{1}$ towards oxygen, addition of ethylene needs to be performed in a glove box. The ethylene was synthesized inside the glove box from the self-metathesis of styrene $(0.3 \mathrm{ml})$, at room temperature, with Piers second-generation catalyst $(15 \mathrm{mg})$ using the gas production apparatus depicted in Figure S2: In the $3 \mathrm{ml}$ vial (1), that contains a small stirring magnet and an amount of the previously mentioned catalyst, styrene is injected from a syringe (3) to start the generation of ethylene. Since the vial is closed off with a septum (2) the gas is forced to rise through a cannula (4) into a glass tube (5) that is, at both sides, closed off with septa. Here possible impurities in the gas (such as vapors from styrene) are adsorbed on a layer of basic alumina (7) kept in place with two wads of quartz wool (6). After purification the gas is led through a long cannula (8) into a solution of $\mathbf{1}$ in benzene- $d_{6}$ contained in a Shigemi NMRtube (9) with J. Young valve. Subsequently, the gas was bubbled through the sample for about 30 seconds. 
A septum (10) on top of the tube prevents loss of ethylene and another cannula (11) is added to vent off excess gas. Analysis through NMR and GC shows that ethylene obtained with the above method has a purity of $>99.99 \%$ (traces of propene were observed with GC).

Table S1. Settings of the GC.

\begin{tabular}{|c|c|}
\hline Inlet port & \\
\hline$T_{\text {inj }}\left({ }^{\circ} \mathrm{C}\right)$ & 200 \\
\hline Type & split/constant pressure \\
\hline Split ratio & $5: 1$ \\
\hline$V_{\text {inj }}(\mu l)$ & 50 \\
\hline Carrier gas & $\mathrm{He}$ \\
\hline $\bar{u}(\mathrm{~cm} / \mathrm{sec})$ & 49 \\
\hline \multicolumn{2}{|l|}{ Column } \\
\hline Type & $\mathrm{PLOT} \mathrm{Al}_{2} \mathrm{O}_{3} / \mathrm{Na}_{2} \mathrm{SO}_{4}$ \\
\hline$L(\mathrm{~m}) \times d_{\mathrm{c}}(\mu \mathrm{m}) \times d_{\mathrm{f}}(\mu \mathrm{m})$ & $50 \times 320 \times 5$ \\
\hline \multicolumn{2}{|l|}{ Oven program } \\
\hline$T_{0}\left({ }^{\circ} \mathrm{C}\right)$ & 130 \\
\hline$t_{\text {hold } 0}(\min )$ & 8 \\
\hline$T_{\text {ramp } 1}\left({ }^{\circ} \mathrm{C} / \mathrm{min}\right)$ & 20 \\
\hline$T_{1}\left({ }^{\circ} \mathrm{C}\right)$ & 190 \\
\hline$t_{\text {hold } 1}$ (min) & 10 \\
\hline \multicolumn{2}{|l|}{ Detector } \\
\hline Type & FID \\
\hline$T_{\text {det }}\left({ }^{\circ} \mathrm{C}\right)$ & 200 \\
\hline $\mathrm{V}_{\mathrm{H}^{2}}(\mathrm{ml} / \mathrm{min})$ & 30 \\
\hline $\mathrm{V}_{\text {Air }}(\mathrm{ml} / \mathrm{min})$ & 300 \\
\hline $\mathrm{V}_{\text {col+mkup }}(\mathrm{ml} / \mathrm{min})$ & 40 \\
\hline
\end{tabular}

Table S2. Comparison of gaseous compounds in solution (benzene- $d_{6}$ ) versus the complete sample.

\begin{tabular}{lccc}
\hline Compound & $\mathrm{mol} \%{ }^{1} \mathrm{H} \mathrm{NMR}(\%)$ & $\mathrm{mol} \% \mathrm{GC}(\%)$ & $\mathrm{Rel}^{\circ}(\%)^{a}$ \\
\hline Ethane & 3.54 & 3.75 & -5.93 \\
Ethylene & 34.37 & 36.64 & -6.60 \\
Propene & 40.64 & 38.95 & 4.16 \\
trans-2-butene & 15.78 & 14.82 & 6.08 \\
cis-2-butene & 5.66 & 5.84 & -3.18 \\
\hline${ }^{a} \mathrm{Rel}^{\circ} \%=\left(\mathrm{mol} \%{ }^{1} \mathrm{H} \mathrm{NMR}-\mathrm{mol} \% \mathrm{GC}\right)$ & $100 / \mathrm{mol}^{1} \%{ }^{1} \mathrm{H} \mathrm{NMR}$ & &
\end{tabular}




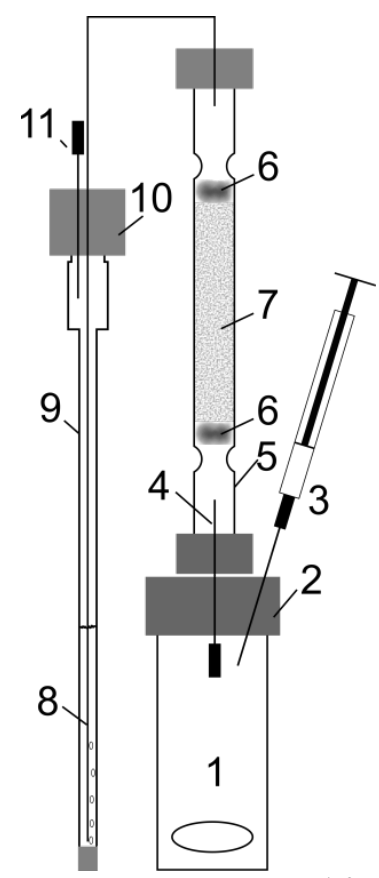

Figure S2. Apparatus used for the generation of ethylene. 1. Reaction vessel (3 ml vial), 2. Septum, 3. Syringe for the addition of styrene, 4. Cannula, 5. Glass tube, 6. Quartz wool, 7. Basic alumina, 8. Long cannula, 9. NMR-tube, 10. Septum, 11. Cannula.

\section{Procedures Catalytic Experiments}

\section{Procedure for the Catalytic Experiment at $298.0 \mathrm{~K}$ without PCy.}

Under inert atmosphere (Ar), in a glove box, $2.6 \mathrm{mg}(3.7 \mu \mathrm{mol})$ of compound 1·0.67(p-cymene) and 2.6 $\mathrm{mg}(22.8 \mu \mathrm{mol})$ of 2,2,3,3-tetramethylbutane (internal standard) were dissolved in $690.2 \mathrm{mg}$ of benzene$d_{6}$. After the aforementioned compounds were completely dissolved, the mixture was transferred to a Shigemi NMR-tube with J. Young valve. Subsequently, ethylene was bubbled through the solution $(t=0$ h) for about 30 seconds, according to the procedure described above. Then, the glass insert and two additional PTFE holders were inserted, the NMR-tube was closed and the sample, protected from light with aluminum foil, was transported to the NMR facility. After tuning, shimming and pulse calibration an initial quantitative ${ }^{1} \mathrm{H}$ NMR spectrum $(850.13 \mathrm{MHz}, 298.0 \mathrm{~K}, \mathrm{D} 1=60 \mathrm{sec})$ was obtained $(t=1.11 \mathrm{~h})$. Following this, one such spectrum was recorded every 30 min using iconNMR software. Subsequently, absolute amounts of compounds in solution were determined against 2,2,3,3-tetramethylbutane (internal standard). In addition, a ${ }^{13} \mathrm{C}\left\{{ }^{1} \mathrm{H}\right\}(213.77 \mathrm{MHz}, 283.0 \mathrm{~K}, \mathrm{D} 1=60 \mathrm{sec})$ NMR spectrum was obtained directly after the final ${ }^{1} \mathrm{H}$ NMR-spectrum had been recorded. The NMR tube was opened and the solution decanted off. Following this, the precipitate remaining in the tube was washed twice with benzene- $d_{6}$, dissolved in chloroform- $d$ and analyzed using ${ }^{1} \mathrm{H}$ NMR. Subsequently, the solution was transferred to a pre-weighed vial $(3 \mathrm{ml})$, the solvent was removed in vacuo and the vial was weighed again to determine the yield of the residue left behind.

\section{Procedure for the Catalytic Experiment at $323.0 \mathrm{~K}$ without $\mathrm{PC} y_{3}$.}

Under inert atmosphere (Ar), in a glove box, $2.6 \mathrm{mg}(3.7 \mu \mathrm{mol})$ of compound $\mathbf{1} \cdot 0.67(p$-cymene) and 3.0 $\mathrm{mg}(26.3 \mu \mathrm{mol})$ of 2,2,3,3-tetramethylbutane (internal standard) were dissolved in $710.0 \mathrm{mg}$ of benzene$d_{6}$. After the compounds were completely dissolved, the mixture was transferred to a Shigemi NMR-tube with J. Young valve. Subsequently, ethylene was bubbled through the solution for about 30 seconds, according to the procedure described above. Then, the glass insert and two additional PTFE holders were inserted, the NMR-tube was closed and the sample, protected from light with aluminum foil, was transported to the NMR facility.

After tuning, shimming and pulse calibration an initial quantitative ${ }^{1} \mathrm{H}$ NMR spectra $(850.13 \mathrm{MHz}, 298.0$ $\mathrm{K}, \mathrm{D} 1=60 \mathrm{sec})$ was obtained $(t=0.0 \mathrm{~h})$. After this, the sample was heated to, and equilibrated at $323.0 \mathrm{~K}$, 
tuned, shimmed and pulse calibrated after which a quantitative ${ }^{1} \mathrm{H}$ NMR spectra $(850.13 \mathrm{MHz}, 323.0 \mathrm{~K}$, $\mathrm{D} 1=60 \mathrm{sec})$ was obtained $(t=1.91 \mathrm{~h})$. Following this, one such spectrum was recorded every $30 \mathrm{~min}$ using iconNMR software. Subsequently, absolute amounts of compounds in solution were determined against 2,2,3,3-tetramethylbutane (internal standard). Th1e NMR tube was opened, and the solution decanted off. Following this, the precipitate remaining in the tube was washed twice with benzene- $d_{6}$, dissolved in chloroform- $d$ and analyzed using ${ }^{1} \mathrm{H}$ NMR. Subsequently, the solution was transferred to a pre-weighed vial $(3 \mathrm{ml})$, the solvent was removed in vacuo and the vial was weighed again to determine the yield of the residue left behind.

Procedure for the Catalytic Experiment at $298.0 \mathrm{~K}$ with 0.57 equiv. of PCy.

Under inert atmosphere (Ar), in a glove box, $80.9 \mathrm{mg}$ of a stock solution, containing $2.5 \mathrm{mg}(8.9 \mu \mathrm{mol})$ of tricyclohexylphosphine dissolved in $348.7 \mathrm{mg}$ benzene- $d_{6}$, is taken and added to $602.4 \mathrm{mg}$ of benzene$d_{6}$. This solution was added to $2.5 \mathrm{mg}(3.6 \mu \mathrm{mol})$ of $\mathbf{1} \cdot 0.67(p$-cymene $)$ and $2.7 \mathrm{mg}(23.6 \mu \mathrm{mol})$ of $2,2,3,3-$ tetramethylbutane (internal standard). After the aforementioned compounds were completely dissolved, the mixture was transferred to a Shigemi NMR-tube with J. Young valve. Subsequently, ethylene was bubbled through the solution $(t=0 \mathrm{~h})$ for about 30 seconds, according to the procedure described above. Then, the glass insert and two additional PTFE holders were inserted, the NMR-tube was closed and the sample, protected from light with aluminum foil, was transported to the NMR facility. After tuning, shimming and pulse calibration an initial quantitative ${ }^{1} \mathrm{H}$ NMR spectrum $(850.13 \mathrm{MHz}, 298.0 \mathrm{~K}, \mathrm{D} 1=60$ sec) was obtained $(t=1.08 \mathrm{~h})$. Following this, one such spectrum was recorded every 30 min using iconNMR software. Subsequently, absolute amounts of compounds in solution were determined against 2,2,3,3-tetramethylbutane (internal standard). In addition, ${ }^{31} \mathrm{P}(242.94 \mathrm{MHz}, 300.0 \mathrm{~K})$ and ${ }^{13} \mathrm{C}\left\{{ }^{1} \mathrm{H}\right\}(213.77$ $\mathrm{MHz}, 283.0 \mathrm{~K}, \mathrm{D} 1=60 \mathrm{sec}) \mathrm{NMR}$ spectra were obtained directly after the final ${ }^{1} \mathrm{H}$ NMR-spectrum had been recorded. The NMR tube was opened and the solution decanted off. Following this, the precipitate remaining in the tube was washed twice with benzene- $d_{6}$, dissolved in chloroform- $d$ and analyzed using ${ }^{1} \mathrm{H}$ NMR. Subsequently, the solution was transferred to a pre-weighed vial $(3 \mathrm{ml})$, the solvent was removed in vacuo and the vial was weighed again to determine the yield of the residue left behind.

Procedure for the Catalytic Experiment at $323.0 \mathrm{~K}$ with 0.58 equiv. of PCy.

Under inert atmosphere (Ar), in a glove box, $93.3 \mathrm{mg}$ of a stock solution, containing $2.5 \mathrm{mg}(8.9 \mu \mathrm{mol})$ of tricyclohexylphosphine dissolved in $348.7 \mathrm{mg}$ benzene- $d_{6}$, is taken and added to $682.6 \mathrm{mg}$ of benzene$d_{6}$. This solution was added to $2.9 \mathrm{mg}(4.1 \mu \mathrm{mol})$ of compound $1 \cdot 0.67(p$-cymene $)$ and $2.4 \mathrm{mg}(21.0 \mu \mathrm{mol})$ of 2,2,3,3-tetramethylbutane (internal standard). After the aforementioned compounds were completely dissolved, the mixture was transferred to a Shigemi NMR-tube with J. Young valve. Subsequently, ethylene was bubbled through the solution for about 30 seconds, according to the procedure described above. Then, the glass insert and two additional PTFE holders were inserted, the NMR-tube was closed and the sample, protected from light with aluminum foil, was transported to the NMR facility. After tuning, shimming and pulse calibration an initial quantitative ${ }^{1} \mathrm{H}$ NMR spectra $(850.13 \mathrm{MHz}, 298.0 \mathrm{~K}$, D1 $=60 \mathrm{sec})$ was obtained $(t=0.0 \mathrm{~h})$. After this, the sample was heated to, and equilibrated at $323.0 \mathrm{~K}$, tuned, shimmed and pulse calibrated after which a quantitative ${ }^{1} \mathrm{H}$ NMR spectra $(850.13 \mathrm{MHz}, 323.0 \mathrm{~K}, \mathrm{D} 1=60$ sec) was obtained $(t=0.43 \mathrm{~h})$. Following this, one such spectrum was recorded every 30 min using iconNMR software. Subsequently, absolute amounts of compounds in solution were determined against 2,2,3,3-tetramethylbutane (internal standard). In addition, ${ }^{31} \mathrm{P}(242.94 \mathrm{MHz}, 300.0 \mathrm{~K})$ and ${ }^{13} \mathrm{C}\left\{{ }^{1} \mathrm{H}\right\}(213.77$ $\mathrm{MHz}, 283.0 \mathrm{~K}, \mathrm{D} 1=60 \mathrm{sec}) \mathrm{NMR}$ spectra were obtained directly after the final ${ }^{1} \mathrm{H}$ NMR-spectrum had been recorded. The NMR tube was opened and the solution decanted off. Following this, the precipitate remaining in the tube was washed twice with benzene- $d_{6}$, dissolved in chloroform- $d$ and analyzed using ${ }^{1} \mathrm{H}$ NMR. Subsequently, the solution was transferred to a pre-weighed vial $(3 \mathrm{ml})$, the solvent was removed in vacuo and the vial was weighed again to determine the yield of the residue left behind. 
Procedure for the Catalytic Experiments with Variable Concentration of 1 (323.0 K with 0.6 equiv. of

$P\left(y_{3}\right)$.

Except for the different amounts of $\mathbf{1}$ and $\mathrm{PCy}_{3}$, the experiments were carried out following the same procedure as described in the previous experiment.

Low concentration of 1 (1/3 of the previous experiment). $18.2 \mathrm{mg}$ of a stock solution, containing 4.6 $\mathrm{mg}(16.4 \mu \mathrm{mol})$ of tricyclohexylphosphine dissolved in $404.7 \mathrm{mg}$ benzene- $d_{6}$, corresponding to 0.55 equiv. of $\mathrm{PCy}_{3}$, was added to $675.5 \mathrm{mg}$ of benzene- $d_{6}$. This solution was added to $0.9 \mathrm{mg}(1.3 \mu \mathrm{mol})$ of compound $1 \cdot 0.67(p$-cymene) and $3.0 \mathrm{mg}(26.3 \mu \mathrm{mol})$ of 2,2,3,3-tetramethylbutane (internal standard).

High concentration of $1.183 .6 \mathrm{mg}$ of a stock solution, containing $4.6 \mathrm{mg}(16.4 \mu \mathrm{mol})$ of tricyclohexylphosphine dissolved in $409.3 \mathrm{mg}$ benzene- $d_{6}$, corresponding to 0.59 equiv. of $\mathrm{PCy}_{3}$, was added to $501 \mathrm{mg}$ of benzene- $d_{6}$. This solution was added to $8.8 \mathrm{mg}(12.5 \mu \mathrm{mol})$ of compound $\mathbf{1} \cdot 0.67(p$ cymene) and $2.6 \mathrm{mg}(22.8 \mu \mathrm{mol})$ of 2,2,3,3-tetramethylbutane (internal standard).

Procedure for the Catalytic Experiment at $323.0 \mathrm{~K}$ with 1.2 equiv. of $\mathrm{PC} y_{3}$.

Under inert atmosphere (Ar), in a glove box, $1.2 \mathrm{mg}(4.3 \mu \mathrm{mol})$ of tricyclohexylphosphine and $3.0 \mathrm{mg}$ $(26.3 \mu \mathrm{mol})$ of 2,2,3,3-tetramethylbutane (internal standard) were dissolved in $683.1 \mathrm{mg}$ benzene- $d_{6}$. This solution was added to $2.5 \mathrm{mg}(3.6 \mu \mathrm{mol})$ of compound $1 \cdot 0.67(p$-cymene) and, after the aforementioned compound was completely dissolved, the mixture was transferred to a Shigemi NMR-tube with J. Young valve. Subsequently, ethylene was bubbled through the solution for about 30 seconds, according to the procedure described above. Then, the glass insert and two additional PTFE holders were inserted, the NMR-tube was closed and the sample, protected from light with aluminum foil, was transported to the NMR facility. After tuning, shimming and pulse calibration an initial quantitative ${ }^{1} \mathrm{H}$ NMR spectra $(600.13 \mathrm{MHz}, 298.0 \mathrm{~K}, \mathrm{D} 1=60 \mathrm{sec})$ was obtained $(t=0.0 \mathrm{~h})$. After this, while being protected from light by aluminum foil, the sample was heated to $323.0 \mathrm{~K}$ in an oil bath and kept at this temperature. At $t=$ $27.25 \mathrm{~h}$ the tube was removed from the oil bath, all oil was removed from the surface of the tube and another quantitative ${ }^{1} \mathrm{H}$ NMR spectra $(600.13 \mathrm{MHz}, 323.0 \mathrm{~K}$, D1 = $60 \mathrm{sec})$ was recorded. Subsequently, absolute amounts of compounds in solution were determined against 2,2,3,3-tetramethylbutane (internal standard). In addition, ${ }^{31} \mathrm{P}(242.94 \mathrm{MHz})$ and ${ }^{13} \mathrm{C}\left\{{ }^{1} \mathrm{H}\right\}(150.90 \mathrm{MHz}, 283.0 \mathrm{~K})$ NMR spectra were obtained directly after the final ${ }^{1} \mathrm{H}$ NMR-spectrum had been recorded.

Procedure for the Catalytic Experiment with 2 (323.0 K with 0.56 equiv. of $\mathrm{PCy}_{3}$ ).

Under inert atmosphere (Ar), in a glove box, $120 \mathrm{mg}$ of a stock solution, containing $2.7 \mathrm{mg}(9.6 \mu \mathrm{mol})$ of tricyclohexylphosphine dissolved in $495 \mathrm{mg}$ benzene- $d_{6}$, is taken and added to $592 \mathrm{mg}$ of benzene- $d_{6}$. This solution was added to $2.5 \mathrm{mg}(4.1 \mu \mathrm{mol})$ of compound 2 and $3.2 \mathrm{mg}(28.0 \mu \mathrm{mol})$ of 2,2,3,3tetramethylbutane (internal standard). After the compounds were completely dissolved, the mixture was transferred to a Shigemi NMR-tube with J. Young valve. Subsequently, ethylene was bubbled through the solution for about 30 seconds, according to the procedure described above. Then, the glass insert and two additional PTFE holders were inserted, the NMR-tube was closed and the sample, protected from light with aluminum foil, was transported to the NMR facility. After tuning, shimming and pulse calibration an initial quantitative ${ }^{1} \mathrm{H}$ NMR spectra $(850.13 \mathrm{MHz}, 298.0 \mathrm{~K}, \mathrm{D} 1=60 \mathrm{sec})$ was obtained $(t=0.0 \mathrm{~h})$. After this, the sample was heated to, and equilibrated at $323.0 \mathrm{~K}$, tuned, shimmed and pulse calibrated after which a quantitative ${ }^{1} \mathrm{H}$ NMR spectra $(850.13 \mathrm{MHz}, 323.0 \mathrm{~K}, \mathrm{D} 1=60 \mathrm{sec})$ was obtained $(t=0.53 \mathrm{~h})$. Following this, one such spectrum was recorded every 30 min using iconNMR software. Subsequently, absolute amounts of compounds in solution were determined against 2,2,3,3-tetramethylbutane (internal standard).

Procedure for the Catalytic Experiment with the Dimer [(p-Cymene) $\left.\mathrm{RuCl}_{2}\right]_{2}$ at $323.0 \mathrm{~K}$.

Under inert atmosphere $(\mathrm{Ar})$, in a glove box, $1.3 \mathrm{mg}(2.1 \mu \mathrm{mol})$ of compound of the dimer $[(p$ cymene $\left.) \mathrm{RuCl}_{2}\right]_{2}$ and $2.6 \mathrm{mg}(22.8 \mu \mathrm{mol})$ of 2,2,3,3-tetramethylbutane (internal standard) were dissolved in $733 \mathrm{mg}$ of benzene- $d_{6}$. After the compounds were completely dissolved, the mixture was transferred to a Shigemi NMR-tube with J. Young valve. Subsequently, ethylene was bubbled through the solution for about 30 seconds, according to the procedure described above. Then, the glass insert and two additional 
PTFE holders were inserted, the NMR-tube was closed and the sample, protected from light with aluminum foil, was transported to the NMR facility (Bruker BioSpin AV500). After this, while being protected from light by aluminum foil, the sample was heated to $323.0 \mathrm{~K}$ in an oil bath and kept at this temperature for six days. After six days heating the tube was removed from the oil bath, all oil was removed from the surface of the tube and another ${ }^{1} \mathrm{H}$ NMR spectra $(500.13 \mathrm{MHz}, 298.0 \mathrm{~K})$ was recorded.

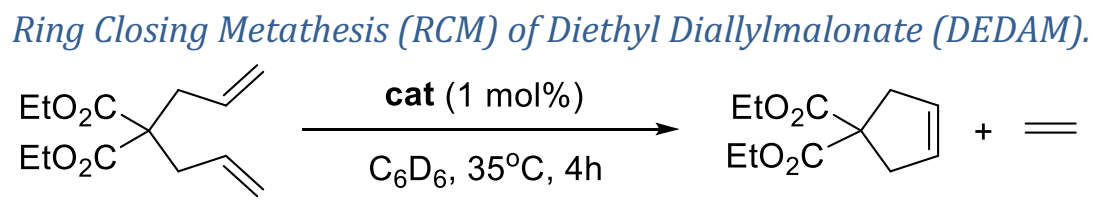

Scheme S1. Ring closing metathesis of diethyl diallylmalonate.

Preparation of a stock solution (A) containing 1 and 0.6 equiv. of PCy3: Under inert atmosphere (Ar), in a glove box, $100.1 \mathrm{mg}$ of a stock solution, containing $2.7 \mathrm{mg}(9.6 \mu \mathrm{mol})$ of tricyclohexylphosphine dissolved in $495 \mathrm{mg}$ benzene- $d_{6}$, corresponding to $0.55 \mathrm{mg}\left(1.9 \mu \mathrm{mol}, 0.6\right.$ equiv.) of $\mathrm{PCy}_{3}$, is taken and added to $3441 \mathrm{mg}$ of benzene- $d_{6}$. This solution was added to $2.4 \mathrm{mg}(3.4 \mu \mathrm{mol})$ of compound $\mathbf{1} \cdot 0.67(p$ cymene) and $1.4 \mathrm{mg}(12.2 \mu \mathrm{mol})$ of 2,2,3,3-tetramethylbutane (internal standard).

$\boldsymbol{R C M}$ with 1: In a glovebox, $15.8 \mathrm{mg}(65 \mu \mathrm{mol})$ of diethyl diallylmalonate and $684 \mathrm{mg}(0.65 \mathrm{ml})$ of stock solution A, corresponding to $0.46 \mathrm{mg}(0.65 \mu \mathrm{mol})$ of $\mathbf{1} \cdot 0.67(p$-cymene $), 0.11 \mathrm{mg}(0.37 \mu \mathrm{mol}, 0.6$ equiv.) of $\mathrm{PCy}_{3}$ and $0.27 \mathrm{mg}(2.36 \mu \mathrm{mol})$ of 2,2,3,3-tetramethylbutane (internal standard), were transferred in a 4 $\mathrm{ml}$ vial equipped with a magnetic stir bar. The vial was capped and transferred to a preheated $\left(35{ }^{\circ} \mathrm{C}\right)$ aluminum block placed on a magnetic stirrer hot plate equipped with a thermostat. After four $h$ the mixture was transferred into a J. Young NMR tube, and a ${ }^{1} \mathrm{H}$ NMR spectrum was immediately recorded on a Bruker Biospin AV500.

RCM with pretreated 1: In the glovebox, $684 \mathrm{mg}(\sim 0.65 \mathrm{ml})$ of stock solution A, corresponding to 0.46 $\mathrm{mg}(0.65 \mu \mathrm{mol})$ of $1 \cdot 0.67$ ( $p$-cymene), $0.11 \mathrm{mg}(0.37 \mu \mathrm{mol}, 0.6$ equiv.) of PCy3, and $0.27 \mathrm{mg}(2.36 \mu \mathrm{mol})$ of 2,2,3,3-tetramethylbutane (internal standard), were transferred in a J. Young NMR tube. Subsequently, ethylene was bubbled through the solution for about 30 seconds, according to the procedure described above. The NMR-tube was closed and the sample, protected from light with aluminum foil was heated at $50{ }^{\circ} \mathrm{C}$ for seven $\mathrm{h}$ in an oil bath. The solvent and volatile compounds (including the internal standard) were removed under vacuum. The residue was dissolved in $665 \mathrm{mg}$ of benzene- $d_{6}$, and to the resulting solution were added $15.8 \mathrm{mg}(65 \mu \mathrm{mol})$ of diethyl diallylmalonate and of $2.9 \mathrm{mg}(25.4 \mu \mathrm{mol})$ of 2,2,3,3tetramethylbutane (internal standard), and the final mixture was transferred in a $4 \mathrm{ml}$ vial equipped with a magnetic stir bar. The vial was capped and transferred to a preheated $\left(35^{\circ} \mathrm{C}\right)$ aluminum block placed on a magnetic stirrer hot plate equipped with a thermostat. After four $\mathrm{h}$ the mixture was transferred into a $\mathrm{J}$. Young NMR tube, and a ${ }^{1} \mathrm{H}$ NMR spectrum was immediately recorded on a Bruker Biospin AV500.

\section{${ }^{1} \mathrm{H}$ and ${ }^{13} \mathrm{C}\left\{{ }^{1} \mathrm{H}\right\}$ NMR Analysis of the Olefinic and Aliphatic Products}

Identification of ethylene, propene, 1-butene, cis- and trans-2-butene and ethane in the ${ }^{1} \mathrm{H}$ and ${ }^{13} \mathrm{C}\left\{{ }^{1} \mathrm{H}\right\}$ NMR spectra was achieved by comparing chemical shifts of individual resonances with those obtained from reference spectra. 1-Butene was identified by comparing the resonance peaks with those experimentally recorded and available in SciFinder. Ethylene and propene were identified by comparison with solutions of commercially available gasses (ethylene $=$ generated as described above and propene $=$ Messer ${ }^{\mathbb{B}}$ CANGas) in benzene- $d_{6}$. From a solution of propene and $6.5 \mathrm{mg}$ of Grubbs's $Z$-selective catalyst in $843.0 \mathrm{mg}$ of benzene- $d_{6}$ cis-2-butene was readily obtained. Subsequently, resonances belonging to trans-2-butene were identified from a mixture of trans- and cis-2-butene obtained from solution of propene and Grubbs second-generation catalyst in benzene- $d_{6}$. Ethane was obtained by suspending 12.2 mg palladium on carbon in a solution of hydrogen and ethylene in benzene- $d_{6}$.

Quantification was performed against 2,2,3,3-tetramethylbutane (internal standard) using the ${ }^{1} \mathrm{H}$ NMR spectra. The ${ }^{13} \mathrm{C}\left\{{ }^{1} \mathrm{H}\right\}$ spectra recorded at the end of each experiment were found to be unsuitable for quantification, even though a D1 of 60 seconds was used. 
Key NMR-shifts are reported below with brown color identifying the resonances used for quantification: Ethylene: ${ }^{1} \mathrm{H}$ NMR $\left(850.13 \mathrm{MHz}, \mathrm{C}_{6} \mathrm{D}_{6}\right): \delta 5.25(\mathrm{~s}, 4 \mathrm{H}) .{ }^{13} \mathrm{C}\left\{{ }^{1} \mathrm{H}\right\}$ NMR $\left(213.77 \mathrm{MHz}, \mathrm{C}_{6} \mathrm{D}_{6}\right): \delta 122.96$. Propene: ${ }^{1} \mathrm{H}$ NMR $\left(850.13 \mathrm{MHz}, \mathrm{C}_{6} \mathrm{D}_{6}\right): \delta 5.72(\mathrm{~m}, 1 \mathrm{H}), 5.00\left(\mathrm{dm},{ }^{3} J_{\mathrm{HH}}=17.0 \mathrm{~Hz}, 1 \mathrm{H}\right), 4.95\left(\mathrm{dm},{ }^{3} J_{\mathrm{HH}}=\right.$ $10.3 \mathrm{~Hz}, 1 \mathrm{H}), 1.55\left(\mathrm{dt},{ }^{3} J_{\mathrm{HH}}=6.4 \mathrm{~Hz},{ }^{3} J_{\mathrm{HH}}=1.5 \mathrm{~Hz}, 3 \mathrm{H}\right) .{ }^{13} \mathrm{C}\left\{{ }^{1} \mathrm{H}\right\} \mathrm{NMR}\left(213.77 \mathrm{MHz}, \mathrm{C}_{6} \mathrm{D}_{6}\right): \delta 133.69$, 115.91, 19.36. 1-Butene: ${ }^{1} \mathrm{H}$ NMR $\left(850.13 \mathrm{MHz}, \mathrm{C}_{6} \mathrm{D}_{6}\right): \delta 5.79(\mathrm{~m}, 1 \mathrm{H}), 5.00\left(\mathrm{dm},{ }^{3} J_{\mathrm{HH}}=17.3 \mathrm{~Hz}, 1 \mathrm{H}\right)$, $4.95\left(\mathrm{dm},{ }^{3} J_{\mathrm{HH}}=10.3 \mathrm{~Hz}, 1 \mathrm{H}\right), 1.92(\mathrm{~m}, 2 \mathrm{H}), 0.89\left(\mathrm{t},{ }^{3} J_{\mathrm{HH}}=7.5 \mathrm{~Hz}, 3 \mathrm{H}\right) .{ }^{13} \mathrm{C}\left\{{ }^{1} \mathrm{H}\right\} \mathrm{NMR}(213.77 \mathrm{MHz}$, $\left.\mathrm{C}_{6} \mathrm{D}_{6}\right): \delta 140.53,113.49,27.04$, 13.26. cis-2-Butene: ${ }^{1} \mathrm{H}$ NMR $\left(850.13 \mathrm{MHz}, \mathrm{C}_{6} \mathrm{D}_{6}\right): \delta 5.48(\mathrm{~m}, 2 \mathrm{H}), 1.51$ $\left(\mathrm{d},{ }^{3} J_{\mathrm{HH}}=4.8 \mathrm{~Hz}, 6 \mathrm{H}\right) \cdot{ }^{13} \mathrm{C}\left\{{ }^{1} \mathrm{H}\right\}$ NMR $\left(213.77 \mathrm{MHz}, \mathrm{C}_{6} \mathrm{D}_{6}\right): \delta$ 124.80, 12.46. trans-2-Butene: ${ }^{1} \mathrm{H}$ NMR $\left(500.13 \mathrm{MHz}, \mathrm{C}_{6} \mathrm{D}_{6}\right): \delta 5.38(\mathrm{~m}, 2 \mathrm{H}), 1.57(\mathrm{~m}, 6 \mathrm{H}) .{ }^{13} \mathrm{C}\left\{{ }^{1} \mathrm{H}\right\} \mathrm{NMR}\left(125.76 \mathrm{MHz}, \mathrm{C}_{6} \mathrm{D}_{6}\right): \delta 126.05,18.03$. Ethane: ${ }^{1} \mathrm{H}$ NMR $\left(850.13 \mathrm{MHz}, \mathrm{C}_{6} \mathrm{D}_{6}\right): \delta 0.80(\mathrm{~s}, 6 \mathrm{H}) .{ }^{13} \mathrm{C}\left\{{ }^{1} \mathrm{H}\right\}$ NMR $\left(213.77 \mathrm{MHz}, \mathrm{C}_{6} \mathrm{D}_{6}\right): \delta 6.95$. 2,2,3,3-Tetramethylbutane (internal standard): ${ }^{1} \mathrm{H}$ NMR $\left(850.13 \mathrm{MHz}, \mathrm{C}_{6} \mathrm{D}_{6}\right): \delta 0.85(\mathrm{~s}, 18 \mathrm{H}) .{ }^{13} \mathrm{C}\left\{{ }^{1} \mathrm{H}\right\}$ NMR (213.77 MHz, $\left.\mathrm{C}_{6} \mathrm{D}_{6}\right): \delta 35.14,25.85$.

\section{${ }^{1} \mathrm{H},{ }^{13} \mathrm{C}\left\{{ }^{1} \mathrm{H}\right\}$ and ${ }^{31} \mathrm{P}$ NMR Analysis of Decomposition Products}

Next to products originating from catalytic processes, an attempt was made to identify possible decomposition products of 1 using ${ }^{1} \mathrm{H}$ NMR spectra recorded during the catalytic reaction and the ${ }^{13} \mathrm{C}\left\{{ }^{1} \mathrm{H}\right\}$ and ${ }^{31} \mathrm{P}$ NMR spectra recorded at the end. Spectra of expected decomposition product $2\left[\left(\mathrm{PCy}_{3}\right)\left(\eta^{6}-p\right.\right.$ cymene) $\mathrm{RuCl}_{2}$ ], that was synthesized according to literature procedures, ${ }^{9}$ are found in Figure S23, Figure S24, and Figure S25. The ${ }^{1} \mathrm{H}$ NMR spectrum of the isolated 1,3-bis(2,4,6-trimethylphenyl)imidazolinium chloride was in agreement with that reported in literature. ${ }^{10}$

Other key NMR-shifts are reported below:

p-Cymene: ${ }^{1} \mathrm{H}$ NMR $\left(850.13 \mathrm{MHz}, \mathrm{C}_{6} \mathrm{D}_{6}\right): \delta 7.05\left(\mathrm{~d},{ }^{3} J_{\mathrm{HH}}=8.4 \mathrm{~Hz}, 2 \mathrm{H}\right), 7.02\left(\mathrm{~d},{ }^{3} J_{\mathrm{HH}}=8.2 \mathrm{~Hz}, 2 \mathrm{H}\right)$, $2.74\left(\mathrm{sept},{ }^{3} J_{\mathrm{HH}}=6.9 \mathrm{~Hz}, 1 \mathrm{H}\right), 2.17(\mathrm{~s}, 3 \mathrm{H}), 1.17\left(\mathrm{~d},{ }^{3} J_{\mathrm{HH}}=7.3 \mathrm{~Hz}, 6 \mathrm{H}\right) .{ }^{13} \mathrm{C}\left\{{ }^{1} \mathrm{H}\right\} \mathrm{NMR}(213.77 \mathrm{MHz}$,

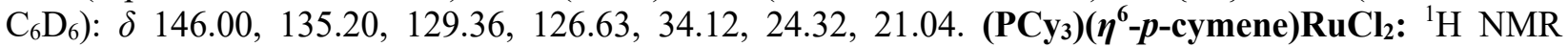
$\left(600.13 \mathrm{MHz}, \mathrm{C}_{6} \mathrm{D}_{6}\right): \delta 5.36\left(\mathrm{~d},{ }^{3} J_{\mathrm{HH}}=6.1 \mathrm{~Hz}, 2 \mathrm{H}\right), 5.23\left(\mathrm{~d},{ }^{3} J_{\mathrm{HH}}=5.9 \mathrm{~Hz}, 2 \mathrm{H}\right), 2.93\left(\mathrm{sept},{ }^{3} J_{\mathrm{HH}}=7.0 \mathrm{~Hz}\right.$, $1 \mathrm{H}), 2.58-2.48(\mathrm{~m}, 3 \mathrm{H}), 2.30-2.19(\mathrm{br}, 6 \mathrm{H}), 1.87$ (s, 3H), 1.76-1.68 (br, 6H), 1.66-1.59 (br, 3H), 1.50-1.38 $(\mathrm{m}, 7 \mathrm{H}), 1.30-1.21(\mathrm{~m}, 5 \mathrm{H}), 1.20\left(\mathrm{~d},{ }^{3} \mathrm{~J}_{\mathrm{HH}}=7.0 \mathrm{~Hz}, 6 \mathrm{H}\right), 1.18-1.09(\mathrm{~m}, 3 \mathrm{H}) .{ }^{13} \mathrm{C}\left\{{ }^{1} \mathrm{H}\right\} \mathrm{NMR}(213.77 \mathrm{MHz}$, $\left.\mathrm{C}_{6} \mathrm{D}_{6}\right): \delta 106.32,94.01,88.90,84.25,35.83,35.72,30.89,30.17,28.02,27.95,26.99,22.68,17.78 .{ }^{31} \mathrm{P}$ NMR (242.94 MHz, $\left.\mathrm{C}_{6} \mathrm{D}_{6}\right): \delta 25.88$.

\section{Calculation of Turnovers and Rates of Product Formation}

Turnovers at a specific reaction time are calculated as the molar yield of the product at that time divided by the initial molar number of $\mathbf{1}$.

Figure 4 (main paper) and Figure S13 illustrate product generation rates. These rates were obtained by plotting the average rate of every $0.5 \mathrm{~h}$ interval. This average, in turn, was calculated by dividing the difference between the concentration of the product $\left(\mu \mathrm{mol} \cdot \mathrm{ml}^{-1}\right)$ at reaction time $\mathrm{t}+0.5(\mathrm{~h})$ and $\mathrm{t}(\mathrm{h})$ by the time interval $0.5(\mathrm{~h})$, as shown in Equation S1. The resulting curves were smoothened using the $\mathrm{T} 4253 \mathrm{H}$ smoothening function which removes spikes and oscillations while retaining the trends. ${ }^{11}$

$$
\text { average product generation rate }(t, t+0.5 h)=\frac{[\text { product }]_{t+0.5}-[\text { product }]_{t}}{0.5 h} \quad \text { (Equation } \mathrm{S} 1 \text { ) }
$$




\section{Results and Discussion}

\section{Reaction of 1 with Ethylene ( $92.0 \mu \mathrm{mol})$ at $298.0 \mathrm{~K}$}

Figure S3 displays the generation of 1-butene, cis- and trans-2-butene, propene and ethane in the course of the experiment. 1-Butene is the first observed product, and is also associated with the highest formation rate during the entire experiment. This compound is presumably obtained via dimerization of ethylene. The rate of formation of 1-butene appears to increase during the first 20-25 h and then slowly decreases until the end of the experiment. In contrast, the rates of fomation of both cis- and trans-2-butene appear constant at the beginning and then slowly increase with time. Interestingly, at the beginning of the reaction, the rates of formation of cis- and trans-2-butene are very similar (the cis/trans ratio is close to unity). However, this ratio decreases with the progress of the reaction and, after a while, the trans-isomer becomes the major product (cis/trans ratio $=0.79$ ). Taken together, these results strongly suggest that, at the beginning of the experiment, 1-butene and 2-butene are generated independently from each other. However, after about 20-25 h some 2-butene stems from double-bond migration in 1-butene, as suggested by the slopes (giving the formation rates) of the product yield curves of 1-butene (decreasing with time) and total butenes (approximately constant with time), see Figure S3. A resonance peak of small intensity appears in the hydride region of the ${ }^{1} \mathrm{H}$ NMR spectrum (at $-11.52 \mathrm{ppm}$ ) after $45 \mathrm{~h}$, and the intensity increases with time for the rest of the experiment. At the end of the experiment $(74 \mathrm{~h})$, the amount of this ruthenium hydride species is about $7 \mathrm{mmol}$. This species is probably involved in the isomerization of 1butene to 2-butene as well as in the cis-trans isomerization of 2-butene, since the isomerization rate appears to increase after the appearance of this peak. The molecular structure of this species is unknown. The isolation of the imidazolium salt (see below) suggests that ruthenium nanoparticles are also formed. Thus, the isomerization of 1-butene to 2-butene can additionally be caused by the formation of $\mathrm{Ru}$ nanoparticles. $^{12}$

Overall, the conversion of ethylene to 1- and 2-butene isomers appears to be a catalytic rather than a stoichiometric process. Furthermore, the conversion of ethylene into butenes proceeds with a rate which remains approximately constant until the end of the experiment. Finally, at the end of the experiment, after $73.5 \mathrm{~h}, 1.43$ turnovers were reached (Table S4). ${ }^{13}$ 


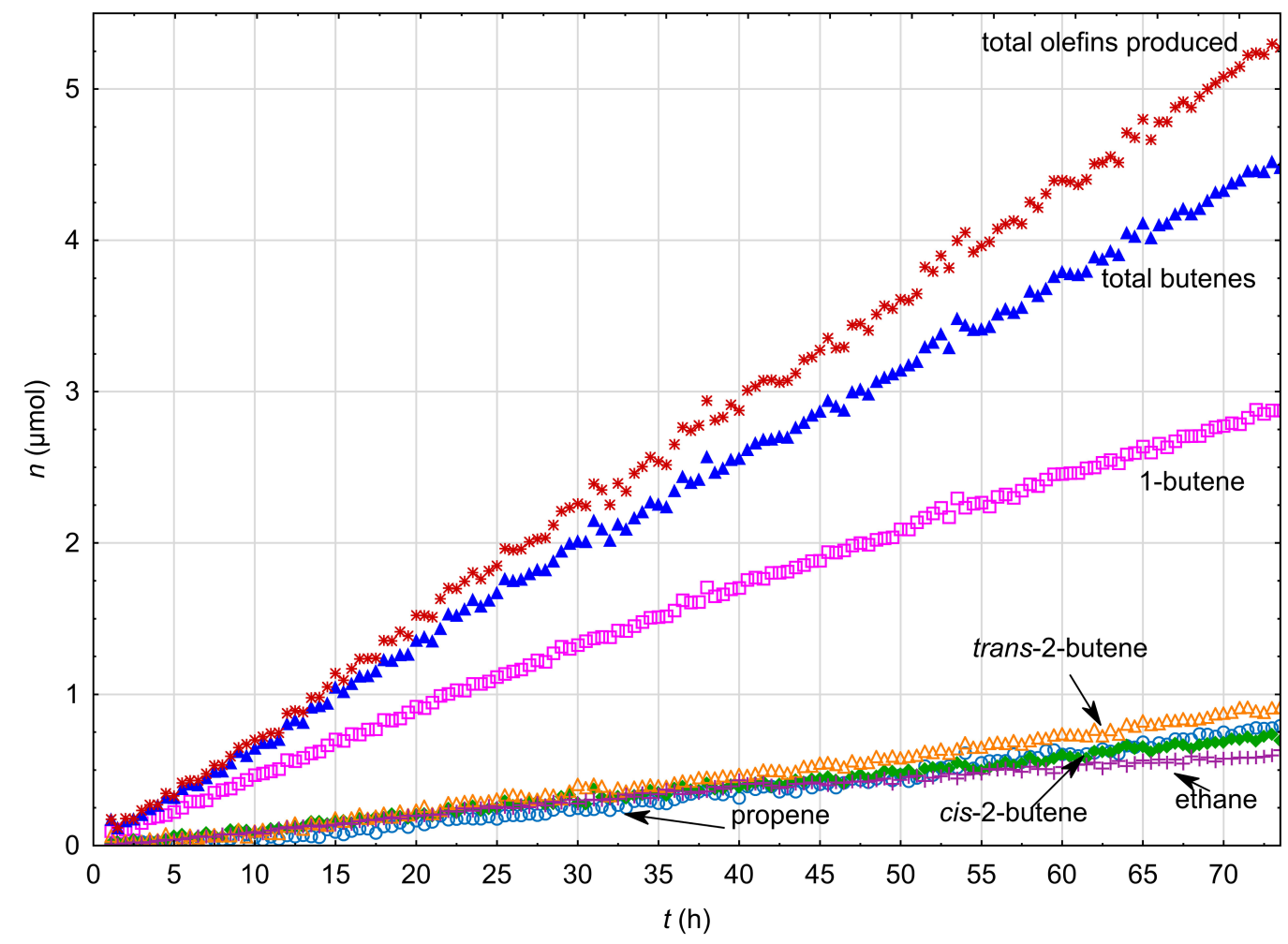

Figure S3. Products obtained with 1 and ethylene $(92 \mu \mathrm{mol})$ at $298.0 \mathrm{~K}$ in $\mathrm{C}_{6} \mathrm{D}_{6}$ : $*$ total olefins produced, $\Delta$ total butenes, $\square 1$ butene, $\triangle$ trans-2-butene, $\bullet$ cis-2-butene, + ethane and $\odot$ propene.

Since ethylene is present in large excess, this behavior suggests that the concentration of the catalytic species remains approximately constant during the experiment. This also suggests that the catalytic activity would probably continue for many additional $\mathrm{h}$ after the last recorded spectrum (i.e. after $73.5 \mathrm{~h}$ ). At the end of the experiment, the amounts of these three olefins are similar and the relative amounts are in the following order: trans-2-butene $>$ propene $>c i s$-2-butene.

The presence of propene in the reaction mixture is consistent with the generation of Ru-alkylidene species. Propene is most likely produced via three different pathways: ethenolysis of 2-butene, ${ }^{14}$ ethylenetriggered decomposition of a previously formed $\mathrm{Ru}$-methylidene complex i.e. via thermal decomposition of the non-substituted metallacyclobutane, and via generation of alkylidenes as shown in the mechanism described in the computational part. ${ }^{8}$ The latter pathway is especially important at the very beginning.

The formation of ethane, which proceeds with a nearly constant rate during the experiment, is a surprising phenomenon and the mechanism behind this transformation is unknown. However, as discussed below in the section "The Origin of Ethane", this reaction is most likely stoichiometric and ethane is probably the product of an ethylene-triggered oxidation of the SIMes ligand.

When the experiment was terminated, $72 \%$ of the ethylene consumed was present in the products, with the remainder presumably having moved to the headspace where it could not be measured.

Scheme S2 illustrates the products resulting from decomposition of $\mathbf{1}$ in the presence of ethylene. In Figure S4, the gradual decrease in intensity of the resonance corresponding to the aliphatic methyl-protons of the bonded $p$-cymene in 1 (at $\delta 1.06\left(\mathrm{~d},{ }^{3} J_{\mathrm{HH}}=7.0 \mathrm{~Hz}, 6 \mathrm{H}\right) \mathrm{ppm}$ is shown. 


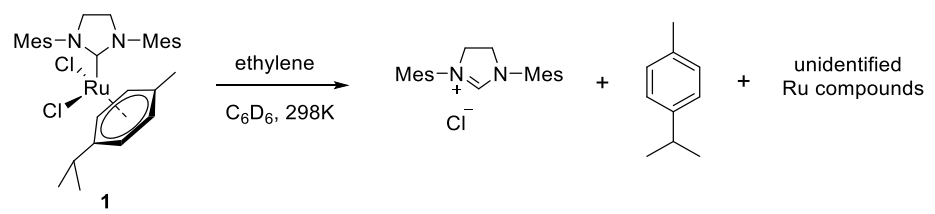

Scheme S2. Decomposition of 1 in the presence of ethylene.

The intensity of the resonances corresponding to the free $p$-cymene increases with time, see Figure S5. At the end of the experiment (i.e. after $73.5 \mathrm{~h}$ ), about $4 \%$ of the initial amount of 1 is present in solution. Furthermore, the analysis of the ${ }^{1} \mathrm{H}$ NMR spectra shows that the decreasing intensity of the singlet at $\delta$ $6.77 \mathrm{ppm}$, corresponding to the four aromatic protons of the SIMes ligand, is, at least in part, replaced by several new resonances of lower intensity in the region 6.26-8.26 ppm (Figure S5). The sum of the integrals of this region of the spectrum (with exclusion of the resonance peaks of the free $p$-cymene and of the NMR solvent $\mathrm{C}_{6} \mathrm{D}_{5} \mathrm{H}$ ) is after $74 \mathrm{~h}$ about $25 \%$ lower than after 1 hour. The main new peaks (all singlets) are at $\delta 6.87,6.84,6.82,6.72$, and $6.56 \mathrm{ppm}$. This observation is consistent with the fact that many Ru-species are formed during this experiment and that a substantial amount of the N-heterocyclic carbene ligand remains bonded to the ruthenium center until the end of the experiment.

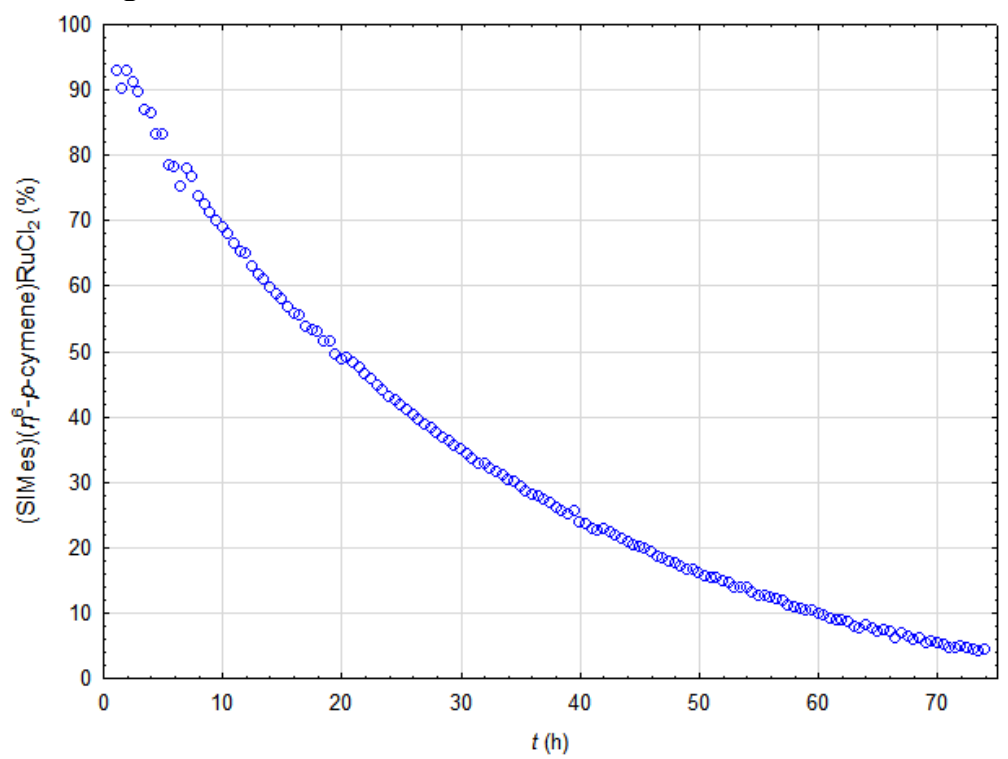

Figure S4. Percentage of 1 present, with 1 and ethylene $(92 \mu \mathrm{mol})$ at $298.0 \mathrm{~K}$ in $\mathrm{C}_{6} \mathrm{D}_{6}$. 


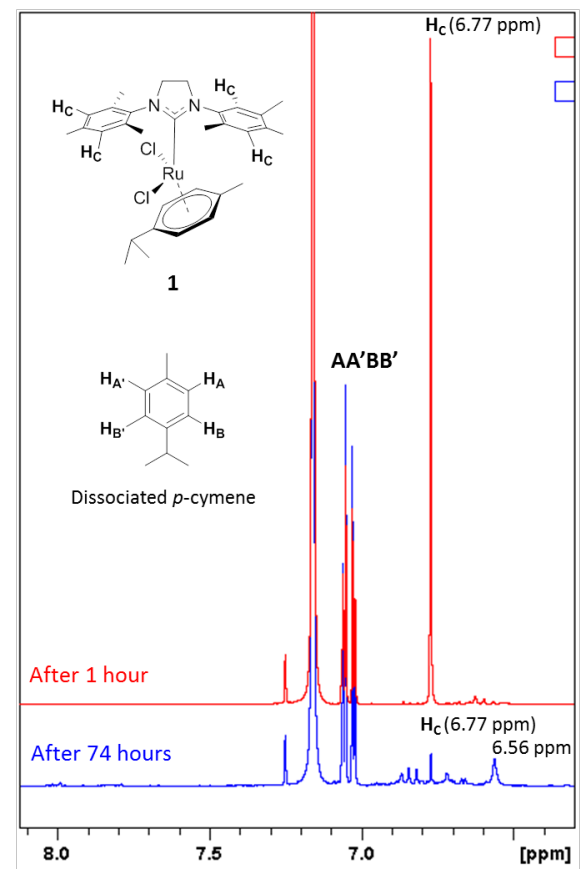

Figure S5. Comparison of the aromatic region of the ${ }^{1} \mathrm{H}$ spectrum of the reaction mixture after $\mathbf{1}$ and $74 \mathrm{~h}$.

After the experiment, a microcrystalline white precipitate was found on the wall of NMR tube. The solid was isolated $(0.4 \mathrm{mg}, 1.1 \mu \mathrm{mol}, 32 \%$ of the initial amount if 1) and identified to be 1,3-bis(2,4,6trimethylphenyl)-imidazolinium chloride. The isolated amount corresponds approximately to the observed reduction of the sum of integrals in the aromatic region. Furthermore, the formation of this product, which was observed also by Janse van Rensburg and cowokers, ${ }^{8}$ indicates that loss of the N-heterocyclic carbene ligand as imidazolium chloride is one of the main decomposition routes for $\mathbf{1}$. The dinuclear ruthenium cyclometalated ethylene complex first isolated by Grubbs and coworkers ${ }^{15}$ and subsequently also by Fogg and coworkers ${ }^{16}$ was not observed.

\section{Reaction of 1 with Ethylene ( $115 \mu \mathrm{mol})$ at $323.0 \mathrm{~K}$}

Whereas in the experiment at $298 \mathrm{~K} 1$-butene was the major product, this experiment is dominated by the production of propene, see Figure S6. At the very beginning, 1-butene is actually the most abundant product, but the rate of formation is decreasing with time. Propene is the second most abundant product, but its rate of formation is, on the contrary, increasing with time. After three $h$, the amount of propene surpasses that of 1-butene, becoming thus the main product of the reaction until the end of the experiment, and after $46 \mathrm{~h}$, propene constitues about $80 \%$ of all organic products. In contrast, at $298 \mathrm{~K}$, propene was detectable only a couple of $\mathrm{h}$ after the appearance of 1- and 2-butenes, and at the end of the experiment $(73.5 \mathrm{~h})$ the amount produced was only $14 \%$ of all organic products.

At the beginning, the amount of 1-butene increases slowly with time, it reaches a maximum at around 20 $\mathrm{h}$, and then slowly declines until the end of the experiment, while propene increases throughout the experiment and its rate of formation does seem to correlate well with the amount of 1-butene present in the reaction mixture. This suggests that 1-butene, after beeing generated, is subsequently converted into propene.

The generation of propene from ethylene is catalytic, with 6.5 turnovers after $46.4 \mathrm{~h}$ (Table S5). ${ }^{13}$

The formation of propene strongly suggests that olefin metathesis active species are generated in the mixture. The rate of propene formation should correlate with the concentration of these active species. Thus, the analysis of the slope of the propene plot (Figure S6) indicates that the concentration of the active species increases during the first $12 \mathrm{~h}$, then remains approximately constant for about $10 \mathrm{~h}$ and then start declining. The amounts of cis- and trans-2-butene increase very slowly during the first 90 minutes of heating, and then start declining and remains very low until at about $22 \mathrm{~h}$. Afterwards, they slowly 
increase until the end of the experiment. The progress of cis- and trans-2-butene with time suggests that up to about $22 \mathrm{~h}$, the concentration of olefin metathesis active species is sufficiently elevated to ethenolyze 2-butene, as soon as it is generated, into propene, while afterwards the concentration of OM active species becomes somewhat lower, thus to allow a partial accumulation of cis- and trans-2-butene.

Similarly to the experiment at room temperature, the cis/trans ratio decreases with time going from 0.65 to about 0.38. This ratio is clearly lower than that at $298 \mathrm{~K}$ (going from about 1 to 0.79), and it demonstrates that at $323 \mathrm{~K}$ olefin isomerization is operative since the beginning of the reaction.

Similarly again to the experiment at $298 \mathrm{~K}$, a resonance peak of small intensity appears in the hydride region of the ${ }^{1} \mathrm{H}$ NMR spectrum (at $-11.78 \mathrm{ppm}$ ) after ca. $20 \mathrm{~h}$, and its intensity slowly increases with time for the rest of the experiment, and the amount of this species reaches $17.5 \mathrm{nmol}$ after $46 \mathrm{~h}$. This species is probably involved in the in the isomerization of 1-butene to 2-butene as well as in the cis-trans isomerization of 2-butene, since these isomerization rates seem to accelerate after the appearance of this peak. The molecular structure of this species is unknown. The isolation of the imidazolium salt (see below) suggests that Ru-nanoparticles are also formed. Thus, olefin isomerization can additionally be caused by the formation of Ru nanoparticles. ${ }^{12}$

Finally, ethane is generated throughout the experiment but the rate of formation decreases with time, while at $298 \mathrm{~K}$, it was appproximately constant. The plot of ethane seems to be uncorrelated with those of propene and 1- and 2-butene. This result strongly suggest that ethane production is not connected to that of the other organic products.

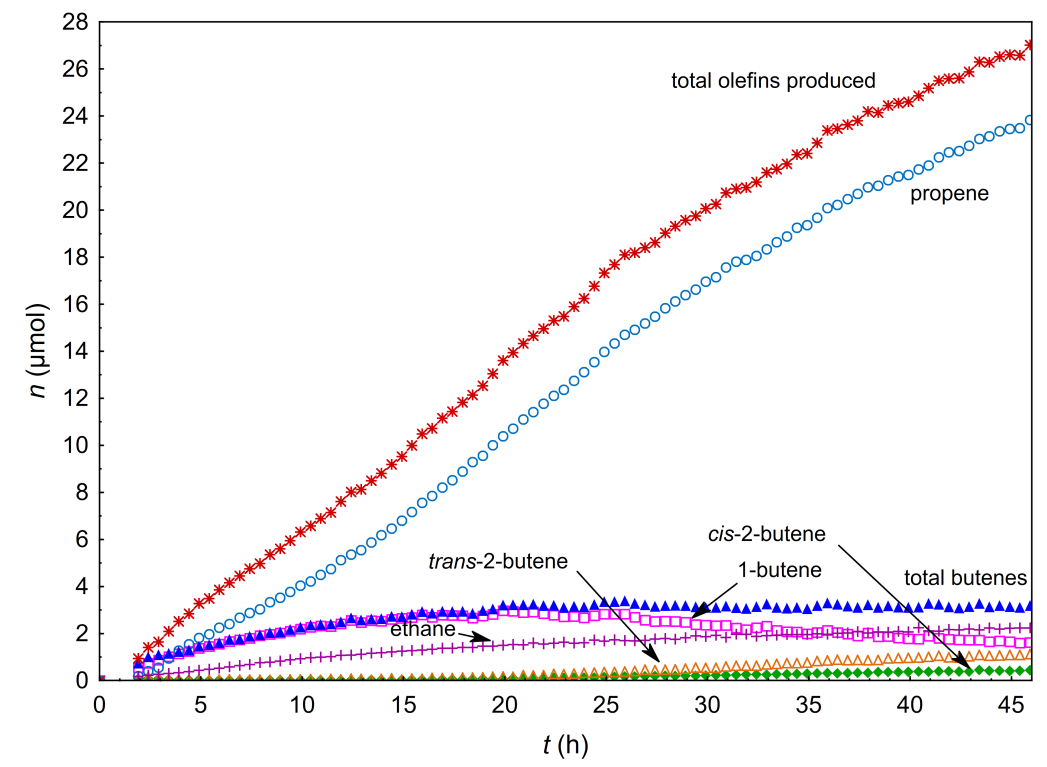

Figure S6. Products obtained with 1 and ethylene $(115 \mu \mathrm{mol})$ at $323.0 \mathrm{~K}$ in $\mathrm{C}_{6} \mathrm{D}_{6}:$ * total olefins produced, $\Delta$ total butenes, $\square$ 1-

butene, $\triangle$ trans-2-butene, $\bullet$ cis-2-butene, + ethane and $\odot$ propene.

When the experiment was terminated, $70 \%$ of the ethylene consumed was present in the products, with the remainder presumably having moved to the headspace where it could not be measured.

Whereas, at room temperature $\mathbf{1}$ is activated very slowly and a residual amount (4\%) was still present at the end of the experiment (i.e. after $73.5 \mathrm{~h}$ ), at $323 \mathrm{~K}$ the peaks of compound 1 disappear within $2.5 \mathrm{~h}$ after heating.

The disappearance of $\mathbf{1}$ is accompanied by the increasing of the intensity of the resonances corresponding to the free $p$-cymene. About $70 \%$ of the $p$-cymene initially coordinated to 1 is present in a dissociated form. The remaining $30 \%$ is presumably coordinated to unknown ruthenium species. The analysis of the aromatic region reveals that, similarly to the experiment at $298 \mathrm{~K}$, many different ruthenium species are generated from 1 after the dissociation of $p$-cymene, see Figure S7. 
Furthermore, the analysis of the ${ }^{1} \mathrm{H}$ NMR spectra shows that the disappearance of the singlet at $\delta 6.78$ ppm $(323 \mathrm{~K})$, corresponding to the four aromatic protons of the SIMes ligand, is, in large part, replaced by several new resonances (all apparently singlets) of lower intensity in the region 6.06-8.35 ppm (Figure S7). After one-hour heating, the most intense of the new peaks is at $6.59 \mathrm{ppm}$. Other relatively large peaks are at $\delta 6.86,6.78,6.79,6.71,6.68$, and 6.65 . The sum of the integrals of this region of the spectrum (with exclusion of the resonance peaks of the free $p$-cymene and of the NMR solvent $\mathrm{C}_{6} \mathrm{D}_{5} \mathrm{H}$ ) is after $73.5 \mathrm{~h}$ about $45 \%$ lower than before heating. This observation is consistent with the fact that many Ru-species are formed during this experiment and that a substantial amount of the N-heterocyclic carbene ligand is lost during the experiment.

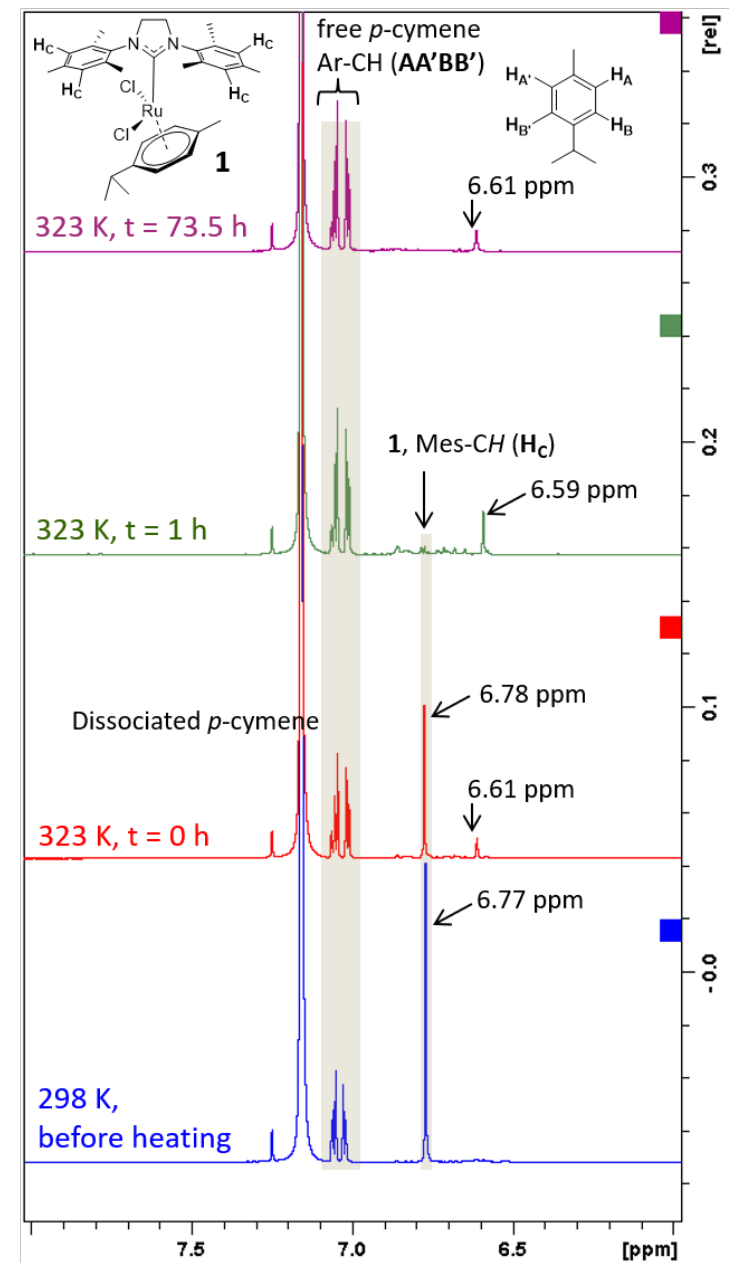

Figure S7. Changes of the aromatic region of the ${ }^{1} \mathrm{H}$ spectrum with the progress of the reaction.

After the experiment, a microcrystalline white precipitate was found on the wall of NMR tube. The solid was isolated $(0.1 \mathrm{mg}, 0.3 \mu \mathrm{mol}, 8 \%)$ and identified to be 1,3-bis(2,4,6-trimethylphenyl)-imidazolinium chloride. The isolated amount corresponds approximately to the observed reduction of the sum of integrals in the aromatic region. Furthermore, the formation of this product, which was observed also by Janse van Rensburg and cowokers, ${ }^{8}$ indicates that loss of the N-heterocyclic carbene ligand as imidazolium chloride is one of the decomposition routes for $\mathbf{1}$. The dinuclear ruthenium cyclometalated ethylene complex first isolated by Grubbs and coworkers ${ }^{15}$ and subsequently also by Fogg and coworkers ${ }^{16}$ was not observed. 


\section{Reaction of 1 with Ethylene ( $115.9 \mu \mathrm{mol})$ at $298.0 \mathrm{~K}$ in the Presence of $\mathrm{PCy}_{3}(0.57$ equiv.)}

Figure S8 shows the generation of 1-butene, cis- and trans-2-butene during the first $72 \mathrm{~h}$. The formation of small amounts of propene and ethane are also detected. 1-Butene is the major product and is the only one clearly detectable by ${ }^{1} \mathrm{H}$ NMR at the beginning of the experiment. The rate of formation of 1-butene increases during the first 10-15 h and then decreases until the end of the experiment. In contrast, for cisand trans-2-butene, the rate of formation seems to increase continuously during the experiment. These results strongly suggest that cis- and trans-2-butene are in part produced from 1-butene via double-bond migration. The cis/trans ratio remains close to unity until about $35 \mathrm{~h}$ and then starts to decrease. At the end of the experiment the cis/trans ratio is equal to 0.86 . This suggests that in the first part of the reaction 2butene is obtained directly via dimerization of ethylene, while from about $35 \mathrm{~h}$ double-bond migration of 1-butene starts to be operative and the contribution of this pathway slowly increases with time. The conversion of ethylene into butene isomers appears to be a catalytic process, and the turnovers, after 72.3 $\mathrm{h}$, are calculated to be 3.1 (see Table S6) ${ }^{13}$ Figure S8 shows that the rate of overall formation of 1 - and 2butene increases rapidly during the first $10-15 \mathrm{~h}$ and then continues incresing slowly until the end of the experiment. This result is consistent with the existence of an induction period (initiation) that seems to correlate well with the dissociation of the $p$-cymene ligand from 1 (Figure S9).

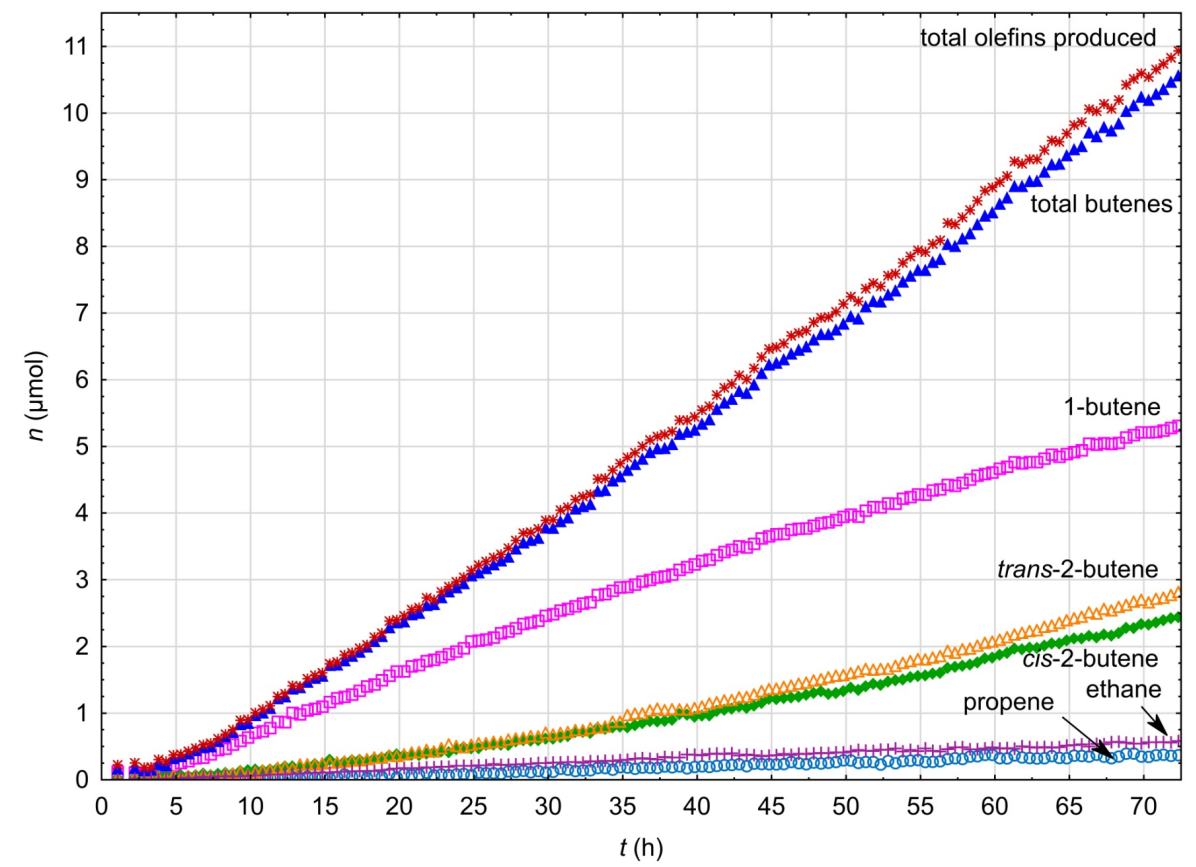

Figure S8. Products obtained with 1, 0.57 equiv. $\mathrm{PCy}_{3}$ and ethylene $(116 \mu \mathrm{mol})$ at $298.0 \mathrm{~K}$ in $\mathrm{C}_{6} \mathrm{D}_{6}$ : $*$ total olefins produced, $\boldsymbol{}$ total butenes, $\square$ 1-butene, $\triangle$ trans-2-butene, $\bullet$ cis-2-butene, + ethane and $\odot$ propene. 


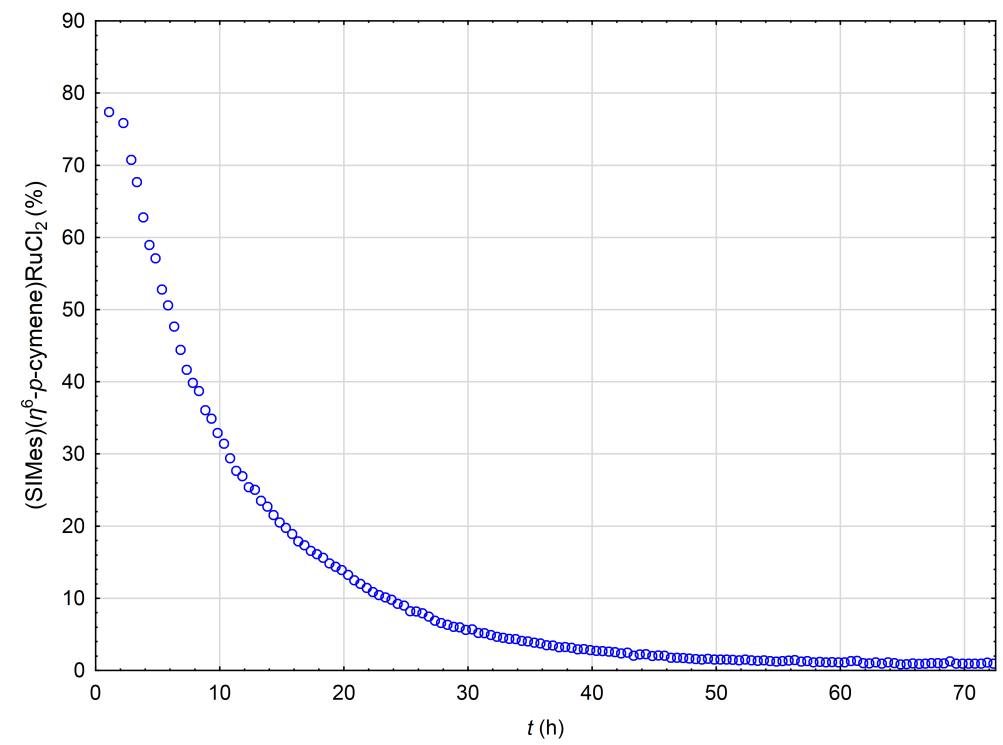

Figure S9. Percentage of 1 present with 1, 0.57 equiv. $\mathrm{PCy}_{3}$ and ethylene $(116 \mu \mathrm{mol})$ at $298.0 \mathrm{~K}$ in $\mathrm{C}_{6} \mathrm{D}_{6}$.

Differently from the butenes, propene is absent at the beginning and starts to be detected only after about $10 \mathrm{~h}$. This observation is consistent with the existence of an induction period for the generation of olefin metathesis active species. The rate of propene formation slowly declines with time. Since the amount of 2butene increases with the progress of reaction, this result is consistent with the fact that the concentration of olefin metathesis active species is decreasing with time. After $72.3 \mathrm{~h}$, the amount of propene is only $0.37 \mu \mathrm{mol}$, which is less than half $(0.77 \mu \mathrm{mol})$ of that obtained in the analogous experiment without phosphine. This may suggest that the presence of $\mathrm{PCy}_{3}$ reduces the catalytic activity by binding the 14electron ruthenium alkylidene species and forming the corresponding 16-electron phosphine-stabilized species. The resonance peaks of latter species should in principle be visible in the alkylidene region of the ${ }^{1} \mathrm{H}$ NMR spectrum, but, most probably due to the very low concentration, we were unable to detect. Similarly, to the experiment without phosphine, ethane grows at a rate which appears to be approximately constant with time. After $72.3 \mathrm{~h}$, the amount of ethane produced $(0.58 \mu \mathrm{mol})$ is very similar to the experiment without phosphine $(0.59 \mu \mathrm{mol})$. This indicates that the addition of phosphine doesn't affect the rate of formation of this product. In contrast, after $72.3 \mathrm{~h}$ the total amounts of butenes is more than twice (10.6 $\mu \mathrm{mol})$ compared to the experiment without phosphine $(10.6 \mu \mathrm{mol})$.

When the experiment was terminated, $82 \%$ of the ethylene consumed was present as products, the remainder presumably having moved to the headspace. Stacked plots of ${ }^{1} \mathrm{H}$ NMR spectra recorded at different intervals of time (Figure S10 and Figure S11) show a gradual increase in intensity of the resonances corresponding to the olefinic products and ethane and a decrease in intensity of the resonances belonging to the ruthenium catalyst $\mathbf{1}$ (as also depicted in Figure S9). Moreover, the intensity of the signals corresponding to the free $p$-cymene increases, while those of the free tricyclohexylphosphine decreases with time. ${ }^{17}$ Furthermore, the analysis of the ${ }^{1} \mathrm{H}$ NMR spectra shows that the decreasing intensity of the singlet peak at $6.77 \mathrm{ppm}$ corresponding to the four aromatic protons of the SIMes ligand is at least in part replaced by several new peaks of lower intensity in the region 6.26-8.26 ppm (Figure S10). The main new resonances (all singlets) are at 6.86 and $6.71 \mathrm{ppm}$. 


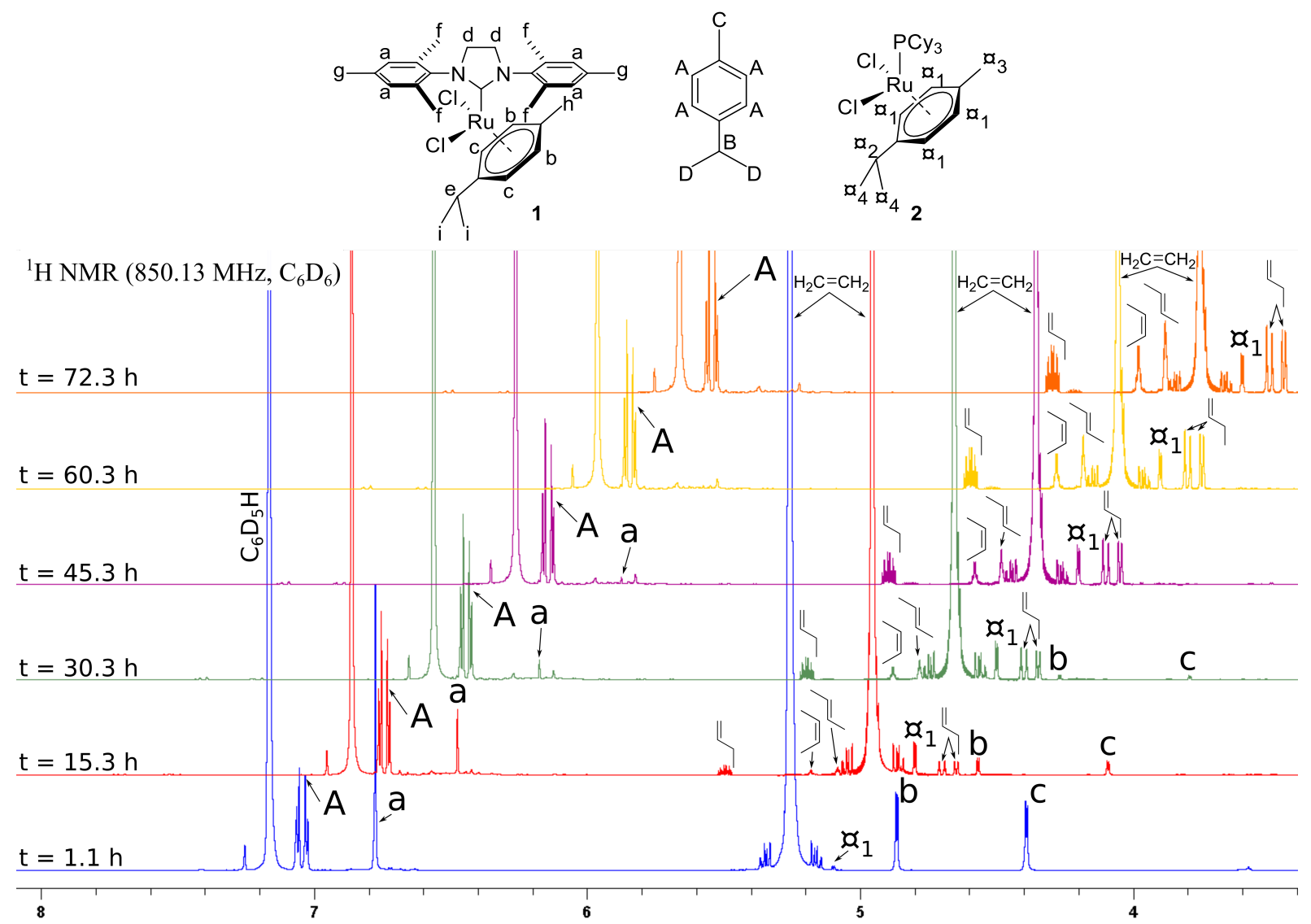

Figure S10. Stacked ${ }^{1} \mathrm{H}$ NMR spectrum $(\delta 8-3.4 \mathrm{ppm})$ of 1, 0.57 equiv. $\mathrm{PCy}_{3}$ and ethylene $(116 \mu \mathrm{mol})$ at $298.0 \mathrm{~K}$ in $\mathrm{C}_{6} \mathrm{D}_{6}$. Capital letters indicate resonances associated with free $p$-cymene, small letters denote resonances associated with 1 and resonances associated with $\left(\mathrm{PCy}_{3}\right)\left(\eta^{6}-p\right.$-cymene $) \mathrm{RuCl}_{2}$ are indicated with $\propto$. The spectra are shifted $\delta 0.3 \mathrm{ppm}$ w.r.t each other.

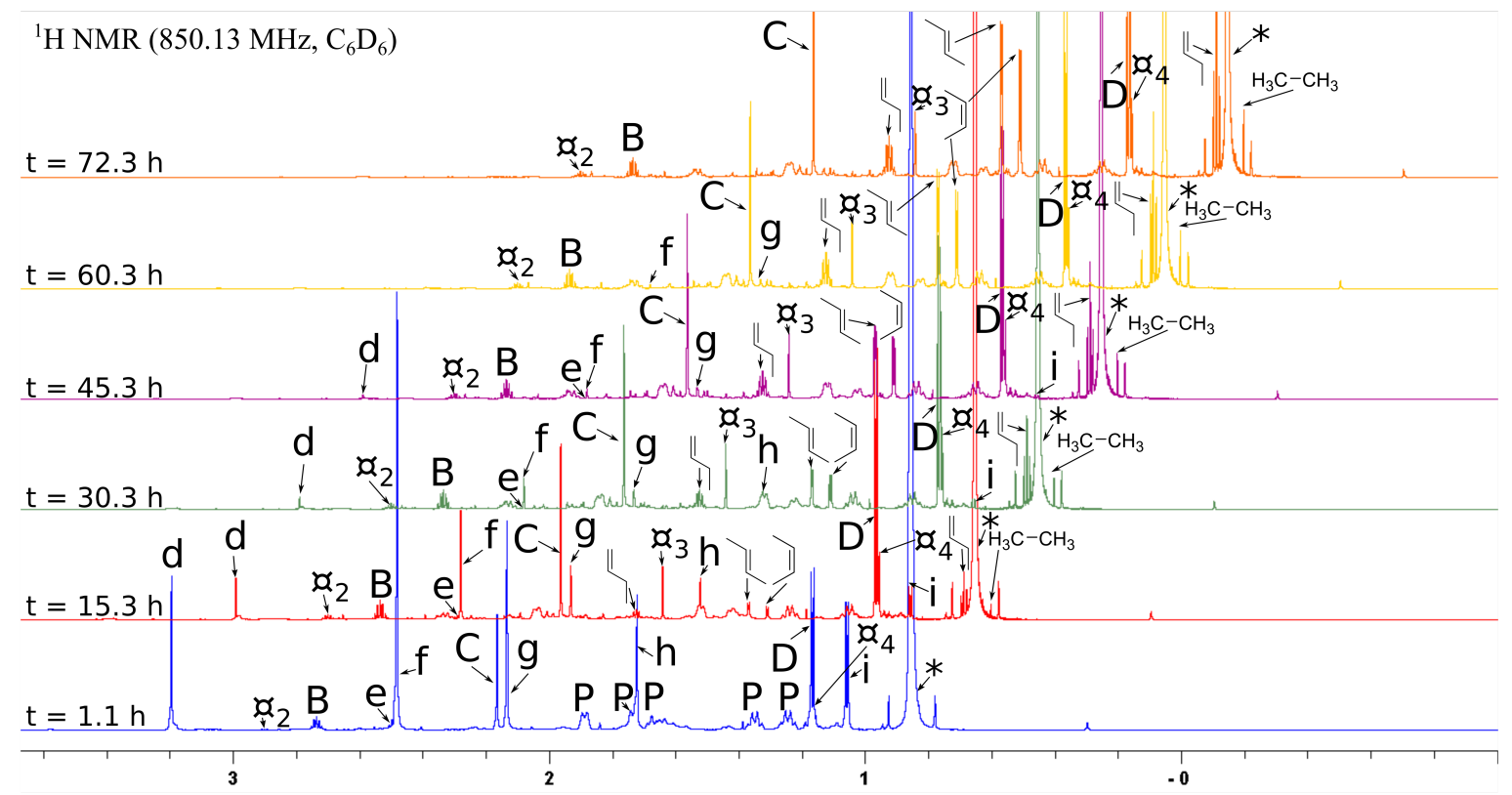

Figure S11. Stacked ${ }^{1} \mathrm{H}$ NMR spectrum $(\delta 3.6-0 \mathrm{ppm})$ of $1,0.57$ equiv. $\mathrm{PCy}_{3}$ and ethylene $(116 \mu \mathrm{mol})$ at $298.0 \mathrm{~K}$ in $\mathrm{C}_{6} \mathrm{D}_{6}$. Capital letters indicate resonances associated with free $p$-cymene, small letters denote resonances associated with 1 , resonances associated with $\left(\mathrm{PCy}_{3}\right)\left(\eta^{6}-p\right.$-cymene $) \mathrm{RuCl}_{2}$ are indicated with $\mathrm{O}, \mathrm{P}$ indicates resonances from free tricyclohexylphosphine and * indicates resonances belonging to the internal standard (2,2,3,3-tetramethylbutane). The spectra are shifted $\delta 0.2$ ppm w.r.t each other. 
These two signals were also present in the experiment carried out in the absence of phosphine ligand. The sum of the integrals of this region of the spectrum (with exclusion of the resonance peaks of the free $p$-cymene and of the NMR solvent $\mathrm{C}_{6} \mathrm{D}_{5} \mathrm{H}$ ) is, after $72.3 \mathrm{~h}$, about $60 \%$ lower than after 1 hour. This result shows that many Ru-species are formed during this experiment and that most of complex $\mathbf{1}$ decomposes via loss of the SIMes ligand. Some resonances of very small intensity are observed in the region from -1 to $-7 \mathrm{ppm}$, indicating the formation of Ru-hydride species.

The complexity of the mixture renders identification of all ruthenium-based species generated from 1 very challenging. However, the analysis of the ${ }^{1} \mathrm{H},{ }^{13} \mathrm{C}$, and ${ }^{31} \mathrm{P}$ spectra shows that the phosphine analogue of 1, i.e. $\left(\mathrm{PCy}_{3}\right)\left(\eta^{6}-p\right.$-cymene) $\mathrm{RuCl}_{2}$ (2) (see Scheme S3; Figure S10, Figure S11 and Figure S23-Figure S25) is generated during the reaction. Tests demonstrate that formation of $\mathbf{2}$ from $\mathbf{1}$ in the presence of $\mathrm{PCy}_{3}$ is relatively easy at room temperature and occurs also in the absence of ethylene. In other words, this decomposition of 1 proceeds via substitution of the SIMes ligand by $\mathrm{PCy}_{3}$ and is independent from the catalytic generation of olefins and ethane.

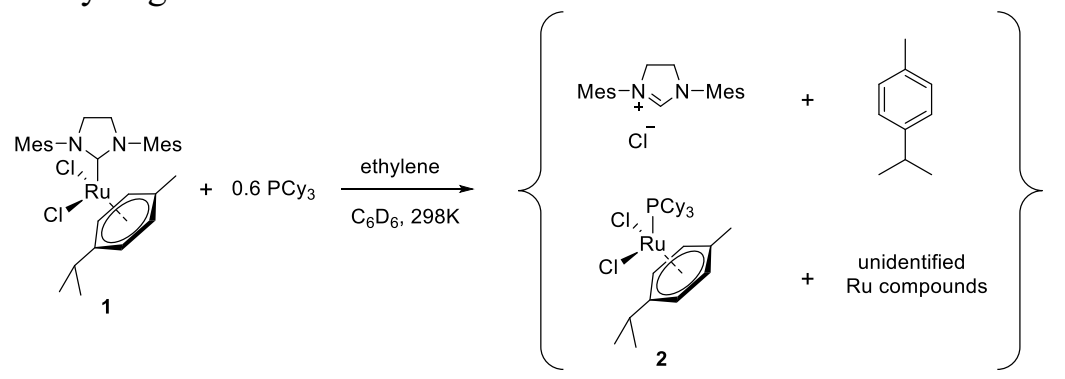

Scheme S3. Decomposition of $\mathbf{1}$ in the presence of $\mathrm{PCy}_{3}$ and ethylene.

The amount of 2 increases during the first $20 \mathrm{~h}$ and then the concentration remains approximately constant until the end of the experiment. This suggests that, in contrast to 1, complex $\mathbf{2}$ is stable under these conditions and does not participate in the catalytic generation of olefins and ethane. The analysis of the ${ }^{31} \mathrm{P}$ NMR spectrum, recorded at the end of the experiment (Figure S30), shows that the signal at 10.9 ppm corresponding to free $\mathrm{PCy}_{3}$ has disappeared and that three new resonances have appeared. The most intense of these signals ( $\delta 25.88 \mathrm{ppm})$ is that of complex 2 . The other ${ }^{31} \mathrm{P}$ NMR resonances, found at $\delta$ 33.79 and $46.42 \mathrm{ppm}$, correspond to unknown compounds, perhaps the one also observed by Janse van Rensburg and coworkers at $\delta 33.8 \mathrm{ppm},{ }^{8}$ and tricyclohexylphosphine oxide $\left((\mathrm{Cy})_{3} \mathrm{P}=\mathrm{O}\right)$, respectively. After the experiment, a microcrystalline white precipitate was found on the wall of NMR tube. The solid was isolated $(0.4 \mathrm{mg}$, yield $=28 \%$ with respect to 1) and identified to be 1,3-bis(2,4,6trimethylphenyl)imidazolinium chloride. The formation of this product, which was observed also by Janse van Rensburg and coworkers, ${ }^{8}$ indicates that loss of the N-heterocyclic carbene ligand as imidazolium chloride is one of the decomposition routes of $\mathbf{1}$. The dinuclear ruthenium cyclometalated ethylene complex first isolated by Grubbs and coworkers, ${ }^{15}$ and subsequently also by Fogg and coworkers, ${ }^{16}$ was not observed.

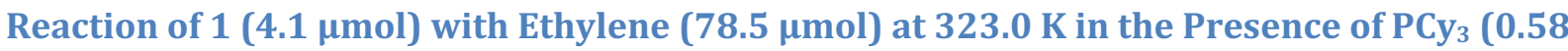 equiv.)}

Upon reacting $1(4.1 \mu \mathrm{mol})$ with ethylene $(78.5 \mu \mathrm{mol})$ at $323.0 \mathrm{~K}$ in the presence of 0.58 equivalents of tricyclohexylphosphine $\left(\mathrm{PCy}_{3}\right)$, a rapid production of 1-butene during the first $3 \mathrm{~h}$ of the reaction is observed ( $1 \mu \mathrm{mol}$, Figure S12 and Table S7). In the corresponding experiment carried out at $298.0 \mathrm{~K}$, discussed above (Figure S8 and Table S6), a comparable yield of 1-butene was reached only after nearly $13 \mathrm{~h}$. In addition, Figure S12 shows that, $13 \mathrm{~h}$ into the experiment, a maximum amount of $2 \mu \mathrm{mol} 1$-butene is obtained, followed by a decrease during the rest of the experiment. Clearly, isomerization of 1-butene to the thermodynamically more stable 2-butene isomers contributes to this decrease. ${ }^{1}$ Indeed, a steep increase in both cis- and trans-2-butene is observed at $t=5 \mathrm{~h}$. Initially, the cis/trans ratio is close to unity. However, rapidly the yield of the thermodynamically more stable trans-2-butene increases versus that of cis-2-butene, giving a final cis/trans ratio of 0.5 at $t=47 \mathrm{~h}$. 


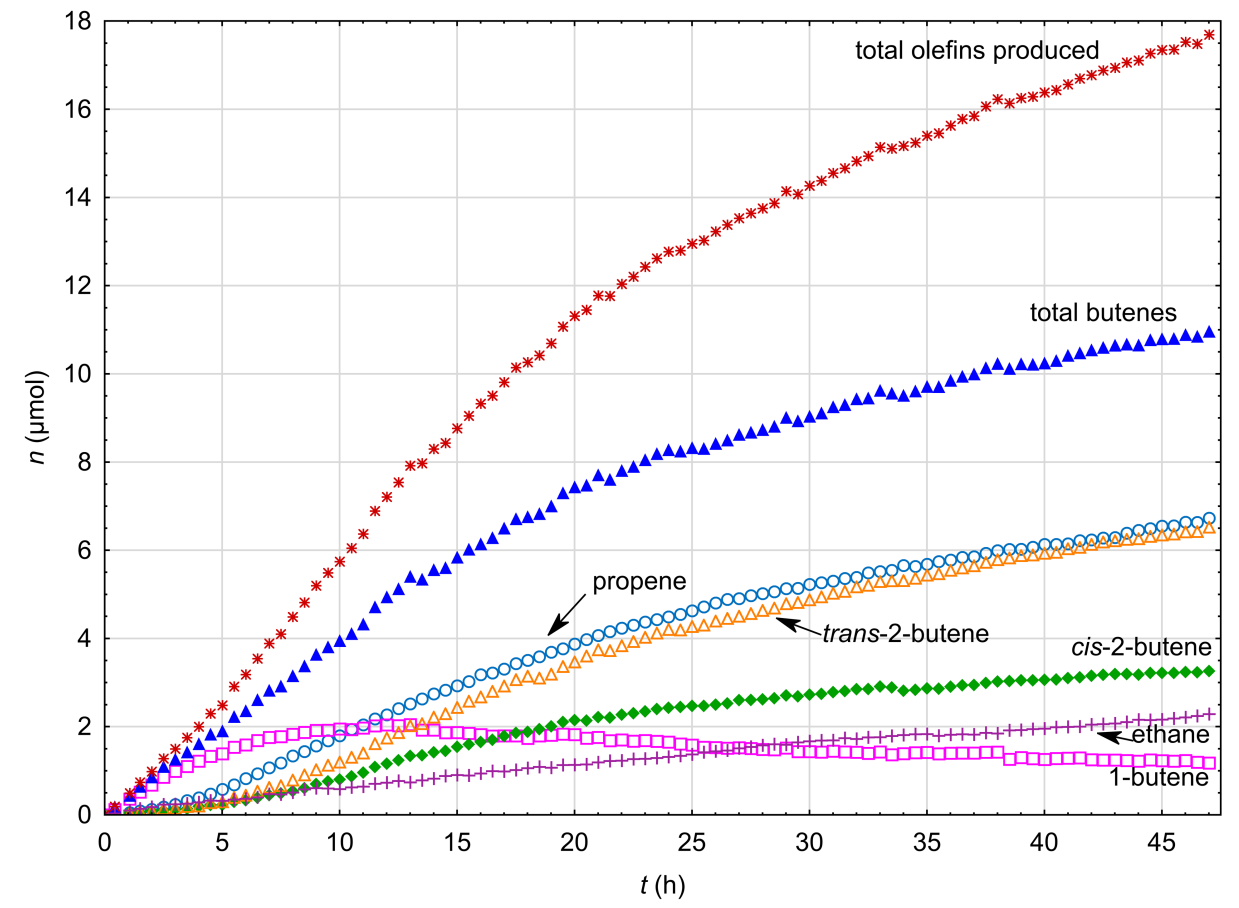

Figure S12. Products obtained with 1, 0.58 equiv. $\mathrm{PCy}_{3}$ and ethylene $(79 \mu \mathrm{mol})$ at $323.0 \mathrm{~K}$ in $\mathrm{C}_{6} \mathrm{D}_{6}$ : $\%$ total olefins produced, $\mathbf{\Delta}$ total butenes, $\square$ 1-butene, $\triangle$ trans-2-butene, $\triangleleft$ cis-2-butene, + ethane and $\circ$ propene.

The propene formation rate increase until about seven $\mathrm{h}$ into the experiment and then starts declining and continues to decrease slowly until the end of the experiment. Interestingly, the steep increasing in propene formation rate coincides with the appearance of a resonance at $18.42 \mathrm{ppm}$ corresponding to the $\mathrm{Ru}-$ methylidene complex GIIm (see Scheme S4 below for the Lewis structure), ${ }^{18}$ and the variation of intensity of this peak over the time correlates well with the rate of propene formation. Indeed, similarly to the rate of propene generation, the intensity of this peak increases in the interval $2.5-7 \mathrm{~h}$ and afterwards slowly decreases until the end of the experiment. This confirms that the generation of propene and the formation of GIIm are tightly connected. Indeed, as discussed above, propene is most likely produced via three different pathways: ethenolysis of 2-butene, ${ }^{14}$ ethylene-triggered decomposition of Ru-methylidene complexes (e.g. GIIm) i.e. via thermal decomposition of the non-substituted metallacyclobutane, and via generation of methylidenes as shown in the mechanism described in the computational part. ${ }^{8}$ The latter pathway is especially important at the very beginning. Figure S13 shows that there is a good correlation between the yield of 1-butene and the generation rate of 2-butenes. This suggests that in this experiment most of 2-butene stems from the isomerization of 1-butene. Furthermore, both the formation of butenes and the total formation of olefins slow down somewhat around $13 \mathrm{~h}$. The correlation between the yield of 1-butene and the generation rate of 2-butenes is shown in Figure S13. Overall, the conversion of ethylene into both butene and propene appears to be a catalytic process, and the turnovers, after $47 \mathrm{~h}$, are calculated to be 2.7 and 1.6 for butenes (the isomers combined) and propene, respectively. ${ }^{13}$

In addition, a steady increase in the amount of ethane is observed. After $27 \mathrm{~h}$, the yield of ethane even begins to surpass that of 1-butene, albeit mainly because 1-butene is consumed. After $47 \mathrm{~h}$, the amount of ethane in solution is $2.3 \mu \mathrm{mol}$, which is slightly more than half equiv. of the initial amount of ruthenium complex $1(4.1 \mu \mathrm{mol})$. Finally, $75 \%$ of the ethylene consumed is present in the products. 


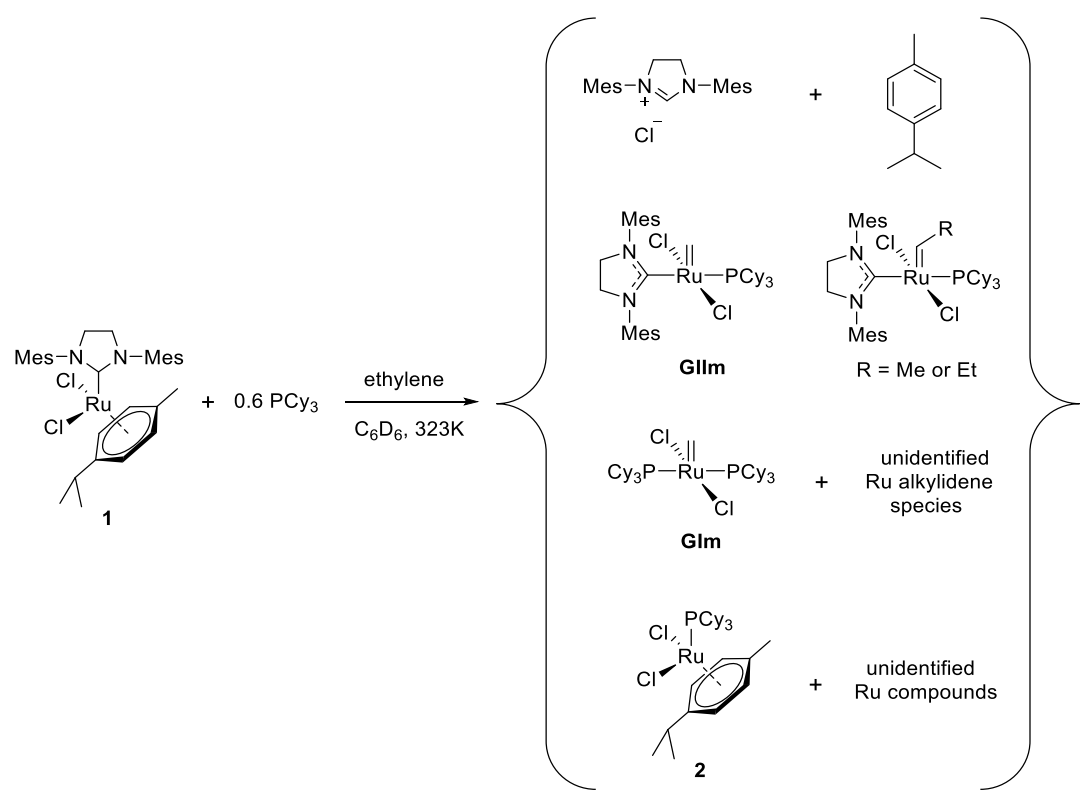

Scheme S4. Decomposition of $\mathbf{1}$ in the presence of $\mathrm{PCy}_{3}$ and ethylene at $323 \mathrm{~K}$.

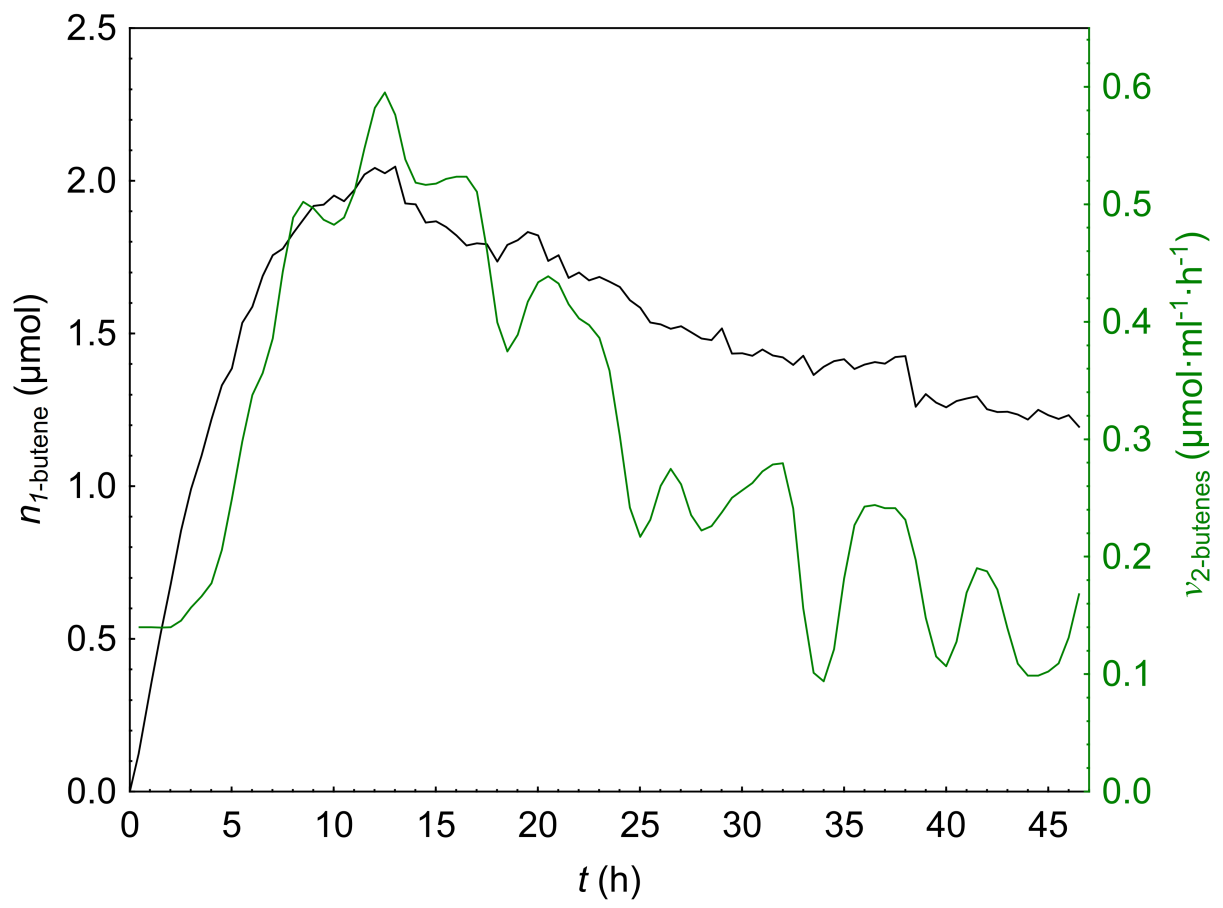

Figure S13. Correlation between the yield of 1-butene (black plot) and the rate of generation of 2-butenes (green plot). The 2butene generation rate was obtained by plotting the average rate of every $0.5 \mathrm{~h}$ interval. This average, in turn, was calculated by dividing the difference between the concentration of the product $\left(\mu \mathrm{mol} \cdot \mathrm{ml}^{-1}\right)$ at reaction time $\mathrm{t}+0.5(\mathrm{~h})$ and $\mathrm{t}(\mathrm{h})$ by the time interval $0.5(\mathrm{~h})$, as shown in Equation S1. The resulting curve was smoothened using the T4253H smoothening function which removes spikes and oscillations while retaining the trends. ${ }^{11}$

Stacked plots of ${ }^{1} \mathrm{H}$ NMR spectra, recorded at different time intervals (Figure S14), show a gradual increase in intensity for the resonances corresponding to the $\mathrm{C} 3$ and $\mathrm{C} 4$ olefinic products and the rapid disappearance (within a couple of $h$ ) of the resonances of $\mathbf{1}$. Moreover, the intensity of the peaks corresponding to the free $p$-cymene increases rapidly during the first two $\mathrm{h}$ of heating, corresponding to ca. 0.35 equiv. with respect to the initial amount of $1,{ }^{17}$ and then continues increasing more slowly until the end of the experiment. After $47 \mathrm{~h}, 67 \%$ of $p$-cymene initially bonded to 1 , has dissociated. These 
trends show that during the first two h, only a minor part (about 35\%) of the $p$-cymene bonded to Ru has dissociated. The remaining $65 \%$ is converted into different Ru-species that retain this ligand. Furthermore, the analysis of the ${ }^{1} \mathrm{H}$ NMR spectra shows that the disappearance of the singlet peak at $6.77 \mathrm{ppm}$ corresponding to the four aromatic protons of the SIMes ligand is accompanied by the formation of a large number of signals in the region 6.26-8.26 ppm, which suggests the conversion into different SIMesbonded Ru-species. The number, the intensity, and the identity of these resonances change over time, and the sum of the integrals of these signals decreases with time, suggesting the formation of insoluble species such as 1,3-bis(2,4,6-trimethylphenyl)imidazolinium chloride $(0.3 \mathrm{mg}$, yield $=21 \%$ with respect to 1$)$, which was isolated as a microcrystalline white precipitate after the experiment.

The catalytic initiation of 1 probably involves dissociation of the $p$-cymene ligand, and the fact that only $35 \%$ of the $p$-cymene has dissociated when the signals of $\mathbf{1}$ have already disappeared suggests that a significant fraction of $\mathbf{1}$ is converted into one or more different species before possibly participating in the catalytic activity. For example, one of the reactions observed from the very beginning is the replacement of SIMes with $\mathrm{PCy}_{3}$ to give $\left(\mathrm{PCy}_{3}\right)\left(\eta^{6}-p\right.$-cymene) $\mathrm{RuCl}_{2}(\mathbf{2})$, the phosphine analogue of $\mathbf{1}$, which is more stable than the latter. As discussed above in the analogous room-temperature experiment, this reaction is relatively easy even at room temperature and does not require the presence of ethylene. The first ${ }^{1} \mathrm{H}$ NMR spectrum recorded at $298 \mathrm{~K}$ shows that about $5 \%$ of 2 has been formed. When the sample is heated at $323 \mathrm{~K}$, the amount of 2 increases significantly and reaches about $34 \%$ after $2.5 \mathrm{~h}$. Then it starts declining and continues a slow decrease to about $3 \%$ at the end of the experiment. The increasing amount of free $p$ cymene between 2.5 and $47 \mathrm{~h}(0.31$ equiv. of the initial amount of $\mathbf{1})$ corresponds to the amount of 2 consumed during this time ( 0.32 equiv. of the initial amount of $\mathbf{1})$. This result demonstrates that, at this temperature, also compound $\mathbf{2}$ loses the $p$-cymene ligand, and therefore could in principle contributing to the catalytic activity observed in the experiment. However, a similar experiment carried out with $\mathbf{2}$ (see below) reveals that the catalytic activity of $\mathbf{2}$ in generating butenes and propene is much lower compared to that of 1 . 


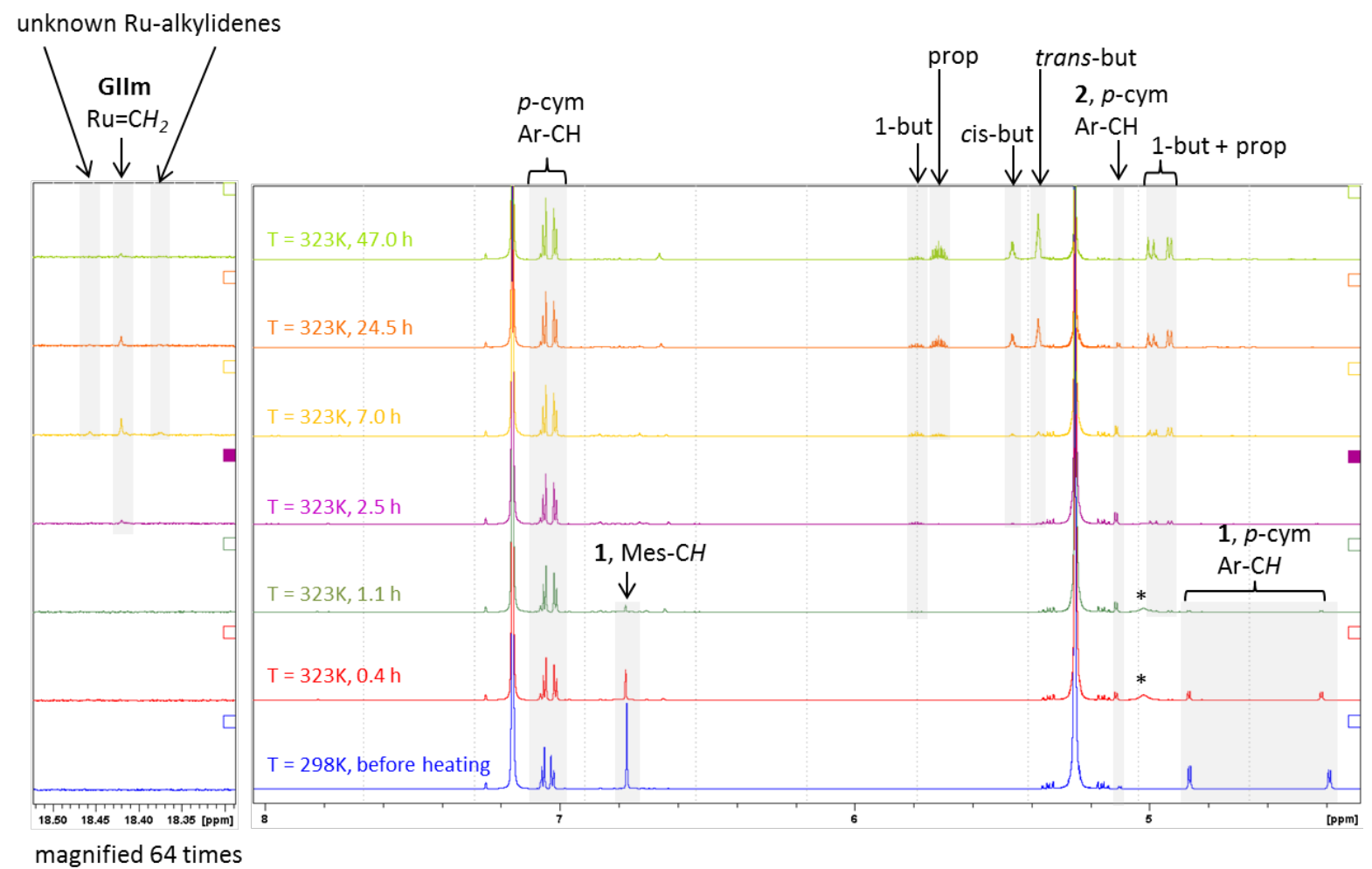

Figure S14. Stacked ${ }^{1} \mathrm{H}$ NMR spectrum $\left(\delta 18.5-18.3\right.$ and 8.0-4.3 ppm) of 1, 0.58 equiv. $\mathrm{PCy}_{3}$ and ethylene (79 $\left.\mu \mathrm{mol}\right)$ at $323.0 \mathrm{~K}$ in $\mathrm{C}_{6} \mathrm{D}_{6}$. Abbreviations: $p$-cym $=p$-cymene, Mes = mesityl, 1-but $=1$-butene, prop $=$ propene, cis-but $=$ cis-2-butene, trans-but $=$ trans-2-butene, $*=$ unknown Ru-species.

Some signals of low intensity, observed in the region from -1 to $-8 \mathrm{ppm}$, demonstrate the formation of ruthenium hydride species. The main resonance is the doublet at $\delta-7.48\left(\mathrm{~d},{ }^{2} J_{\mathrm{PH}}=51 \mathrm{~Hz}, 1 \mathrm{H}\right)$. It appears in the spectrum after about $10 \mathrm{~h}$, and the intensity increases until about $25 \mathrm{~h}$ and then decreases, see Figure S15.

The chemical shift and the peak multiplicity of this signal are compatible with those of the rutheniumhydride complex $\mathrm{PCy}_{3}(\mathrm{Cl}) \mathrm{HRu}(p$-cymene $) .{ }^{19}$ The reactivity of this species is investigated in a separate experiment (see next section "Reaction of $2(4.1 \mu \mathrm{mol})$ with ethylene $(89.5 \mu \mathrm{mol})$ at $323.0 \mathrm{~K}$ in the presence of PCy3 (0.58 equiv.)"). Finally, the analysis of the plot suggests that there is no correlation between ethane formation and formation of hydride species. Moreover, the amount of ruthenium hydrides observed during this experiment are ca. two order of magnitude smaller than ethane. Thus, ruthenium hydrides appear not to be an important source of hydrogen for the generation of ethane. 


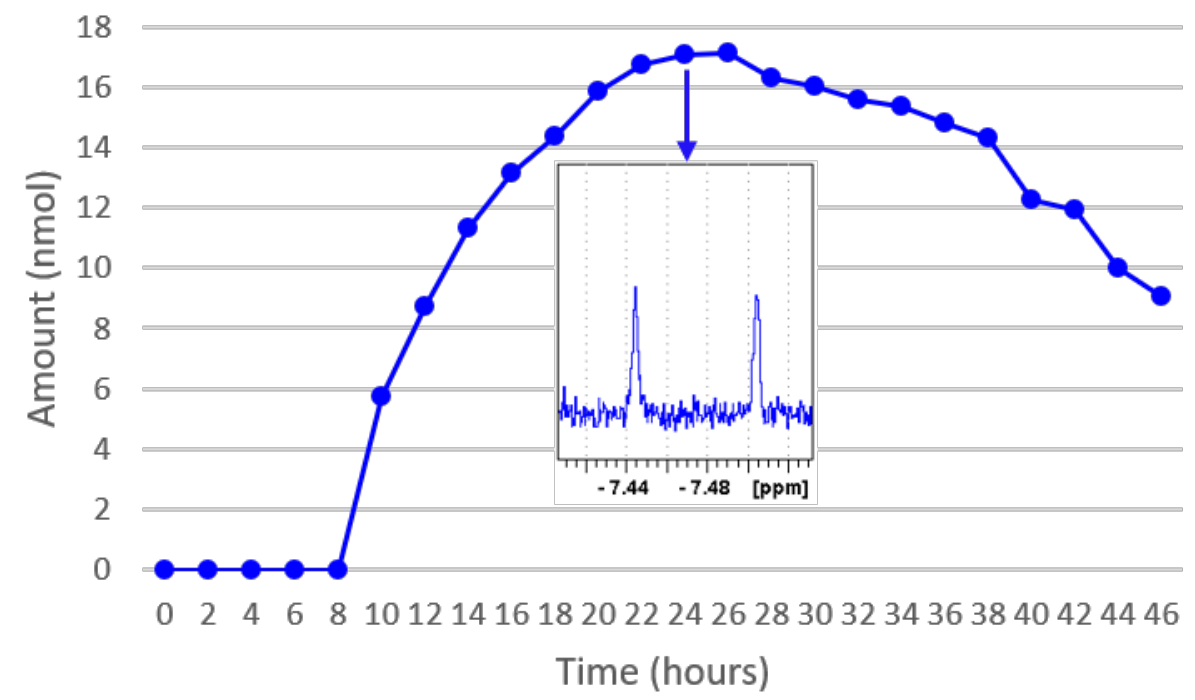

Figure S15. Evolution of the Ru-hydride peak at $-7.48 \mathrm{ppm}\left(\mathrm{d},{ }^{2} J_{\mathrm{PH}}=51 \mathrm{~Hz}, 1 \mathrm{H}\right)$ in the experiment of 1, 0.58 equiv. PCy 3 and ethylene $(79 \mu \mathrm{mol})$ at $323.0 \mathrm{~K}$ in $\mathrm{C}_{6} \mathrm{D}_{6}$.

The left side of Figure S14 shows a magnified portion of the alkylidene region of the ${ }^{1} \mathrm{H}$ NMR spectrum, which reveals the generation of Ru-alkylidene species during the experiment. The resonance of the main peak at $\delta 18.42 \mathrm{ppm}$, is characteristic for the methylidene analog of Grubbs second-generation catalyst (GIIm, Scheme S4). ${ }^{18}$ This compound begins to appear at $t=2.5 \mathrm{~h}$ and reaches its maximum amount of $\approx$ $10.4 \mathrm{nmol}(0.25 \%$ w.r.t. 1) at $t=7$ h. Following this, decomposition causes the signal to decrease to a value corresponding to $\approx 2 \mathrm{nmol}$ when the reaction was terminated at $t=47 \mathrm{~h}$. Between $t=4 \mathrm{~h}$ and $t=27$ $\mathrm{h}$, two other resonances are present, at $\delta 18.46$ and $18.38 \mathrm{ppm}$. The structure of these Ru-alkylidene species is unknown. Subsequently, in the spectrum recorded at $t=9.5 \mathrm{~h}$, a resonance of relative low intensity, at $\delta 19.00 \mathrm{ppm}$ was observed. This species is still present at $t=10 \mathrm{~h}$, but subsequently disappears. The chemical shift of this resonance agrees with that reported for the ethylidene and propylidene analogues of Grubbs second-generation catalyst. ${ }^{20}$ Finally, at $t=25.5 \mathrm{~h}$, a small peak at $\delta$ $19.42 \mathrm{ppm}$ indicates the presence of a miniscule amount of the methylidene analog of Grubbs firstgeneration catalyst (GIm, Scheme S4). ${ }^{18}$

In the ${ }^{31} \mathrm{P}$ NMR spectrum, the resonance found at $\delta 25.90 \mathrm{ppm}$ was identified as $\left(\mathrm{PCy}_{3}\right)\left(\eta^{6}-p\right.$ cymene) $\mathrm{RuCl}_{2}$ (Figure S33). Furthermore, a small amount of free $\mathrm{PCy}_{3}$ was present $(\delta 10.95 \mathrm{ppm})$ together with the corresponding oxide $\left.(\mathrm{Cy})_{3} \mathrm{P}=\mathrm{O}\right)$ at $\delta 46.38 \mathrm{ppm}$. Moreover, the resonance at $\delta 34.6 \mathrm{ppm}$ is very close to that reported for the phosphonium salt $\left[\mathrm{CH}_{3} \mathrm{PCy}_{3}\right] \mathrm{Cl}$. However the reported doublet associated with the methyl protons ${ }^{15}$ was not observed in the ${ }^{1} \mathrm{H}$ NMR spectrum. Janse van Rensburg et al. observed resonances at: $\delta 40.3,40.0$ and $33.8 \mathrm{ppm}$ in the ${ }^{31} \mathrm{P}$ spectrum. These could be the resonances we obtained at $\delta 41.26,40.79$ and $33.69 \mathrm{ppm}$ (Figure S33). ${ }^{8}$ For the resonances at $\delta 81.06,35.74$ and 12.56 ppm no satisfactory identification could be obtained. Finally, the methylidene analog of Grubbs secondgeneration catalyst $(\mathbf{G I I m})$, expected at $\delta 38.6 \mathrm{ppm},{ }^{21}$ is most likely not observed due to its low concentration.

\section{Reaction of $2(4.1 \mu \mathrm{mol})$ with Ethylene ( $89.5 \mu \mathrm{mol})$ at $323.0 \mathrm{~K}$ in the Presence of $\mathrm{PCу}(0.58$ equiv.)}

To better understand the influence of the phosphine ligand in the experiments with $\mathbf{1}$, we have repeated the previous experiment using 2 (i.e. the $\mathrm{PCy}_{3}$ analogue of $\mathbf{1}$ ) instead of $\mathbf{1}$, and applying otherwise the same reaction conditions. Indeed, complex $\mathbf{1}$ is in part converted into $\mathbf{2}$ when $\mathrm{PCy}_{3}$ is used as an additive.

A stacked plot of ${ }^{1} \mathrm{H}$ NMR spectra recorded at different time is shown in Figure S16. The analysis of these data demonstrates that $\mathbf{2}$ is much more stable than $\mathbf{1}$. Whereas, the reasonance peaks of $\mathbf{1}$ disappear 
after a couple of h, only $12 \%$ of $p$-cymene results dissociated from 2 after $6.75 \mathrm{~h}$, and about $40 \%$ of unreacted 2 is still present in the reaction mixture at the end of the experiment $(47.25 \mathrm{~h})$.

Similarly to $\mathbf{1}$, the reaction of $\mathbf{2}$ with ethylene produces the corresponding Ru-methylidene complex (GIm, $19.42 \mathrm{ppm}) .^{18}$ The generation of GIm from $\mathbf{2}$ appears significantly slower compared to GIIm from 1, but the former reaction seems to be more productive. The singlet of GIm at $19.42 \mathrm{ppm}$ is clearly detected only after about five $h$ heating. The amount of GIm slowly increases over time during nearly all experiment and reaches a maximum at $38.25 \mathrm{~h}$, and then slowly declines. At $38.25 \mathrm{~h}$, the conversion of 2 is $55 \%$ whereas the yield of $\mathbf{G I m}$ is $0.55 \%$. Differently from $\mathbf{1}$, the reaction of $\mathbf{2}$ with ethylene produces very small amounts of $\mathrm{C} 3$ and $\mathrm{C} 4$ olefins. At the end of reaction, the amount of propene is 0.03 equivalents of the initial amount of 2, while 1-butene is 0.02 equivalents, trans-2-butene is 0.009 equiv. and cis-2-butene $8 \cdot 10^{-4}$ equiv..

On the contrary of the experiments with $\mathbf{1}$, propene is detected before 1-butene. The multiplet at 5.71 ppm $(1 \mathrm{H})$ of propene starts to be distinguished from the baseline noise after about $12 \mathrm{~h}$, while that of 1butene $(5.79 \mathrm{ppm}, \mathrm{m}, 1 \mathrm{H})$ after about $22 \mathrm{~h}$. After $22 \mathrm{~h}$ it begins to be detected also the multiplet of trans-2butene $(1.56 \mathrm{ppm}, 6 \mathrm{H})$, while the doublet of cis-2-butene at $1.51 \mathrm{ppm}(6 \mathrm{H})$ is distinguishable from the baseline noise only about $36 \mathrm{~h}$. Since the amount of 2-butene is essentially negligible during all experiment, the main route to propene, especially at the beginning, would most likely not be via ethenolysis of 2-butene. DFT calculations (see Scheme 4 in the main paper) predicts that propene is released during the formation of the ruthenium-methylidene complex. On the other hand, it is known that in the presence of ethylene GIm decomposes by releasing an equivalent of propene. Thus, propene must be regarded as a marker for both formation and decomposition of the methylidene complex. Figure S16 shows that the amount of GIm increases with time during most of the experiment. This means that its generation from $\mathbf{2}$ and ethylene happens faster than its decomposition.

Finally, also the reaction of $\mathbf{2}$ with ethylene produces ethane. Figure S16 show that similarly to the experiments conducted with 1 this product is generated from the beginning of the reaction and the amount increases with time. After 47 the amount of ethane is $4.1 \mu \mathrm{mol}$, which corresponds to 0.26 equiv. of the initial amount of $\mathbf{2}$. Thus, the main conversion of ethylene into other hydrocarbons is its reduction to ethane.

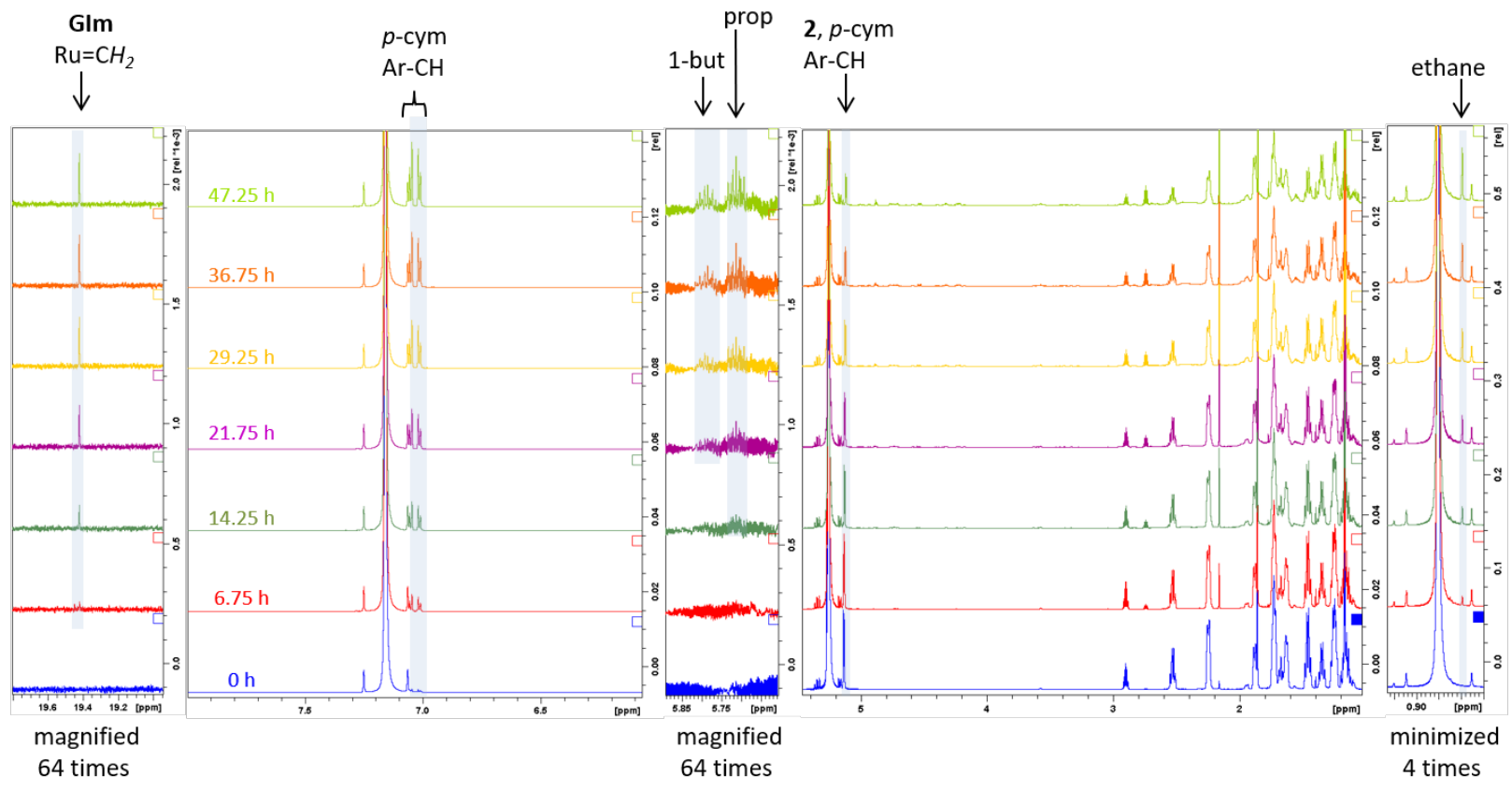

Figure S16. Stacked ${ }^{1} \mathrm{H}$ NMR spectrum $(\delta$ 19.8-19.0, 8.0-6.0, 5.90-5.60, 5.5-1, and 1.0-0.6 ppm) of 2 (4.1 $\mu \mathrm{mol}), 0.58$ equiv. $\mathrm{PCy}_{3}$ and ethylene $(89.5 \mu \mathrm{mol})$ at $323.0 \mathrm{~K}$ in $\mathrm{C}_{6} \mathrm{D}_{6}$. Abbreviations: $p$-cym $=p$-cymene, 1 -but $=1$-butene, prop $=$ propene. 


\section{Reaction of 1 (3.6 $\mu \mathrm{mol})$ with Ethylene $(84.3 \mu \mathrm{mol})$ at $323.0 \mathrm{~K}$ in the Presence of РСУз $(1.2$ equiv.)}

To better understand the influence of the phosphine ligand, the experiment with 1 at $323 \mathrm{~K}$ was repeated using twice the original amount of $\mathrm{PCy}_{3}$ (1.2 equiv. instead of 0.58 equiv.). The NMR sample was, while protected from light with aluminum foil, heated in an oil bath and, after $47.25 \mathrm{~h}$ at $323.0 \mathrm{~K},{ }^{1} \mathrm{H},{ }^{13} \mathrm{C}\left\{{ }^{1} \mathrm{H}\right\}$ and ${ }^{31} \mathrm{P}$ NMR spectra were recorded. Comparison of the ${ }^{1} \mathrm{H}$ NMR spectrum of this experiment with that obtained after $47 \mathrm{~h}$ of heating with 0.58 equiv. of $\mathrm{PCy}_{3}$, (Figure S17) shows that less $\mathrm{C} 3$ and $\mathrm{C} 4$ olefinic products are formed: $8.6 \mu \mathrm{mol}(2.4 \text { turnovers })^{13}$ vs. $17.6 \mu$ mol $(4.3 \text { turnovers })^{13}$ using 0.58 equiv. of PCy ${ }^{2}$ The lower yield of olefins is mainly due to propene ( $0.2 \mathrm{vs} .1 .6$ turnovers $).{ }^{13}$

Remarkably, with 1.2 equiv. of $\mathrm{PCy}_{3}$, more 1-butene was obtained (2.1 vs. $\left.1.2 \mu \mathrm{mol}\right)$ while both 2butene isomers were produced in lower yields $(2.3 v s .3 .3 \mu \mathrm{mol}$ and $3.7 v s .6 .5 \mu \mathrm{mol}$ for cis and trans-2butene, respectively), with a cis/trans ratio of 0.62 , vs. 0.49 with 0.58 equiv. of $\mathrm{PCy}_{3}$. These results show a reduced tendency to olefin isomerization at higher concentration of $\mathrm{PCy}_{3}$. When the experiment was terminated, $64 \%$ of the ethylene consumed was present in the products, with the remainder presumably having moved to the headspace where it could not be measured. ${ }^{22}$

Less ethane is also produced compared to the experiment conducted with 0.58 equiv. of $\mathrm{PCy}_{3}(1.2 v s .2 .3$ $\mu \mathrm{mol}$ corresponding to 0.35 and 0.55 equivalents with respect to the initial amount of $\mathbf{1}$, respectively. The amount of dissociated $p$-cymene is similar to that of the previous experiment $(68 \% \mathrm{vs} .67 \%$ with 0.58 equiv. of $\mathrm{PCy}_{3}$ ). Moreover, as in the original experiment, no residual signals of $\mathbf{1}$ are visible in the ${ }^{1} \mathrm{H}$ spectrum, whereas the amount of 2 is significantly higher (13\% of the initial amount of 1 vs. 3\% in the previous experiment). These results, together with those obtained in the experiment with 2 as catalyst, strongly suggest that the lower observed catalytic activity is caused by more of $\mathbf{1}$ being converted to $\mathbf{2}$ when more $\mathrm{PCy}_{3}$ is added. Indeed, as discussed in the previous experiments, 2 reacts more slowly with ethylene and produces significantly less $\mathrm{C} 3$ and $\mathrm{C} 4$ olefins than 1. Investigation of the alkylidene region reveals the presence of two resonances at $\delta 19.41$ and $18.42 \mathrm{ppm}$, belonging to Grubbs first- (GIm) and second-generation methylidene catalysts (GIIm), respectively. Remarkably, the amount of GIm (6.0 $\mathrm{nmol})$ is about three times larger than that of GIIm $(2.1 \mathrm{nmol})$, while no visible traces of GIm was present after 47 hour in the experiment with 0.58 equiv. of $\mathrm{PCy}_{3}$, and the yield of GIIm was slightly lower than that of the experiment carried out with 1.2 equiv. of $\mathrm{PCy}_{3}$ (i.e. $0.05 \%$ vs. $0.06 \%$ ). The fact that less propene and more $\mathrm{Ru}$ methylidene species are found in the reaction conducted with a double amount of $\mathrm{PCy}_{3}$ suggests that a higher concentration of $\mathrm{PCy}_{3}$ slows down the dissociation of the phosphine ligand from GIm and GIIm, which, in turn, slows down the olefin metathesis activity of these complexes (e.g. ethenolysis of 2-butene) and suppresses the thermal decomposition of the unsubstituted ruthenacyclobutane intermediate $\mathbf{R u - 2}$.

In the ${ }^{31} \mathrm{P}$ NMR spectrum, the resonance at $\delta 25.93 \mathrm{ppm}$ was identified as $\left(\mathrm{PCy}_{3}\right)\left(\eta^{6}-p\right.$-cymene $) \mathrm{RuCl}_{2}(\mathbf{2}$, Figure S18). In addition, quite some free $\mathrm{PCy}_{3}$ was still present $(\delta 10.91 \mathrm{ppm})$ together with the corresponding oxide $\left.(\mathrm{Cy})_{3} \mathrm{P}=\mathrm{O}\right)$ at $\delta 46.44 \mathrm{ppm}$. Moreover, the signals at $\delta 41.23,40.73$ and $33.79 \mathrm{ppm}$ could be those connected to unidentified species found by Janse van Rensburg et al. at: $\delta 40.3,40.0$ and $33.8 \mathrm{ppm} .{ }^{8}$ The peaks corresponding to the Ru methylidene complexes $\mathbf{G I m}(\delta 43.5 \mathrm{ppm})$ and $\mathbf{G I I m}(\delta$ $38.6 \mathrm{ppm}$ ) are not observed, most likely because of the low concentration of these species. 


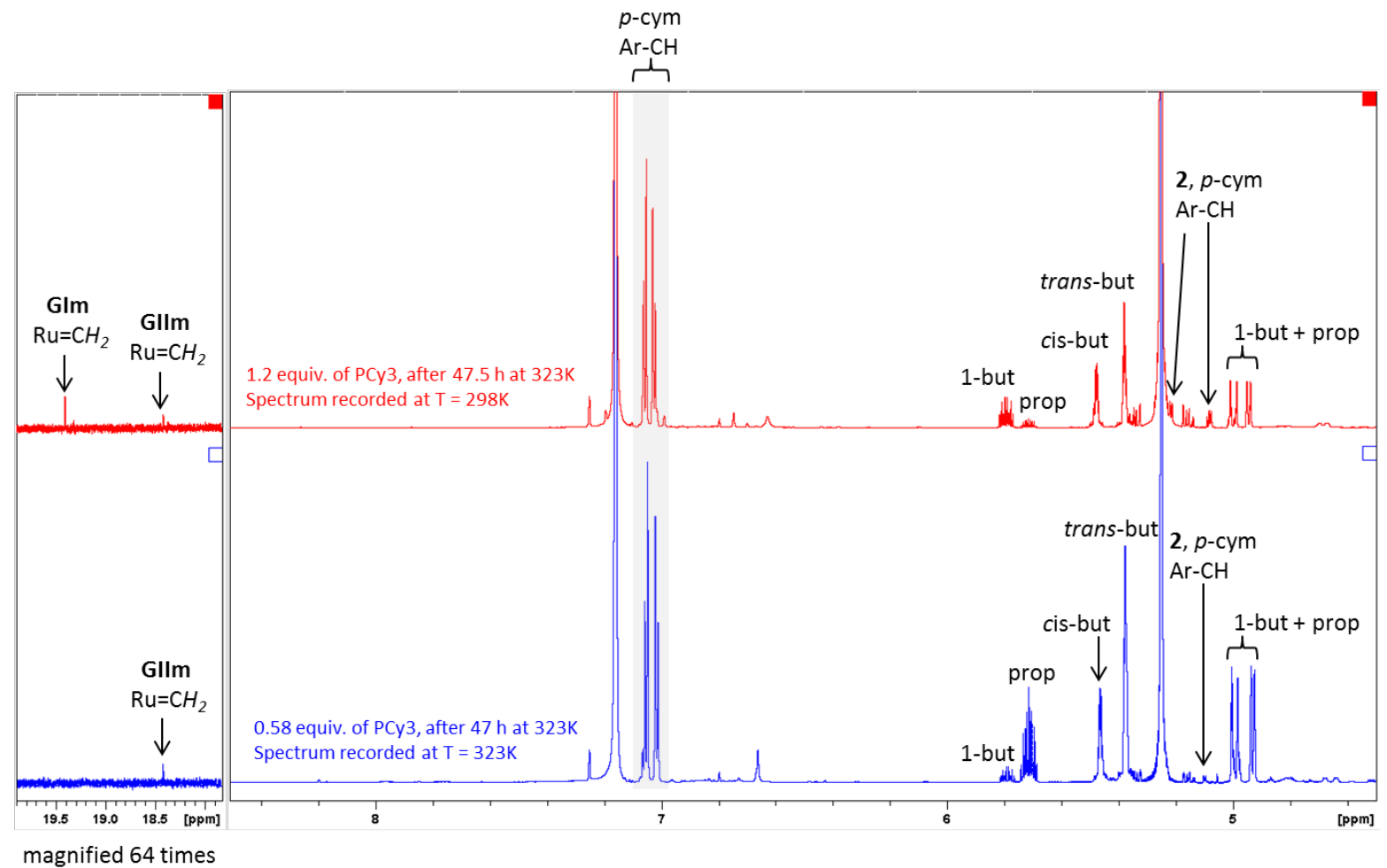

Figure S17. ${ }^{1} \mathrm{H}$ NMR spectra in $\mathrm{C}_{6} \mathrm{D}_{6}(\delta 20-18$ and 8.5-4.5 ppm) of the reaction of 1 with an excess of ethylene at $323 \mathrm{~K}$, in the presence of 0.58 equiv. (blue plot) and 1.2 equiv. (red plot) of $\mathrm{PCy}_{3}$. Abbreviations: $p$-cym $=p$-cymene, Mes $=$ mesityl, 1 -but $=1$ butene, prop $=$ propene, cis-but $=$ cis-2-butene, trans-but $=$ trans-2-butene.

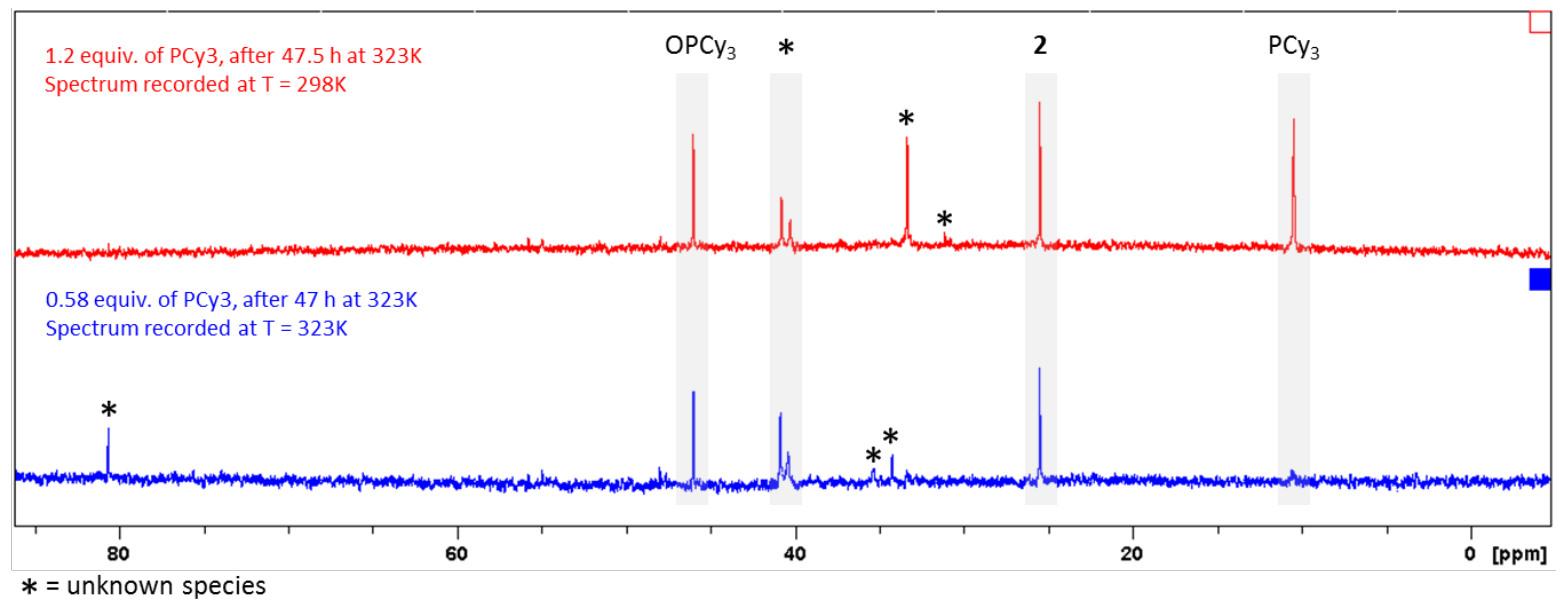

Figure S18. ${ }^{31} \mathrm{P}$ NMR spectra in $\mathrm{C}_{6} \mathrm{D}_{6}(\delta 100-0 \mathrm{ppm})$ of the reaction of 1 with an excess of ethylene at $323 \mathrm{~K}$, in the presence of 0.58 equiv. (blue plot) and 1.2 equiv. (red plot) of $\mathrm{PCy}_{3}$.

\section{The Origin of Ethane}

The results in Table S3 suggest that the reduction of ethylene to ethane, detected in all reactions of ethylene with either $\mathbf{1}$ or $\mathbf{2}$, is stoichiometric rather than catalytic. Indeed, none of the experiments produces more than one equivalent of ethane with respect to ruthenium. Moreover, the hydrogen source does not seem to be ethylene. Indeed, resonances suggestive of oxidized organic products such as acetylene or butadiene were not observed in the NMR spectra. In addition, when the dimer $[(p$ cymene) $\left.\mathrm{RuCl}_{2}\right]_{2}$, the precursor of both $\mathbf{1}$ and $\mathbf{2}$, was heated with ethylene at $323 \mathrm{~K}$, no traces of ethane was observed even after six days. The latter experiment also rules out $p$-cymene as the potential reductant. 
Overall, these experiments suggest that the most probable source of hydrogen for the reduction of ethylene to ethane are the two donor ligands SIMes and $\mathrm{PCy}_{3}$. In other words, ethylene oxidizes these two electron-rich ligands and is, itself, reduced to ethane.

Table S3. Generation of ethane in the various experiments of $\mathbf{1}$ and $\mathbf{2}$ with ethylene.

\begin{tabular}{|l|l|l|l|l|l|l|l|}
\hline Entry & $\mathrm{T}, \mathrm{K}$ & $\begin{array}{l}\mathbf{1}, \\
\mu \mathrm{mol}\end{array}$ & $\begin{array}{l}\mathbf{2}, \\
\mu \mathrm{mol}\end{array}$ & $\begin{array}{l}\mathrm{PC} \mathrm{y}_{3}, \mu \mathrm{mol} \\
\text { (equiv. to Ru) }\end{array}$ & $\begin{array}{l}\mathrm{C}_{2} \mathrm{H}_{4}, \\
\text { equiv. }\end{array}$ & $\begin{array}{l}\text { After } 19 \mathrm{~h}, \\
\mathrm{C}_{2} \mathrm{H}_{6}, \mu \mathrm{mol} \\
\text { (equiv. to Ru) }\end{array}$ & $\begin{array}{l}\text { After } 45 \mathrm{~h}, \\
\mathrm{C}_{2} \mathrm{H}_{6}, \mu \mathrm{mol} \\
\text { (equiv. to Ru) }\end{array}$ \\
\hline 1 & 323 & 1.3 & & $0.71(0.55)$ & 91 & $0.66(0.51)$ & $1.20(0.92)$ \\
\hline 2 & 323 & 4.1 & & $2.37(0.58)$ & 31 & $1.07(0.26)$ & $2.16(0.55)$ \\
\hline 3 & 323 & 12.3 & & $7.27(0.59)$ & 11 & $2.26(0.13)$ & \\
\hline 4 & 323 & 3.6 & & $4.3(1.2)$ & 32 & & $0.92(0.26)$ \\
\hline 5 & 323 & 3.7 & & 0 & 38 & $1.69(0.46)$ & $2.57(0.69)$ \\
\hline 6 & 323 & & 4.1 & $2.29(0.56)$ & 22 & $0.40(0.10)$ & $1.24(0.30)$ \\
\hline 7 & 298 & 3.7 & & 0 & 44 & $0.18(0.05)$ & $0.41(0.11)$ \\
\hline 8 & 298 & 3.6 & & $2.05(0.57)$ & 29 & $0.15(0.04)$ & $0.36(0.10)$ \\
\hline
\end{tabular}

\section{Reaction Order and Molecularity of the Alkylidene Generation.}

To shed light on the reaction mechanism of the alkylidene generation we have repeated the reaction of $\mathbf{1}$ with ethylene at $323 \mathrm{~K}$ in the presence of 0.6 equiv. of $\mathrm{PCy}_{3}$ by using both a three-fold lower and a threefold higher concentration of 1. Figure S19, Figure S20, and Figure S21 display the progress of the concentration of GIIm, determined by ${ }^{1} \mathrm{H}$ NMR, during the first eight $\mathrm{h}$ for the experiments done with an initial concentration of 1 of $1.78 \mathrm{mmol} / \mathrm{L}, 5.02 \mathrm{mmol} / \mathrm{L}$, and $17.35 \mathrm{mmol} / \mathrm{L}$ respectively. The initial concentration of ethylene was $173 \mathrm{mmol} / \mathrm{L}, 96 \mathrm{mmol} / \mathrm{L}$, and $152 \mathrm{mmol} / \mathrm{L}$ respectively. The initial rates of GIIm generation were estimated from the slope of linear trend (red line in Figure S19, Figure S20, and Figure S21) fitting the profile of the curve [GIIm] vs. time (dots in Figure S19, Figure S20, and Figure S21). Since the profiles display an induction period, the fitted curved were allowed a non-zero intercept. Moreover, the fitting considered the experimental points that dysplay a linear trend after the induction time, and yield the highest slope (i.e., post-induction maximum recorded rate). Namely, points between and including 1.9 and $3.4 \mathrm{~h}, 2.5$ and $4 \mathrm{~h}$, and 1.7 and $3.2 \mathrm{~h}$ respectively for the low, mid and high concentration of $\mathbf{1}$. This some what arbitrary procedure is clearly a compromize dictated by the complexity of the reaction under study, the scarcity of experimental data and the unambiguous induction observed for the concentration of GIIm. Nevertheless, we believe the fitted lines reflect faitfully the trend displayed by the experimental values. Sensitivity analysis, in fact, shows that the numerical effect on the estimated reaction rates is negligible with respect to the overall relation between intial rates at different concentration of $\mathbf{1}$.

We found that going from the lowest $(1.94 \mathrm{mmol} / \mathrm{L})$ to the middle $(5.02 \mathrm{mmol} / \mathrm{L})$ concentration in ruthenium the initial rate increases from about 2 to about $4 \mu \mathrm{mol} \cdot \mathrm{L}^{-1} \cdot \mathrm{h}^{-1}$, while going from the middle concentration to the highest $(17.35 \mathrm{mmol} / \mathrm{L})$ the initial rate essentially remained the same (i.e., ca. 4 $\mu \mathrm{mol} \cdot \mathrm{L}^{-1} \cdot \mathrm{h}^{-1}$ ), see Figure S22. These results seem to suggest that order in $\mathbf{1}$ is decreasing with increasing concentration of $\mathbf{1}$. It is also plausible that the lower rate than expected obtained in the experiment with the highest concentration may be caused by the the lack of stirring. However, although the number of data available are insufficient to unambiguously determine the reaction order in $\mathbf{1}$ with high accuracy, these results are consistent with a reaction order lower or equal to one and are certainly not compatible with a second-order in $\mathbf{1}$. In contrast, a reaction order higher than one was suggested by a different experiment done with 1 in neat allylbenzene, where the ratio olefin isomerization/olefin metathesis was evaluated at increasing concentration of $\mathbf{1}$ and the role of this approximation could not be evaluated. ${ }^{1}$ 


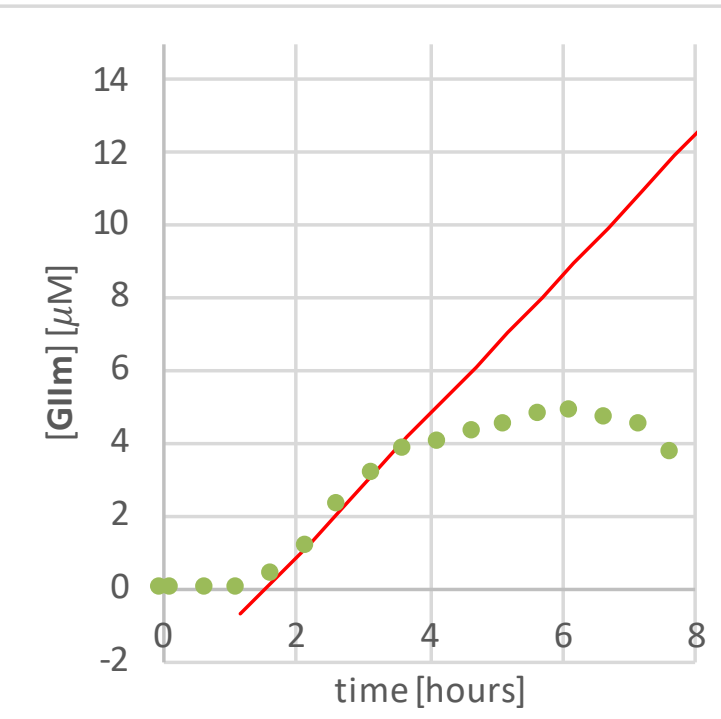

Figure S19. Concentration of GIIm vs time for $[\mathbf{1}]=1.78 \mathrm{mmol} / \mathrm{L}$. Initial rate of $\mathbf{G I I m}$ formation $=1.94 \mu \mathrm{mol} \cdot \mathrm{L}^{-1} \cdot \mathrm{h}^{-1}$, determinated as the slope of the best fit line (red line) of the first five measurements with [GIIm] $>0$.

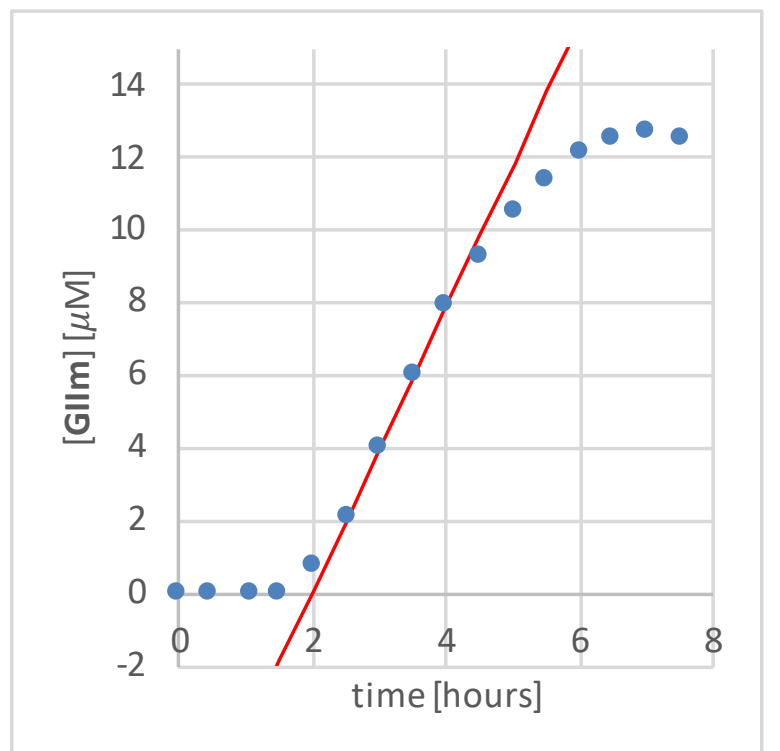

Figure S20. Concentration of GIIm vs time for $[\mathbf{1}]=5.02 \mathrm{mmol} / \mathrm{L}$. Initial rate of GIIm formation $=3.92 \mu \mathrm{mol} \cdot \mathrm{L}^{-1} \cdot \mathrm{h}^{-1}$, determinated as the slope of the best fit line (red line) of the first five measurements with [GIIm] $>0$. 


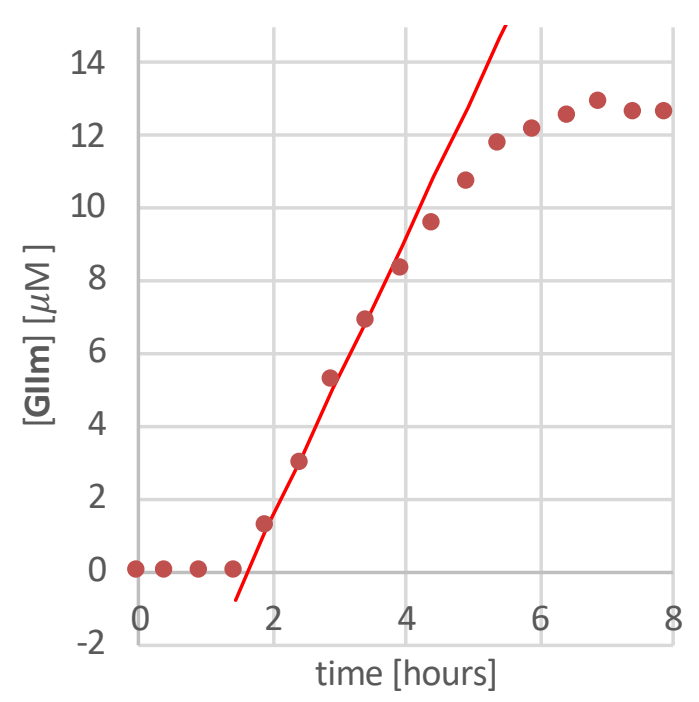

Figure S21. Concentration of GIIm vs time for $[\mathbf{1}]=17.35 \mathrm{mmol} / \mathrm{L}$. Initial rate of $\mathbf{G I I m}$ formation $=3.86 \mu \mathrm{mol} \cdot \mathrm{L}^{-1} \cdot \mathrm{h}^{-1}$, determinated as the slope of the best fit line (red line) of the first five measurements with [GIIm] $>0$.

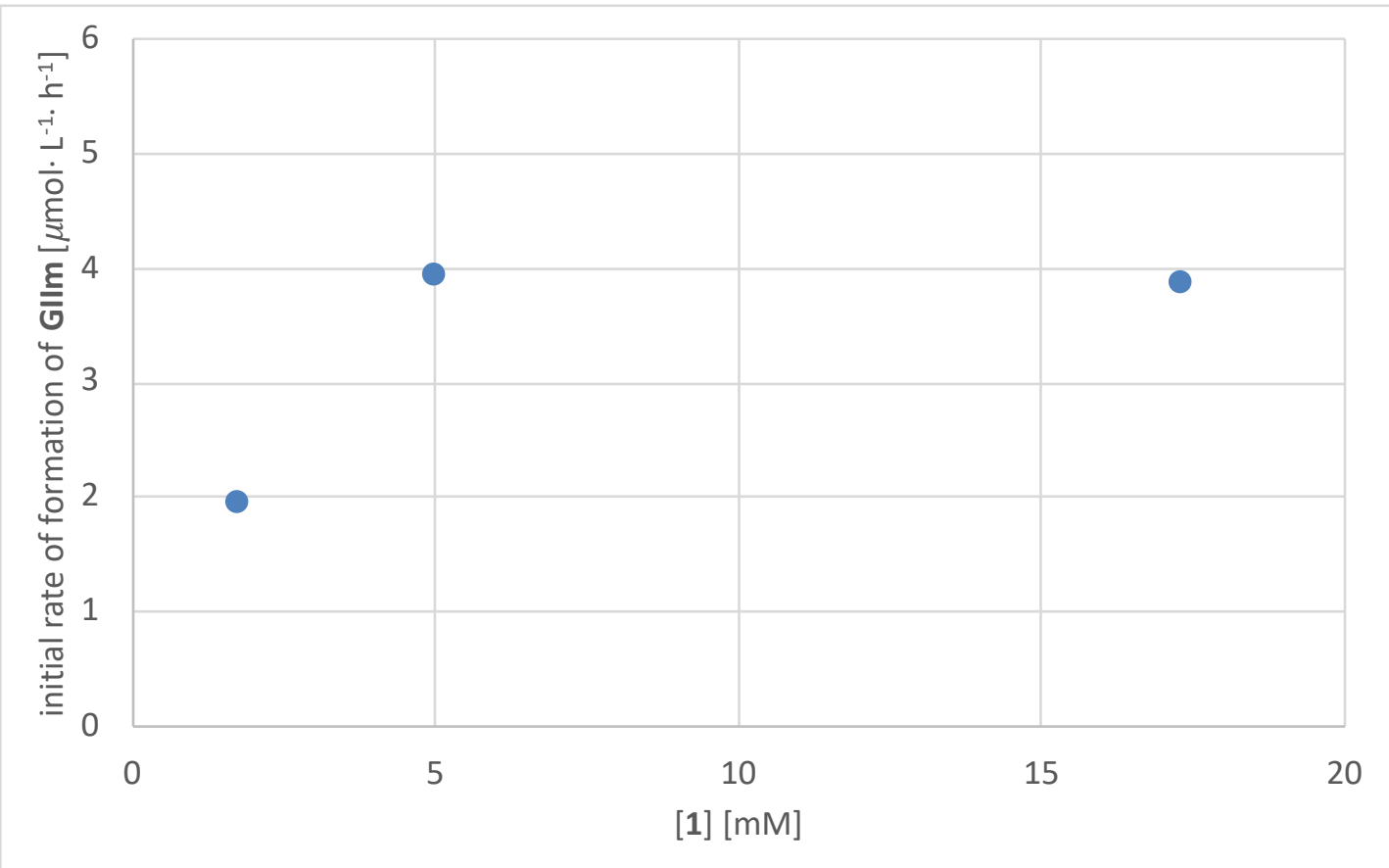

Figure S22. Initial rate of GIIm formation $\left(\mu \mathrm{mol} \cdot \mathrm{L}^{-1} \cdot \mathrm{h}^{-1}\right)$ vs. concentration of $\mathbf{1}\left(\mathrm{mmol} \cdot \mathrm{L}^{-1}\right)$ at $t=0 \mathrm{~h}$. 


\section{Additional NMR Spectra}

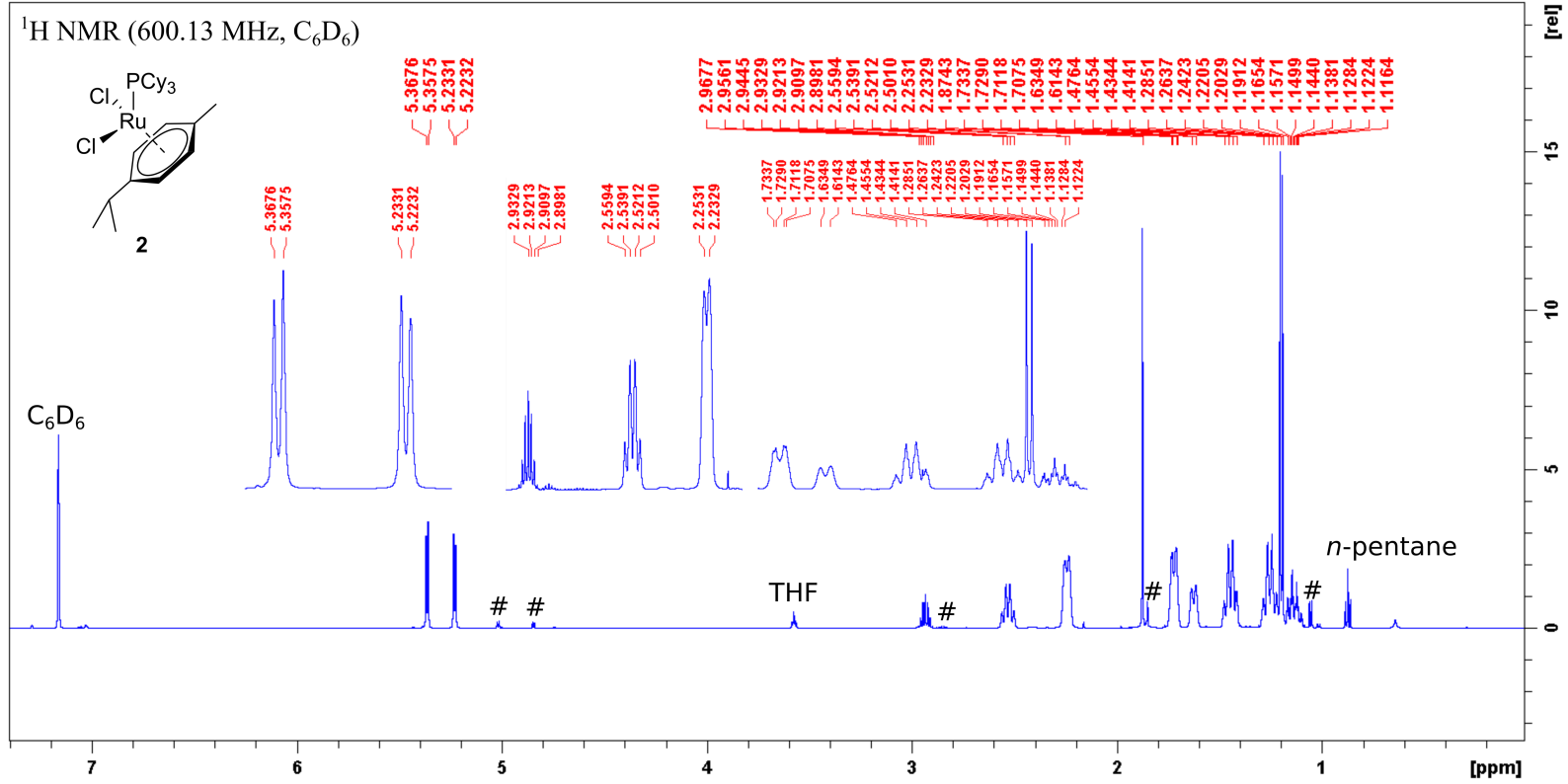

Figure S23. ${ }^{1} \mathrm{H}$ NMR spectrum of $\left(\mathrm{PCy}_{3}\right)\left(\eta^{6}-\mathrm{p}\right.$-cymene $) \mathrm{RuCl}_{2}(2)$ in $\mathrm{C}_{6} \mathrm{D}_{6}$, THF $=$ tetrahydrofuran and impurities coming from starting compound $\left[\mathrm{RuCl}_{2}(p \text {-cymene })\right]_{2}$ are indicated with \#.

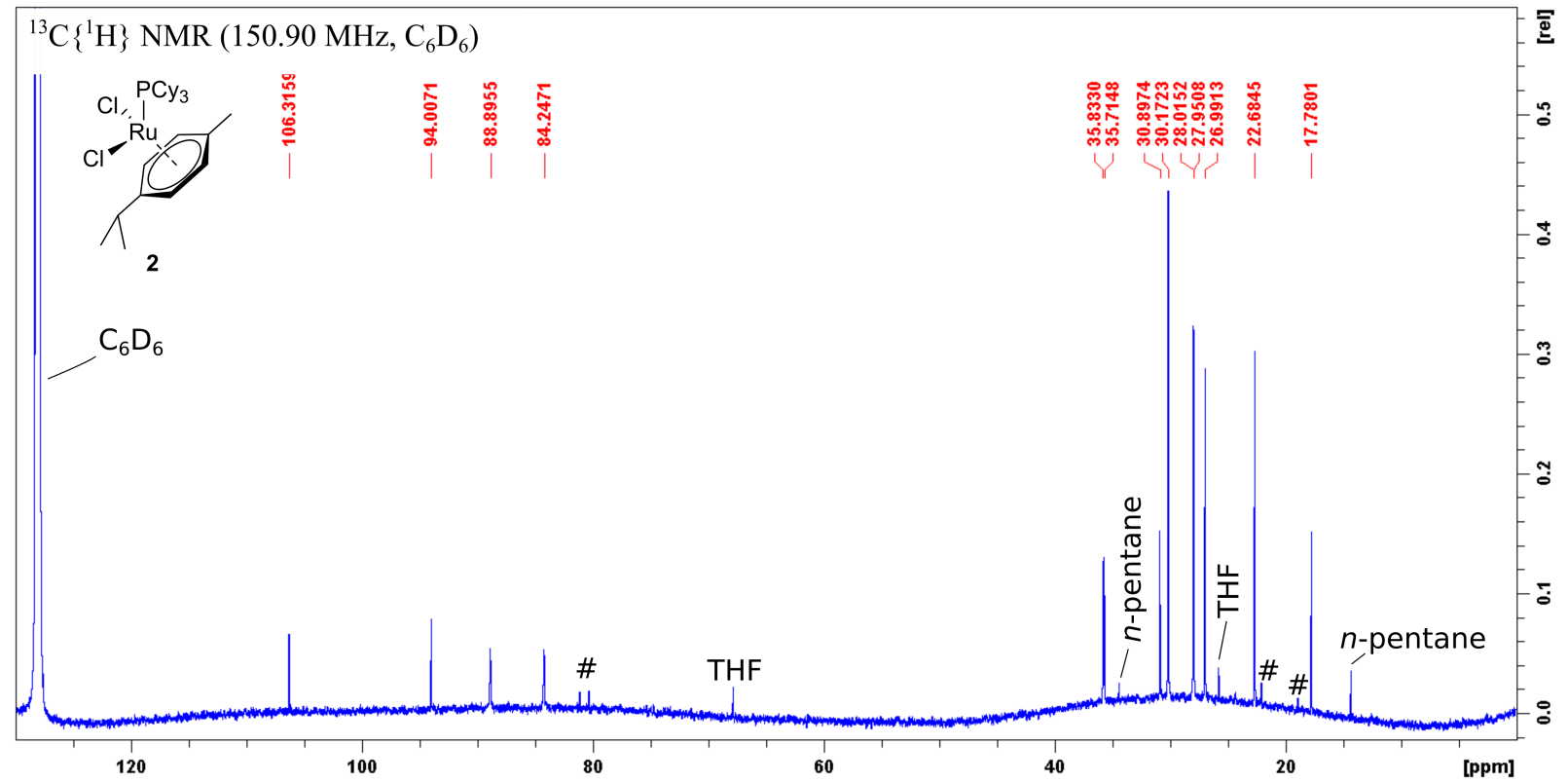

Figure S24. ${ }^{13} \mathrm{C}\left\{{ }^{1} \mathrm{H}\right\}$ NMR spectrum of $\left(\mathrm{PCy}_{3}\right)\left(\eta^{6}\right.$ - $p$-cymene $) \mathrm{RuCl}_{2}(2)$ in $\mathrm{C}_{6} \mathrm{D}_{6}$, , THF $=$ tetrahydrofuran and impurities coming from starting compound $\left[\mathrm{RuCl}_{2}(p \text {-cymene })\right]_{2}$ are indicated with \#. 


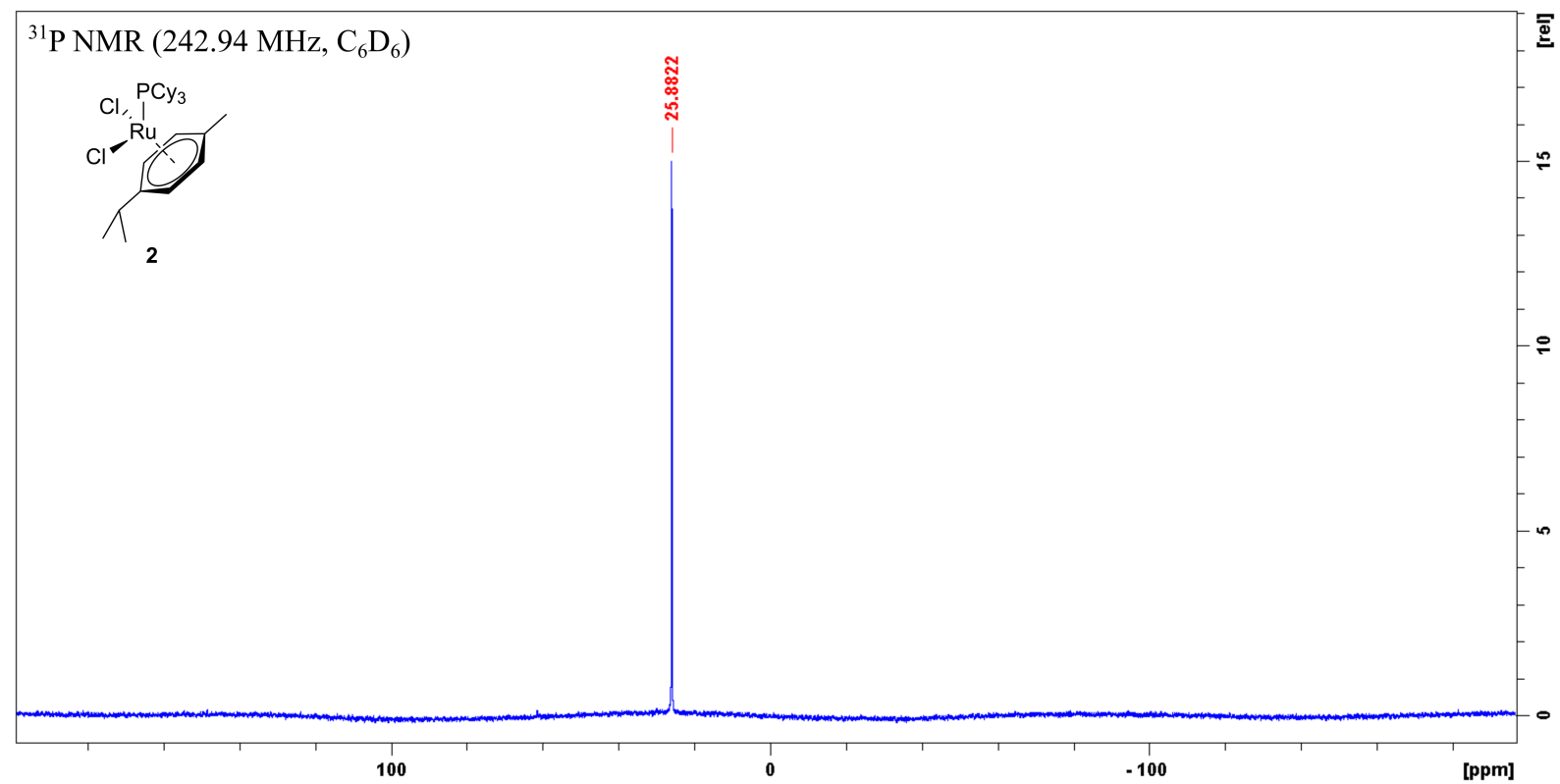

Figure S25. ${ }^{31} \mathrm{P} \mathrm{NMR}$ spectrum of $\left(\mathrm{PCy}_{3}\right)\left(\eta^{6}-p\right.$-cymene $) \mathrm{RuCl}_{2}(2)$ in $\mathrm{C}_{6} \mathrm{D}_{6}$.

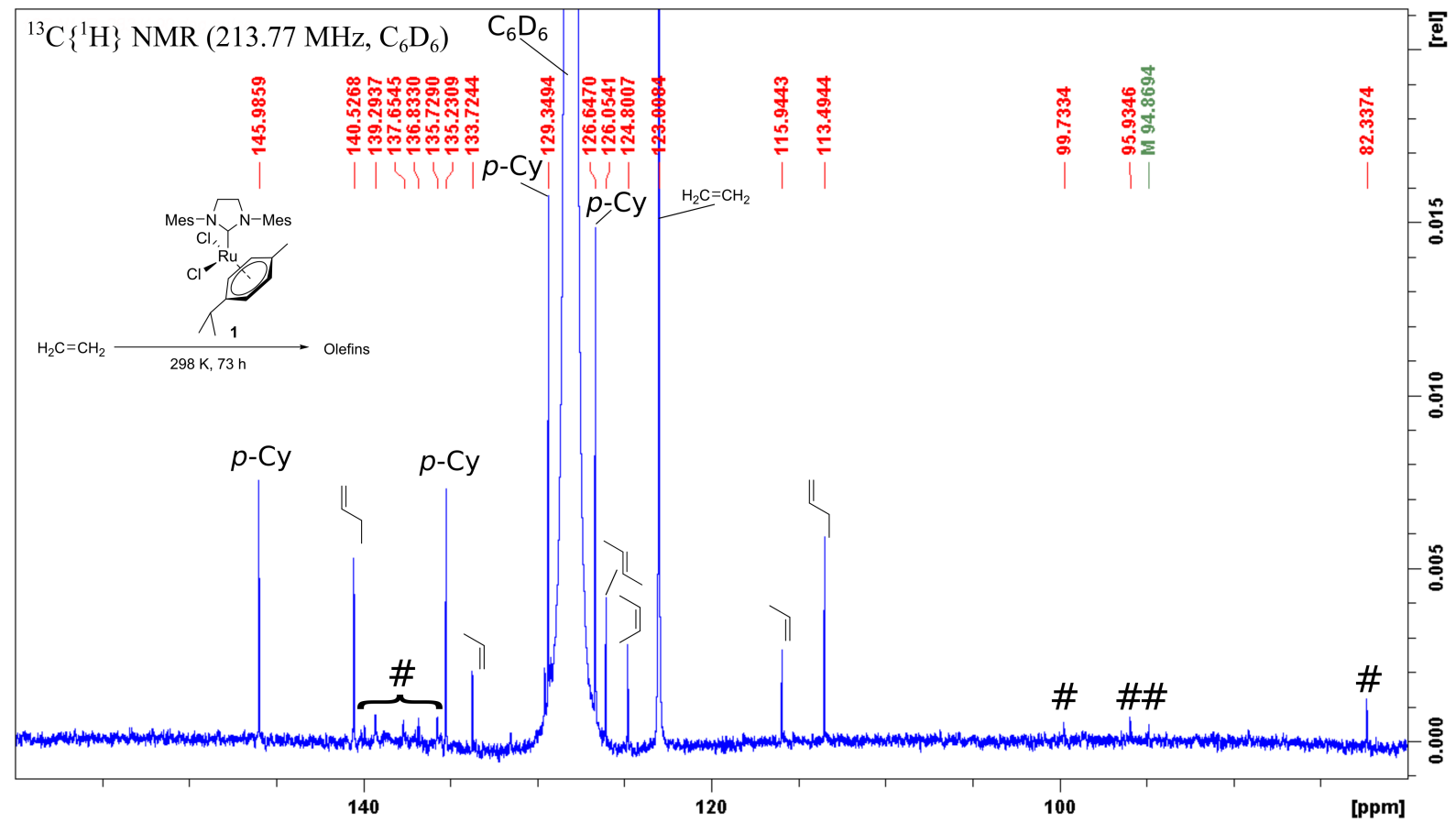

Figure S26. ${ }^{13} \mathrm{C}\left\{{ }^{1} \mathrm{H}\right\}$ NMR spectrum $(\delta 160-80 \mathrm{ppm})$ of $\mathbf{1}$ and ethylene $(92 \mu \mathrm{mol})$ after $73 \mathrm{~h}$ at $298.0 \mathrm{~K}$ in $\mathrm{C}_{6} \mathrm{D}_{6}$. With \# indicating resonances belonging to unknown decomposition products and $p$-Cy indicates resonances belong to free $p$-cymene. 


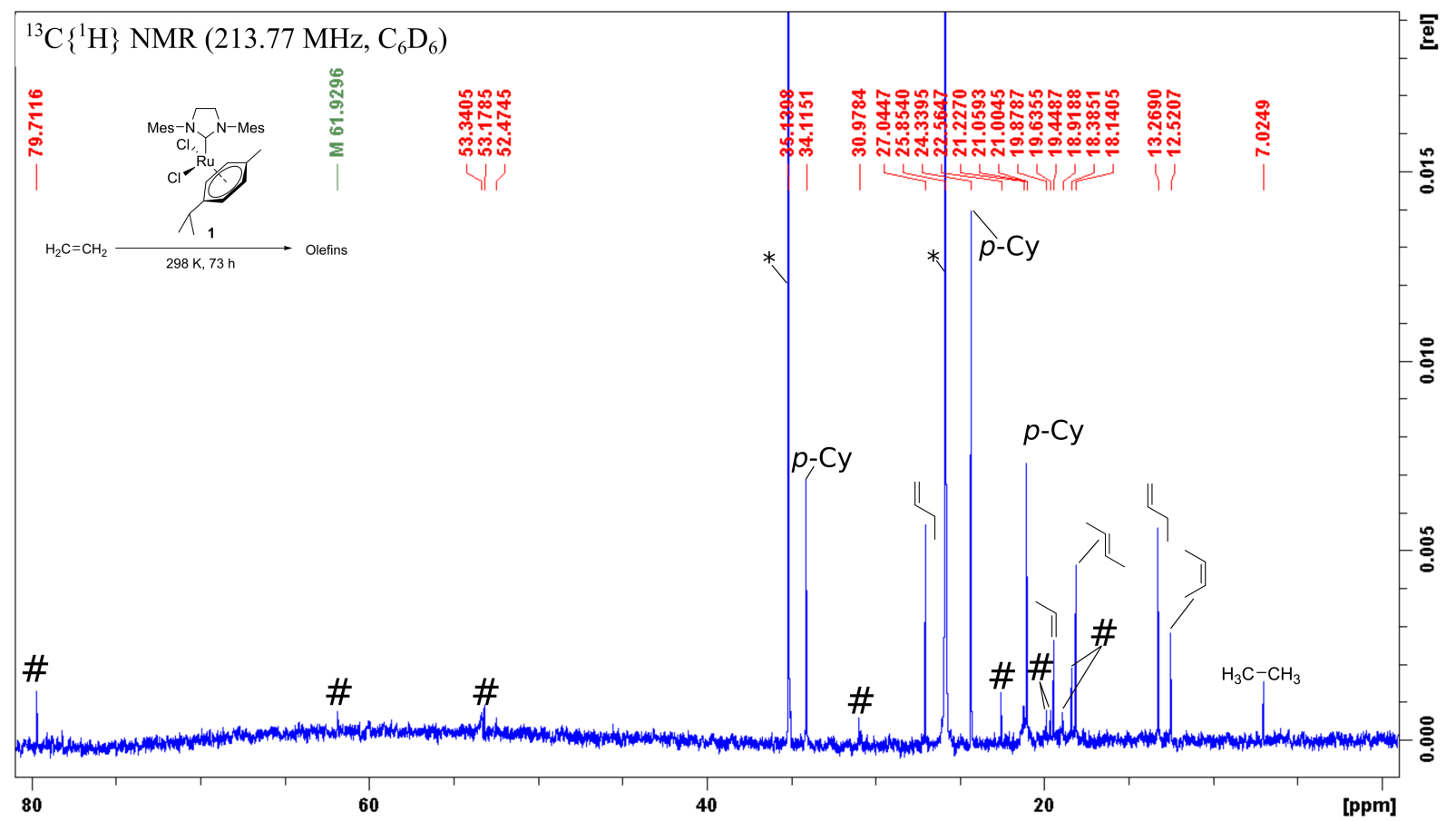

Figure S27. ${ }^{13} \mathrm{C}\left\{{ }^{1} \mathrm{H}\right\}$ NMR spectrum $(\delta 80-0 \mathrm{ppm})$ of 1 and ethylene $(92 \mu \mathrm{mol})$ after $73 \mathrm{~h}$ at $298.0 \mathrm{~K}$ in $\mathrm{C}_{6} \mathrm{D}_{6}$. With \# indicating resonances belonging to unknown decomposition products, $p$-Cy indicates resonances belong to free $p$-cymene and $*$ denotes resonances belonging to the internal standard (2,2,3,3-tetramethylbutane).

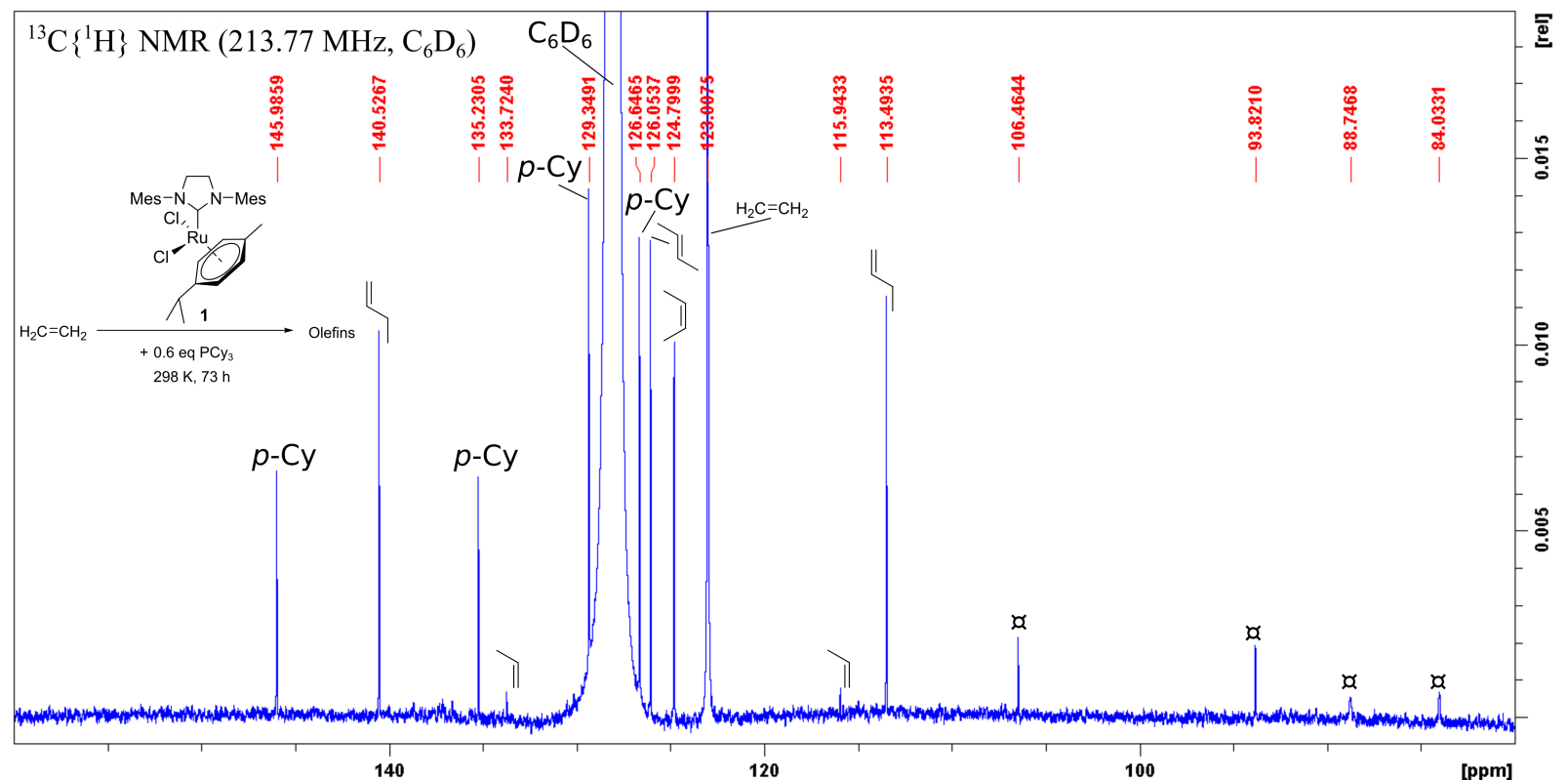

Figure S28. ${ }^{13} \mathrm{C}\left\{{ }^{1} \mathrm{H}\right\}$ NMR spectrum $(\delta 160-80 \mathrm{ppm})$ of $\mathbf{1}, 0.57$ equiv. $\mathrm{PCy}_{3}$ and ethylene $(116 \mu \mathrm{mol})$ after $73 \mathrm{~h}$ at $298.0 \mathrm{~K}$ in $\mathrm{C}_{6} \mathrm{D}_{6}$. With $\propto$ indicating resonances identified as belonging to $\left(\mathrm{PCy}_{3}\right)\left(\eta^{6}-p\right.$-cymene $) \mathrm{RuCl}_{2}$ and $p$-Cy indicates resonances belong to free $p$-cymene. 


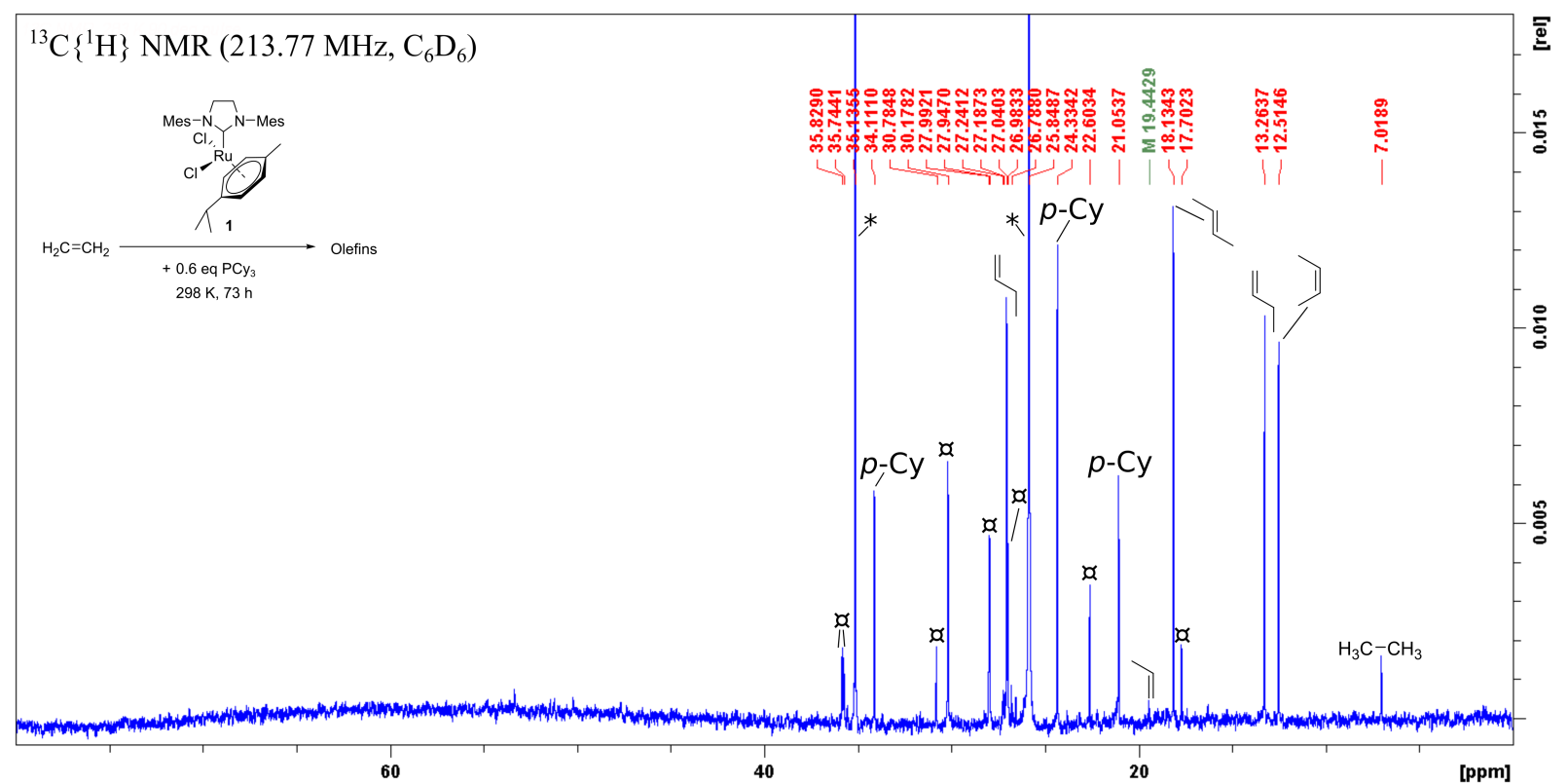

Figure S29. ${ }^{13} \mathrm{C}\left\{{ }^{1} \mathrm{H}\right\}$ NMR spectrum $(\delta 80-0 \mathrm{ppm})$ of $1,0.57$ equiv. PCy 3 and ethylene $(116 \mu \mathrm{mol})$ after $73 \mathrm{~h}$ at $298.0 \mathrm{~K}$ in $\mathrm{C}_{6} \mathrm{D}_{6}$. With $\mathrm{x}$ indicating resonances identified as belonging to $\left(\mathrm{PCy}_{3}\right)\left(\eta^{6}-p\right.$-cymene $) \mathrm{RuCl}_{2}, p$-Cy indicates resonances belong to free $p$ cymene and $*$ denoting resonances belonging to the internal standard (2,2,3,3-tetramethylbutane).

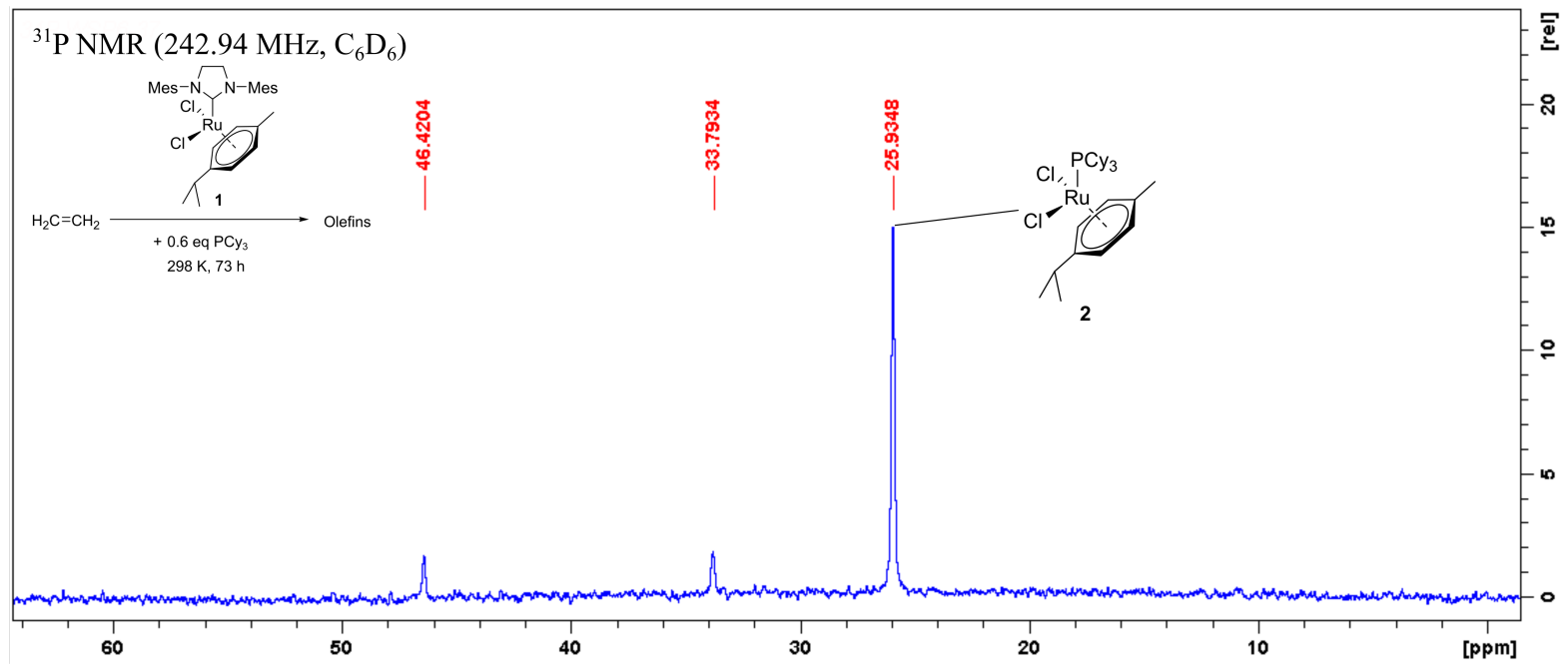

Figure S30. ${ }^{31} \mathrm{P}$ NMR spectrum of $1,0.57$ equiv. $\mathrm{PCy}_{3}$ and ethylene $(116 \mu \mathrm{mol})$ after $73 \mathrm{~h}$ at $298.0 \mathrm{~K}^{\text {in }} \mathrm{C}_{6} \mathrm{D}_{6}$. 


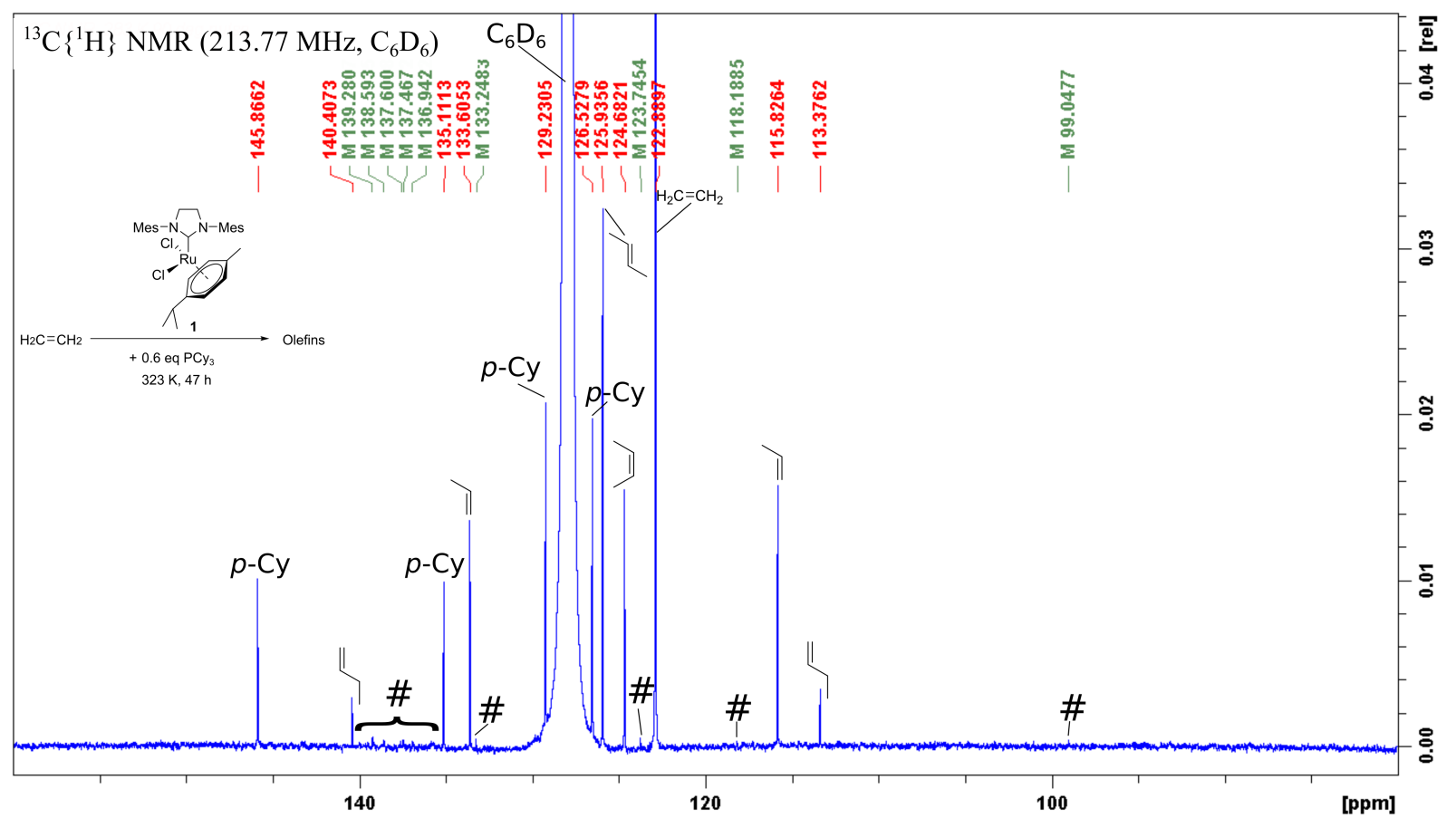

Figure S31. ${ }^{13} \mathrm{C}\left\{{ }^{1} \mathrm{H}\right\}$ NMR spectrum $(\delta 160-80 \mathrm{ppm})$ of $1,0.58$ equiv. $\mathrm{PCy}$ and ethylene $(79 \mu \mathrm{mol})$ after $47 \mathrm{~h}$ at $323.0 \mathrm{~K}$ in $\mathrm{C}_{6} \mathrm{D}_{6}$. With \# indicating resonances belonging to unknown decomposition products and $p$-Cy indicates resonances belong to free $p$ cymene.

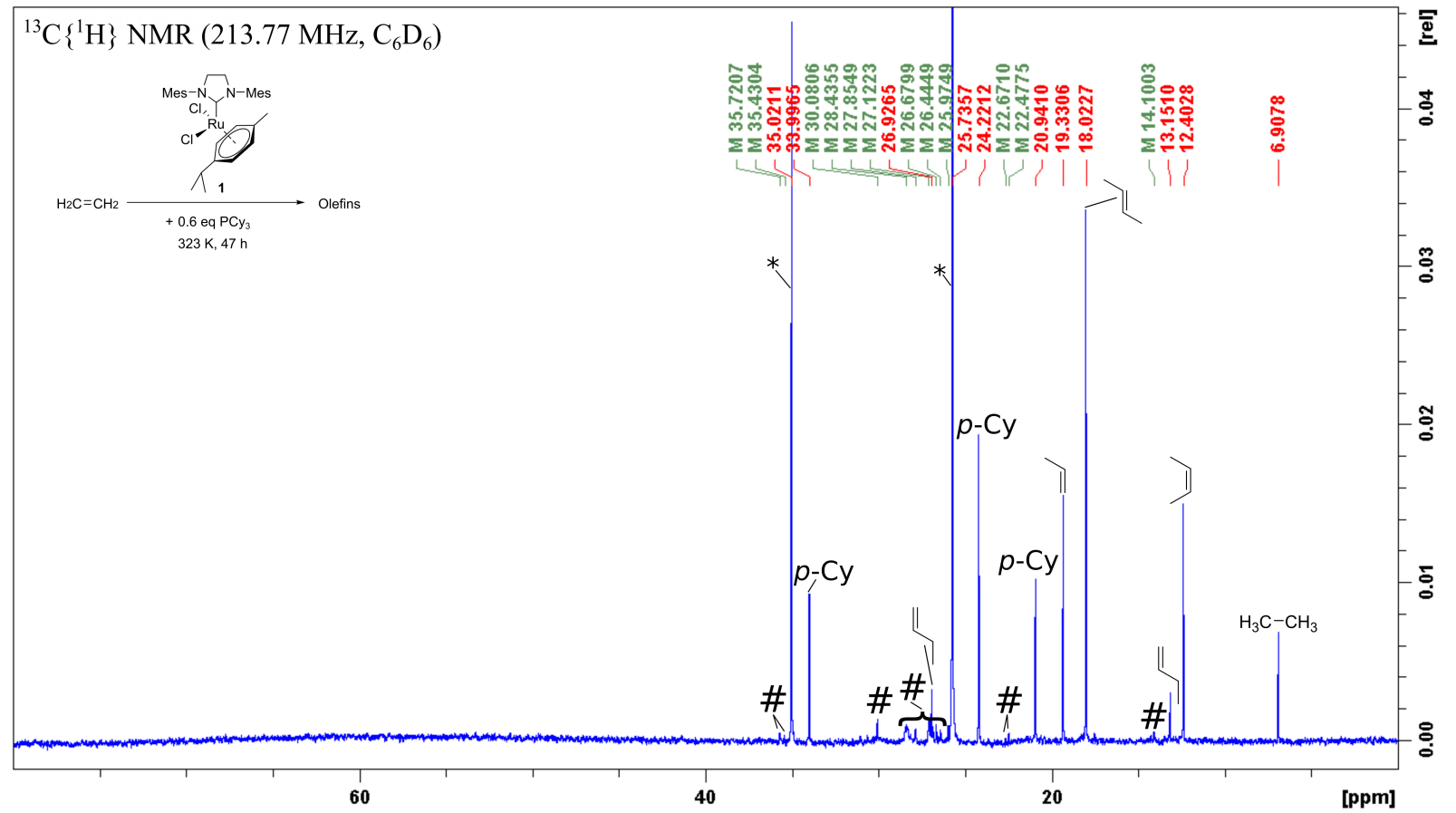

Figure S32. ${ }^{13} \mathrm{C}\left\{{ }^{1} \mathrm{H}\right\}$ NMR spectrum $(\delta 80-0 \mathrm{ppm})$ of 1, 0.58 equiv. $\mathrm{PCy}_{3}$ and ethylene $(79 \mu \mathrm{mol})$ after $47 \mathrm{~h}$ at $323.0 \mathrm{~K}$ in $\mathrm{C}_{6} \mathrm{D}_{6}$. With \# indicating resonances belonging to unknown decomposition products, $p$-Cy indicates resonances belong to free $p$-cymene and $*$ denotes resonances belonging to the internal standard (2,2,3,3-tetramethylbutane). 


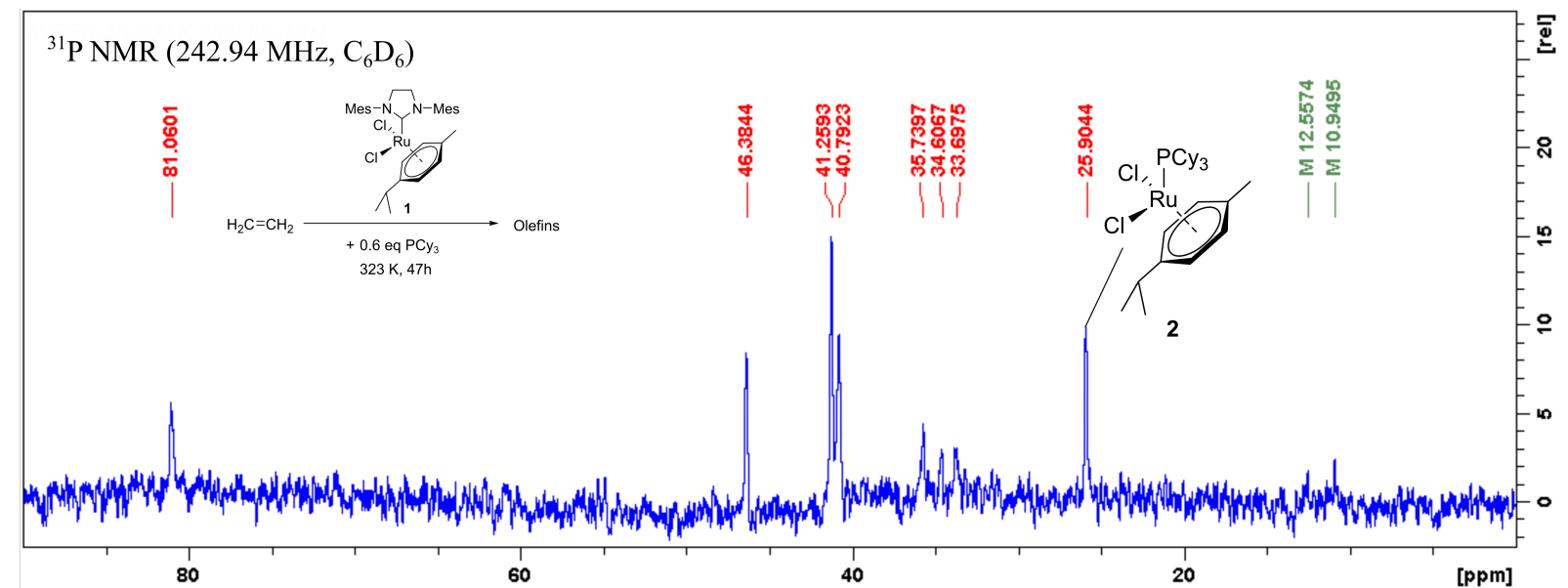

Figure S33. ${ }^{31} \mathrm{P}$ NMR spectrum of $\mathbf{1}, 0.58$ equiv. $\mathrm{PCy}_{3}$ and ethylene $(79 \mu \mathrm{mol})$ after $47 \mathrm{~h}$ at $323.0 \mathrm{~K}$ in $\mathrm{C}_{6} \mathrm{D}_{6}$.
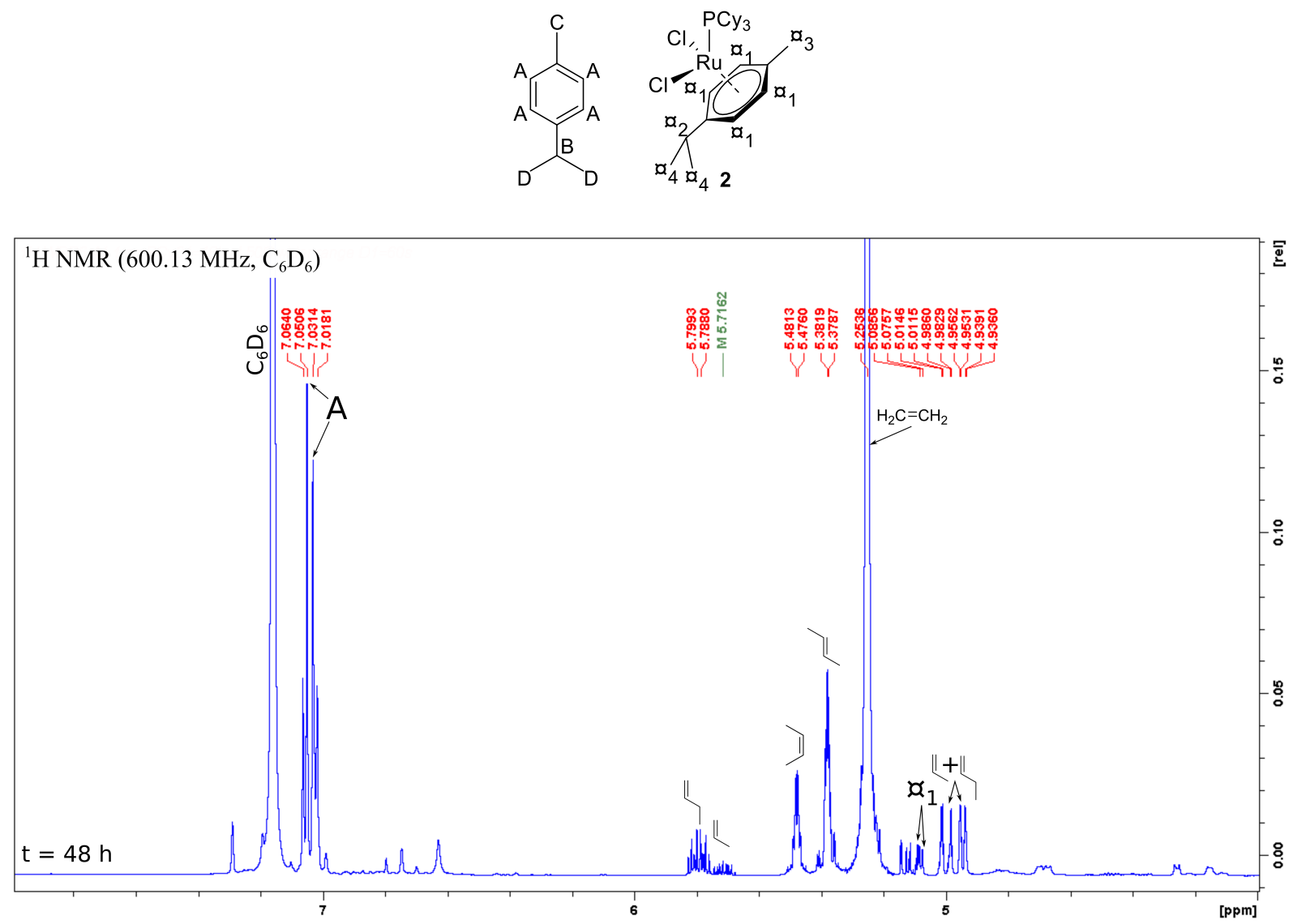

Figure S34. ${ }^{1} \mathrm{H}$ NMR spectrum $(\delta 8-4 \mathrm{ppm})$ of 1, 1.2 equiv. $\mathrm{PCy}_{3}$ and ethylene $(84 \mu \mathrm{mol})$ after $48 \mathrm{~h}$ at $323.0 \mathrm{~K}$ in $\mathrm{C}_{6} \mathrm{D}_{6}$. Capital letters indicate resonances associated with free $p$-cymene and resonances associated with $\left(\mathrm{PCy}_{3}\right)\left(\eta^{6}-p\right.$-cymene $) \mathrm{RuCl}_{2}$ are indicated with $\propto$. Other, unidentified resonances most likely correspond to unknown decomposition products. 


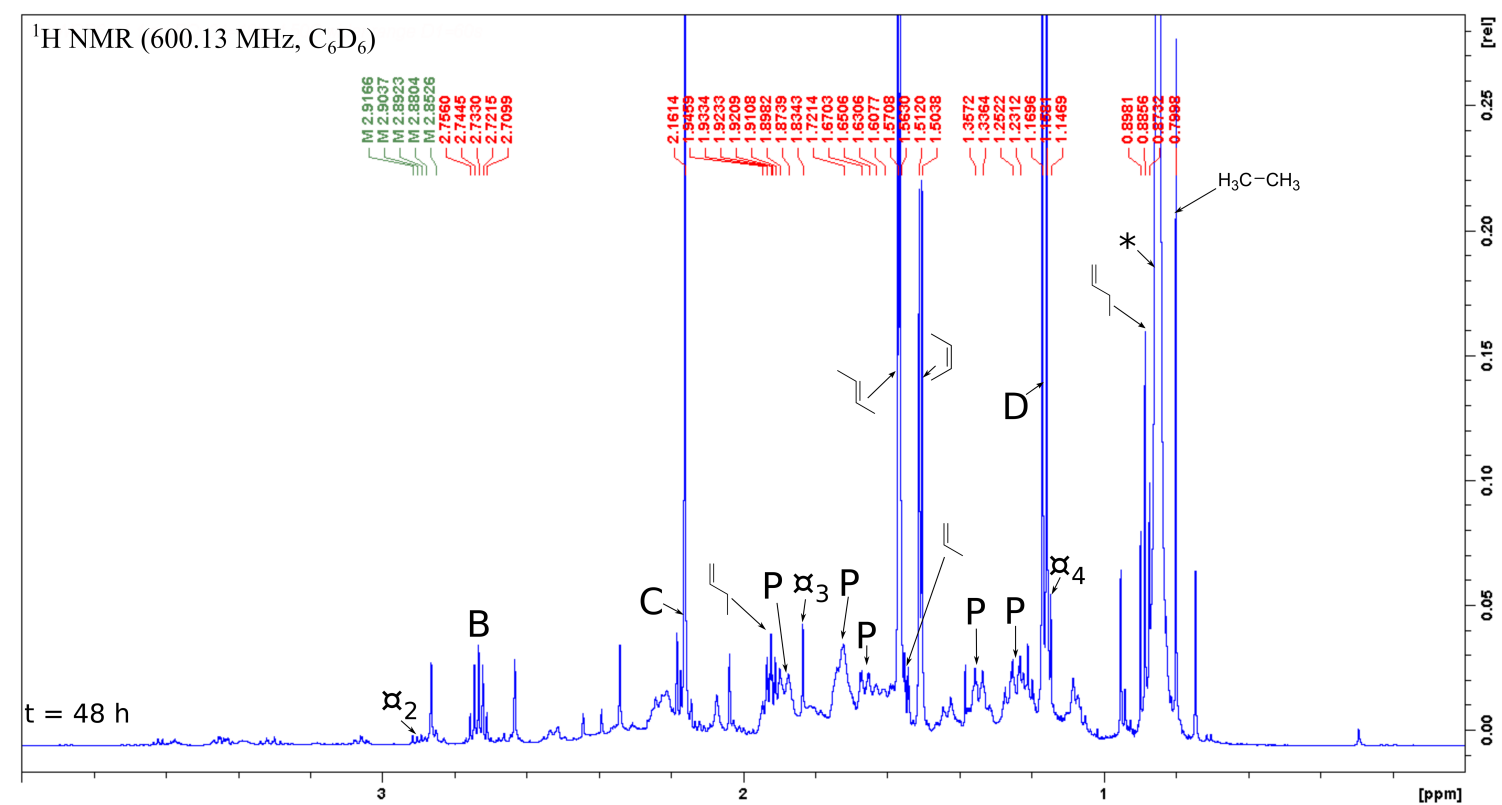

Figure S35. ${ }^{1} \mathrm{H}$ NMR spectrum $(\delta 4-0 \mathrm{ppm})$ of 1, 1.2 equiv. $\mathrm{PCy}_{3}$ and ethylene $(84 \mu \mathrm{mol})$ after $48 \mathrm{~h}$ at $323.0 \mathrm{~K}$ in $\mathrm{C}_{6} \mathrm{D}_{6}$. Capital letters indicate resonances associated with free $p$-cymene, $\mathrm{P}$ refers to $\mathrm{PCy}_{3}$, resonances associated with $\left(\mathrm{PCy}_{3}\right)\left(\eta^{6}-p\right.$ cymene $) \mathrm{RuCl}_{2}$ are indicated with $\mathrm{a}$ and $*$ denotes resonances belonging to the internal standard (2,2,3,3-tetramethylbutane). Other, unidentified resonances most likely correspond to unknown decomposition products.

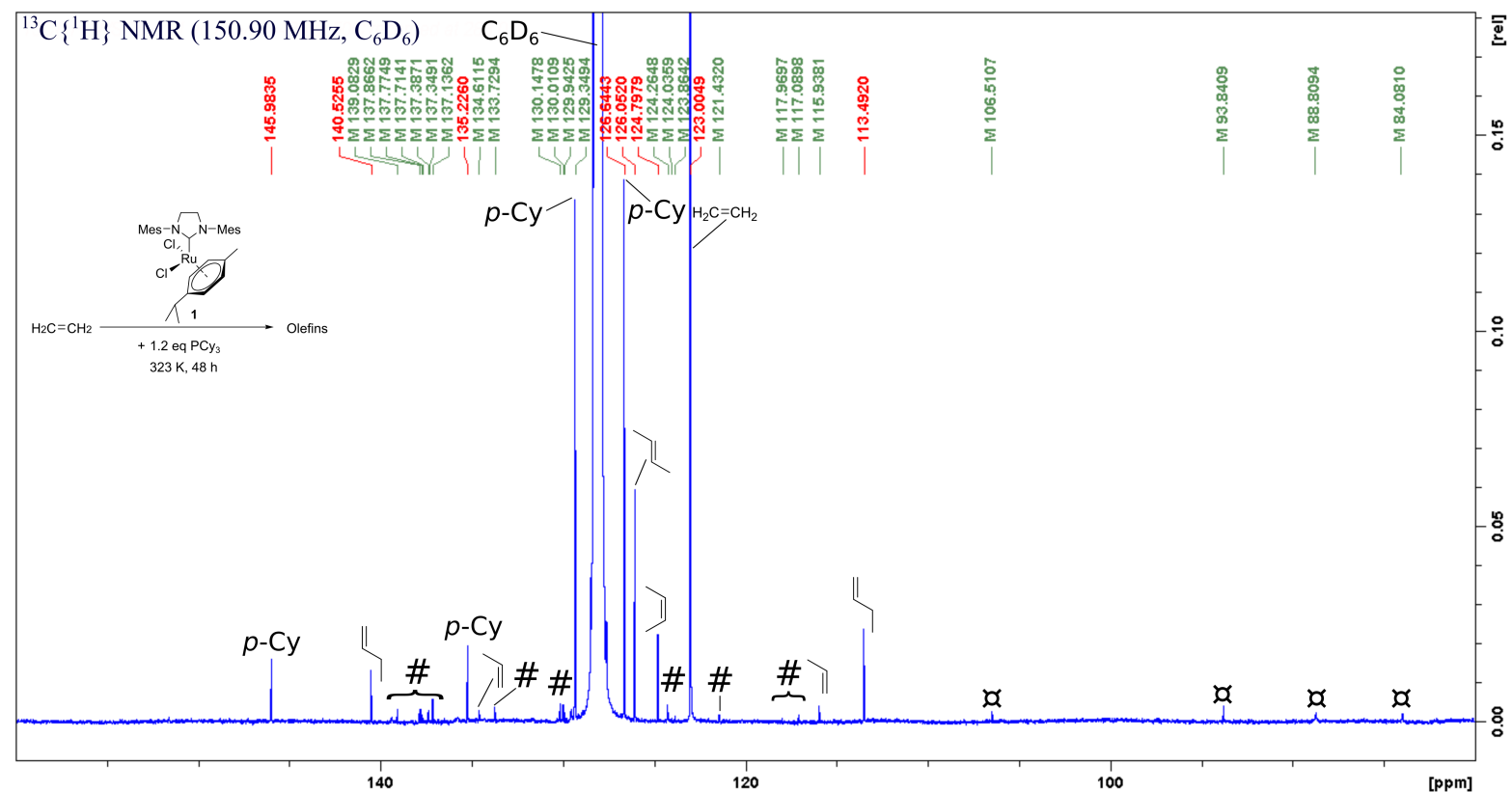

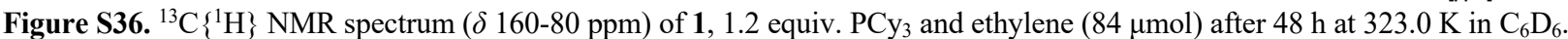
With $\propto$ indicating resonances identified as belonging to $\left(\mathrm{PCy}_{3}\right)\left(\eta^{6}-p\right.$-cymene $) \mathrm{RuCl}_{2}, p$-Cy indicates resonances belong to free $p$ cymene and \# denotes resonances belonging to unknown decomposition products. 


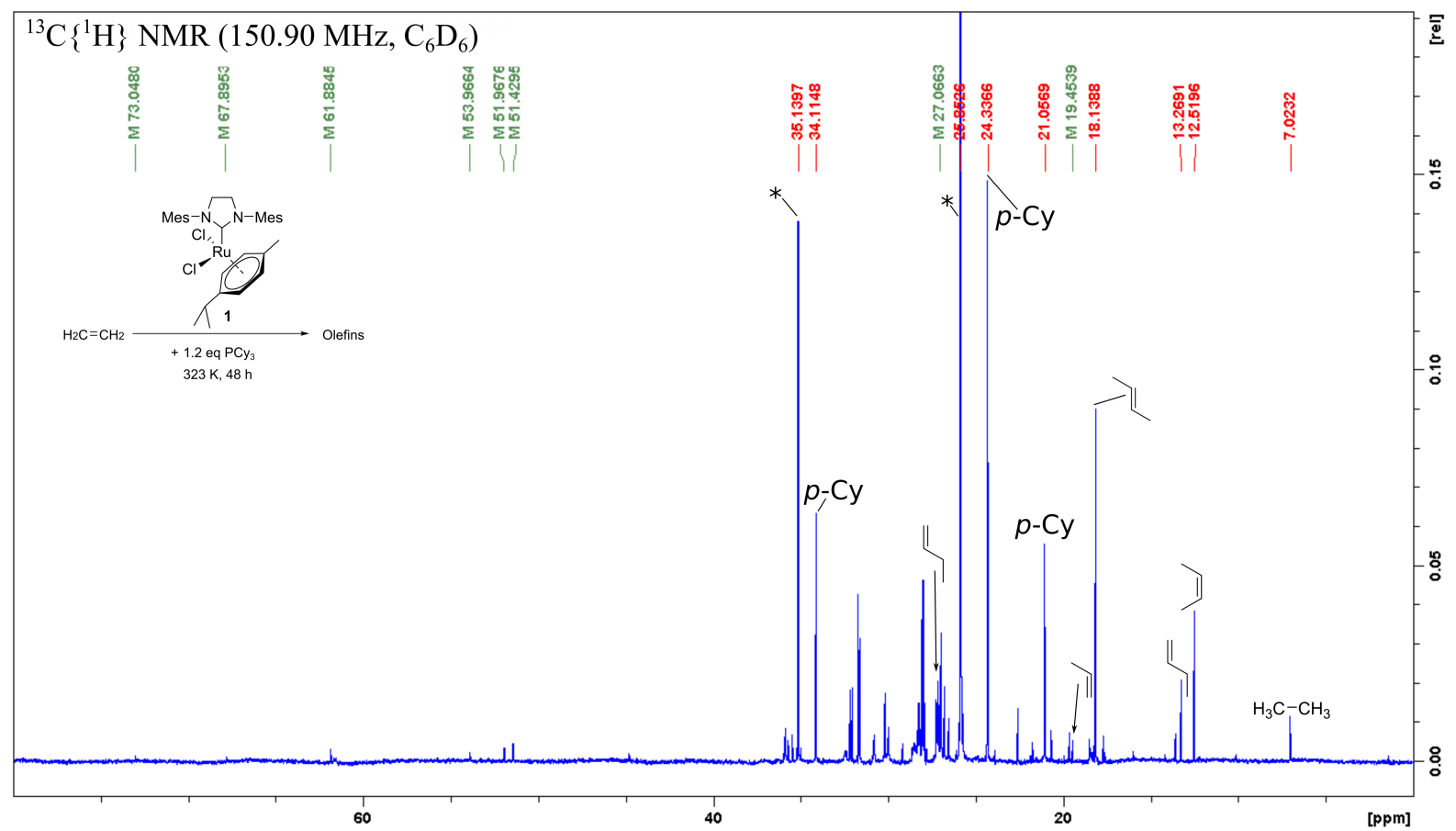

Figure S37. ${ }^{13} \mathrm{C}\left\{{ }^{1} \mathrm{H}\right\}$ NMR spectrum $(\delta 80-0 \mathrm{ppm})$ of $1,1.2$ equiv. $\mathrm{PCy}_{3}$ and ethylene $(84 \mu \mathrm{mol})$ after $48 \mathrm{~h}$ at $323.0 \mathrm{~K}$ in $\mathrm{C}_{6} \mathrm{D}_{6}$. With $p$-Cy indicating resonances belong to free $p$-cymene and * denotes resonances belonging to the internal standard $(2,2,3,3-$ tetramethylbutane). A detailed view of the region between $\delta 37-13.5 \mathrm{ppm}$ can be found in Figure S38. Non-indicated resonances, found in the current figure outside the aforementioned region, are presumed to belong to unknown decomposition products.

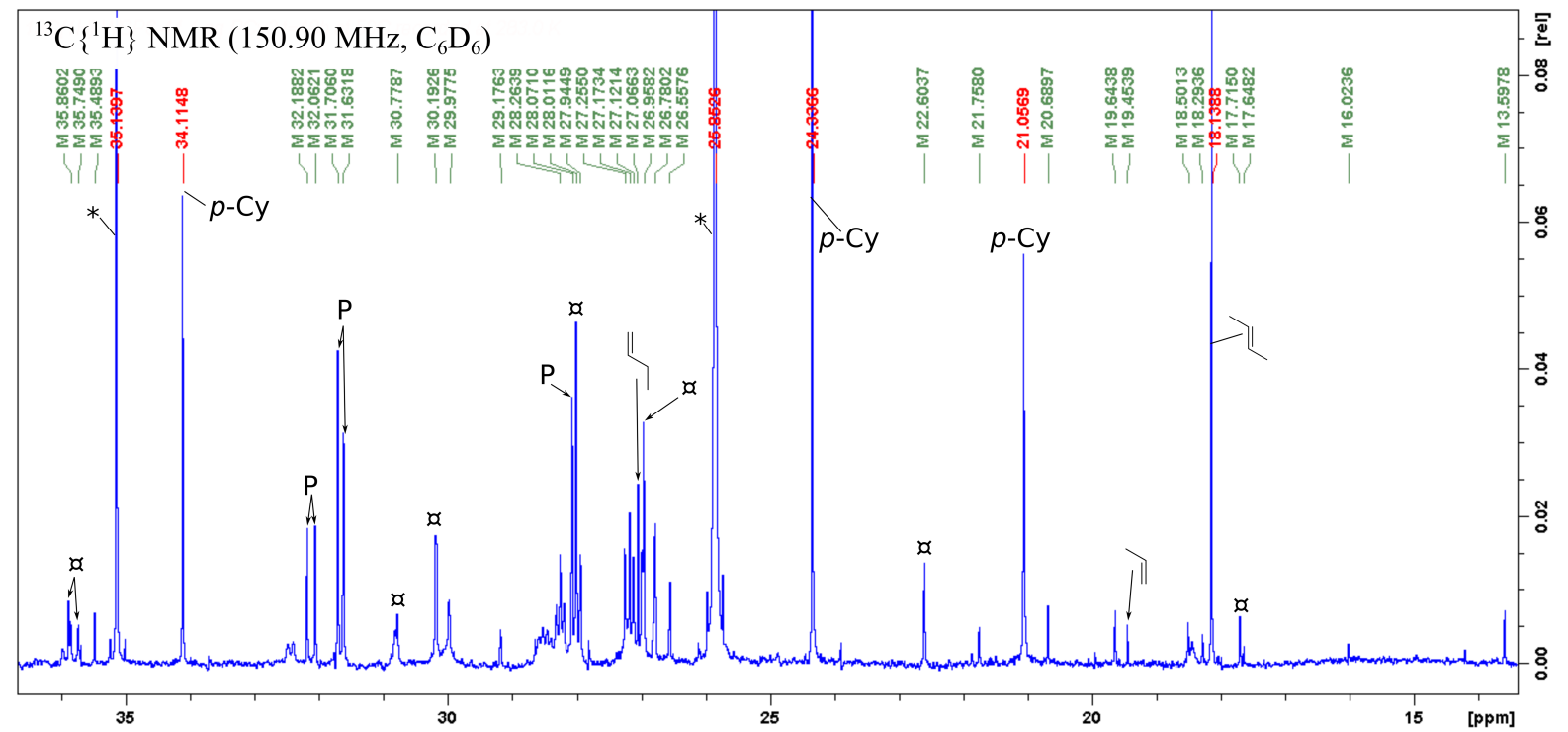

Figure S38. ${ }^{13} \mathrm{C}\left\{{ }^{1} \mathrm{H}\right\}$ NMR spectrum (details $\left.\delta 37-13.5 \mathrm{ppm}\right)$ of $1,1.2$ equiv. $\mathrm{PCy}$ and ethylene $(84 \mu \mathrm{mol})$ after $48 \mathrm{~h}$ at $323.0 \mathrm{~K}$ in $\mathrm{C}_{6} \mathrm{D}_{6}$. With $\alpha$ indicating resonances identified as belonging to $\left(\mathrm{PCy}_{3}\right)\left(\eta^{6}-p\right.$-cymene $) \mathrm{RuCl}_{2}, p-\mathrm{Cy}$ indicates resonances belong to free $p$-cymene, $*$ denotes resonances belonging to the internal standard $(2,2,3,3$-tetramethylbutane $)$ and resonances belonging to free $\mathrm{PCy}_{3}$ are indicated with $\mathrm{P}$. Non-indicated resonances are presumed to belong to unknown decomposition products. 


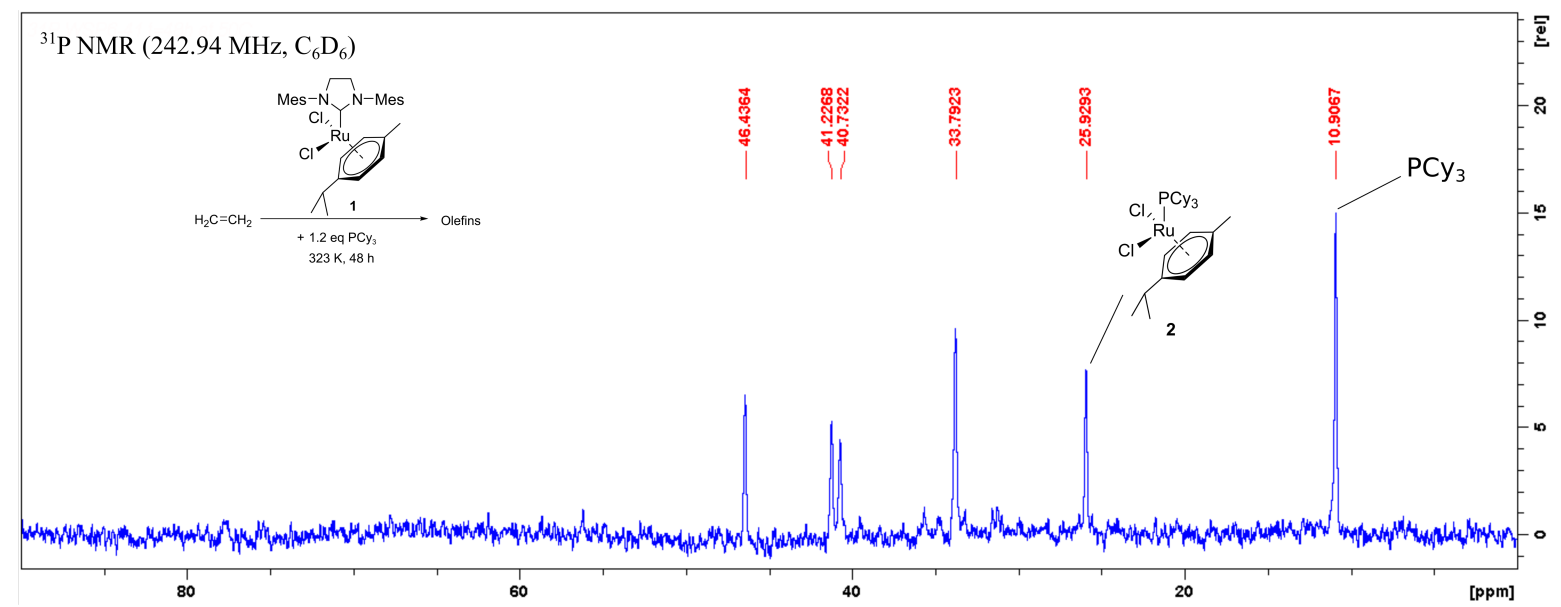

Figure S39. ${ }^{31} \mathrm{P}$ NMR spectrum of $1,1.2$ equiv. $\mathrm{PCy}_{3}$ and ethylene $(84 \mu \mathrm{mol})$ after $48 \mathrm{~h}$ at $323.0 \mathrm{~K}$ in $\mathrm{C}_{6} \mathrm{D}_{6}$. 


\section{Tables with Catalytic Data}

Table S4. Results obtained with 1 and ethylene $(92 \mu \mathrm{mol})$ at $298.0 \mathrm{~K}$ in $\mathrm{C}_{6} \mathrm{D}_{6}$.

\begin{tabular}{|c|c|c|c|c|c|c|c|c|c|c|c|}
\hline entry & $\begin{array}{c}t \\
(\mathrm{~h})\end{array}$ & $\begin{array}{l}n_{\text {propene }} \\
(\mu \mathrm{mol})\end{array}$ & $\begin{array}{l}n_{1 \text {-butene }} \\
(\mu \mathrm{mol})\end{array}$ & $\begin{array}{c}n_{c i s-2-} \\
\text { butene } \\
(\mu \mathrm{mol})\end{array}$ & $\begin{array}{c}n_{\text {trans }-2-} \\
\text { butene } \\
(\mu \mathrm{mol})\end{array}$ & $\begin{array}{c}n_{\text {total }}{ }^{a}{ }^{a}{ }^{2}{ }^{2} \text { atenol } \\
(\mu \mathrm{mos}\end{array}$ & $\begin{array}{c}n_{\text {total olefins }}^{b} \\
(\mu \mathrm{mol})\end{array}$ & $\begin{array}{c}n_{\text {ethane }} \\
(\mu \mathrm{mol})\end{array}$ & $\begin{array}{l}1^{c} \\
(\%)\end{array}$ & turnovers $^{d}$ & $\begin{array}{l}n_{\text {ethylene }} \\
(\mu \mathrm{mol})\end{array}$ \\
\hline 1 & 1.11 & 0.000 & 0.097 & 0.015 & 0.063 & 0.175 & 0.175 & 0.009 & 93.0 & 0.05 & 92.045 \\
\hline 2 & 1.51 & 0.000 & 0.088 & 0.024 & 0.006 & 0.119 & 0.119 & 0.008 & 90.2 & 0.03 & 91.898 \\
\hline 3 & 1.99 & 0.015 & 0.107 & 0.027 & 0.031 & 0.164 & 0.179 & 0.016 & 92.8 & 0.05 & 92.107 \\
\hline 4 & 2.49 & 0.000 & 0.111 & 0.039 & 0.026 & 0.176 & 0.176 & 0.022 & 91.3 & 0.05 & 92.046 \\
\hline 5 & 2.99 & 0.026 & 0.151 & 0.028 & 0.028 & 0.206 & 0.233 & 0.018 & 89.6 & 0.06 & 91.940 \\
\hline 6 & 3.49 & 0.025 & 0.182 & 0.019 & 0.044 & 0.245 & 0.269 & 0.024 & 86.8 & 0.07 & 91.918 \\
\hline 7 & 3.99 & 0.004 & 0.191 & 0.038 & 0.038 & 0.267 & 0.271 & 0.031 & 86.5 & 0.07 & 91.776 \\
\hline 8 & 4.49 & 0.025 & 0.202 & 0.041 & 0.077 & 0.320 & 0.346 & 0.038 & 83.3 & 0.09 & 91.757 \\
\hline 9 & 4.99 & 0.011 & 0.223 & 0.063 & 0.032 & 0.318 & 0.329 & 0.036 & 83.1 & 0.09 & 91.588 \\
\hline 10 & 5.49 & 0.037 & 0.251 & 0.074 & 0.054 & 0.378 & 0.415 & 0.042 & 78.5 & 0.11 & 91.430 \\
\hline 11 & 5.99 & 0.022 & 0.292 & 0.047 & 0.068 & 0.408 & 0.430 & 0.051 & 78.2 & 0.12 & 91.404 \\
\hline 12 & 6.49 & 0.029 & 0.291 & 0.060 & 0.051 & 0.402 & 0.431 & 0.062 & 75.2 & 0.12 & 91.109 \\
\hline 13 & 6.99 & 0.026 & 0.300 & 0.085 & 0.064 & 0.449 & 0.475 & 0.059 & 77.9 & 0.13 & 90.963 \\
\hline 14 & 7.49 & 0.038 & 0.351 & 0.069 & 0.072 & 0.491 & 0.530 & 0.059 & 76.8 & 0.14 & 90.864 \\
\hline 15 & 7.99 & 0.040 & 0.368 & 0.061 & 0.064 & 0.494 & 0.534 & 0.075 & 73.8 & 0.14 & 90.727 \\
\hline 16 & 8.49 & 0.044 & 0.378 & 0.104 & 0.065 & 0.547 & 0.591 & 0.076 & 72.4 & 0.16 & 90.574 \\
\hline 17 & 8.99 & 0.025 & 0.409 & 0.107 & 0.107 & 0.623 & 0.648 & 0.075 & 71.3 & 0.18 & 90.438 \\
\hline 18 & 9.49 & 0.074 & 0.436 & 0.102 & 0.058 & 0.596 & 0.670 & 0.085 & 70.1 & 0.18 & 90.333 \\
\hline 19 & 9.99 & 0.054 & 0.467 & 0.083 & 0.096 & 0.645 & 0.699 & 0.089 & 68.9 & 0.19 & 90.167 \\
\hline 20 & 10.49 & 0.037 & 0.477 & 0.099 & 0.107 & 0.683 & 0.720 & 0.081 & 68.0 & 0.19 & 90.159 \\
\hline 21 & 10.99 & 0.059 & 0.492 & 0.111 & 0.080 & 0.683 & 0.742 & 0.100 & 66.5 & 0.20 & 89.912 \\
\hline 22 & 11.49 & 0.043 & 0.507 & 0.115 & 0.080 & 0.702 & 0.745 & 0.114 & 65.2 & 0.20 & 89.753 \\
\hline 23 & 11.99 & 0.067 & 0.566 & 0.113 & 0.129 & 0.807 & 0.874 & 0.123 & 65.1 & 0.24 & 89.656 \\
\hline 24 & 12.49 & 0.060 & 0.559 & 0.125 & 0.148 & 0.832 & 0.892 & 0.115 & 62.9 & 0.24 & 89.450 \\
\hline 25 & 12.99 & 0.068 & 0.581 & 0.125 & 0.109 & 0.815 & 0.883 & 0.127 & 61.9 & 0.24 & 89.329 \\
\hline 26 & 13.49 & 0.061 & 0.609 & 0.158 & 0.149 & 0.916 & 0.977 & 0.133 & 61.0 & 0.26 & 89.260 \\
\hline 27 & 13.99 & 0.056 & 0.623 & 0.159 & 0.143 & 0.925 & 0.981 & 0.126 & 59.8 & 0.27 & 89.122 \\
\hline 28 & 14.49 & 0.107 & 0.664 & 0.143 & 0.135 & 0.942 & 1.049 & 0.133 & 58.9 & 0.28 & 88.988 \\
\hline 29 & 14.99 & 0.091 & 0.702 & 0.165 & 0.181 & 1.049 & 1.139 & 0.138 & 57.9 & 0.31 & 88.821 \\
\hline 30 & 15.49 & 0.074 & 0.692 & 0.165 & 0.162 & 1.019 & 1.094 & 0.148 & 56.7 & 0.30 & 88.647 \\
\hline 31 & 15.99 & 0.097 & 0.739 & 0.150 & 0.183 & 1.071 & 1.169 & 0.142 & 55.8 & 0.32 & 88.561 \\
\hline 32 & 16.49 & 0.112 & 0.735 & 0.179 & 0.207 & 1.122 & 1.234 & 0.162 & 55.6 & 0.33 & 88.419 \\
\hline 33 & 16.99 & 0.112 & 0.768 & 0.176 & 0.180 & 1.124 & 1.235 & 0.171 & 53.8 & 0.33 & 88.335 \\
\hline 34 & 17.49 & 0.084 & 0.770 & 0.191 & 0.194 & 1.155 & 1.239 & 0.169 & 53.2 & 0.33 & 88.280 \\
\hline 35 & 17.99 & 0.127 & 0.832 & 0.185 & 0.213 & 1.230 & 1.356 & 0.180 & 53.0 & 0.37 & 88.156 \\
\hline 36 & 18.49 & 0.128 & 0.828 & 0.208 & 0.189 & 1.225 & 1.353 & 0.187 & 51.4 & 0.37 & 88.095 \\
\hline 37 & 18.99 & 0.152 & 0.841 & 0.194 & 0.227 & 1.262 & 1.414 & 0.180 & 51.5 & 0.38 & 87.970 \\
\hline 38 & 19.49 & 0.122 & 0.882 & 0.180 & 0.203 & 1.265 & 1.387 & 0.198 & 49.6 & 0.37 & 87.855 \\
\hline 39 & 19.99 & 0.164 & 0.920 & 0.204 & 0.234 & 1.358 & 1.522 & 0.196 & 48.8 & 0.41 & 87.760 \\
\hline 40 & 20.49 & 0.140 & 0.909 & 0.210 & 0.260 & 1.380 & 1.520 & 0.200 & 48.9 & 0.41 & 87.580 \\
\hline
\end{tabular}




\begin{tabular}{|c|c|c|c|c|c|c|c|c|c|c|c|}
\hline 41 & 20.99 & 0.157 & 0.947 & 0.195 & 0.211 & 1.353 & 1.510 & 0.203 & 48.3 & 0.41 & 87.451 \\
\hline 42 & 21.49 & 0.197 & 0.990 & 0.212 & 0.232 & 1.435 & 1.631 & 0.207 & 47.5 & 0.44 & 87.395 \\
\hline 43 & 21.99 & 0.171 & 1.004 & 0.246 & 0.282 & 1.532 & 1.702 & 0.228 & 46.6 & 0.46 & 87.234 \\
\hline 44 & 22.49 & 0.175 & 1.028 & 0.217 & 0.278 & 1.523 & 1.698 & 0.226 & 45.8 & 0.46 & 87.154 \\
\hline 45 & 22.99 & 0.183 & 1.025 & 0.249 & 0.290 & 1.564 & 1.747 & 0.246 & 44.8 & 0.47 & 86.924 \\
\hline 46 & 23.49 & 0.178 & 1.069 & 0.261 & 0.297 & 1.626 & 1.805 & 0.231 & 44.0 & 0.49 & 86.763 \\
\hline 47 & 23.99 & 0.178 & 1.069 & 0.232 & 0.283 & 1.584 & 1.762 & 0.247 & 43.1 & 0.48 & 86.627 \\
\hline 48 & 24.49 & 0.192 & 1.085 & 0.244 & 0.295 & 1.624 & 1.815 & 0.263 & 42.6 & 0.49 & 86.585 \\
\hline 49 & 24.99 & 0.177 & 1.115 & 0.250 & 0.307 & 1.672 & 1.849 & 0.248 & 41.8 & 0.50 & 86.487 \\
\hline 50 & 25.49 & 0.199 & 1.136 & 0.294 & 0.335 & 1.765 & 1.964 & 0.255 & 41.2 & 0.53 & 86.401 \\
\hline 51 & 25.99 & 0.200 & 1.152 & 0.266 & 0.336 & 1.753 & 1.953 & 0.248 & 40.4 & 0.53 & 86.254 \\
\hline 52 & 26.49 & 0.200 & 1.165 & 0.269 & 0.327 & 1.761 & 1.960 & 0.268 & 39.6 & 0.53 & 86.128 \\
\hline 53 & 26.99 & 0.210 & 1.194 & 0.294 & 0.310 & 1.798 & 2.008 & 0.255 & 38.9 & 0.54 & 86.004 \\
\hline 54 & 27.49 & 0.201 & 1.225 & 0.294 & 0.306 & 1.825 & 2.026 & 0.282 & 38.3 & 0.55 & 85.909 \\
\hline 55 & 27.99 & 0.211 & 1.216 & 0.270 & 0.337 & 1.824 & 2.034 & 0.265 & 37.5 & 0.55 & 85.806 \\
\hline 56 & 28.49 & 0.237 & 1.272 & 0.277 & 0.332 & 1.881 & 2.117 & 0.291 & 36.9 & 0.57 & 85.730 \\
\hline 57 & 28.99 & 0.262 & 1.315 & 0.324 & 0.309 & 1.947 & 2.210 & 0.283 & 36.4 & 0.60 & 85.678 \\
\hline 58 & 29.49 & 0.236 & 1.302 & 0.336 & 0.360 & 1.998 & 2.234 & 0.303 & 35.6 & 0.60 & 85.518 \\
\hline 59 & 29.99 & 0.247 & 1.326 & 0.283 & 0.404 & 2.013 & 2.260 & 0.279 & 35.2 & 0.61 & 85.458 \\
\hline 60 & 30.49 & 0.235 & 1.352 & 0.275 & 0.383 & 2.010 & 2.245 & 0.299 & 34.4 & 0.61 & 85.329 \\
\hline 61 & 30.99 & 0.241 & 1.371 & 0.373 & 0.405 & 2.149 & 2.391 & 0.304 & 33.5 & 0.65 & 85.109 \\
\hline 62 & 31.49 & 0.257 & 1.380 & 0.322 & 0.392 & 2.094 & 2.351 & 0.302 & 32.9 & 0.64 & 84.954 \\
\hline 63 & 31.99 & 0.234 & 1.378 & 0.312 & 0.329 & 2.019 & 2.253 & 0.310 & 32.9 & 0.61 & 84.928 \\
\hline 64 & 32.49 & 0.268 & 1.422 & 0.324 & 0.380 & 2.126 & 2.395 & 0.310 & 32.1 & 0.65 & 84.745 \\
\hline 65 & 32.99 & 0.250 & 1.420 & 0.314 & 0.359 & 2.092 & 2.342 & 0.339 & 31.5 & 0.63 & 84.667 \\
\hline 66 & 33.49 & 0.293 & 1.451 & 0.331 & 0.385 & 2.167 & 2.461 & 0.324 & 31.0 & 0.66 & 84.581 \\
\hline 67 & 33.99 & 0.298 & 1.479 & 0.337 & 0.391 & 2.207 & 2.505 & 0.346 & 30.4 & 0.68 & 84.477 \\
\hline 68 & 34.49 & 0.295 & 1.507 & 0.365 & 0.401 & 2.272 & 2.567 & 0.314 & 30.1 & 0.69 & 84.469 \\
\hline 69 & 34.99 & 0.281 & 1.511 & 0.351 & 0.395 & 2.257 & 2.538 & 0.340 & 29.3 & 0.69 & 84.278 \\
\hline 70 & 35.49 & 0.278 & 1.517 & 0.332 & 0.390 & 2.239 & 2.517 & 0.370 & 28.6 & 0.68 & 84.154 \\
\hline 71 & 35.99 & 0.305 & 1.555 & 0.379 & 0.414 & 2.347 & 2.653 & 0.361 & 28.1 & 0.72 & 84.090 \\
\hline 72 & 36.49 & 0.325 & 1.623 & 0.390 & 0.427 & 2.440 & 2.765 & 0.341 & 27.9 & 0.75 & 84.050 \\
\hline 73 & 36.99 & 0.341 & 1.607 & 0.368 & 0.426 & 2.401 & 2.742 & 0.357 & 27.3 & 0.74 & 83.969 \\
\hline 74 & 37.49 & 0.356 & 1.610 & 0.363 & 0.448 & 2.421 & 2.777 & 0.347 & 26.8 & 0.75 & 83.871 \\
\hline 75 & 37.99 & 0.370 & 1.707 & 0.412 & 0.452 & 2.571 & 2.940 & 0.359 & 26.2 & 0.79 & 83.803 \\
\hline 76 & 38.49 & 0.343 & 1.649 & 0.373 & 0.446 & 2.468 & 2.811 & 0.389 & 25.6 & 0.76 & 83.647 \\
\hline 77 & 38.99 & 0.337 & 1.661 & 0.374 & 0.460 & 2.494 & 2.831 & 0.377 & 25.2 & 0.77 & 83.536 \\
\hline 78 & 39.49 & 0.361 & 1.695 & 0.400 & 0.457 & 2.552 & 2.913 & 0.391 & 25.6 & 0.79 & 83.401 \\
\hline 79 & 39.99 & 0.315 & 1.704 & 0.386 & 0.470 & 2.561 & 2.875 & 0.432 & 23.9 & 0.78 & 83.204 \\
\hline 80 & 40.49 & 0.391 & 1.757 & 0.398 & 0.464 & 2.618 & 3.009 & 0.407 & 23.6 & 0.81 & 83.166 \\
\hline 81 & 40.99 & 0.373 & 1.772 & 0.414 & 0.477 & 2.662 & 3.035 & 0.409 & 23.0 & 0.82 & 83.067 \\
\hline 82 & 41.49 & 0.389 & 1.765 & 0.422 & 0.499 & 2.686 & 3.075 & 0.416 & 22.6 & 0.83 & 83.013 \\
\hline 83 & 41.99 & 0.391 & 1.804 & 0.388 & 0.496 & 2.687 & 3.078 & 0.398 & 22.8 & 0.83 & 82.952 \\
\hline 84 & 42.49 & 0.356 & 1.805 & 0.406 & 0.495 & 2.705 & 3.061 & 0.381 & 22.4 & 0.83 & 82.825 \\
\hline 85 & 42.99 & 0.375 & 1.812 & 0.407 & 0.479 & 2.699 & 3.074 & 0.388 & 21.9 & 0.83 & 82.769 \\
\hline
\end{tabular}




\begin{tabular}{|c|c|c|c|c|c|c|c|c|c|c|c|}
\hline 86 & 43.49 & 0.357 & 1.840 & 0.435 & 0.491 & 2.765 & 3.122 & 0.409 & 21.3 & 0.84 & 82.574 \\
\hline 87 & 43.99 & 0.413 & 1.855 & 0.420 & 0.523 & 2.798 & 3.211 & 0.410 & 20.8 & 0.87 & 82.497 \\
\hline 88 & 44.49 & 0.383 & 1.882 & 0.440 & 0.525 & 2.846 & 3.229 & 0.417 & 20.4 & 0.87 & 82.381 \\
\hline 89 & 44.99 & 0.405 & 1.884 & 0.443 & 0.545 & 2.871 & 3.276 & 0.411 & 20.1 & 0.89 & 82.320 \\
\hline 90 & 45.49 & 0.411 & 1.941 & 0.455 & 0.547 & 2.943 & 3.354 & 0.400 & 19.8 & 0.91 & 82.224 \\
\hline 91 & 45.99 & 0.383 & 1.938 & 0.432 & 0.536 & 2.905 & 3.288 & 0.417 & 19.2 & 0.89 & 82.093 \\
\hline 92 & 46.49 & 0.415 & 1.951 & 0.409 & 0.519 & 2.879 & 3.295 & 0.415 & 18.6 & 0.89 & 81.885 \\
\hline 93 & 46.99 & 0.441 & 1.984 & 0.455 & 0.560 & 2.999 & 3.440 & 0.424 & 18.3 & 0.93 & 81.889 \\
\hline 94 & 47.49 & 0.433 & 2.001 & 0.471 & 0.545 & 3.017 & 3.450 & 0.425 & 17.9 & 0.93 & 81.771 \\
\hline 95 & 47.99 & 0.420 & 1.990 & 0.446 & 0.549 & 2.986 & 3.405 & 0.423 & 17.6 & 0.92 & 81.705 \\
\hline 96 & 48.49 & 0.442 & 2.022 & 0.459 & 0.588 & 3.069 & 3.511 & 0.431 & 17.2 & 0.95 & 81.618 \\
\hline 97 & 48.99 & 0.472 & 2.031 & 0.493 & 0.572 & 3.096 & 3.568 & 0.443 & 16.7 & 0.96 & 81.477 \\
\hline 98 & 49.49 & 0.431 & 2.037 & 0.490 & 0.590 & 3.118 & 3.550 & 0.409 & 16.6 & 0.96 & 81.493 \\
\hline 99 & 49.99 & 0.467 & 2.091 & 0.474 & 0.579 & 3.144 & 3.611 & 0.436 & 16.2 & 0.98 & 81.385 \\
\hline 100 & 50.49 & 0.426 & 2.090 & 0.499 & 0.589 & 3.178 & 3.604 & 0.453 & 15.7 & 0.97 & 81.134 \\
\hline 101 & 50.99 & 0.447 & 2.137 & 0.466 & 0.597 & 3.201 & 3.648 & 0.461 & 15.4 & 0.99 & 81.082 \\
\hline 102 & 51.49 & 0.527 & 2.168 & 0.507 & 0.622 & 3.298 & 3.824 & 0.427 & 15.4 & 1.03 & 81.163 \\
\hline 103 & 51.99 & 0.467 & 2.196 & 0.515 & 0.615 & 3.327 & 3.794 & 0.449 & 14.8 & 1.03 & 80.948 \\
\hline 104 & 52.49 & 0.515 & 2.233 & 0.513 & 0.637 & 3.383 & 3.898 & 0.459 & 14.7 & 1.05 & 80.980 \\
\hline 105 & 52.99 & 0.527 & 2.171 & 0.510 & 0.610 & 3.291 & 3.818 & 0.474 & 13.8 & 1.03 & 80.701 \\
\hline 106 & 53.49 & 0.515 & 2.296 & 0.547 & 0.640 & 3.484 & 3.999 & 0.444 & 13.7 & 1.08 & 80.745 \\
\hline 107 & 53.99 & 0.612 & 2.234 & 0.527 & 0.679 & 3.441 & 4.052 & 0.469 & 13.8 & 1.09 & 80.706 \\
\hline 108 & 54.49 & 0.511 & 2.260 & 0.502 & 0.651 & 3.412 & 3.924 & 0.482 & 13.2 & 1.06 & 80.527 \\
\hline 109 & 54.99 & 0.548 & 2.268 & 0.497 & 0.651 & 3.415 & 3.963 & 0.476 & 12.7 & 1.07 & 80.398 \\
\hline 110 & 55.49 & 0.560 & 2.241 & 0.515 & 0.674 & 3.431 & 3.991 & 0.506 & 12.5 & 1.08 & 80.295 \\
\hline 111 & 55.99 & 0.562 & 2.305 & 0.526 & 0.682 & 3.513 & 4.075 & 0.502 & 12.3 & 1.10 & 80.316 \\
\hline 112 & 56.49 & 0.561 & 2.322 & 0.552 & 0.675 & 3.548 & 4.109 & 0.510 & 12.1 & 1.11 & 80.205 \\
\hline 113 & 56.99 & 0.605 & 2.299 & 0.532 & 0.693 & 3.525 & 4.130 & 0.501 & 11.8 & 1.12 & 80.149 \\
\hline 114 & 57.49 & 0.552 & 2.347 & 0.542 & 0.670 & 3.558 & 4.110 & 0.489 & 11.1 & 1.11 & 80.099 \\
\hline 115 & 57.99 & 0.589 & 2.389 & 0.583 & 0.693 & 3.665 & 4.254 & 0.522 & 11.0 & 1.15 & 79.908 \\
\hline 116 & 58.49 & 0.581 & 2.377 & 0.548 & 0.711 & 3.636 & 4.217 & 0.507 & 10.7 & 1.14 & 79.809 \\
\hline 117 & 58.99 & 0.627 & 2.421 & 0.554 & 0.708 & 3.682 & 4.309 & 0.506 & 10.4 & 1.16 & 79.767 \\
\hline 118 & 59.49 & 0.632 & 2.455 & 0.577 & 0.731 & 3.762 & 4.395 & 0.480 & 10.4 & 1.19 & 79.779 \\
\hline 119 & 59.99 & 0.603 & 2.457 & 0.598 & 0.739 & 3.794 & 4.397 & 0.519 & 10.0 & 1.19 & 79.580 \\
\hline 120 & 60.49 & 0.606 & 2.462 & 0.587 & 0.733 & 3.781 & 4.387 & 0.511 & 9.7 & 1.19 & 79.533 \\
\hline 121 & 60.99 & 0.593 & 2.464 & 0.578 & 0.732 & 3.774 & 4.367 & 0.534 & 9.1 & 1.18 & 79.313 \\
\hline 122 & 61.49 & 0.606 & 2.494 & 0.577 & 0.726 & 3.797 & 4.403 & 0.537 & 8.8 & 1.19 & 78.955 \\
\hline 123 & 61.99 & 0.614 & 2.501 & 0.623 & 0.768 & 3.891 & 4.506 & 0.549 & 8.8 & 1.22 & 79.076 \\
\hline 124 & 62.49 & 0.639 & 2.530 & 0.620 & 0.726 & 3.876 & 4.516 & 0.510 & 8.5 & 1.22 & 78.978 \\
\hline 125 & 62.99 & 0.624 & 2.550 & 0.614 & 0.766 & 3.930 & 4.554 & 0.544 & 7.8 & 1.23 & 78.890 \\
\hline 126 & 63.49 & 0.608 & 2.528 & 0.642 & 0.737 & 3.906 & 4.514 & 0.560 & 7.7 & 1.22 & 78.632 \\
\hline 127 & 63.99 & 0.661 & 2.586 & 0.664 & 0.802 & 4.052 & 4.712 & 0.526 & 8.1 & 1.27 & 78.736 \\
\hline 128 & 64.49 & 0.651 & 2.596 & 0.642 & 0.789 & 4.027 & 4.678 & 0.546 & 7.7 & 1.26 & 78.641 \\
\hline 129 & 64.99 & 0.684 & 2.638 & 0.653 & 0.824 & 4.116 & 4.800 & 0.563 & 7.1 & 1.30 & 78.587 \\
\hline 130 & 65.49 & 0.648 & 2.599 & 0.619 & 0.800 & 4.017 & 4.665 & 0.546 & 7.3 & 1.26 & 78.389 \\
\hline
\end{tabular}




\begin{tabular}{l|llllllllll|l}
131 & 65.99 & 0.679 & 2.658 & 0.641 & 0.805 & 4.103 & 4.782 & 0.569 & 7.0 & 1.29 & 78.311 \\
132 & 66.49 & 0.670 & 2.633 & 0.652 & 0.829 & 4.114 & 4.784 & 0.550 & 6.2 & 1.29 & 78.183 \\
133 & 66.99 & 0.705 & 2.674 & 0.675 & 0.826 & 4.175 & 4.880 & 0.569 & 6.8 & 1.32 & 78.201 \\
134 & 67.49 & 0.705 & 2.706 & 0.666 & 0.839 & 4.210 & 4.915 & 0.543 & 6.3 & 1.33 & 78.222 \\
135 & 67.99 & 0.702 & 2.707 & 0.640 & 0.828 & 4.176 & 4.878 & 0.590 & 5.9 & 1.32 & 78.038 \\
136 & 68.49 & 0.738 & 2.708 & 0.666 & 0.838 & 4.212 & 4.950 & 0.568 & 6.1 & 1.34 & 77.985 \\
137 & 68.99 & 0.735 & 2.744 & 0.674 & 0.848 & 4.266 & 5.000 & 0.583 & 5.5 & 1.35 & 77.866 \\
138 & 69.49 & 0.722 & 2.766 & 0.683 & 0.870 & 4.319 & 5.041 & 0.557 & 5.6 & 1.36 & 77.876 \\
139 & 69.99 & 0.750 & 2.774 & 0.683 & 0.874 & 4.331 & 5.081 & 0.574 & 5.5 & 1.37 & 77.684 \\
140 & 70.49 & 0.728 & 2.793 & 0.705 & 0.882 & 4.380 & 5.108 & 0.574 & 5.2 & 1.38 & 77.440 \\
141 & 70.99 & 0.751 & 2.786 & 0.701 & 0.911 & 4.399 & 5.149 & 0.582 & 4.7 & 1.39 & 77.590 \\
142 & 71.49 & 0.763 & 2.828 & 0.718 & 0.914 & 4.460 & 5.223 & 0.580 & 4.7 & 1.41 & 77.469 \\
143 & 71.99 & 0.778 & 2.881 & 0.691 & 0.889 & 4.461 & 5.240 & 0.582 & 5.0 & 1.42 & 77.343 \\
144 & 72.49 & 0.773 & 2.854 & 0.722 & 0.880 & 4.455 & 5.229 & 0.588 & 4.8 & 1.41 & 77.209 \\
145 & 72.99 & 0.777 & 2.876 & 0.735 & 0.910 & 4.521 & 5.298 & 0.597 & 4.3 & 1.43 & 77.099 \\
146 & 73.49 & 0.793 & 2.875 & 0.694 & 0.913 & 4.482 & 5.275 & 0.631 & 4.2 & 1.43 & 76.970 \\
\hline
\end{tabular}

${ }^{a} n_{\text {total butenes }}=n_{1 \text {-butene }}+n_{\text {cis-2-butene }}+n_{\text {trans-2-butene. }}{ }^{b} n_{\text {total olefins }}=n_{1 \text {-butene }}+n_{\text {cis-2-butene }}+n_{\text {trans-2-butene }}+n_{\text {propene. }}{ }^{c}$ Percentage of compound $\mathbf{1}$ present in solution determined from the ${ }^{1} \mathrm{H}$ resonances of the coordinated $p$-cymene. ${ }^{d}$ Moles of total olefins produced $\left(n_{\text {total olefins }}\right)$ divided by the initial molar number of $\mathbf{1}$. 
Table S5. Results obtained with 1 and ethylene $(115 \mu \mathrm{mol})$ at $323.0 \mathrm{~K}$ in $\mathrm{C}_{6} \mathrm{D}_{6}$.

\begin{tabular}{|c|c|c|c|c|c|c|c|c|c|}
\hline entry & $\begin{array}{c}t \\
(\mathrm{~h})\end{array}$ & $\begin{array}{l}n_{\text {propene }} \\
(\mu \mathrm{mol})\end{array}$ & $\begin{array}{l}n_{1 \text {-butene }} \\
(\mu \mathrm{mol})\end{array}$ & $\begin{array}{c}n_{c i s-2-} \\
\text { butene } \\
(\mu \mathrm{mol})\end{array}$ & $\begin{array}{c}n_{\text {trans-2- }} \\
\text { butene } \\
(\mu \mathrm{mol})\end{array}$ & $\begin{array}{c}n_{\text {total butenes }}{ }^{a} \\
(\mu \mathrm{mol})\end{array}$ & $\begin{array}{c}n_{\text {total olefins }}^{b} \\
(\mu \mathrm{mol})\end{array}$ & $\begin{array}{c}n_{\text {ethane }} \\
(\mu \mathrm{mol})\end{array}$ & turnovers $^{c}$ \\
\hline 1 & 0.00 & 0.000 & 0.000 & 0.000 & 0.000 & 0.000 & 0.000 & 0.000 & 0.00 \\
\hline 2 & 1.91 & 0.195 & 0.549 & 0.084 & 0.108 & 0.741 & 0.936 & 0.208 & 0.25 \\
\hline 3 & 2.41 & 0.401 & 0.747 & 0.085 & 0.172 & 1.005 & 1.406 & 0.261 & 0.38 \\
\hline 4 & 2.91 & 0.553 & 0.877 & 0.088 & 0.133 & 1.097 & 1.650 & 0.307 & 0.45 \\
\hline 5 & 3.41 & 0.943 & 1.015 & 0.055 & 0.084 & 1.154 & 2.097 & 0.348 & 0.57 \\
\hline 6 & 3.91 & 1.282 & 1.141 & 0.027 & 0.066 & 1.234 & 2.516 & 0.396 & 0.68 \\
\hline 7 & 4.41 & 1.549 & 1.244 & 0.022 & 0.031 & 1.297 & 2.846 & 0.447 & 0.77 \\
\hline 8 & 4.91 & 1.819 & 1.374 & 0.033 & 0.051 & 1.458 & 3.277 & 0.510 & 0.89 \\
\hline 9 & 5.41 & 1.970 & 1.460 & 0.018 & 0.039 & 1.517 & 3.487 & 0.558 & 0.94 \\
\hline 10 & 5.91 & 2.249 & 1.552 & 0.021 & 0.038 & 1.612 & 3.861 & 0.607 & 1.04 \\
\hline 11 & 6.41 & 2.410 & 1.671 & 0.031 & 0.047 & 1.750 & 4.159 & 0.682 & 1.12 \\
\hline 12 & 6.91 & 2.675 & 1.731 & 0.010 & 0.033 & 1.774 & 4.449 & 0.732 & 1.20 \\
\hline 13 & 7.41 & 2.875 & 1.809 & 0.030 & 0.037 & 1.876 & 4.751 & 0.798 & 1.28 \\
\hline 14 & 7.91 & 3.029 & 1.908 & 0.009 & 0.033 & 1.950 & 4.979 & 0.880 & 1.35 \\
\hline 15 & 8.41 & 3.328 & 1.974 & 0.032 & 0.028 & 2.034 & 5.361 & 0.912 & 1.45 \\
\hline 16 & 8.91 & 3.527 & 2.023 & 0.019 & 0.032 & 2.073 & 5.600 & 0.958 & 1.51 \\
\hline 17 & 9.41 & 3.775 & 2.103 & 0.017 & 0.054 & 2.174 & 5.950 & 1.022 & 1.61 \\
\hline 18 & 9.91 & 4.039 & 2.198 & 0.028 & 0.049 & 2.275 & 6.314 & 1.070 & 1.71 \\
\hline 19 & 10.41 & 4.218 & 2.267 & 0.034 & 0.060 & 2.361 & 6.580 & 1.128 & 1.78 \\
\hline 20 & 10.91 & 4.495 & 2.331 & 0.030 & 0.029 & 2.389 & 6.884 & 1.156 & 1.86 \\
\hline 21 & 11.41 & 4.743 & 2.310 & 0.026 & 0.067 & 2.403 & 7.145 & 1.176 & 1.93 \\
\hline 22 & 11.91 & 5.122 & 2.422 & 0.021 & 0.051 & 2.494 & 7.616 & 1.239 & 2.06 \\
\hline 23 & 12.41 & 5.352 & 2.561 & 0.023 & 0.081 & 2.665 & 8.017 & 1.254 & 2.17 \\
\hline 24 & 12.91 & 5.554 & 2.476 & 0.032 & 0.070 & 2.578 & 8.132 & 1.293 & 2.20 \\
\hline 25 & 13.41 & 5.872 & 2.551 & 0.038 & 0.031 & 2.620 & 8.492 & 1.343 & 2.29 \\
\hline 26 & 13.91 & 6.185 & 2.530 & 0.035 & 0.068 & 2.632 & 8.816 & 1.379 & 2.38 \\
\hline 27 & 14.41 & 6.461 & 2.608 & 0.031 & 0.093 & 2.732 & 9.194 & 1.409 & 2.48 \\
\hline 28 & 14.91 & 6.794 & 2.649 & 0.040 & 0.037 & 2.726 & 9.520 & 1.453 & 2.57 \\
\hline 29 & 15.41 & 7.173 & 2.688 & 0.049 & 0.086 & 2.823 & 9.995 & 1.472 & 2.70 \\
\hline 30 & 15.91 & 7.560 & 2.804 & 0.052 & 0.072 & 2.929 & 10.488 & 1.498 & 2.83 \\
\hline 31 & 16.41 & 7.853 & 2.695 & 0.054 & 0.116 & 2.866 & 10.719 & 1.532 & 2.90 \\
\hline 32 & 16.91 & 8.212 & 2.788 & 0.057 & 0.102 & 2.947 & 11.159 & 1.592 & 3.02 \\
\hline 33 & 17.41 & 8.516 & 2.769 & 0.056 & 0.104 & 2.929 & 11.444 & 1.588 & 3.09 \\
\hline 34 & 17.91 & 8.884 & 2.760 & 0.062 & 0.126 & 2.948 & 11.832 & 1.623 & 3.20 \\
\hline 35 & 18.41 & 9.294 & 2.662 & 0.066 & 0.121 & 2.848 & 12.142 & 1.637 & 3.28 \\
\hline 36 & 18.91 & 9.564 & 2.757 & 0.079 & 0.139 & 2.975 & 12.539 & 1.697 & 3.39 \\
\hline 37 & 19.41 & 10.012 & 2.817 & 0.068 & 0.155 & 3.040 & 13.052 & 1.691 & 3.53 \\
\hline 38 & 19.91 & 10.393 & 2.975 & 0.068 & 0.170 & 3.213 & 13.606 & 1.733 & 3.68 \\
\hline 39 & 20.41 & 10.715 & 2.955 & 0.101 & 0.167 & 3.223 & 13.938 & 1.777 & 3.77 \\
\hline 40 & 20.91 & 11.108 & 2.900 & 0.091 & 0.230 & 3.221 & 14.329 & 1.755 & 3.87 \\
\hline 41 & 21.41 & 11.420 & 2.923 & 0.105 & 0.212 & 3.239 & 14.659 & 1.851 & 3.96 \\
\hline 42 & 21.91 & 11.767 & 2.851 & 0.113 & 0.226 & 3.191 & 14.958 & 1.795 & 4.04 \\
\hline
\end{tabular}




\begin{tabular}{|c|c|c|c|c|c|c|c|c|c|}
\hline 43 & 22.41 & 12.104 & 2.791 & 0.121 & 0.287 & 3.199 & 15.303 & 1.837 & 4.14 \\
\hline 44 & 22.91 & 12.370 & 2.722 & 0.119 & 0.269 & 3.110 & 15.480 & 1.882 & 4.18 \\
\hline 45 & 23.41 & 12.745 & 2.732 & 0.129 & 0.295 & 3.155 & 15.900 & 1.839 & 4.30 \\
\hline 46 & 23.91 & 13.116 & 2.678 & 0.128 & 0.326 & 3.132 & 16.248 & 1.857 & 4.39 \\
\hline 47 & 24.41 & 13.540 & 2.768 & 0.127 & 0.337 & 3.233 & 16.772 & 1.990 & 4.53 \\
\hline 48 & 24.91 & 13.981 & 2.835 & 0.150 & 0.367 & 3.352 & 17.333 & 1.942 & 4.68 \\
\hline 49 & 25.41 & 14.335 & 2.829 & 0.156 & 0.366 & 3.351 & 17.686 & 2.001 & 4.78 \\
\hline 50 & 25.91 & 14.706 & 2.837 & 0.168 & 0.385 & 3.390 & 18.096 & 1.961 & 4.89 \\
\hline 51 & 26.41 & 14.914 & 2.703 & 0.168 & 0.406 & 3.277 & 18.190 & 1.951 & 4.92 \\
\hline 52 & 26.91 & 15.178 & 2.578 & 0.182 & 0.462 & 3.222 & 18.400 & 1.984 & 4.97 \\
\hline 53 & 27.41 & 15.469 & 2.520 & 0.187 & 0.446 & 3.153 & 18.621 & 2.040 & 5.03 \\
\hline 54 & 27.91 & 15.836 & 2.525 & 0.205 & 0.462 & 3.192 & 19.028 & 2.069 & 5.14 \\
\hline 55 & 28.41 & 16.124 & 2.520 & 0.200 & 0.478 & 3.197 & 19.321 & 2.033 & 5.22 \\
\hline 56 & 28.91 & 16.388 & 2.483 & 0.202 & 0.505 & 3.190 & 19.578 & 2.105 & 5.29 \\
\hline 57 & 29.41 & 16.624 & 2.382 & 0.208 & 0.545 & 3.135 & 19.759 & 2.194 & 5.34 \\
\hline 58 & 29.91 & 16.955 & 2.357 & 0.218 & 0.541 & 3.116 & 20.071 & 2.156 & 5.42 \\
\hline 59 & 30.41 & 17.146 & 2.341 & 0.224 & 0.543 & 3.108 & 20.254 & 2.228 & 5.47 \\
\hline 60 & 30.91 & 17.568 & 2.349 & 0.230 & 0.594 & 3.173 & 20.741 & 2.118 & 5.60 \\
\hline 61 & 31.41 & 17.809 & 2.237 & 0.236 & 0.626 & 3.099 & 20.908 & 2.271 & 5.65 \\
\hline 62 & 31.91 & 17.894 & 2.177 & 0.262 & 0.624 & 3.064 & 20.957 & 2.274 & 5.66 \\
\hline 63 & 32.41 & 18.051 & 2.248 & 0.263 & 0.632 & 3.143 & 21.194 & 2.180 & 5.73 \\
\hline 64 & 32.91 & 18.340 & 2.318 & 0.255 & 0.686 & 3.259 & 21.599 & 2.204 & 5.84 \\
\hline 65 & 33.41 & 18.642 & 2.141 & 0.266 & 0.703 & 3.109 & 21.751 & 2.224 & 5.88 \\
\hline 66 & 33.91 & 18.884 & 2.079 & 0.266 & 0.736 & 3.081 & 21.965 & 2.260 & 5.94 \\
\hline 67 & 34.41 & 19.250 & 2.073 & 0.286 & 0.745 & 3.105 & 22.355 & 2.261 & 6.04 \\
\hline 68 & 34.91 & 19.360 & 1.991 & 0.292 & 0.765 & 3.048 & 22.408 & 2.263 & 6.06 \\
\hline 69 & 35.41 & 19.673 & 2.050 & 0.311 & 0.834 & 3.194 & 22.868 & 2.301 & 6.18 \\
\hline 70 & 35.91 & 20.090 & 2.135 & 0.314 & 0.851 & 3.300 & 23.390 & 2.278 & 6.32 \\
\hline 71 & 36.41 & 20.227 & 2.053 & 0.306 & 0.884 & 3.243 & 23.470 & 2.321 & 6.34 \\
\hline 72 & 36.91 & 20.467 & 1.982 & 0.313 & 0.864 & 3.160 & 23.627 & 2.336 & 6.38 \\
\hline 73 & 37.41 & 20.693 & 1.899 & 0.328 & 0.876 & 3.104 & 23.797 & 2.396 & 6.43 \\
\hline 74 & 37.91 & 20.976 & 2.026 & 0.327 & 0.868 & 3.220 & 24.196 & 2.336 & 6.54 \\
\hline 75 & 38.41 & 21.038 & 1.872 & 0.362 & 0.877 & 3.111 & 24.149 & 2.388 & 6.53 \\
\hline 76 & 38.91 & 21.271 & 1.882 & 0.356 & 0.936 & 3.174 & 24.446 & 2.365 & 6.61 \\
\hline 77 & 39.41 & 21.433 & 1.811 & 0.356 & 0.955 & 3.122 & 24.555 & 2.412 & 6.64 \\
\hline 78 & 39.91 & 21.497 & 1.781 & 0.372 & 0.962 & 3.115 & 24.612 & 2.390 & 6.65 \\
\hline 79 & 40.41 & 21.734 & 1.766 & 0.377 & 0.977 & 3.120 & 24.855 & 2.590 & 6.72 \\
\hline 80 & 40.91 & 21.904 & 1.891 & 0.373 & 1.013 & 3.278 & 25.182 & 2.446 & 6.80 \\
\hline 81 & 41.41 & 22.252 & 1.803 & 0.394 & 1.050 & 3.248 & 25.499 & 2.427 & 6.89 \\
\hline 82 & 41.91 & 22.461 & 1.757 & 0.392 & 0.984 & 3.133 & 25.594 & 2.512 & 6.92 \\
\hline 83 & 42.41 & 22.517 & 1.731 & 0.381 & 0.978 & 3.089 & 25.606 & 2.493 & 6.92 \\
\hline 84 & 42.91 & 22.737 & 1.729 & 0.386 & 1.031 & 3.147 & 25.883 & 2.529 & 6.99 \\
\hline 85 & 43.41 & 23.035 & 1.726 & 0.454 & 1.093 & 3.273 & 26.307 & 2.496 & 7.11 \\
\hline 86 & 43.91 & 23.145 & 1.663 & 0.421 & 1.053 & 3.137 & 26.282 & 2.584 & 7.10 \\
\hline 87 & 44.41 & 23.349 & 1.695 & 0.420 & 1.070 & 3.185 & 26.534 & 2.525 & 7.17 \\
\hline
\end{tabular}




\begin{tabular}{l|lllllllll}
88 & 44.91 & 23.453 & 1.653 & 0.405 & 1.091 & 3.149 & 26.603 & 2.571 & 7.19 \\
89 & 45.41 & 23.487 & 1.596 & 0.422 & 1.086 & 3.104 & 26.590 & 2.580 & 7.19 \\
90 & 45.91 & 23.830 & 1.621 & 0.444 & 1.125 & 3.190 & 27.020 & 2.586 & 7.30 \\
91 & 46.41 & 23.991 & 1.611 & 0.430 & 1.140 & 3.181 & 27.172 & 2.607 & 7.34
\end{tabular}

${ }^{a} n_{\text {total butenes }}=n_{1 \text {-butene }}+n_{\text {cis-2-butene }}+n_{\text {trans-2-butene. }}{ }^{b} n_{\text {total olefins }}=n_{1 \text {-butene }}+n_{\text {cis-2-butene }}+n_{\text {trans-2-butene }}+n_{\text {propene. }}{ }^{c}$ Moles of total olefins produced $\left(n_{\text {total olefins }}\right)$ divided by the initial molar number of $\mathbf{1}$. 
Table S6. Results obtained with 1, 0.57 equiv. $\mathrm{PCy}_{3}$ and ethylene $(116 \mu \mathrm{mol})$ at $298.0 \mathrm{~K}$ in $\mathrm{C}_{6} \mathrm{D}_{6}$.

\begin{tabular}{|c|c|c|c|c|c|c|c|c|c|c|c|}
\hline entry & $\begin{array}{c}t \\
\text { (h) }\end{array}$ & $\begin{array}{l}n_{\text {propene }} \\
(\mu \mathrm{mol})\end{array}$ & $\begin{array}{l}n_{1 \text {-butene }} \\
(\mu \mathrm{mol})\end{array}$ & $\begin{array}{c}n_{\text {cis-2- }} \\
\text { butene } \\
(\mu \mathrm{mol})\end{array}$ & $\begin{array}{c}n_{\text {trans-2- }} \\
\text { butene } \\
(\mu \mathrm{mol})\end{array}$ & $\begin{array}{c}n_{\text {total butenes }}{ }^{a} \\
(\mu \mathrm{mol})\end{array}$ & $\begin{array}{c}n_{\text {total olefins }}{ }^{b} \\
(\mu \mathrm{mol})\end{array}$ & $\begin{array}{c}n_{\text {ethane }} \\
(\mu \mathrm{mol})\end{array}$ & $\begin{array}{l}1^{c} \\
(\%)\end{array}$ & turnovers $^{d}$ & $\begin{array}{l}n_{\text {ethylene }} \\
(\mu \mathrm{mol})\end{array}$ \\
\hline 1 & 1.08 & 0.057 & 0.124 & 0.023 & 0.021 & 0.169 & 0.226 & 0.006 & 77.4 & 0.06 & 115.879 \\
\hline 2 & 2.23 & 0.075 & 0.109 & 0.031 & 0.039 & 0.179 & 0.254 & 0.018 & 75.9 & 0.07 & 116.072 \\
\hline 3 & 2.85 & 0.039 & 0.092 & 0.019 & 0.034 & 0.145 & 0.184 & 0.011 & 70.8 & 0.05 & 116.528 \\
\hline 4 & 3.33 & 0.035 & 0.098 & 0.041 & 0.015 & 0.153 & 0.188 & 0.014 & 67.7 & 0.05 & 116.544 \\
\hline 5 & 3.83 & 0.063 & 0.163 & 0.046 & 0.033 & 0.241 & 0.304 & 0.011 & 62.8 & 0.09 & 116.389 \\
\hline 6 & 4.33 & 0.057 & 0.144 & 0.058 & 0.017 & 0.219 & 0.276 & 0.019 & 59.0 & 0.08 & 116.115 \\
\hline 7 & 4.83 & 0.045 & 0.214 & 0.052 & 0.061 & 0.327 & 0.372 & 0.021 & 57.1 & 0.10 & 117.035 \\
\hline 8 & 5.33 & 0.029 & 0.252 & 0.071 & 0.031 & 0.354 & 0.384 & 0.022 & 52.8 & 0.11 & 116.071 \\
\hline 9 & 5.83 & 0.032 & 0.281 & 0.058 & 0.058 & 0.397 & 0.429 & 0.033 & 50.6 & 0.12 & 115.954 \\
\hline 10 & 6.33 & 0.027 & 0.323 & 0.068 & 0.055 & 0.446 & 0.473 & 0.034 & 47.7 & 0.13 & 115.873 \\
\hline 11 & 6.83 & 0.037 & 0.333 & 0.091 & 0.065 & 0.489 & 0.526 & 0.047 & 44.4 & 0.15 & 116.582 \\
\hline 12 & 7.33 & 0.044 & 0.383 & 0.068 & 0.051 & 0.502 & 0.546 & 0.035 & 41.7 & 0.15 & 115.425 \\
\hline 13 & 7.83 & 0.034 & 0.446 & 0.089 & 0.047 & 0.582 & 0.616 & 0.052 & 39.9 & 0.17 & 115.384 \\
\hline 14 & 8.33 & 0.032 & 0.466 & 0.103 & 0.083 & 0.652 & 0.684 & 0.054 & 38.8 & 0.19 & 115.044 \\
\hline 15 & 8.83 & 0.033 & 0.530 & 0.096 & 0.093 & 0.719 & 0.753 & 0.048 & 36.1 & 0.21 & 115.106 \\
\hline 16 & 9.33 & 0.057 & 0.585 & 0.131 & 0.120 & 0.836 & 0.892 & 0.051 & 34.9 & 0.25 & 114.631 \\
\hline 17 & 9.82 & 0.050 & 0.635 & 0.143 & 0.076 & 0.854 & 0.904 & 0.060 & 32.9 & 0.25 & 114.547 \\
\hline 18 & 10.32 & 0.033 & 0.705 & 0.152 & 0.115 & 0.972 & 1.005 & 0.066 & 31.5 & 0.28 & 114.364 \\
\hline 19 & 10.82 & 0.048 & 0.728 & 0.143 & 0.139 & 1.010 & 1.058 & 0.065 & 29.4 & 0.30 & 114.114 \\
\hline 20 & 11.32 & 0.031 & 0.777 & 0.155 & 0.147 & 1.079 & 1.110 & 0.080 & 27.7 & 0.31 & 114.083 \\
\hline 21 & 11.83 & 0.026 & 0.871 & 0.174 & 0.180 & 1.225 & 1.251 & 0.080 & 26.9 & 0.35 & 113.597 \\
\hline 22 & 12.33 & 0.040 & 0.863 & 0.201 & 0.187 & 1.252 & 1.292 & 0.084 & 25.4 & 0.36 & 113.542 \\
\hline 23 & 12.83 & 0.039 & 1.006 & 0.201 & 0.164 & 1.371 & 1.410 & 0.086 & 25.1 & 0.40 & 113.355 \\
\hline 24 & 13.33 & 0.015 & 0.982 & 0.178 & 0.228 & 1.389 & 1.403 & 0.093 & 23.5 & 0.39 & 113.224 \\
\hline 25 & 13.83 & 0.048 & 1.042 & 0.198 & 0.230 & 1.470 & 1.517 & 0.100 & 22.7 & 0.43 & 112.763 \\
\hline 26 & 14.33 & 0.037 & 1.068 & 0.237 & 0.226 & 1.531 & 1.567 & 0.099 & 21.6 & 0.44 & 112.668 \\
\hline 27 & 14.83 & 0.044 & 1.098 & 0.218 & 0.244 & 1.561 & 1.604 & 0.110 & 20.5 & 0.45 & 112.627 \\
\hline 28 & 15.33 & 0.020 & 1.168 & 0.299 & 0.255 & 1.722 & 1.742 & 0.111 & 19.8 & 0.49 & 112.334 \\
\hline 29 & 15.82 & 0.037 & 1.225 & 0.245 & 0.261 & 1.730 & 1.767 & 0.120 & 18.9 & 0.50 & 112.257 \\
\hline 30 & 16.32 & 0.079 & 1.246 & 0.272 & 0.275 & 1.793 & 1.872 & 0.121 & 17.9 & 0.53 & 111.948 \\
\hline 31 & 16.82 & 0.046 & 1.279 & 0.294 & 0.287 & 1.860 & 1.906 & 0.130 & 17.4 & 0.54 & 111.779 \\
\hline 32 & 17.32 & 0.052 & 1.336 & 0.298 & 0.288 & 1.922 & 1.973 & 0.133 & 16.6 & 0.55 & 111.315 \\
\hline 33 & 17.82 & 0.027 & 1.401 & 0.288 & 0.308 & 1.998 & 2.024 & 0.135 & 16.2 & 0.57 & 111.354 \\
\hline 34 & 18.32 & 0.057 & 1.430 & 0.321 & 0.332 & 2.083 & 2.140 & 0.136 & 15.6 & 0.60 & 111.409 \\
\hline 35 & 18.82 & 0.044 & 1.494 & 0.330 & 0.331 & 2.155 & 2.200 & 0.147 & 14.8 & 0.62 & 110.918 \\
\hline 36 & 19.32 & 0.092 & 1.561 & 0.365 & 0.363 & 2.288 & 2.380 & 0.154 & 14.4 & 0.67 & 110.636 \\
\hline 37 & 19.83 & 0.037 & 1.625 & 0.382 & 0.356 & 2.364 & 2.400 & 0.171 & 13.9 & 0.67 & 110.664 \\
\hline 38 & 20.33 & 0.085 & 1.629 & 0.368 & 0.380 & 2.377 & 2.462 & 0.176 & 13.3 & 0.69 & 110.319 \\
\hline 39 & 20.83 & 0.072 & 1.702 & 0.373 & 0.407 & 2.482 & 2.554 & 0.172 & 12.5 & 0.72 & 110.185 \\
\hline 40 & 21.33 & 0.076 & 1.722 & 0.371 & 0.414 & 2.506 & 2.582 & 0.176 & 12.0 & 0.73 & 110.009 \\
\hline 41 & 21.83 & 0.107 & 1.781 & 0.393 & 0.448 & 2.622 & 2.729 & 0.187 & 11.4 & 0.77 & 109.822 \\
\hline 42 & 22.33 & 0.064 & 1.801 & 0.411 & 0.424 & 2.636 & 2.701 & 0.188 & 10.9 & 0.76 & 109.509 \\
\hline
\end{tabular}




\begin{tabular}{|c|c|c|c|c|c|c|c|c|c|c|c|}
\hline 43 & 22.83 & 0.079 & 1.839 & 0.426 & 0.474 & 2.739 & 2.818 & 0.196 & 10.5 & 0.79 & 109.384 \\
\hline 44 & 23.33 & 0.069 & 1.885 & 0.412 & 0.530 & 2.827 & 2.896 & 0.196 & 10.1 & 0.81 & 108.973 \\
\hline 45 & 23.83 & 0.082 & 1.924 & 0.457 & 0.507 & 2.887 & 2.969 & 0.212 & 9.8 & 0.83 & 108.927 \\
\hline 46 & 24.33 & 0.088 & 1.974 & 0.490 & 0.487 & 2.950 & 3.038 & 0.211 & 9.3 & 0.85 & 108.711 \\
\hline 47 & 24.83 & 0.071 & 2.075 & 0.469 & 0.522 & 3.067 & 3.137 & 0.208 & 9.0 & 0.88 & 108.553 \\
\hline 48 & 25.33 & 0.108 & 2.089 & 0.475 & 0.548 & 3.112 & 3.220 & 0.218 & 8.2 & 0.90 & 108.247 \\
\hline 49 & 25.83 & 0.099 & 2.105 & 0.523 & 0.547 & 3.176 & 3.275 & 0.218 & 8.2 & 0.92 & 108.077 \\
\hline 50 & 26.33 & 0.101 & 2.136 & 0.528 & 0.568 & 3.232 & 3.333 & 0.238 & 8.0 & 0.94 & 107.881 \\
\hline 51 & 26.83 & 0.091 & 2.199 & 0.524 & 0.567 & 3.290 & 3.381 & 0.225 & 7.5 & 0.95 & 107.738 \\
\hline 52 & 27.33 & 0.126 & 2.224 & 0.521 & 0.601 & 3.347 & 3.473 & 0.227 & 6.9 & 0.98 & 107.510 \\
\hline 53 & 27.82 & 0.121 & 2.275 & 0.575 & 0.619 & 3.469 & 3.589 & 0.232 & 6.6 & 1.01 & 107.298 \\
\hline 54 & 28.32 & 0.137 & 2.338 & 0.593 & 0.630 & 3.561 & 3.698 & 0.237 & 6.3 & 1.04 & 107.103 \\
\hline 55 & 28.82 & 0.115 & 2.358 & 0.586 & 0.655 & 3.599 & 3.714 & 0.241 & 6.1 & 1.04 & 106.929 \\
\hline 56 & 29.32 & 0.133 & 2.386 & 0.594 & 0.649 & 3.630 & 3.763 & 0.252 & 6.0 & 1.06 & 106.601 \\
\hline 57 & 29.82 & 0.108 & 2.461 & 0.617 & 0.705 & 3.783 & 3.891 & 0.252 & 5.6 & 1.09 & 106.416 \\
\hline 58 & 30.32 & 0.120 & 2.487 & 0.617 & 0.677 & 3.781 & 3.900 & 0.258 & 5.7 & 1.10 & 106.275 \\
\hline 59 & 30.82 & 0.165 & 2.538 & 0.639 & 0.702 & 3.880 & 4.045 & 0.261 & 5.2 & 1.14 & 106.017 \\
\hline 60 & 31.32 & 0.152 & 2.565 & 0.655 & 0.715 & 3.936 & 4.088 & 0.265 & 5.2 & 1.15 & 105.817 \\
\hline 61 & 31.83 & 0.134 & 2.603 & 0.719 & 0.745 & 4.068 & 4.202 & 0.269 & 4.9 & 1.18 & 105.613 \\
\hline 62 & 32.33 & 0.140 & 2.645 & 0.719 & 0.743 & 4.107 & 4.247 & 0.257 & 4.7 & 1.19 & 105.460 \\
\hline 63 & 32.83 & 0.142 & 2.664 & 0.702 & 0.771 & 4.137 & 4.279 & 0.278 & 4.5 & 1.20 & 105.155 \\
\hline 64 & 33.33 & 0.177 & 2.772 & 0.779 & 0.782 & 4.333 & 4.510 & 0.274 & 4.4 & 1.27 & 105.120 \\
\hline 65 & 33.83 & 0.175 & 2.786 & 0.764 & 0.798 & 4.348 & 4.523 & 0.286 & 4.4 & 1.27 & 104.820 \\
\hline 66 & 34.33 & 0.152 & 2.837 & 0.784 & 0.869 & 4.490 & 4.641 & 0.281 & 4.1 & 1.30 & 104.627 \\
\hline 67 & 34.83 & 0.192 & 2.885 & 0.797 & 0.871 & 4.554 & 4.746 & 0.295 & 4.0 & 1.33 & 104.436 \\
\hline 68 & 35.33 & 0.184 & 2.894 & 0.786 & 0.970 & 4.650 & 4.834 & 0.301 & 3.9 & 1.36 & 104.243 \\
\hline 69 & 35.82 & 0.167 & 2.928 & 0.817 & 0.992 & 4.737 & 4.904 & 0.317 & 3.8 & 1.38 & 103.937 \\
\hline 70 & 36.32 & 0.183 & 2.974 & 0.850 & 0.994 & 4.817 & 5.000 & 0.324 & 3.5 & 1.41 & 103.798 \\
\hline 71 & 36.82 & 0.182 & 2.998 & 0.879 & 1.040 & 4.917 & 5.098 & 0.316 & 3.5 & 1.43 & 103.625 \\
\hline 72 & 37.32 & 0.176 & 3.034 & 0.873 & 1.065 & 4.972 & 5.148 & 0.306 & 3.3 & 1.45 & 103.475 \\
\hline 73 & 37.82 & 0.201 & 3.051 & 0.883 & 1.042 & 4.977 & 5.178 & 0.342 & 3.3 & 1.46 & 103.310 \\
\hline 74 & 38.32 & 0.188 & 3.077 & 0.900 & 1.058 & 5.035 & 5.224 & 0.334 & 3.2 & 1.47 & 102.221 \\
\hline 75 & 38.82 & 0.203 & 3.150 & 0.984 & 1.054 & 5.189 & 5.392 & 0.334 & 2.9 & 1.52 & 102.792 \\
\hline 76 & 39.32 & 0.172 & 3.193 & 0.976 & 1.052 & 5.221 & 5.393 & 0.380 & 3.0 & 1.52 & 102.490 \\
\hline 77 & 39.82 & 0.193 & 3.232 & 0.944 & 1.075 & 5.251 & 5.443 & 0.376 & 2.8 & 1.53 & 102.333 \\
\hline 78 & 40.32 & 0.202 & 3.284 & 0.961 & 1.097 & 5.343 & 5.545 & 0.366 & 2.7 & 1.56 & 102.203 \\
\hline 79 & 40.82 & 0.194 & 3.302 & 0.964 & 1.142 & 5.407 & 5.601 & 0.387 & 2.7 & 1.57 & 101.900 \\
\hline 80 & 41.32 & 0.208 & 3.370 & 1.031 & 1.162 & 5.563 & 5.771 & 0.394 & 2.6 & 1.62 & 101.738 \\
\hline 81 & 41.82 & 0.220 & 3.417 & 1.051 & 1.191 & 5.658 & 5.879 & 0.388 & 2.6 & 1.65 & 101.596 \\
\hline 82 & 42.32 & 0.222 & 3.452 & 1.067 & 1.196 & 5.715 & 5.937 & 0.399 & 2.4 & 1.67 & 101.371 \\
\hline 83 & 42.82 & 0.233 & 3.502 & 1.096 & 1.234 & 5.832 & 6.066 & 0.364 & 2.5 & 1.70 & 101.195 \\
\hline 84 & 43.32 & 0.204 & 3.484 & 1.078 & 1.242 & 5.804 & 6.007 & 0.379 & 2.1 & 1.69 & 100.804 \\
\hline 85 & 43.83 & 0.242 & 3.541 & 1.111 & 1.279 & 5.930 & 6.173 & 0.368 & 2.2 & 1.73 & 100.691 \\
\hline 86 & 44.33 & 0.237 & 3.622 & 1.152 & 1.328 & 6.102 & 6.339 & 0.352 & 2.3 & 1.78 & 100.549 \\
\hline 87 & 44.83 & 0.234 & 3.654 & 1.215 & 1.358 & 6.227 & 6.460 & 0.362 & 2.0 & 1.82 & 100.315 \\
\hline
\end{tabular}




\begin{tabular}{|c|c|c|c|c|c|c|c|c|c|c|c|}
\hline 88 & 45.33 & 0.231 & 3.683 & 1.208 & 1.365 & 6.257 & 6.488 & 0.370 & 2.1 & 1.82 & 100.106 \\
\hline 89 & 45.83 & 0.237 & 3.689 & 1.224 & 1.394 & 6.308 & 6.544 & 0.389 & 2.1 & 1.84 & 99.835 \\
\hline 90 & 46.33 & 0.267 & 3.750 & 1.233 & 1.410 & 6.393 & 6.660 & 0.375 & 1.8 & 1.87 & 99.690 \\
\hline 91 & 46.83 & 0.251 & 3.767 & 1.245 & 1.439 & 6.451 & 6.701 & 0.392 & 1.8 & 1.88 & 99.360 \\
\hline 92 & 47.33 & 0.235 & 3.771 & 1.274 & 1.455 & 6.499 & 6.734 & 0.385 & 1.8 & 1.89 & 99.018 \\
\hline 93 & 47.83 & 0.259 & 3.812 & 1.303 & 1.489 & 6.604 & 6.863 & 0.396 & 1.7 & 1.93 & 98.780 \\
\hline 94 & 48.33 & 0.246 & 3.849 & 1.328 & 1.508 & 6.685 & 6.930 & 0.404 & 1.6 & 1.95 & 98.590 \\
\hline 95 & 48.83 & 0.256 & 3.863 & 1.286 & 1.537 & 6.685 & 6.941 & 0.398 & 1.5 & 1.95 & 98.215 \\
\hline 96 & 49.33 & 0.267 & 3.896 & 1.299 & 1.559 & 6.754 & 7.021 & 0.411 & 1.6 & 1.97 & 98.118 \\
\hline 97 & 49.83 & 0.293 & 3.944 & 1.330 & 1.568 & 6.842 & 7.135 & 0.410 & 1.5 & 2.01 & 97.978 \\
\hline 98 & 50.33 & 0.292 & 3.977 & 1.380 & 1.598 & 6.956 & 7.248 & 0.424 & 1.5 & 2.04 & 97.685 \\
\hline 99 & 50.83 & 0.253 & 3.947 & 1.347 & 1.625 & 6.920 & 7.173 & 0.449 & 1.5 & 2.02 & 97.496 \\
\hline 100 & 51.33 & 0.267 & 4.038 & 1.400 & 1.663 & 7.101 & 7.368 & 0.436 & 1.5 & 2.07 & 97.389 \\
\hline 101 & 51.83 & 0.268 & 4.093 & 1.439 & 1.649 & 7.181 & 7.449 & 0.433 & 1.4 & 2.09 & 97.155 \\
\hline 102 & 52.33 & 0.228 & 4.088 & 1.422 & 1.663 & 7.172 & 7.401 & 0.452 & 1.5 & 2.08 & 96.924 \\
\hline 103 & 52.83 & 0.283 & 4.145 & 1.470 & 1.664 & 7.279 & 7.562 & 0.451 & 1.4 & 2.13 & 96.729 \\
\hline 104 & 53.33 & 0.250 & 4.141 & 1.491 & 1.707 & 7.339 & 7.589 & 0.472 & 1.4 & 2.13 & 96.506 \\
\hline 105 & 53.83 & 0.282 & 4.216 & 1.503 & 1.753 & 7.472 & 7.754 & 0.427 & 1.4 & 2.18 & 96.277 \\
\hline 106 & 54.33 & 0.287 & 4.242 & 1.540 & 1.779 & 7.561 & 7.847 & 0.447 & 1.3 & 2.21 & 96.127 \\
\hline 107 & 54.83 & 0.297 & 4.282 & 1.556 & 1.808 & 7.646 & 7.943 & 0.430 & 1.3 & 2.23 & 95.918 \\
\hline 108 & 55.33 & 0.269 & 4.278 & 1.564 & 1.803 & 7.646 & 7.915 & 0.473 & 1.3 & 2.22 & 95.644 \\
\hline 109 & 55.83 & 0.270 & 4.342 & 1.592 & 1.833 & 7.767 & 8.037 & 0.434 & 1.4 & 2.26 & 95.508 \\
\hline 110 & 56.33 & 0.279 & 4.357 & 1.603 & 1.853 & 7.813 & 8.092 & 0.427 & 1.5 & 2.27 & 95.375 \\
\hline 111 & 56.83 & 0.314 & 4.425 & 1.678 & 1.933 & 8.036 & 8.350 & 0.460 & 1.2 & 2.35 & 95.053 \\
\hline 112 & 57.33 & 0.324 & 4.411 & 1.693 & 1.903 & 8.007 & 8.331 & 0.488 & 1.3 & 2.34 & 94.773 \\
\hline 113 & 57.83 & 0.314 & 4.455 & 1.711 & 1.956 & 8.122 & 8.435 & 0.488 & 1.1 & 2.37 & 94.628 \\
\hline 114 & 58.33 & 0.350 & 4.503 & 1.726 & 1.969 & 8.198 & 8.548 & 0.456 & 1.2 & 2.40 & 94.519 \\
\hline 115 & 58.83 & 0.353 & 4.562 & 1.759 & 2.010 & 8.331 & 8.685 & 0.457 & 1.1 & 2.44 & 94.366 \\
\hline 116 & 59.33 & 0.373 & 4.589 & 1.820 & 2.053 & 8.461 & 8.834 & 0.470 & 1.2 & 2.48 & 94.085 \\
\hline 117 & 59.83 & 0.374 & 4.609 & 1.831 & 2.071 & 8.511 & 8.885 & 0.487 & 1.2 & 2.50 & 93.810 \\
\hline 118 & 60.33 & 0.323 & 4.667 & 1.874 & 2.100 & 8.641 & 8.965 & 0.469 & 1.1 & 2.52 & 93.509 \\
\hline 119 & 60.83 & 0.321 & 4.696 & 1.902 & 2.137 & 8.734 & 9.056 & 0.476 & 1.3 & 2.54 & 93.301 \\
\hline 120 & 61.33 & 0.367 & 4.763 & 1.968 & 2.176 & 8.907 & 9.274 & 0.466 & 1.3 & 2.61 & 93.144 \\
\hline 121 & 61.83 & 0.334 & 4.748 & 1.955 & 2.204 & 8.906 & 9.240 & 0.503 & 1.0 & 2.60 & 92.868 \\
\hline 122 & 62.33 & 0.327 & 4.768 & 1.982 & 2.230 & 8.980 & 9.307 & 0.493 & 1.0 & 2.62 & 92.784 \\
\hline 123 & 62.83 & 0.319 & 4.768 & 1.976 & 2.243 & 8.987 & 9.306 & 0.481 & 1.1 & 2.62 & 92.592 \\
\hline 124 & 63.33 & 0.322 & 4.818 & 2.010 & 2.294 & 9.122 & 9.444 & 0.491 & 1.0 & 2.65 & 92.358 \\
\hline 125 & 63.82 & 0.356 & 4.877 & 2.045 & 2.310 & 9.232 & 9.587 & 0.483 & 1.1 & 2.69 & 92.237 \\
\hline 126 & 64.32 & 0.328 & 4.845 & 2.044 & 2.352 & 9.241 & 9.569 & 0.490 & 1.0 & 2.69 & 91.989 \\
\hline 127 & 64.82 & 0.325 & 4.887 & 2.088 & 2.393 & 9.368 & 9.693 & 0.516 & 0.9 & 2.72 & 91.743 \\
\hline 128 & 65.32 & 0.364 & 4.916 & 2.119 & 2.423 & 9.457 & 9.821 & 0.520 & 0.9 & 2.76 & 91.522 \\
\hline 129 & 65.82 & 0.358 & 4.942 & 2.119 & 2.445 & 9.506 & 9.864 & 0.531 & 1.0 & 2.77 & 91.278 \\
\hline 130 & 66.32 & 0.350 & 5.042 & 2.157 & 2.510 & 9.709 & 10.059 & 0.500 & 0.9 & 2.83 & 91.143 \\
\hline 131 & 66.82 & 0.375 & 5.027 & 2.134 & 2.491 & 9.653 & 10.028 & 0.541 & 1.0 & 2.82 & 90.853 \\
\hline 132 & 67.32 & 0.347 & 5.050 & 2.177 & 2.563 & 9.790 & 10.137 & 0.534 & 1.0 & 2.85 & 90.643 \\
\hline
\end{tabular}




\begin{tabular}{l|llllllllll|l}
133 & 67.83 & 0.325 & 5.039 & 2.161 & 2.538 & 9.739 & 10.064 & 0.563 & 1.0 & 2.83 & 90.469 \\
134 & 68.33 & 0.350 & 5.054 & 2.199 & 2.592 & 9.845 & 10.195 & 0.572 & 1.0 & 2.87 & 90.211 \\
135 & 68.83 & 0.392 & 5.136 & 2.276 & 2.622 & 10.034 & 10.426 & 0.556 & 1.3 & 2.93 & 90.038 \\
136 & 69.33 & 0.395 & 5.171 & 2.275 & 2.671 & 10.117 & 10.512 & 0.543 & 1.0 & 2.95 & 89.887 \\
137 & 69.83 & 0.350 & 5.213 & 2.334 & 2.697 & 10.244 & 10.593 & 0.544 & 1.0 & 2.98 & 89.716 \\
138 & 70.33 & 0.343 & 5.212 & 2.327 & 2.660 & 10.198 & 10.542 & 0.564 & 1.0 & 2.96 & 89.500 \\
139 & 70.83 & 0.368 & 5.215 & 2.358 & 2.714 & 10.287 & 10.655 & 0.564 & 1.0 & 2.99 & 89.203 \\
140 & 71.33 & 0.375 & 5.238 & 2.394 & 2.729 & 10.361 & 10.736 & 0.565 & 1.0 & 3.02 & 89.027 \\
141 & 71.83 & 0.361 & 5.278 & 2.421 & 2.771 & 10.470 & 10.831 & 0.560 & 1.1 & 3.04 & 88.842 \\
142 & 72.33 & 0.369 & 5.310 & 2.439 & 2.815 & 10.564 & 10.933 & 0.582 & 1.0 & 3.07 & 88.718 \\
\hline
\end{tabular}

${ }^{a} n_{\text {total butenes }}=n_{1 \text {-butene }}+n_{\text {cis-2-butene }}+n_{\text {trans-2-butene. }}{ }^{b} n_{\text {total olefins }}=n_{1 \text {-butene }}+n_{\text {cis-2-butene }}+n_{\text {trans-2-butene }}+n_{\text {propene. }}{ }^{c}$ Percentage of compound 1 present in solution determined from the ${ }^{1} \mathrm{H}$ resonances of the coordinated $p$-cymene. ${ }^{d}$ Moles of total olefins produced $\left(n_{\text {total olefins }}\right)$ divided by the initial molar number of $\mathbf{1}$. 
Table S7. Results obtained with $1,0.58$ equiv. $\mathrm{PCy}_{3}$ and ethylene $(79 \mu \mathrm{mol})$ at $323.0 \mathrm{~K}$ in $\mathrm{C}_{6} \mathrm{D}_{6}$.

\begin{tabular}{|c|c|c|c|c|c|c|c|c|c|c|c|}
\hline entry & $\begin{array}{c}t \\
(\mathrm{~h})\end{array}$ & $\begin{array}{l}n_{\text {propene }} \\
(\mu \mathrm{mol})\end{array}$ & $\begin{array}{l}n_{1 \text {-butene }} \\
(\mu \mathrm{mol})\end{array}$ & $\begin{array}{c}n_{\text {cis-2- }} \\
\text { butene } \\
(\mu \mathrm{mol})\end{array}$ & $\begin{array}{c}n_{\text {trans-2- }} \\
\text { butene } \\
(\mu \mathrm{mol})\end{array}$ & $\begin{array}{c}n_{\text {total butenes }}{ }^{a} \\
(\mu \mathrm{mol})\end{array}$ & $\begin{array}{c}n_{\text {total olefins }}^{b} \\
(\mu \mathrm{mol})\end{array}$ & $\begin{array}{c}n_{\text {ethane }} \\
(\mu \mathrm{mol})\end{array}$ & $\begin{array}{c}1^{c} \\
(\%)\end{array}$ & turnovers $^{d}$ & $\begin{array}{l}n_{\text {ethylene }} \\
(\mu \mathrm{mol})\end{array}$ \\
\hline 1 & 0.00 & 0.000 & 0.000 & 0.000 & 0.000 & 0.000 & 0.000 & 0.011 & 68.4 & 0.00 & 78.541 \\
\hline 2 & 0.43 & 0.007 & 0.124 & 0.028 & 0.029 & 0.181 & 0.188 & 0.034 & 27.9 & 0.05 & 78.052 \\
\hline 3 & 1.07 & 0.052 & 0.358 & 0.048 & 0.036 & 0.441 & 0.493 & 0.099 & 7.1 & 0.12 & 76.334 \\
\hline 4 & 1.51 & 0.091 & 0.516 & 0.055 & 0.074 & 0.645 & 0.736 & 0.137 & 1.4 & 0.17 & 75.137 \\
\hline 5 & 2.01 & 0.126 & 0.678 & 0.090 & 0.089 & 0.856 & 0.982 & 0.179 & 0.4 & 0.24 & 74.051 \\
\hline 6 & 2.51 & 0.179 & 0.854 & 0.105 & 0.137 & 1.096 & 1.275 & 0.236 & 0.2 & 0.31 & 72.988 \\
\hline 7 & 3.01 & 0.235 & 0.991 & 0.123 & 0.146 & 1.260 & 1.495 & 0.242 & 0.0 & 0.37 & 72.077 \\
\hline 8 & 3.51 & 0.322 & 1.099 & 0.141 & 0.187 & 1.427 & 1.749 & 0.255 & 0.0 & 0.42 & 71.228 \\
\hline 9 & 4.01 & 0.387 & 1.220 & 0.193 & 0.200 & 1.612 & 1.999 & 0.285 & 0.0 & 0.49 & 70.418 \\
\hline 10 & 4.51 & 0.471 & 1.330 & 0.225 & 0.271 & 1.826 & 2.297 & 0.323 & 0.0 & 0.57 & 69.621 \\
\hline 11 & 5.01 & 0.579 & 1.386 & 0.224 & 0.295 & 1.905 & 2.484 & 0.319 & 0.0 & 0.60 & 68.781 \\
\hline 12 & 5.51 & 0.681 & 1.535 & 0.298 & 0.394 & 2.227 & 2.908 & 0.347 & 0.0 & 0.72 & 67.999 \\
\hline 13 & 6.01 & 0.823 & 1.587 & 0.328 & 0.445 & 2.360 & 3.183 & 0.365 & 0.0 & 0.78 & 67.188 \\
\hline 14 & 6.51 & 0.932 & 1.688 & 0.389 & 0.535 & 2.612 & 3.544 & 0.398 & 0.0 & 0.88 & 66.504 \\
\hline 15 & 7.01 & 1.067 & 1.756 & 0.446 & 0.620 & 2.822 & 3.889 & 0.473 & 0.0 & 0.96 & 65.649 \\
\hline 16 & 7.51 & 1.179 & 1.777 & 0.485 & 0.659 & 2.921 & 4.100 & 0.464 & 0.0 & 1.01 & 64.828 \\
\hline 17 & 8.01 & 1.338 & 1.829 & 0.538 & 0.786 & 3.153 & 4.491 & 0.506 & 0.0 & 1.10 & 63.965 \\
\hline 18 & 8.51 & 1.433 & 1.872 & 0.604 & 0.903 & 3.379 & 4.813 & 0.578 & 0.0 & 1.19 & 63.097 \\
\hline 19 & 9.01 & 1.561 & 1.918 & 0.698 & 1.022 & 3.637 & 5.198 & 0.607 & 0.0 & 1.28 & 62.255 \\
\hline 20 & 9.51 & 1.678 & 1.921 & 0.760 & 1.125 & 3.807 & 5.485 & 0.600 & 0.0 & 1.35 & 61.445 \\
\hline 21 & 10.01 & 1.794 & 1.952 & 0.800 & 1.196 & 3.949 & 5.743 & 0.585 & 0.0 & 1.41 & 60.593 \\
\hline 22 & 10.51 & 1.938 & 1.933 & 0.871 & 1.304 & 4.108 & 6.046 & 0.623 & 0.0 & 1.47 & 59.798 \\
\hline 23 & 11.01 & 2.042 & 1.971 & 0.957 & 1.398 & 4.326 & 6.367 & 0.641 & 0.0 & 1.56 & 58.996 \\
\hline 24 & 11.51 & 2.176 & 2.020 & 1.084 & 1.611 & 4.715 & 6.891 & 0.707 & 0.0 & 1.69 & 58.185 \\
\hline 25 & 12.01 & 2.268 & 2.042 & 1.160 & 1.737 & 4.940 & 7.207 & 0.731 & 0.0 & 1.78 & 57.426 \\
\hline 26 & 12.51 & 2.410 & 2.024 & 1.238 & 1.865 & 5.128 & 7.537 & 0.768 & 0.0 & 1.85 & 56.680 \\
\hline 27 & 13.01 & 2.513 & 2.047 & 1.337 & 2.022 & 5.405 & 7.918 & 0.738 & 0.0 & 1.95 & 55.994 \\
\hline 28 & 13.51 & 2.629 & 1.925 & 1.350 & 2.066 & 5.341 & 7.970 & 0.782 & 0.0 & 1.95 & 55.286 \\
\hline 29 & 14.01 & 2.740 & 1.923 & 1.418 & 2.216 & 5.557 & 8.297 & 0.824 & 0.0 & 2.03 & 54.576 \\
\hline 30 & 14.51 & 2.826 & 1.863 & 1.450 & 2.292 & 5.605 & 8.431 & 0.884 & 0.0 & 2.06 & 53.892 \\
\hline 31 & 15.01 & 2.925 & 1.867 & 1.540 & 2.433 & 5.841 & 8.766 & 0.904 & 0.0 & 2.14 & 53.234 \\
\hline 32 & 15.51 & 3.022 & 1.849 & 1.597 & 2.577 & 6.023 & 9.046 & 0.894 & 0.0 & 2.21 & 52.598 \\
\hline 33 & 16.01 & 3.177 & 1.821 & 1.652 & 2.671 & 6.145 & 9.322 & 0.938 & 0.0 & 2.26 & 51.984 \\
\hline 34 & 16.51 & 3.218 & 1.788 & 1.700 & 2.799 & 6.287 & 9.505 & 0.992 & 0.0 & 2.31 & 51.367 \\
\hline 35 & 17.01 & 3.307 & 1.795 & 1.786 & 2.921 & 6.501 & 9.809 & 0.986 & 0.0 & 2.39 & 50.804 \\
\hline 36 & 17.51 & 3.428 & 1.792 & 1.857 & 3.065 & 6.714 & 10.142 & 1.017 & 0.0 & 2.47 & 50.237 \\
\hline 37 & 18.01 & 3.504 & 1.736 & 1.883 & 3.142 & 6.761 & 10.264 & 1.059 & 0.0 & 2.50 & 49.676 \\
\hline 38 & 18.51 & 3.584 & 1.790 & 1.941 & 3.100 & 6.831 & 10.416 & 1.090 & 0.0 & 2.53 & 49.138 \\
\hline 39 & 19.01 & 3.688 & 1.805 & 2.008 & 3.189 & 7.002 & 10.690 & 1.070 & 0.0 & 2.61 & 49.030 \\
\hline 40 & 19.51 & 3.766 & 1.832 & 2.105 & 3.363 & 7.301 & 11.067 & 1.131 & 0.0 & 2.70 & 48.141 \\
\hline 41 & 20.01 & 3.872 & 1.821 & 2.149 & 3.468 & 7.437 & 11.309 & 1.133 & 0.0 & 2.75 & 47.658 \\
\hline 42 & 20.51 & 3.973 & 1.738 & 2.136 & 3.601 & 7.475 & 11.448 & 1.145 & 0.0 & 2.78 & 47.436 \\
\hline
\end{tabular}




\begin{tabular}{|c|c|c|c|c|c|c|c|c|c|c|c|}
\hline 43 & 21.01 & 4.067 & 1.756 & 2.215 & 3.739 & 7.710 & 11.777 & 1.190 & 0.0 & 2.86 & 46.974 \\
\hline 44 & 21.51 & 4.156 & 1.682 & 2.204 & 3.722 & 7.608 & 11.764 & 1.223 & 0.0 & 2.83 & 46.409 \\
\hline 45 & 22.01 & 4.234 & 1.700 & 2.272 & 3.834 & 7.806 & 12.040 & 1.228 & 0.0 & 2.90 & 45.970 \\
\hline 46 & 22.51 & 4.302 & 1.674 & 2.300 & 3.928 & 7.903 & 12.204 & 1.264 & 0.0 & 2.94 & 45.540 \\
\hline 47 & 23.01 & 4.373 & 1.686 & 2.345 & 4.023 & 8.053 & 12.426 & 1.273 & 0.0 & 3.00 & 45.125 \\
\hline 48 & 23.51 & 4.426 & 1.670 & 2.399 & 4.120 & 8.190 & 12.616 & 1.290 & 0.0 & 3.05 & 44.715 \\
\hline 49 & 24.01 & 4.490 & 1.653 & 2.426 & 4.200 & 8.279 & 12.769 & 1.315 & 0.0 & 3.09 & 44.322 \\
\hline 50 & 24.51 & 4.545 & 1.609 & 2.446 & 4.193 & 8.248 & 12.793 & 1.340 & 0.0 & 3.08 & 43.939 \\
\hline 51 & 25.01 & 4.626 & 1.584 & 2.470 & 4.270 & 8.324 & 12.950 & 1.374 & 0.0 & 3.11 & 43.552 \\
\hline 52 & 25.51 & 4.717 & 1.536 & 2.470 & 4.302 & 8.308 & 13.025 & 1.419 & 0.0 & 3.12 & 43.181 \\
\hline 53 & 26.01 & 4.807 & 1.530 & 2.497 & 4.393 & 8.420 & 13.227 & 1.445 & 0.0 & 3.16 & 42.827 \\
\hline 54 & 26.51 & 4.884 & 1.515 & 2.527 & 4.454 & 8.496 & 13.380 & 1.462 & 0.0 & 3.19 & 42.475 \\
\hline 55 & 27.01 & 4.901 & 1.523 & 2.602 & 4.501 & 8.627 & 13.528 & 1.511 & 0.0 & 3.23 & 42.131 \\
\hline 56 & 27.51 & 4.969 & 1.504 & 2.606 & 4.565 & 8.675 & 13.644 & 1.539 & 0.0 & 3.25 & 41.765 \\
\hline 57 & 28.01 & 5.016 & 1.484 & 2.618 & 4.634 & 8.735 & 13.751 & 1.578 & 0.0 & 3.28 & 41.448 \\
\hline 58 & 28.51 & 5.059 & 1.478 & 2.644 & 4.687 & 8.809 & 13.869 & 1.604 & 0.0 & 3.31 & 41.125 \\
\hline 59 & 29.01 & 5.131 & 1.516 & 2.710 & 4.785 & 9.011 & 14.142 & 1.592 & 0.0 & 3.38 & 40.832 \\
\hline 60 & 29.51 & 5.139 & 1.435 & 2.686 & 4.814 & 8.934 & 14.073 & 1.643 & 0.0 & 3.36 & 40.516 \\
\hline 61 & 30.01 & 5.227 & 1.435 & 2.726 & 4.877 & 9.038 & 14.266 & 1.672 & 0.0 & 3.40 & 40.225 \\
\hline 62 & 30.51 & 5.267 & 1.427 & 2.742 & 4.941 & 9.110 & 14.377 & 1.688 & 0.0 & 3.43 & 39.920 \\
\hline 63 & 31.01 & 5.300 & 1.448 & 2.782 & 5.022 & 9.252 & 14.552 & 1.692 & 0.0 & 3.48 & 39.641 \\
\hline 64 & 31.51 & 5.359 & 1.428 & 2.806 & 5.068 & 9.302 & 14.661 & 1.738 & 0.0 & 3.51 & 39.360 \\
\hline 65 & 32.01 & 5.385 & 1.422 & 2.847 & 5.167 & 9.436 & 14.821 & 1.725 & 0.0 & 3.56 & 39.088 \\
\hline 66 & 32.51 & 5.486 & 1.397 & 2.852 & 5.204 & 9.453 & 14.938 & 1.758 & 0.0 & 3.57 & 38.822 \\
\hline 67 & 33.01 & 5.517 & 1.428 & 2.908 & 5.289 & 9.624 & 15.141 & 1.761 & 0.0 & 3.63 & 38.567 \\
\hline 68 & 33.51 & 5.547 & 1.364 & 2.890 & 5.308 & 9.562 & 15.109 & 1.789 & 0.0 & 3.62 & 38.288 \\
\hline 69 & 34.01 & 5.655 & 1.391 & 2.813 & 5.309 & 9.513 & 15.167 & 1.813 & 0.0 & 3.62 & 38.148 \\
\hline 70 & 34.51 & 5.635 & 1.410 & 2.837 & 5.363 & 9.609 & 15.244 & 1.826 & 0.0 & 3.66 & 37.903 \\
\hline 71 & 35.01 & 5.682 & 1.416 & 2.865 & 5.434 & 9.715 & 15.397 & 1.833 & 0.0 & 3.70 & 37.654 \\
\hline 72 & 35.51 & 5.743 & 1.384 & 2.859 & 5.467 & 9.710 & 15.453 & 1.794 & 0.0 & 3.71 & 37.408 \\
\hline 73 & 36.01 & 5.778 & 1.399 & 2.907 & 5.546 & 9.851 & 15.629 & 1.819 & 0.0 & 3.76 & 37.187 \\
\hline 74 & 36.51 & 5.836 & 1.406 & 2.937 & 5.597 & 9.941 & 15.777 & 1.826 & 0.0 & 3.79 & 36.956 \\
\hline 75 & 37.01 & 5.851 & 1.401 & 2.945 & 5.650 & 9.995 & 15.847 & 1.820 & 0.0 & 3.82 & 36.726 \\
\hline 76 & 37.51 & 5.929 & 1.423 & 2.988 & 5.725 & 10.136 & 16.065 & 1.860 & 0.0 & 3.87 & 36.507 \\
\hline 77 & 38.01 & 5.992 & 1.426 & 3.022 & 5.785 & 10.233 & 16.225 & 1.849 & 0.0 & 3.90 & 36.286 \\
\hline 78 & 38.51 & 6.016 & 1.260 & 3.034 & 5.825 & 10.119 & 16.135 & 1.914 & 0.0 & 3.85 & 36.048 \\
\hline 79 & 39.01 & 6.023 & 1.301 & 3.050 & 5.878 & 10.229 & 16.253 & 1.925 & 0.0 & 3.92 & 35.855 \\
\hline 80 & 39.51 & 6.067 & 1.274 & 3.051 & 5.892 & 10.217 & 16.284 & 1.945 & 0.0 & 3.93 & 35.645 \\
\hline 81 & 40.01 & 6.133 & 1.258 & 3.061 & 5.928 & 10.247 & 16.381 & 1.956 & 0.0 & 3.94 & 35.438 \\
\hline 82 & 40.51 & 6.132 & 1.279 & 3.069 & 5.953 & 10.302 & 16.434 & 1.990 & 0.0 & 3.96 & 35.245 \\
\hline 83 & 41.01 & 6.146 & 1.288 & 3.101 & 6.031 & 10.420 & 16.566 & 1.995 & 0.0 & 4.00 & 35.044 \\
\hline 84 & 41.51 & 6.217 & 1.295 & 3.117 & 6.066 & 10.477 & 16.695 & 2.003 & 0.0 & 4.03 & 34.853 \\
\hline 85 & 42.01 & 6.235 & 1.252 & 3.155 & 6.133 & 10.541 & 16.775 & 2.047 & 0.0 & 4.05 & 34.658 \\
\hline 86 & 42.51 & 6.277 & 1.243 & 3.173 & 6.184 & 10.600 & 16.878 & 2.059 & 0.0 & 4.08 & 34.471 \\
\hline 87 & 43.01 & 6.290 & 1.244 & 3.193 & 6.214 & 10.651 & 16.941 & 2.074 & 0.0 & 4.10 & 34.284 \\
\hline
\end{tabular}




\begin{tabular}{l|llllllllll|l}
88 & 43.51 & 6.386 & 1.235 & 3.191 & 6.248 & 10.675 & 17.060 & 2.105 & 0.0 & 4.12 & 34.106 \\
89 & 44.01 & 6.451 & 1.219 & 3.178 & 6.258 & 10.654 & 17.105 & 2.156 & 0.0 & 4.11 & 33.914 \\
90 & 44.51 & 6.490 & 1.250 & 3.214 & 6.311 & 10.775 & 17.265 & 2.152 & 0.0 & 4.16 & 33.740 \\
91 & 45.01 & 6.548 & 1.233 & 3.217 & 6.346 & 10.796 & 17.344 & 2.162 & 0.0 & 4.17 & 33.563 \\
92 & 45.51 & 6.553 & 1.220 & 3.215 & 6.364 & 10.799 & 17.352 & 2.186 & 0.0 & 4.18 & 33.393 \\
93 & 46.01 & 6.636 & 1.233 & 3.240 & 6.414 & 10.887 & 17.523 & 2.209 & 0.0 & 4.21 & 33.220 \\
94 & 46.51 & 6.631 & 1.195 & 3.231 & 6.425 & 10.851 & 17.482 & 2.241 & 0.0 & 4.20 & 33.047 \\
95 & 47.01 & 6.728 & 1.179 & 3.261 & 6.522 & 10.961 & 17.689 & 2.286 & 0.0 & 4.25 & 32.555 \\
\hline
\end{tabular}

${ }^{a} n_{\text {total butenes }}=n_{1 \text {-butene }}+n_{\text {cis-2-butene }}+n_{\text {trans-2-butene. }}{ }^{b} n_{\text {total olefins }}=n_{1 \text {-butene }}+n_{\text {cis-2-butene }}+n_{\text {trans-2-butene }}+n_{\text {propene. }}{ }^{c}$ Percentage of compound $\mathbf{1}$ present in solution determined from the ${ }^{1} \mathrm{H}$ resonances of the coordinated $p$-cymene. ${ }^{d}$ Moles of total olefins produced ( $\left.n_{\text {total olefins }}\right)$ divided by the initial molar number of $\mathbf{1}$.

Table S8. Amount of GIIm obtained with 1, 0.58 equiv. $\mathrm{PCy}_{3}$ and ethylene $(79 \mu \mathrm{mol})$ at $323.0 \mathrm{~K}$ in $\mathrm{C}_{6} \mathrm{D}_{6}$.

\begin{tabular}{|c|c|c|c|c|c|c|c|c|}
\hline entry & $\begin{array}{c}t \\
\text { (h) }\end{array}$ & $\begin{array}{c}n_{\text {GIIm }} \\
(\mu \mathrm{mol})\end{array}$ & entry & $\begin{array}{c}t \\
\text { (h) }\end{array}$ & $\begin{array}{c}n_{\mathrm{GIIm}} \\
(\mu \mathrm{mol})\end{array}$ & entry & $\begin{array}{c}t \\
\text { (h) }\end{array}$ & $\begin{array}{c}n_{\mathrm{GII}} \\
(\mu \mathrm{mol})\end{array}$ \\
\hline 1 & 0.00 & 0.000 & 33 & 16.01 & 8.184 & 65 & 32.01 & 4.555 \\
\hline 2 & 0.43 & 0.000 & 34 & 16.51 & 7.976 & 66 & 32.51 & 4.479 \\
\hline 3 & 1.07 & 0.000 & 35 & 17.01 & 7.919 & 67 & 33.01 & 4.139 \\
\hline 4 & 1.51 & 0.000 & 36 & 17.51 & 7.730 & 68 & 33.51 & 4.045 \\
\hline 5 & 2.01 & 0.000 & 37 & 18.01 & 7.466 & 69 & 34.01 & 4.082 \\
\hline 6 & 2.51 & 1.682 & 38 & 18.51 & 7.390 & 70 & 34.51 & 3.572 \\
\hline 7 & 3.01 & 3.667 & 39 & 19.01 & 7.390 & 71 & 35.01 & 3.497 \\
\hline 8 & 3.51 & 5.179 & 40 & 19.51 & 7.239 & 72 & 35.51 & 3.308 \\
\hline 9 & 4.01 & 6.464 & 41 & 20.01 & 6.880 & 73 & 36.01 & 3.251 \\
\hline 10 & 4.51 & 7.522 & 42 & 20.51 & 6.747 & 74 & 36.51 & 3.364 \\
\hline 11 & 5.01 & 8.600 & 43 & 21.01 & 6.691 & 75 & 37.01 & 3.232 \\
\hline 12 & 5.51 & 9.299 & 44 & 21.51 & 6.558 & 76 & 37.51 & 3.119 \\
\hline 13 & 6.01 & 9.904 & 45 & 22.01 & 6.464 & 77 & 38.01 & 3.156 \\
\hline 14 & 6.51 & 10.244 & 46 & 22.51 & 6.407 & 78 & 38.51 & 3.062 \\
\hline 15 & 7.01 & 10.357 & 47 & 23.01 & 6.350 & 79 & 39.01 & 3.100 \\
\hline 16 & 7.51 & 10.244 & 48 & 23.51 & 6.143 & 80 & 39.51 & 2.911 \\
\hline 17 & 8.01 & 10.206 & 49 & 24.01 & 6.124 & 81 & 40.01 & 2.684 \\
\hline 18 & 8.51 & 10.130 & 50 & 24.51 & 6.010 & 82 & 40.51 & 2.627 \\
\hline 19 & 9.01 & 10.036 & 51 & 25.01 & 5.708 & 83 & 41.01 & 2.400 \\
\hline 20 & 9.51 & 9.941 & 52 & 25.51 & 5.840 & 84 & 41.51 & 2.419 \\
\hline 21 & 10.01 & 9.847 & 53 & 26.01 & 5.708 & 85 & 42.01 & 2.778 \\
\hline 22 & 10.51 & 9.752 & 54 & 26.51 & 5.708 & 86 & 42.51 & 2.363 \\
\hline 23 & 11.01 & 9.620 & 55 & 27.01 & 5.311 & 87 & 43.01 & 2.306 \\
\hline 24 & 11.51 & 9.431 & 56 & 27.51 & 5.349 & 88 & 43.51 & 2.400 \\
\hline 25 & 12.01 & 9.356 & 57 & 28.01 & 5.330 & 89 & 44.01 & 2.363 \\
\hline 26 & 12.51 & 9.185 & 58 & 28.51 & 5.122 & 90 & 44.51 & 2.155 \\
\hline 27 & 13.01 & 9.053 & 59 & 29.01 & 4.990 & 91 & 45.01 & 2.136 \\
\hline 28 & 13.51 & 9.053 & 60 & 29.51 & 4.820 & 92 & 45.51 & 2.155 \\
\hline 29 & 14.01 & 8.978 & 61 & 30.01 & 4.763 & 93 & 46.01 & 2.098 \\
\hline 30 & 14.51 & 8.807 & 62 & 30.51 & 4.631 & 94 & 46.51 & 1.928 \\
\hline 31 & 15.01 & 8.656 & 63 & 31.01 & 4.649 & 95 & 47.01 & 2.003 \\
\hline 32 & 15.51 & 8.335 & 64 & 31.51 & 4.649 & & & \\
\hline
\end{tabular}


Table S9. Results obtained with 1, 1.2 equiv. $\mathrm{PCy}_{3}$ and ethylene $(84.3 \mu \mathrm{mol})$ at $323.0 \mathrm{~K}$ in $\mathrm{C}_{6} \mathrm{D}_{6}$.

\begin{tabular}{|c|c|c|c|c|c|c|c|c|c|c|c|}
\hline entry & $\begin{array}{c}t \\
\text { (h) }\end{array}$ & $\begin{array}{l}n_{\text {propene }} \\
(\mu \mathrm{mol})\end{array}$ & $\begin{array}{l}n_{1 \text {-butene }} \\
(\mu \mathrm{mol})\end{array}$ & $\begin{array}{c}n_{\text {cis-2- }} \\
\text { butene } \\
(\mu \mathrm{mol})\end{array}$ & $\begin{array}{c}n_{\text {trans-2- }} \\
\text { butene } \\
(\mu \mathrm{mol})\end{array}$ & $\begin{array}{c}n_{\text {total butenes }}^{a} \\
(\mu \mathrm{mol})\end{array}$ & $\begin{array}{c}n_{\text {total olefins }}{ }^{b} \\
(\mu \mathrm{mol})\end{array}$ & $\begin{array}{c}n_{\text {ethane }} \\
(\mu \mathrm{mol})\end{array}$ & $\begin{array}{l}1^{c} \\
(\%)\end{array}$ & turnovers $^{d}$ & $\begin{array}{l}n_{\text {ethylene }} \\
(\mu \mathrm{mol})\end{array}$ \\
\hline 1 & 0.00 & 0.000 & 0.000 & 0.000 & 0.000 & 0.000 & 0.000 & 0.000 & 71.8 & 0 & 84.346 \\
\hline 2 & 47.25 & 0.534 & 2.092 & 2.278 & 3.730 & 8.101 & 8.634 & 1.230 & 0.0 & 2.43 & 55.762 \\
\hline
\end{tabular}

present in solution determined from the ${ }^{1} \mathrm{H}$ resonances of the coordinated $p$-cymene. ${ }^{d}$ Moles of total olefins produced $\left(n_{\text {total olefins }}\right)$ divided by the initial molar number of $\mathbf{1}$. 


\section{Computational Part}

\section{Computational Methods}

Molecular structures were built, visualized, analyzed, and edited via the combined use of ChemAxon's Marvin package (version 6.2.1), ${ }^{23}$ UCSF Chimera (version 1.11) ${ }^{24}$ and Molden (version 5.0). ${ }^{25}$ When needed, conformational searches and preliminary geometry relaxations were performed with Spartan $16^{26}$ using its implementation of Merck's force field (MMFF94) ${ }^{27}$ and of the semi-empirical method PM6, ${ }^{28}$ usually coupled with manually set geometrical constraints in the surrounding of the metal center. Finally, all the DFT calculations described below, were performed with version d 01 of the Gaussian 09 suite of programs. $^{29}$

\section{Geometry Optimization}

Molecular geometries were optimized using Head-Gordon's long-range- and dispersion-corrected hybrid density functional $\omega \mathrm{B} 97 \mathrm{XD} .{ }^{30}$ The geometries produced by this functional for ruthenium-based olefin metathesis catalysts and other homogeneous catalysts are in good agreement with those from X-ray diffraction. ${ }^{31}$ All elements but ruthenium were described by Dunning's correlation-consistent valence double- $\zeta$ plus polarization basis sets (cc-pVDZ) $)^{32,33}$ obtained from the EMSL basis set exchange database. ${ }^{34,35}$ Ruthenium atoms were described by combining the Stuttgart 28-electron relativistic effective core potential (ECP28MDF ${ }^{36}$ retrieved from the Stuttgart/Cologne Group website) ${ }^{37}$ with the correlationconsistent valence double- $\zeta$ plus polarization basis set (cc-pVDZ-PP) ${ }^{36}$ obtained from the EMSL basis set exchange database. ${ }^{34,35}$ Numerical integration was performed using Gaussian's "ultrafine" grid for the SCF energies and the gradients, whereas the "finegrid" was used for CPHF analytical Hessian calculations. All electronic states were checked for internal instability before optimizing the geometry. Instable solutions were re-optimized to a real, spin-restricted or unrestricted solution for spin singlet or triplet states, respectively. Next, geometries were optimized using tight convergence criteria (max. force $1.5 \cdot 10^{-5}$, RMS force $1.0 \cdot 10^{-5}$, max. displacement $6.0 \cdot 10^{-5}$, RMS displacement $4.0 \cdot 10^{-5}$ ), without symmetry constraints, using default convergence criteria for the self-consistent field (SCF) procedure (RMS change in density matrix $<1.0 \cdot 10^{-8}$, max. change in density matrix $=1.0 \cdot 10^{-6}$ ). A sample input file is provided below (see page 131).

The eigenvalues of the analytically calculated Hessian matrix were used to characterize each stationary point, confirming only a single (for transition states) or no imaginary frequency (for minima).

Thermal corrections to free energies were obtained from the analytical Hessian matrices calculated at the geometry optimization level of theory. The translational, rotational, and vibrational components of the thermal corrections to enthalpies and Gibbs free energies were calculated within the ideal-gas, rigid-rotor, and harmonic oscillator approximations, except that all frequencies below $100 \mathrm{~cm}^{-1}$ were shifted to 100 $\mathrm{cm}^{-1}$ when calculating the vibrational component of the entropy. This approach prevents the asymptotic behavior of the harmonic approximation with very low-frequency modes, and is known as the quasiharmonic oscillator approximation. ${ }^{38,39}$

\section{Minimum Energy Crossing Points}

Geometries corresponding to minimum energy crossing points (MECPs) were located along the seam of crossing between spin-singlet and spin-triplet surfaces using Gaussian via the interface program developed by Harvey and co-workers (version: November 2009). ${ }^{40}$ All parameters defining the DFT model were the same as described above for general geometry optimization. The convergence criterion for the electronic energy gradient was set to $1.0 \cdot 10^{-4}$ a.u., and the energy difference between the two spin state energy calculations was required to be lower than 0.00050 a.u.. The curvature along the seam was confirmed by the eigenvalues of state-average Hessian matrix obtained by the Glowfreq program (version November2015). ${ }^{41}$ 


\section{Intrinsic Reaction Coordinate Calculations}

The minimum energy reaction paths descending from the most important transition states were explored, in Gaussian, using intrinsic reaction coordinate (IRC) calculations, thereby confirming the connection between such transition states and the enveloping local minima. All IRC calculations used the initial force constants obtained analytically at the end of the transition-state optimization and were required to compute the force constants at every point ("CalcAll" option). The algorithm used for the predictor step was, in the majority of the calculations, the local quadratic approximation (LQA) ${ }^{42,43}$ With the present DFT model, the LQA algorithm was found to often outperform the Hessian-based predictor-corrector integrator (HPC) ${ }^{44,45}$ method in tracing the IRCs. We also explored different step sizes, seeking compromises between accuracy and computational cost. The step size thus varied from case to case. The case-specific details are given in the IRC figure captions.

Several IRC calculations terminated prematurely, with the gradient falling below the convergence criteria. In other words, the reaction paths often encountered flat regions on the potential energy surfaces (PESs). Despite this flatness, the convergence criteria for local minimum optimization were most of the time not satisfied and the IRC calculations were typically restarted. These restarts were performed by taking a step in the direction of the imaginary mode. For cases where this procedure still did not escape the flat region, the last geometry on the prematurely terminated IRC path was used as a starting point for a geometry optimization. Small maximum optimization step sizes (MaxStep between 1 and 5) were used in the latter optimizations to ensure convergence to the closest local minima.

\section{Single-Point (SP) Energy Calculations}

The energy of each stationary point was calculated using a generalized gradient approximation (GGA) functional (PBE) developed by Perdew, Burke and Ernzerhof ${ }^{46}$ in combination with Grimme's D3 empirical dispersion term with Becke-Johnson (BJ) damping. ${ }^{47}$ When corrected for basis set superposition errors (BSSE) using the counterpoise method, this combination of functional and empirical dispersion gave the best overall accuracy in a validation study of density functionals for ruthenium-catalyzed olefin metathesis. $^{48}$ The PBE-D3BJ combination is expected to perform well also in the present study of ruthenium-mediated reactions related to olefin metathesis, and the large single-point (SP) basis sets (see below) should eliminate the need for BSSE corrections and contribute to accurate relative energies. The BJ damping parameters used were those of Smith and co-workers, which were shown to increase the accuracy substantially compared to the original parameters. ${ }^{49}$ Electrostatic and non-electrostatic solvation effects in benzene were accounted for by using the polarizable continuum model (PCM) in combination with the "Dis", "Rep", and "Cav" keywords. ${ }^{50-52}$ The solvent, i.e., benzene, was described by the built-in value for the dielectric constant, and the solute cavity was constructed using the united atom topological model with atomic radii optimized for Hartree-Fock (keyword "UAHF"). For brevity this computational model is labeled PBE-D3MBJ-PCM in the following.

To estimate the sensitivity of the relative energies to changes of the computational model, we also report SP energies, calculated at the same geometries, using a meta-GGA functional (M06L) ${ }^{53}$ and a hybrid meta-GGA (M06) ${ }^{54} \mathrm{We}$ are thus able to compare results from functionals belonging to three different rungs of Jacob's ladder ${ }^{55}$ and to assess the robustness of the relative energies with respect to changing the density functional (cf., section Sensitivity to the DFT Method). Comparison with the M06L results appears to be particularly relevant, since this functional partly was designed for transition-metal chemistry $^{54}$ and has been validated for ruthenium-catalyzed olefin metathesis. ${ }^{48,56}$ The M06 and M06L functionals were combined with PCM-calculated solvent corrections as described above, thus giving overall computational models labeled M06-PCM and M06L-PCM, respectively.

Each of the computational models PBE-D3MBJ-PCM, M06-PCM, and M06L-PCM was coupled with the same basis sets for SP calculations. Ruthenium was described by the ECP28MDF relativistic effective core potential ${ }^{36}$ accompanied by a correlation-consistent valence quadruple- $\zeta$ plus polarization basis set (ECP28MDF_VQZ) $)^{36}$ obtained from the Stuttgart/Cologne Group website. ${ }^{37}$ Carbon and hydrogen atoms were described by valence quadruple- $\zeta$ plus polarization (cc-pVQZ) ${ }^{32}$ obtained from the EMSL basis sets 
exchange website. ${ }^{34,35}$ For all other atoms, the augmented valence quadruple- $\zeta$ plus polarization (aug-ccpVQZ) ${ }^{32,33,57}$ basis sets were taken from the EMSL basis set repository. ${ }^{34,35}$

Finally, numerical integrations were performed with the "ultrafine" grid of Gaussian 09, the selfconsistent field (SCF) density-based convergence criterion was set to RMS $<1.0 \cdot 10^{-5}$ and maximum change $<1.0 \cdot 10^{-3}$. A sample input file is provided below (see page 136).

\section{Calculation of Gibbs Free Energies}

Gibbs free energies for model X, where X = PBE-D3MBJ-PCM, M06-PCM, and M06L-PCM, were calculated at $298.15 \mathrm{~K}$ according to Equation $\mathrm{S} 2$

$$
G_{X}^{C_{6} H_{6}}=E_{X}^{C_{6} H_{6}}+\Delta G_{\omega B 97 X D, q h}^{T=298.15 K}+\Delta G_{1 a t m \rightarrow 1 M}^{T=298.15 K}
$$

where $E_{X}^{C_{6} H_{6}}$ is the SP energy including corrections from implicit solvation and, if applicable, also dispersion corrections; $\Delta G_{\omega B 97 X D, q h}^{T=298}$ is the thermal correction to give the Gibbs free energy, calculated at the geometry optimization level using the quasi-harmonic approximation, and $\Delta G_{1 \mathrm{~atm} \rightarrow 1 \mathrm{M}}^{T=298.15 \mathrm{~K}}$ is the standard state correction corresponding to $1 \mathrm{M}$ solution (but exhibiting infinite-dilution, ideal-gas-like behavior), which is equal to $1.89 \mathrm{kcal} \mathrm{mol}^{-1}\left(=\mathrm{RT} \cdot \ln (24.46)=3.019 \cdot 10^{-3}\right.$ a.u.). The resulting Gibbs free energies relative to M1 (i.e., $\Delta G_{X}^{C_{6} H_{6}}$ ) are collected in Table S10 (page S104) of the Computational Data section. Unless otherwise stated, schemes report the relative free energy as obtained using the PBE-D3MBJ-PCM model.

\section{Variational Transition States}

Dissociative elementary reaction steps (i.e., A-B $\rightarrow$ A + B) that require small or no geometrical strain sometimes do not involve enthalpic barriers on the PES. In such cases, attempts to explore the dissociation of the two fragments typically lead to minimum energy pathways with monotonously increasing potential energy, with the barrier corresponding to complete separation of the two fragments A and B. However, even without a maximum on the PES, the free energy surface along such elementary reactions in solution may still be associated with a maximum corresponding to a variational transition state (TS $\mathbf{T S A R}_{\mathbf{V A R}}$ ). The free energy of the latter can be estimated by reversing the Eyring equation using a typical rate constant for diffusion-controlled reactions. ${ }^{58}$

For instance, the free energy barrier for dissociation of $p$-cymene from M1 has been estimated by adding $4.4 \mathrm{kcal} / \mathrm{mol}$ (due to diffusion control) to (at $298 \mathrm{~K}$ ) free energy of the dissociated system. ${ }^{1}$ We here apply the same approximation in all case in which no barrier could be found on the PES of dissociative steps.

\section{Minimum Energy Crossing Points}

The free energy of each MECP was calculated by i) taking the average of the SP energy calculated at the MECP geometry with each spin multiplicity, ii) taking the average of the thermochemical corrections obtained for the two minima on either side of the spin-crossing seam, and, to account for a lower-than-1 spin-crossing probability, ${ }^{59}$ iii) adding a penalty $\left(\Delta G_{p \text { Xover }}\right)$ that Schneider and co-workers have evaluated to $1-5 \mathrm{kcal} / \mathrm{mol} .^{60}$ To summarize, the effective free energy range for an MECP was calculated at $298.15 \mathrm{~K}$ for a DFT model X, where $\mathrm{X}=$ PBE-D3MBJ-PCM, M06-PCM, and M06L-PCM, as:

$$
G_{X}^{C_{6} H_{6}}=\bar{E}_{X}^{C_{6} H_{6}}+\overline{\Delta G}_{\omega B 97 X D, q h}^{T=298.15 K}+\Delta G_{p X o v e r}+\Delta G_{1 a t m \rightarrow 1 M}^{T=298.15 K}
$$

where:

$$
\bar{E}_{X}^{C_{6} H_{6}}=\frac{E(S=0)_{X}^{C_{6} H_{6}}+E(S=1)_{X}^{C_{6} H_{6}}}{2}
$$

where $E(S=0)_{X}^{C_{6} H_{6}}$ and $E(S=1)_{X}^{C_{6} H_{6}}$ are the SP energies calculated for the MECP geometry, with singlet and triplet spin-state multiplicity, respectively (values in Table S11, page S114), and

$$
\overline{\Delta G}_{\omega B 97 X D, q h}^{T=298.15 K}=\frac{\Delta G(o p t, S=0))_{\omega B 97 X D, q h}^{T=298.15 K}+\Delta G(o p t, S=1)_{\omega B 97 X D, q h}^{T=298.15 K}}{2}
$$


where $\Delta G(o p t, S=0)_{\omega B 97 X D, q h}^{T=298.15}$ and $\Delta G(o p t, S=1)_{\omega B 97 X D, q h}^{T=298.15}$ are the thermal corrections to the Gibbs free energy calculated at the geometry optimization level with the quasi-harmonic approximation for the two geometry optimized minima on the spin-singlet and spin-triplet surface, respectively (values in Table $\mathrm{S} 10$, page S104). The resulting relative free energy ranges calculated with respect to $\mathbf{M} 1$ are collected in Table S12 (page S114).

\section{Calculation of Enthalpies}

Enthalpies for model X, where X = PBE-D3MBJ-PCM, M06-PCM, and M06L-PCM, were calculated at 298.15 K according to Equation S6

$$
H_{X}^{C_{6} H_{6}}=E_{X}^{C_{6} H_{6}}+\Delta H_{\omega B}^{T=298.15 K}+\Delta H_{1 a t m \rightarrow 1 M}^{T=298.15 K}
$$

where $E_{X}^{C_{6} H_{6}}$ is the SP energy including corrections from implicit solvation and, if applicable, also dispersion corrections; $\Delta H_{\omega B}^{T=298.15 K}$ is the thermal correction to the enthalpy, calculated at the geometry optimization level using the quasi-harmonic approximation, and $\Delta H_{1 a t m \rightarrow 1 M}^{T=298.15 K}$ is the standard state correction corresponding to $1 \mathrm{M}$ solution (but exhibiting infinite-dilution, ideal-gas-like behavior), which is equal to $1.89 \mathrm{kcal} \mathrm{mol}^{-1}\left(=\mathrm{RT} \cdot \ln (24.46)=3.019 \cdot 10^{-3}\right.$ a.u.). The resulting enthalpies relative to M1 (i.e., $\Delta H_{X}^{C_{6} H_{6}}$ ) are collected in Table S10 (page S104) of the Computational Data section.

\section{Reaction Mechanisms}

The computational exploration of the reaction mechanisms focused on the processes involving NHCbound $\mathrm{Ru}$ complexes that could be derived from 1 (represented by the molecular model M1, see Scheme S5). In particular, we explored alternative activation pathways for 1 (i.e., model M1) that led to replacement of the $p$-cymene ligand with ethylene $(\mathbf{M 1} \rightarrow \mathbf{M 1 8}$ in Scheme S5), possibly involving also cyclometallation of the NHC ligand $(\mathbf{M 1} \rightarrow \mathbf{M} 24 \rightarrow \mathbf{M 1 8})$. Next, we searched for pathways leading to Rucatalyzed transformation of ethylene into 1-butene, trans- and cis-2-butene, and considered the isomerization of these $\mathrm{C} 4$ products. $\mathrm{Ru}$ (II)-catalyzed hydrogenation of ethylene to ethane was also modeled. Finally, we considered the generation of methyl-substituted metallacyclobutane M303 from various intermediates along the reaction paths mentioned above, and the consequential access to alkylidenes and other olefin metathesis-related species that were detected experimentally (M310 and M313). Each of these reactions is described in detail in dedicated sections below.

As described in the main paper, the role of $\mathrm{PCy}_{3}$ has, in the computational investigation, been limited to that of "trapping" alkylidenes such as GIIm. This is motivated by the same organic products being formed with and without $\mathrm{PCy}_{3} .1$ and $\mathbf{2}$ (the $\mathrm{PCy}_{3}$ analogue) give the same kinds of products, but $\mathbf{1}$ has a richer reactivity, and this reactivity is the one studied in the following.

Finally, we here consider the energies resulting from the DFT method PBE-D3MBJ-PCM. Discussions about the sensitivity of the mechanistic results on the DFT method used to calculate the relative free energies are collected in a dedicated section (see page 100). 


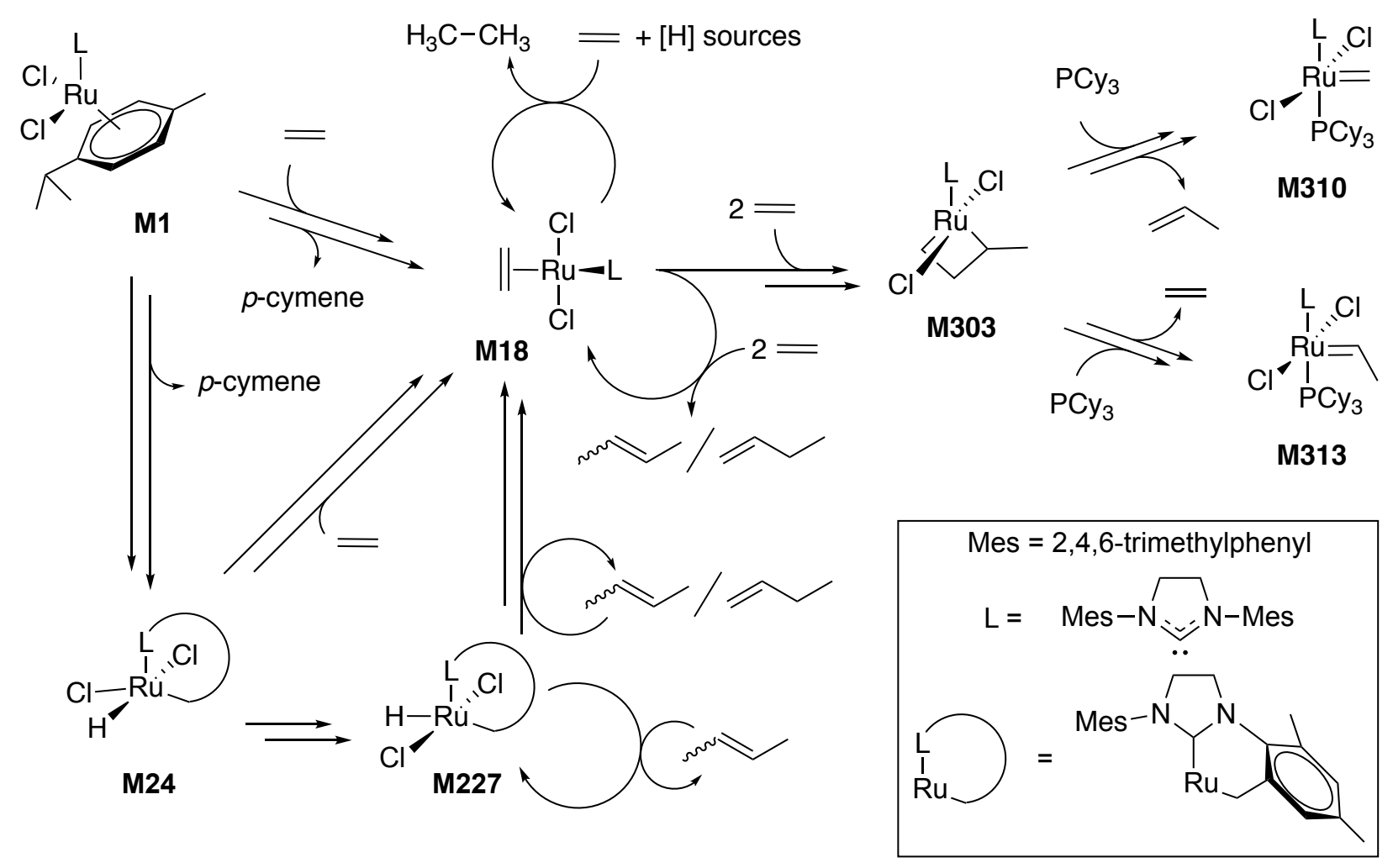

Scheme S5. Concise overview of the reaction network.

Whereas Scheme S5 offers a brief summary of the reactions modelled in this study, Scheme S6 provides a more detailed map of the reaction network. The network starts from model M1 that represents the experimental $p$-cymene complex 1, and that was built starting from the published crystallographic structure. ${ }^{1}$ The reaction network was divided into sections (labelled $P \#$ ) to facilitate the identification and referencing of different portions of the vast and complex reaction network. 


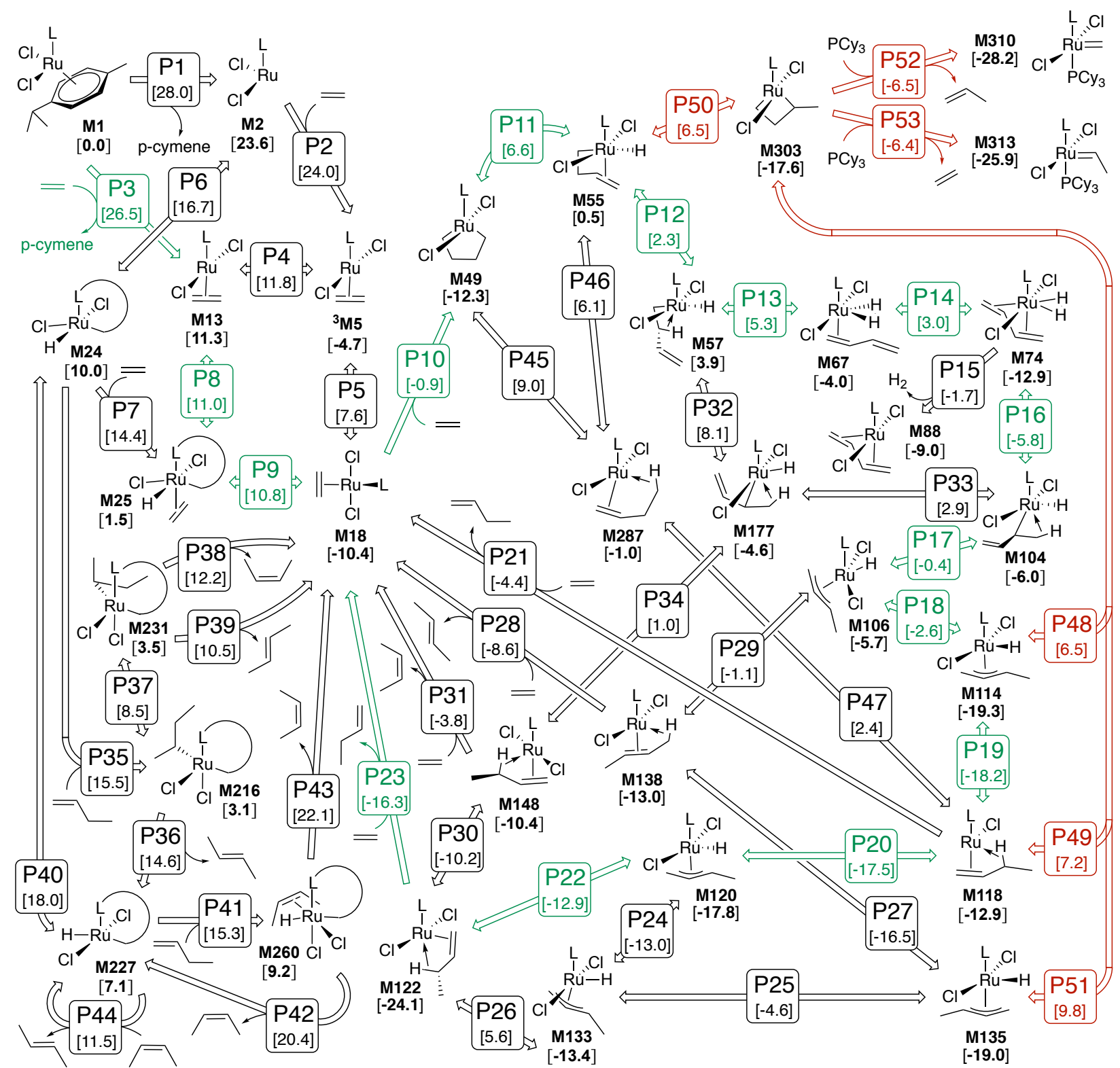

Scheme S6. Overview of the reaction network explored in this contribution. Values between square brackets represent free energies ( $\mathrm{kcal} / \mathrm{mol}$ with respect to M1). Arrows represent sections (a.k.a. parcels, and labelled "P\#") of the reaction network that contain all the steps needed to connect the species at either end of the arrow, in addition to the pathways of potential side reactions. Each arrow is labelled with section name $(\mathrm{P} \#)$ and the highest free energy (relative to M1) encountered along that connection. The pathway leading to 1-butene is highlighted in green, the one leading to alkylidenes in red.

\section{Activation of M1: Exchange of $p$-Cymene with Ethylene}

M1 is an 18-electron complex with no vacant coordination site on the metal. Therefore, any reaction involving the central metal of M1 and ethylene should involve dissociation of a ligand or reduction of hapticity. The most labile of the ligand in M1 is expected to be $p$-cymene, in agreement with previous work on similar $p$-cymene complexes of $\mathrm{Ru}^{61}$ The direct dissociation of $p$-cymene (section P1 in Scheme S7) was investigated computationally in previous work ${ }^{1}$ and was found to proceed via a pathway for which no pronounced transition state could be found on the potential energy surface. The estimation of the free energy barrier from the variational transition state ( $\mathbf{T S}_{\mathbf{V A R}}$, see Computational Methods section) with the present PBE-D3MBJ-PCM model produces a barrier of $28 \mathrm{kcal} / \mathrm{mol}$ from M1. After dissociation of $p$ cymene, the resulting 12-electron species $\mathbf{M} 2$ can gain stability by crossing to the spin-triplet surface via 
the minimum energy crossing point (MECP) M3 to yield ${ }^{3} \mathbf{M} 4$ (the superscript is used in figures and text to indicate an electronic state with spin multiplicity different from one. For instance, model ${ }^{3} \mathbf{M} 4$ has spintriplet state). The solvent used for the experiments, i.e., benzene- $d_{6}$, may bind $\mathrm{Ru}$ in and $\eta^{6}$ fashion. Thus, electron deficient Ru centers $\mathbf{M} 2$ and ${ }^{3} \mathbf{M} 4$ are expected to be short-lived. Benzene should be more labile than $p$-cymene owing to its lower electron donation capability, and is most likely exchanged back via pathways that are likely very similar to those described here for $p$-cymene. ${ }^{3} \mathbf{M} 4$ can eventually bind ethylene trans to the NHC to form the square planar ${ }^{3}$ M5. The cis approach is much less stable than the trans one, as highlighted by the energy of spin-triplet cis analogue ${ }^{3} \mathbf{M 1 6}$ (see Scheme S8).
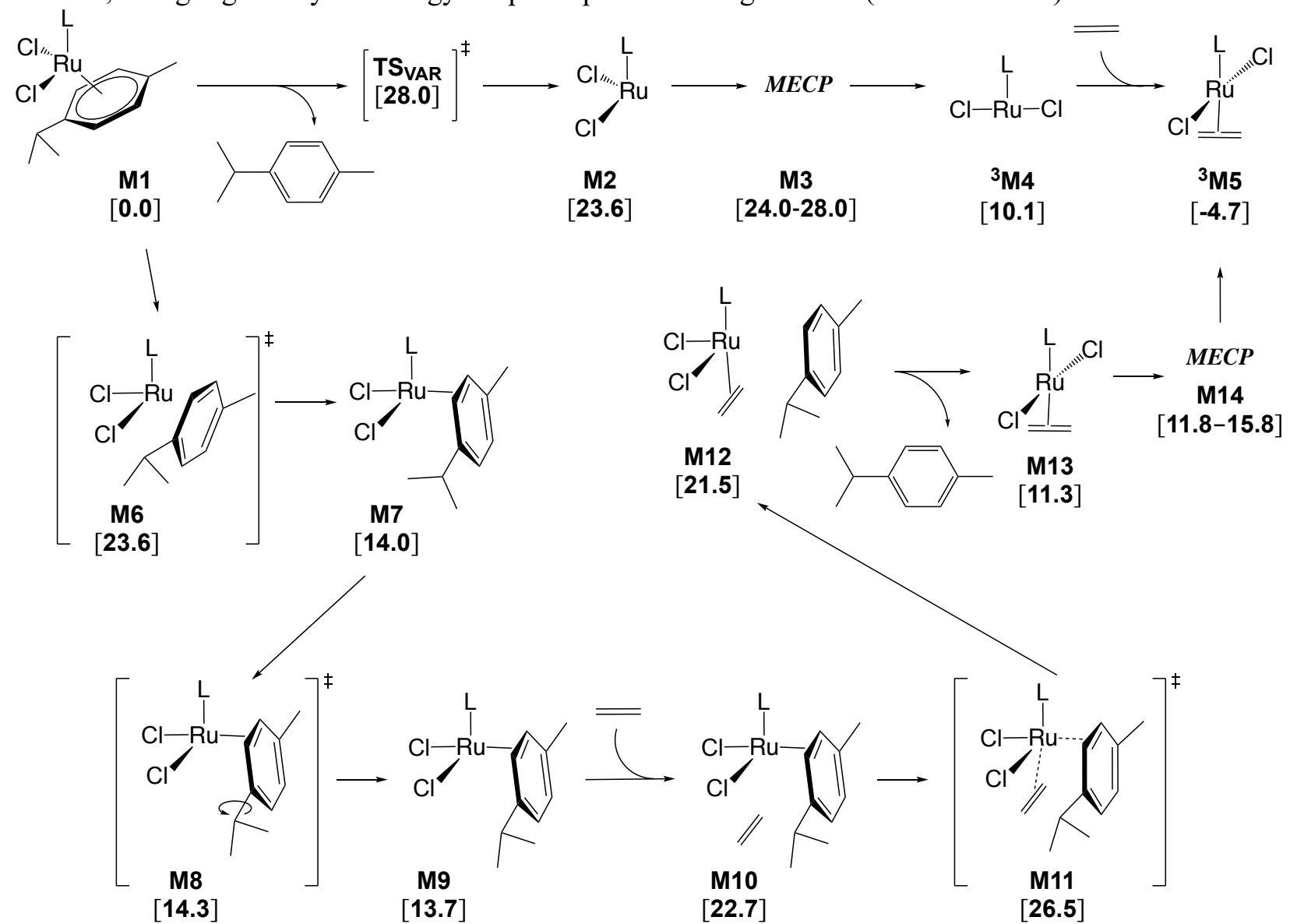

Scheme S7. Free energies (kcal/mol with respect to M1) of species along sections P1 (M1-M2), P2 (M2- ${ }^{3}$ M5), P3 (M1-M13), and P4 (M13- ${ }^{3}$ M5) of the reaction network. The superscript in ${ }^{3} \mathbf{M} 4$ and ${ }^{3} \mathbf{M 5}$ indicates spin triplet state.

${ }^{3} \mathbf{M 5}$ can be generated also via an associative interchange type of mechanism for the exchange of $p$ cymene with ethylene (section P3 and P4 of the reaction network, Scheme S7). Along this pathway the hapticity of $p$-cymene is reduced from $\eta^{6}$ of $\mathbf{M 1}$ to $\eta^{2}$ of $\mathbf{M} 7$. In this way a vacant coordination site becomes available for uptake of ethylene. According to the PBE-D3MBJ-PCM model, the barrier for the reduction of hapticity $(23.6 \mathrm{kcal} / \mathrm{mol}$ at M6) and those about conformational adaptation (M8) and binding of ethylene (M11) are smaller than the estimate of the variational transition state leading to M2. This model favors the interchange over the dissociative pathway, but this result is highly sensitive to the DFT model used to calculate the relative free energies (see dedicated section on page 100).

The square planar ${ }^{3} \mathbf{M 5}$ can rearrange to reduce the angle between the NHC and the $\eta^{2}$-ethylene ligand (section P5 in Scheme S8). While the result is a loss of stability $\left({ }^{3} \mathbf{M 1 6}\right.$ is more than $10 \mathrm{kcal} / \mathrm{mol}$ less stable than ${ }^{3}$ M5), this change allows facile electron pairing via MECP M17 and thus geometrical relaxation into the spin singlet $\mathbf{M 1 8}$. The latter has a saw-horse geometry with the $\eta^{2}$-ethylene cis to the NHC. This geometry avoids the trans influence between ethylene and NHC, and is ca. $6 \mathrm{kcal} / \mathrm{mol}$ more 
stable than ${ }^{3} \mathbf{M 5}$, and ca. $10 \mathrm{kcal} / \mathrm{mol}$ more stable than M1. Species M18 is the most stable Ru-ethylene complex found, and is expected to be more stable than any benzene-based analogue of M1 owing to the better orbital overlap between the dative ligand and the metal combined with the lack of trans influence.

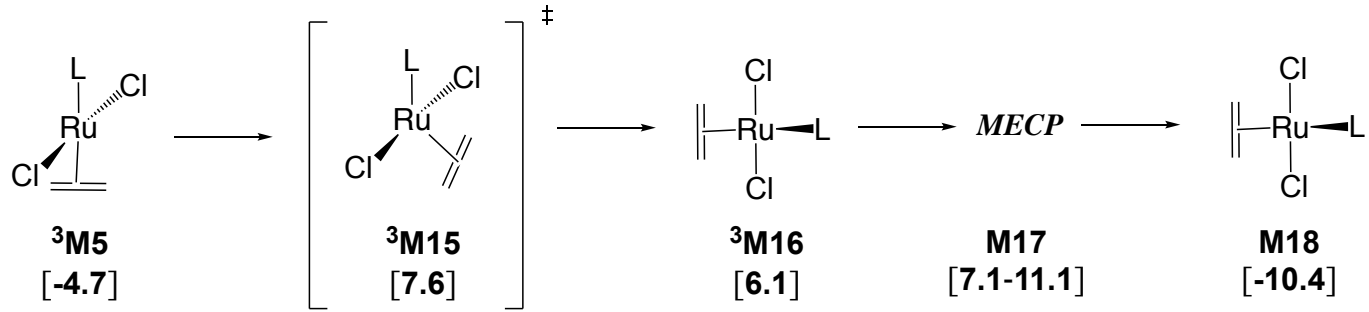

Scheme S8. Free energies ( $\mathrm{kcal} / \mathrm{mol}$ with respect to M1) of species along section P5 of the reaction network. The superscript in ${ }^{3}$ M5, ${ }^{3}$ M15 and ${ }^{3}$ M16 indicates spin triplet state.

The dissociative pathway described in Scheme S7 yields the electron-deficient (12-electrons) species M2 that, in alternative to rearranging the electronic state to cross into the spin-triplet surface (via MECP M3), can perform an intramolecular $\mathrm{C}-\mathrm{H}$ activation of any one of the close-by methyl groups from the NHC ligand (see Scheme S9). Such cyclometallation occurs after barrier-less formation of the hapto-two interaction in $\mathbf{M 2 0}$ via transition state M19, formation of a $\mathrm{C}-\mathrm{H}$ agostic interaction in $\mathbf{M 2 2}$, and finally rupture of the $\mathrm{C}-\mathrm{H}$ bond at transition state $\mathbf{M 2 3}$ with formation of Ru-hydride M24. The three transition states have energy significantly lower than that of MECP M3. Thus, within the PBE-D3MBJ-PCM model, the cyclometallation is preferred over the spin-crossover pathway presented above. As reported before, ${ }^{1}$ the resulting hydride (M24) contributes explaining the isomerization of olefinic compounds by double bond migration and cis-trans isomerization (see below). However, since the most abundant olefin in the present system, especially at the beginning of the reaction, is ethylene, hydride M24 is most likely coordinating this olefin to form M25 (Scheme S9). No potential energy barrier was found for this step, so we report the variational estimate for the transition state free energy. Notably, the ethylene-coordinated complex M25 can be obtained easily also by cyclometallation of ethylene-bound complex M13 (Scheme S9).

The sole energetically feasible reaction found for M25 is the insertion leading to alkyl complex M32 (see Scheme S10). The conformational flexibility of the ethyl ligand creates some complexity and various possibilities for further reactivity. In fact, two completely different isomerization pathways were found to reach the lowest energy, cyclometallated alkyl complex M34: either directly via M33, or via M35-M39. The direct pathway via transition state $\mathbf{M 3 3}$ is about $8 \mathrm{kcal} / \mathrm{mol}$ less demanding of the walkaround via M35. The resulting cyclometallated ethyl complex M34 is characterized by a cis relation between the ethyl ligand and either ends of the cyclometallated NHC ligand. This reduces the interaction between the trans-influencing ligands and explains the stability of this configuration with respect to the known alternatives M32 and M36. In addition, the conformation of M34 places the methyl end of the ethyl ligand relatively close to the metal center and properly oriented to proceed with the formal return of a hydride to the cyclometallated end of the NHC ligand. This occurs via formation of the $\mathrm{C}-\mathrm{H}$ agostic complex M41 and direct transfer of the hydride from the agostic feature onto the mesityl group. The corresponding transition state, i.e., M42, sees also a concerted change of configuration that brings one of the chloride ligands cis to the NHC ligand. The resulting $\eta^{2}$-ethylene complex, i.e., M43, has an intact NHC ligand and can easily isomerize via the configurational changes in M44 and M46 leading to the lowest energy configuration M18, that was previously identified as the result of pathways involving non-cyclometallated Ru species.

To summarize this section, these DFT calculations identify as the lowest energy pathway the dissociative release of $p$-cymene followed by intramolecular cyclometallation leading to Ru-hydride $\mathbf{M 2 4}$ that reacts with ethylene to generate a $\eta^{2}$-ethylene complex with an intact (i.e., non-cyclometallated) NHC ligand. With energies provided by the PBE-D3MBJ-PCM model, this pathway is less demanding than the two other alternatives presented above. Namely, the interchange of $p$-cymene with ethylene, and the 
dissociative release of $p$-cymene followed by unpairing of electrons to form the 12-electon, spin-triplet species ${ }^{3} \mathbf{M} 4$.
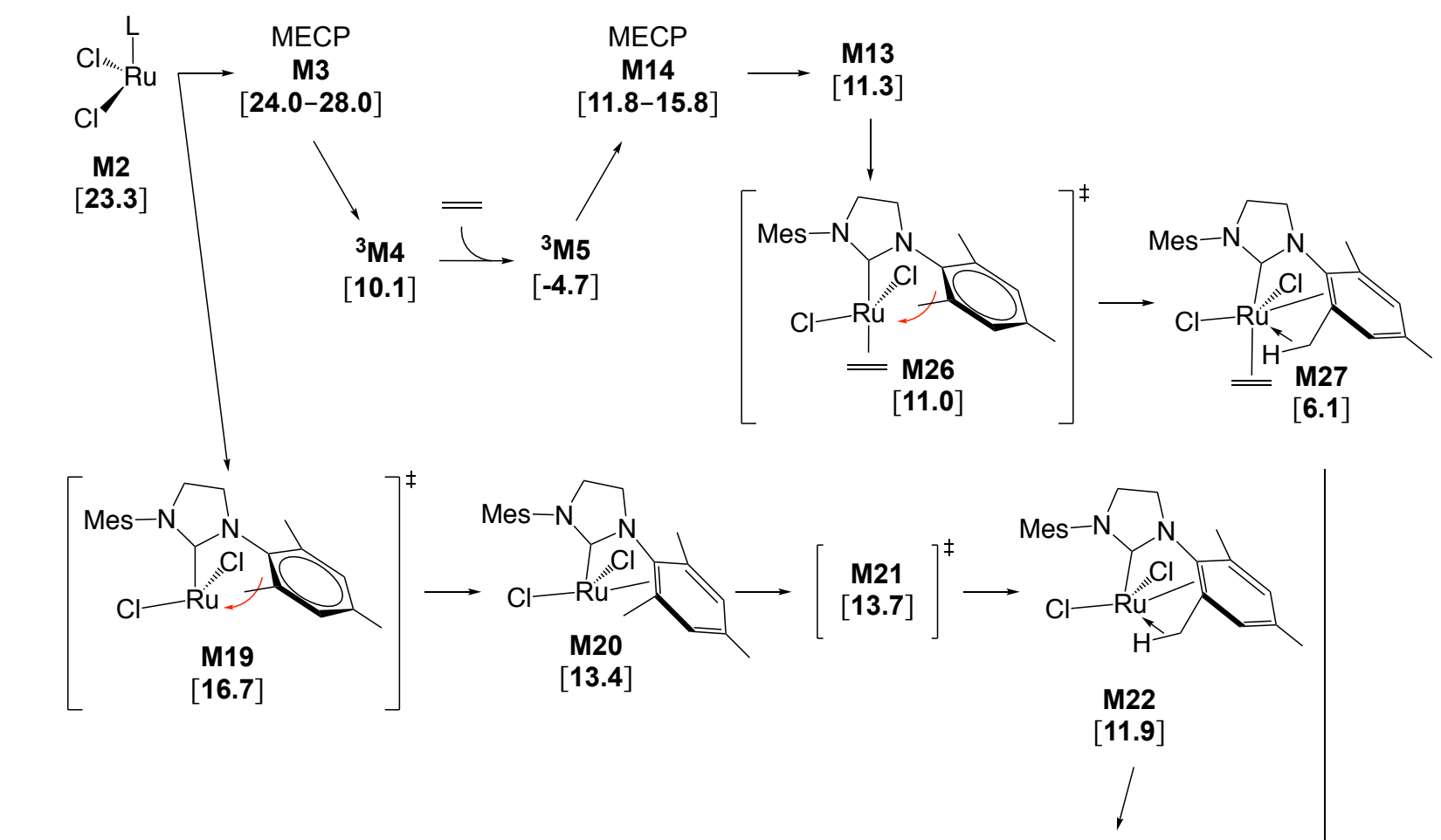

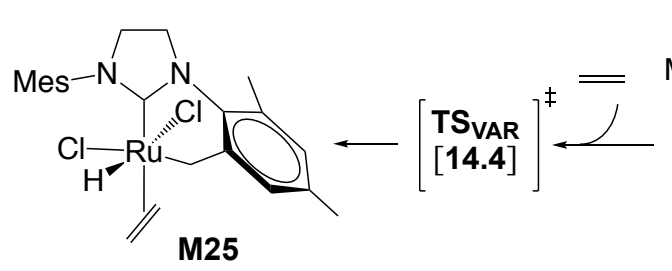

[1.5]
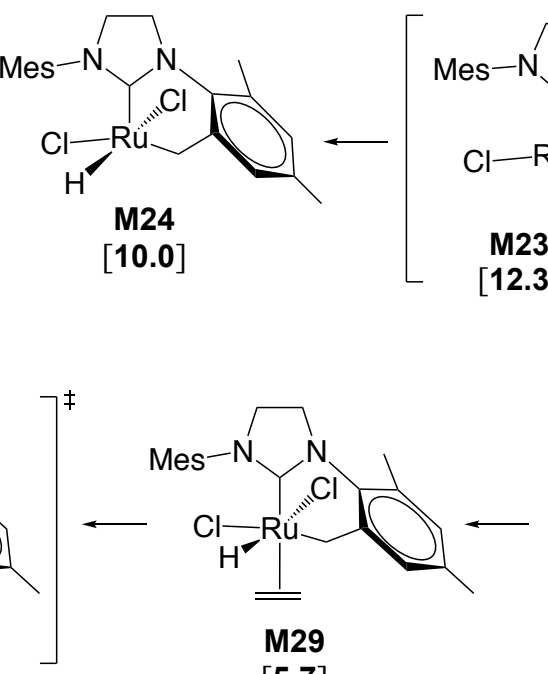

[12.3]
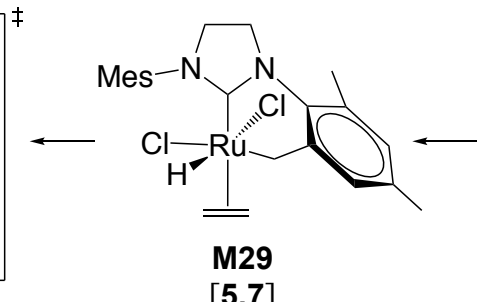
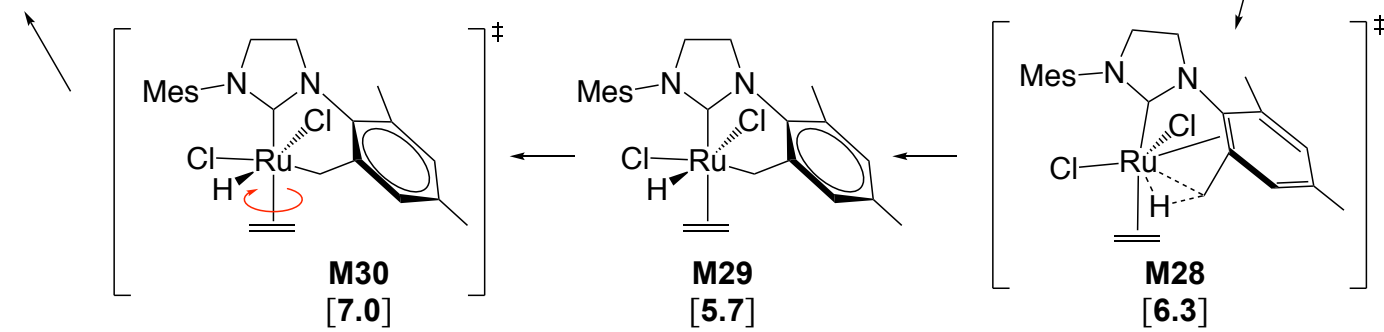

Scheme S9. Free energies (kcal/mol with respect to M1) of species along sections P2 (M2- ${ }^{3}$ M5), P4 ( ${ }^{3}$ M5-M13), P6 (M2-M24), P7 (M24-M25), and P8 (M13-M25) of the reaction network. The superscript in ${ }^{3} \mathbf{M} 4$ and ${ }^{3} \mathbf{M} 5$ indicates spin triplet state. 


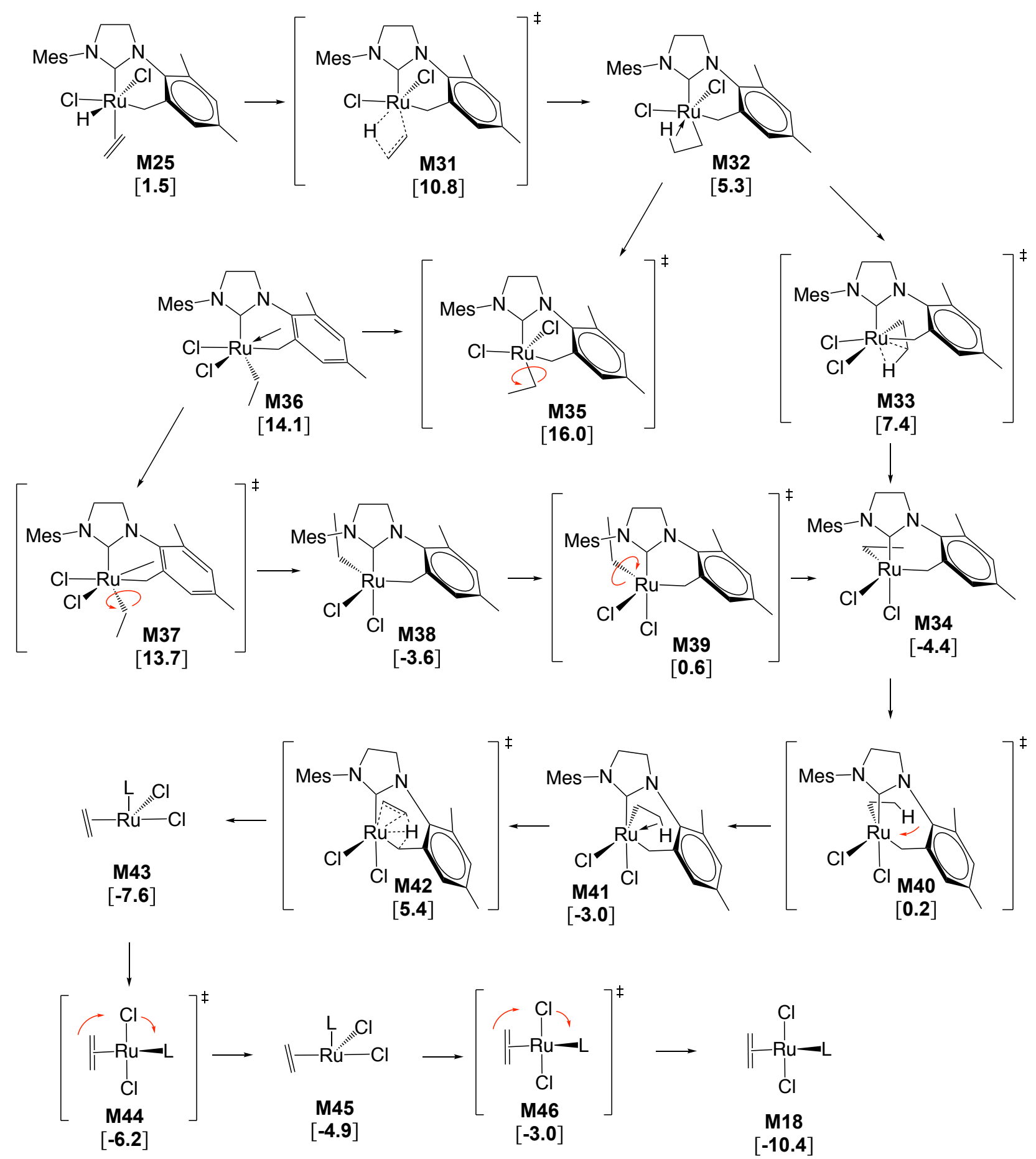

Scheme S10. Free energies ( $\mathrm{kcal} / \mathrm{mol}$ with respect to M1) of species along section P9 of the reaction network.

\section{Coupling of Ethylene}

The relatively high concentration of ethylene pushes towards formation of ethylene-bound species. M18 has two formal vacant coordination sites that can be used for further uptake of ethylene (Scheme S11). Among the bis- $\eta^{2}$-ethylene complexes that have been modeled in this study, M50 is the only one more stable than M18. M50 has the two ethylene ligands rotated almost perpendicularly to each other. This arrangement is not ideal for coupling of the two olefinic substrates with formation of $\mathrm{C}-\mathrm{C}$ bond; a step that was speculated in our previous work for allylbenzene. ${ }^{1}$ Propeller rotation of one $\eta^{2}$-ethylene is 
expected to be easy as there is no steric impediment, and aligns the two ethylene ligands so that the dihedral along the shortest $\mathrm{C}-\mathrm{C}$ distance if ca. 32 degrees in M47. With such alignment, the previously mentioned $\mathrm{C}-\mathrm{C}$ bond formation can occur via the relatively small barrier at M48 (ca. $8 \mathrm{kcal} / \mathrm{mol}$ from M47, and ca. $11 \mathrm{kcal} / \mathrm{mol}$ from M50) yielding the metallacyclopentane M49.

The hypothetical cis- $\mathrm{Cl}$ configuration analogues M51, $\mathbf{M 5 2}$ and M53 are significantly more demanding and are thus not further considered in the following.

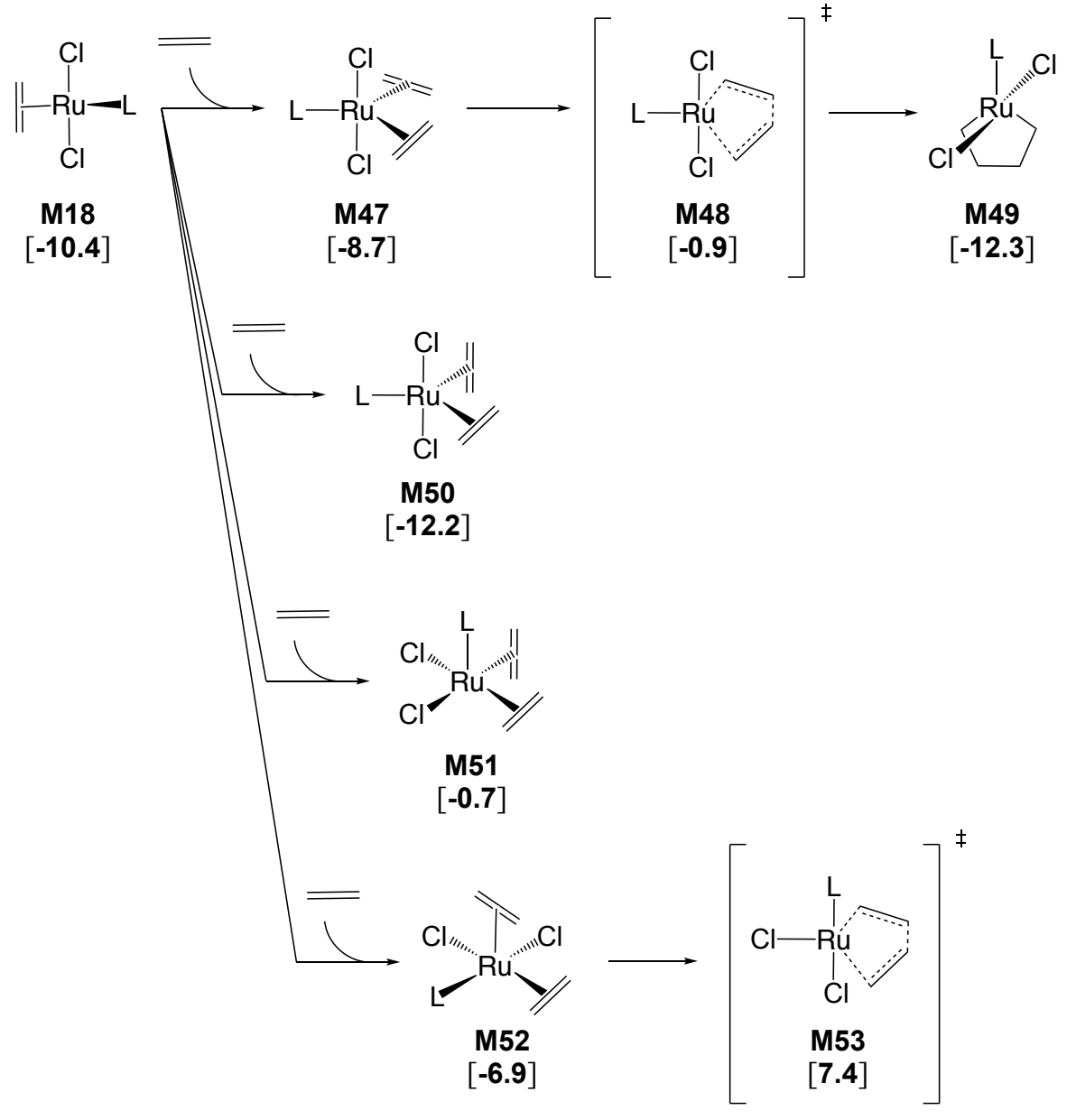

Scheme S11. Free energies (kcal/mol with respect to M1) of species along section P10 of the reaction network.

\section{Formation of 1 -Butene}

Metallacyclopentane M49 is the most stable species encountered in the reaction network so far, but such species has not been detected experimentally. On the contrary, organic products with four carbon atoms, i.e., the so-called C4 products, were experimentally identified in the reaction mixture. In particular, 1- and 2-butenes contain the same atoms of two ethylene molecules that were incorporated into M49 (i.e., 4 carbon atoms, and 8 hydrogen atoms). This suggest the existence of reaction pathways involving a rearrangement of $\mathbf{M 4 9}$ that eventually releases the entire $\mathrm{C} 4$ unit with all the hydrogen atoms connected to each of these carbons, and originally deriving from the ethylene substrate. As we have found in precious studies, ${ }^{1}$ metallacyclopentanes can undergo stepwise 1,2-H shift and 1,3-H shift. These alternatives are explored below for the specific case of unsubstituted metallacyclobutane M49. The hypothetical possibility of reductive elimination releasing cyclobutene was discarded because such a peculiar organic product is not detected in the reaction mixture.

The stepwise 1,2-H shift path begins with $\beta-\mathrm{H}$ elimination via transition state M54 and produces M55, which is a Ru-hydride with $\eta^{2}$-butenyl ligand (Scheme S12). Starting from this species there is a pathway 
that would lead directly to the metallacyclobutane and, thus, to the olefin metathesis species. Such a pathway, which has been suggested previously for allylbenzene as the olefinic substrate, ${ }^{1}$ is included in the present work as section P50 and will be discussed later (see Scheme S45).

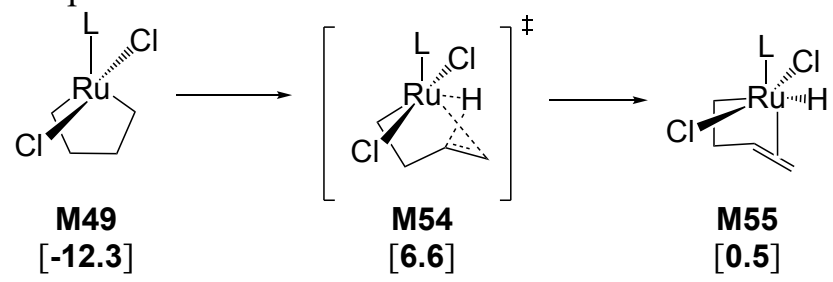

Scheme S12. Free energies ( $\mathrm{kcal} / \mathrm{mol}$ with respect to M1) of species along section P11 of the reaction network.

Instead, the rearrangement shown in Scheme S13 requires a much smaller barrier $(2.8 \mathrm{kcal} / \mathrm{mol}$ at $\mathbf{M 5 6}$ with respect to M55) than the first step along the pathway eventually leading to the metallacyclobutane $(6.4 \mathrm{kcal} / \mathrm{mol}$ at $\mathbf{M 2 9 6}$ with respect to $\mathbf{M 5 5}$, see P50 in Scheme S45). The olefinic component of M55 is thus very labile and can easily be replaced by the $\mathrm{CH}$-agostic interaction characterizing M57. This involves torsion around one of the $\mathrm{C}-\mathrm{C}$ single bonds of the butenyl ligand.

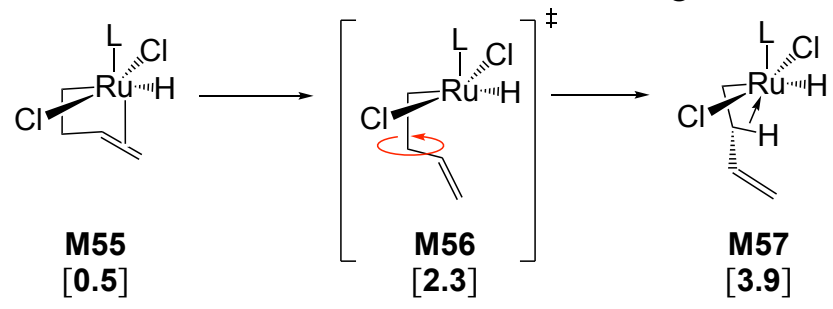

Scheme S13. Free energies (kcal/mol with respect to M1) of species along section P12 of the reaction network.

M57 is less stable than M55, but can easily proceed to further conformational adaptations that involve only negligible energy barriers lower than $2 \mathrm{kcal} / \mathrm{mol}$ (Scheme S14). The conformer resulting from the multistep rearrangement (i.e., M65) undergoes facile $\beta-\mathrm{H}$ elimination (transition state M66 is only 3 $\mathrm{kcal} / \mathrm{mol}$ above M65) with formation of $\eta^{2}$-s-trans-butadiene and $\eta^{2}$ molecular hydrogen at M67. This second elimination is somewhat surprising, but, as shown in Scheme S14, the energy profile leaves no doubt on the facility of this rearrangement. Moreover, attempts to identify alternative pathways departing from any Ru- $\eta^{2}$-butenyl complexes (M55, M57, M59, M61, M63, and M65) produced only pathways with activation barriers higher than the few $\mathrm{kcal} / \mathrm{mol}$ associated with the formation of M67. In particular, among such attempts, the pathways via $\mathbf{M 6 8}$ and $\mathbf{M 7 1}$ could result in species that would allow reductive elimination and formation of 1-butene. The first, via $\mathbf{M 6 8}$ (or M70, which has a different configuration), involves movement of the hydride ligand across the metal center by passing in between the $\mathrm{Cl}$ and NHC ligands. The second, i.e., via M71, proceeds by twisting of the carbon skeleton roughly along the $\mathrm{Ru}-$ NHC bond axis, and produces practically the same result as the path via M68 (there are only by small conformational differences between $\mathbf{M 6 9}$ and M72). In both cases the energy of the transition states is substantially higher than that of the $\beta$-H elimination that produces butadiene-bound complex M67. It should be pointed out that the overall pathway from M55 to M67 involves transition states with maximum free energy that is about $1 \mathrm{kcal} / \mathrm{mol}$ lower than the overall path leading to metallacyclobutane, which is described below (see P50 in Scheme S45). However, the stability of M67 increases the effective barrier to return back and reach the metallacyclobutane (ca. $10 \mathrm{kcal} / \mathrm{mol}$ from $\mathbf{M 6 7}$ to $\mathbf{M 2 9 6}$ and ca. $10.5 \mathrm{kcal} / \mathrm{mol}$ from M67 to M302), thus disfavoring the return from M67 back to M55 and into the alternative P50 pathway eventually reaching to metallacyclobutane. This effect becomes even stronger with the further rearrangement of $\mathbf{M 6 7}$ into $\mathbf{M 7 4}$ that gains about $9 \mathrm{kcal} / \mathrm{mol}$. 


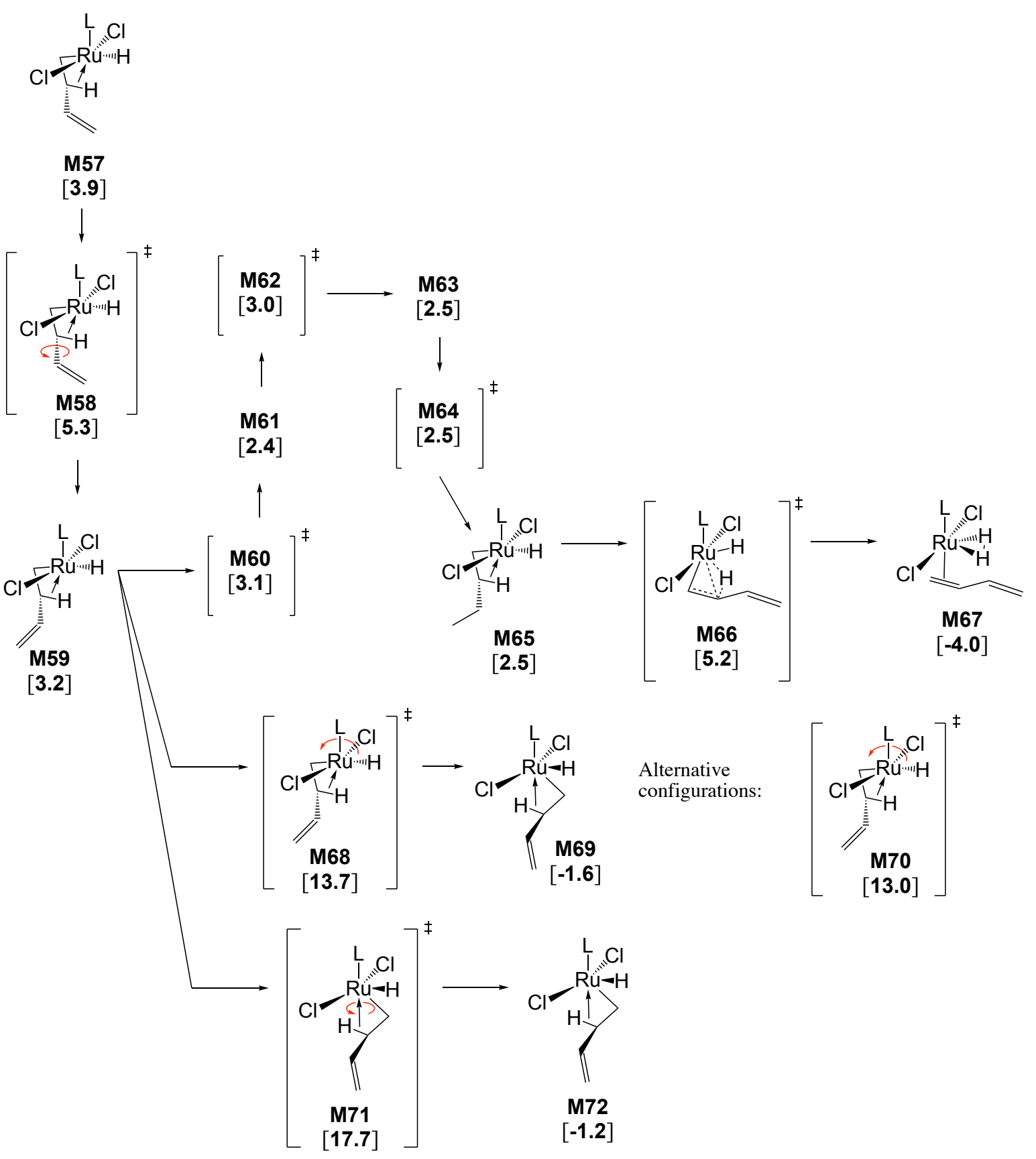

Scheme S14. Free energies (kcal/mol with respect to M1) of species along section P13 of the reaction network.

The $\eta^{2}$-butadiene ligand in M67 coordinates the metal as a 2-electron donor using half of its $\pi$-electrons, and the propeller-like rotation along the axis of such connection can occur via transition state $\mathbf{M 7 3}$ (Scheme S15). After M73 the free double bond of butadiene moves close enough to the metal to form the tetra-hapto coordination mode of M74 placing the second half of the $\pi$-electrons $c i s$ to the NHC. In this step the coordinated molecular hydrogen is twisted by $90^{\circ}$. Thanks to these rearrangements, M74 is substantially more stable than M67 and, if fact, M74 is the most stable species in the reaction network among those described so far. The relative ease of formation of the $\eta^{4}$ coordination mode from M67 is most likely the reason limiting release of butadiene in the reaction mixture and, therefore, detection of such $\mathrm{C} 4$ product. However, it should be noted that similar experiments previously performed by van 
Rensburg and co-workers could detect traces of butadiene in reactions performed with second-generation methylidene and ethylene in benzene- $d_{6}$ at $40{ }^{\circ} \mathrm{C} .{ }^{8}$ These experiments are compatible with decomposition of unsubstituted metallacyclobutane with formation of $\mathrm{Ru}(\mathrm{II})$ species like $\mathbf{M} 2$ that can bind ethylene and enter the reaction path that forma butadiene as described up to this point.

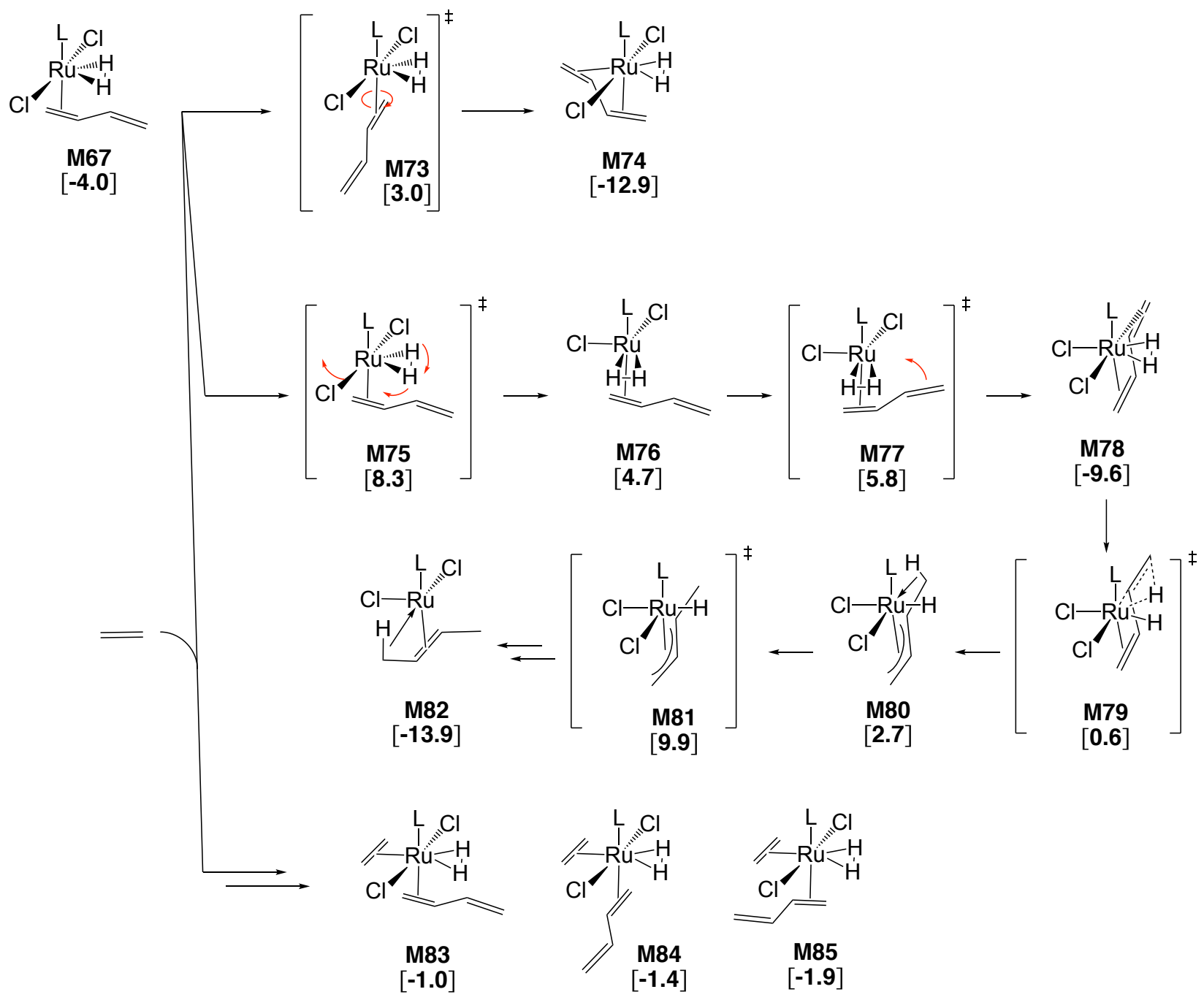

Scheme S15. Free energies (kcal/mol with respect to M1) of species along section P14 of the reaction network.

When trying to identify alternative pathways we explored the possibility of changing the configuration of the $\mathrm{Ru}$ center by formation of cis-Cl species $\mathbf{M 7 6}$, which could allow hapto-four coordination of $s$ trans-butadiene with both of its terminal carbon atoms cis to the coordinated molecular hydrogen (M78). The latter is a configuration that could allow formation of trans-2-butene at M82, but the energies of transition states M75 (change of configuration), M77 (increase of hapticity), and M81 (final reductive elimination), are significantly higher than those along the of the most preferred pathway generating trans2-butene and that is described later in the text.

Molecular hydrogen can dissociate from M74 with a barrier of only $11.3 \mathrm{kcal} / \mathrm{mol}$ at $\mathbf{M 8 6}$. The resulting $\mathrm{Ru}$ complex, M88, which is unstable with respect to M74, is formally a source of butadiene. The hypothetical dissociation of butadiene would require rupture of the hapto-four system, possibly stepwise via an hapto-two intermediate. All attempts to model an $\eta^{2}$-butadiene coordinating cis to the NHC failed returning the hapto-four coordination mode. Instead, the configurations with $\eta^{2}$-butadiene trans to the 
NHC (i.e., M93) were found significantly unstable with respect to M88 even in the spin-triplet state (i.e., ${ }^{3}$ M92), which is most likely a mandatory step along the dissociation of butadiene from M88. This hypothetical dissociation of butadiene would result in an overall catalytic conversion of two ethylene molecules into molecular hydrogen and butadiene. This reaction is endoergonic (reaction free energy 2.7 $\mathrm{kcal} / \mathrm{mol}$ ), thus butadiene and hydrogen are not expected to accumulate.

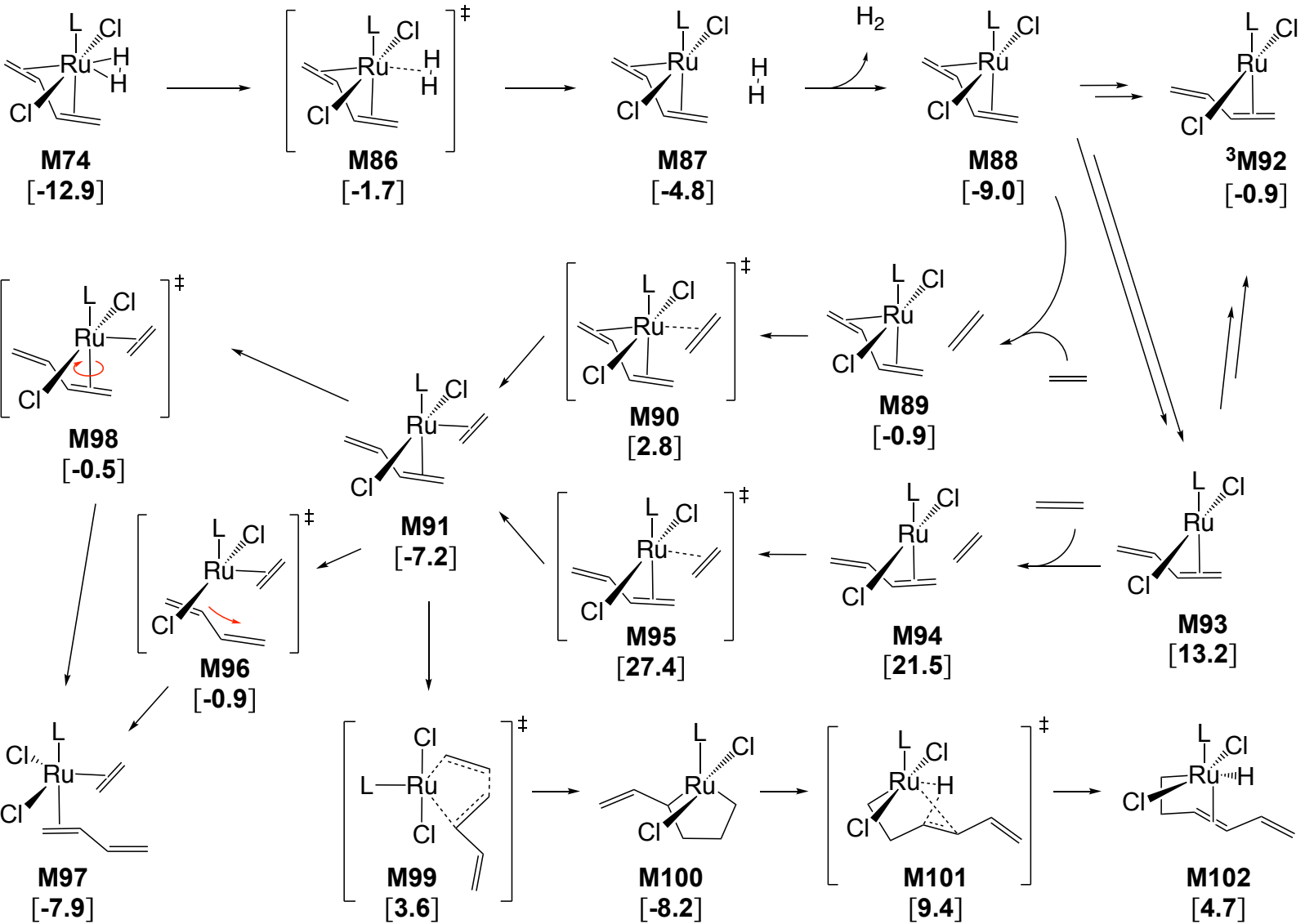

Scheme S16. Free energies ( $\mathrm{kcal} / \mathrm{mol}$ with respect to M1) of species related to P15, i.e., the liberation of molecular hydrogen and butadiane.

In the quest of a hypothetical process transforming the Ru-bound butadiene of $\mathbf{M 8 8}$ in a thermodynamically favored product, we considered alternative scenarios. Attempts to identify a cyclometallation pathway involving $\mathrm{CH}$-activation of a methyl group from the mesityl could not find any system with reasonable energy. Next, given the overwhelming concentration of ethylene, the possibility of reacting with ethylene was also evaluated. However, the instability of trans-coordinated $\eta^{2}$-butadiene configuration (M93) prevents a dissociative mechanism for exchange of one $\eta^{2}$ end of butadiene with an incoming ethylene (i.e., via M95). Instead, the associative analogue of this exchange, i.e., via M90, requires only ca. $12 \mathrm{kcal} / \mathrm{mol}$ from $\mathbf{M 8 8}$, and describes a trajectory in which the release of the $\eta^{2}$ component of butadiene occurs at a very late stage along the reaction coordinate, thus providing the metal with sufficient electron donation. The resulting complex (M91) can also rearrange to its more stable isomer M97 by sliding of the butadiene ligand (via M96) rather than twisting it (via M98). M91 was also tested for the $\mathrm{C}-\mathrm{C}$ bond formation reaction similar to the one described above for the coupling of ethylene to form a metallacyclopentane. This type of reactivity, via M99, can generate a C6 backbone at M100, but the subsequent $\beta-\mathrm{H}$ elimination via M101 is too demanding for this pathway to play a central role.

Overall, despite the liability of molecular hydrogen, the calculated stability of M74 and the absence of experimental traces of free molecular hydrogen and butadiene, or butadiene-derived products, suggest that even if molecular hydrogen can be liberated and can possibly by a minor contribute to the formation of 
hydrogenated substrates (regarding formation of ethane, see page 98), most of such molecular hydrogen will further react with $\mathrm{Ru}$-bound butadiene as described in the following paragraphs, thus eventually be incorporated in the 1- and 2-butene products.

In fact, the presence of both molecular hydrogen and the unsaturated $\mathrm{C} 4$ skeleton on the same Ru center suggests hydrogenation reactions. However, only one end of the $\eta^{4}$-butadiene is close enough and properly oriented towards the molecular hydrogen to proceed with the insertion step shown in Scheme S17. This step requires only ca. $7 \mathrm{kcal} / \mathrm{mol}$ to reach transition state $\mathbf{M 1 0 3}$ from $\mathbf{M 7 4}$, which is a lower barrier than that for the release of molecular hydrogen. $\mathbf{M 1 0 3}$ sees also the concerted rupture of the multihapto bonding originally characterizing the butadiene complex. This is most likely due to the trans influence produced by the hydride ligand that is generated trans to the coordination site previously occupied by one of the $\eta^{2}$ components of the once $\eta^{4}$-butadiene. The resulting species (M104) has a mono-hapto allyl ligand with a $\beta$-CH-agostic interaction involving the hydrogen atom previously belonging to the molecular hydrogen ligand.

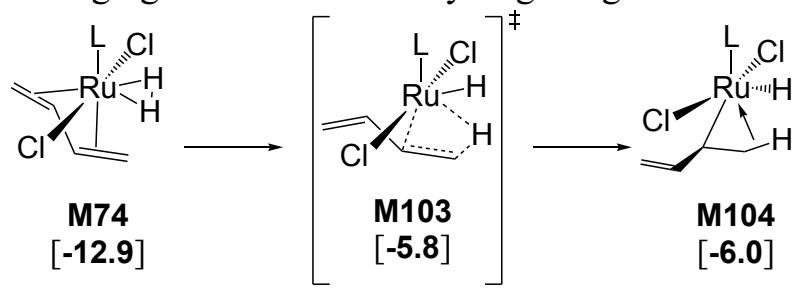

Scheme S17. Free energies (kcal/mol with respect to M1) of species along section P16 of the reaction network.

M104 has still one hydride ligand that could be used to further hydrogenate the C4 carbon skeleton, which originated from two ethylene molecules. However, such hydride does not have any open coordination site in cis, so it cannot bind the olefinic function of the butenyl ligand. The most direct elementary step would be the reductive elimination involving the hydride and the alkyl ligand. This hypothetical step was searched without success possibly due to the lack of a suitable electron donor able to replace the $\mathrm{CH}$-agostic interaction during the rupture of the latter that makes room for the reductive elimination.

Instead, exploration of the potential energy surface in the surroundings of M104 could identify in transition state M105 (see Scheme S18) a feasible step involving the concerted movement of both the hydride ligand and the olefinic fragment of M104. This peculiar rearrangement allows formation of intermediate M106: an unusual configuration displaying the $\eta^{3}$-allylic coordination mode for the butenyl ligand. 


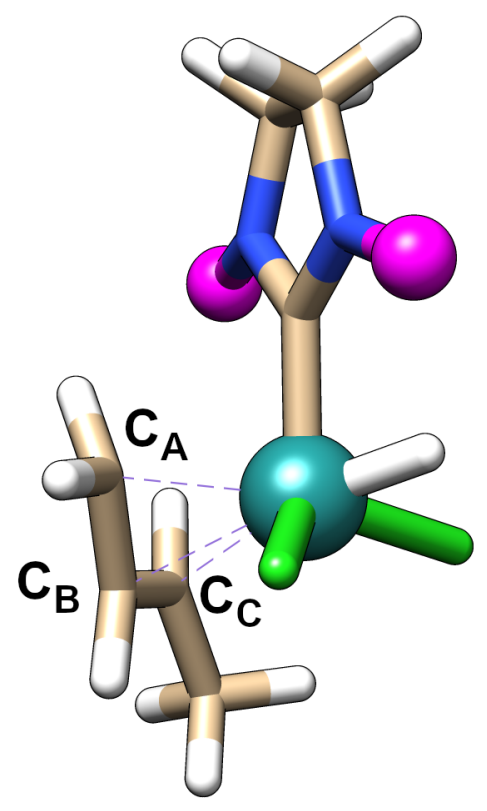

Figure S40: Three-dimensional representation of M106. For clarity, the mesityl groups are represented by magenta spheres.

In this configuration the hydride is trans to the $\eta^{3}$-allylic $\left(\mathrm{C}_{\mathrm{i}}-\mathrm{Ru}-\mathrm{H}\right.$ angles are $137^{\circ}, 162^{\circ}, 160^{\circ}$ respectively for $\mathrm{i}=\mathrm{A}, \mathrm{B}$, and $\mathrm{C}$ as in Figure $\mathrm{S} 40$ ) and $\mathrm{cis}$ to the $\mathrm{NHC}$ ligand (NHC-Ru-H angle $73^{\circ}$ ). The $\mathrm{Ru}-\mathrm{NHC}$ bond is twisted to the point that the smallest dihedral angle $\mathrm{N}-\mathrm{C}-\mathrm{Ru}-\mathrm{Cl}$ is about $33^{\circ}$. This, combined with bending at the NHC's nitrogen atom, allows the coordination of the $\eta^{3}$-allylic fragment cis to the $\mathrm{NHC}$ and with a small angle $\mathrm{C}_{\mathrm{A}}-\mathrm{Ru}-\mathrm{NHC}\left(83^{\circ}\right)$. The geometry of $\mathrm{C}_{\mathrm{A}}$ in Figure $\mathrm{S} 40$ has bond angles with the hydrogen atoms that suggests a significant $\mathrm{sp}^{3}$ character for this carbon. On the contrary, $\mathrm{C}_{\mathrm{B}}$ and $\mathrm{C}_{\mathrm{C}}$ are almost planar in agreement with an $\mathrm{sp}^{2}$ hybridization. Moreover, the $\mathrm{Ru}-\mathrm{C}$ distances $\left(\mathrm{C}_{\mathrm{i}}-\mathrm{Ru} 2.16\right.$, 2.30, and $2.53 \AA$ respectively for $\mathrm{i}=\mathrm{A}, \mathrm{B}$, and $\mathrm{C}$ ) are consistent with a dominant alkyl-type character for $\mathrm{C}_{\mathrm{A}}$ while $\mathrm{C}_{\mathrm{B}}$ and $\mathrm{C}_{\mathrm{C}}$ display olefin-like features and suffer more from the trans influence of the hydride.

In alternative to the formation of M106, two alternative pathways were explore trying to bring the alkyl ligand and the hydride cis to each other, thus allowing reductive elimination eventually releasing 1-butene: i) the formal movement of the hydride across the metal center via transition state M109 (achieved by moving the hydride practically around one $\mathrm{Ru}-\mathrm{Cl}$ bond), and ii) twisting of the butenyl ligand along the major axis of the Ru-(butenyl) interaction, that is, via M107 or M112. However, the energies of the transition states are too high to compete with the isomerization via M105 and formation of M106. 


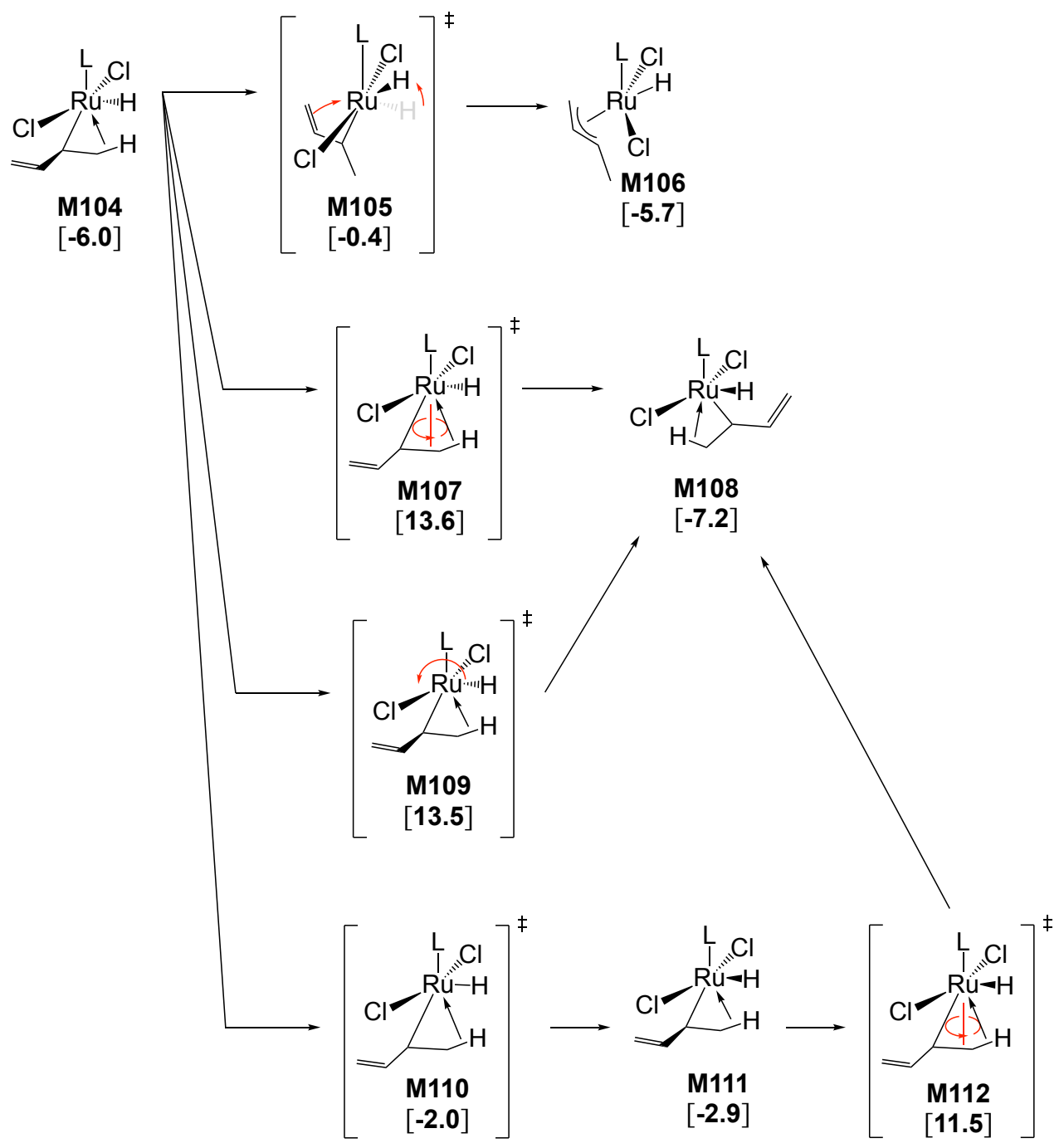

Scheme S18. Free energies (kcal/mol with respect to M1) of species along section P17 of the reaction network.

As anticipated, the peculiar geometry of $\mathbf{M 1 0 6}$ allows two peculiar rearrangements. In one case, the formal reductive elimination between the hydride and the alkyl ligand of M104. This first alternative eventually leads to the formation of 1-butene. In the other case, the pathway describes the movement of the hydride to the opposite side of the $\mathrm{Ru}$ center and further onto the terminal methylene end of the allyl group of M106. This second alternative, which will be described in more detail further below, leads to the formation of trans-2-butene and is slightly more demanding (via $\mathbf{M 1 4 0}$ at $-1.1 \mathrm{kcal} / \mathrm{mol}$ with respect to M1) than the first one that leads to 1-butene (via $\mathbf{M 1 1 3}$ at $-2.6 \mathrm{kcal} / \mathrm{mol}$ with respect to M1), and that is described in the next paragraph.

To perform a reductive elimination that produces 1-butene, the relative position between the hydride and $\mathrm{C}_{\mathrm{C}}$ must change from almost trans as in $\mathbf{M 1 0 6}\left(\mathrm{C}_{\mathrm{C}}-\mathrm{Ru}-\mathrm{H}\right.$ angle $\left.160^{\circ}\right)$ to cis as in M114 $\left(\mathrm{C}_{\mathrm{C}}-\mathrm{Ru}-\mathrm{H}\right.$ angle $64^{\circ}$ ). This occurs via transition state $\mathbf{M 1 1 3}$ by concerted and coherent bending of angles centered on the metal atom. The result is a complex (M114) where the $\eta^{3}$-ligand occupies the coordination site trans to the $\mathrm{NHC}\left(\mathrm{C}_{\mathrm{i}}-\mathrm{Ru}-\mathrm{NHC}\right.$ angles are $163^{\circ}, 161^{\circ}, 127^{\circ}$ respectively for $\mathrm{i}=\mathrm{A}, \mathrm{B}$, and $\left.\mathrm{C}\right)$, and the hydride is cis to both $\mathrm{NHC}$ and $\eta^{3}$-ligand ( $\mathrm{H}-\mathrm{Ru}-\mathrm{NHC} 76^{\circ}$ ). $\mathbf{M 1 1 4}$ is more than $6 \mathrm{kcal} / \mathrm{mol}$ more stable than the previous lowest energy point in the path: the $\eta^{4}$-butadiene complex M74. Reaching M114 from M74 involves a barrier of $12.5 \mathrm{kcal} / \mathrm{mol}$, which implies fast reactivity already at room temperature (half-life lower than one second). 


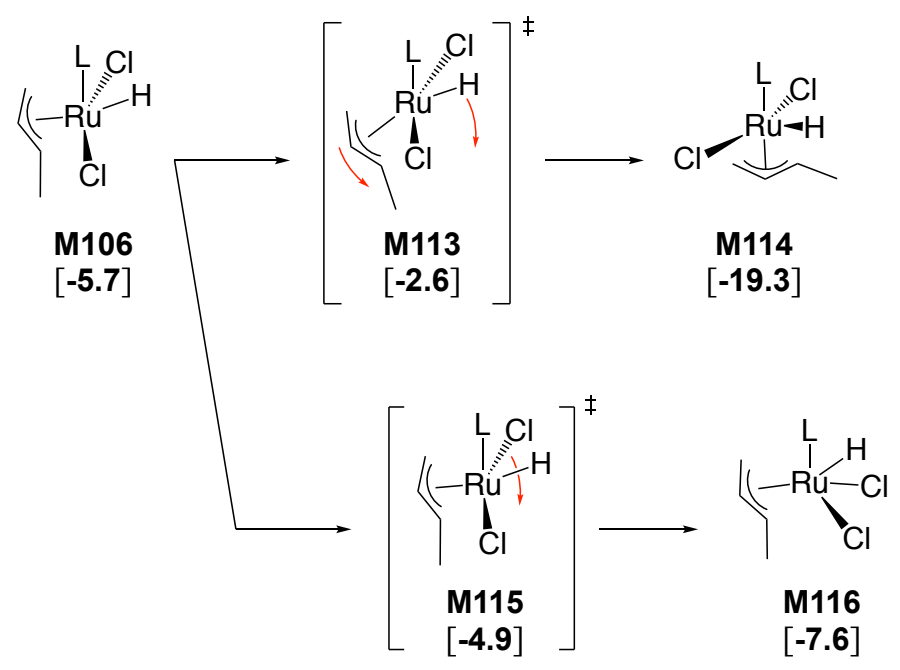

Scheme S19. Free energies (kcal/mol with respect to M1) of species along section P18 of the reaction network.

As displayed in Scheme S19 the path via M113 does not involve the lowest energy transition state among those found departing from M106. In fact, bending of the $\mathrm{Cl}-\mathrm{Ru}-\mathrm{Cl}$ angle via M115 is less demanding than the step via M113. The resulting complex M116 has $97^{\circ}$ between the $\mathrm{Ru}-\mathrm{Cl}$ bonds and sees the methylene end of the allyl ligand approaching the space between the two mesityl substituents on the NHC. The hydride is still trans to the $\eta^{3}$-ligand system, but even more bent towards the NHC than in M106 (NHC-Ru-H angle $63^{\circ}$ and $73^{\circ}$, respectively). Intermediate M116 is more stable than M106. However, any attempt to find pathways departing from M116 did not identify any other alternative with sufficiently low barrier to compete with M113. Thus, M116 is here considered an off-path intermediate that is more stable than M106, though not sufficiently stable to represent a substantial perturbation to the kinetics.

Finally, from M114 reductive elimination to form metal-coordinated 1-butene is facile via transition state M117, which has an imaginary mode characterized by the complementary bending of the two Cl-Ru$\mathrm{H}$ angles that produce an overall change of configuration of the metal center. However, no hydride-like minimum was found as the reductive elimination follows along the same downhill energy profile (see IRC profile in Figure S69). The resulting product (M118) sees the $\eta^{2}$-ligand almost trans to the NHC with an additional $\mathrm{CH}$-agostic interaction clearly deriving from the previous hydride.

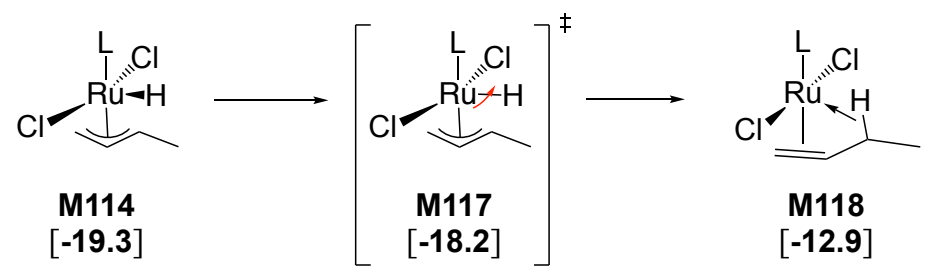

Scheme S20. Free energies ( $\mathrm{kcal} / \mathrm{mol}$ with respect to M1) of species along section P19 of the reaction network.

To release 1-butene without generating the highly coordinatively unsaturated M2, or better, its spintriplet analogue ${ }^{3} \mathbf{M} 4$, we looked for associative product release pathways involving coordination of ethylene prior to release of 1-butene. Direct coordination of ethylene on M118 via transition state M123 (Scheme S21) would generate a complex with $\eta^{2}$-ethylene cis to NHC (M124), which is preferable when trying to approach M18 because trans-analogue M13 has higher energy and requires crossing into the spin-triplet surface to relax (see Scheme S7). However, M118 is much less stable than most of its isomers 
bearing a Ru-coordinated, fully formed 1-butene ligand. Thus, further rearrangement is preferred and kinetically favored.

The oxidative addition of the allylic hydrogen via M119, which is a slightly different pathway than the reverse of M117, yields a Ru-hydride complex (M120, Scheme S21) that is formally very similar to the opposite configuration of M114, but differs from the latter by the conformation of the NHC-mesityl bond and by the $\mathrm{Cl}-\mathrm{Ru}-\mathrm{Cl}$ angle. Yet another conceptually similar step, though with different configuration (via M121) results in the 1-butene complex M122. This, like M118 is a $\eta^{2}$-complex with an additional CHagostic interaction, but in $\mathbf{M 1 2 2}$ the $\mathrm{CH}$-agostic bond is trans to the NHC, which allows lower trans influence between the NHC and the $\eta^{2}$-system. The latter is roughly cis to the NHC in M122 (NHC-Ru$\mathrm{CH}_{2}$ angle $99^{\circ}$ ), and is significantly closer to the metal (C-Ru distances 2.15 and $2.06 \AA$ ) than in M118 (2.28 and $2.16 \AA$ ). The overall effect is that $\mathbf{M 1 2 2}$ is about $12 \mathrm{kcal} / \mathrm{mol}$ more stable than its isomer M118.
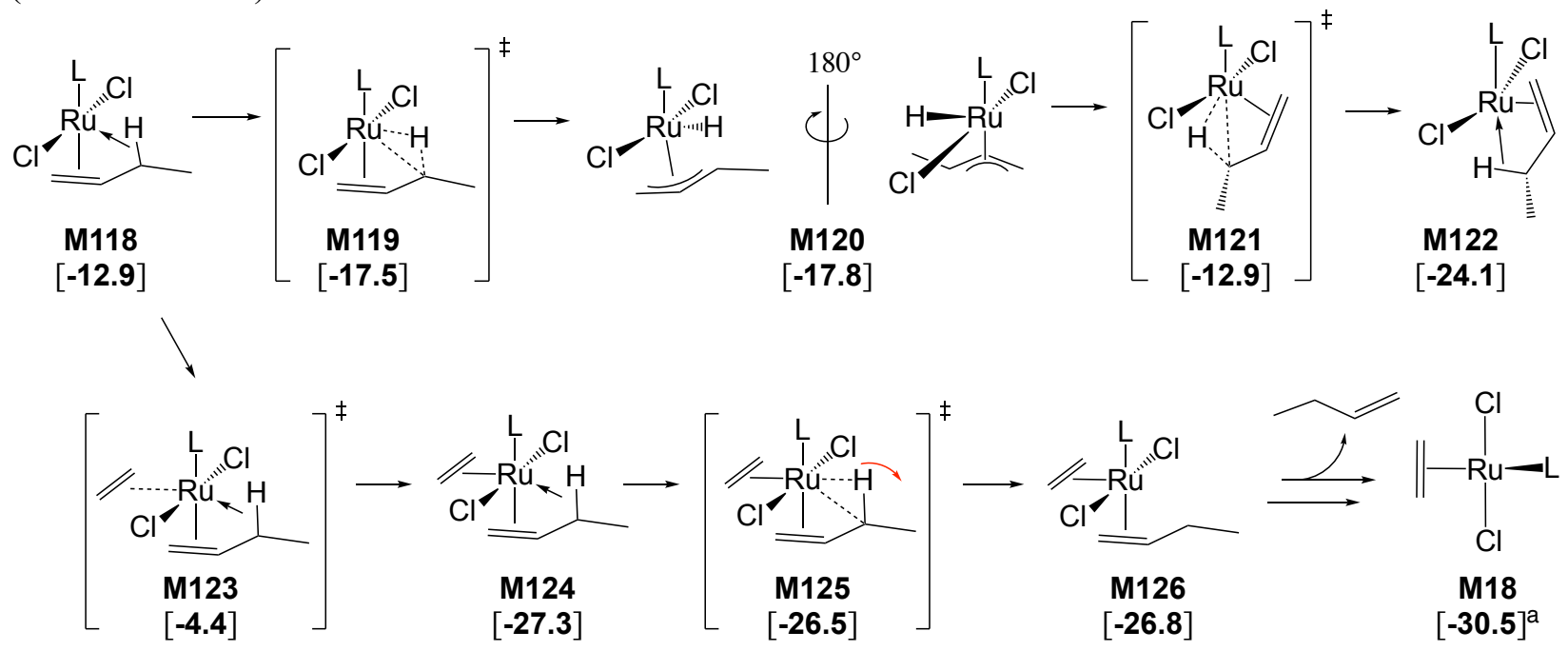

Scheme S21. Free energies (kcal/mol with respect to M1) of species along section P20 (M118-M120), P21 (M118-M18), and

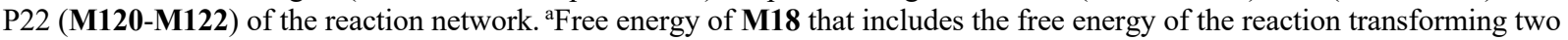
ethylene molecules into 1-butene.
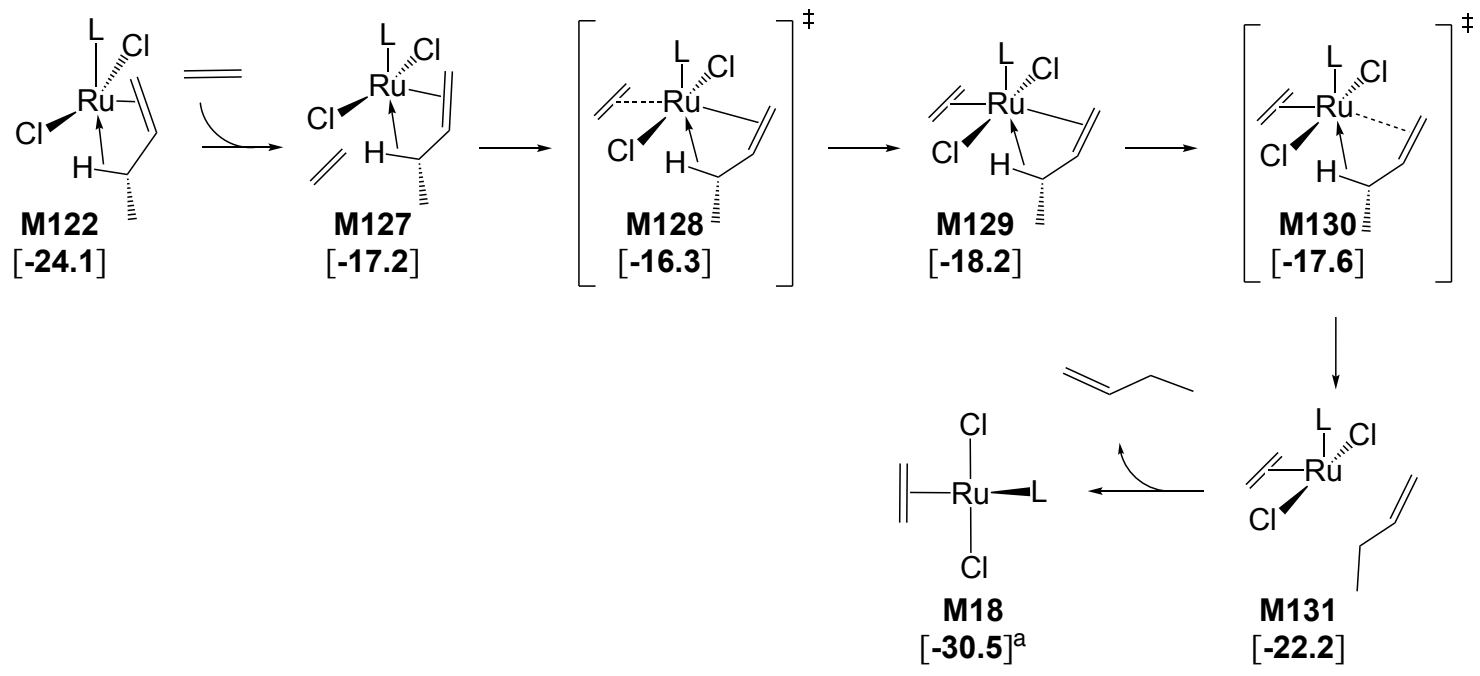

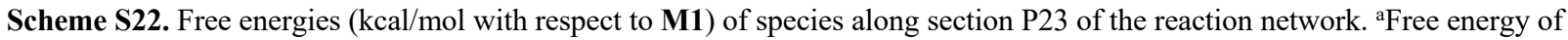
$\mathbf{M 1 8}$ that includes the free energy of the reaction transforming two ethylene molecules into 1-butene.

Due to the $\eta^{2}$-component practically cis to the NHC, the vacant coordination site of $\mathbf{M 1 2 2}$ is more accessible than in M118. This permits easy uptake of a new ethylene molecule to give the 18-electron 
compound M129 (Scheme S22). This complex can release 1-butene generating the initial M18, which is now pre-loaded with a new molecule of ethylene. Such dissociation of 1-butene is found to be barrier-less in the potential energy surface of the SP energy model, despite the identification of a proper transition state at the geometry optimization level.

Finally, with the regeneration of M18 this collection of steps defines a catalytic cycle for the coupling of two ethylene molecules to form 1-butene using M18 as catalyst. The overall energy profile of this catalytic cycle is depicted in Scheme S23. 


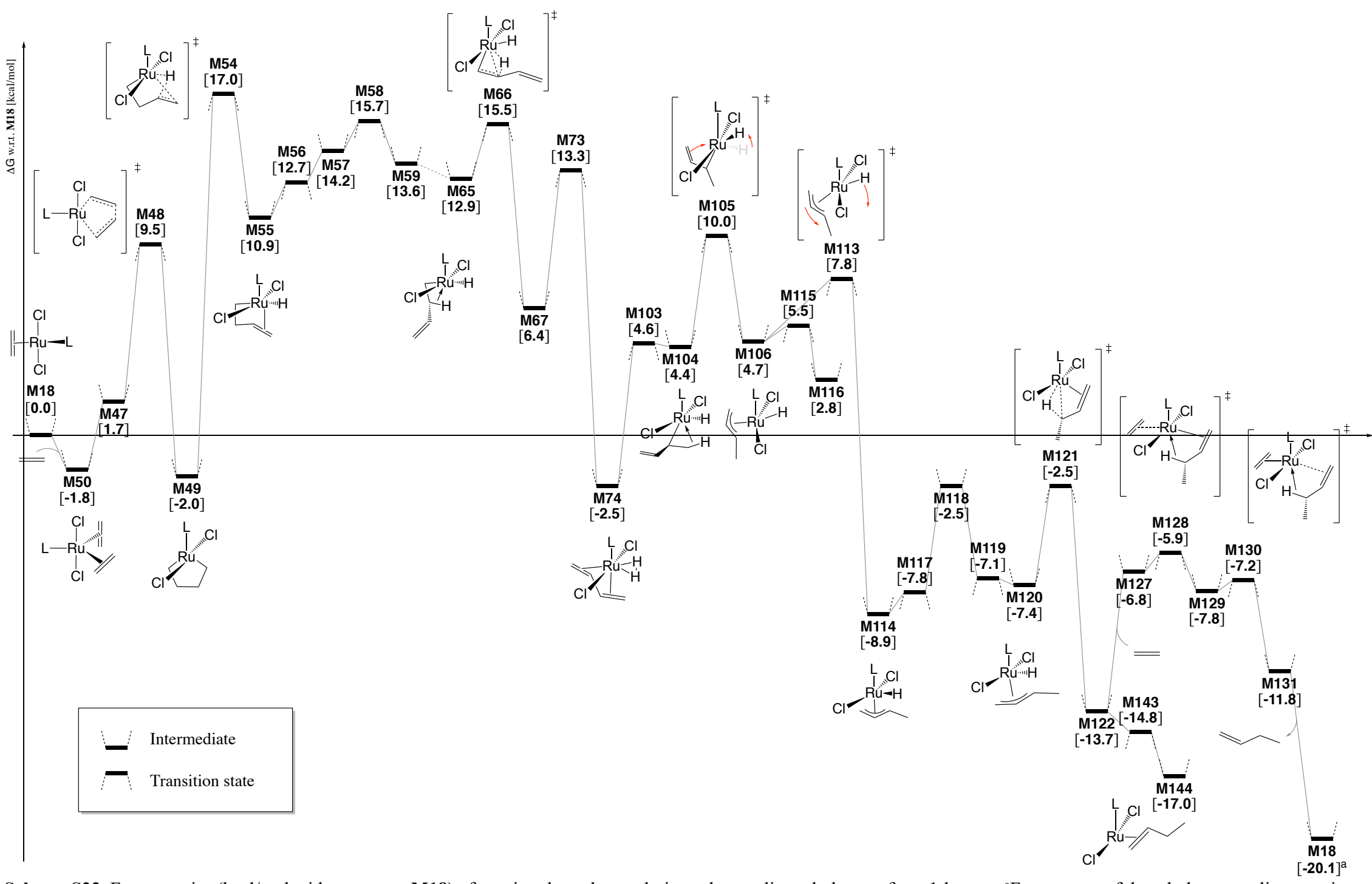

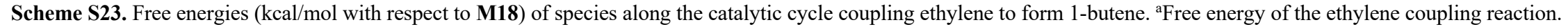




\section{Formation of trans-2-Butene}

The portion of the reaction network that is characterized by Ru-hydrides and a fully formed $\mathrm{C} 4$ backbone includes also pathways leading to isomerization of the 1- and 2-butenes. In particular, M120 was introduced as part of the isomerization steps of the Ru- $\eta^{2}$-(1-butene) complexes (see Scheme S21). However, as shown in Scheme S24, M120 can also rearrange by bending of the NHC-(Ru- $\eta^{3}$-allyl) angle to give isomer M133, which then undergoes $180^{\circ}$ twisting of the $\eta^{3}$-allyl group thus bringing the methylene end of the allyl group cis to the hydride (M135). An alternative pathway connecting M122 with M133 (P26, via M136) has a significant barrier and is thus excluded. From the configuration of M135 the reductive elimination that generates Ru-bound trans-2-butene requires only $2.5 \mathrm{kcal} / \mathrm{mol}$ via M137 (Scheme S25).
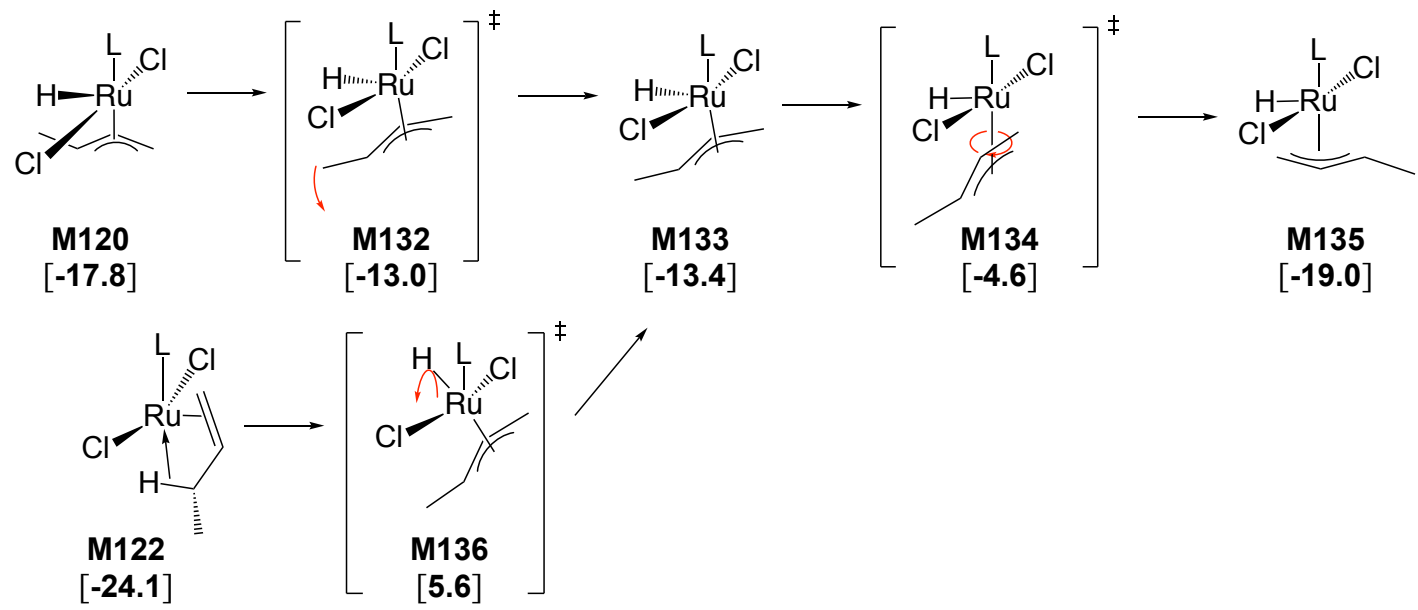

Scheme S24. Free energies (kcal/mol with respect to M1) of species along section P24 (M120-M133), P25 (M133-M135), and P26 (M122-M133) of the reaction network.

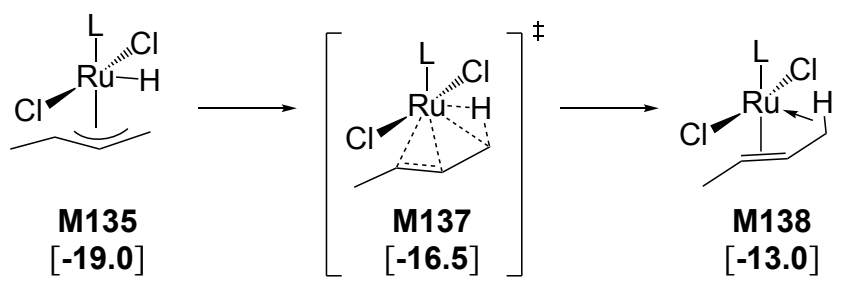

Scheme S25. Free energies (kcal/mol with respect to M1) of species along section P27 of the reaction network.

As above, we considered an associative mechanism for the final exchange of the product (trans-2butene) with a new ethylene molecule thus avoiding the dissociative generation of the electron deficient pair M2 ${ }^{3}$ M4 (Scheme S26).

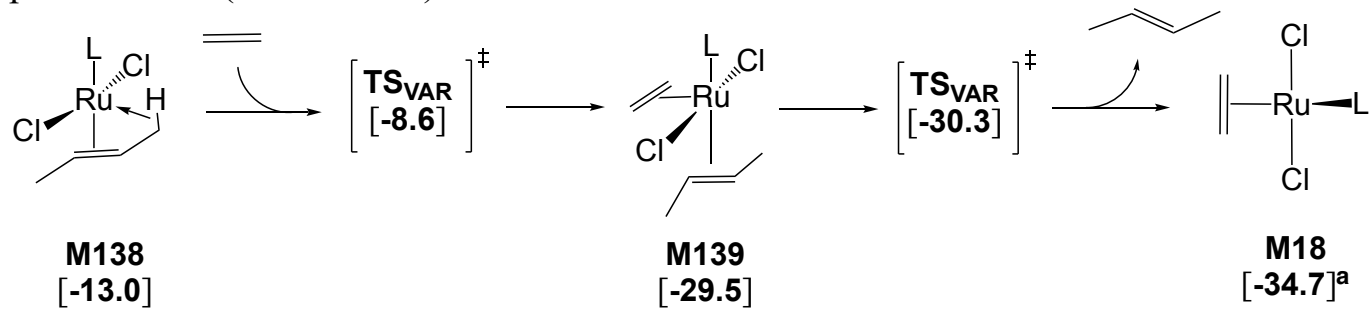

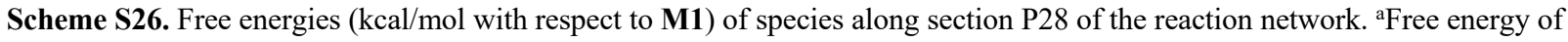
M18 that includes the free energy of the reaction transforming two ethylene molecules into trans-2-butene. 
The above connection identifies a pathway catalyzed by M18 for the isomerization of 1-butene into trans-2-butene. In fact, rather than producing M120 from ethylene, such species can be obtained from M122 and, in turn, from binding 1-butene onto M18. These steps are collected in Scheme S27 where the free energies are reported using M18 as reference to highlight the overall barrier of this catalytic cycle $(25.9 \mathrm{kcal} / \mathrm{mol})$. Such isomerization by double bond migration is practically the same mechanism identified in our previous contribution for the isomerization of allylbenzene by the $\mathrm{Ru}(\mathrm{II})$ species ${ }^{3} \mathbf{M} 4{ }^{1}$ extended by the additional associative exchange of ethylene with butene to avoid 12-electron species.

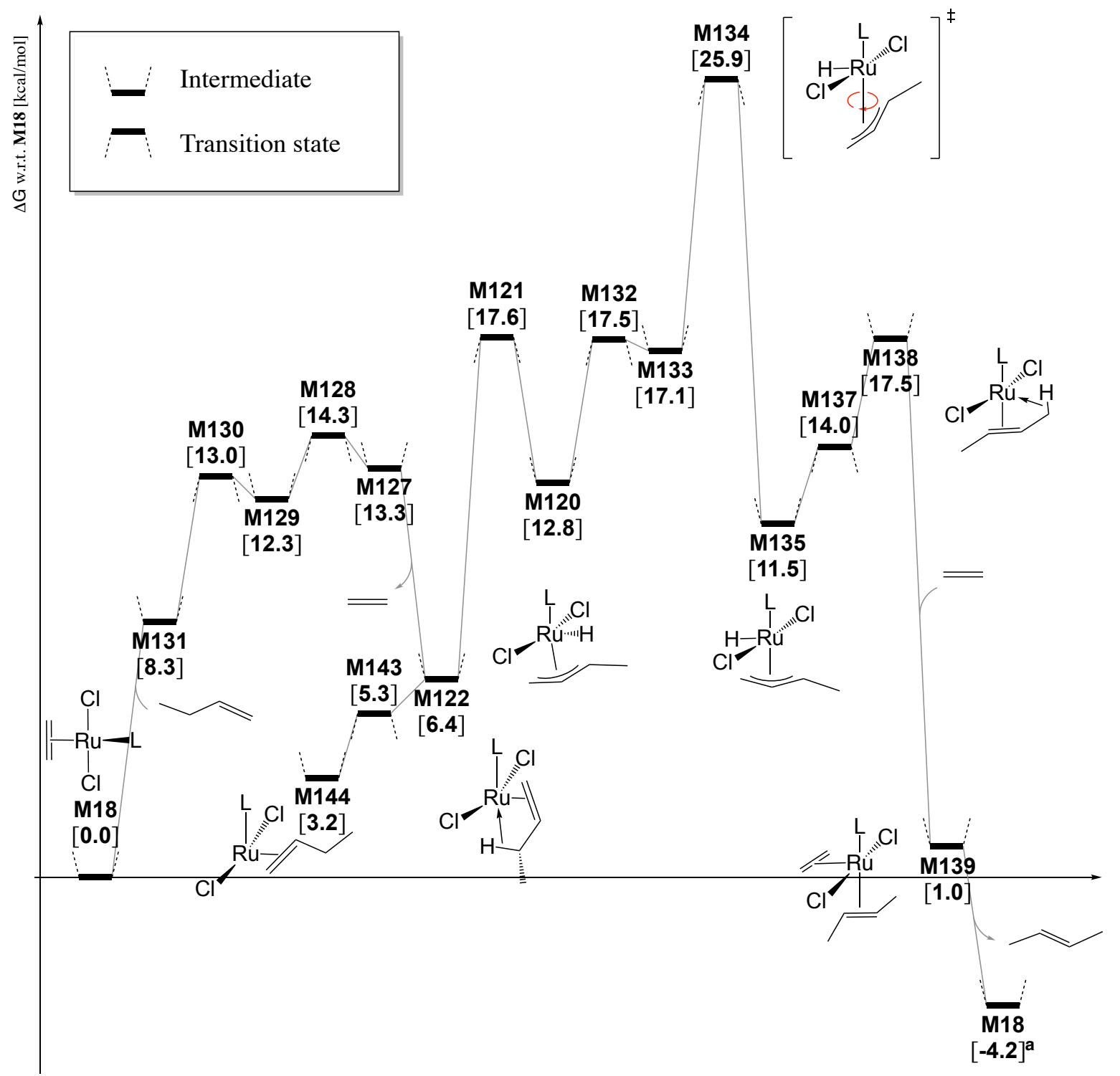

Scheme S27. Free energies ( $\mathrm{kcal} / \mathrm{mol}$ with respect to M18) of species along the isomerization cycle converting 1-butene to trans2-butene. ${ }^{\text {a }}$ Free energy of the isomerization of 1-butene to trans-2-butene.

Along the pathway producing 1-butene from two ethylene we anticipated the existence of an alternative possibility departing from the peculiar M106 complex and producing trans-2-butene. M106 was introduced in Scheme S18 as deriving from the rearrangement of the 3-but-2-enyl hydride species M104, which originated by partial hydrogenation of butadiene, which, in turn, comes from coupling of ethylene.

The path allowing direct production of trans-2-butene from Ru-bund butadiene is shown in Scheme S28. The barrier at $\mathbf{M 1 4 0}$ only $1.5 \mathrm{kcal} / \mathrm{mol}$ higher than M113, which leads to release of 1-butene (see Scheme 
$\mathrm{S} 19)$. In M140, the hydride moves practically around the $\mathrm{Ru}-\mathrm{Cl}$ bond ending up into the opposite side of the Ru center with respect to its starting location. This movement is coupled with the concerted one of the $\eta^{3}$-allyl moiety, and assisted by the metal-coordinating carbene atom of the NHC ligand. The latter interaction, though not as evident at the transition state, is clearly identified along the minimum energy pathway (see IRC profile in Figure S74 and attached XYZ file) departing from M140. Along the pathway, in fact, the hydrogen atom transits at only $1.29 \AA$ from the carbene atom, which displays tetrahedral distortion (i.e., improper dihedral $\mathrm{NC}(\mathrm{N}) \mathrm{Ru}$ bends by about $10^{\circ}$ ) compatible with an increased $\mathrm{sp}^{3}$ character. While this interaction plays a role in the surroundings of the transition state, the path does not involve an intermediate with a sigma bond between the hydrogen and the carbene. Nevertheless, the existence of this interaction along the present pathway suggests a novel catalyst decomposition mechanism by proton transfer from the metal center to the NHC. This hypothesis is corroborated also by another local minimum (see M279 in Scheme S41). Such type of decomposition path, which is not further investigate in this contribution, could explain the generation of imidazolium cation, which has been observed in the experiments described above (see page 11).

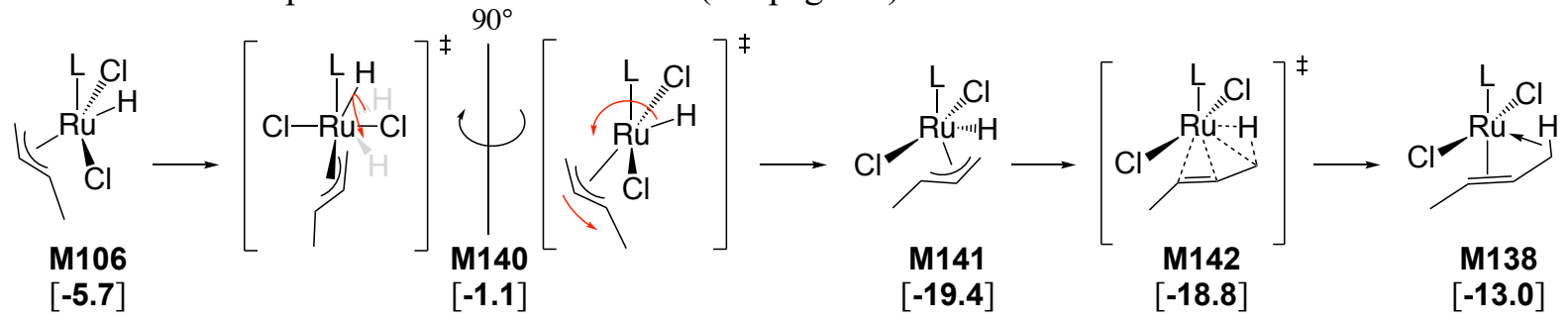

Scheme S28. Free energies (kcal/mol with respect to M1) of species along section P29 of the reaction network.

This direct formation of trans-2-butene from ethylene is consistent with the kinetics reported by the experiments. In fact, the difference in initial rates of formation for 1-butene (fast) and trans-2-butene (slow) is compatible with the $1.5 \mathrm{kcal} / \mathrm{mol}$ free energy difference between $\mathbf{M 1 1 3}$, which leads to 1-butene, and M140, which leads to trans-2-butene. Moreover, the practically constant production rate of trans-2butene in (see Figure S3) implies a rate law that does not include a dependency on the concentration of 1butene. Such dependency would suggest isomerization of 1-butene as the main source of trans-2-butene. The presence of the phosphine seems to alter the ratio between direct production of trans-2-butene and isomerization of 1-butene to trans-2-butene. In fact, there is a significant increase in the production rate of trans-2-butene (see Figure S8) that suggests a rate law including a dependency on the concentration of 1butene.

\section{Formation of cis-2-Butene}

The strategies identified in the above mechanisms that produce trans-2-butene from methyl substituted $\eta^{3}$-allyl species were tentatively investigated as candidates for the formation of cis-2-butene. In fact, $\eta^{3}$ allyl species can be generated from 1-butene, but depending on the conformation of the latter, the resulting $\eta^{3}$-allyl species can be syn like all the $\eta^{3}$-allyl species encountered so far (e.g., M106, M114, M116, M120, M133, M135, M141) or anti (e.g., M150, M157, M160 in Scheme S29). Upon reductive elimination between the hydride and the unsubstituted end of the allyl system, the syn stereoisomers generate trans-2-butene, while the anti generates cis-2-butene. 

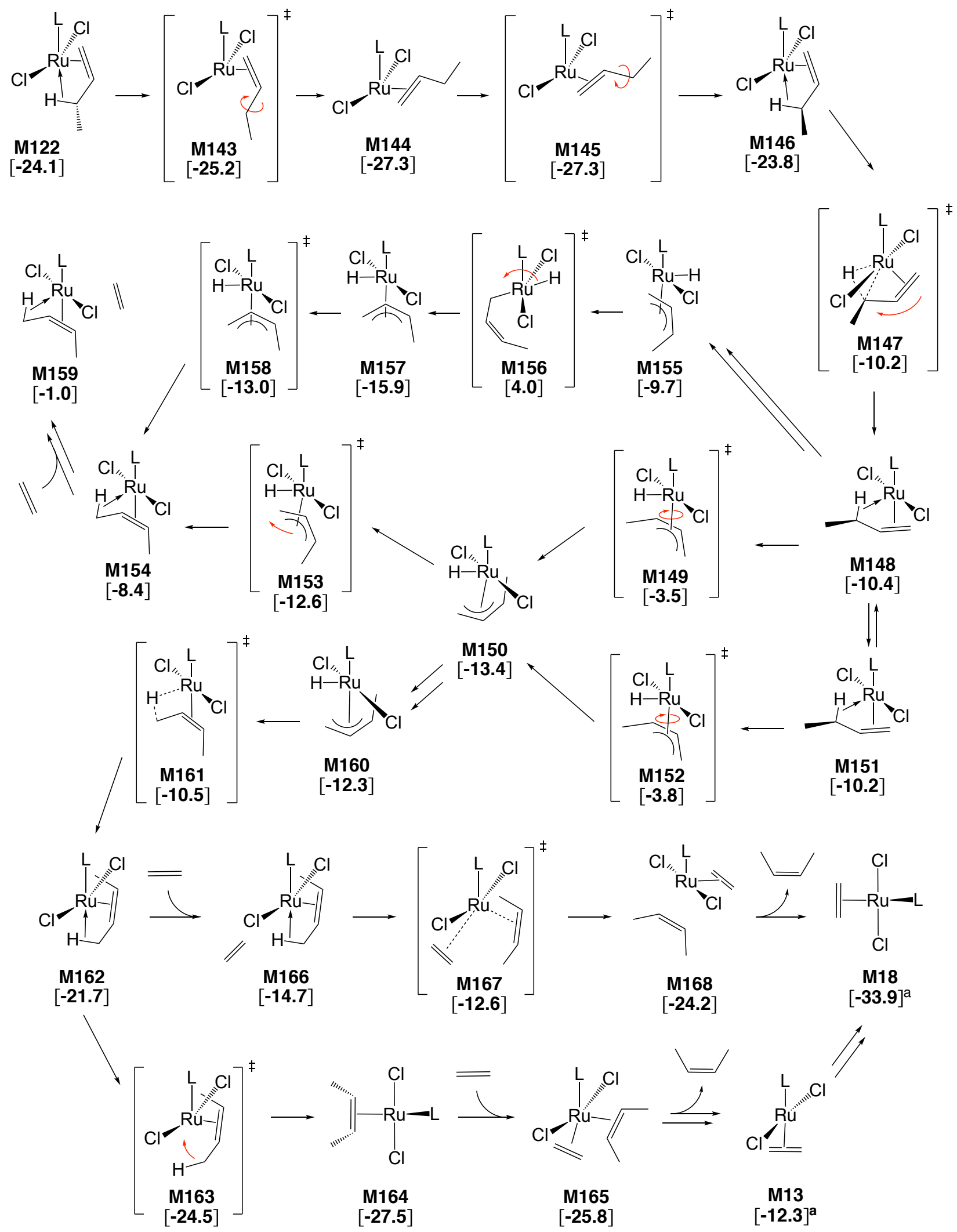

Scheme S29. Free energies (kcal/mol with respect to M1) of species along section P30 (M122-M148) and P31 (M148-M18) of the reaction network. 
To obtain an anti-form allyl ligand we need to coordinate 1-butene with the proper conformation. In particular, M146 has a C-C-C-C dihedral angle of $46^{\circ}$, is only slightly less stable than its analogue M122, and is connected to its other isomers by negligible barriers (M143 and M145). While the conformation of the carbon skeleton is suitable to give anti $\eta^{3}$-allyl species, the orientation with respect to the metal center requires a further adjustment (via M147) that gives M148 or, by further twisting of the Ru-NHC bond, M151. These two are both analogues of M118 with a different conformation of the hapto-two 1-butene ligand. The hypothetical Ru-hydrides with anti- $\eta^{3}$-(methyl)allyl ligand resulting from oxidative addition of M148 and M151 bearing the hydride and the methyl-substituted carbon on the same side of the metal center were not found. However, the isomer obtained by twisting of the whole anti- $\eta^{3}$-(methyl)allyl group along the $\mathrm{Ru}-\left(\eta^{3}\right.$-allyl) axis (i.e., M150) can be reached from both M148 and M151 in a single step that combines i) the oxidative addition and ii) the isomerization (i.e., twisting along hapto-three bond). Alternative pathways that would lead to the same or closely related species were sought by twisting the hydride on the other side of the metal center, but the barrier at M156 is significantly higher than those identified by M149 and M152.

Reductive elimination on M150 yields Ru-coordinated cis-2-butene (M159), however the trans relation between the latter and NHC makes the resulting complex unstable with respect to other isomers, and the associative exchange with ethylene via M159 becomes too demanding. Instead, further twisting along the hapto-three bond of M150 to give M160, and the following reductive elimination yield a much more stable hapto-two complex with cis-2-butene (M162). In the latter, the $\eta^{3}$ ligand is roughly cis to the NHC while the $\mathrm{CH}$-agostic bond is trans to it and can easily be broken (i.e., barrier-less step at the SP energy level) allowing further twisting of the butene and access to the most stable cis-2-butene complex M164. Once again, we consider the associative exchange of the product with a new molecule of ethylene to generate M18. The exchange can be done already from M162, via the interchange transition state M167, to obtain the Ru- $\eta^{2}$-ethylene intermediate with the most stable stereochemistry (M18). This alternative avoids the dissociation of the cis-coordinated olefin (i.e., cis-2-butene, see M165) resulting in a transcoordinated Ru- $\eta^{2}$-ethylene 14-electron complex, which is less stable than the cis analogue, and would require either further uptake of ethylene or spin-crossover into the spin-triplet state to gain stability as previously discussed in Scheme S11. Overall, these pathways define a catalytic cycle for the isomerization of 1-butene to cis-2-butene (Scheme S27). 


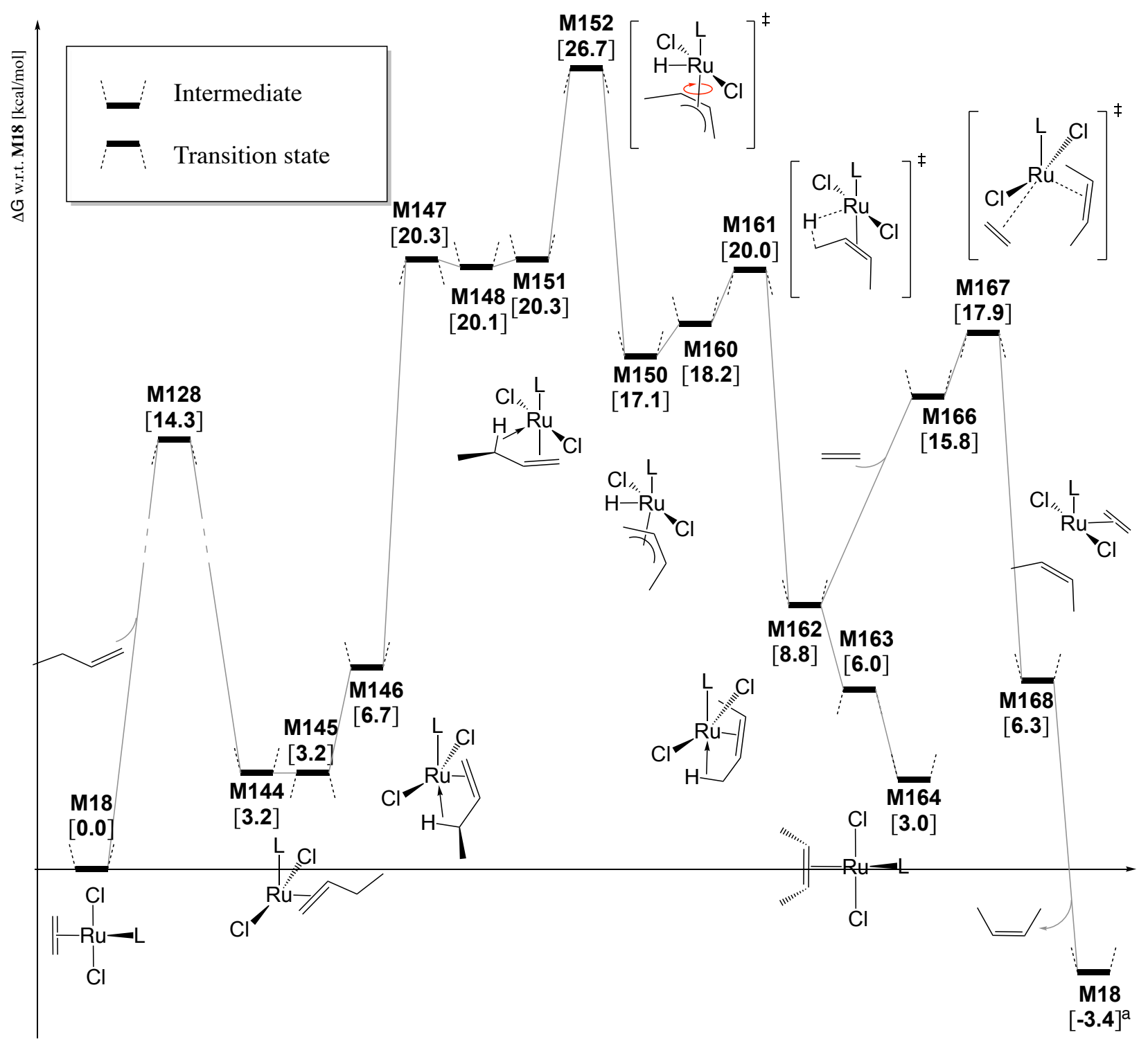

Scheme S30. Free energies ( $\mathrm{kcal} / \mathrm{mol}$ with respect to M18) of species along the isomerization cycle converting 1-butene to $c i s-2-$

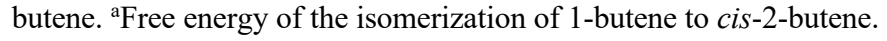

As observed above for trans-2-butene, the production rate of cis-2-butene in Figure S3 is practically constant at the beginning of the experiment. The isomerization of 1-butene would most likely imply a dependency of the production rate on the concentration of 1-butene and, therefore, a significant increase in the cis-2-butene formation rate. The experiments without phosphine do not show such dependency and, thus, suggest that the main source of cis-2-butene is a direct formation from ethylene rather than isomerization of 1-butene.

In the attempt to identify a pathway describing the direct formation of cis-2-butene from two ethylene molecules, we considered the hypothetical formation of $s$-cis-butadiene. In fact, the reaction network described above for direct formation of trans-2-butene involves Ru-bound s-trans-butadiene which evolves into 1-butene or trans-2-butene. An analogous reaction network based on s-cis-butadiene could hypothetically produce 1-butene and cis-2-butene directly from ethylene, which would be in agreement with the trends observed experimentally. Such pathway remains elusive, and the following text describes the most promising, yet failed attempts to identify this or other alternative pathways leading to direct production of cis-2-butene. 
We tested the possibility to change the conformation of butadiene by twisting the $\mathrm{C}-\mathrm{C}$ bond in the $\mathrm{C} 4$ skeleton when it is still a simple single bond. This conformational change is easy on M57 (just 1.4 $\mathrm{kcal} / \mathrm{mol}$ barrier from M57 to M169), but the subsequent $\beta$-H elimination in M171 (3.8 kcal/mol barrier from M170) is not as easy as the one for $s$-trans analogue $\mathbf{M 6 6}(2.9 \mathrm{kcal} / \mathrm{mol}$ barrier with respect to M65). After propeller-style rotation of $\eta^{2}$-butadiene in M172, we obtain the hapto-four complex of $s$-cisbutadiene $\mathbf{M 1 7 5}$ that is $1.3 \mathrm{kcal} / \mathrm{mol}$ less stable than the s-trans-analogue M74 (Scheme S15).

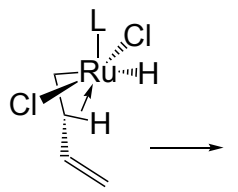

M57

[3.9]

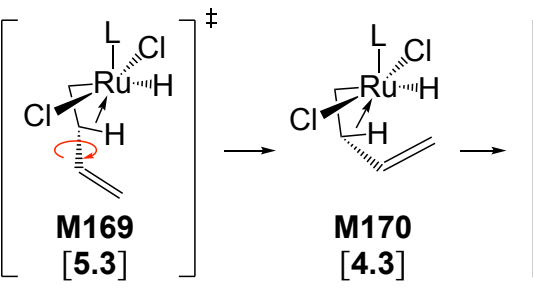

[4.3]

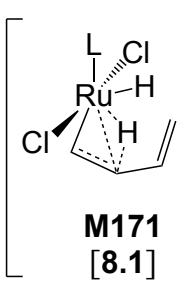

[8.1]

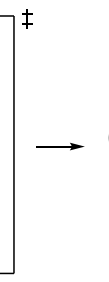

C

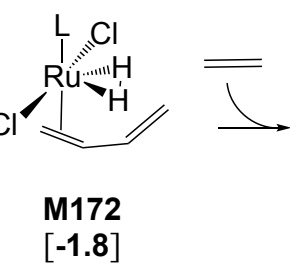

$[-1.8]$

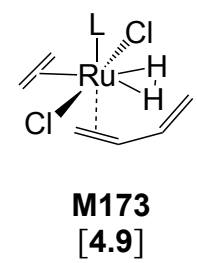

[4.9]
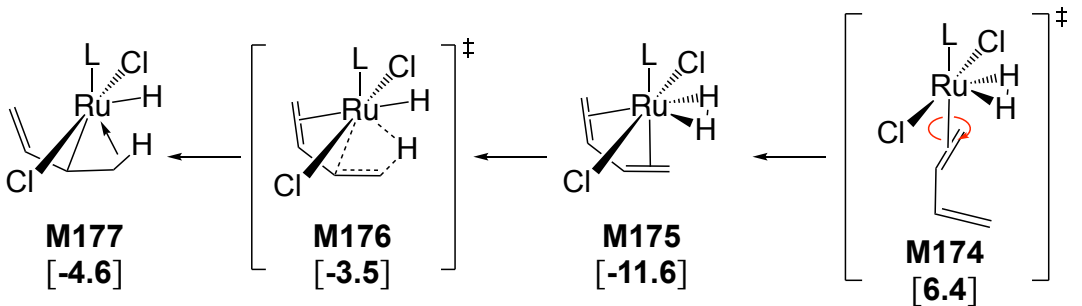

Scheme S31. Free energies ( $\mathrm{kcal} / \mathrm{mol}$ with respect to M1) of species along section P32 of the reaction network.

Transfer of one hydrogen onto the carbon skeleton of the $s$-cis-butadiene of M175 generates the butenyl ligand M177, but this latter species is more conveniently generated via M178 by the conformational change on the $\mathrm{C}-\mathrm{C}$ skeleton of $\mathbf{M 1 0 4}$ (Scheme S32), a species deriving from $s$-trans-butadiene. Therefore, formation of $s$-cis-butene is not required in order to access M177.

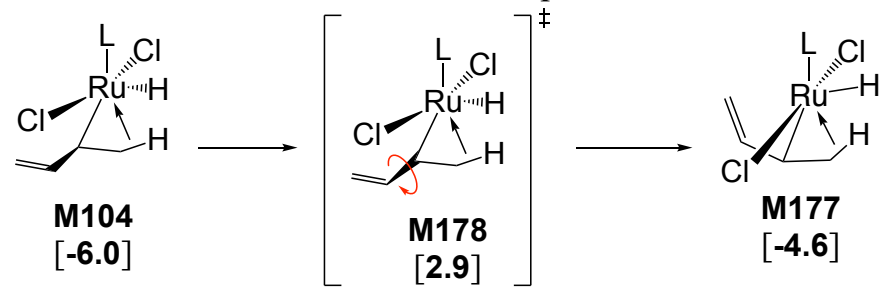

Scheme S32. Free energies ( $\mathrm{kcal} / \mathrm{mol}$ with respect to M1) of species along section P33 of the reaction network.

From M177 a more stable isomer (M179) can be obtained by relaxing the conformation of the NHC ligand (see Scheme S33), which is expected to be an easy step. Next, the increase of hapticity yields the hapto-three intermediate M181. The next step via $\mathbf{M 1 8 2}$ includes first the rupture of the CH-agostic bond, and then the reductive elimination to obtains M148, which was encountered before along the pathway leading to cis-2-butene (see Scheme S29).

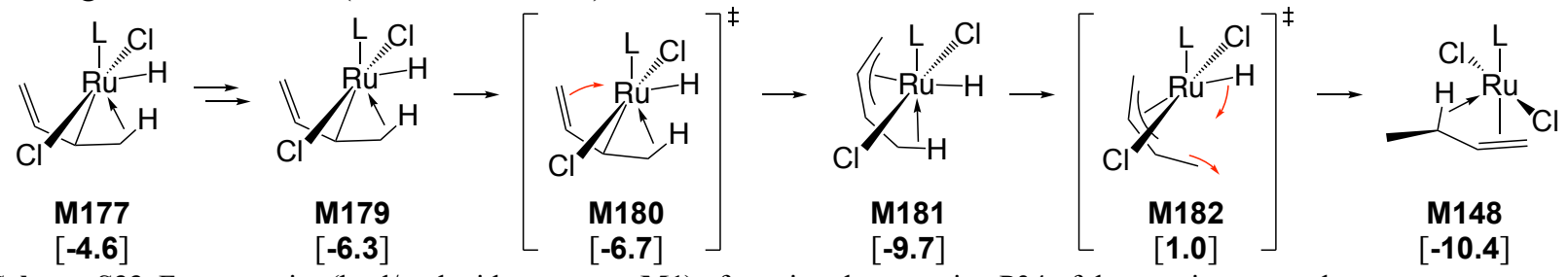

Scheme S33. Free energies ( $\mathrm{kcal} / \mathrm{mol}$ with respect to M1) of species along section P34 of the reaction network. 
This pathway for direct production of cis-2-butene goes via transition state M178 that implies a slower formation of cis-2-butene than of trans-2-butene. On the contrary, in the experiment performed without phosphine and at room temperature (Figure S3 and Table S4) the concentration of cis-2-butene at the beginning of the reaction seems slightly higher than that of the trans isomer. This implies a slightly faster formation of cis-2-butene than trans-2-butene, in addition to cis-to-trans isomerization reaction (see below). This is in contrast with the energy of M178 (2.9 kcal/mol w.r.t. M1) higher than that of M105 ($0.4 \mathrm{kcal} / \mathrm{mol}$ w.r.t. M1), and suggests the existence of faster alternative pathways for the direct conversion of ethylene into cis-2-butene.

To test the existence of other pathway connecting s-cis-butadiene with formation of cis-2-butene we considered the formation of cis-2-butene by stepwise hydrogenation of s-cis-butadiene on NHCcoordinated $\mathrm{Ru}$-species with cis-Cl configuration (Scheme S34). Two similar paths were found from M197, which holds molecular hydrogen and s-cis-butadiene, to M201 and M199, which are two different Ru-complexes of cis-2-butene characterized by, respectively, one or two $\mathrm{CH}$-agostic interactions between the methyl groups of cis-2-butene and the metal. The free energy of the transition states involved in each of these alternative steps (M200 $-2.5 \mathrm{kcal} / \mathrm{mol}$, and $\mathbf{M 1 9 8}-3.3 \mathrm{kcal} / \mathrm{mol}$, both with $\mathbf{M 1}$ as reference) are lower than those encountered for the direct formation of trans-2-butene from Ru-bound s-trans-butadiene (i.e., M105 $-0.4 \mathrm{kcal} / \mathrm{mol}, \mathbf{M 1 4 0}-1.1 \mathrm{kcal} / \mathrm{mol}$, with M1 as reference), thus promising with respect to faster production of cis-2-butene.

Trying to proceed backwards, we searched for pathways reaching M197 from the cis-Cl species binding s-trans-butadiene like M187 and M189. However, the energy of M188, M194, and M196 are too high, and, moreover, we could not find any suitable pathway able to connect such cis-Cl species with the rest of the reaction network, which is mostly characterized by trans- $\mathrm{Cl}$ configuration. 

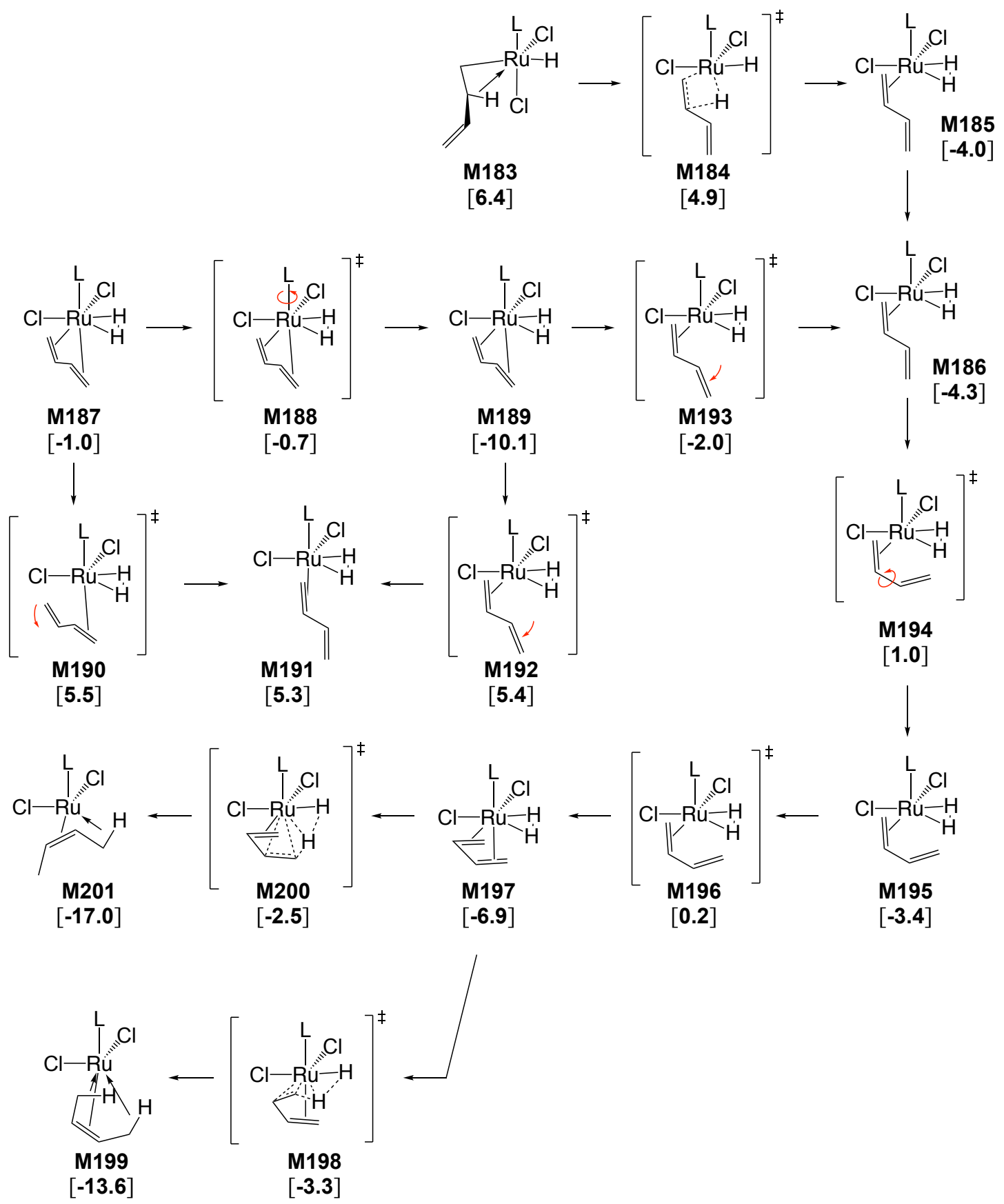

Scheme S34. Free energies (kcal/mol with respect to M1) of cis-Cl Ru-species related to butadiene.

The change of configuration via $\mathbf{M 7 5}$ (8.3 $\mathrm{kcal} / \mathrm{mol}$ relative to M1, see Scheme S15) is too demanding to be consistent with faster formation of cis-2-butene than trans-2-butene. Moreover, among the attempts to identify cis- $\mathrm{Cl}$ analogues for the coupling of ethylene molecules (i.e., M53 in Scheme S11 and in Scheme S35, and M202, M203 in Scheme S35) the lowest energy transition state, i.e., M53 (7.4 kcal/ mol relative to M1), leads to a metallacyclopentane that still requires too much energy for the $\beta-\mathrm{H}$ elimination via $\mathbf{M 2 0 5}$ (Scheme S35). The same issue affects yet another configuration tested as M207, which is thus not compatible with faster production of cis-2-butene than trans-2-butene. 

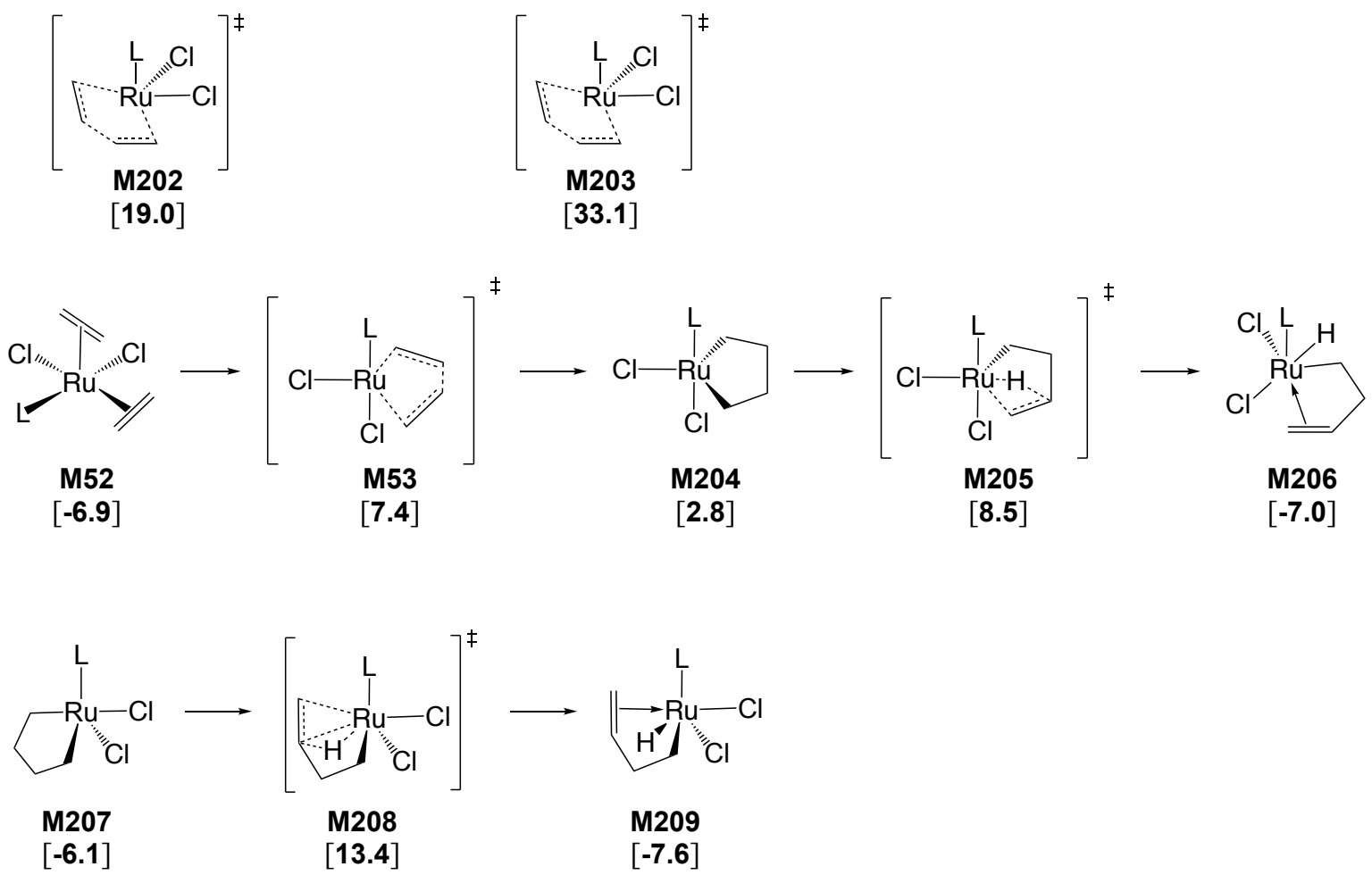

Scheme S35. Free energies ( $\mathrm{kcal} / \mathrm{mol}$ with respect to $\mathbf{M 1}$ ) of cis-Cl Ru-species related to metallacyclopentane formation and rupture.

In conclusion, though the experimental results in Figure S3 and Table S4 suggests direct production of cis-2-butene from ethylene to be slightly faster than that of trans-2-butene, a consistent pathway for the cis configuration is still to be found.

\section{Isomerization of Butenes by Cyclometallated Ru Centers}

The cyclometallation step described above in Scheme S9 produces a hydride (M24) that was previously suggested to catalyze isomerization of allylbenzene. ${ }^{1}$ In the present context, the most abundant olefin in the system is ethylene, and Scheme S10 has shown how coordination of such non-isomerizable olefin can trigger the regeneration of the original, non-cylometallated NHC ligand and thus M18. However, as their concentration increases, 1- and cis-2-butenes can be isomerized to their most thermodynamically stable isomer trans-2-butene. In this section we explore the possibility of reacting Ru-hydrides generated from cyclometallation with the butenes searching for double bond migration and cis-trans isomerization pathways.

Coordination of 1-butene on M24 proceeded in analogy with the mechanism previously published for allylbenzene (Scheme S36). ${ }^{1}$ After obtaining a suitable arrangement between the hydride and the haptotwo ligand in M212, insertion at M213 forms the isobutyl ligand in M214. This alkyl ligand is trans to the $\mathrm{NHC}$ and the system suffers from trans influence. Isomerization to the triangular bipyramid system M216 relieves more than $11 \mathrm{kcal} / \mathrm{mol}$ from M214. The small and flexible isobutyl chain can easily explore alternative conformations M218, M220, M222, M224, M229, and M231. $\beta$-H elimination on different conformations can generate butenes with cis or trans stereochemistry. Trans-2-butene could be formed by elimination from M224 that would generate hydride M227, but the energy of transition state M225 is higher than other alternatives that become accessible via conformer M231. 

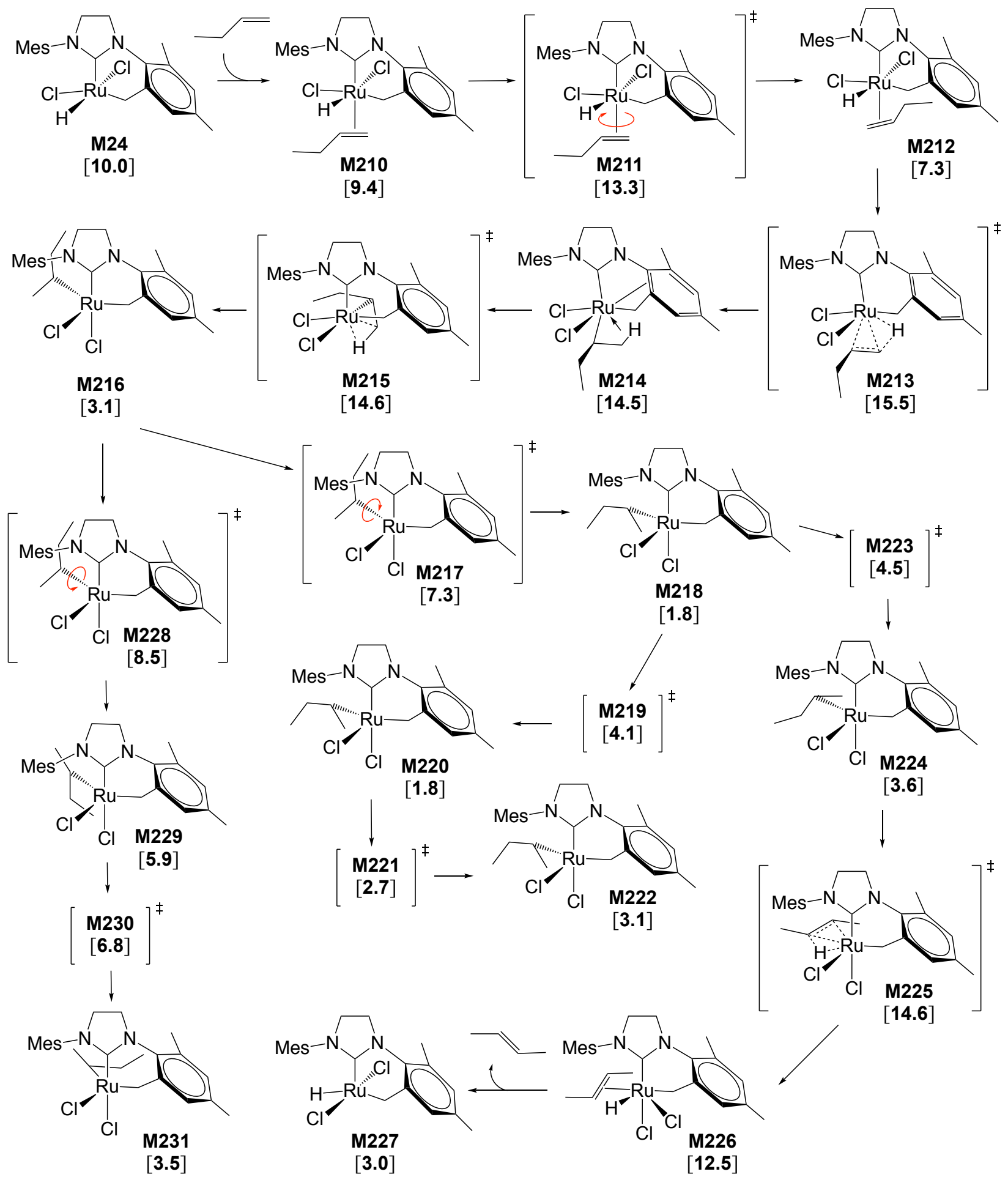

Scheme S36. Free energies (kcal/mol with respect to M1) of species along section P35 (M24-M216), P36 (M216-M227), and P37 (M216-M231) of the reaction network.

The conformation of the isobutyl chain in M231 is suitable for producing cis-2-butene by $\beta-\mathrm{H}$ elimination, and this pathway (Scheme S37) rather than forming an intermediate Ru-hydride complex proceeds via transition state $\mathbf{M 2 3 4}$, where the metal center mediates the transfer of the hydride atom from the isobutyl ligand to the cyclometallated end of the mesityl fragment. This results in the regeneration of 
the intact NHC ligand, though still involved in $\mathrm{CH}$-agostic interaction at $\mathbf{M 2 3 5}$. Two alternatives were found to liberate the mesityl from such $\mathrm{CH}$-agostic bond and form cis-2-butene complexes $\mathbf{M 2 3 7}$ and M239. These two are both characterized by cis- $\mathrm{Cl}$ configuration and differ by the conformation along the hapto-two bond. In particular M239 sees the two methyl groups of cis-2-butene pointing away from the NHC ligand, thus reducing the interaction with such ligand and allowing further conformational change yielding M240 most likely without significant free energy barrier. The cis-Cl configuration of M240 can easily be converted into trans-Cl via M241. The resulting trans-Cl $\eta^{2}$-cis-2-butene complex M164 was already connected to M18 in Scheme S29.

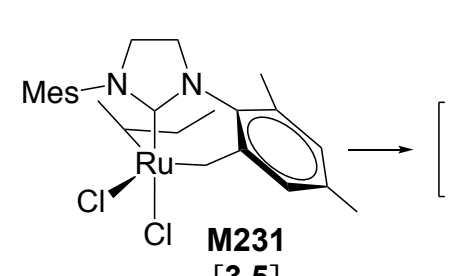

[3.5]

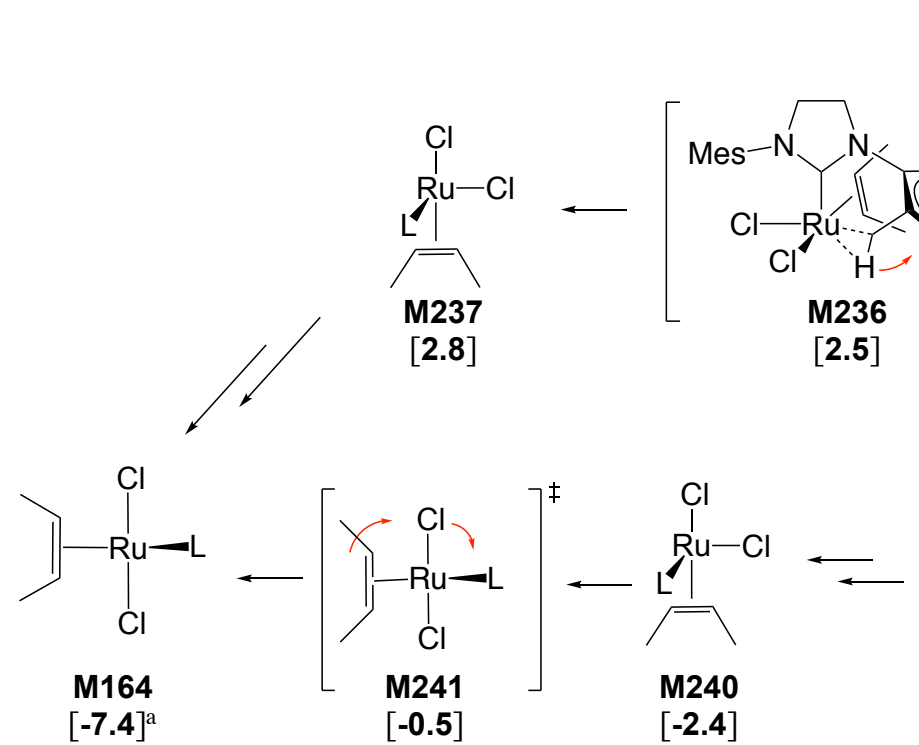

$[-7.4]^{a}$

$[-0.5]$

$[-2.4]$
M232 [8.2]

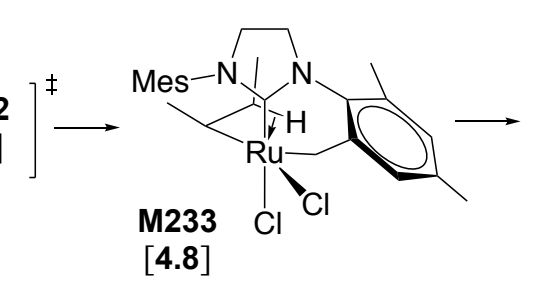

L

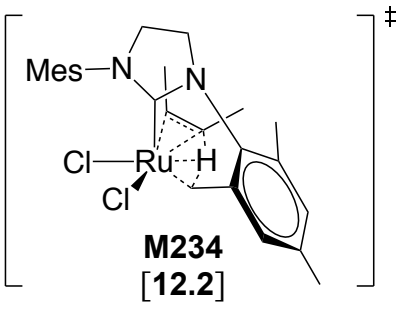

[12.2]

[1.2]

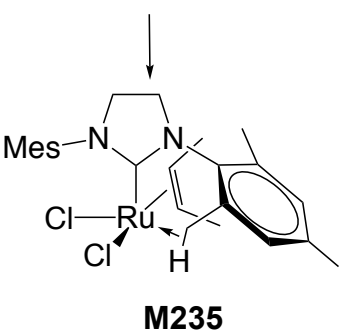

[2.7]

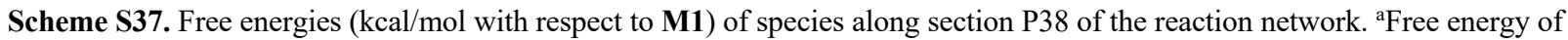
M164 that includes the free energy of the reaction transforming 1-butene into cis-2-butene.

The geometry of M231 allows to form $\mathrm{CH}$-agostic interaction with both hydrogen atoms of the methylene in the isobutyl chain. The pathway just described starts with formation of agostic interaction with one such hydrogen atoms and yields cis-2-butene. Instead, formation of the $\mathrm{CH}$-agostic interaction with the other hydrogen atom leads to trans-2-butene as shown in Scheme S38. This latter pathway involves, after formation of $\mathrm{CH}$-agostic interaction with the isobutyl (in M243), Ru-mediated transfer of hydride from the isobutyl to the cyclometallated mesityl group (transition state M244). This transition state leads to trans-2-butene and has lower free energy than the corresponding one leading to cis-2-butene (M234) due to the methyl groups belonging to the forming butene conveniently places away from the cyclometallated mesityl. Like in the previous case, the result is a $\mathrm{CH}$-agostic bond with an intact mesityl in $\mathbf{M 2 4 5}$, and the subsequent release of such interaction with formation of cis-Cl $\eta^{2}$-trans-2-butene complex M247. Small conformational changes lead to M248, and then the configuration is altered by easy and concerted twisting of the butene ligand and of one chloride ligand (via M249). This results in the trans-Cl $\eta^{2}$-trans-2-butene complex M250. The cis arrangement between the hapto-two trans-2-butene ligand and $\mathrm{NHC}$ is novel in this reaction network, but the exchange of this ligand with ethylene to form M18, though not further investigated, is expected to be closely related to the analogue pathway for cis-2butene in Scheme S29. 


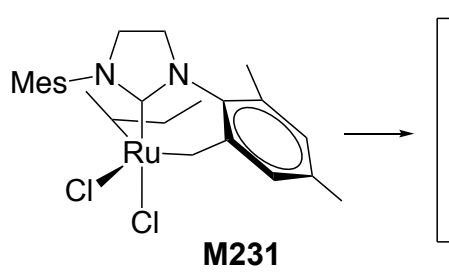

[3.5]<smiles>Cc1cc(C)cc(C2N(C)CCN2C(Cl)=C(Cl)Cl)c1</smiles>

[6.4]

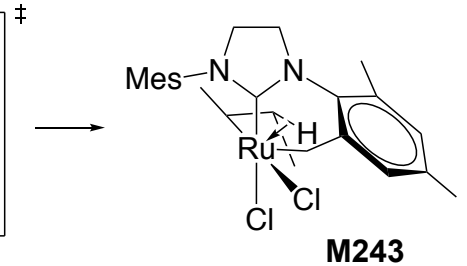

[4.3]

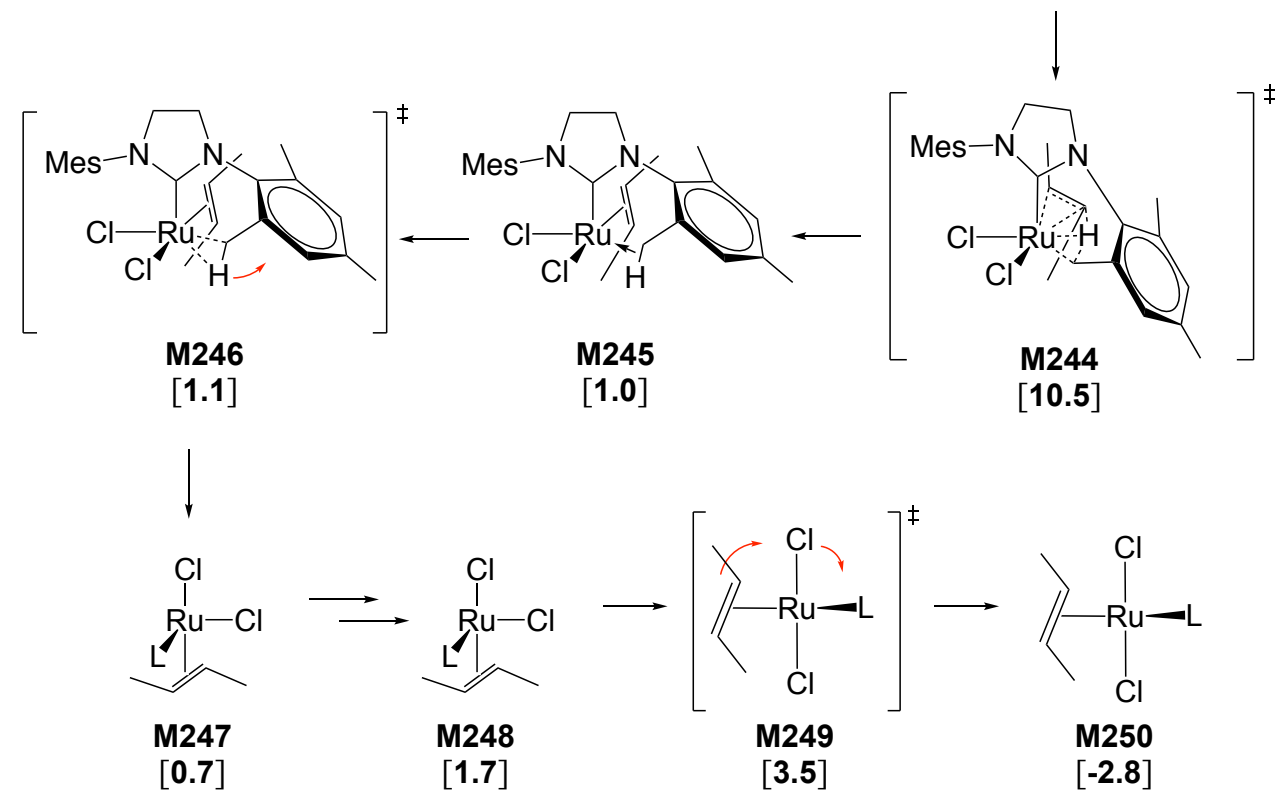

Scheme S38. Free energies ( $\mathrm{kcal} / \mathrm{mol}$ with respect to M1) of species along section P39 of the reaction network.
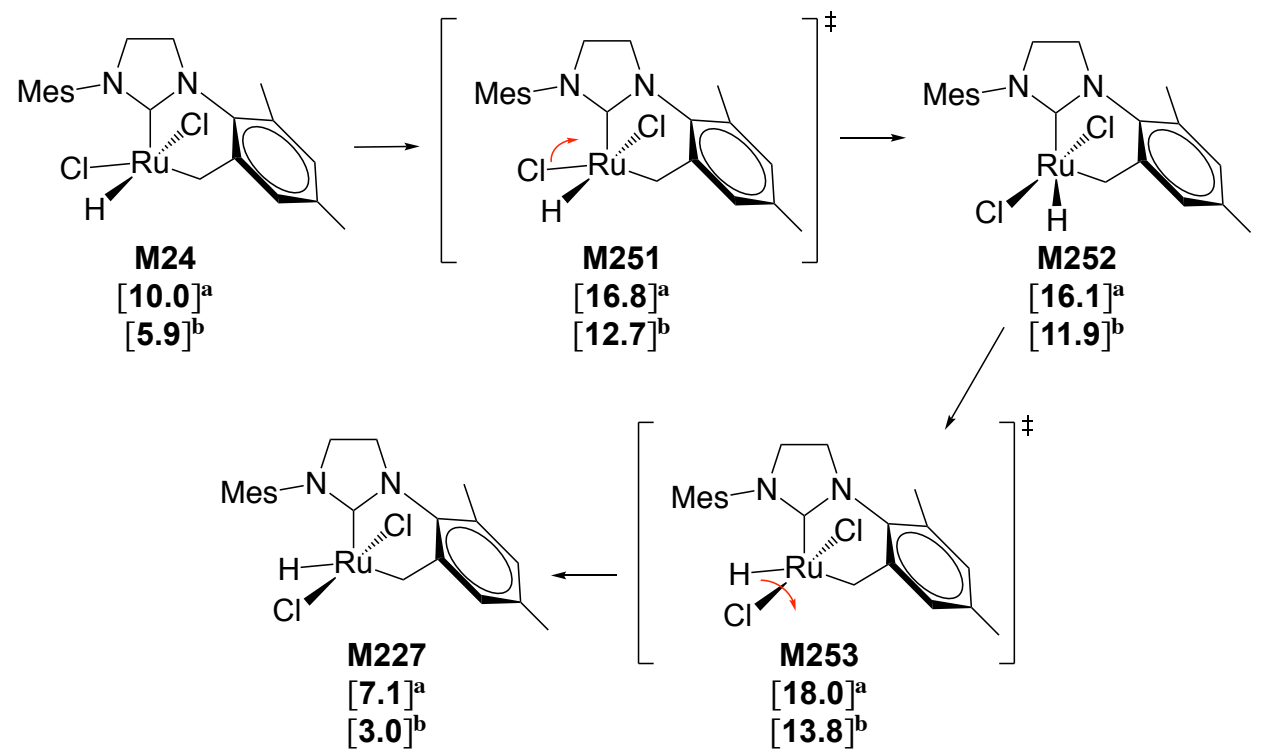

Scheme S39. Free energies ( $\mathrm{kcal} / \mathrm{mol}$ with respect to M1) of species along section P40 of the reaction network. ${ }^{\text {a Free energy }}$ calculated for direct formation from M1 via P1 and P6. ${ }^{b}$ Values including the free energy of the reaction transforming 1-butene into trans-2-butene.

Let's now recall the Ru-hydride complex M227 that result from path P36 (Scheme S36) because this species can regenerate M24 (Scheme S39). This isomerization closes the 1-butene isomerization cycle catalyzed by $\mathbf{M 2 4}$, and represents the turnover determining step of such catalytic cycle, as shown in the previous publication for isomerization of allylbenzene. ${ }^{1}$ However, we have now discovered than rather 
than regenerating $\mathbf{M 2 4}$ and close this catalytic cycle, M227 can act as catalyst for isomerization of 2butenes.

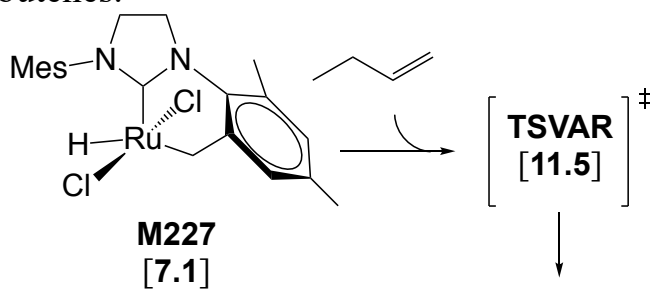

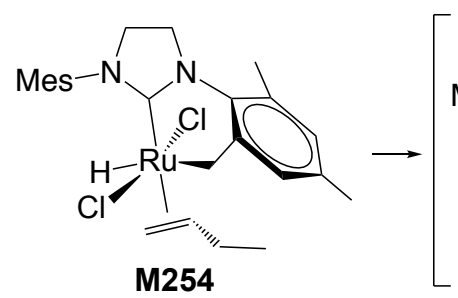

[5.9]

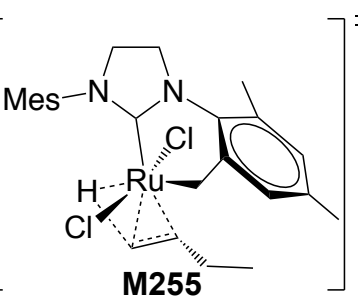

[15.3]

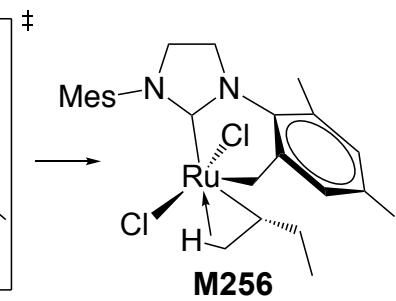

[8.9]

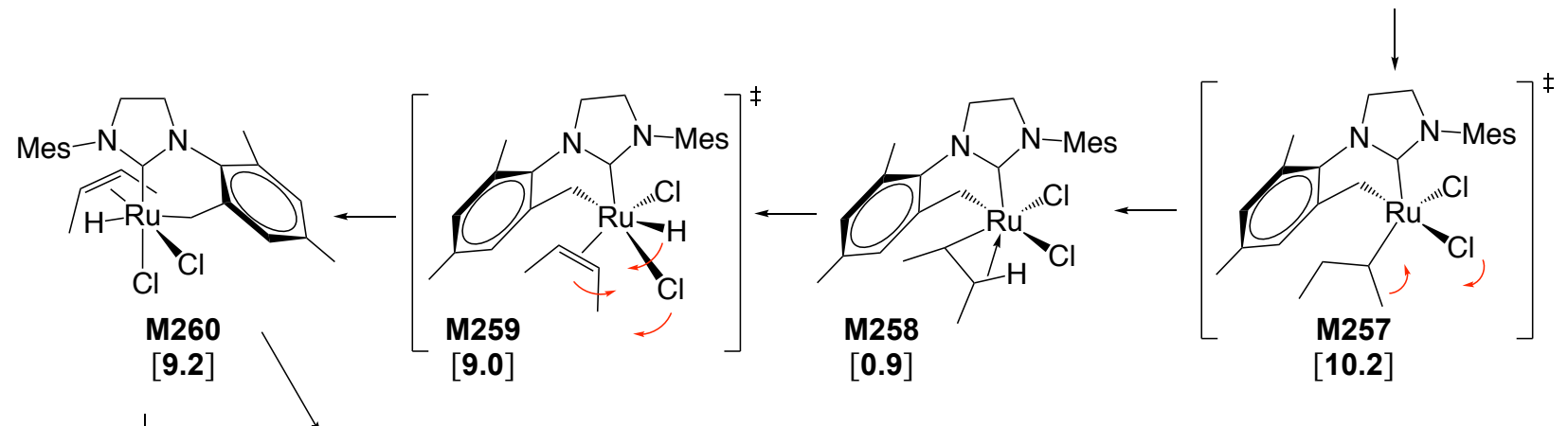

$[9.2$
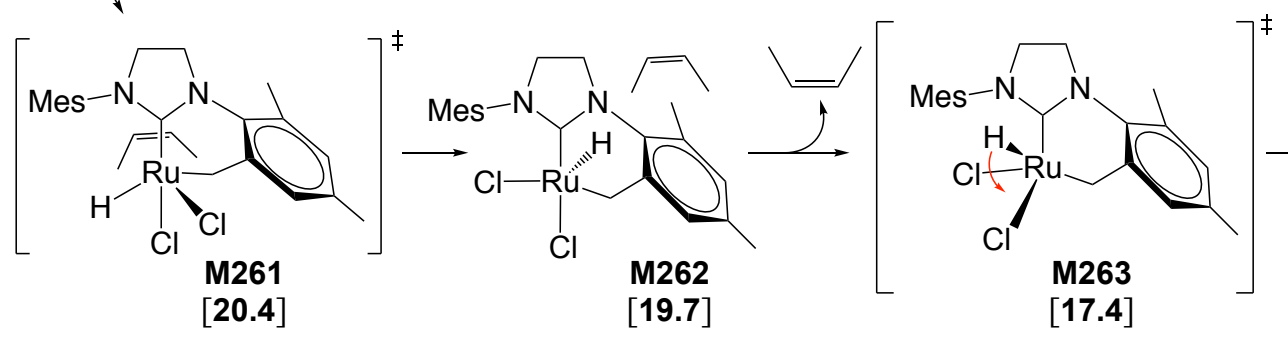

M227

$[1.1]^{\mathrm{a}}$

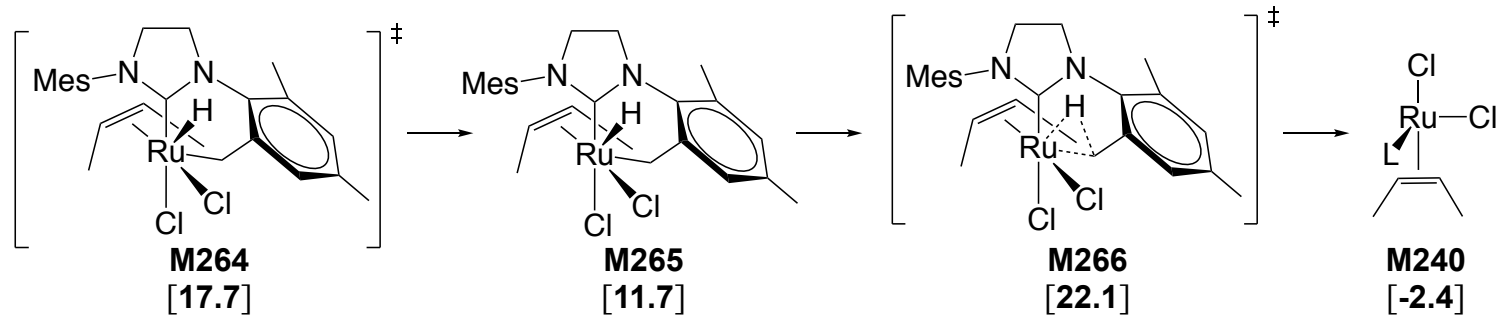

Scheme S40. Free energies (kcal/mol with respect to M1) of species along section P41 (M227-M260), P42 (M260-M227), and

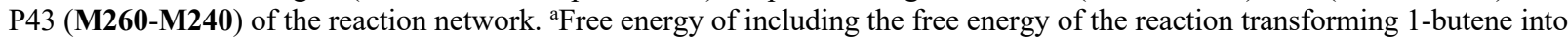
cis-2-butene.

Coordination of butenes on $\mathbf{M 2 2 7}$ is expected to be diffusion controlled as any attempt to strip away such ligands from the bound complexes M254, M267, and M283 resulted in monotonously increasing potential energy. No significant geometrical change in the two fragments was observed, and no pronounced potential energy barrier (i.e., transition state) could be identified. This is plausible because M227 is already reasonably stable, it gains stability by binding a dative ligand, and the coordination site 
used to bind the butenes is wide and comfortable for such substrates. Thus, we used the variational transition state approximation as described in the Computational Methods section.

After coordination of 1-butene onto M227, insertion leads to isobutyl ligand in $\mathbf{M 2 5 6}$ where the ethyl chain coils in the pocket generated by the cyclometallated NHC ligand (Scheme S40). Such folding of the chain determines the stereochemistry of the $\mathrm{C} 4$ chain resulting from $\beta-\mathrm{H}$ elimination. After rearrangement to form an alternative $\mathrm{CH}$-agostic interaction at M258, $\beta$-H elimination yields the hapto-two cis-2-butene complex M260. Attempts to model analogues pathway leading to trans-2-butene failed because of the incompatibility of the latter stereochemistry with the small pocket defined by the cyclometallated $\mathrm{Ru}$ center in M227. While the highest energy transition state between M227 and M260 is M255 (i.e., olefin insertion), the release of cis-2-butene from M260 is described as challenging by the present DFT model. Two possibilities were found and are depicted in Scheme S40. The first is about direct release of cis-2butene via transition states $\mathbf{M 2 6 1}$ and is followed by regeneration of Ru-hydride M227. This path defines a catalytic cycle for the isomerization of 1-butene to cis-2-butene by double bond migration catalyzed by M227. However, this catalytic cycle is not very effective due to the generation of a hydride at M262 (and the related transition states) that has a configuration sensibly less stable than its isomer M227. The second, though more energetically demanding possibility sees the isomerization of hydride M260 followed by reductive elimination in $\mathbf{M 2 6 6}$ that regenerates an intact NHC ligand at M240. As described in Scheme S37, the hapto-two species M240 can further evolve to exchange cis-2-butene with ethylene, thus hampering the catalytic cycle M227-M260-M262-M227.

Besides these pathways catalyzing double bond migration, $\mathbf{M 2 2 7}$ catalyzes cis-trans isomerization with extreme efficiency. As anticipated, coordination of cis-2-butene on M227 is expected to be diffusion controlled (Scheme S41). The bound olefin in M267 is then transformed into an isobutyl ligand. This allows to change the configuration of the originally loaded olefin by twisting the conformation of the alkyl chain (via M270 and M274). Then, a new olefin is generated by $\beta-\mathrm{H}$ elimination on the new conformation of the isobutyl chain. If such elimination occurs on the methyl end of the isobutyl fragment (via M276), the result is 1-butene. However, this reaction would be endoergonic, and the direct release of 1-butene is unlikely due to the high energy of $\mathbf{M 2 8 0}$ that generates the high energy hydride M281, which is similar to the one at M262. Moreover, attempt to move the hydride as to saturate the cyclometallated end of the NHC ligand requires much more energy (via M278) than proceeding from $\mathbf{M 2 7 5}$ with $\beta-\mathrm{H}$ elimination of the methylene group (via M282). This path generates the thermodynamically favored trans-2-butene that is easily released (i.e., no barrier found in the potential energy surface), thus closing a very efficient cistrans isomerization cycle catalyzed by $\mathbf{M 2 2 7}$. 

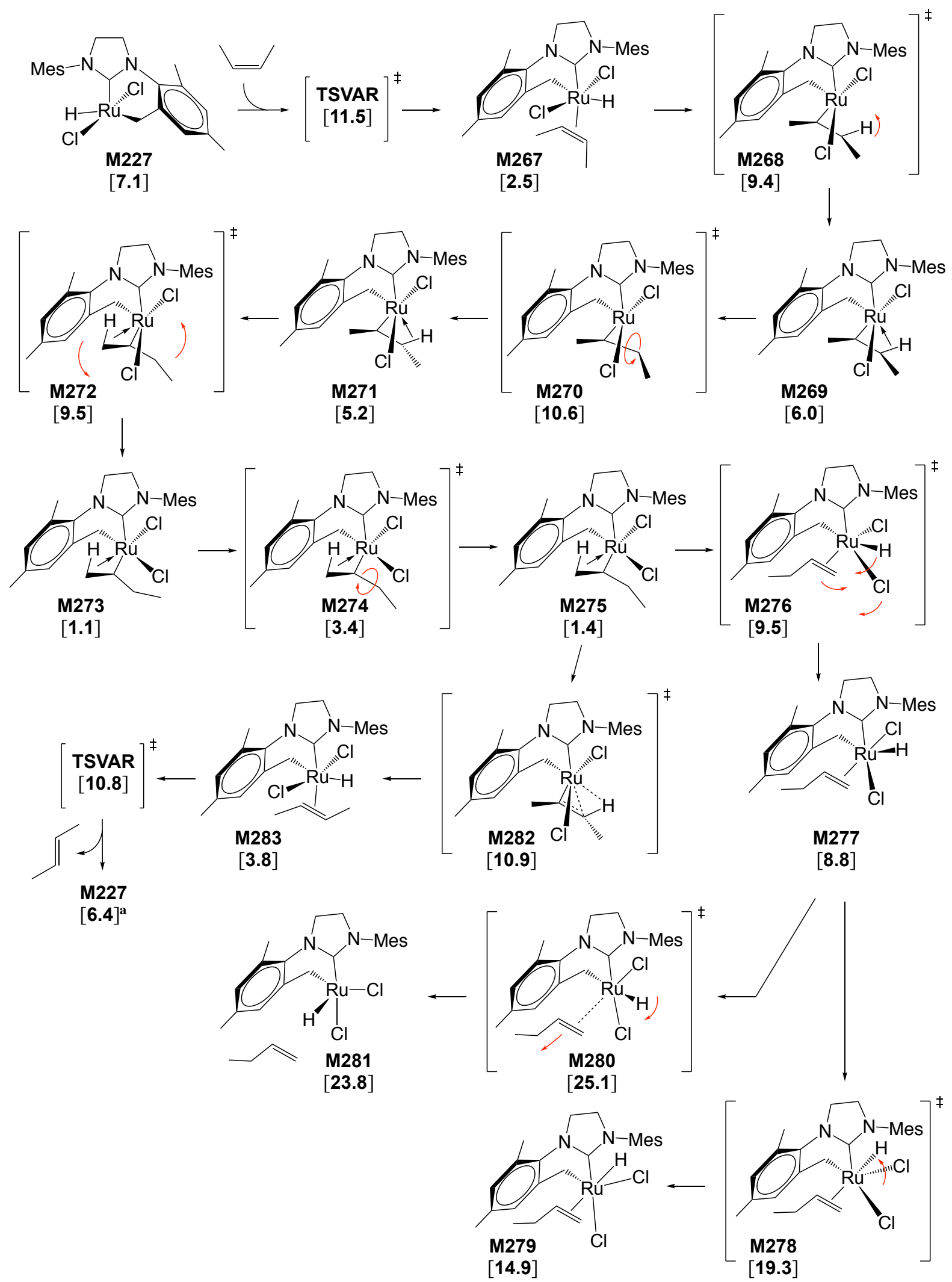

Scheme S41. Free energies ( $\mathrm{kcal} / \mathrm{mol}$ with respect to M1) of species along section P44 of the reaction network. ${ }^{\text {a Value including }}$ the free energy of the isomerization of cis-2-butene to trans-2-butene. 


\section{1,3-H Shift on Metallacyclopentane}

It is worth considering the previously speculated possibility of performing $1,3-\mathrm{H}$ shift on the metallacyclopentane (Scheme S42).

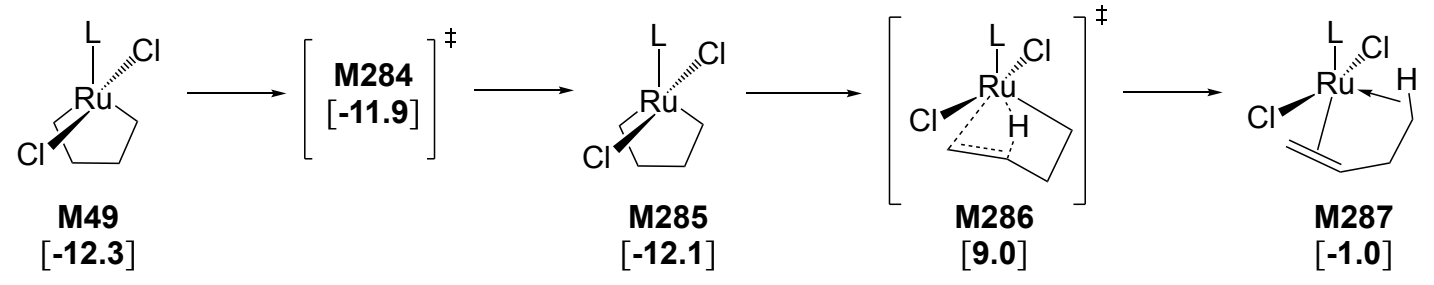

Scheme S42. Free energies (kcal/mol with respect to M1) of species along section P45 of the reaction network.

This path generates 1-butene in one step from the metallacyclopentane $\mathbf{M 2 8 5}$, which is a conformer of the more stable M49. The barrier at $\mathbf{M 2 8 6}$ is, however, higher than the competing path proceeding via M54 by $2.4 \mathrm{kcal} / \mathrm{mol}$. The resulting 1-butene complex M287 can also be obtained from transferring the hydride of $\mathbf{M 5 5}$ on the opposite side of the metal center (i.e., via M288, that is, moving $\mathrm{H}$ around the $\mathrm{Ru}-\mathrm{Cl}$ bond, Scheme S43). The energy of transition state M288 is higher than that of M56 (see Scheme $\mathrm{S} 13$ ) where the coordination mode of the organic $\mathrm{C} 4$ skeleton is changed by twisting of one single $\mathrm{C}-\mathrm{C}$ bond as to decoordinate the olefinic function in favor of the $\beta-\mathrm{CH}$ agostic bond of M57 (see Scheme S13). Once reached, the 1-butene complex $\mathbf{M 2 8 7}$ is unstable with respect to its isomers M118, M122, and M144, and can easily evolve to reach M118 (Scheme S44). However, both pathways producing M287 involve barriers higher than the lowest energy one (via M55 and M56), and are therefore less likely than the latter.

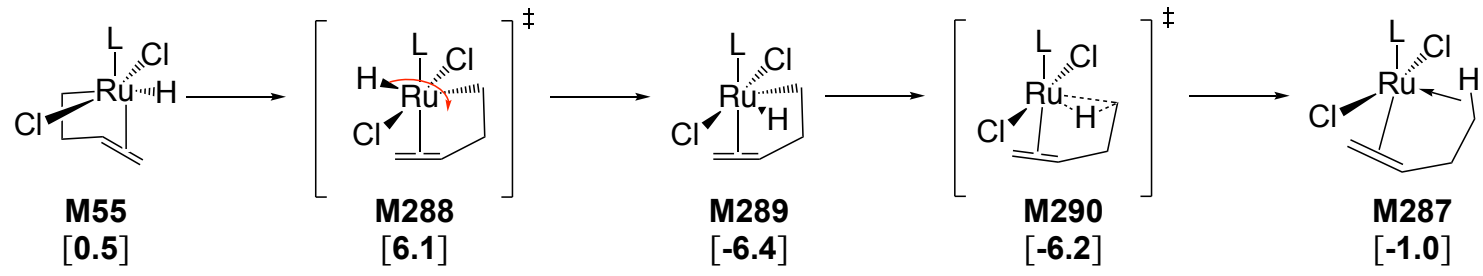

Scheme S43. Free energies (kcal/mol with respect to M1) of species along section P46 of the reaction network.

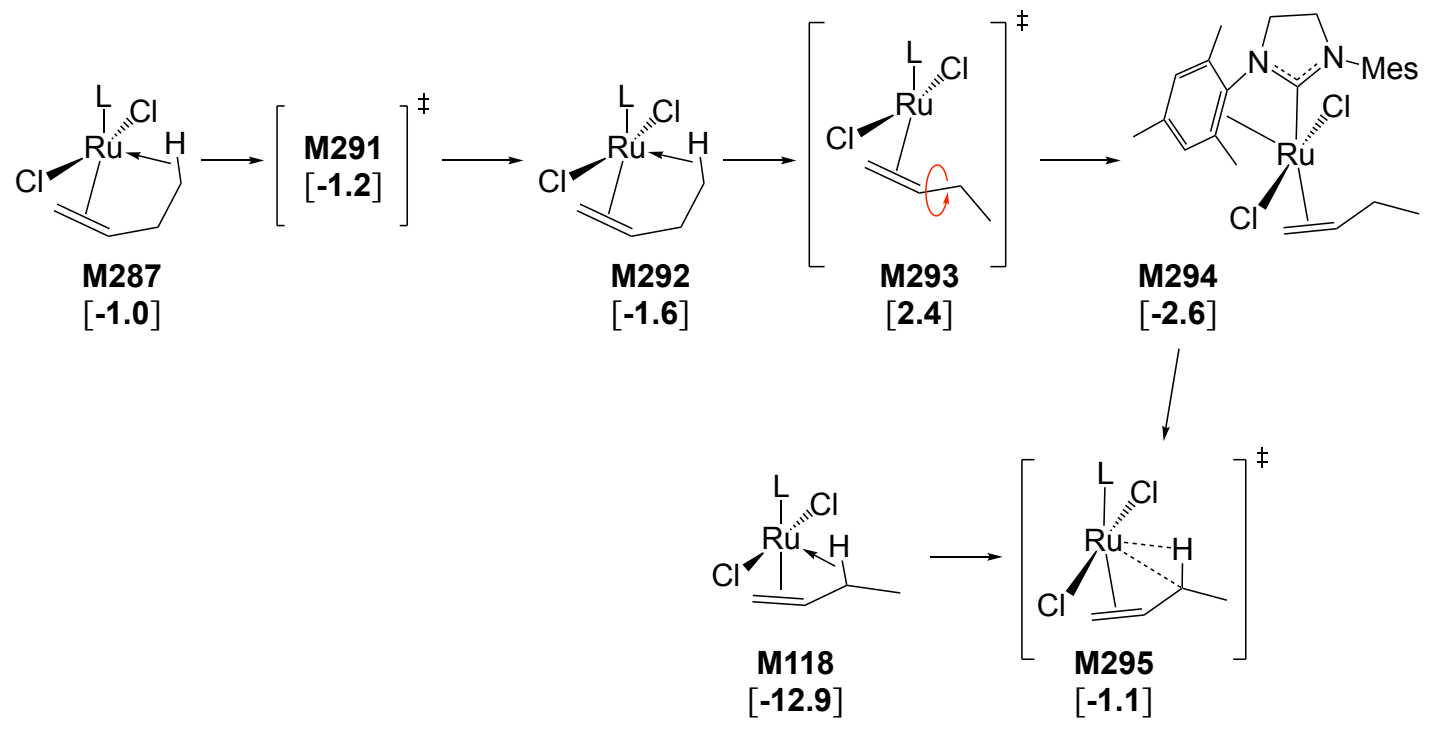

Scheme S44. Free energies (kcal/mol with respect to M1) of species along section P47 of the reaction network. 


\section{Formation of Alkylidenes}

Our calculations suggest that alkylidenes are obtained via formation of metallacyclobutane (MCB) from the $\eta^{3}$-allyl and olefinic complexes that result from the reaction of M1 with ethylene. Overall, these pathways represent the reverse of metallacyclobutane decomposition by $\beta-\mathrm{H}$ elimination. The latter can involve $\mathrm{C}-\mathrm{H}$ bonds either internal or external to the metallacyclobutane ring (see below). In either case these pathways eventually produce an olefin and a $\mathrm{Ru}(\mathrm{II})$ center, ${ }^{1}$ which are the reactants of the experiments performed in the present study.

Methyl substituted MCB (M303) could be reached from several of the species mentioned before (Scheme S45). Among the others, the lowest energy transition state M304 involves H-shift onto the median carbon of $\eta^{3}$-allyl fragment of Ru-hydride M114, which can be connected to M1 by the pathways described in the previous sections. In particular, M114 is along the lowest energy pathway for the direct production of 1-butene from ethylene.

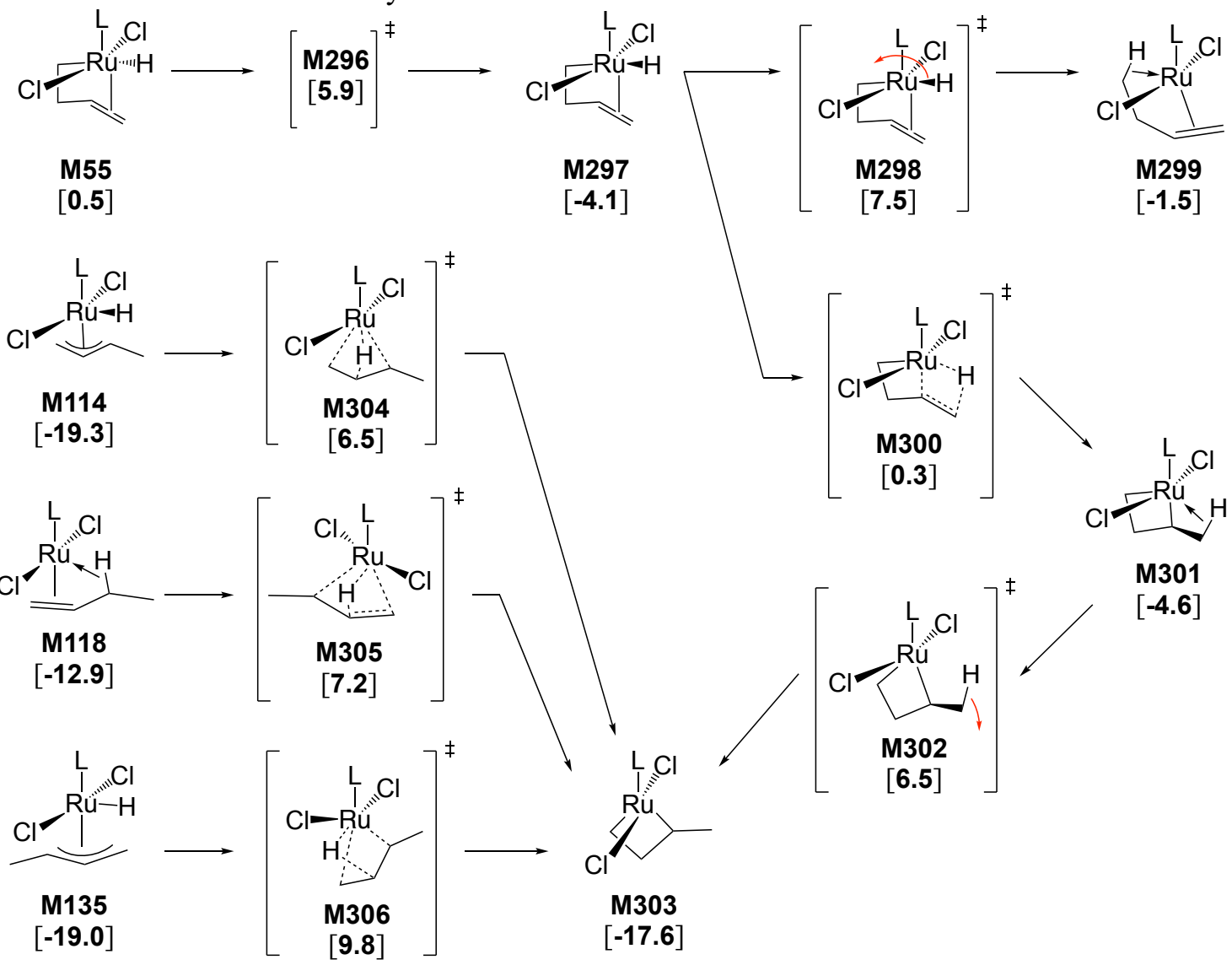

Scheme S45. Free energies (kcal/mol with respect to M1) of species along sections P48 (M114-M303), P49 (M118-M303), P50 (M55-M303), and P51 (M135-M303).

Though slightly more demanding, the path via transition state M305 incorporates the same type of reactivity of M304 and P19 (i.e., the connection between M114 and M118) in a single step. Instead, the transition state describing H-shift from the position external to the forming MCB (M300), is much lower in energy than M304 and M305, but relaxation of the resulting $\mathrm{CH}$-agostic complex M301 requires crossing transition state $\mathbf{M 3 0 2}$ that has the same energy of M304. The external H-shift is, however, dependent on the preliminary formation of the 1-but-3-enyl Ru-hydride M297 that, to date, has been obtained only from isomerization of M55 via the M296, which has energy comparable to M302 and M304. All these pathways have energies higher than the sequence of steps leading from M55 to the production of butenes. Therefore, the reaction network is consistent with the relative rates observed 
experimentally, that is, formation of 1-butene faster than that of metathesis related species, like alkylidenes, and that of the marker for alkylidene formation and decomposition, that is, propene.

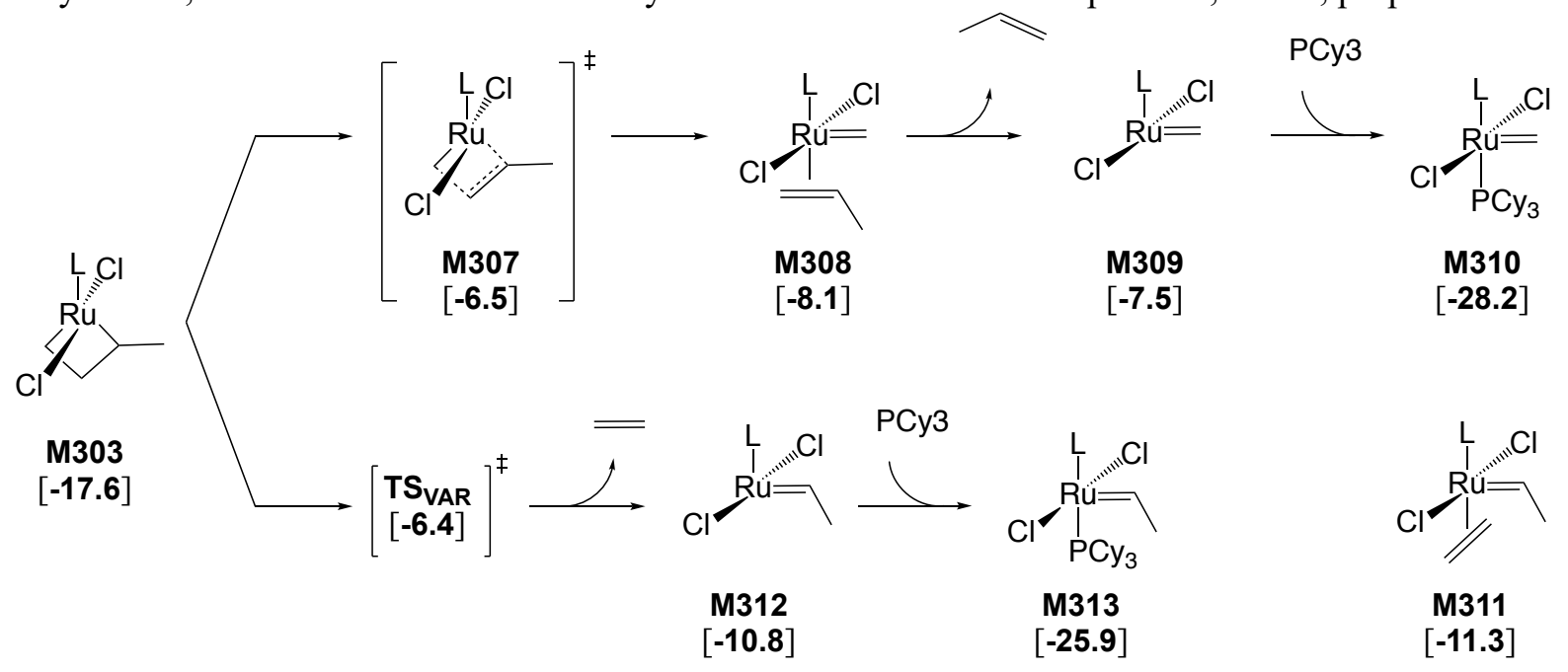

Scheme S46. Free energies (kcal/mol with respect to M1) of species along sections P52 (M303-M310) and P53 (M303-M313).

MCB M303 is already part of the olefin metathesis catalytic cycle, though it is not observable, but two different alkylidenes can be formed by each cycloreversion path. Methylidene M309 is formed by release of propene from $\pi$-complex M308. Binding the resulting methylidene M309 with the phosphine gives the 16-electron methylidene detected experimentally (M310). The latter is thermodynamically more stable than ethylidene analogue M313. As known, the 14-electron ethylidene (M312) is more stable than the corresponding methylidene (M309), and should react instantaneously with ethylene as no pronounced transition state could be found along the cycloreversion reaction coordinate in the potential energy surface. The sole $\pi$-complex containing both ethylidene and ethylene that was found is M311 where the olefin is twisted by $90^{\circ}$ with respect to the $\mathrm{Ru}$-ethylidene bond, and thus is not ideally oriented to form a metallacyclobutane.

\section{Homocoupling of Ethylene}

Ethylene can react with methylidene M309 to form metallacyclopropane M314. Exploration of the potential energy surface along this reaction coordinates did not find any barrier. In particular, no attempt could identify a $\pi$-complex with ethylene coordinated parallel to the $\mathrm{Ru}=\mathrm{CH}_{2}$ bond, either directly built or obtained by forcing cycloreversion of M314. Instead, we could identify the alternative $\pi$-complex M315, which does not have the proper arrangement for direct formation of the metallacyclobutane, and the associated pathway proceeding via torsion of the methylidene (M316) and formation of metallacyclobutane M314 via transition state M318. The latter shows formation of metallacyclobutane in a single step. Thus, in agreement with the experience describe above, we conclude that the potential energy surface defined by the present DFT method does not show any local minimum corresponding to a $\pi$-complex with ethylene coordinated parallel to the $\mathrm{Ru}=\mathrm{CH}_{2}$ bond. Therefore, we provide the variational transition state for the coordination of ethylene onto M309 as an estimate of the barrier for homocoupling of ethylene. 


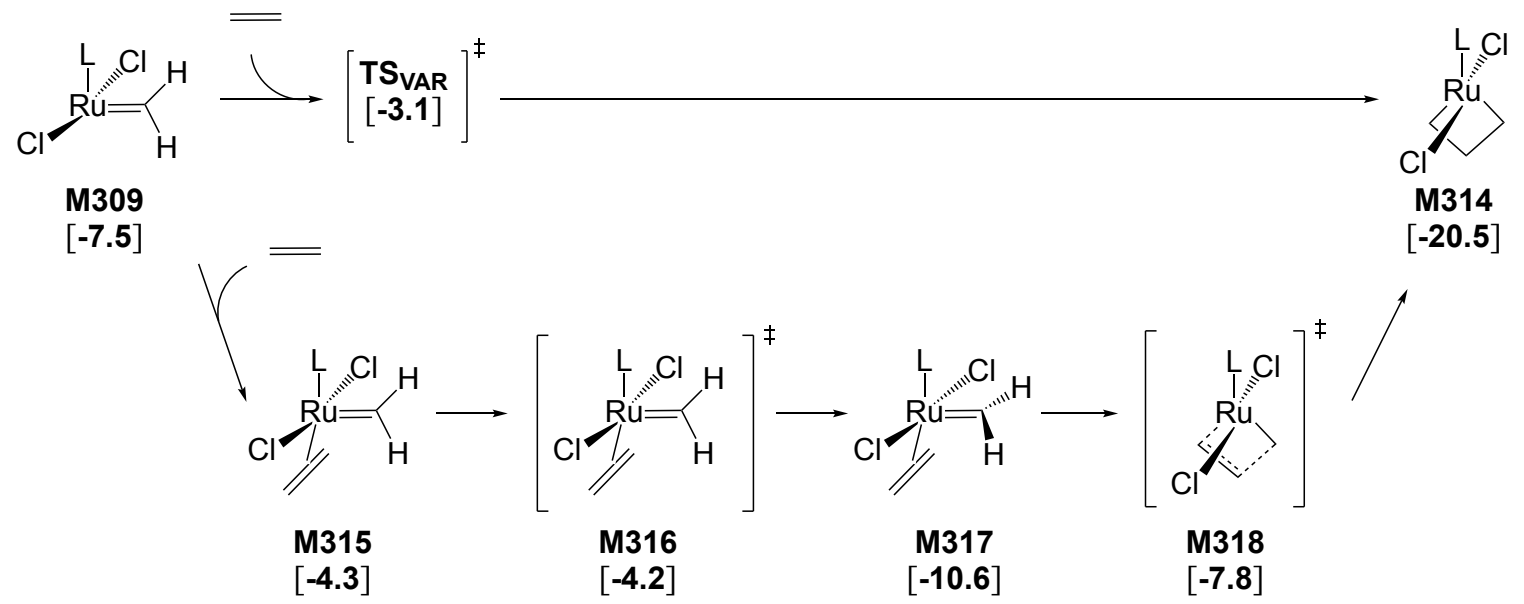

Scheme S47. Free energies (kcal/mol with respect to M1) of species along ethylene homocoupling pathways.

\section{Formation of Propene from Decomposition of Unsubstituted Metallacyclobutane}

The $\beta$-H elimination on unsubstituted metallacyclobutane M314 was suggested as a major path for decomposition of olefin metathesis catalyst. ${ }^{8}$ This step forms Ru-hydride M320 that evolves into the $\mathrm{Ru}(\mathrm{II})$ complex M322, which coordinates propene hapto-two trans to the NHC ligand. Overall, in this reaction one $\mathrm{H}$ atom is moved from the $\beta$ carbon of the $\mathrm{C} 3$ backbone of metallacyclobutane $\mathbf{M 3 1 4}$ to the $\alpha$ position, and this allows release of propene as shown in Scheme S48. As observed before for analogous olefin-coordinating systems (i.e., M118, M138, and M148), this configuration is unstable with respect to certain hydrides, like, in the case of propene, M324. Therefore, the existence of a pathway analogous to the sequence P20-P22-P23 (see Scheme S21 and Scheme S22), which describes the exchange of 1-butene in $\mathbf{M 1 1 8}$ with ethylene, is highly likely to lead to easy release of propene from M324, but such hypothesis is not further explored in the present study. In any case, the high concentration of ethylene can also trigger the release of propene returning the Ru(II) M18 via an associative pathway even from $\mathbf{M 3 2 2}$ as shown in Scheme S48.
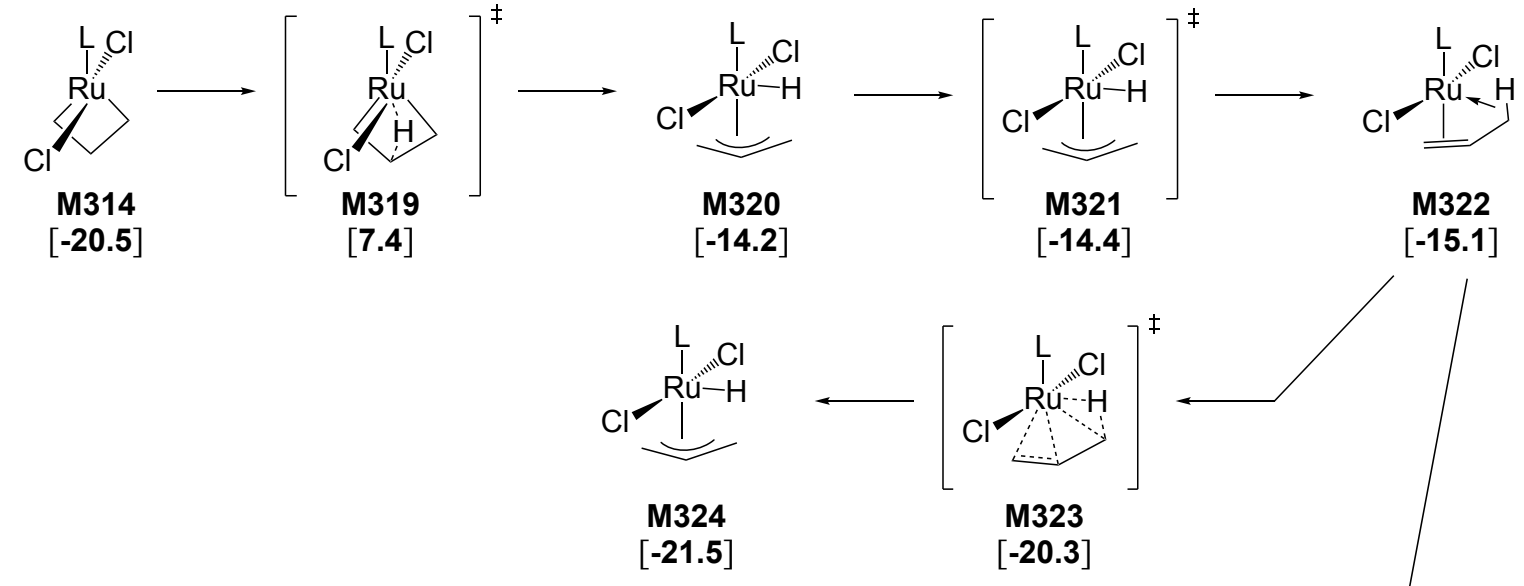

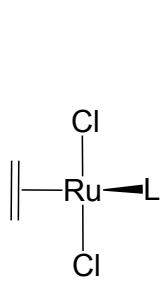

M18

$[-35.4]^{\mathrm{a}}$
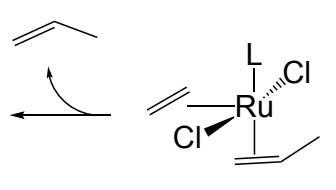

M325

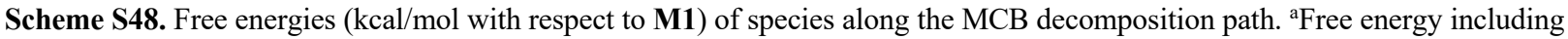
the free energy of the reaction transforming ethylene into propene. 


\section{Hydrogenation of Ethylene}

The experiments detected ethane in the reaction mixture. Thus, we evaluate a reaction mechanism for the hydrogenation of ethylene by molecular hydrogen, though the actual source of the hydrogen atoms remains unknown. In fact, the NMR characterization of the products in the reaction medium did not identify hydrogen-depleted species that could provide hints on the identity of the source of either molecular hydrogen or single hydrogen atoms. This might imply that such products are masked (e.g., under the benzene and benzene- $d_{n<6}$ signals), insoluble, or paramagnetic.

As shown in the above reaction network, the DFT model suggests that molecular hydrogen is formed from two ethylene molecules, which are first collected as metallacyclopentane, then yielding Ru-bound butadiene (Scheme S15 and Scheme S16). In fact, Ru-bound molecular hydrogen was encountered in Scheme S15, but neither s-trans-butadiene nor molecular hydrogen were released from $\mathbf{M 7 4}$ as all the $\mathrm{H}$ atoms of these species make their way into the experimentally observed products: mostly in the C4 products 1- or 2-butenes, and, in a minor fraction, into the metallacyclobutane, alkylidene and olefin metathesis related products. These transformations are thermodynamically favored over release of both molecular hydrogen and s-trans-butene, which, instead, is endoergonic.

Given the easy formation of M74, potential pathway combining coupling of olefins and elimination were considered as candidates for production of molecular hydrogen. In particular, release of molecular hydrogen from M74 or M67 would allow binding of a new ethylene molecule to form M91 (see Scheme S16), which can couple with butadiene to form a C6 unit, and possibly lead to benzene. This hypothetical reaction could explain the presence of molecular hydrogen and the apparent lack of a hydrogen-depleted organic product (i.e., benzene), which would be masked by the proton-containing impurity of the deuterated solvent. However, the attempt to explore this possibility is described in Scheme S16 and shows that $\beta$-H elimination from the linear C6 backbone is too energy demanding via M101 to yield molecular hydrogen.

In contrast, the possibility of abstracting $\mathrm{H}$ from the dative ligands (i.e., SIMes and $\mathrm{PCy}_{3}$ ) is known, in particular for $\mathrm{PCy}_{3}$. Considering phosphine-free experiments allows to exclude the known role of the phosphine as $\mathrm{H}$ atom donor. Thus, the SIMes remains the most plausible, yet hypothetical source of $\mathrm{H}$ atoms in phosphine-free experiments. However, we did not explore the details on the reaction mechanism yielding $\mathrm{H}$ atoms from SIMes.

In the absence of an unambiguously proven source of $\mathrm{H}$ atoms, the ethylene hydrogenation mechanism is here modelled under the assumption that molecular hydrogen is somehow provided by the reaction mixture by an unknown source. Exchange of ethylene for molecular hydrogen (see M326) Instead, the coordination of molecular hydrogen on M18 would give M327, which has both ethylene and $\mathrm{H}_{2}$ cis to the NHC. This system is significantly less stable than M328, and does not allow direct hydrogenation of Rubound ethylene. Thus, M327 is expected to rearrange into M328 before proceeding. The next sequence of steps resembles the one described above for the generation of 1-butene. In fact, after a first insertion (in Scheme S49, after transition state M329) the system is characterized by a hydride and an alkyl ligand that are too far from each other for direct reductive elimination (in Scheme S49, M330 and M331, which differ by small conformational changes). Two alternatives were evaluated for bringing the hydride and alkyl ligand cis to each other and proceed with the reductive elimination. In one case, twisting of the overall C2 fragment via transition state $\mathbf{M 3 3 2}$ would results in concerted reductive elimination to give the ethanebound species M333. In the second case, the same outcome (M337 and M333 differ only by small conformational changes) can be achieved by moving the hydride around the $\mathrm{Ru}-\mathrm{Cl}$ bond and, thus, from one side (as in M331) to the other side of the Ru center (as in M335). The barrier associated with this step via $\mathbf{M 3 3 4}$ is $4.1 \mathrm{kcal} / \mathrm{mol}$ less demanding than the twisting occurring via M332. Next, the resulting intermediate M335, which has the hydride and the alkyl end of the C2 unit cis to each other, can easily proceed to reductively eliminate ethane via transition state M336; a step that is barrier less in the SP energy model. Finally, an incoming ethylene molecule can coordinate the Ru center providing much more electron-donating ligand than ethane. Thus, this coordination triggers the release of ethane, and brings the metal center back into Ru(II) species M18, thus closing the catalytic cycle for hydrogenation of ethylene. 


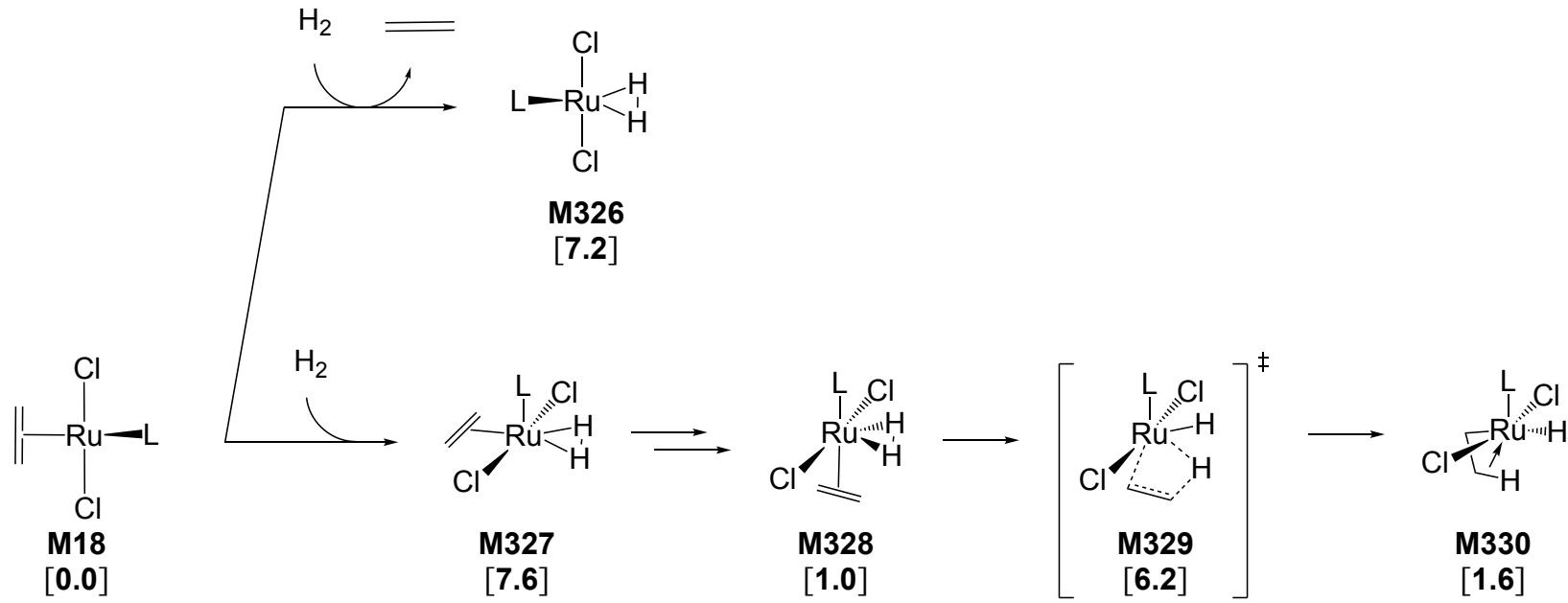

[1.6]

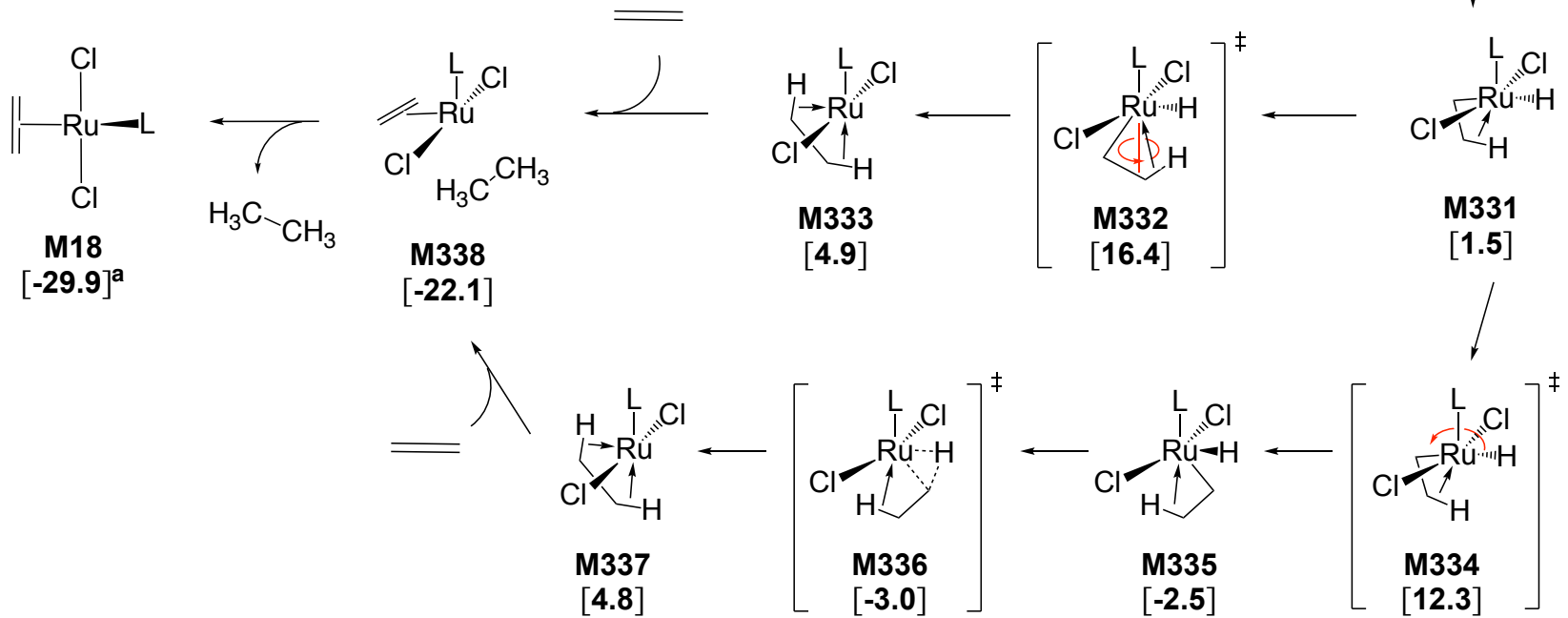

Scheme S49. Free energies ( $\mathrm{kcal} / \mathrm{mol}$ with respect to M1) of species along the ethylene hydrogenation pathway. ${ }^{\mathrm{a}}$ The free energy of $\mathbf{M 1 8}$ includes the free energy of the hypothetical reaction transforming of ethylene into ethane and $s$-trans-butadiene.

\section{Ethylene Insertion into Ruthenacycloalkanes}

The insertion of ethylene into metallacyclopentane M49 was suggested by the reviewers as a hypothetical pathway leading to higher olefins. The barrier for the insertion of ethylene into M49 via transition states M341 (Scheme S50) involves an activation energy of more than $34 \mathrm{kcal} / \mathrm{mol}$. In agreement with this barrier, no experimental evidence suggests the presence of higher olefins such as the hexenes that could hypothetically be generated from ruthenacycloheptane $\mathbf{M 3 4 2}$ upon $\beta-\mathrm{H}$ elimination followed by reductive elimination.

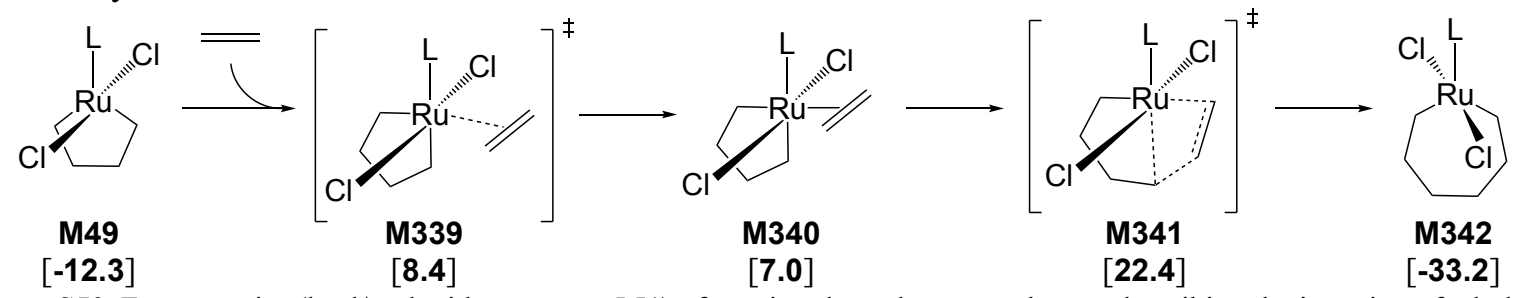

Scheme S50. Free energies ( $\mathrm{kcal} / \mathrm{mol}$ with respect to M1) of species along the two pathways describing the insertion of ethylene in ruthenacyclopentane M49. 
A similar reaction pathway is the insertion of ethylene into metallacyclobutane M314. The energy barrier calculated with the PBE-D3MBJ model is surprisingly low (i.e., ca. $28 \mathrm{kcal} / \mathrm{mol}$ from M314). However, as further discussed in the Sensitivity to the DFT Method section, the energy of transition state M345 changes significantly with the DFT model, and PBE-D3MBJ underestimates this value. The energy required to cross $\mathbf{M 3 4 5}$ is most likely higher than what depicted in Scheme S51. In fact, the barrier from M314 to M345 grows to almost $36 \mathrm{kcal} / \mathrm{mol}$ when the energies are calculated with the M06L-PCM model. Therefore, this reaction pathway is much less promising than what suggested by the PBE-D3MBJ energy model. As such, we did not model further evolutions from the ruthenacyclohexane M346.

The energy of the latter species (i.e., M346) is substantially lower than previous models of this compound. This is mostly due to the absence, in M346, of the constraining C-agostic interactions that characterize the geometry considered in the work of Webster. ${ }^{62}$ Notably, the geometry described therein is the correct result of a specific ethylene exchange mechanism, but should not be mistaken to be a general assessment of the relative stability of a ruthenacyclohexane. Nevertheless, the stability of M346 does not change the above considerations concerning the energy demands of the pathway displayed in Scheme S51.

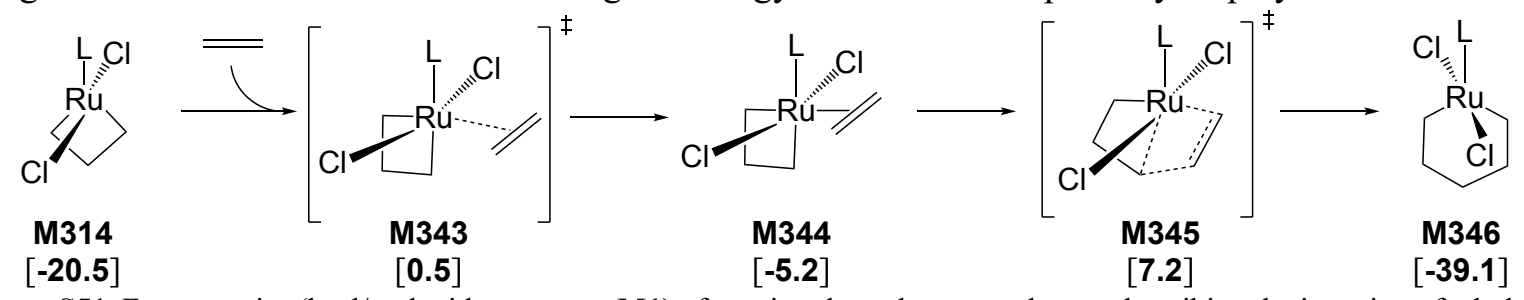

Scheme S51. Free energies ( $\mathrm{kcal} / \mathrm{mol}$ with respect to M1) of species along the two pathways describing the insertion of ethylene in ruthenacyclobutane $\mathbf{M 3 1 4}$.

\section{Sensitivity to the DFT Method}

As described in the Computational Methods section above, the SP energies were calculated using the M06 and M06L functionals in addition to our workhorse functional, PBE-D3MBJ, to gauge the sensitivity of the calculated relative energies to the density functional used. All the three functionals were combined with PCM-estimates of the solvent effects, resulting in the three overall computational models PBED3MBJ-PCM, M06-PCM, and M06L-PCM.

First, although the two Minnesota functionals M06-PCM and M06L-PCM belong to different rungs on Jacob's ladder, their free energies calculated relative to M1 correlate well and are distributed fairly symmetrically on both sides of the diagonal (Figure S41). This correlation even includes the spin-triplet complexes (indicated as labeled, blue points in the correlation plot), which may be surprising given the very different contributions of exact exchange in these two functionals, $27 \%$ and $0 \%$ for M06 and M06L, respectively.

Next, the corresponding correlation plot between M06L-PCM and PBE-D3MBJ-PCM reveals that PBE predicts relative energies that are fairly systematically shifted downward by ca. $10 \mathrm{kcal} / \mathrm{mol}$ (Figure S42). In other words, PBE predicts the energy of most of the complexes to be ca. $10 \mathrm{kcal} / \mathrm{mol}$ more stable relative to M1 than M06L (and thereby, given the excellent correlation in Figure S41, also M06) does. The origin of the systematic difference is the reference complex, the precatalyst M1, which the Minnesota functionals (M06L and M06) predict to be more stable (relative to the other complexes) than PBE-D3MBJ does. M1 contains, as the only compound in the study, a tightly bound (via $\eta^{6}$-coordination) $p$-cymene ligand, and it is tempting to ascribe the added stability of this complex to the parameterization of midrange correlation effects in $\mathrm{M} 06 \mathrm{~L}$ and $\mathrm{M} 06 .{ }^{53,54}$

Conversely, the destabilization (by ca. $15 \mathrm{kcal} / \mathrm{mol}$ ) of the large PCy M313 predicted by M06L and M06 compared to PBE-D3MBJ is presumably due to the lack of long-range dispersion in the Minnesota functionals. 


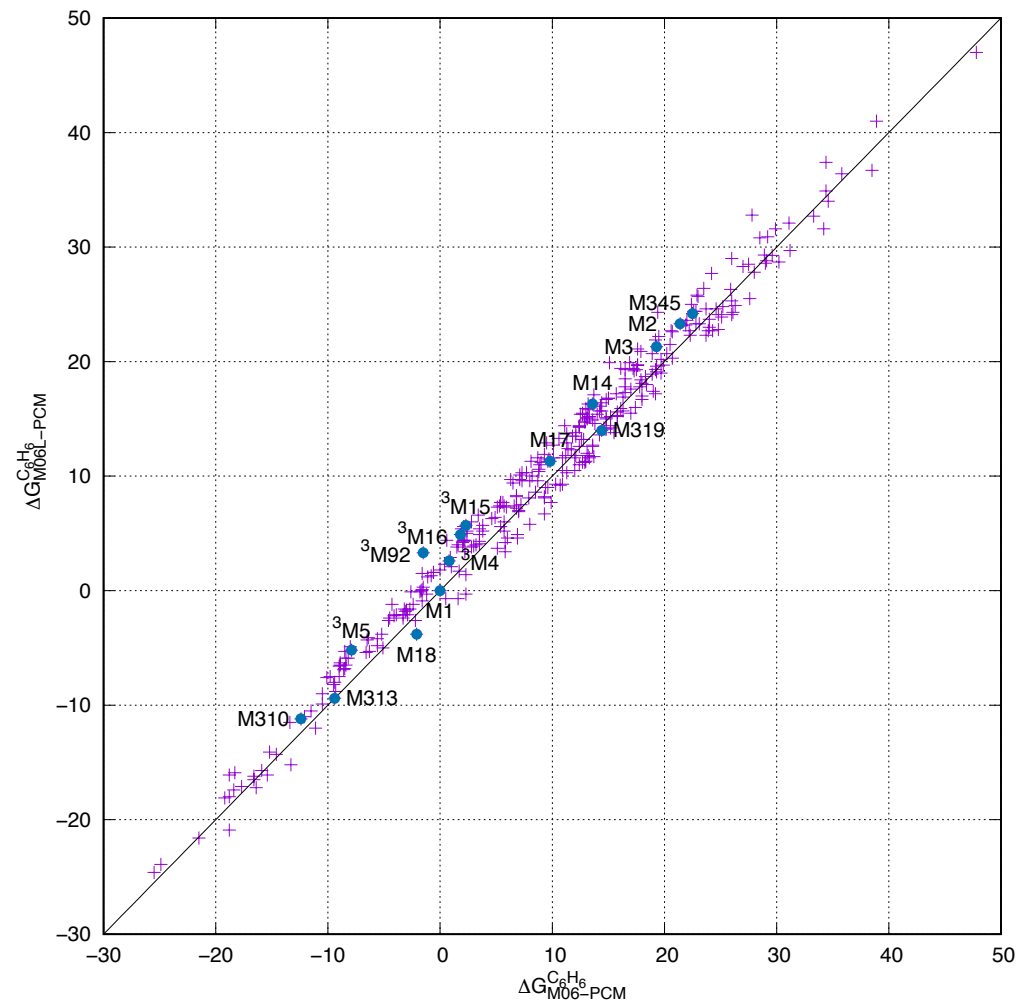

Figure S41: Correlation between relative free energies calculated with M06-PCM and M06L-PCM. Blue points are used to improve the visibility of the species identified by a label.

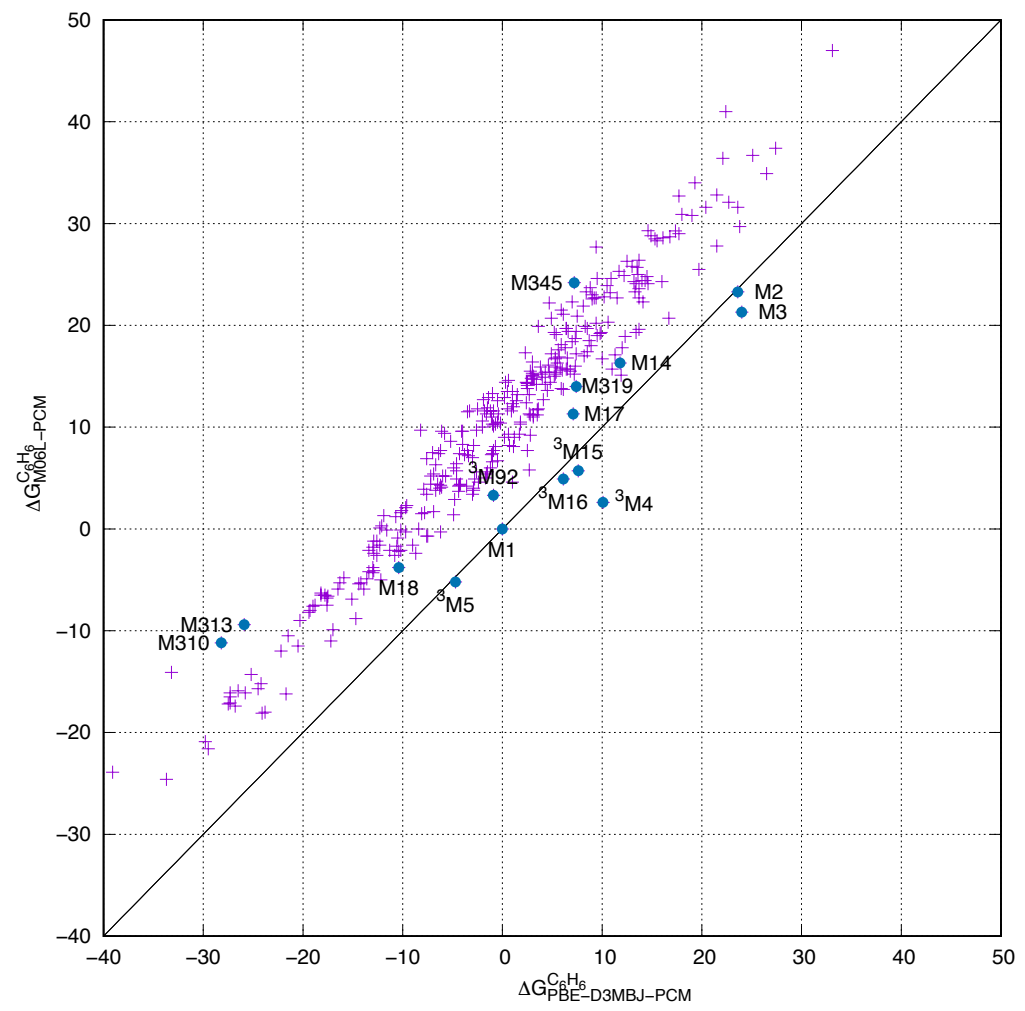

Figure S42: Correlation between relative free energies calculated with PBE-D3MBJ-PCM and M06L-PCM. Blue points are used to improve the visibility of the species identified by a label. 
In contrast, some smaller molecules (such as M2 and M3), including several with spin-triplet states $\left({ }^{3} \mathbf{M} 4,{ }^{3} \mathbf{M 5},{ }^{3} \mathbf{M 1 5},{ }^{3} \mathbf{M 1 6}\right.$, and $\left.{ }^{3} \mathbf{M 9 2}\right)$ either deviate less from, or are even below, the diagonal. The Minnesota functionals thus predict somewhat higher stability for the spin-triplet states than does PBED3MBJ.

This and the other systematic differences between the relative energies obtained by PBE-D3MBJ-PCM and M06-PCM and M06L-PCM have surprisingly little effect on the predicted reaction mechanisms. Most importantly, the computational models consistently prefer the pathway sequence P10-P11-P12-P13-P14 (see Scheme S6) to the competing reactions P32, P45, P46, and, critically, P50. This implies that, at room temperature, formation of the $\mathrm{C} 4$ skeleton (butadiene and thus also butene) is faster than formation of metallacyclobutane, which evolves into ruthenium methylidene and propene. This is consistent with the experimentally observed initial rates of formation at room temperature. Moreover, the DFT methods agree on the ratio between the rates of direct formation of 1-butene (via pathway P18) and trans-2-butene (via pathway P29). Both these pathways depart from $\mathbf{M 1 0 6}$ and the density functionals agree on P18 being preferred by ca. $1.5 \mathrm{kcal} / \mathrm{mol}$, consistent with the faster formation of 1-butene than of trans-2-butene at room temperature (Figure $\mathrm{S} 3$ ).

The mechanistically most relevant disagreements between the density functionals pertain to the pathways for exchange of $p$-cymene with ethylene and to the formation of M18. This exchange mechanism is sensitive to the computational model partly because of the involvement of a handful of relatively small molecules (M2, the variational transition state leading to M2 (the free energy of which depends on that of M2), and ${ }^{3} \mathbf{M} 4$, the spin-triplet analogue of M2) which are predicted to be more stable (relative to M1) by the Minnesota functionals than by PBE-D3MBJ (Figure S42) and partly because of the involvement of a relatively large transition state (M11 predicted to be more stable by PBE-D3MBJ than by the Minnesota functionals, presumably due to the inclusion of long-range dispersion in the former) on the interchange pathway P3. Together, due to these differences for individual intermediates and transition states, PBE-D3MBJ predicts, in contrast to the Minnesota functionals, lower barriers for the interchange (P3) than for the dissociative release of $p$-cymene (P1). Similarly, for the pathways continuing from M2, the Minnesota functionals give lower energies for the spin-triplet states involved in the P2 pathway than does PBE-D3MBJ and, for this reason, predict overall barriers of the same magnitude for P2 and P6. In contrast, PBE-D3MBJ prefers the low-spin intramolecular cyclometallation pathway P6 via M24. Concluding, the differences between the barriers of the pathways leading from M1 to M18 are small and the functionals disagree on the ranking of these pathways. However, even if an agreed-upon route from M1 to M18 cannot be identified, the main message given by all the methods is that M18 is a key intermediate for which the stability is higher than that of the 18-electron precatalyst M1.

The choice of the density functional also affects the details of the pathways involved in the (hypothetical) release of molecular hydrogen from M74. Whereas, according to the Minnesota functionals, $\mathrm{H}_{2}$-complex $\mathbf{M 7 4}$ and $\mathrm{H}_{2}$-free $\mathbf{M 8 8}$ on pathway P15 are equally stable, PBE-D3MBJ suggests that M74 is ca. $4 \mathrm{kcal} / \mathrm{mol}$ more stable and that $\mathbf{M 8 8}$ should therefore bind $\mathrm{H}_{2}$. Moreover, in contrast to PBE-D3MBJ, the Minnesota functionals rank pathway P16, i.e., the first transfer of hydrogen onto butadiene, as more demanding than that of $\mathrm{H}_{2}$ release (P15). Despite these differences, PBE-D3MBJ and the Minnesota functionals agree on the single most important feature of this part of the overall mechanism: Conversion of ethylene to butadiene and molecular hydrogen is endergonic, consistent with the lack of experimental observation of these products.

Finally, the choice of the DFT model has a significant impact on the energy barrier of the insertion of ethylene into metallacyclobutane M314 via transition state M345 (Scheme S51). The PBE-D3MBJ model estimates a surprisingly low barrier (i.e., $28 \mathrm{kcal} / \mathrm{mol}$ from M314) which grows to almost $36 \mathrm{kcal} / \mathrm{mol}$ with the M06L-PCM model. Notably, PBE-D3MBJ suggests that transition state M345 is as stable as transition state (i.e., M319) of the well-known $\beta$-H elimination of unsubtituted metallacyclobutane. ${ }^{8}$ This estimate implies that both decomposition pathways (i.e., $\beta$-H elimination at M314, and insertion of ethylene into M314) are equally operative at room temperature. Moreover, it is also reasonable to assume that the ruthenacyclohexane resulting from the insertion (i.e., M346) can decompose to release C5 byproducts, such as pentene, via $\beta-\mathrm{H}$ elimination followed by reductive elimination. According to this hypothesis, the 
C5 byproducts should then be detected in experiments where unsubstituted metallacyclobutane is exposed to ethylene, and should appear alongside the propene resulting from the well-established $\beta-\mathrm{H}$ elimination of unsubstituted ruthenacyclobutane (via M319). ${ }^{8}$ However, experimental observation of the reactivity of ruthenacyclobutane M314 in presence of ethylene detected propene, which supports the $\beta-\mathrm{H}$ elimination pathway via M319, ${ }^{8,63}$ and butenes, which derive from metathesis of propene and coupling of ethylene, but no C5 byproducts. While the disappearance of ${ }^{13} \mathrm{C}$ labelled $\mathbf{M 3 1 4}$ does follows and associative mechanisms at low temperature and in excess of ethylene, ${ }^{64} \mathrm{C} 5$ byproducts were not detected in the reaction mixture. Therefore, in light of the above experimental evidence we conclude that the relative energies of transition state M345 (i.e., insertion) and M319 (i.e., $\beta$-H elimination) are more accurate with M06L-PCM than with PBE-D3MBJ. While PBE-D3MBJ is misleadingly suggesting that ethylene insertion is doable, M06L-PCM excludes such pathway from those populated at room temperature, and is thus in better agreement with the experimental evidence than PBE-D3MBJ. 


\section{Computational Data}

Energies of Minima and Transition States

Table S10: energy components for minima and transition states. Relative free energies are calculated with respect to M1.

\begin{tabular}{|c|c|c|c|c|c|c|c|c|c|c|c|c|c|}
\hline 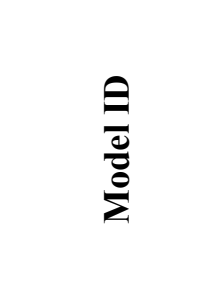 & 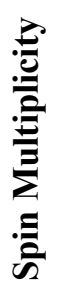 & 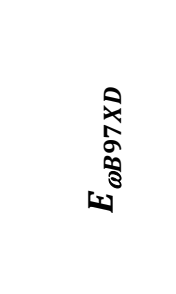 & 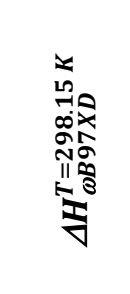 & 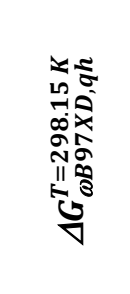 & 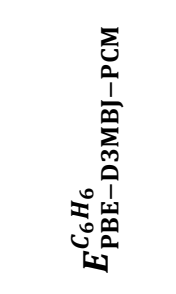 & 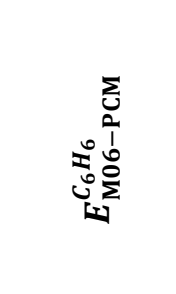 & 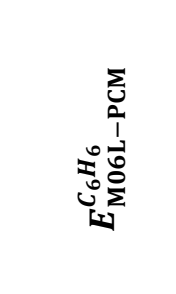 & 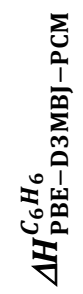 & 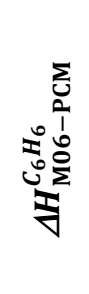 & 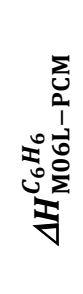 & 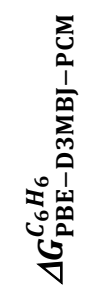 & 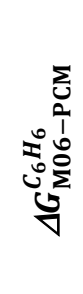 & 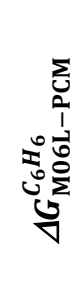 \\
\hline ethylene & 1 & -78.560878 & 0.055158 & 0.028980 & -78.507429 & -78.553486 & -78.607919 & - & - & - & - & - & - \\
\hline propene & 1 & -117.870108 & 0.084886 & 0.054850 & -117.790950 & -117.858220 & -117.939794 & - & - & - & - & - & - \\
\hline$p$-cymene & 1 & -389.405093 & 0.224446 & 0.178186 & -389.163322 & -389.357108 & -389.616395 & - & - & - & - & - & - \\
\hline $\mathrm{PCy}_{3}$ & 1 & -1047.026249 & 0.505834 & 0.441427 & -1046.409675 & -1046.919909 & -1047.390757 & - & - & - & - & - & - \\
\hline s-trans-butadiene & 1 & -155.941994 & 0.091085 & 0.058999 & -155.846683 & -155.928113 & -156.033542 & - & - & - & - & - & - \\
\hline 1-butene & 1 & -157.173880 & 0.114934 & 0.081232 & -157.067187 & -157.155964 & -157.263658 & - & - & - & - & - & - \\
\hline cis-2-butene & 1 & -157.176991 & 0.114695 & 0.080375 & -157.071750 & -157.160112 & -157.269186 & - & - & - & - & - & - \\
\hline trans-2-butene & 1 & -157.178574 & 0.114532 & 0.080704 & -157.073288 & -157.161850 & -157.270431 & - & - & - & - & - & - \\
\hline $\mathbf{H}_{2}$ & 1 & -1.171574 & 0.013345 & -0.001487 & -1.163456 & -1.169059 & -1.170407 & - & - & - & - & - & - \\
\hline ethane & 1 & -79.803363 & 0.079186 & 0.051663 & -79.739706 & -79.791840 & -79.850794 & - & - & - & - & - & - \\
\hline M1 & 1 & -2329.756339 & 0.685018 & 0.581983 & -2328.700788 & -2329.584993 & -2330.527297 & 0.0 & 0.0 & 0.0 & 0.0 & 0.0 & 0.0 \\
\hline M2 & 1 & -1940.284280 & 0.457414 & 0.371585 & -1939.470702 & -1940.164629 & -1940.844544 & 41.8 & 39.6 & 41.6 & 23.6 & 21.4 & 23.3 \\
\hline M4 & 3 & -1940.310425 & 0.457588 & 0.370385 & -1939.490976 & -1940.196266 & -1940.876399 & 29.2 & 19.9 & 21.7 & 10.1 & 0.8 & 2.6 \\
\hline M5 & 3 & -2018.909080 & 0.515247 & 0.423218 & -2018.042750 & -2018.784336 & -2019.517607 & 1.0 & -2.2 & 0.5 & -4.7 & -7.9 & -5.2 \\
\hline M6 & 1 & -2329.716041 & 0.683078 & 0.577367 & -2328.658608 & -2329.532780 & -2330.472374 & 25.3 & 31.5 & 33.2 & 23.6 & 29.9 & 31.6 \\
\hline M7 & 1 & -2329.723359 & 0.683688 & 0.577291 & -2328.673716 & -2329.543985 & -2330.483698 & 16.2 & 24.9 & 26.5 & 14.0 & 22.8 & 24.4 \\
\hline M8 & 1 & -2329.722923 & 0.682511 & 0.577272 & -2328.673225 & -2329.544234 & -2330.483719 & 15.7 & 24.0 & 25.8 & 14.3 & 22.6 & 24.4 \\
\hline M9 & 1 & -2329.723041 & 0.683523 & 0.576848 & -2328.673836 & -2329.544750 & -2330.484497 & 16.0 & 24.3 & 25.9 & 13.7 & 22.0 & 23.6 \\
\hline M10 & 1 & -2408.293098 & 0.741041 & 0.625142 & -2407.183137 & -2408.100051 & -2409.095184 & 14.4 & 22.8 & 23.8 & 22.7 & 31.1 & 32.1 \\
\hline M11 & 1 & -2408.291235 & 0.740575 & 0.628033 & -2407.179989 & -2408.097696 & -2409.093655 & 16.1 & 23.9 & 24.4 & 26.5 & 34.4 & 34.9 \\
\hline M12 & 1 & -2408.301292 & 0.741905 & 0.628863 & -2407.188773 & -2408.108758 & -2409.105853 & 11.4 & 17.8 & 17.6 & 21.5 & 28.0 & 27.8 \\
\hline M13 & 1 & -2018.879045 & 0.514986 & 0.423842 & -2018.017998 & -2018.750652 & -2019.482633 & 16.4 & 18.8 & 22.2 & 11.3 & 13.7 & 17.1 \\
\hline M15 & 3 & -2018.890244 & 0.514084 & 0.422465 & -2018.022528 & -2018.767343 & -2019.499350 & 13.0 & 7.8 & 11.2 & 7.6 & 2.3 & 5.7 \\
\hline M16 & 3 & -2018.890467 & 0.514757 & 0.421076 & -2018.023456 & -2018.766779 & -2019.499383 & 12.8 & 8.5 & 11.6 & 6.1 & 1.8 & 4.9 \\
\hline M18 & 1 & -2018.905404 & 0.515708 & 0.426660 & -2018.055336 & -2018.778576 & -2019.518751 & -6.6 & 1.7 & 0.0 & -10.4 & -2.1 & -3.8 \\
\hline
\end{tabular}




\begin{tabular}{|c|c|c|c|c|c|c|c|c|c|c|c|c|c|}
\hline M19 & 1 & -1940.280920 & 0.455906 & 0.371751 & -1939.481805 & -1940.166689 & -1940.848824 & 33.9 & 37.4 & 37.9 & 16.7 & 20.2 & 20.7 \\
\hline M20 & 1 & -1940.281098 & 0.456544 & 0.371252 & -1939.486598 & -1940.167746 & -1940.850615 & 31.3 & 37.1 & 37.2 & 13.4 & 19.2 & 19.3 \\
\hline M21 & 1 & -1940.281076 & 0.455627 & 0.371920 & -1939.486763 & -1940.168255 & -1940.850831 & 30.6 & 36.2 & 36.5 & 13.7 & 19.3 & 19.6 \\
\hline M22 & 1 & -1940.286510 & 0.456492 & 0.373096 & -1939.490782 & -1940.176688 & -1940.859220 & 28.6 & 31.5 & 31.8 & 11.9 & 14.8 & 15.1 \\
\hline M23 & 1 & -1940.275363 & 0.452773 & 0.370022 & -1939.487134 & -1940.166957 & -1940.850105 & 28.6 & 35.2 & 35.2 & 12.3 & 18.9 & 18.9 \\
\hline M24 & 1 & -1940.278335 & 0.454746 & 0.372078 & -1939.492807 & -1940.170524 & -1940.855554 & 26.3 & 34.2 & 33.0 & 10.0 & 18.0 & 16.7 \\
\hline M25 & 1 & -2018.868396 & 0.513001 & 0.424950 & -2018.034736 & -2018.753462 & -2019.489956 & 4.7 & 15.8 & 16.4 & 1.5 & 12.6 & 13.2 \\
\hline M26 & 1 & -2018.873703 & 0.513545 & 0.424656 & -2018.019257 & -2018.750571 & -2019.485608 & 14.7 & 18.0 & 19.5 & 11.0 & 14.2 & 15.7 \\
\hline M27 & 1 & -2018.874810 & 0.514573 & 0.425244 & -2018.027706 & -2018.755869 & -2019.489525 & 10.1 & 15.3 & 17.7 & 6.1 & 11.3 & 13.7 \\
\hline M28 & 1 & -2018.862090 & 0.511044 & 0.423289 & -2018.025311 & -2018.745631 & -2019.481029 & 9.3 & 19.5 & 20.8 & 6.3 & 16.5 & 17.8 \\
\hline M29 & 1 & -2018.862364 & 0.512689 & 0.423987 & -2018.026949 & -2018.746683 & -2019.483116 & 9.4 & 19.9 & 20.5 & 5.7 & 16.3 & 16.9 \\
\hline M30 & 1 & -2018.861110 & 0.511736 & 0.424593 & -2018.025622 & -2018.744713 & -2019.481280 & 9.6 & 20.5 & 21.1 & 7.0 & 17.9 & 18.4 \\
\hline M31 & 1 & -2018.855442 & 0.511141 & 0.423714 & -2018.018606 & -2018.737436 & -2019.472833 & 13.6 & 24.7 & 26.0 & 10.8 & 21.9 & 23.2 \\
\hline M32 & 1 & -2018.865330 & 0.514534 & 0.427353 & -2018.031071 & -2018.750762 & -2019.489105 & 7.9 & 18.5 & 17.9 & 5.3 & 15.8 & 15.2 \\
\hline M33 & 1 & -2018.855734 & 0.513584 & 0.426254 & -2018.026546 & -2018.744193 & -2019.484856 & 10.2 & 22.0 & 20.0 & 7.4 & 19.2 & 17.2 \\
\hline M34 & 1 & -2018.886724 & 0.514886 & 0.426273 & -2018.045404 & -2018.766670 & -2019.506423 & -0.8 & 8.7 & 7.3 & -4.4 & 5.1 & 3.7 \\
\hline M35 & 1 & -2018.849023 & 0.513030 & 0.424967 & -2018.011635 & -2018.731923 & -2019.472323 & 19.2 & 29.3 & 27.5 & 16.0 & 26.1 & 24.3 \\
\hline M36 & 1 & -2018.853694 & 0.513957 & 0.425819 & -2018.015420 & -2018.736714 & -2019.476253 & 17.4 & 26.9 & 25.6 & 14.1 & 23.7 & 22.3 \\
\hline M37 & 1 & -2018.850992 & 0.513272 & 0.426426 & -2018.016632 & -2018.736254 & -2019.476305 & 16.2 & 26.8 & 25.1 & 13.7 & 24.3 & 22.7 \\
\hline M38 & 1 & -2018.884204 & 0.514861 & 0.426592 & -2018.044380 & -2018.766022 & -2019.505875 & -0.2 & 9.1 & 7.6 & -3.6 & 5.8 & 4.2 \\
\hline M39 & 1 & -2018.877359 & 0.514107 & 0.426968 & -2018.038151 & -2018.758140 & -2019.498181 & 3.2 & 13.6 & 11.9 & 0.6 & 10.9 & 9.3 \\
\hline M40 & 1 & -2018.879872 & 0.513859 & 0.427310 & -2018.039130 & -2018.760558 & -2019.499079 & 2.4 & 11.9 & 11.2 & 0.2 & 9.6 & 9.0 \\
\hline M41 & 1 & -2018.882807 & 0.514944 & 0.428392 & -2018.045269 & -2018.765752 & -2019.503304 & -0.7 & 9.3 & 9.2 & -3.0 & 7.0 & 7.0 \\
\hline M42 & 1 & -2018.861609 & 0.511461 & 0.424778 & -2018.028307 & -2018.742539 & -2019.480311 & 7.7 & 21.7 & 21.5 & 5.4 & 19.3 & 19.1 \\
\hline M43 & 1 & -2018.896606 & 0.515240 & 0.427086 & -2018.051286 & -2018.773053 & -2019.514275 & -4.3 & 4.9 & 2.5 & -7.6 & 1.6 & -0.7 \\
\hline M44 & 1 & -2018.896454 & 0.513994 & 0.426252 & -2018.048181 & -2018.771215 & -2019.512838 & -3.2 & 5.3 & 2.7 & -6.2 & 2.3 & -0.3 \\
\hline M45 & 1 & -2018.899887 & 0.515663 & 0.427290 & -2018.047183 & -2018.772140 & -2019.511042 & -1.5 & 5.8 & 4.8 & -4.9 & 2.3 & 1.4 \\
\hline M46 & 1 & -2018.897426 & 0.514460 & 0.426601 & -2018.043493 & -2018.765968 & -2019.507163 & 0.1 & 8.9 & 6.5 & -3.0 & 5.8 & 3.4 \\
\hline M47 & 1 & -2097.489444 & 0.574065 & 0.478296 & -2096.579733 & -2097.353641 & -2098.144097 & -17.1 & -11.7 & -10.8 & -8.7 & -3.3 & -2.4 \\
\hline M48 & 1 & -2097.473667 & 0.573428 & 0.479673 & -2096.568604 & -2097.338479 & -2098.125490 & -10.5 & -2.6 & 0.5 & -0.9 & 7.1 & 10.1 \\
\hline M49 & 1 & -2097.494179 & 0.574926 & 0.481020 & -2096.588240 & -2097.353647 & -2098.142815 & -21.9 & -11.2 & -9.4 & -12.3 & -1.6 & 0.1 \\
\hline M50 & 1 & -2097.491529 & 0.573643 & 0.478545 & -2096.585528 & -2097.356816 & -2098.148505 & -21.0 & -13.9 & -13.8 & -12.2 & -5.1 & -5.0 \\
\hline M51 & 1 & -2097.466278 & 0.574092 & 0.480174 & -2096.568851 & -2097.337590 & -2098.129150 & -10.3 & -1.6 & -1.4 & -0.7 & 7.9 & 8.1 \\
\hline M52 & 1 & -2097.478341 & 0.574542 & 0.480903 & -2096.579424 & -2097.348271 & -2098.140233 & -16.6 & -8.0 & -8.1 & -6.9 & 1.7 & 1.7 \\
\hline M53 & 1 & -2097.455437 & 0.574074 & 0.481238 & -2096.556921 & -2097.325674 & -2098.112226 & -2.8 & 5.9 & 9.2 & 7.4 & 16.1 & 19.4 \\
\hline M54 & 1 & -2097.451233 & 0.570929 & 0.479065 & -2096.556120 & -2097.321746 & -2098.110116 & -4.2 & 6.4 & 8.6 & 6.6 & 17.2 & 19.4 \\
\hline M55 & 1 & -2097.467523 & 0.572178 & 0.477146 & -2096.563876 & -2097.328460 & -2098.117611 & -8.3 & 2.9 & 4.6 & 0.5 & 11.8 & 13.5 \\
\hline M56 & 1 & -2097.463416 & 0.569955 & 0.474823 & -2096.558765 & -2097.318536 & -2098.109196 & -6.5 & 7.8 & 8.5 & 2.3 & 16.5 & 17.3 \\
\hline M57 & 1 & -2097.468381 & 0.570924 & 0.475090 & -2096.556493 & -2097.323976 & -2098.112906 & -4.5 & 5.0 & 6.8 & 3.9 & 13.3 & 15.2 \\
\hline
\end{tabular}




\begin{tabular}{|c|c|c|c|c|c|c|c|c|c|c|c|c|c|}
\hline M58 & 1 & -2097.466095 & 0.569671 & 0.475394 & -2096.554451 & -2097.321854 & -2098.110737 & -4.0 & 5.5 & 7.4 & 5.3 & 14.8 & 16.7 \\
\hline M59 & 1 & -2097.470299 & 0.571026 & 0.475660 & -2096.558167 & -2097.325251 & -2098.114235 & -5.5 & 4.2 & 6.0 & 3.2 & 12.9 & 14.7 \\
\hline M60 & 1 & -2097.470020 & 0.569952 & 0.475578 & -2096.558185 & -2097.324835 & -2098.113617 & -6.2 & 3.8 & 5.8 & 3.1 & 13.1 & 15.0 \\
\hline M61 & 1 & -2097.470047 & 0.570736 & 0.474522 & -2096.558173 & -2097.324810 & -2098.113590 & -5.7 & 4.3 & 6.3 & 2.4 & 12.4 & 14.4 \\
\hline M62 & 1 & -2097.470013 & 0.569834 & 0.475450 & -2096.558221 & -2097.324722 & -2098.113325 & -6.3 & 3.8 & 5.9 & 3.0 & 13.1 & 15.1 \\
\hline M63 & 1 & -2097.470037 & 0.570785 & 0.474928 & -2096.558540 & -2097.325235 & -2098.114038 & -5.9 & 4.1 & 6.0 & 2.5 & 12.4 & 14.3 \\
\hline M64 & 1 & -2097.470018 & 0.569635 & 0.475040 & -2096.558568 & -2097.325300 & -2098.114204 & -6.6 & 3.3 & 5.2 & 2.5 & 12.4 & 14.3 \\
\hline M65 & 1 & -2097.470346 & 0.570676 & 0.475184 & -2096.558712 & -2097.325916 & -2098.115096 & -6.0 & 3.6 & 5.3 & 2.5 & 12.1 & 13.8 \\
\hline M66 & 1 & -2097.460867 & 0.566294 & 0.470810 & -2096.550151 & -2097.314620 & -2098.101952 & -3.4 & 7.9 & 10.8 & 5.2 & 16.5 & 19.3 \\
\hline M67 & 1 & -2097.475693 & 0.568264 & 0.472006 & -2096.565929 & -2097.329390 & -2098.118693 & -12.1 & -0.1 & 1.5 & -4.0 & 8.0 & 9.6 \\
\hline M68 & 1 & -2097.446498 & 0.570074 & 0.475355 & -2096.541111 & -2097.307970 & -2098.095321 & 4.6 & 14.5 & 17.3 & 13.7 & 23.5 & 26.4 \\
\hline M69 & 1 & -2097.474613 & 0.571203 & 0.474731 & -2096.564799 & -2097.331908 & -2098.118722 & -9.5 & 0.2 & 3.3 & -1.6 & 8.1 & 11.3 \\
\hline M70 & 1 & -2097.447460 & 0.569826 & 0.474866 & -2096.541622 & -2097.308489 & -2098.095792 & 4.2 & 14.0 & 16.9 & 13.0 & 22.9 & 25.8 \\
\hline M71 & 1 & -2097.440314 & 0.569176 & 0.475546 & -2096.534878 & -2097.304244 & -2098.091363 & 8.0 & 16.2 & 19.2 & 17.7 & 26.0 & 29.0 \\
\hline M72 & 1 & -2097.474688 & 0.571230 & 0.475329 & -2096.564782 & -2097.331521 & -2098.118749 & -9.5 & 0.4 & 3.3 & -1.2 & 8.7 & 11.6 \\
\hline M73 & 1 & -2097.460334 & 0.566711 & 0.471353 & -2096.554191 & -2097.318438 & -2098.107115 & -5.7 & 5.8 & 7.8 & 3.0 & 14.4 & 16.4 \\
\hline M74 & 1 & -2097.491295 & 0.570362 & 0.476976 & -2096.585142 & -2097.353951 & -2098.140868 & -22.8 & -14.2 & -11.1 & -12.9 & -4.3 & -1.2 \\
\hline M75 & 1 & -2097.457130 & 0.567957 & 0.472626 & -2096.546966 & -2097.315722 & -2098.102841 & -0.4 & 8.3 & 11.3 & 8.3 & 16.9 & 19.9 \\
\hline M76 & 1 & -2097.459578 & 0.568916 & 0.471917 & -2096.551909 & -2097.322061 & -2098.109339 & -2.9 & 4.9 & 7.8 & 4.7 & 12.5 & 15.4 \\
\hline M77 & 1 & -2097.457703 & 0.568447 & 0.474750 & -2096.553067 & -2097.323867 & -2098.111462 & -3.9 & 3.5 & 6.2 & 5.8 & 13.2 & 15.8 \\
\hline M78 & 1 & -2097.477805 & 0.570599 & 0.477286 & -2096.580164 & -2097.346714 & -2098.135588 & -19.5 & -9.5 & -7.6 & -9.6 & 0.4 & 2.3 \\
\hline M79 & 1 & -2097.453119 & 0.567420 & 0.473421 & -2096.559942 & -2097.321460 & -2098.112104 & -8.9 & 4.3 & 5.1 & 0.6 & 13.8 & 14.6 \\
\hline M80 & 1 & -2097.454515 & 0.570468 & 0.476371 & -2096.559564 & -2097.326123 & -2098.117330 & -6.7 & 3.3 & 3.7 & 2.7 & 12.8 & 13.2 \\
\hline M81 & 1 & -2097.446772 & 0.570145 & 0.476870 & -2096.548578 & -2097.319020 & -2098.108073 & 0.0 & 7.6 & 9.4 & 9.9 & 17.5 & 19.3 \\
\hline M82 & 1 & -2097.503148 & 0.573439 & 0.478261 & -2096.587979 & -2097.361721 & -2098.149669 & -22.7 & -17.2 & -14.7 & -13.9 & -8.4 & -5.9 \\
\hline M83 & 1 & -2176.050121 & 0.628633 & 0.527000 & -2175.091514 & -2175.901676 & -2176.743678 & -22.1 & -10.5 & -7.8 & -1.0 & 10.6 & 13.3 \\
\hline M84 & 1 & -2176.049738 & 0.628352 & 0.527936 & -2175.093123 & -2175.904370 & -2176.745337 & -23.3 & -12.4 & -9.0 & -1.4 & 9.5 & 12.9 \\
\hline M85 & 1 & -2176.049756 & 0.628629 & 0.528210 & -2175.094220 & -2175.903656 & -2176.745782 & -23.8 & -11.8 & -9.1 & -1.9 & 10.1 & 12.7 \\
\hline M86 & 1 & -2097.472834 & 0.567124 & 0.472922 & -2096.563155 & -2097.339211 & -2098.126943 & -11.1 & -7.0 & -4.4 & -1.7 & 2.4 & 5.0 \\
\hline M87 & 1 & -2097.475452 & 0.567200 & 0.469609 & -2096.564741 & -2097.338217 & -2098.126946 & -12.0 & -6.3 & -4.3 & -4.8 & 0.9 & 2.9 \\
\hline M88 & 1 & -2096.300661 & 0.552072 & 0.459940 & -2095.399910 & -2096.167671 & -2096.955576 & -10.4 & -4.6 & -3.0 & -9.0 & -3.2 & -1.6 \\
\hline M89 & 1 & -2174.871192 & 0.609180 & 0.507134 & -2173.909676 & -2174.722250 & -2175.563813 & -12.5 & -6.0 & -3.8 & -0.9 & 5.6 & 7.7 \\
\hline M90 & 1 & -2174.863593 & 0.608930 & 0.510979 & -2173.907584 & -2174.720633 & -2175.562196 & -11.3 & -5.1 & -3.0 & 2.8 & 9.0 & 11.2 \\
\hline M91 & 1 & -2174.874693 & 0.609947 & 0.511037 & -2173.923617 & -2174.731655 & -2175.573034 & -20.8 & -11.4 & -9.1 & -7.2 & 2.1 & 4.4 \\
\hline M92 & 3 & -2096.291411 & 0.551120 & 0.454596 & -2095.381637 & -2096.159613 & -2096.942420 & 0.5 & -0.1 & 4.7 & -0.9 & -1.5 & 3.3 \\
\hline M93 & 1 & -2096.263376 & 0.550754 & 0.455241 & -2095.359775 & -2096.126943 & -2096.909532 & 14.0 & 20.1 & 25.1 & 13.2 & 19.4 & 24.3 \\
\hline M94 & 1 & -2174.835338 & 0.608130 & 0.503407 & -2173.870257 & -2174.683187 & -2175.520123 & 11.6 & 17.9 & 22.9 & 21.5 & 27.8 & 32.8 \\
\hline M95 & 1 & -2174.827099 & 0.607342 & 0.504283 & -2173.861652 & -2174.673521 & -2175.513662 & 16.5 & 23.5 & 26.5 & 27.4 & 34.4 & 37.4 \\
\hline M96 & 1 & -2174.865971 & 0.608016 & 0.508563 & -2173.911059 & -2174.713498 & -2175.559057 & -14.1 & -1.2 & -1.6 & -0.9 & 12.0 & 11.6 \\
\hline
\end{tabular}

S106 


\begin{tabular}{|c|c|c|c|c|c|c|c|c|c|c|c|c|c|}
\hline M97 & 1 & -2174.874976 & 0.609699 & 0.511075 & -2173.924765 & -2174.730363 & -2175.573916 & -21.6 & -10.7 & -9.8 & -7.9 & 3.0 & 3.9 \\
\hline M98 & 1 & -2174.862540 & 0.608497 & 0.510123 & -2173.911917 & -2174.714443 & -2175.559037 & -14.3 & -1.5 & -1.3 & -0.5 & 12.4 & 12.6 \\
\hline M99 & 1 & -2174.853715 & 0.609658 & 0.512277 & -2173.907585 & -2174.712218 & -2175.549528 & -10.9 & 0.6 & 5.4 & 3.6 & 15.1 & 19.9 \\
\hline M100 & 1 & -2174.875747 & 0.610893 & 0.512933 & -2173.927062 & -2174.726915 & -2175.566425 & -22.3 & -7.8 & -4.4 & -8.2 & 6.3 & 9.7 \\
\hline M101 & 1 & -2174.834483 & 0.606177 & 0.508785 & -2173.894812 & -2174.694183 & -2175.533599 & -5.1 & 9.8 & 13.2 & 9.4 & 24.2 & 27.7 \\
\hline M102 & 1 & -2174.851466 & 0.608004 & 0.508780 & -2173.902311 & -2174.701703 & -2175.542464 & -8.6 & 6.2 & 8.8 & 4.7 & 19.5 & 22.2 \\
\hline M103 & 1 & -2097.473835 & 0.566967 & 0.473017 & -2096.569785 & -2097.332717 & -2098.120000 & -15.3 & -3.0 & -0.1 & -5.8 & 6.5 & 9.4 \\
\hline M104 & 1 & -2097.479679 & 0.571043 & 0.475738 & -2096.572823 & -2097.339700 & -2098.129452 & -14.7 & -4.8 & -3.5 & -6.0 & 3.8 & 5.2 \\
\hline M105 & 1 & -2097.463711 & 0.570953 & 0.477537 & -2096.565768 & -2097.332399 & -2098.121527 & -10.3 & -0.3 & 1.4 & -0.4 & 9.6 & 11.3 \\
\hline M106 & 1 & -2097.478972 & 0.572869 & 0.478755 & -2096.575392 & -2097.339738 & -2098.132461 & -15.1 & -3.7 & -4.2 & -5.7 & 5.7 & 5.2 \\
\hline M107 & 1 & -2097.443429 & 0.569716 & 0.476563 & -2096.542360 & -2097.310951 & -2098.098746 & 3.6 & 12.4 & 14.9 & 13.6 & 22.4 & 25.0 \\
\hline M108 & 1 & -2097.482684 & 0.571807 & 0.476134 & -2096.575163 & -2097.342330 & -2098.129761 & -15.6 & -6.0 & -3.2 & -7.2 & 2.4 & 5.2 \\
\hline M109 & 1 & -2097.445403 & 0.570034 & 0.475646 & -2096.541679 & -2097.309070 & -2098.096659 & 4.2 & 13.8 & 16.4 & 13.5 & 23.0 & 25.7 \\
\hline M110 & 1 & -2097.472487 & 0.569896 & 0.475188 & -2096.565919 & -2097.332161 & -2098.121293 & -11.0 & -0.8 & 0.9 & -2.0 & 8.2 & 10.0 \\
\hline M111 & 1 & -2097.474381 & 0.570891 & 0.475238 & -2096.567335 & -2097.334435 & -2098.124183 & -11.3 & -1.6 & -0.3 & -2.9 & 6.8 & 8.2 \\
\hline M112 & 1 & -2097.446744 & 0.569255 & 0.475783 & -2096.545074 & -2097.312967 & -2098.101510 & 1.6 & 10.8 & 12.9 & 11.5 & 20.6 & 22.7 \\
\hline M113 & 1 & -2097.469647 & 0.570953 & 0.477131 & -2096.568810 & -2097.335825 & -2098.123564 & -12.2 & -2.5 & 0.1 & -2.6 & 7.1 & 9.7 \\
\hline M114 & 1 & -2097.503028 & 0.572379 & 0.477443 & -2096.595777 & -2097.362465 & -2098.152112 & -28.2 & -18.3 & -16.9 & -19.3 & -9.4 & -8.0 \\
\hline M115 & 1 & -2097.478580 & 0.571388 & 0.478339 & -2096.573691 & -2097.339398 & -2098.130820 & -15.0 & -4.4 & -4.1 & -4.9 & 5.7 & 6.0 \\
\hline M116 & 1 & -2097.481328 & 0.572131 & 0.477413 & -2096.577047 & -2097.344053 & -2098.133883 & -16.6 & -6.9 & -5.6 & -7.6 & 2.2 & 3.4 \\
\hline M117 & 1 & -2097.502860 & 0.571005 & 0.477424 & -2096.593913 & -2097.360915 & -2098.149746 & -27.9 & -18.2 & -16.3 & -18.2 & -8.4 & -6.5 \\
\hline M118 & 1 & -2097.505397 & 0.572982 & 0.478057 & -2096.586095 & -2097.358284 & -2098.146563 & -21.8 & -15.3 & -13.0 & -12.9 & -6.4 & -4.1 \\
\hline M119 & 1 & -2097.504249 & 0.570468 & 0.476034 & -2096.591533 & -2097.360487 & -2098.148535 & -26.8 & -18.2 & -15.8 & -17.5 & -9.0 & -6.6 \\
\hline M120 & 1 & -2097.504283 & 0.572138 & 0.476929 & -2096.592777 & -2097.361183 & -2098.149316 & -26.5 & -17.6 & -15.3 & -17.8 & -8.9 & -6.5 \\
\hline M121 & 1 & -2097.495540 & 0.570752 & 0.476492 & -2096.584625 & -2097.353759 & -2098.142224 & -22.2 & -13.8 & -11.7 & -12.9 & -4.5 & -2.4 \\
\hline M122 & 1 & -2097.521495 & 0.574826 & 0.480354 & -2096.606275 & -2097.380964 & -2098.171097 & -33.3 & -28.4 & -27.3 & -24.1 & -19.2 & -18.1 \\
\hline M123 & 1 & -2176.076949 & 0.629172 & 0.526594 & -2175.096514 & -2175.915026 & -2176.757709 & -24.9 & -18.6 & -16.3 & -4.4 & 2.0 & 4.2 \\
\hline M124 & 1 & -2176.105786 & 0.633049 & 0.533819 & -2175.140248 & -2175.955294 & -2176.797415 & -49.9 & -41.4 & -38.8 & -27.3 & -18.8 & -16.1 \\
\hline M125 & 1 & -2176.105725 & 0.632314 & 0.534806 & -2175.139954 & -2175.955506 & -2176.798009 & -50.2 & -42.0 & -39.6 & -26.5 & -18.3 & -15.9 \\
\hline M126 & 1 & -2176.108201 & 0.633726 & 0.533464 & -2175.139223 & -2175.954398 & -2176.799036 & -48.8 & -40.4 & -39.4 & -26.8 & -18.4 & -17.4 \\
\hline M127 & 1 & -2176.096485 & 0.631891 & 0.527867 & -2175.118235 & -2175.938718 & -2176.783214 & -36.8 & -31.7 & -30.6 & -17.2 & -12.1 & -11.0 \\
\hline M128 & 1 & -2176.090324 & 0.632032 & 0.533489 & -2175.122372 & -2175.938661 & -2176.779893 & -39.3 & -31.6 & -28.4 & -16.3 & -8.5 & -5.3 \\
\hline M129 & 1 & -2176.091076 & 0.633540 & 0.533732 & -2175.125753 & -2175.939558 & -2176.781607 & -40.5 & -31.2 & -28.5 & -18.2 & -8.9 & -6.3 \\
\hline M130 & 1 & -2176.090985 & 0.632582 & 0.534202 & -2175.125164 & -2175.939291 & -2176.782849 & -40.7 & -31.7 & -29.9 & -17.6 & -8.5 & -6.8 \\
\hline M131 & 1 & -2176.094222 & 0.632444 & 0.529043 & -2175.127434 & -2175.938297 & -2176.786116 & -42.2 & -31.1 & -32.1 & -22.2 & -11.1 & -12.0 \\
\hline M132 & 1 & -2097.493514 & 0.571127 & 0.477573 & -2096.585869 & -2097.355965 & -2098.145584 & -22.8 & -15.0 & -13.6 & -13.0 & -5.2 & -3.8 \\
\hline M133 & 1 & -2097.493514 & 0.572126 & 0.476993 & -2096.585865 & -2097.355966 & -2098.145588 & -22.2 & -14.4 & -12.9 & -13.4 & -5.6 & -4.2 \\
\hline M134 & 1 & -2097.476476 & 0.570816 & 0.477765 & -2096.572638 & -2097.341729 & -2098.130619 & -14.7 & -6.3 & -4.4 & -4.6 & 3.8 & 5.7 \\
\hline M135 & 1 & -2097.504963 & 0.572061 & 0.476793 & -2096.594679 & -2097.363010 & -2098.150826 & -27.7 & -18.8 & -16.3 & -19.0 & -10.1 & -7.6 \\
\hline
\end{tabular}




\begin{tabular}{|c|c|c|c|c|c|c|c|c|c|c|c|c|c|}
\hline M136 & 1 & -2097.455299 & 0.570968 & 0.476823 & -2096.555439 & -2097.319827 & -2098.110724 & -3.8 & 7.6 & 8.2 & 5.6 & 17.0 & 17.6 \\
\hline M137 & 1 & -2097.503887 & 0.570505 & 0.475668 & -2096.589495 & -2097.358842 & -2098.147073 & -25.5 & -17.2 & -14.9 & -16.5 & -8.2 & -5.9 \\
\hline M138 & 1 & -2097.505563 & 0.572808 & 0.477156 & -2096.585405 & -2097.357570 & -2098.146037 & -21.4 & -14.9 & -12.8 & -13.0 & -6.5 & -4.3 \\
\hline M139 & 1 & -2176.110722 & 0.633223 & 0.532506 & -2175.142468 & -2175.958277 & -2176.804767 & -51.2 & -43.2 & -43.3 & -29.5 & -21.5 & -21.6 \\
\hline M140 & 1 & -2097.468098 & 0.571155 & 0.477355 & -2096.566624 & -2097.333293 & -2098.121749 & -10.7 & -0.7 & 1.4 & -1.1 & 8.9 & 11.0 \\
\hline M141 & 1 & -2097.502768 & 0.572406 & 0.477474 & -2096.596005 & -2097.362651 & -2098.152513 & -28.4 & -18.4 & -17.1 & -19.4 & -9.5 & -8.2 \\
\hline M142 & 1 & -2097.502704 & 0.571225 & 0.477326 & -2096.594820 & -2097.361677 & -2098.151194 & -28.3 & -18.5 & -17.0 & -18.8 & -9.0 & -7.5 \\
\hline M143 & 1 & -2097.517178 & 0.574288 & 0.480391 & -2096.608118 & -2097.373810 & -2098.165196 & -34.8 & -24.2 & -23.9 & -25.2 & -14.6 & -14.3 \\
\hline M144 & 1 & -2097.520130 & 0.575299 & 0.480007 & -2096.611130 & -2097.376516 & -2098.168237 & -36.0 & -25.3 & -25.2 & -27.3 & -16.6 & -16.5 \\
\hline M145 & 1 & -2097.518776 & 0.574066 & 0.480066 & -2096.611150 & -2097.378408 & -2098.169263 & -36.8 & -27.2 & -26.6 & -27.3 & -17.7 & -17.1 \\
\hline M146 & 1 & -2097.520792 & 0.574967 & 0.480099 & -2096.605616 & -2097.380096 & -2098.170797 & -32.8 & -27.7 & -27.0 & -23.8 & -18.8 & -18.0 \\
\hline M147 & 1 & -2097.490039 & 0.570574 & 0.476251 & -2096.580063 & -2097.349154 & -2098.138377 & -19.5 & -11.1 & -9.4 & -10.2 & -1.8 & -0.1 \\
\hline M148 & 1 & -2097.502815 & 0.573160 & 0.478126 & -2096.582277 & -2097.354805 & -2098.143556 & -19.3 & -13.0 & -11.0 & -10.4 & -4.1 & -2.2 \\
\hline M149 & 1 & -2097.473775 & 0.571693 & 0.478970 & -2096.572020 & -2097.340449 & -2098.128632 & -13.7 & -4.9 & -2.6 & -3.5 & 5.4 & 7.7 \\
\hline M150 & 1 & -2097.490487 & 0.572429 & 0.477224 & -2096.586097 & -2097.352564 & -2098.142545 & -22.1 & -12.0 & -10.8 & -13.4 & -3.3 & -2.1 \\
\hline M151 & 1 & -2097.502788 & 0.573339 & 0.478462 & -2096.582271 & -2097.354759 & -2098.143812 & -19.1 & -12.8 & -11.1 & -10.2 & -3.9 & -2.1 \\
\hline M152 & 1 & -2097.473992 & 0.571756 & 0.478903 & -2096.572495 & -2097.340926 & -2098.129204 & -14.0 & -5.2 & -2.9 & -3.8 & 5.1 & 7.3 \\
\hline M153 & 1 & -2097.490469 & 0.571473 & 0.478313 & -2096.585966 & -2097.352204 & -2098.142145 & -22.6 & -12.4 & -11.2 & -12.6 & -2.4 & -1.2 \\
\hline M154 & 1 & -2097.496608 & 0.573037 & 0.477836 & -2096.578725 & -2097.350550 & -2098.139722 & -17.1 & -10.4 & -8.7 & -8.4 & -1.7 & 0.0 \\
\hline M155 & 1 & -2097.486105 & 0.572273 & 0.477771 & -2096.580758 & -2097.349841 & -2098.140275 & -18.9 & -10.4 & -9.5 & -9.7 & -1.2 & -0.3 \\
\hline M156 & 1 & -2097.460788 & 0.571218 & 0.476913 & -2096.558026 & -2097.325293 & -2098.115144 & -5.3 & 4.3 & 5.6 & 4.0 & 13.6 & 14.9 \\
\hline M157 & 1 & -2097.494945 & 0.572766 & 0.477801 & -2096.590754 & -2097.356844 & -2098.147472 & -24.8 & -14.5 & -13.7 & -15.9 & -5.6 & -4.8 \\
\hline M158 & 1 & -2097.493252 & 0.571080 & 0.477266 & -2096.585565 & -2097.352296 & -2098.142077 & -22.6 & -12.7 & -11.4 & -13.0 & -3.1 & -1.8 \\
\hline M159 & 1 & -2176.070714 & 0.630633 & 0.526031 & -2175.090655 & -2175.908478 & -2176.752176 & -20.3 & -13.5 & -11.9 & -1.0 & 5.7 & 7.4 \\
\hline M160 & 1 & -2097.493273 & 0.572283 & 0.478199 & -2096.585340 & -2097.352507 & -2098.142666 & -21.7 & -12.1 & -11.0 & -12.3 & -2.7 & -1.6 \\
\hline M161 & 1 & -2097.490418 & 0.570448 & 0.476368 & -2096.580641 & -2097.348997 & -2098.139745 & -19.9 & -11.0 & -10.3 & -10.5 & -1.6 & -0.9 \\
\hline M162 & 1 & -2097.518412 & 0.574800 & 0.480614 & -2096.602712 & -2097.377084 & -2098.168416 & -31.1 & -25.9 & -25.6 & -21.7 & -16.6 & -16.2 \\
\hline M163 & 1 & -2097.517398 & 0.573816 & 0.480589 & -2096.607257 & -2097.376064 & -2098.167623 & -34.5 & -25.9 & -25.7 & -24.5 & -15.9 & -15.7 \\
\hline M164 & 1 & -2097.519081 & 0.574928 & 0.479851 & -2096.611297 & -2097.376143 & -2098.169217 & -36.4 & -25.3 & -26.0 & -27.5 & -16.4 & -17.2 \\
\hline M164 & 1 & -2097.519081 & 0.574928 & 0.479851 & -2096.611297 & -2097.376143 & -2098.169217 & -4.5 & 4.5 & 3.0 & -7.4 & 1.6 & 0.1 \\
\hline M165 & 1 & -2176.102360 & 0.633208 & 0.532932 & -2175.137014 & -2175.949080 & -2176.796539 & -47.8 & -37.4 & -38.1 & -25.8 & -15.4 & -16.1 \\
\hline M166 & 1 & -2176.092428 & 0.631925 & 0.527820 & -2175.114247 & -2175.934139 & -2176.779795 & -34.3 & -28.8 & -28.4 & -14.7 & -9.3 & -8.8 \\
\hline M167 & 1 & -2176.083851 & 0.632185 & 0.533937 & -2175.116963 & -2175.932790 & -2176.775973 & -35.8 & -27.8 & -25.9 & -12.6 & -4.6 & -2.6 \\
\hline M168 & 1 & -2176.099074 & 0.632663 & 0.530698 & -2175.132205 & -2175.943491 & -2176.792808 & -45.1 & -34.2 & -36.1 & -24.2 & -13.3 & -15.2 \\
\hline M169 & 1 & -2097.466863 & 0.569903 & 0.475788 & -2096.554845 & -2097.321936 & -2098.110945 & -4.1 & 5.6 & 7.4 & 5.3 & 15.0 & 16.8 \\
\hline M170 & 1 & -2097.467389 & 0.570960 & 0.475332 & -2096.555965 & -2097.323110 & -2098.112756 & -4.1 & 5.5 & 6.9 & 4.3 & 14.0 & 15.4 \\
\hline M171 & 1 & -2097.455923 & 0.566487 & 0.471169 & -2096.545770 & -2097.310653 & -2098.098203 & -0.5 & 10.5 & 13.3 & 8.1 & 19.2 & 21.9 \\
\hline M172 & 1 & -2097.469933 & 0.568023 & 0.471328 & -2096.561747 & -2097.325306 & -2098.114736 & -9.6 & 2.3 & 3.8 & -1.8 & 10.1 & 11.6 \\
\hline M173 & 1 & -2176.045703 & 0.628279 & 0.526216 & -2175.081385 & -2175.892712 & -2176.736744 & -16.0 & -5.1 & -3.7 & 4.9 & 15.7 & 17.2 \\
\hline
\end{tabular}




\begin{tabular}{|c|c|c|c|c|c|c|c|c|c|c|c|c|c|}
\hline M174 & 1 & -2097.458581 & 0.567303 & 0.472521 & -2096.549852 & -2097.314611 & -2098.103064 & -2.6 & 8.6 & 10.7 & 6.4 & 17.6 & 19.7 \\
\hline M175 & 1 & -2097.487946 & 0.570432 & 0.477143 & -2096.583109 & -2097.351395 & -2098.139337 & -21.5 & -12.6 & -10.1 & -11.6 & -2.6 & -0.1 \\
\hline M176 & 1 & -2097.468386 & 0.567948 & 0.474390 & -2096.567537 & -2097.328996 & -2098.117974 & -13.3 & -0.1 & 1.8 & -3.5 & 9.7 & 11.5 \\
\hline M177 & 1 & -2097.478117 & 0.571561 & 0.476307 & -2096.571126 & -2097.338637 & -2098.128058 & -13.3 & -3.8 & -2.3 & -4.6 & 4.9 & 6.4 \\
\hline M178 & 1 & -2097.469108 & 0.569690 & 0.474843 & -2096.557793 & -2097.324431 & -2098.113277 & -6.1 & 3.9 & 5.8 & 2.9 & 12.9 & 14.8 \\
\hline M179 & 1 & -2097.480273 & 0.571475 & 0.476770 & -2096.574316 & -2097.341701 & -2098.132306 & -15.3 & -5.8 & -5.0 & -6.3 & 3.2 & 4.0 \\
\hline M180 & 1 & -2097.479808 & 0.571225 & 0.478915 & -2096.577160 & -2097.344499 & -2098.134813 & -17.3 & -7.7 & -6.7 & -6.7 & 2.8 & 3.8 \\
\hline M181 & 1 & -2097.482154 & 0.572314 & 0.479035 & -2096.582047 & -2097.347538 & -2098.137682 & -19.7 & -9.0 & -7.9 & -9.7 & 1.0 & 2.1 \\
\hline M182 & 1 & -2097.462382 & 0.570954 & 0.476361 & -2096.562349 & -2097.327117 & -2098.116883 & -8.1 & 3.0 & 4.3 & 1.0 & 12.1 & 13.5 \\
\hline M183 & 1 & -2097.458907 & 0.570399 & 0.475352 & -2096.552667 & -2097.317396 & -2098.105932 & -2.4 & 8.8 & 10.9 & 6.4 & 17.6 & 19.7 \\
\hline M184 & 1 & -2097.452760 & 0.567451 & 0.473089 & -2096.552802 & -2097.313061 & -2098.102008 & -4.4 & 9.6 & 11.5 & 4.9 & 18.9 & 20.7 \\
\hline M185 & 1 & -2097.471922 & 0.569780 & 0.474829 & -2096.568712 & -2097.334074 & -2098.123608 & -12.9 & -2.1 & -0.6 & -4.0 & 6.8 & 8.3 \\
\hline M186 & 1 & -2097.472507 & 0.569958 & 0.475189 & -2096.569560 & -2097.334771 & -2098.125303 & -13.3 & -2.4 & -1.6 & -4.3 & 6.6 & 7.4 \\
\hline M187 & 1 & -2097.465486 & 0.569786 & 0.475619 & -2096.564708 & -2097.334047 & -2098.121225 & -10.4 & -2.1 & 0.9 & -1.0 & 7.3 & 10.3 \\
\hline M188 & 1 & -2097.464769 & 0.569024 & 0.477293 & -2096.565939 & -2097.335096 & -2098.122823 & -11.6 & -3.2 & -0.6 & -0.7 & 7.7 & 10.3 \\
\hline M189 & 1 & -2097.477788 & 0.570206 & 0.476524 & -2096.580206 & -2097.346661 & -2098.135552 & -19.8 & -9.7 & -7.9 & -10.1 & 0.0 & 1.8 \\
\hline M190 & 1 & -2097.457642 & 0.568024 & 0.472975 & -2096.551772 & -2097.322088 & -2098.108973 & -3.3 & 4.3 & 7.5 & 5.5 & 13.2 & 16.3 \\
\hline M191 & 1 & -2097.459575 & 0.569040 & 0.472501 & -2096.551648 & -2097.321803 & -2098.109100 & -2.6 & 5.1 & 8.0 & 5.3 & 13.0 & 15.9 \\
\hline M192 & 1 & -2097.457689 & 0.568095 & 0.473958 & -2096.552969 & -2097.323756 & -2098.111374 & -4.1 & 3.3 & 6.0 & 5.4 & 12.7 & 15.4 \\
\hline M193 & 1 & -2097.467987 & 0.569014 & 0.475400 & -2096.566140 & -2097.331472 & -2098.120431 & -11.7 & -0.9 & 0.9 & -2.0 & 8.8 & 10.6 \\
\hline M194 & 1 & -2097.463460 & 0.568816 & 0.475078 & -2096.561065 & -2097.326524 & -2098.117385 & -8.7 & 2.0 & 2.7 & 1.0 & 11.7 & 12.3 \\
\hline M195 & 1 & -2097.469011 & 0.569891 & 0.474677 & -2096.567662 & -2097.333892 & -2098.125173 & -12.1 & -1.9 & -1.5 & -3.4 & 6.8 & 7.2 \\
\hline M196 & 1 & -2097.464195 & 0.569079 & 0.475406 & -2096.562620 & -2097.327512 & -2098.116848 & -9.5 & 1.6 & 3.2 & 0.2 & 11.3 & 12.9 \\
\hline M197 & 1 & -2097.471466 & 0.570352 & 0.476840 & -2096.575311 & -2097.341335 & -2098.130268 & -16.7 & -6.3 & -4.4 & -6.9 & 3.5 & 5.4 \\
\hline M198 & 1 & -2097.459773 & 0.567834 & 0.474982 & -2096.567780 & -2097.327760 & -2098.118472 & -13.5 & 0.6 & 1.4 & -3.3 & 10.9 & 11.6 \\
\hline M199 & 1 & -2097.499903 & 0.572618 & 0.478602 & -2096.587853 & -2097.361459 & -2098.148405 & -23.1 & -17.5 & -14.4 & -13.6 & -8.0 & -4.9 \\
\hline M200 & 1 & -2097.458964 & 0.567453 & 0.474731 & -2096.566199 & -2097.327001 & -2098.117892 & -12.8 & 0.9 & 1.5 & -2.5 & 11.2 & 11.8 \\
\hline M201 & 1 & -2097.503637 & 0.573865 & 0.479486 & -2096.594134 & -2097.366270 & -2098.157215 & -26.3 & -19.7 & -19.1 & -17.0 & -10.5 & -9.9 \\
\hline M202 & 1 & -2097.437170 & 0.572990 & 0.480445 & -2096.537688 & -2097.305075 & -2098.093287 & 8.6 & 18.1 & 20.4 & 19.0 & 28.5 & 30.8 \\
\hline M203 & 1 & -2097.399936 & 0.569238 & 0.474381 & -2096.509171 & -2097.268319 & -2098.061458 & 24.1 & 38.8 & 38.0 & 33.1 & 47.8 & 47.0 \\
\hline M204 & 1 & -2097.467550 & 0.575147 & 0.481981 & -2096.565010 & -2097.331776 & -2098.119190 & -7.2 & 2.7 & 5.5 & 2.8 & 12.7 & 15.5 \\
\hline M205 & 1 & -2097.452138 & 0.572229 & 0.479679 & -2096.553614 & -2097.318048 & -2098.110318 & -1.9 & 9.5 & 9.3 & 8.5 & 19.9 & 19.7 \\
\hline M206 & 1 & -2097.473951 & 0.573447 & 0.480965 & -2096.579597 & -2097.339592 & -2098.130934 & -17.4 & -3.3 & -2.9 & -7.0 & 7.2 & 7.5 \\
\hline M207 & 1 & -2097.479154 & 0.574289 & 0.480522 & -2096.577844 & -2097.336815 & -2098.127184 & -15.8 & -1.0 & 0.0 & -6.1 & 8.7 & 9.6 \\
\hline M208 & 1 & -2097.446115 & 0.572046 & 0.478557 & -2096.544769 & -2097.309162 & -2098.102104 & 3.6 & 15.0 & 14.3 & 13.4 & 24.8 & 24.1 \\
\hline M209 & 1 & -2097.474960 & 0.573037 & 0.480160 & -2096.579853 & -2097.339039 & -2098.131071 & -17.8 & -3.2 & -3.3 & -7.6 & 7.0 & 6.9 \\
\hline M210 & 1 & -2097.478121 & 0.572592 & 0.478609 & -2096.583291 & -2097.346090 & -2098.135904 & 11.6 & 21.9 & 22.4 & 9.4 & 19.7 & 20.2 \\
\hline M211 & 1 & -2097.472069 & 0.571351 & 0.477626 & -2096.576097 & -2097.339601 & -2098.130070 & 15.3 & 25.2 & 25.3 & 13.3 & 23.1 & 23.3 \\
\hline M212 & 1 & -2097.480632 & 0.572689 & 0.478680 & -2096.586693 & -2097.348380 & -2098.138443 & 9.5 & 20.5 & 20.9 & 7.3 & 18.3 & 18.7 \\
\hline
\end{tabular}




\begin{tabular}{|c|c|c|c|c|c|c|c|c|c|c|c|c|c|}
\hline M213 & 1 & -2097.468322 & 0.570976 & 0.477941 & -2096.572797 & -2097.333746 & -2098.122382 & 17.1 & 28.6 & 29.9 & 15.5 & 27.0 & 28.3 \\
\hline M214 & 1 & -2097.473859 & 0.573848 & 0.481165 & -2096.577697 & -2097.339826 & -2098.131112 & 15.9 & 26.6 & 26.2 & 14.5 & 25.2 & 24.8 \\
\hline M215 & 1 & -2097.468812 & 0.573090 & 0.480610 & -2096.577043 & -2097.338029 & -2098.131749 & 15.8 & 27.2 & 25.3 & 14.6 & 26.0 & 24.1 \\
\hline M216 & 1 & -2097.493987 & 0.574835 & 0.481344 & -2096.595969 & -2097.360367 & -2098.153298 & 5.0 & 14.3 & 12.9 & 3.1 & 12.4 & 11.0 \\
\hline M217 & 1 & -2097.488232 & 0.574466 & 0.482984 & -2096.590980 & -2097.354037 & -2098.147089 & 7.9 & 18.1 & 16.6 & 7.3 & 17.4 & 16.0 \\
\hline M218 & 1 & -2097.497769 & 0.575075 & 0.481376 & -2096.598097 & -2097.362148 & -2098.154566 & 3.8 & 13.3 & 12.3 & 1.8 & 11.3 & 10.3 \\
\hline M219 & 1 & -2097.494104 & 0.574057 & 0.482527 & -2096.595569 & -2097.359601 & -2098.151822 & 4.8 & 14.3 & 13.4 & 4.1 & 13.6 & 12.7 \\
\hline M220 & 1 & -2097.494785 & 0.574670 & 0.481404 & -2096.598140 & -2097.361089 & -2098.154217 & 3.6 & 13.8 & 12.2 & 1.8 & 12.0 & 10.5 \\
\hline M221 & 1 & -2097.494375 & 0.573737 & 0.481482 & -2096.596795 & -2097.359657 & -2098.153014 & 3.8 & 14.1 & 12.4 & 2.7 & 13.0 & 11.3 \\
\hline M222 & 1 & -2097.495884 & 0.575126 & 0.481936 & -2096.596608 & -2097.360217 & -2098.153631 & 4.8 & 14.6 & 12.9 & 3.1 & 12.9 & 11.2 \\
\hline M223 & 1 & -2097.492960 & 0.573909 & 0.482246 & -2096.594660 & -2097.358415 & -2098.150093 & 5.3 & 15.0 & 14.4 & 4.5 & 14.2 & 13.6 \\
\hline M224 & 1 & -2097.495771 & 0.575098 & 0.482742 & -2096.596620 & -2097.360553 & -2098.153518 & 4.8 & 14.4 & 12.9 & 3.6 & 13.2 & 11.8 \\
\hline M225 & 1 & -2097.462502 & 0.570561 & 0.478412 & -2096.574704 & -2097.331227 & -2098.121306 & 15.7 & 29.9 & 30.3 & 14.6 & 28.9 & 29.3 \\
\hline M226 & 1 & -2097.467332 & 0.571757 & 0.477871 & -2096.577542 & -2097.335448 & -2098.125489 & 14.7 & 28.0 & 28.4 & 12.5 & 25.9 & 26.3 \\
\hline M227 & 1 & -1940.279571 & 0.455099 & 0.372554 & -1939.497847 & -1940.168005 & -1940.850954 & 19.2 & 32.1 & 31.6 & 3.0 & 15.8 & 15.3 \\
\hline M228 & 1 & -2097.484663 & 0.573771 & 0.482253 & -2096.588345 & -2097.350762 & -2098.144000 & 9.1 & 19.7 & 18.1 & 8.5 & 19.0 & 17.4 \\
\hline M229 & 1 & -2097.490998 & 0.575003 & 0.482173 & -2096.592437 & -2097.356266 & -2098.149757 & 7.3 & 17.0 & 15.2 & 5.9 & 15.5 & 13.8 \\
\hline M230 & 1 & -2097.490233 & 0.573807 & 0.482220 & -2096.591018 & -2097.353979 & -2098.147110 & 7.5 & 17.7 & 16.2 & 6.8 & 17.0 & 15.5 \\
\hline M231 & 1 & -2097.495207 & 0.574823 & 0.481625 & -2096.595654 & -2097.358555 & -2098.152459 & 5.2 & 15.4 & 13.4 & 3.5 & 13.7 & 11.7 \\
\hline M232 & 1 & -2097.486990 & 0.573477 & 0.482448 & -2096.589070 & -2097.352620 & -2098.144955 & 8.5 & 18.3 & 17.3 & 8.2 & 18.0 & 17.0 \\
\hline M233 & 1 & -2097.489572 & 0.574399 & 0.483214 & -2096.595132 & -2097.357744 & -2098.149746 & 5.3 & 15.7 & 14.9 & 4.8 & 15.2 & 14.4 \\
\hline M234 & 1 & -2097.472398 & 0.571508 & 0.480751 & -2096.580868 & -2097.337656 & -2098.130636 & 12.4 & 26.5 & 25.1 & 12.2 & 26.3 & 24.9 \\
\hline M235 & 1 & -2097.505286 & 0.574778 & 0.481778 & -2096.597075 & -2097.367920 & -2098.162143 & 4.3 & 9.5 & 7.3 & 2.7 & 8.0 & 5.8 \\
\hline M236 & 1 & -2097.503716 & 0.574001 & 0.481611 & -2096.597249 & -2097.364582 & -2098.158831 & 3.7 & 11.1 & 8.9 & 2.5 & 9.9 & 7.7 \\
\hline M237 & 1 & -2097.504930 & 0.575000 & 0.480478 & -2096.595578 & -2097.362303 & -2098.155277 & 5.4 & 13.2 & 11.8 & 2.8 & 10.7 & 9.2 \\
\hline M238 & 1 & -2097.490434 & 0.573839 & 0.482066 & -2096.582506 & -2097.352451 & -2098.143241 & 12.9 & 18.7 & 18.6 & 12.0 & 17.8 & 17.8 \\
\hline M239 & 1 & -2097.503596 & 0.574648 & 0.478755 & -2096.596387 & -2097.362646 & -2098.153892 & 4.6 & 12.8 & 12.4 & 1.2 & 9.4 & 9.0 \\
\hline M240 & 1 & -2097.513442 & 0.575061 & 0.480038 & -2096.603473 & -2097.369311 & -2098.162238 & 0.5 & 8.8 & 7.5 & -2.4 & 6.0 & 4.6 \\
\hline M241 & 1 & -2097.511336 & 0.573710 & 0.479495 & -2096.599876 & -2097.363443 & -2098.158333 & 1.9 & 11.7 & 9.1 & -0.5 & 9.3 & 6.7 \\
\hline M242 & 1 & -2097.490631 & 0.573548 & 0.482347 & -2096.591712 & -2097.355120 & -2098.146801 & 6.9 & 16.8 & 16.2 & 6.4 & 16.3 & 15.7 \\
\hline M243 & 1 & -2097.491631 & 0.574212 & 0.482130 & -2096.594881 & -2097.357637 & -2098.148964 & 5.3 & 15.6 & 15.2 & 4.3 & 14.6 & 14.2 \\
\hline M244 & 1 & -2097.474041 & 0.570694 & 0.478704 & -2096.581522 & -2097.337579 & -2098.130165 & 11.5 & 26.0 & 24.8 & 10.5 & 25.1 & 23.9 \\
\hline M245 & 1 & -2097.507570 & 0.574679 & 0.481422 & -2096.599473 & -2097.369240 & -2098.163573 & 2.7 & 8.6 & 6.4 & 1.0 & 6.9 & 4.6 \\
\hline M246 & 1 & -2097.505107 & 0.573445 & 0.479571 & -2096.597369 & -2097.363527 & -2098.156237 & 3.3 & 11.5 & 10.2 & 1.1 & 9.3 & 8.1 \\
\hline M247 & 1 & -2097.506183 & 0.574525 & 0.479267 & -2096.597812 & -2097.363262 & -2098.155764 & 3.7 & 12.3 & 11.2 & 0.7 & 9.3 & 8.2 \\
\hline M248 & 1 & -2097.506146 & 0.574970 & 0.480395 & -2096.597366 & -2097.362766 & -2098.155117 & 4.2 & 12.9 & 11.9 & 1.7 & 10.3 & 9.3 \\
\hline M249 & 1 & -2097.505374 & 0.574156 & 0.481429 & -2096.595499 & -2097.360020 & -2098.153122 & 4.9 & 14.1 & 12.6 & 3.5 & 12.7 & 11.2 \\
\hline M250 & 1 & -2097.511561 & 0.575259 & 0.480748 & -2096.604871 & -2097.368634 & -2098.162445 & -0.3 & 9.4 & 7.4 & -2.8 & 6.9 & 4.9 \\
\hline M251 & 1 & -1940.267734 & 0.453899 & 0.372558 & -1939.482442 & -1940.151616 & -1940.837001 & 28.2 & 41.6 & 39.6 & 12.7 & 26.1 & 24.1 \\
\hline
\end{tabular}




\begin{tabular}{|c|c|c|c|c|c|c|c|c|c|c|c|c|c|}
\hline M252 & 1 & -1940.268054 & 0.454655 & 0.371579 & -1939.482658 & -1940.152511 & -1940.836137 & 28.5 & 41.5 & 40.6 & 11.9 & 24.9 & 24.0 \\
\hline M253 & 1 & -1940.264317 & 0.453707 & 0.372001 & -1939.480042 & -1940.152557 & -1940.832815 & 29.5 & 40.9 & 42.1 & 13.8 & 25.2 & 26.4 \\
\hline M254 & 1 & -2097.482904 & 0.572575 & 0.478953 & -2096.589095 & -2097.349030 & -2098.139703 & 7.9 & 20.0 & 20.0 & 5.9 & 18.0 & 18.1 \\
\hline M255 & 1 & -2097.464866 & 0.571398 & 0.478801 & -2096.573967 & -2097.333735 & -2098.122958 & 16.7 & 28.9 & 29.8 & 15.3 & 27.5 & 28.5 \\
\hline M256 & 1 & -2097.487697 & 0.575222 & 0.482784 & -2096.588275 & -2097.352344 & -2098.143654 & 10.1 & 19.6 & 19.2 & 8.9 & 18.4 & 18.0 \\
\hline M257 & 1 & -2097.480828 & 0.574247 & 0.481980 & -2096.585335 & -2097.341276 & -2098.135233 & 11.3 & 25.9 & 23.9 & 10.2 & 24.8 & 22.8 \\
\hline M258 & 1 & -2097.497061 & 0.574543 & 0.482083 & -2096.600314 & -2097.359655 & -2098.153057 & 2.1 & 14.6 & 12.9 & 0.9 & 13.3 & 11.6 \\
\hline M259 & 1 & -2097.471681 & 0.571278 & 0.479581 & -2096.584898 & -2097.340191 & -2098.132379 & 9.7 & 24.7 & 23.8 & 9.0 & 24.0 & 23.0 \\
\hline M260 & 1 & -2097.471887 & 0.572775 & 0.480195 & -2096.585176 & -2097.340997 & -2098.133558 & 10.5 & 25.2 & 24.0 & 9.2 & 23.9 & 22.7 \\
\hline M261 & 1 & -2097.460412 & 0.570336 & 0.475930 & -2096.563074 & -2097.320245 & -2098.115142 & 22.8 & 36.7 & 34.0 & 20.4 & 34.2 & 31.6 \\
\hline M262 & 1 & -2097.468078 & 0.571484 & 0.474558 & -2096.562821 & -2097.329413 & -2098.123424 & 23.7 & 31.6 & 29.6 & 19.7 & 27.6 & 25.5 \\
\hline M263 & 1 & -1940.256557 & 0.453052 & 0.371113 & -1939.474722 & -1940.146051 & -1940.828201 & 33.5 & 45.8 & 45.5 & 17.4 & 29.6 & 29.3 \\
\hline M264 & 1 & -2097.453171 & 0.570958 & 0.478662 & -2096.570030 & -2097.324359 & -2098.116065 & 18.9 & 34.5 & 33.9 & 17.7 & 33.3 & 32.7 \\
\hline M265 & 1 & -2097.455964 & 0.572661 & 0.480116 & -2096.581151 & -2097.337508 & -2098.129291 & 13.0 & 27.3 & 26.6 & 11.7 & 26.0 & 25.3 \\
\hline M266 & 1 & -2097.452778 & 0.571436 & 0.479885 & -2096.564261 & -2097.321653 & -2098.111452 & 22.8 & 36.5 & 37.0 & 22.1 & 35.8 & 36.4 \\
\hline M267 & 1 & -2097.486809 & 0.572434 & 0.477981 & -2096.593696 & -2097.353152 & -2098.145002 & 4.9 & 17.3 & 16.6 & 2.5 & 14.8 & 14.1 \\
\hline M268 & 1 & -2097.468633 & 0.570646 & 0.477213 & -2096.581838 & -2097.340718 & -2098.130557 & 11.3 & 24.0 & 24.6 & 9.4 & 22.2 & 22.7 \\
\hline M269 & 1 & -2097.491775 & 0.575195 & 0.482907 & -2096.592903 & -2097.356468 & -2098.147583 & 7.2 & 17.0 & 16.7 & 6.0 & 15.8 & 15.6 \\
\hline M270 & 1 & -2097.484637 & 0.574803 & 0.483437 & -2096.586192 & -2097.349339 & -2098.140602 & 11.1 & 21.2 & 20.9 & 10.6 & 20.7 & 20.3 \\
\hline M271 & 1 & -2097.493654 & 0.575310 & 0.482953 & -2096.594362 & -2097.358154 & -2098.148579 & 6.3 & 16.0 & 16.2 & 5.2 & 14.8 & 15.0 \\
\hline M272 & 1 & -2097.486313 & 0.573499 & 0.481599 & -2096.586153 & -2097.349063 & -2098.140807 & 10.3 & 20.6 & 19.9 & 9.5 & 19.7 & 19.0 \\
\hline M273 & 1 & -2097.498038 & 0.574477 & 0.482327 & -2096.600172 & -2097.360342 & -2098.152728 & 2.2 & 14.1 & 13.1 & 1.1 & 13.1 & 12.0 \\
\hline M274 & 1 & -2097.493962 & 0.573515 & 0.482794 & -2096.597025 & -2097.357426 & -2098.149690 & 3.5 & 15.3 & 14.4 & 3.4 & 15.2 & 14.2 \\
\hline M275 & 1 & -2097.496057 & 0.574154 & 0.481406 & -2096.598872 & -2097.358584 & -2098.150879 & 2.8 & 15.0 & 14.0 & 1.4 & 13.6 & 12.6 \\
\hline M276 & 1 & -2097.472732 & 0.571740 & 0.480714 & -2096.585190 & -2097.340314 & -2098.131000 & 9.8 & 25.0 & 25.0 & 9.5 & 24.6 & 24.6 \\
\hline M277 & 1 & -2097.473177 & 0.572919 & 0.480631 & -2096.586309 & -2097.341208 & -2098.132442 & 9.9 & 25.1 & 24.8 & 8.8 & 24.0 & 23.7 \\
\hline M278 & 1 & -2097.453586 & 0.571448 & 0.480201 & -2096.569058 & -2097.323836 & -2098.115598 & 19.8 & 35.1 & 34.5 & 19.3 & 34.6 & 34.0 \\
\hline M279 & 1 & -2097.456152 & 0.572877 & 0.481070 & -2096.577019 & -2097.333566 & -2098.124666 & 15.7 & 29.9 & 29.7 & 14.9 & 29.1 & 28.8 \\
\hline M280 & 1 & -2097.456051 & 0.570864 & 0.477266 & -2096.556890 & -2097.314735 & -2098.108250 & 27.1 & 40.5 & 38.7 & 25.1 & 38.5 & 36.7 \\
\hline M281 & 1 & -2097.463983 & 0.571835 & 0.475742 & -2096.557389 & -2097.324831 & -2098.117901 & 27.4 & 34.7 & 33.3 & 23.8 & 31.2 & 29.7 \\
\hline M282 & 1 & -2097.466573 & 0.570688 & 0.477705 & -2096.579957 & -2097.338713 & -2098.127968 & 12.5 & 25.3 & 26.2 & 10.9 & 23.7 & 24.6 \\
\hline M283 & 1 & -2097.485464 & 0.572281 & 0.477940 & -2096.591544 & -2097.351065 & -2098.142206 & 6.2 & 18.5 & 18.3 & 3.8 & 16.1 & 15.9 \\
\hline M284 & 1 & -2097.493732 & 0.573837 & 0.481424 & -2096.587971 & -2097.352851 & -2098.141315 & -22.4 & -11.3 & -9.2 & -11.9 & -0.8 & 1.3 \\
\hline M285 & 1 & -2097.494085 & 0.574754 & 0.481060 & -2096.587862 & -2097.353609 & -2098.142593 & -21.8 & -11.2 & -9.4 & -12.1 & -1.5 & 0.3 \\
\hline M286 & 1 & -2097.452412 & 0.570433 & 0.477409 & -2096.550537 & -2097.314541 & -2098.103341 & -1.1 & 10.6 & 12.5 & 9.0 & 20.7 & 22.6 \\
\hline M287 & 1 & -2097.491394 & 0.574512 & 0.479635 & -2096.568708 & -2097.344309 & -2098.131136 & -9.9 & -5.6 & -2.4 & -1.0 & 3.4 & 6.6 \\
\hline M288 & 1 & -2097.455257 & 0.570876 & 0.477524 & -2096.555369 & -2097.319569 & -2098.105865 & -3.8 & 7.7 & 11.2 & 6.1 & 17.6 & 21.1 \\
\hline M289 & 1 & -2097.479538 & 0.572466 & 0.477933 & -2096.575710 & -2097.339607 & -2098.128106 & -15.6 & -3.9 & -1.8 & -6.4 & 5.3 & 7.4 \\
\hline M290 & 1 & -2097.479524 & 0.571106 & 0.477620 & -2096.575060 & -2097.339111 & -2098.127379 & -16.0 & -4.4 & -2.2 & -6.2 & 5.4 & 7.7 \\
\hline
\end{tabular}

S111 


\begin{tabular}{|c|c|c|c|c|c|c|c|c|c|c|c|c|c|}
\hline M291 & 1 & -2097.491370 & 0.573156 & 0.478883 & -2096.568420 & -2097.344450 & -2098.131287 & -10.6 & -6.5 & -3.3 & -1.2 & 2.8 & 6.0 \\
\hline M292 & 1 & -2097.494062 & 0.574547 & 0.479634 & -2096.569723 & -2097.346699 & -2098.132992 & -10.5 & -7.0 & -3.5 & -1.6 & 1.9 & 5.4 \\
\hline M293 & 1 & -2097.477736 & 0.573943 & 0.479677 & -2096.563469 & -2097.331659 & -2098.121890 & -7.0 & 2.0 & 3.1 & 2.4 & 11.4 & 12.4 \\
\hline M294 & 1 & -2097.481337 & 0.574933 & 0.479989 & -2096.571606 & -2097.341463 & -2098.133007 & -11.5 & -3.5 & -3.3 & -2.6 & 5.4 & 5.6 \\
\hline M295 & 1 & -2097.480021 & 0.573610 & 0.480168 & -2096.569548 & -2097.340904 & -2098.130539 & -11.0 & -4.0 & -2.6 & -1.1 & 5.9 & 7.3 \\
\hline M296 & 1 & -2097.456011 & 0.570450 & 0.474821 & -2096.553016 & -2097.312304 & -2098.102576 & -2.6 & 12.0 & 13.0 & 5.9 & 20.5 & 21.5 \\
\hline M297 & 1 & -2097.474213 & 0.572181 & 0.477875 & -2096.572029 & -2097.336274 & -2098.124517 & -13.4 & -2.0 & 0.3 & -4.1 & 7.3 & 9.6 \\
\hline M298 & 1 & -2097.454813 & 0.570780 & 0.476822 & -2096.552497 & -2097.318446 & -2098.105503 & -2.1 & 8.3 & 11.4 & 7.5 & 17.9 & 20.9 \\
\hline M299 & 1 & -2097.494067 & 0.574609 & 0.480022 & -2096.569904 & -2097.346776 & -2098.133088 & -10.6 & -7.0 & -3.5 & -1.5 & 2.1 & 5.6 \\
\hline M300 & 1 & -2097.466890 & 0.570381 & 0.476871 & -2096.563989 & -2097.329342 & -2098.115852 & -9.5 & 1.2 & 4.6 & 0.3 & 11.1 & 14.4 \\
\hline M301 & 1 & -2097.479949 & 0.574271 & 0.480815 & -2096.575694 & -2097.345383 & -2098.135026 & -14.4 & -6.4 & -5.0 & -4.6 & 3.5 & 4.9 \\
\hline M302 & 1 & -2097.460244 & 0.572716 & 0.479135 & -2096.556347 & -2097.325327 & -2098.114363 & -3.3 & 5.2 & 7.0 & 6.5 & 15.0 & 16.8 \\
\hline M303 & 1 & -2097.504765 & 0.574054 & 0.479625 & -2096.595199 & -2097.365284 & -2098.153607 & -26.8 & -19.0 & -16.8 & -17.6 & -9.8 & -7.5 \\
\hline M304 & 1 & -2097.455111 & 0.570224 & 0.475877 & -2096.553076 & -2097.323051 & -2098.112879 & -2.8 & 5.1 & 6.4 & 6.5 & 14.4 & 15.7 \\
\hline M305 & 1 & -2097.455570 & 0.570586 & 0.476923 & -2096.552942 & -2097.322786 & -2098.114735 & -2.5 & 5.5 & 5.4 & 7.2 & 15.2 & 15.2 \\
\hline M306 & 1 & -2097.453326 & 0.569978 & 0.475985 & -2096.547843 & -2097.318516 & -2098.107370 & 0.3 & 7.8 & 9.7 & 9.8 & 17.3 & 19.2 \\
\hline M307 & 1 & -2097.483910 & 0.571295 & 0.476368 & -2096.574331 & -2097.345433 & -2098.131341 & -15.4 & -8.3 & -4.5 & -6.5 & 0.6 & 4.4 \\
\hline M308 & 1 & -2097.485062 & 0.571844 & 0.474610 & -2096.575142 & -2097.347285 & -2098.134239 & -15.6 & -9.1 & -6.0 & -8.1 & -1.6 & 1.5 \\
\hline M309 & 1 & -1979.586563 & 0.484124 & 0.396028 & -1978.762444 & -1979.465018 & -1980.177104 & -1.8 & 6.1 & 5.0 & -7.5 & 0.5 & -0.7 \\
\hline M310 & 1 & -3026.686456 & 0.994165 & 0.868643 & -3025.233336 & -3026.433536 & -3027.612826 & -39.5 & -23.6 & -22.5 & -28.2 & -12.4 & -11.2 \\
\hline M311 & 1 & -2097.486289 & 0.572370 & 0.475814 & -2096.581305 & -2097.350507 & -2098.141067 & -19.1 & -10.8 & -10.0 & -11.3 & -2.9 & -2.1 \\
\hline M312 & 1 & -2018.903019 & 0.514233 & 0.422110 & -2018.051500 & -2018.774166 & -2019.512349 & -5.1 & 3.6 & 3.1 & -10.8 & -2.2 & -2.6 \\
\hline M313 & 1 & -3065.999819 & 1.024650 & 0.897031 & -3064.515686 & -3065.736064 & -3066.944371 & -38.3 & -21.8 & -21.8 & -25.9 & -9.4 & -9.4 \\
\hline M314 & 1 & -2058.195612 & 0.544556 & 0.453289 & -2057.315805 & -2058.065850 & -2058.827631 & -29.3 & -22.2 & -20.3 & -20.5 & -13.4 & -11.5 \\
\hline M315 & 1 & -2058.161834 & 0.542554 & 0.448976 & -2057.285678 & -2058.036833 & -2058.798020 & -11.6 & -5.2 & -3.0 & -4.3 & 2.1 & 4.3 \\
\hline M316 & 1 & -2058.161589 & 0.541385 & 0.448588 & -2057.285187 & -2058.036361 & -2058.797598 & -12.0 & -5.7 & -3.5 & -4.2 & 2.2 & 4.4 \\
\hline M317 & 1 & -2058.171306 & 0.542515 & 0.449354 & -2057.296084 & -2058.045384 & -2058.808019 & -18.2 & -10.6 & -9.3 & -10.6 & -3.0 & -1.7 \\
\hline M318 & 1 & -2058.169249 & 0.541265 & 0.448854 & -2057.291172 & -2058.041017 & -2058.802304 & -15.9 & -8.7 & -6.5 & -7.8 & -0.6 & 1.6 \\
\hline M319 & 1 & -2058.143460 & 0.541340 & 0.450600 & -2057.268705 & -2058.018893 & -2058.784280 & -1.7 & 5.3 & 4.9 & 7.4 & 14.4 & 14.0 \\
\hline M320 & 1 & -2058.181854 & 0.542616 & 0.450374 & -2057.302969 & -2058.051596 & -2058.814850 & -22.4 & -14.5 & -13.5 & -14.2 & -6.3 & -5.3 \\
\hline M321 & 1 & -2058.181779 & 0.541640 & 0.450577 & -2057.303456 & -2058.052296 & -2058.815122 & -23.3 & -15.5 & -14.3 & -14.4 & -6.6 & -5.4 \\
\hline M322 & 1 & -2058.195372 & 0.543334 & 0.450813 & -2057.304846 & -2058.055806 & -2058.817818 & -23.1 & -16.6 & -14.9 & -15.1 & -8.6 & -6.9 \\
\hline M323 & 1 & -2058.191174 & 0.541133 & 0.449484 & -2057.311790 & -2058.057438 & -2058.819793 & -28.9 & -19.1 & -17.6 & -20.3 & -10.5 & -9.0 \\
\hline M324 & 1 & -2058.192162 & 0.542798 & 0.450555 & -2057.314771 & -2058.060071 & -2058.823280 & -29.7 & -19.7 & -18.7 & -21.5 & -11.5 & -10.5 \\
\hline M325 & 1 & -2136.802636 & 0.603538 & 0.505293 & -2135.864323 & -2136.658607 & -2137.476378 & -54.5 & -46.3 & -45.4 & -33.7 & -25.5 & -24.6 \\
\hline M326 & 1 & -1941.500960 & 0.474080 & 0.386997 & -1940.690677 & -1941.373565 & -1942.060775 & 5.8 & 17.2 & 16.8 & -0.5 & 10.9 & 10.5 \\
\hline M327 & 1 & -2020.084802 & 0.533775 & 0.442726 & -2019.221290 & -2019.954129 & -2020.692575 & -7.8 & 1.2 & 2.7 & -0.2 & 8.8 & 10.4 \\
\hline M328 & 1 & -2020.094597 & 0.532460 & 0.440280 & -2019.229317 & -2019.958388 & -2020.696623 & -13.7 & -2.3 & -0.6 & -6.7 & 4.6 & 6.3 \\
\hline M329 & 1 & -2020.085568 & 0.530552 & 0.439411 & -2019.220103 & -2019.950032 & -2020.686799 & -9.1 & 1.7 & 4.3 & -1.5 & 9.3 & 11.9 \\
\hline
\end{tabular}

S112 


\begin{tabular}{|c|c|c|c|c|c|c|c|c|c|c|c|c|c|}
\hline M330 & 1 & -2020.098232 & 0.535435 & 0.444335 & -2019.232363 & -2019.964266 & -2020.704143 & -13.7 & -4.1 & -3.5 & -6.1 & 3.5 & 4.1 \\
\hline M331 & 1 & -2020.098200 & 0.535512 & 0.444452 & -2019.232604 & -2019.964371 & -2020.704037 & -13.8 & -4.1 & -3.4 & -6.2 & 3.5 & 4.3 \\
\hline M332 & 1 & -2020.068338 & 0.533890 & 0.444440 & -2019.208930 & -2019.943600 & -2020.681307 & 0.0 & 7.9 & 9.9 & 8.7 & 16.5 & 18.5 \\
\hline M333 & 1 & -2020.106962 & 0.537207 & 0.444901 & -2019.227761 & -2019.967984 & -2020.705141 & -9.7 & -5.4 & -3.0 & -2.9 & 1.5 & 3.8 \\
\hline M334 & 1 & -2020.074954 & 0.534581 & 0.444346 & -2019.215321 & -2019.947037 & -2020.684981 & -3.5 & 6.1 & 8.0 & 4.6 & 14.3 & 16.1 \\
\hline M335 & 1 & -2020.102528 & 0.535785 & 0.443831 & -2019.238400 & -2019.970186 & -2020.707586 & -17.3 & -7.6 & -5.4 & -10.2 & -0.6 & 1.6 \\
\hline M336 & 1 & -2020.102475 & 0.533787 & 0.442609 & -2019.237927 & -2019.969850 & -2020.706990 & -18.2 & -8.7 & -6.3 & -10.7 & -1.1 & 1.2 \\
\hline M337 & 1 & -2020.106888 & 0.537246 & 0.444672 & -2019.227555 & -2019.967675 & -2020.704687 & -9.5 & -5.1 & -2.7 & -2.9 & 1.6 & 4.0 \\
\hline M338 & 1 & -2098.720667 & 0.596765 & 0.497793 & -2097.798965 & -2098.574635 & -2099.373339 & -48.9 & -37.8 & -40.0 & -29.8 & -18.8 & -20.9 \\
\hline M339 & 1 & -2176.033292 & 0.632773 & 0.535546 & -2175.085112 & -2175.890741 & -2176.736239 & -15.5 & -1.1 & -0.6 & 8.4 & 22.8 & 23.3 \\
\hline M340 & 1 & -2176.033655 & 0.633885 & 0.535593 & -2175.087425 & -2175.891581 & -2176.737889 & -16.2 & -0.9 & -0.9 & 7.0 & 22.3 & 22.3 \\
\hline M341 & 1 & -2176.006133 & 0.632556 & 0.535261 & -2175.062523 & -2175.864810 & -2176.707738 & -1.4 & 15.1 & 17.2 & 22.4 & 38.9 & 41.0 \\
\hline M342 & 1 & -2176.113650 & 0.634966 & 0.537411 & -2175.153348 & -2175.953175 & -2176.797790 & -56.9 & -38.9 & -37.8 & -33.2 & -15.2 & -14.1 \\
\hline M343 & 1 & -2136.736110 & 0.601824 & 0.505685 & -2135.810231 & -2136.599085 & -2137.418784 & -21.7 & -10.0 & -10.4 & 0.5 & 12.1 & 11.8 \\
\hline M344 & 1 & -2136.739323 & 0.603568 & 0.507824 & -2135.821364 & -2136.606968 & -2137.426029 & -27.6 & -13.9 & -13.8 & -5.2 & 8.5 & 8.6 \\
\hline M345 & 1 & -2136.717834 & 0.602330 & 0.506956 & -2135.800732 & -2136.583744 & -2137.400216 & -15.4 & -0.1 & 1.6 & 7.2 & 22.5 & 24.2 \\
\hline M346 & 1 & -2136.813641 & 0.604837 & 0.509445 & -2135.877038 & -2136.661834 & -2137.479443 & -61.7 & -47.5 & -46.5 & -39.1 & -24.9 & -23.9 \\
\hline
\end{tabular}


Energies of Minimum Energy Crossing Points

Table S11: SP energy components of minimum energy crossing points (MECPs).

\begin{tabular}{|c|c|c|c|c|c|c|}
\hline 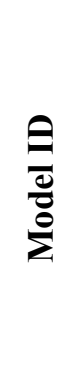 & 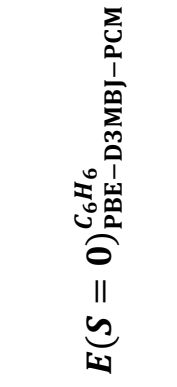 & 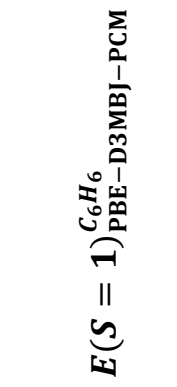 & 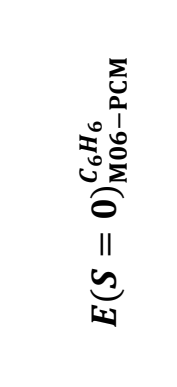 & 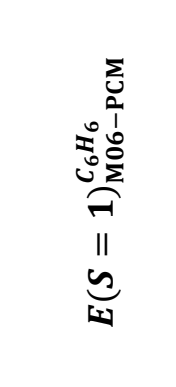 & 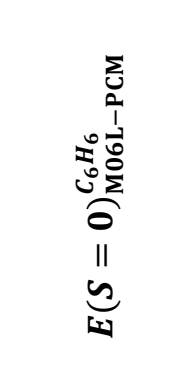 & 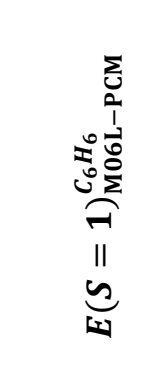 \\
\hline M3 & -1939.471602 & -1939.470280 & -1940.164199 & -1940.173516 & -1940.845026 & -1940.852380 \\
\hline M14 & -2018.019713 & -2018.017144 & -2018.747815 & -2018.756323 & -2019.482420 & -2019.487935 \\
\hline M17 & -2018.029771 & -2018.022699 & -2018.756643 & -2018.760105 & -2019.492666 & -2019.494334 \\
\hline
\end{tabular}

Table S12: MECP's range of Gibbs free energy relative to M1 calculated with different DFT models.

\begin{tabular}{|c|c|c|c|c|c|c|}
\hline \multirow[t]{2}{*}{$\frac{\theta}{\frac{0}{0}}$} & \multicolumn{2}{|c|}{ 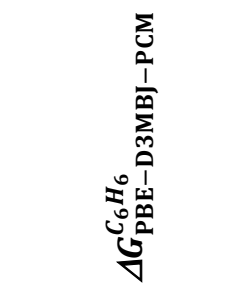 } & \multicolumn{2}{|c|}{ 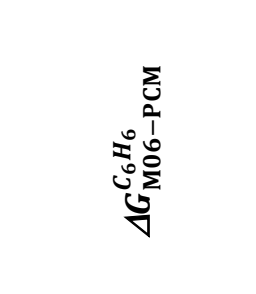 } & \multicolumn{2}{|c|}{ 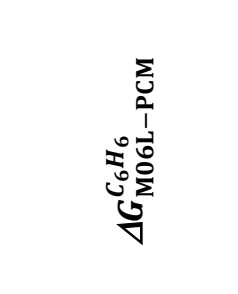 } \\
\hline & Min. & Max. & Min. & Max. & Min. & Max. \\
\hline M3 & 24.0 & 28.0 & 19.3 & 23.3 & 21.3 & 25.3 \\
\hline M14 & 11.8 & 15.8 & 13.6 & 17.6 & 16.3 & 20.3 \\
\hline M17 & 7.1 & 11.1 & 9.8 & 13.8 & 11.3 & 15.3 \\
\hline
\end{tabular}




\section{Geometries}

Molecular geometries are collected in the attached XYZ file.

\section{IRC Trajectories}

In the following we provide a selection of the IRC trajectories confirming the connection between the most relevant transition state and the surrounding local minima.

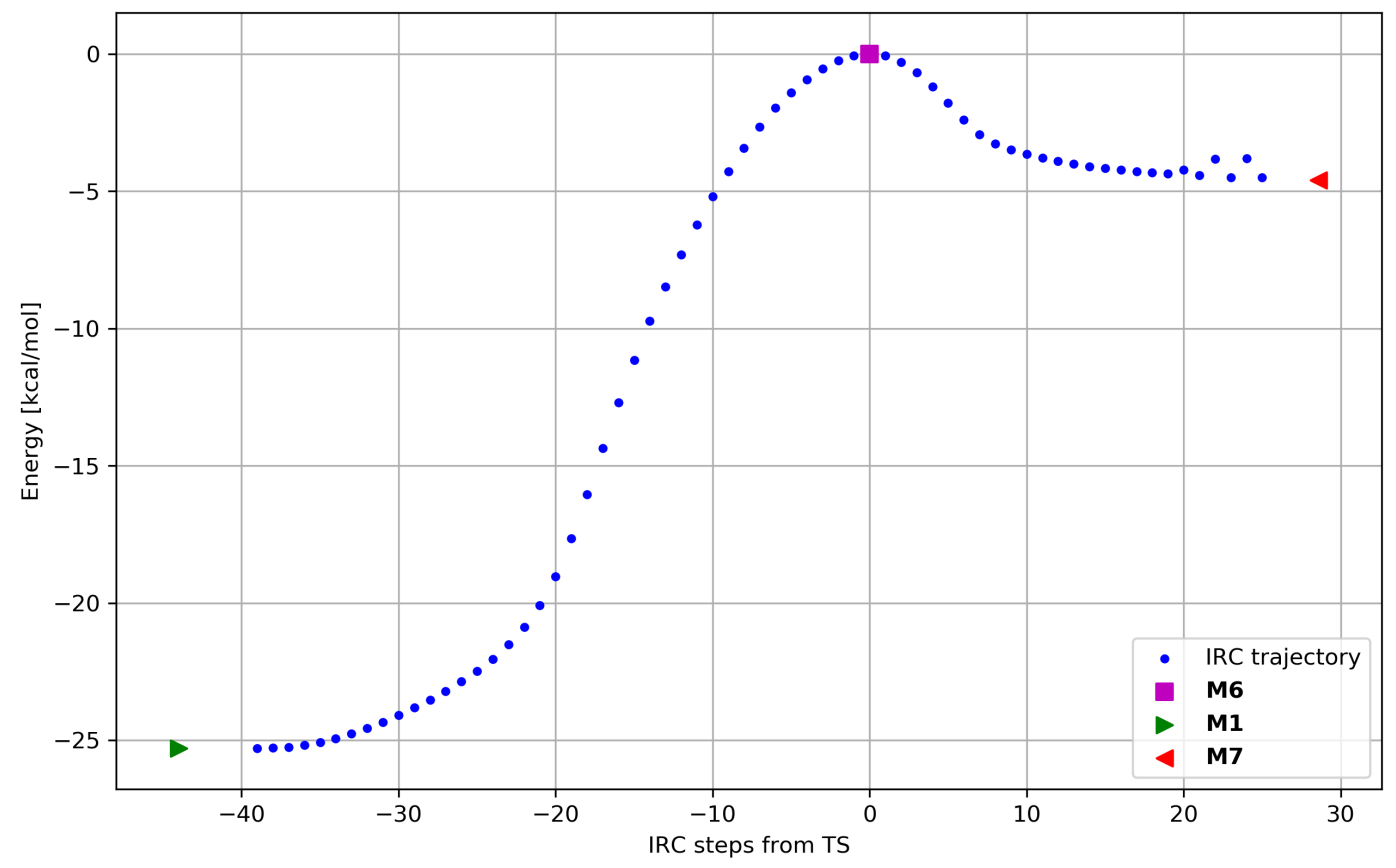

Figure S43: IRC trajectory for transition state M6. Predictor step size for LQA algorithm: 0.30 Bohr. The end of the trajectory is polluted by partial torsion of one $o$-methyl group in a mesityl group. 


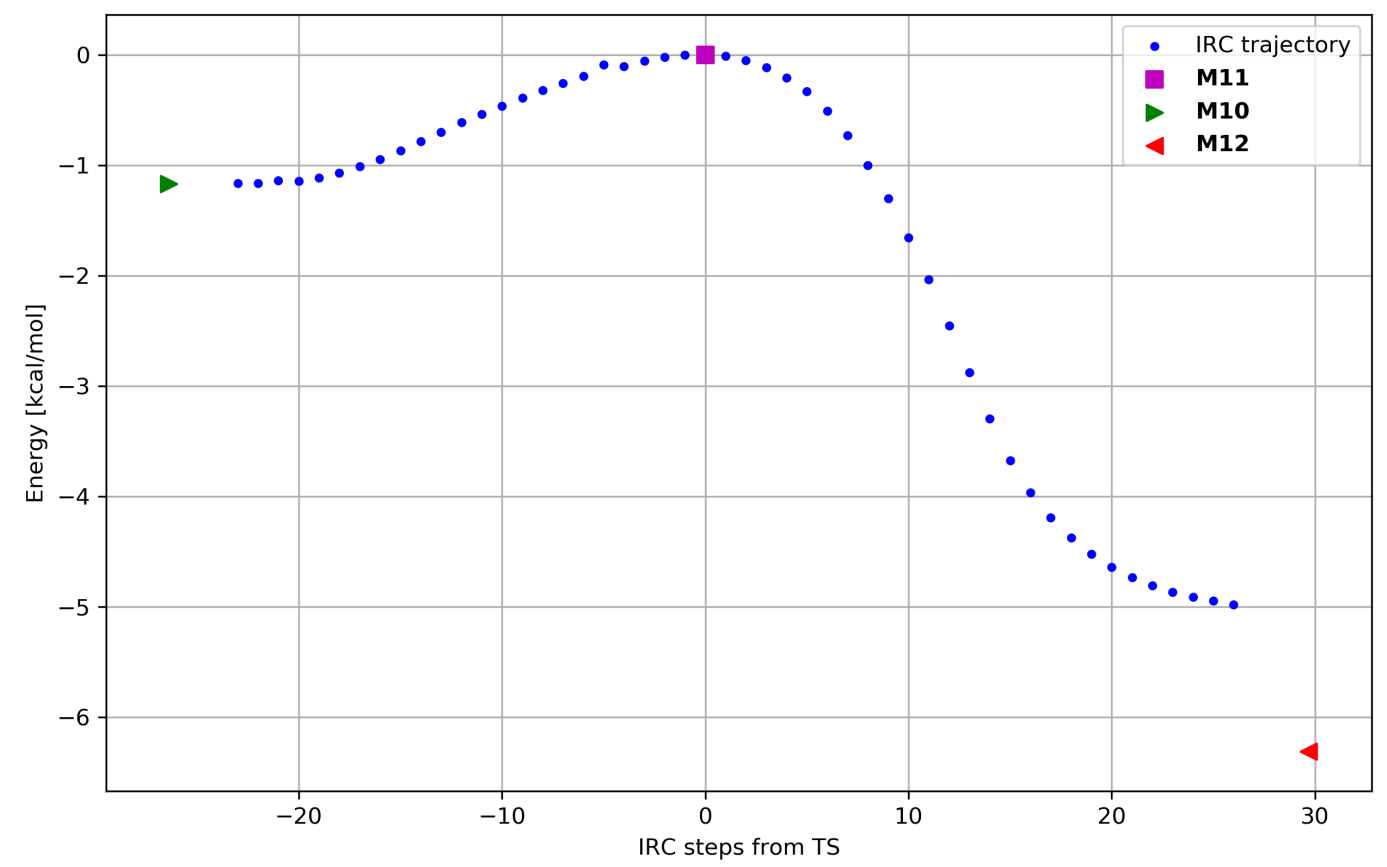

Figure S44: IRC trajectory for transition state M11. Predictor step size for LQA algorithm: 0.30 Bohr.

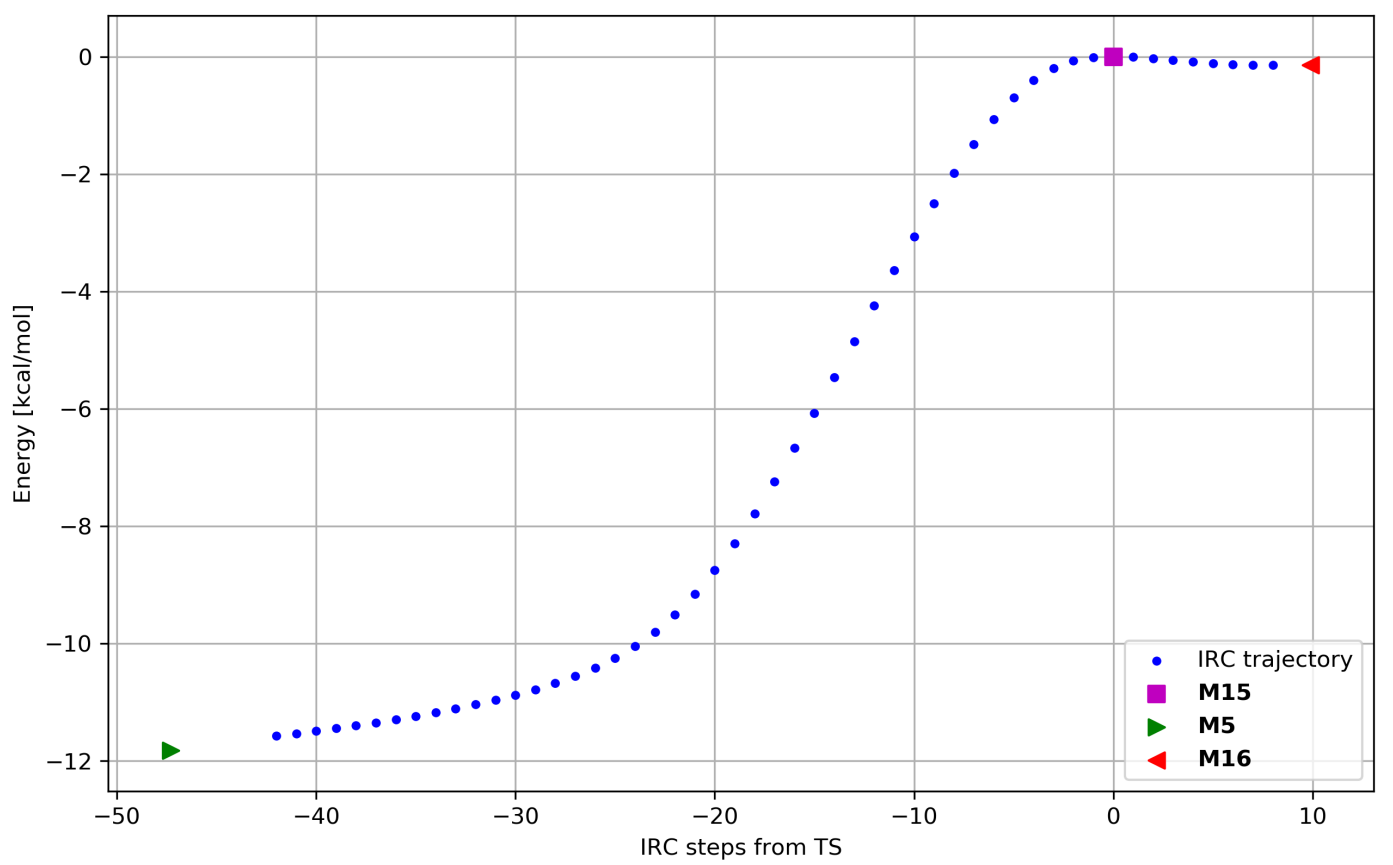

Figure S45: IRC trajectory for transition state M15. Predictor step size for LQA algorithm: 0.30 Bohr. 


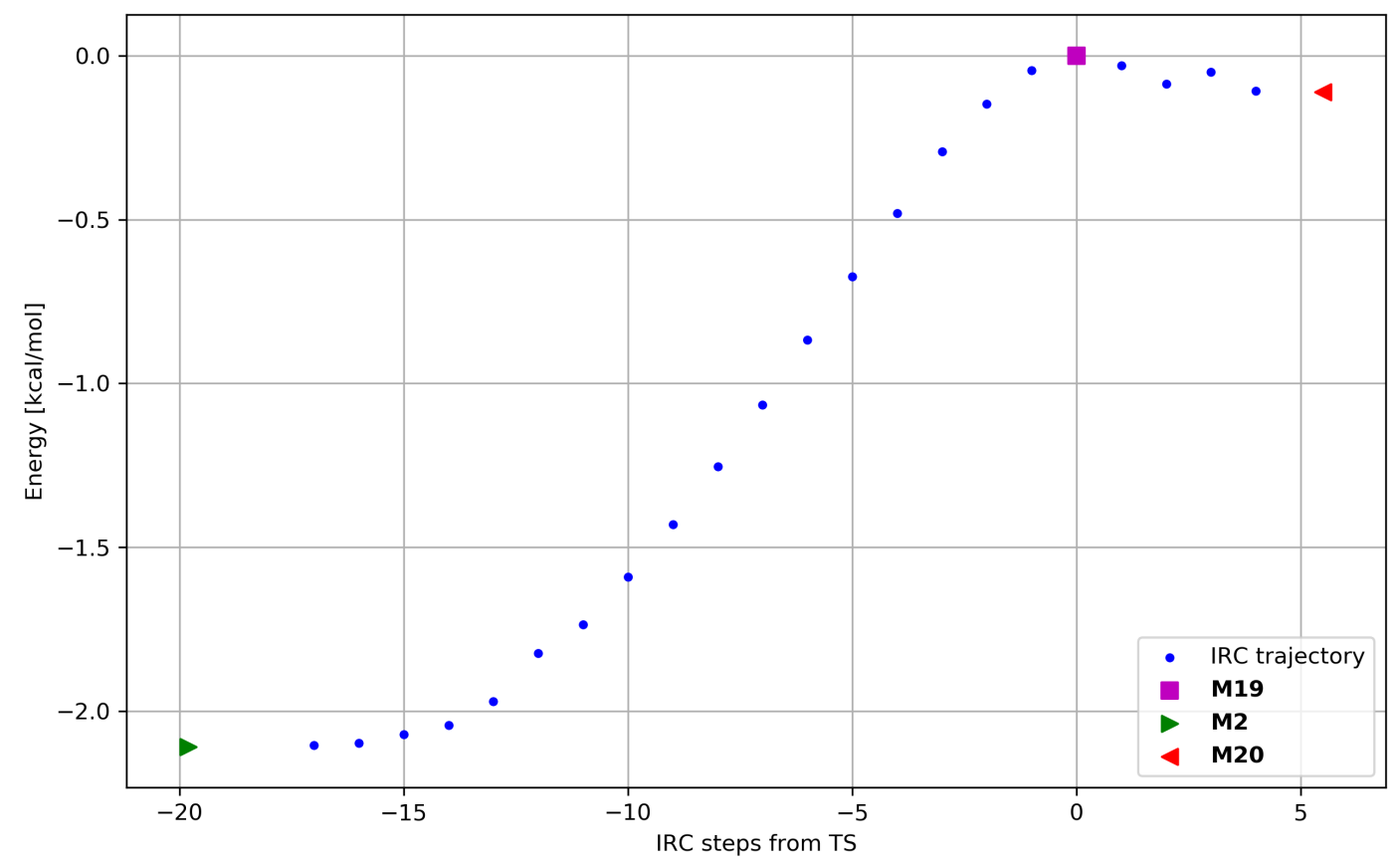

Figure S46: IRC trajectory for transition state M19. Predictor step size for LQA algorithm: 0.45 Bohr.

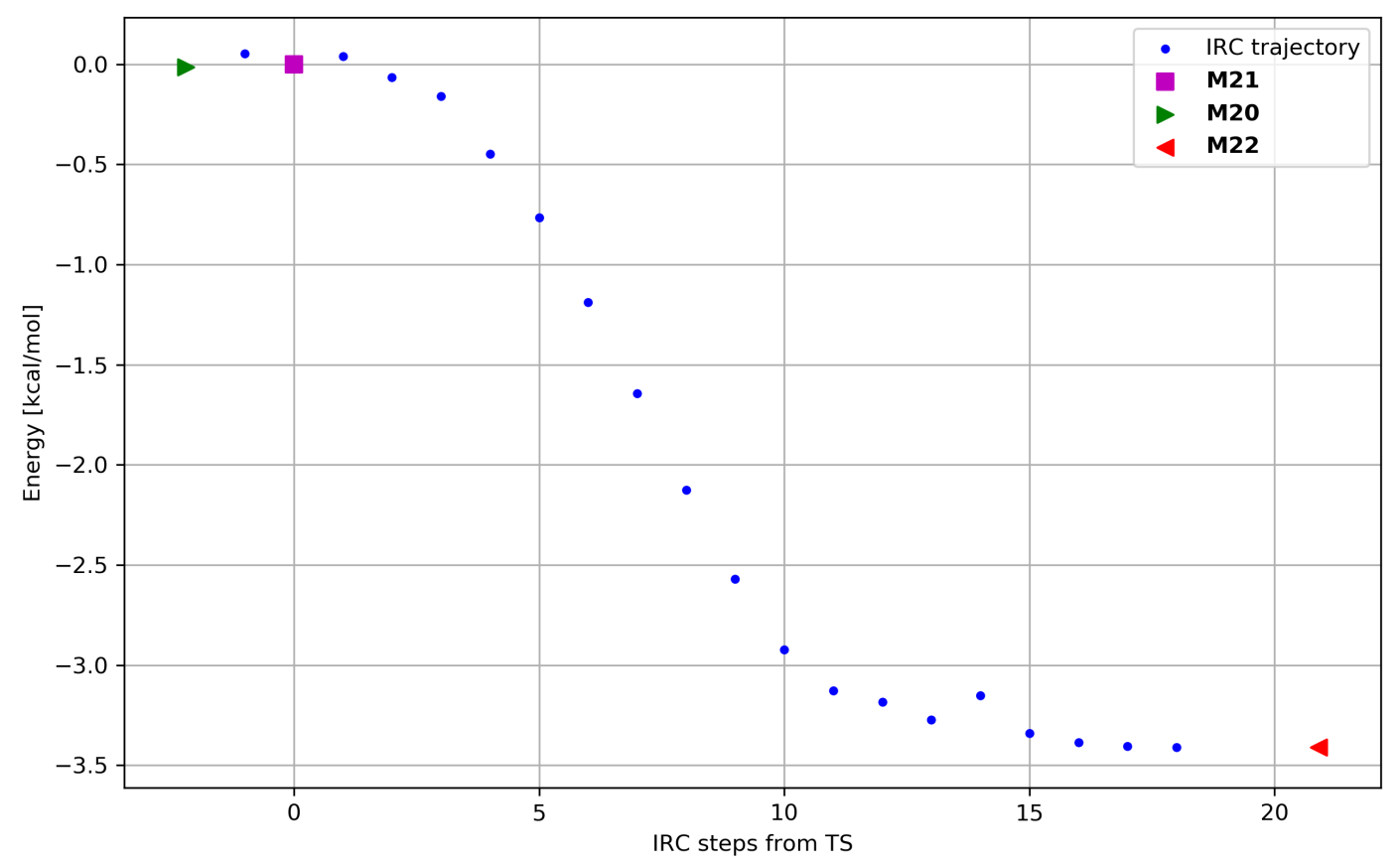

Figure S47: IRC trajectory for transition state M21. Predictor step size for LQA algorithm: 0.45 Bohr. 


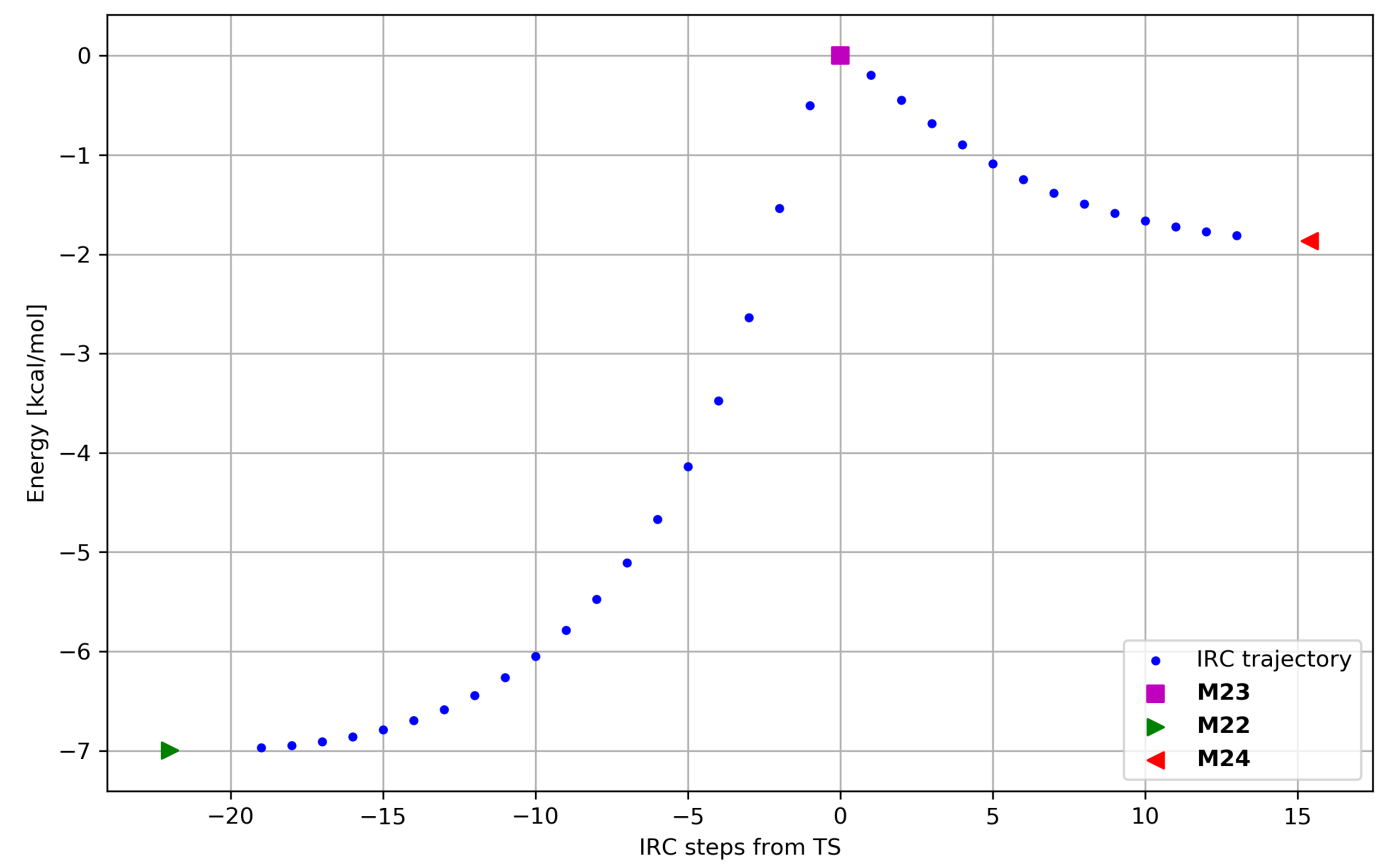

Figure S48: IRC trajectory for transition state M23. Predictor step size for LQA algorithm: 0.35 Bohr.

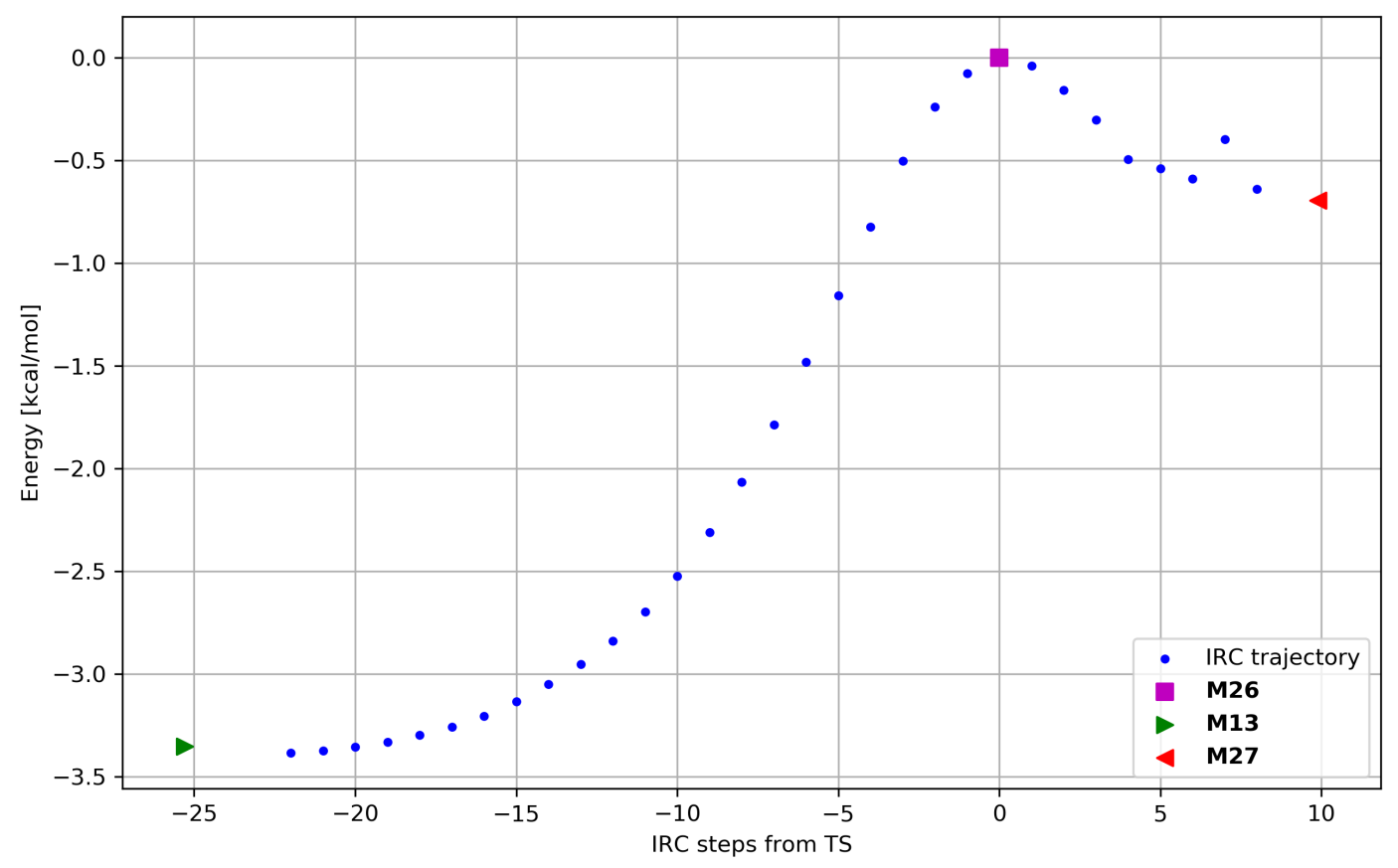

Figure S49: IRC trajectory for transition state M26. Predictor step size for LQA algorithm: 0.35 Bohr. 


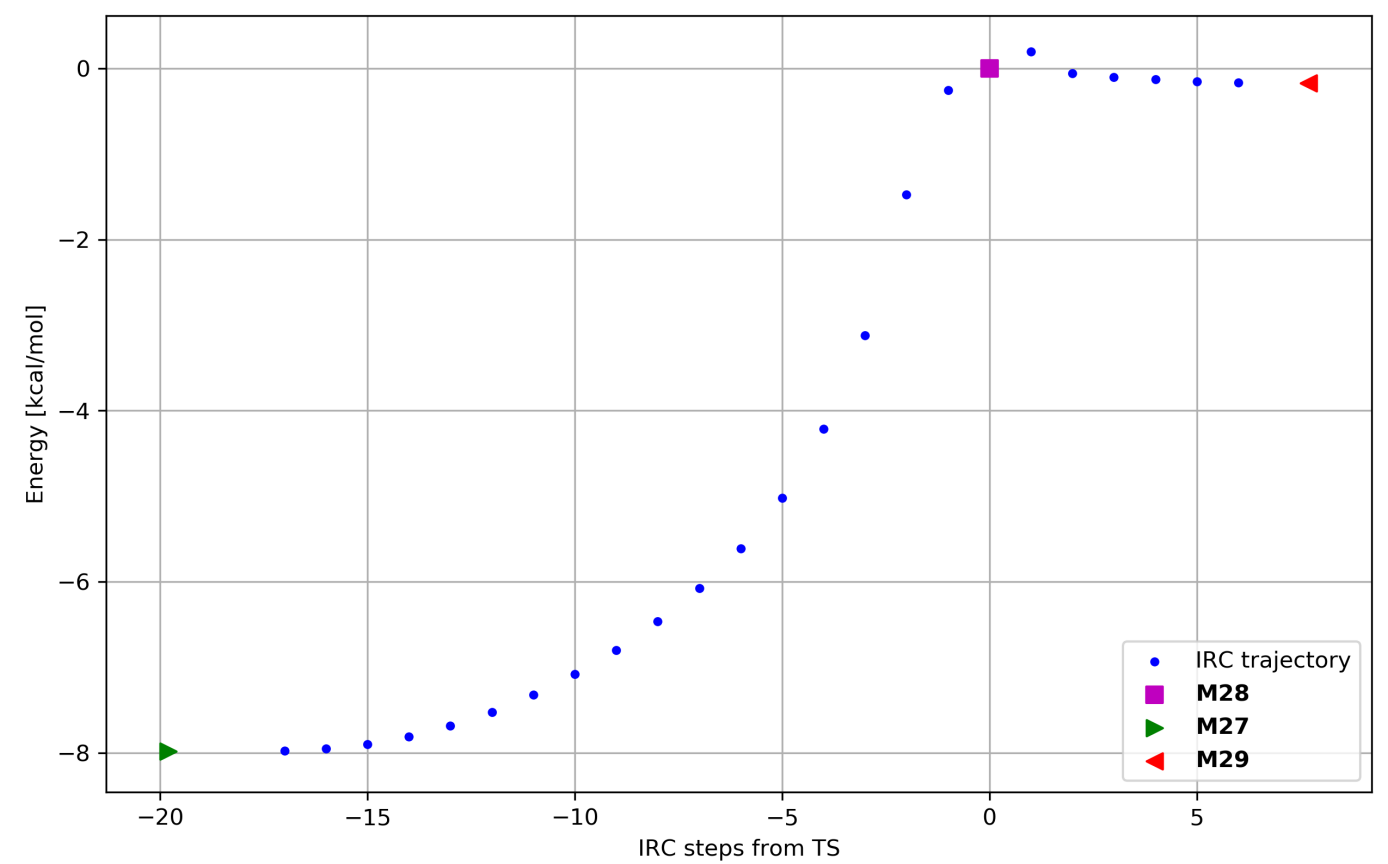

Figure S50: IRC trajectory for transition state M28. Predictor step size for LQA algorithm: 0.35 Bohr.

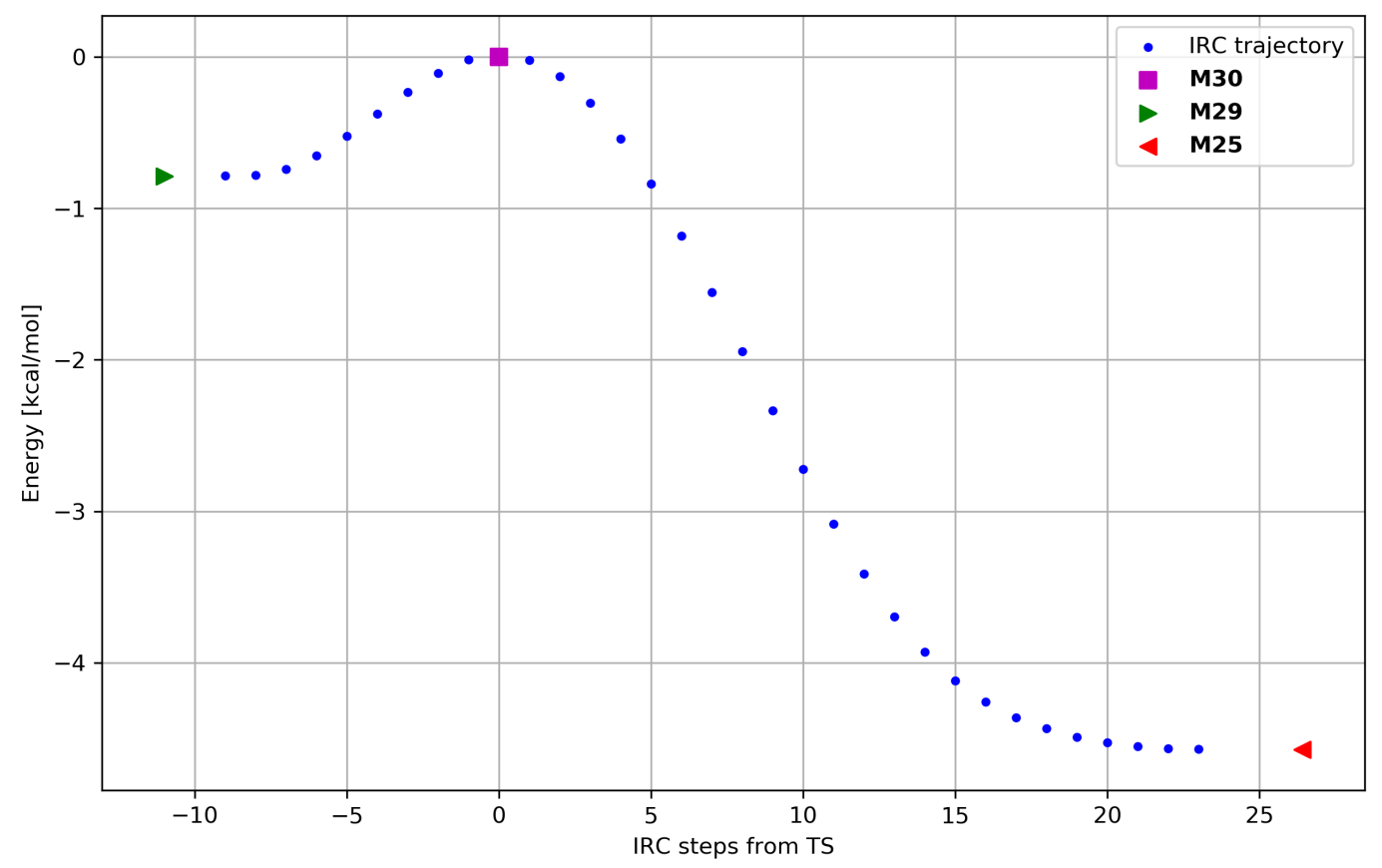

Figure S51: IRC trajectory for transition state M30. Predictor step size for LQA algorithm: 0.40 Bohr. 


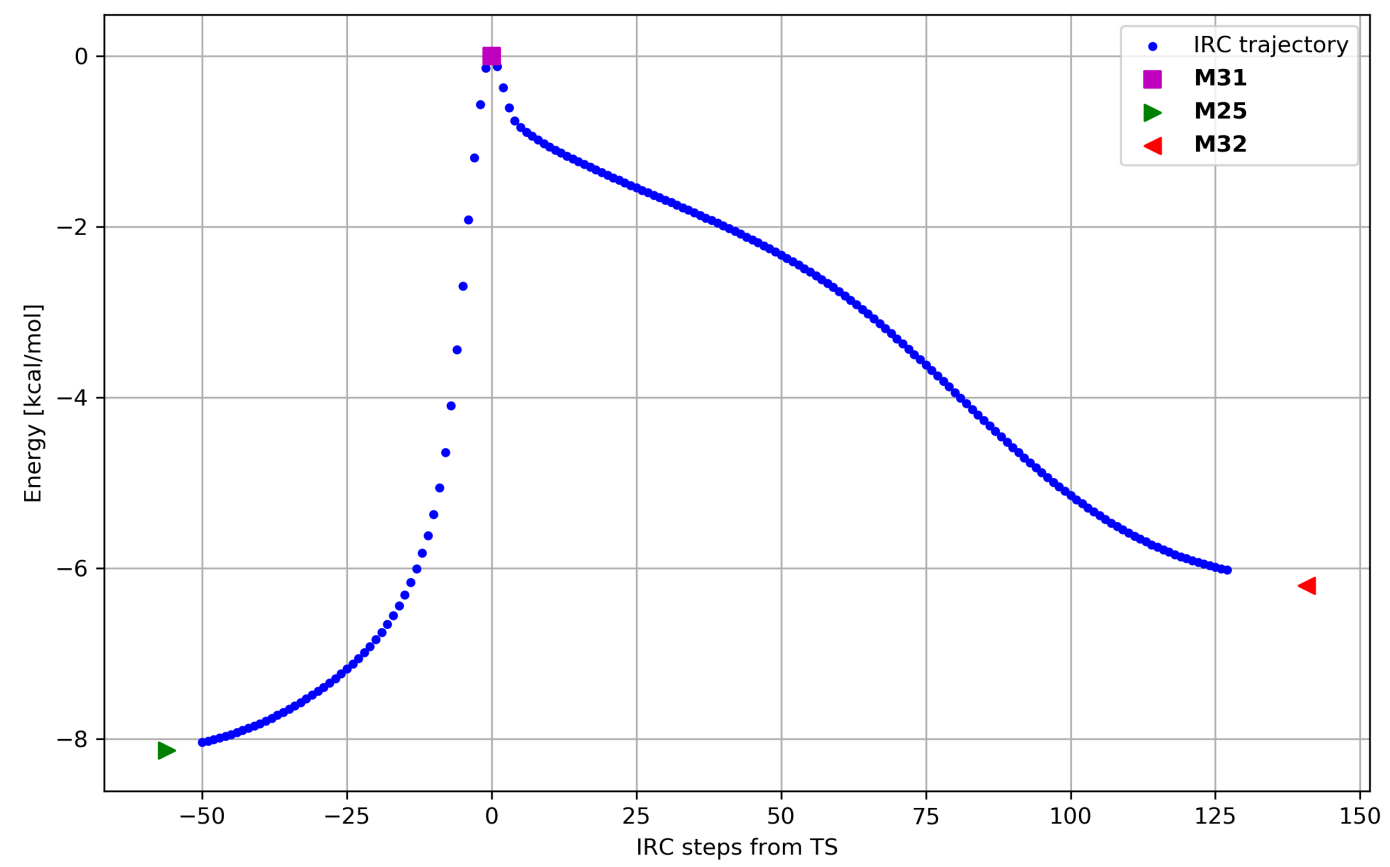

Figure S52: IRC trajectory for transition state M31. Predictor step size for LQA algorithm: 0.15 Bohr.

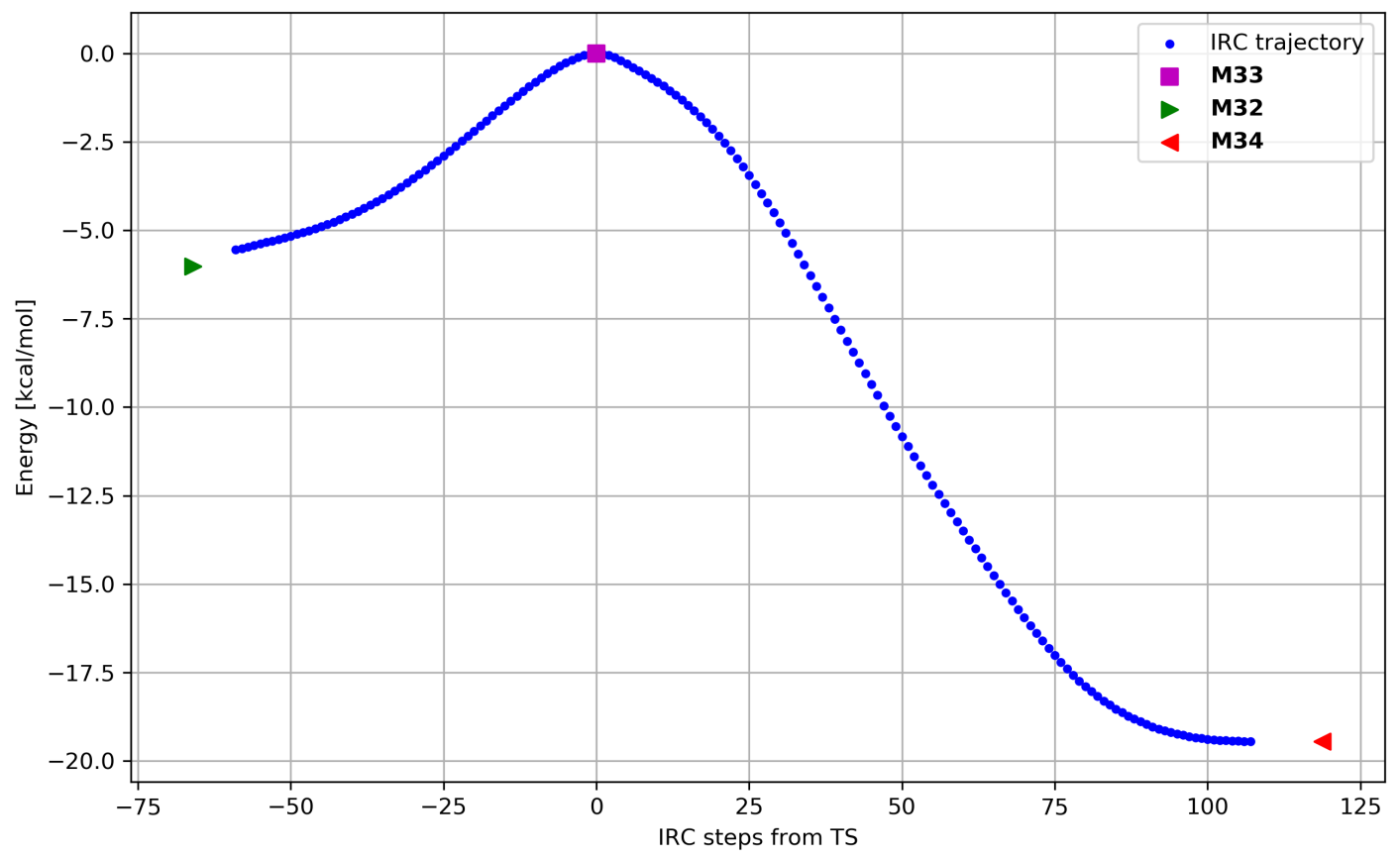

Figure S53: IRC trajectory for transition state M33. Predictor step size for LQA algorithm: 0.30 Bohr. 


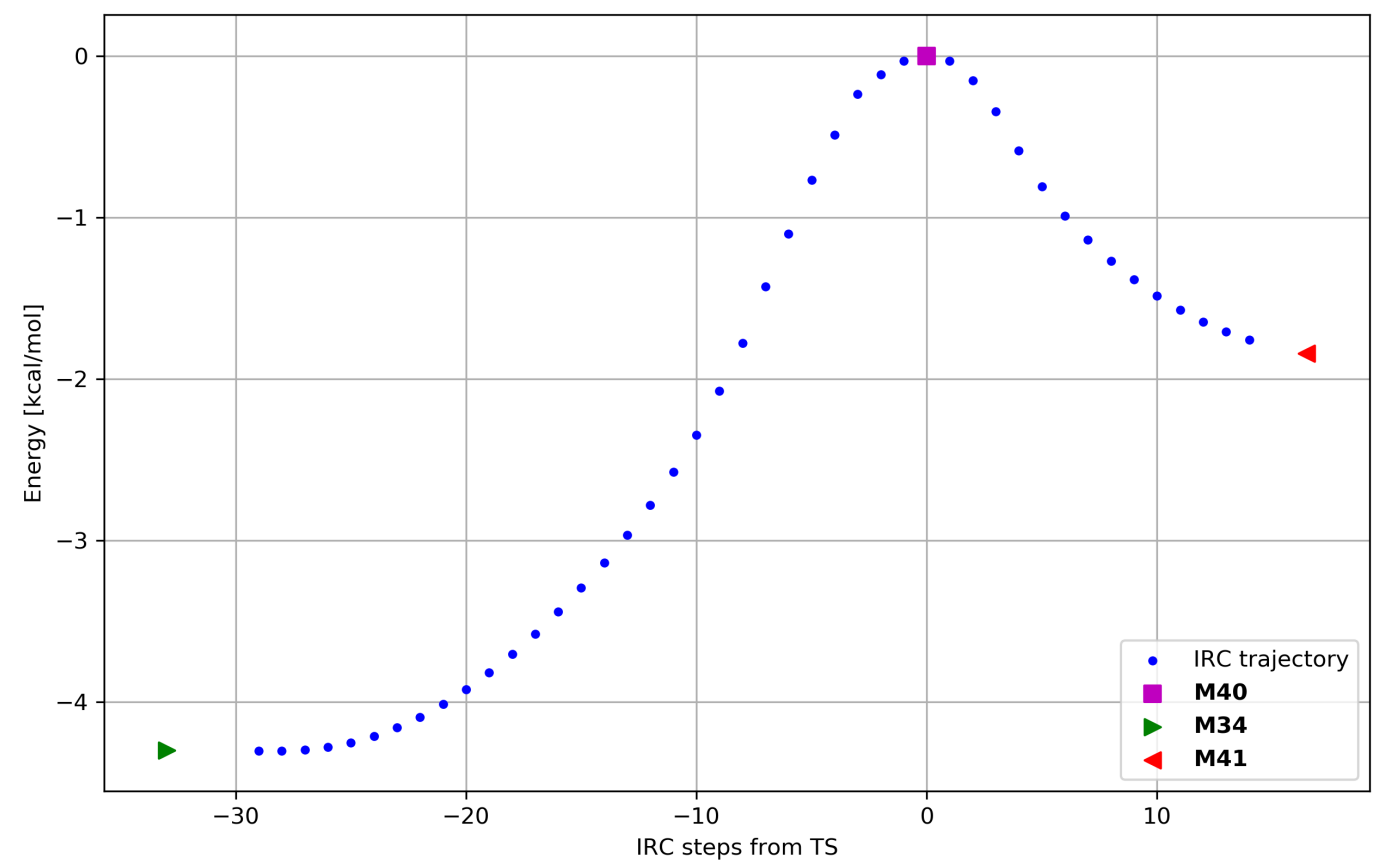

Figure S54: IRC trajectory for transition state M40. Predictor step size for LQA algorithm: 0.30 Bohr.

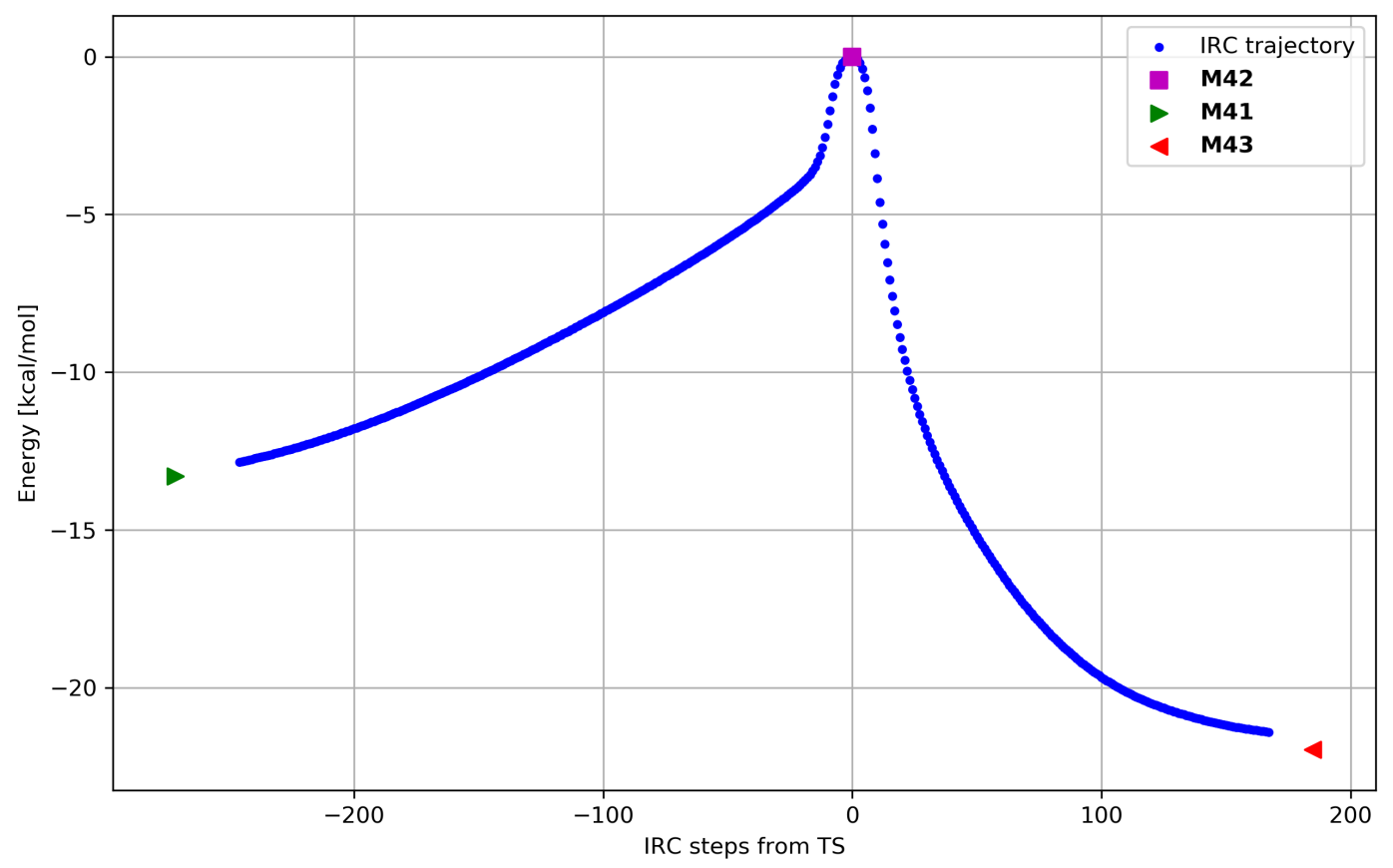

Figure S55: IRC trajectory for transition state M42. Predictor step size for LQA algorithm: 0.10 Bohr. 


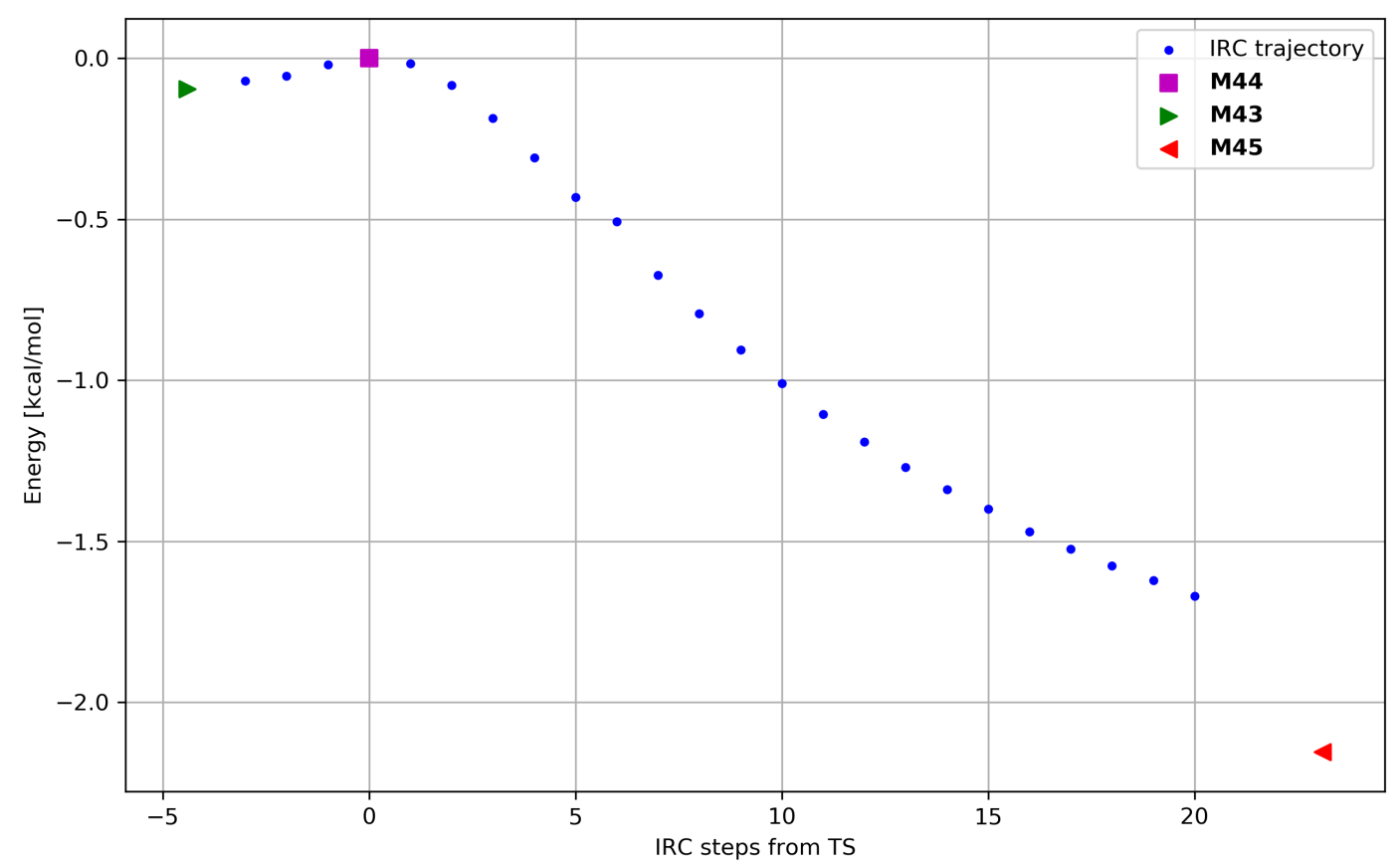

Figure S56: IRC trajectory for transition state M44. Predictor step size for LQA algorithm: 0.45 Bohr.

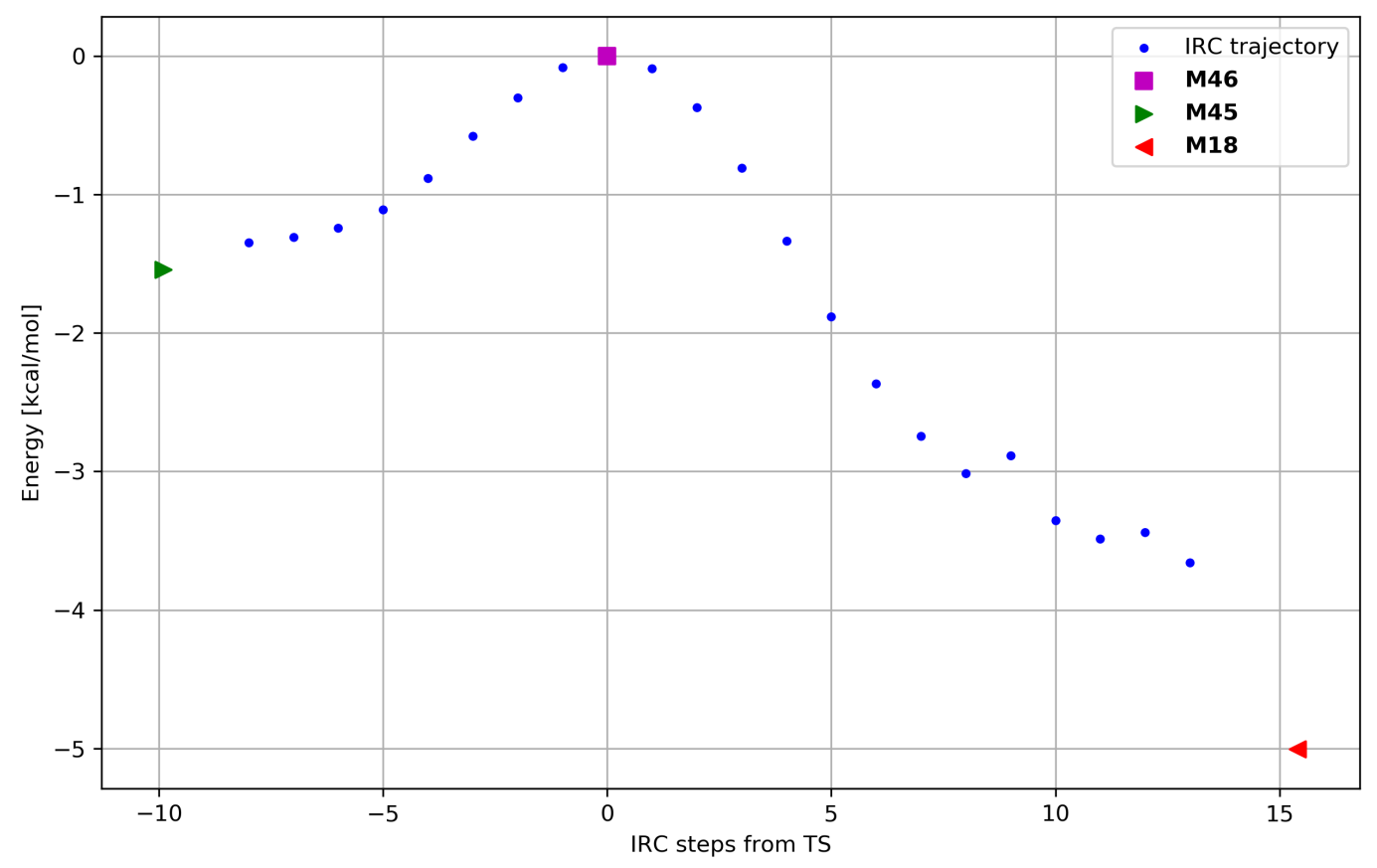

Figure S57: IRC trajectory for transition state M46. Predictor step size for LQA algorithm: 0.40 Bohr. 


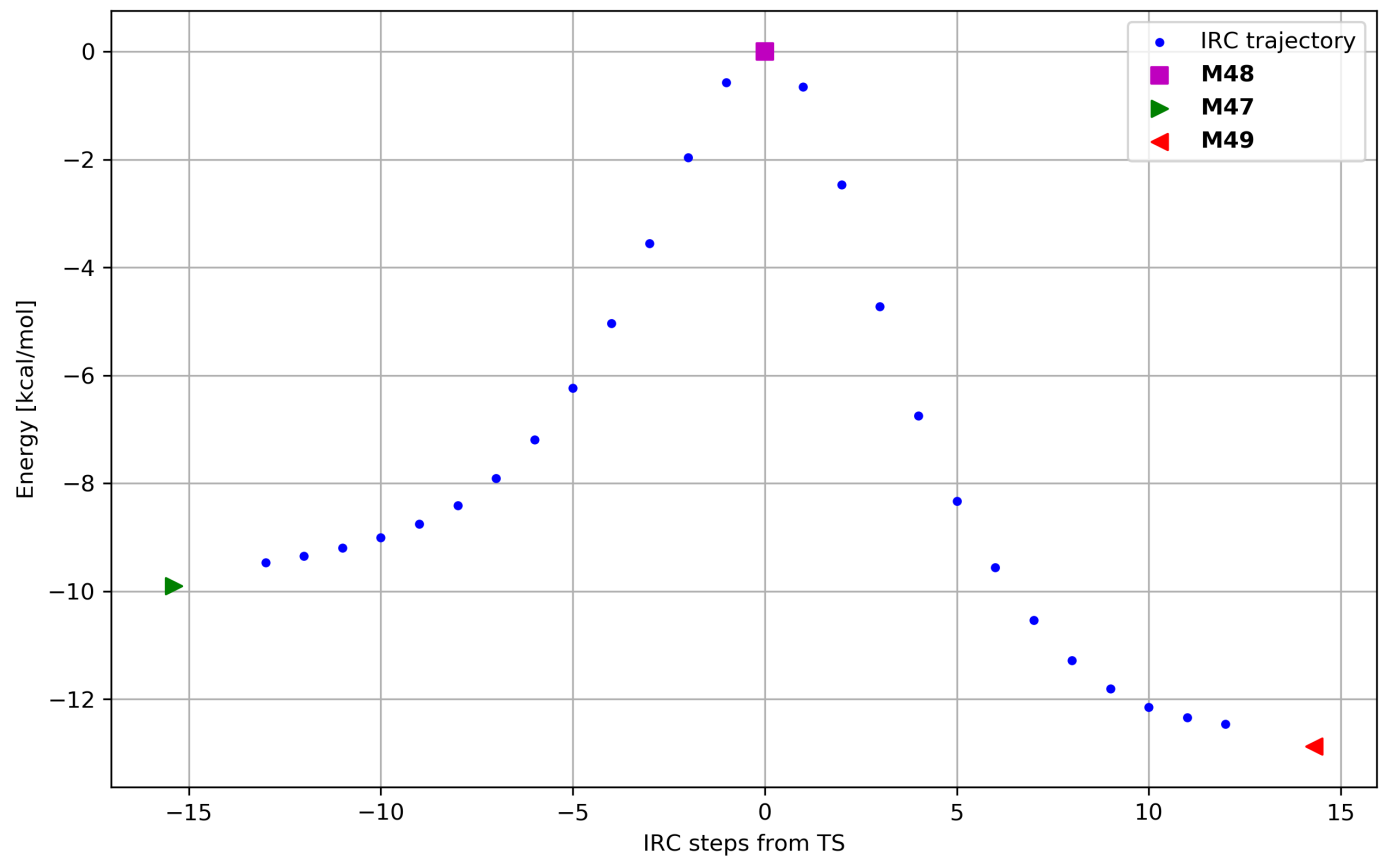

Figure S58: IRC trajectory for transition state M48. Hessian-based predictor-corrector algorithm, step size 0.30 Bohr.

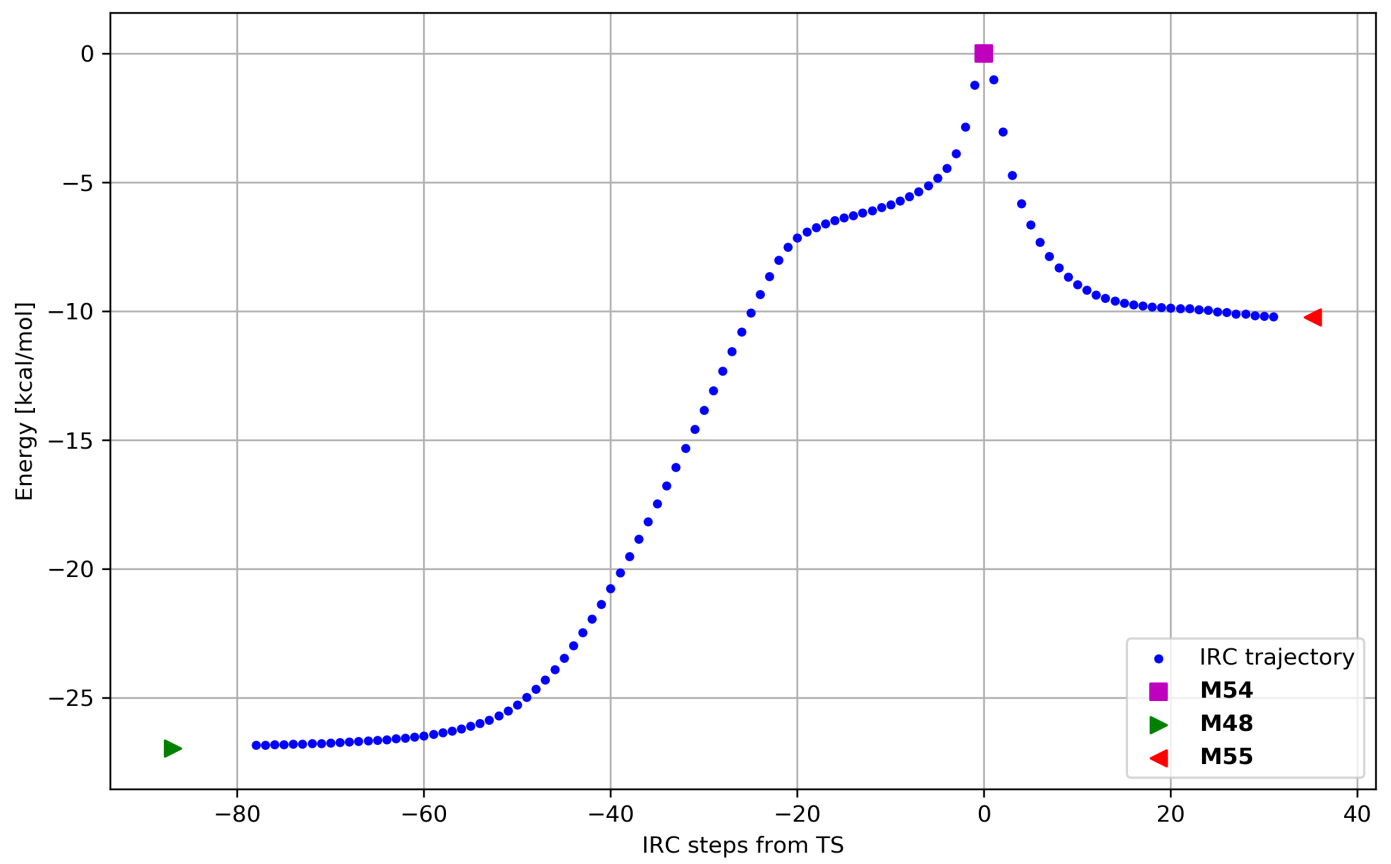

Figure S59: IRC trajectory for transition state M54. Predictor step size for LQA algorithm: 0.40 Bohr. 


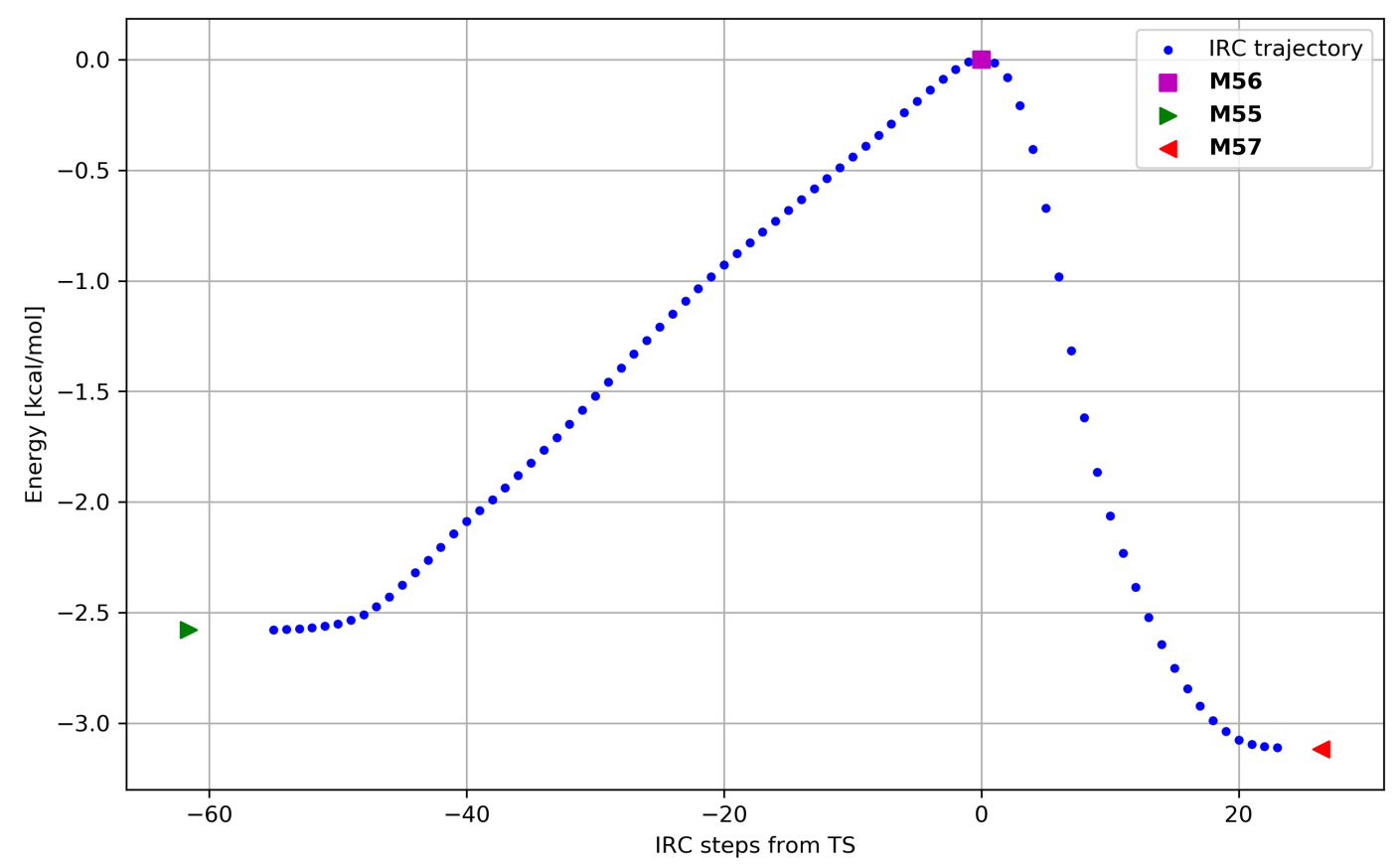

Figure S60: IRC trajectory for transition state M56. Predictor step size for LQA algorithm: 0.30 Bohr.

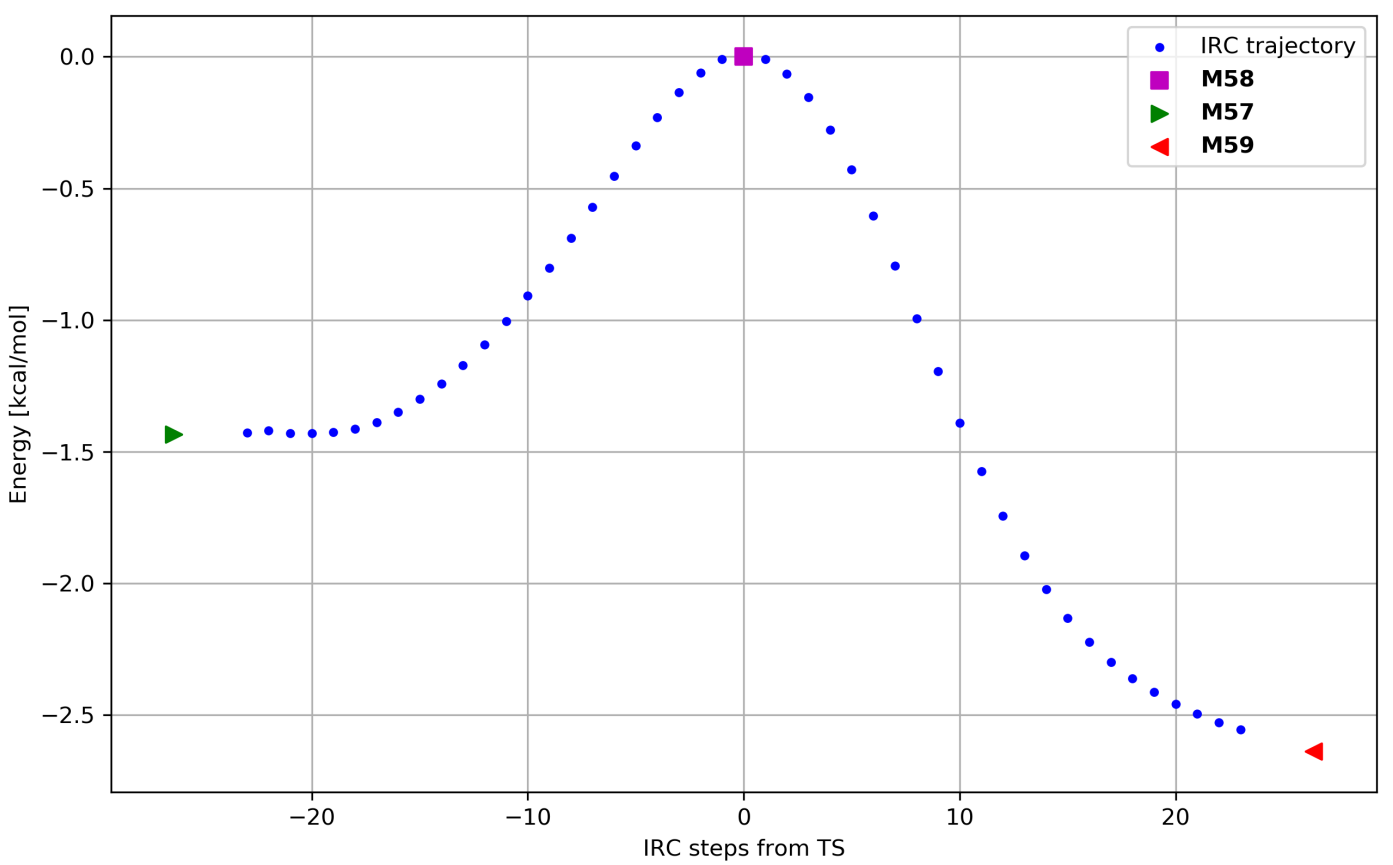

Figure S61: IRC trajectory for transition state M58. Predictor step size for LQA algorithm: 0.30 Bohr. 


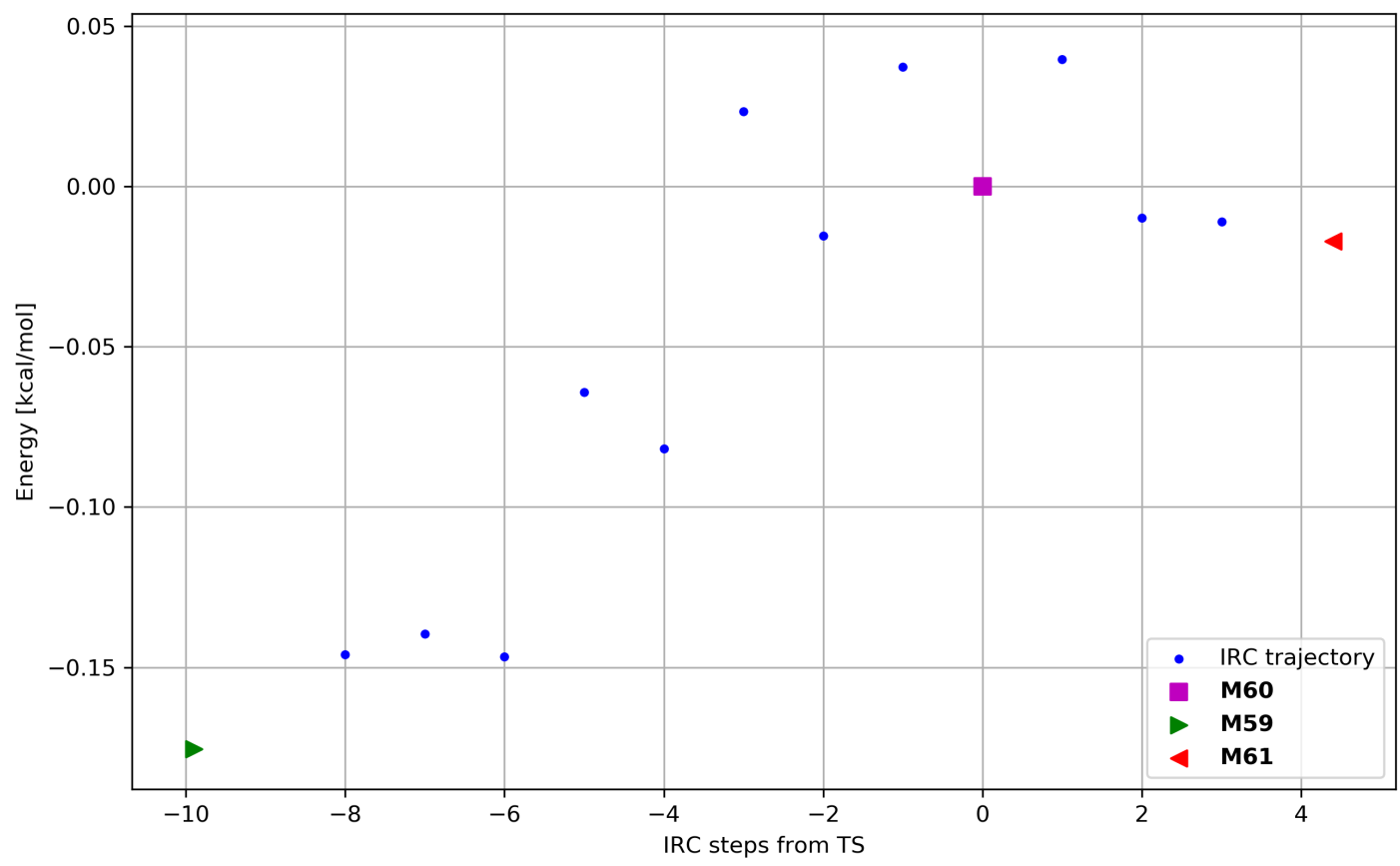

Figure S62: IRC trajectory for transition state M60. Predictor step size for LQA algorithm: 0.45 Bohr. The trajectory is polluted by partial torsion of one $o$-methyl group in a mesityl.

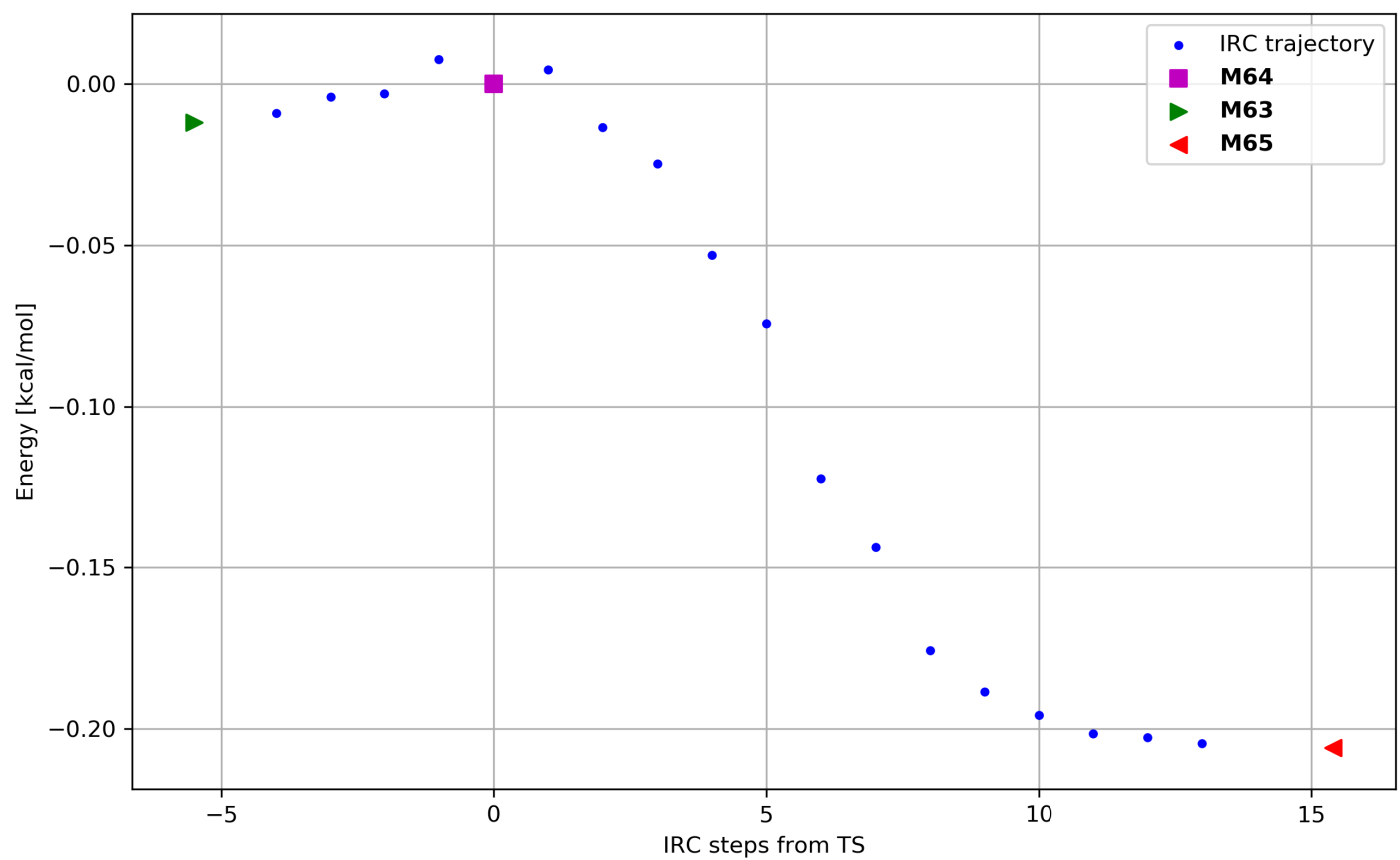

Figure S63: IRC trajectory for transition state M64. Predictor step size for LQA algorithm: 0.35 and 0.30 Bohr towards M63 and M65, respectively. 


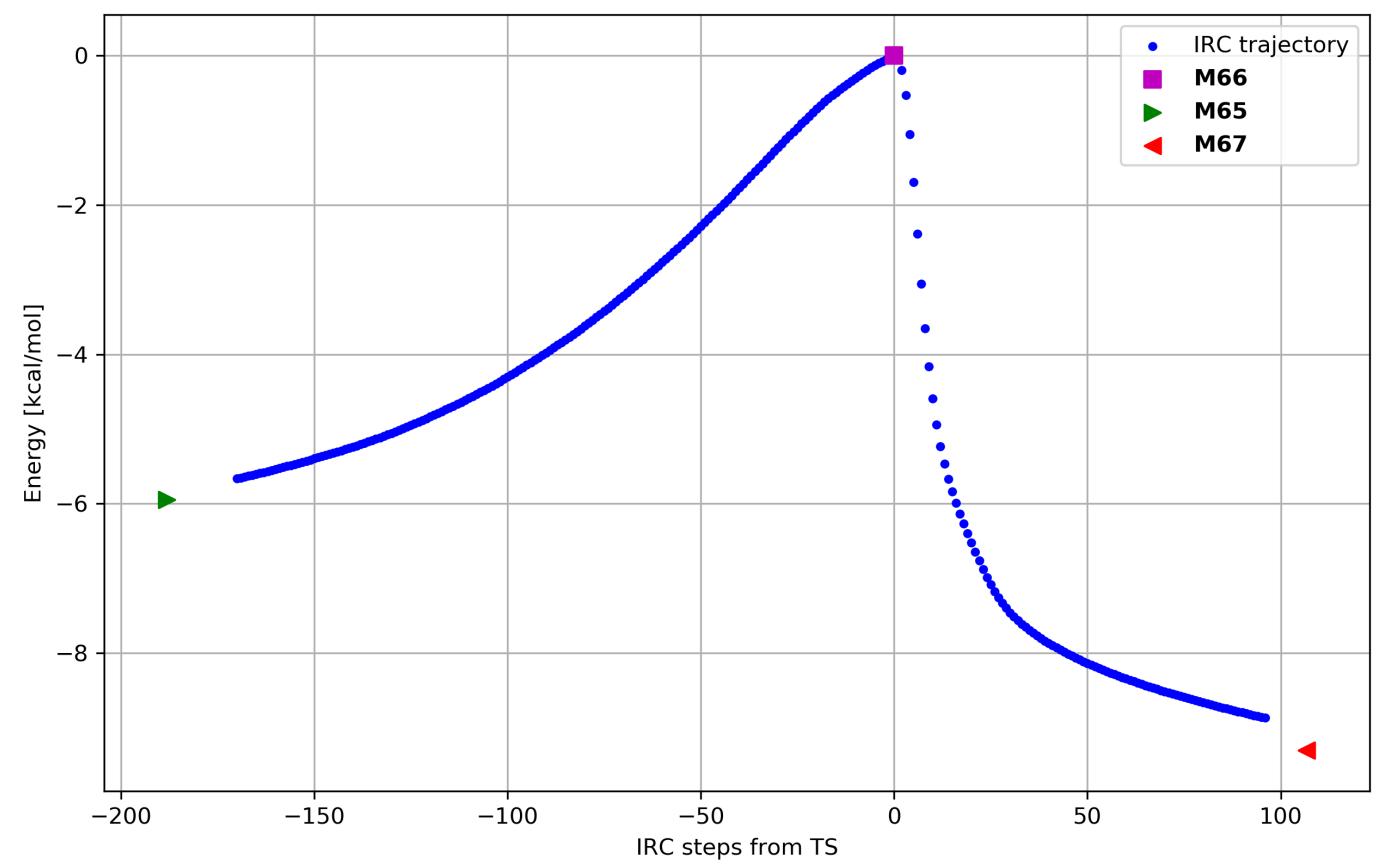

Figure S64: IRC trajectory for transition state M66. Predictor step size for LQA algorithm: 0.30 Bohr.

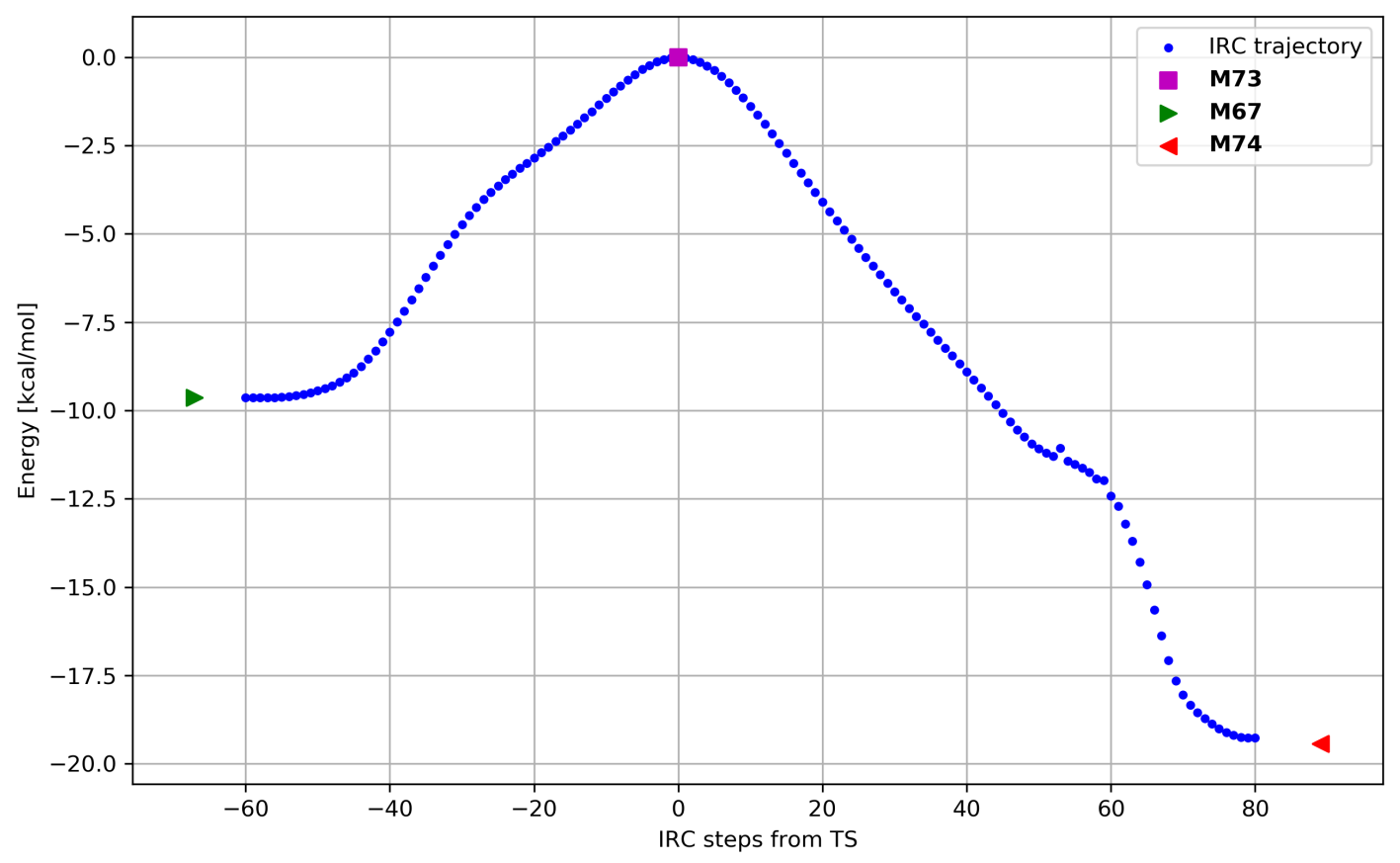

Figure S65: IRC trajectory for transition state M73. Predictor step size for LQA algorithm: 0.45 Bohr. 


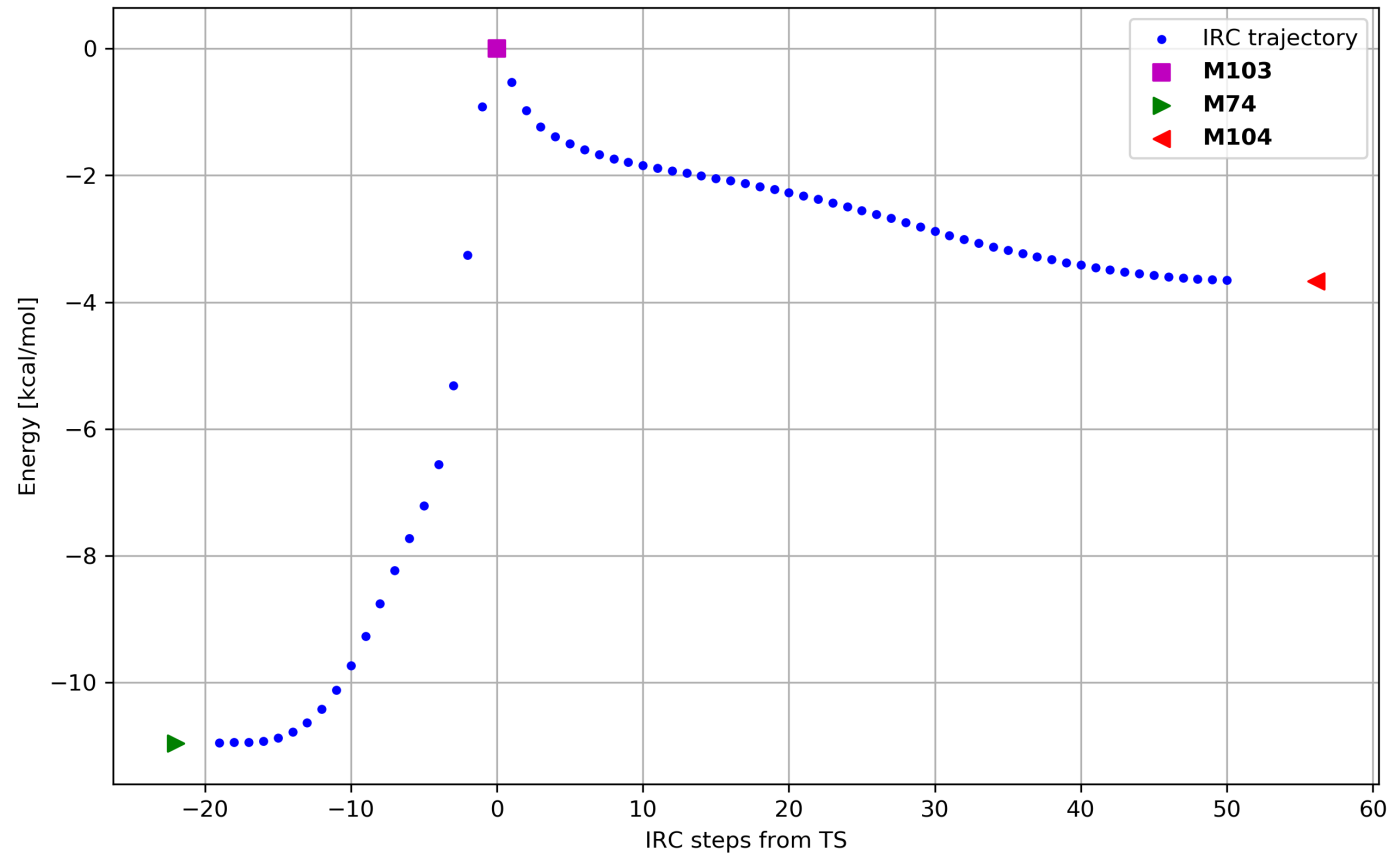

Figure S66: IRC trajectory for transition state M103. Predictor step size for LQA algorithm: 0.30 Bohr.

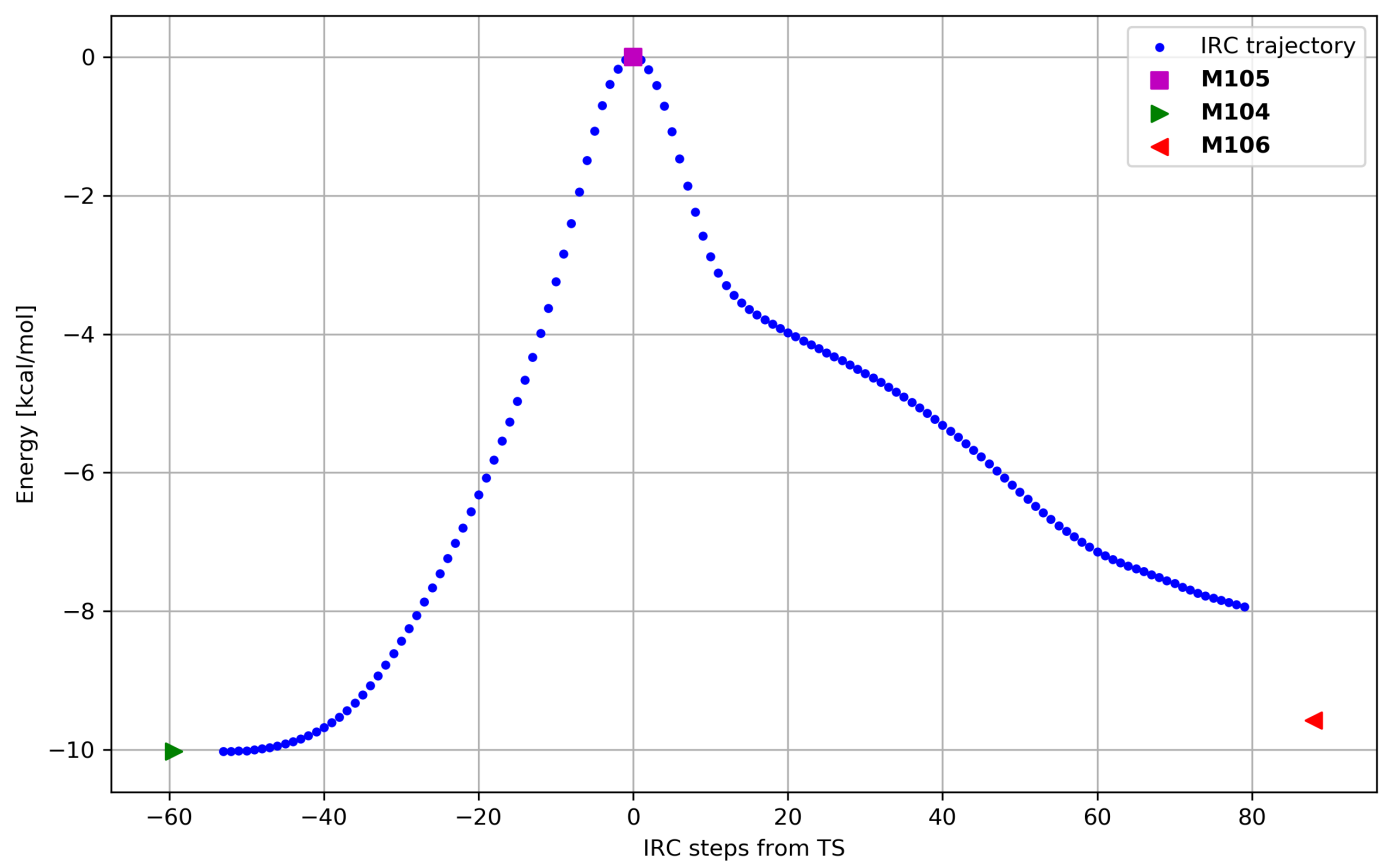

Figure S67: IRC trajectory for transition state M105. Predictor step size for LQA algorithm: 0.30 Bohr. Connection between end of trajectory and M106 has been verifies by short step-size energy minimization. 


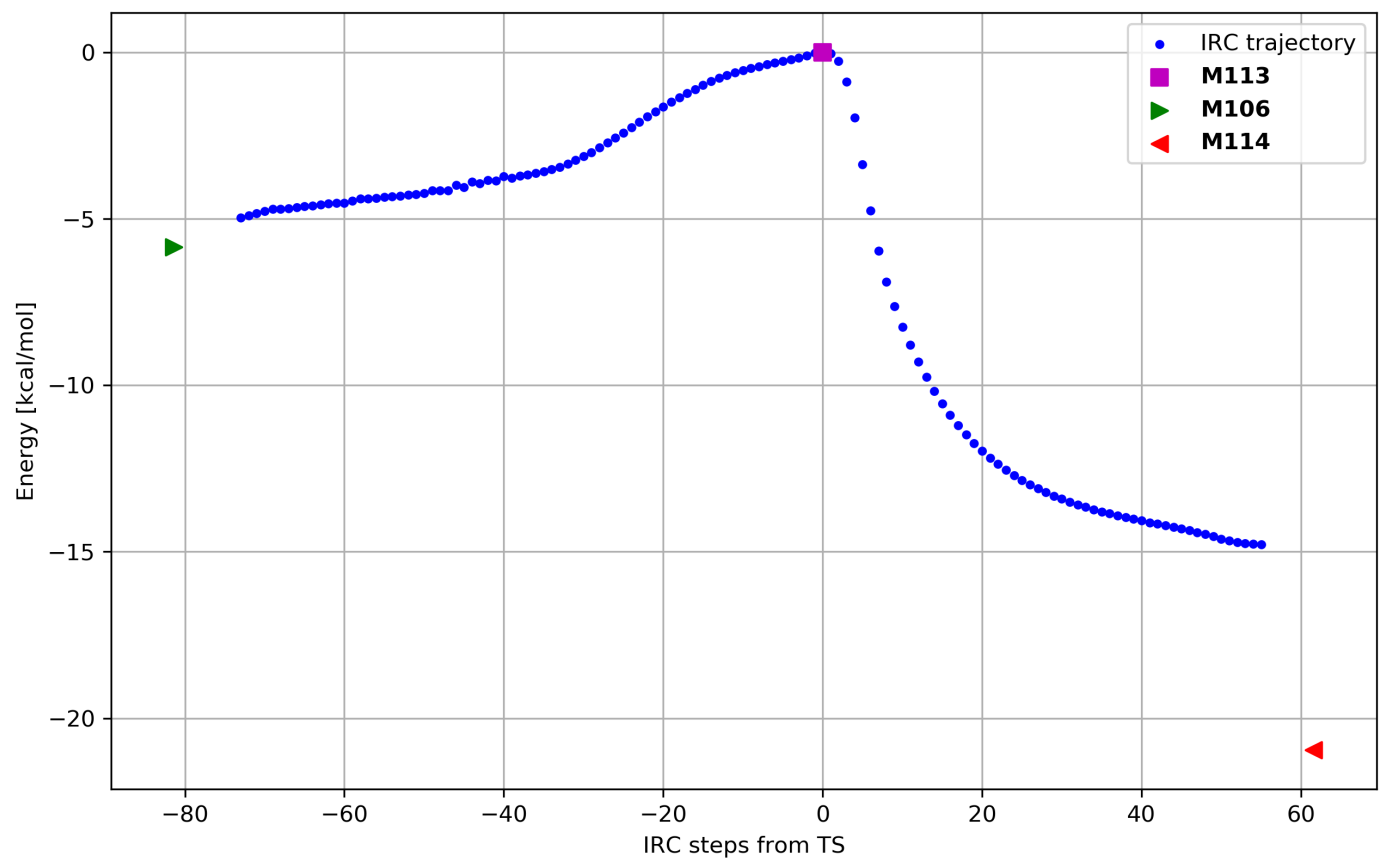

Figure S68: IRC trajectory for transition state M113. Predictor step size for LQA algorithm: 0.30 Bohr. Connection between end of trajectory and M114 has been verifies by short step-size energy minimization.

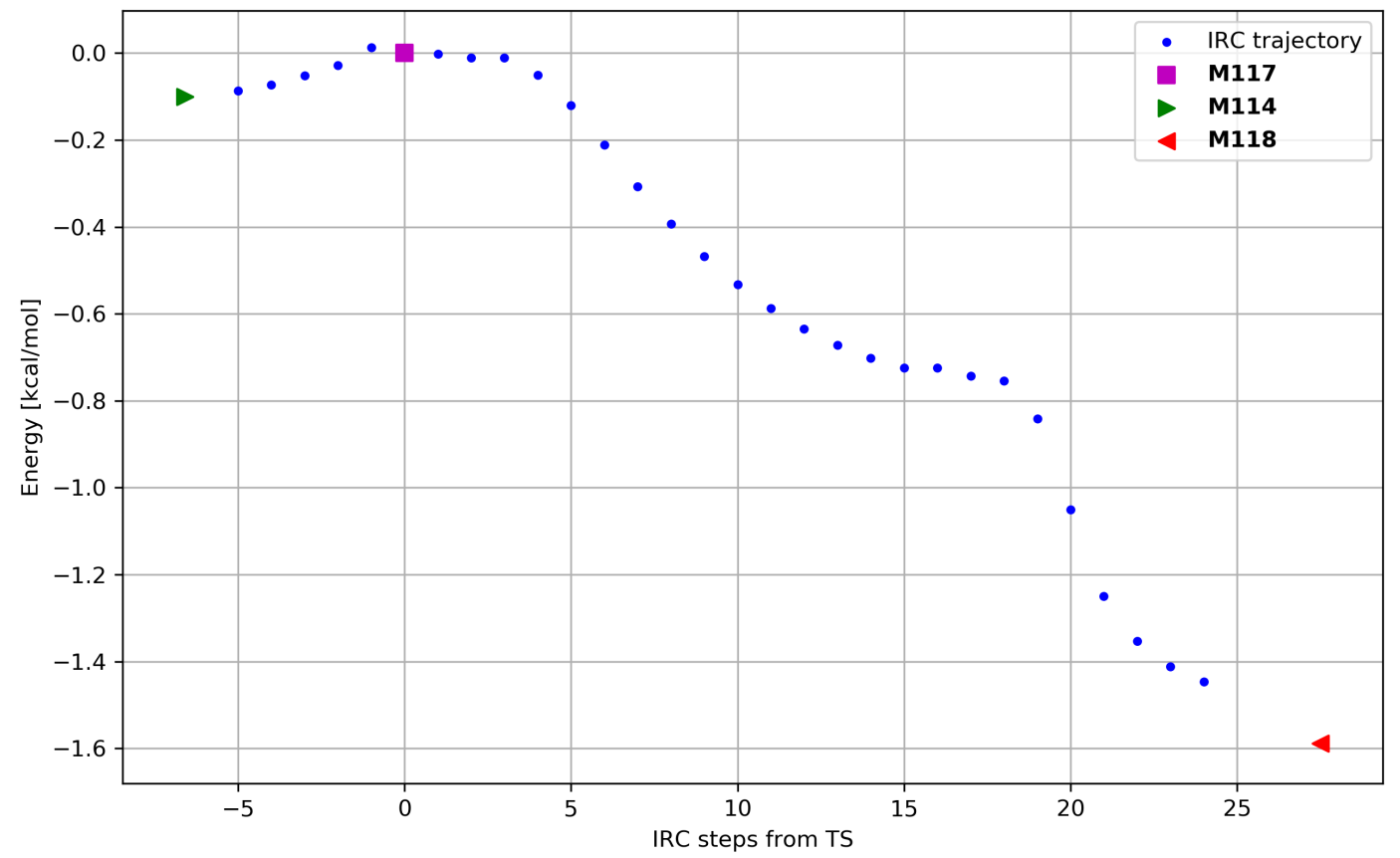

Figure S69: IRC trajectory for transition state M117. Predictor step size for LQA algorithm: 0.30 and 0.10 Bohr respectively towards M114 and M118. 


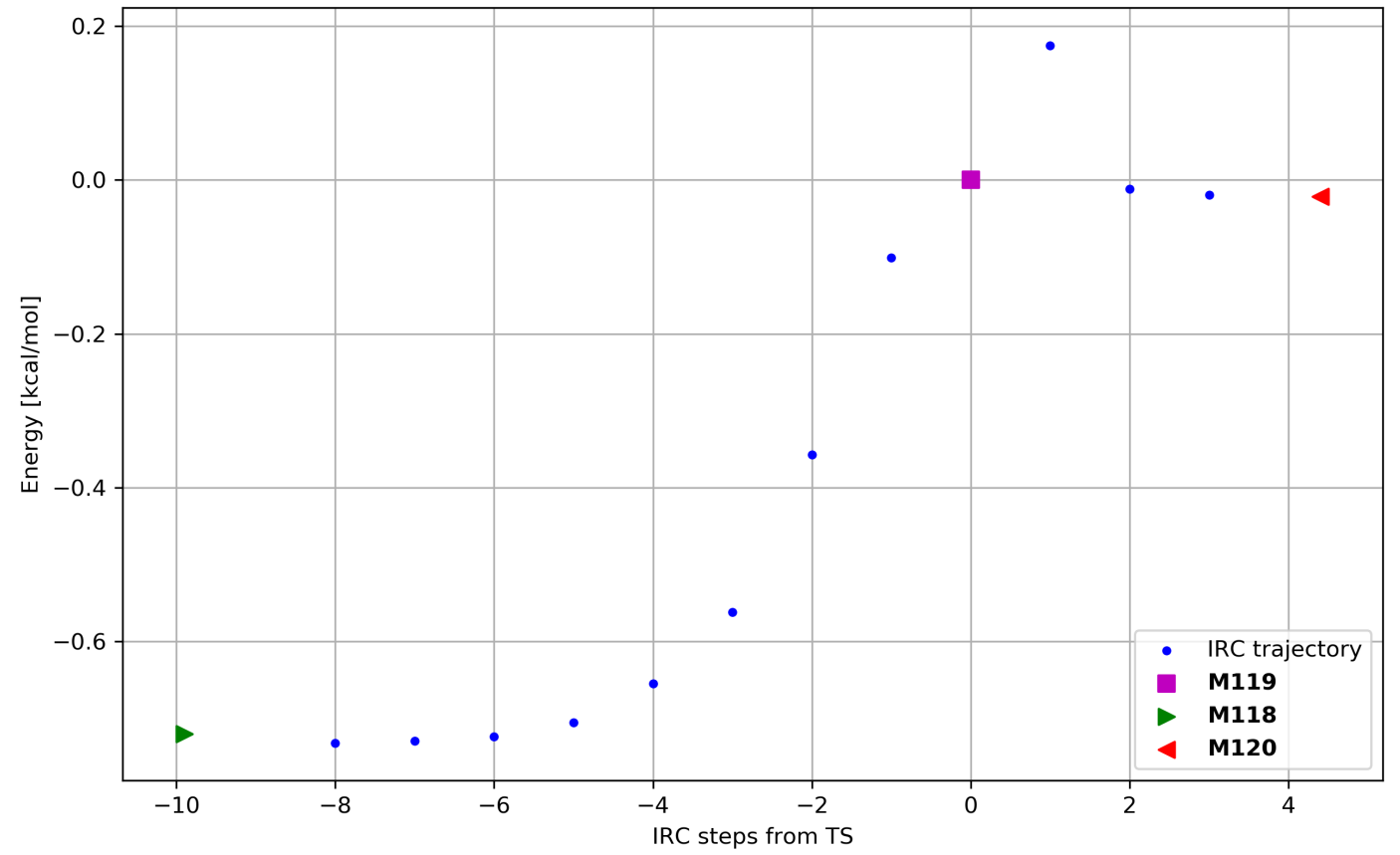

Figure S70: IRC trajectory for transition state M119. Predictor step size for LQA algorithm: 0.35 and 0.30 Bohr respectively towards M118 and M120.

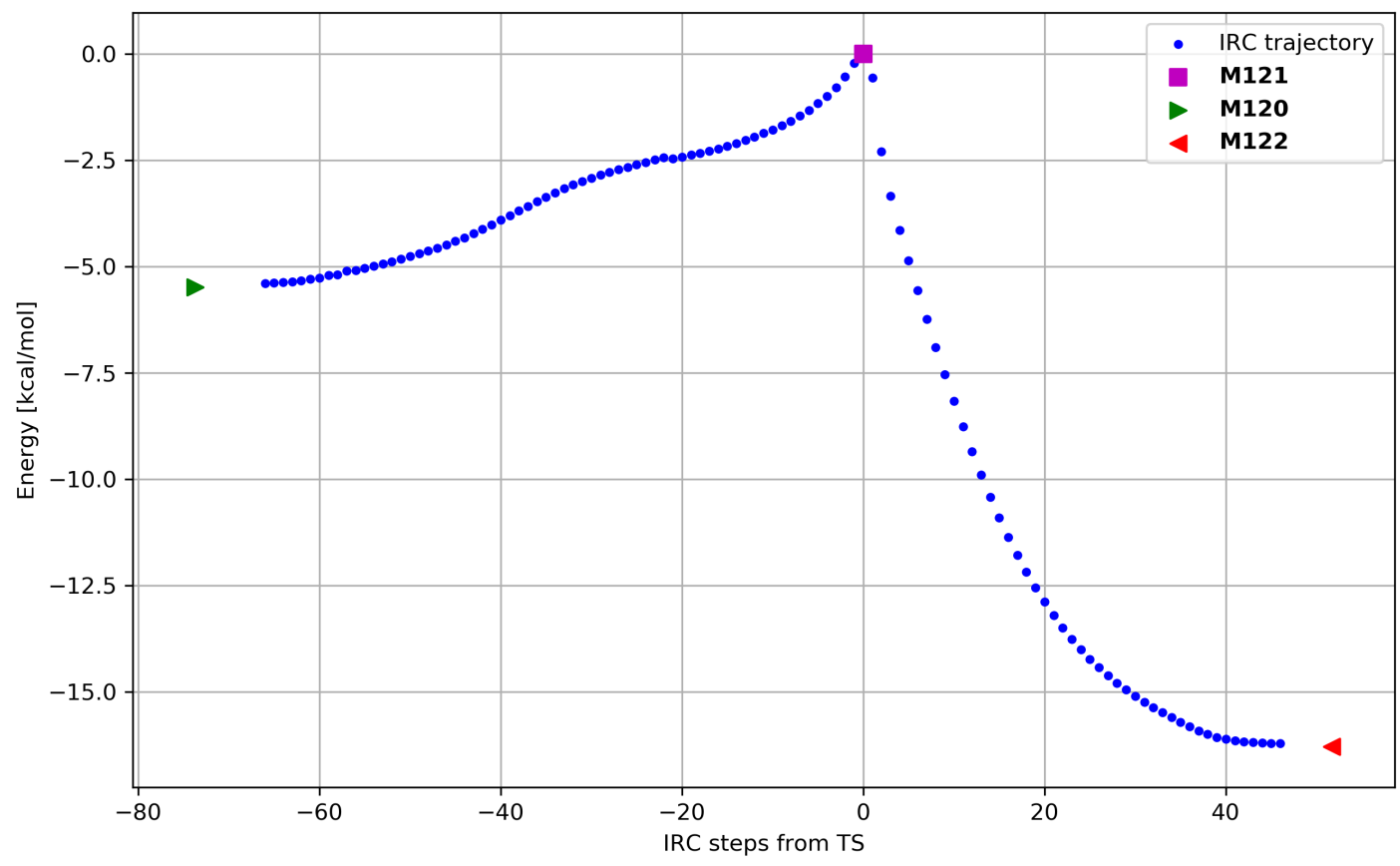

Figure S71: IRC trajectory for transition state M121. Predictor step size for LQA algorithm: 0.35 Bohr. 


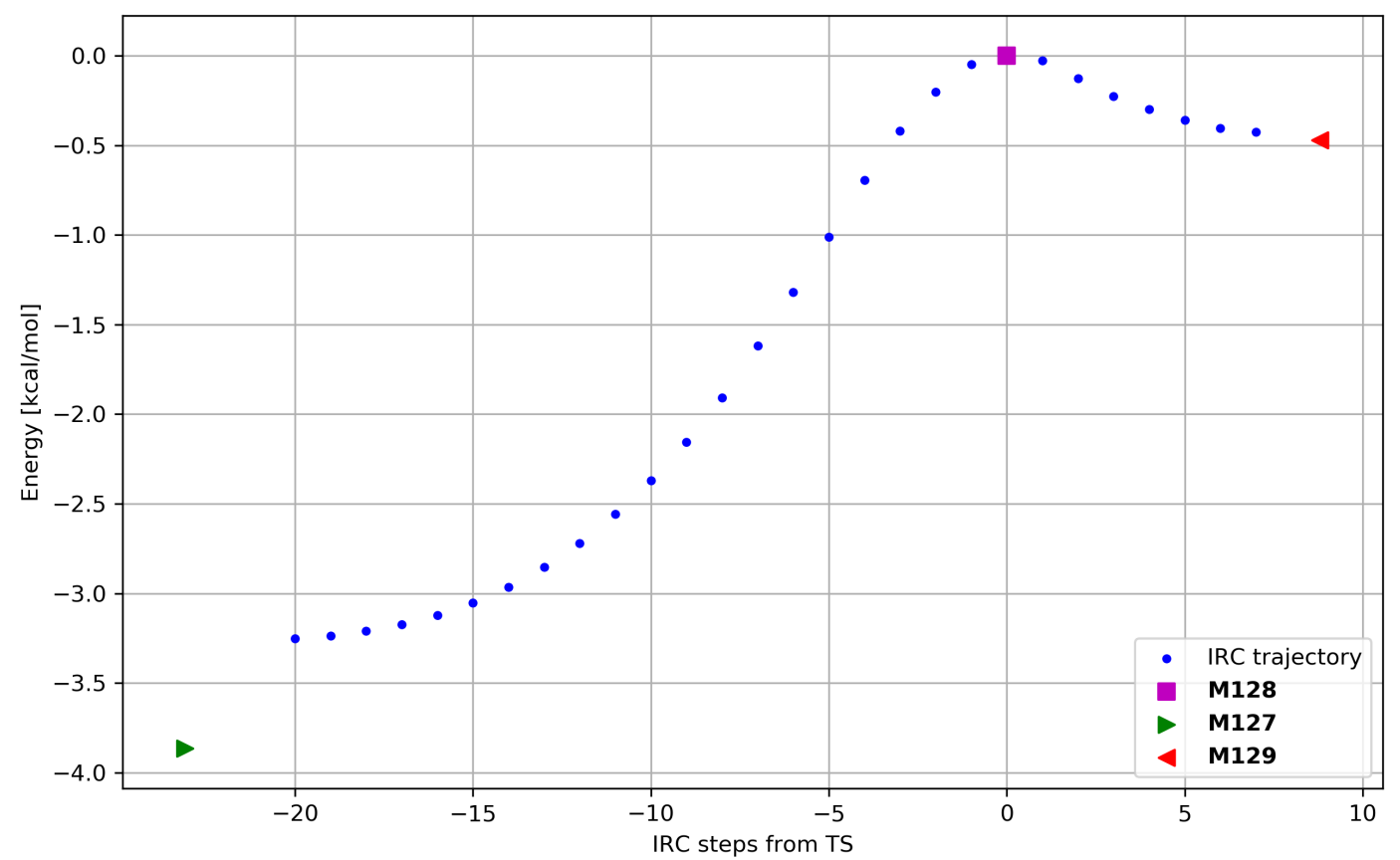

Figure S72: IRC trajectory for transition state M128. Predictor step size for LQA algorithm: 0.35 Bohr.

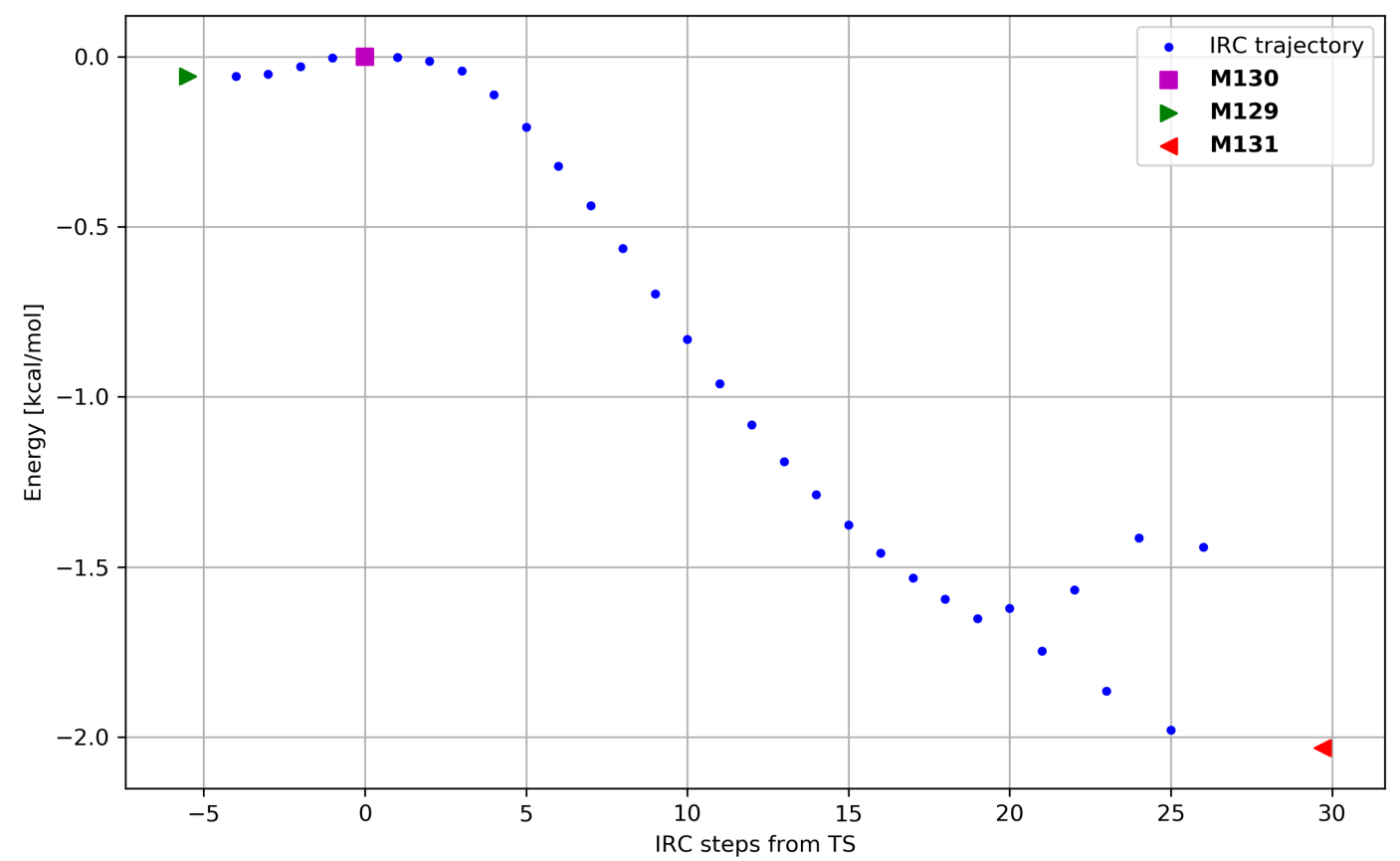

Figure S73: IRC trajectory for transition state M130. Predictor step size for LQA algorithm: 0.35 Bohr. The final part of the trajectory is polluted by partial torsion of one $o$-methyl group in a mesityl. 


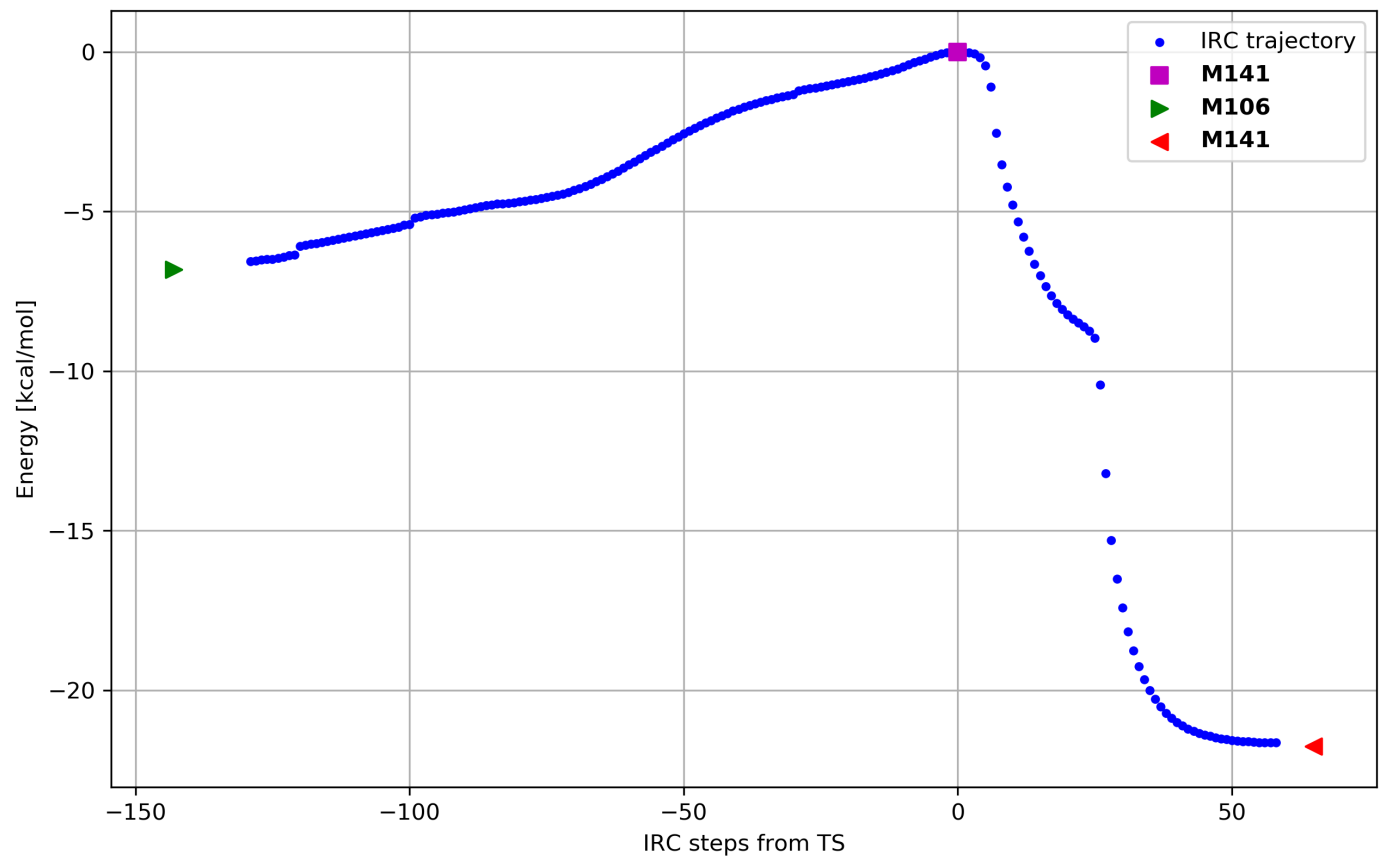

Figure S74: IRC trajectory for transition state M141. Predictor step size for LQA algorithm: 0.30 Bohr. The connection between IRC step -30 and M106 has been confirmed by small step-size energy minimization. The rest of the trajectory in the direction of M106 was obtained by restarting the IRC calculation three times in correspondence of the discontinuities visible in the figure. Each time performing between 1 and 3 steps with the geometry optimization protocol and restarting the IRC trajectory after updating the imaginary vector.

\title{
Sample Gaussian Input Files
}

\author{
Geometry Optimization \\ $\% \mathrm{CHK}=\mathrm{M} 18$ \\ \#P \\ \# HF/GENECP 5D 7F \\ \# NOSYMM \\ \# SCF $=($ VSHIFT $=1000$, NOSYM, NOVARACC, MAXCYCLE $=180$, CONVER $=4)$ \\ \# SP
}

Step 1 - protocol 1

01

C $+2.11692968 \mathrm{E}+00-3.03932430 \mathrm{E}+00-3.57977049 \mathrm{E}-01$

Ru -9.27226213E-02 +6.21734648E-01 -8.86568419E-02

C $+1.00502803 \mathrm{E}+00-2.65282801 \mathrm{E}+00-1.11381219 \mathrm{E}+00$

C $+1.04136866 \mathrm{E}+00-2.60215614 \mathrm{E}+00-2.51657648 \mathrm{E}+00$

C $+2.23985493 \mathrm{E}+00-2.91874940 \mathrm{E}+00-3.15301915 \mathrm{E}+00$

C $+3.38031223 E+00-3.28618454 E+00-2.43064040 E+00$

C $+3.29889253 \mathrm{E}+00-3.34388544 \mathrm{E}+00-1.03955366 \mathrm{E}+00$

N $-2.34163933 E-01-2.39116030 E+00-4.60673320 E-01$

C $-7.70427757 \mathrm{E}-01-1.19768564 \mathrm{E}+00-1.10764886 \mathrm{E}-01$

N $-2.01880346 E+00-1.45074079 E+00+3.67292124 \mathrm{E}-01$

C $-2.41476791 \mathrm{E}+00-2.84992129 \mathrm{E}+00+2.47102920 \mathrm{E}-01$

C $-1.09805640 \mathrm{E}+00-3.52671483 \mathrm{E}+00-1.26568096 \mathrm{E}-01$

C $-2.99092095 \mathrm{E}+00-4.54234771 \mathrm{E}-01+7.02260953 \mathrm{E}-01$

C $-3.80329568 \mathrm{E}+00+7.84026427 \mathrm{E}-02-3.16693105 \mathrm{E}-01$

C $-4.71829677 \mathrm{E}+00+1.07166602 \mathrm{E}+00+2.52779319 \mathrm{E}-02$

C $-4.86711246 \mathrm{E}+00+1.51520706 \mathrm{E}+00+1.34154828 \mathrm{E}+00$

C $-4.11081539 \mathrm{E}+00+9.00233245 \mathrm{E}-01+2.33827075 \mathrm{E}+00$

C $-3.18109926 \mathrm{E}+00-1.00859853 \mathrm{E}-01+2.04796747 \mathrm{E}+00$

C $-3.76898432 \mathrm{E}+00-4.50936510 \mathrm{E}-01-1.72632230 \mathrm{E}+00$ 
C $-2.48952403 \mathrm{E}+00-8.19028398 \mathrm{E}-01+3.17516326 \mathrm{E}+00$

C $-5.81928118 \mathrm{E}+00+2.63499946 \mathrm{E}+00+1.67000478 \mathrm{E}+00$

$\mathrm{Cl}-1.18507448 \mathrm{E}+00+1.52463459 \mathrm{E}+00-1.95539735 \mathrm{E}+00$

C $-1.75530378 \mathrm{E}-01-2.17946419 \mathrm{E}+00-3.29704179 \mathrm{E}+00$

C $+4.66789688 \mathrm{E}+00-3.59434840 \mathrm{E}+00-3.15000213 \mathrm{E}+00$

$+2.04756551 \mathrm{E}+00-3.11140539 \mathrm{E}+00+1.14596084 \mathrm{E}+00$

$+2.43845559 \mathrm{E}+00+1.17294025 \mathrm{E}+00+4.50002976 \mathrm{E}-01$

$+1.99709574 \mathrm{E}+00+3.48371787 \mathrm{E}-01-1.19672620 \mathrm{E}-01$

$\mathrm{C}+1.53767041 \mathrm{E}+00+5.68971465 \mathrm{E}-01-1.43146799 \mathrm{E}+00$

$\mathrm{Cl}+2.84910836 \mathrm{E}-01+6.37536277 \mathrm{E}-01+2.22496558 \mathrm{E}+00$

$\mathrm{H}+1.62431692 \mathrm{E}+00+1.56457516 \mathrm{E}+00-1.87805235 \mathrm{E}+00$

$-2.83024450 \mathrm{E}+00-3.21483793 \mathrm{E}+00+1.19685160 \mathrm{E}+00$

$-3.18479216 \mathrm{E}+00-2.96196998 \mathrm{E}+00-5.34141958 \mathrm{E}-01$

$-6.66481311 E-01-4.09466750 E+00+7.13164844 E-01$

$-1.18815758 \mathrm{E}+00-4.20378000 \mathrm{E}+00-9.87751977 \mathrm{E}-01$

$+4.18177713 E+00-3.62970335 E+00-4.62043666 E-01$

$+2.28816908 \mathrm{E}+00-2.87300400 \mathrm{E}+00-4.24421954 \mathrm{E}+00$

$-5.33450687 \mathrm{E}+00+1.51166796 \mathrm{E}+00-7.62933560 \mathrm{E}-01$

$-4.24826684 \mathrm{E}+00+1.20047932 \mathrm{E}+00+3.38032064 \mathrm{E}+00$

$-2.12269975 \mathrm{E}+00-1.06434816 \mathrm{E}-01+3.92599589 \mathrm{E}+00$

$-1.62812746 \mathrm{E}+00-1.40021120 \mathrm{E}+00+2.82897562 \mathrm{E}+00$

$-3.20418669 E+00-1.49752571 E+00+3.67076426 E+00$

$-2.79411701 E+00-8.75796419 E-01-1.99218755 E+00$

$-3.98236040 E+00+3.48558444 E-01-2.44725217 E+00$

$-4.53883167 E+00-1.23236219 E+00-1.84824911 E+00$

$+1.57774654 \mathrm{E}+00-4.05301362 \mathrm{E}+00+1.47489773 \mathrm{E}+00$

$+1.46106898 \mathrm{E}+00-2.28092137 \mathrm{E}+00+1.56753727 \mathrm{E}+00$

$+3.05394965 E+00-3.07913047 E+00+1.58496529 E+00$

$-1.05188271 \mathrm{E}+00-2.79600630 \mathrm{E}+00-3.04161812 \mathrm{E}+00$

$-3.53142200 \mathrm{E}-04-2.27023797 \mathrm{E}+00-4.37709614 \mathrm{E}+00$

$-4.45116314 E-01-1.13265030 E+00-3.07834979 E+00$

$-6.13495499 E+00+2.60131836 E+00+2.72231274 E+00$

$-5.33662105 E+00+3.61062929 E+00+1.49813133 E+00$

$-6.71811937 E+00+2.59690397 E+00+1.03750448 E+00$

$+4.50220501 \mathrm{E}+00-4.30481257 \mathrm{E}+00-3.97383130 \mathrm{E}+00$

$+5.41661473 E+00-4.02569925 E+00-2.47149139 E+00$

$+5.09764879 E+00-2.67957395 E+00-3.58798749 E+00$

$+2.32274390 \mathrm{E}+00-6.39396867 \mathrm{E}-01+2.02100323 \mathrm{E}-01$

H +1.50077503E+00 $-2.44765990 E-01-2.15419440 E+00$
Ru $\quad 0$
S 71.000
$1.593720000 \mathrm{E}+02$
2.09016000E+01
1. $30741000 \mathrm{E}+01$
$4.7147400 \mathrm{E}+00$
$1.1361200 \mathrm{E}+00$
$5.154640 \mathrm{E}-01$
$1.067670 \mathrm{E}-01$
S 71.000
$1.593720000 \mathrm{E}+02$
$2.09016000 \mathrm{E}+01$
1. 30741000E+01
$4.7147400 \mathrm{E}+00$
$1.1361200 \mathrm{E}+00$
$5.154640 \mathrm{E}-01$
1.067670E-01
71.000
$1.593720000 \mathrm{E}+02$
$2.09016000 \mathrm{E}+01$
1. $30741000 \mathrm{E}+01$
4. $7147400 \mathrm{E}+00$
$1.1361200 \mathrm{E}+00$
5.154640E-01
1.067670E-01
S 11.000
$3.92180 \mathrm{E}-02$
P 1.000
1. $28738000 \mathrm{E}+01$
$6.730 \mathrm{E}-04$
$-3.71610 E-02$
$1.665170 \mathrm{E}-01$
$-6.159540 E-01$
8. 428300E-01
$4.477630 \mathrm{E}-01$
$1.84450 \mathrm{E}-02$
$-1.690 E-04$
$9.3920 \mathrm{E}-03$
$-4.77130 \mathrm{E}-02$
2. $011130 \mathrm{E}-01$
$-3.734210 \mathrm{E}-01$
$-2.738590 \mathrm{E}-01$
$6.093840 \mathrm{E}-01$
8.950E-04
$-3.96350 \mathrm{E}-02$
1. 442430E-01
$-5.192870 \mathrm{E}-01$
$1.5854040 \mathrm{E}+00$
$-9.082030 \mathrm{E}-01$
$-1.4720020 E+00$
$1.0000000 \mathrm{E}+00$
$2.99680 \mathrm{E}-02$ 


\begin{tabular}{|c|c|}
\hline & $-1.879300 \mathrm{E}-01$ \\
\hline $1.5652400 \mathrm{E}+00$ & 4. $999900 \mathrm{E}-01$ \\
\hline 7. $361940 \mathrm{E}-01$ & $4.957320 E-01$ \\
\hline $3.263170 \mathrm{E}-01$ & $1.475150 \mathrm{E}-01$ \\
\hline $1.124070 \mathrm{E}-01$ & $6.0880 E-03$ \\
\hline \multirow{2}{*}{$\begin{array}{lcc}\mathrm{P} & 6 & 1.000 \\
1.28738000 \mathrm{E}+01\end{array}$} & \\
\hline & $-7.8010 E-03$ \\
\hline $6.1475000 \mathrm{E}+00$ & $5.36560 \mathrm{E}-02$ \\
\hline $1.5652400 \mathrm{E}+00$ & $-1.719400 \mathrm{E}-01$ \\
\hline 7.361940E-01 & $-1.991700 \mathrm{E}-01$ \\
\hline $3.263170 \mathrm{E}-01$ & $7.44110 \mathrm{E}-02$ \\
\hline $1.124070 \mathrm{E}-01$ & $5.971370 \mathrm{E}-01$ \\
\hline $\begin{array}{cc}6 & 1.000 \\
1 & \end{array}$ & \\
\hline \multirow{2}{*}{$\begin{array}{l}1.28738000 \mathrm{E}+01 \\
6.1475000 \mathrm{E}+00\end{array}$} & $-1.35020 \mathrm{E}-02$ \\
\hline & $9.37330 \mathrm{E}-02$ \\
\hline 1. $5652400 \mathrm{E}+00$ & $-3.159100 \mathrm{E}-01$ \\
\hline $7.361940 \mathrm{E}-01$ & $3000 E-01$ \\
\hline $3.263170 \mathrm{E}-01$ & $4.008770 \mathrm{E}-01$ \\
\hline $1.124070 \mathrm{E}-01$ & $7.537660 \mathrm{E}-01$ \\
\hline \multirow{2}{*}{$\begin{array}{l}P \\
3.91720 \mathrm{E}-02\end{array}$} & \\
\hline & $1.0000000 \mathrm{E}+00$ \\
\hline D 51.000 & \\
\hline $6.2126500 \mathrm{E}+00$ & $-2.45970 E-02$ \\
\hline $2.7853900 E+00$ & $1.273340 \mathrm{E}-01$ \\
\hline $1.2685100 \mathrm{E}+00$ & $3.570810 \mathrm{E}-01$ \\
\hline $5.517900 \mathrm{E}-01$ & $4.082070 \mathrm{E}-01$ \\
\hline $2.247310 \mathrm{E}-01$ & $2.812400 \mathrm{E}-01$ \\
\hline $\begin{array}{lll}D & 5 & 1.000\end{array}$ & \\
\hline $6.2126500 \mathrm{E}+00$ & $2.61160 \mathrm{E}-02$ \\
\hline $2.7853900 \mathrm{E}+00$ & $-1.459400 E-01$ \\
\hline $1.2685100 \mathrm{E}+00$ & $-4.425210 E-01$ \\
\hline $5.517900 \mathrm{E}-01$ & $-1.690200 \mathrm{E}-01$ \\
\hline $2.247310 \mathrm{E}-01$ & $5.052370 \mathrm{E}-01$ \\
\hline 1.000 & \\
\hline $8.24110 \mathrm{E}-02$ & $1.0000000 \mathrm{E}+00$ \\
\hline 11.000 & \\
\hline $8.500000 \mathrm{E}-01$ & $1.0000000 \mathrm{E}+00$ \\
\hline
\end{tabular}

$.0000000 \mathrm{E}+00$

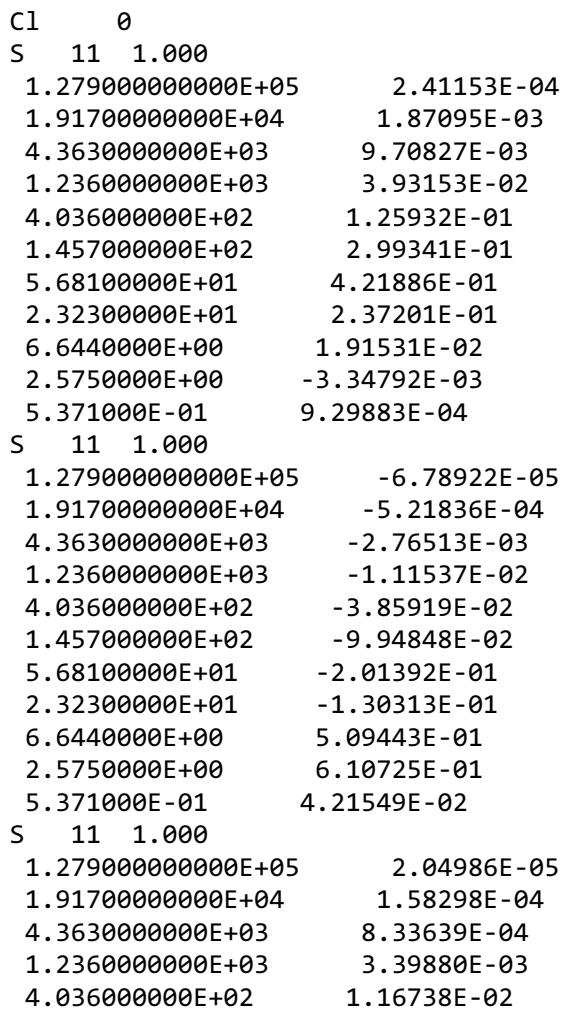


$1.457000000 \mathrm{E}+02$

$5.68100000 \mathrm{E}+01$

2. $32300000 \mathrm{E}+01$

$6.6440000 \mathrm{E}+00$

$2.5750000 \mathrm{E}+00$

5. $371000 \mathrm{E}-01$

S 11.000

1.938000E-01

P $7 \quad 1.000$

4.176000000E+02

$9.83300000 \mathrm{E}+01$

$3.10400000 \mathrm{E}+01$

1. $11900000 \mathrm{E}+01$

4. $2490000 \mathrm{E}+00$

$1.6240000 \mathrm{E}+00$

$5.322000 \mathrm{E}-01$

P $7 \quad 1.000$

4.176000000E+02

$9.83300000 \mathrm{E}+01$

$3.10400000 \mathrm{E}+01$

1. $11900000 \mathrm{E}+01$

4. $2490000 \mathrm{E}+00$

$1.6240000 \mathrm{E}+00$

5.322000E-01

P 11.000

1.620000E-01

D 11.000

$6.000000 \mathrm{E}-01$

$* * * *$

\begin{tabular}{lll}
$\mathrm{N}$ & \multicolumn{2}{l}{0} \\
$\mathrm{~S}$ & 8 & 1.000
\end{tabular}

$9.0460000000 \mathrm{E}+03$

$1.3570000000 \mathrm{E}+03$

$3.093000000 \mathrm{E}+02$

$8.77300000 \mathrm{E}+01$

$2.85600000 \mathrm{E}+01$

$1.02100000 \mathrm{E}+01$

$3.8380000 \mathrm{E}+00$

$7.466000 \mathrm{E}-01$

S 81.000

$9.0460000000 \mathrm{E}+03$

$1.3570000000 \mathrm{E}+03$

$3.093000000 \mathrm{E}+02$

$8.77300000 \mathrm{E}+01$

$2.85600000 \mathrm{E}+01$

$1.02100000 \mathrm{E}+01$

$3.8380000 \mathrm{E}+00$

$7.466000 \mathrm{E}-01$

S 11.000

2. 248000E- 01

P 31.000

1. $35500000 \mathrm{E}+01$

2. $9170000 \mathrm{E}+00$

7.973000E-01

P 11.000

2.185000E-01

D $1 \quad 1.000$

8.170000E-01

$* * * *$

$\mathrm{C}$

S 81.000

$6.6650000000 \mathrm{E}+03$

1. $0000000000 \mathrm{E}+03$

2. $280000000 \mathrm{E}+02$

$6.47100000 \mathrm{E}+01$

2.10600000E+01

$7.4950000 \mathrm{E}+00$

$2.7970000 \mathrm{E}+00$

5. $215000 \mathrm{E}-01$
$3.09622 \mathrm{E}-02$

$6.29533 \mathrm{E}-02$

4.60257E-02

$-2.19312 \mathrm{E}-01$

$-4.08773 \mathrm{E}-01$

$6.38465 \mathrm{E}-01$

$1.0000000 \mathrm{E}+00$

$5.25982 \mathrm{E}-03$

3. $98332 \mathrm{E}-02$

1. $64655 \mathrm{E}-01$

3. $87322 \mathrm{E}-01$

4. $57072 \mathrm{E}-01$

1. $51636 \mathrm{E}-01$

1.81615E-03

$-1.43570 \mathrm{E}-03$

$-1.07796 \mathrm{E}-02$

$-4.70075 E-02$

$-1.11030 \mathrm{E}-01$

$-1.53275 \mathrm{E}-01$

8.94609E-02

5.79444E-01

$1.0000000 \mathrm{E}+00$

$1.0000000 \mathrm{E}+00$

$7.000 E-04$
$5.3890 E-03$
$2.74060 E-02$
$1.032070 E-01$
$2.787230 E-01$
$4.485400 E-01$
$2.782380 E-01$
$1.54400 E-02$

$-1.530 \mathrm{E}-04$

$-1.2080 \mathrm{E}-03$

$-5.9920 \mathrm{E}-03$

$-2.45440 \mathrm{E}-02$

$-6.74590 \mathrm{E}-02$

$-1.580780 \mathrm{E}-01$

$-1.218310 \mathrm{E}-01$

$5.490030 \mathrm{E}-01$

$1.0000000 \mathrm{E}+00$

3.99190E-02

2.171690E-01

5.103190E-01

$1.0000000 \mathrm{E}+00$

$1.0000000 \mathrm{E}+00$
$6.920 \mathrm{E}-04$
$5.3290 \mathrm{E}-03$
2.70770E-02
$1.017180 \mathrm{E}-01$
2.747400E- 01
4. $485640 \mathrm{E}-01$
2.850740E- 01

$1.52040 \mathrm{E}-02$ 


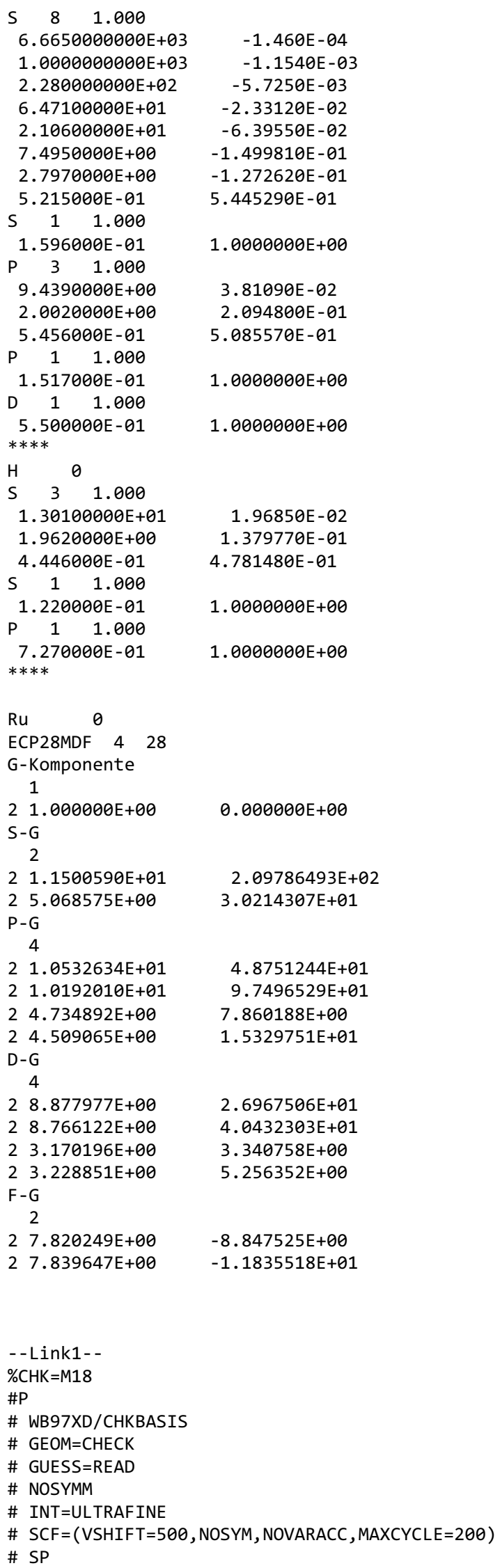




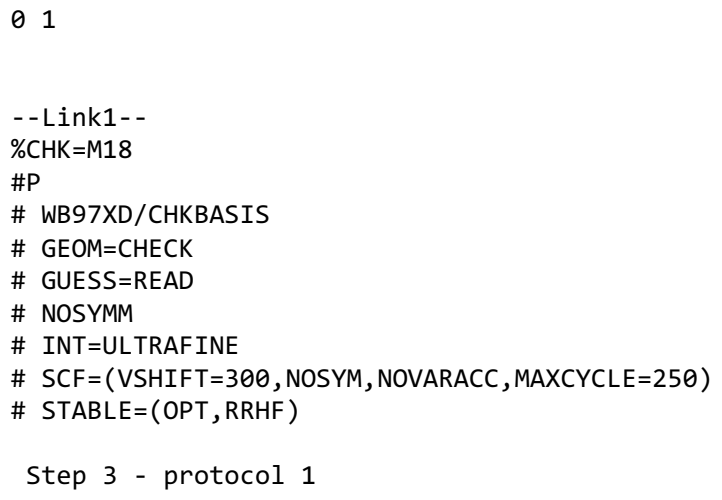

01

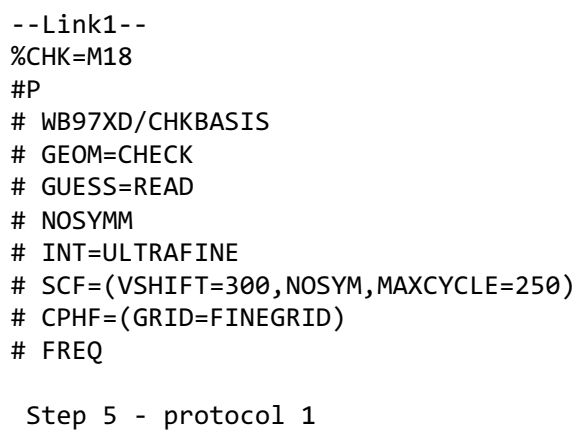

\section{Single-Point (SP) Energy Calculations}

Below we report a sample Gaussian input file for calculating the SP energy using the computational model PBE-D3MBJ-PCM. In order to reproduce the calculations with Gaussian 09, the following environmental variables must be set to obtain the modified BJ empirical dispersion correction: GAUSS_DFTD3_S6=1000000, GAUSS_DFTD3_S8=358940, GAUSS_DFTD3_ABJ1=12092, and GAUSS_DFTD3_ABJ2=5938951.

$\% \mathrm{CHK}=\mathrm{M} 18$ 


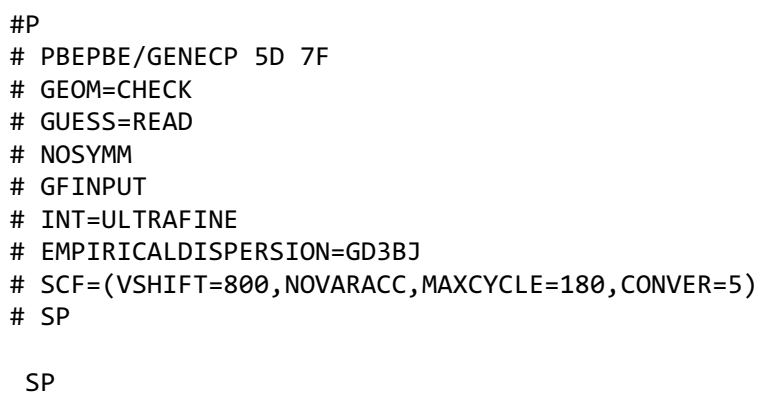




\begin{tabular}{|c|c|}
\hline $3.1705900 \mathrm{E}+00$ & 4.0155200E-01 \\
\hline $1.4948700 \mathrm{E}+00$ & $-2.1997860 E+00$ \\
\hline $8.0053000 \mathrm{E}-01$ & $-4.3552200 \mathrm{E}-01$ \\
\hline $4.1163800 \mathrm{E}-01$ & $3.4276070 \mathrm{E}+00$ \\
\hline $1.4241700 \mathrm{E}-01$ & $-2.4454790 \mathrm{E}+00$ \\
\hline $6.3563000 \mathrm{E}-02$ & $-1.4994300 \mathrm{E}-01$ \\
\hline $2.8296000 \mathrm{E}-02$ & $1.0823060 \mathrm{E}+00$ \\
\hline \multirow{2}{*}{$\begin{array}{lcc}S & 14 & 1.000 \\
7.2698900 \mathrm{E}+03\end{array}$} & \\
\hline & $-1.6000000 E-05$ \\
\hline $1.0878400 \mathrm{E}+03$ & $-1.8100000 \mathrm{E}-04$ \\
\hline $2.4178700 \mathrm{E}+02$ & $-3.3200000 \mathrm{E}-04$ \\
\hline $3.5452500 \mathrm{E}+01$ & $-2.5429000 \mathrm{E}-02$ \\
\hline $2.2141500 \mathrm{E}+01$ & $1.4439700 \mathrm{E}-01$ \\
\hline 1. 3829200E+01 & $-3.8364400 \mathrm{E}-01$ \\
\hline $5.1321300 \mathrm{E}+00$ & $1.3902860 \mathrm{E}+00$ \\
\hline $3.1705900 \mathrm{E}+00$ & $1.2715400 \mathrm{E}-01$ \\
\hline $1.4948700 \mathrm{E}+00$ & $-6.8551520 E+00$ \\
\hline $8.0053000 \mathrm{E}-01$ & $9.6961050 \mathrm{E}+00$ \\
\hline $3800 \mathrm{E}-01$ & +00 \\
\hline $00 \mathrm{E}-01$ & $=-01$ \\
\hline $6.3563000 \mathrm{E}-02$ & $570 E+00$ \\
\hline $2.8296000 \mathrm{E}-02$ & -1.85 \\
\hline 1.000 & \\
\hline $2.8296000 \mathrm{E}-02$ & $1.0000000 \mathrm{E}+00$ \\
\hline 111.000 & \\
\hline $1.7430700 \mathrm{E}+02$ & $1.0300000 \mathrm{E}-04$ \\
\hline $1.1504000 \mathrm{E}+01$ & $5.3994000 \mathrm{E}-02$ \\
\hline $7.1917600 \mathrm{E}+00$ & $-1.6101200 \mathrm{E}-01$ \\
\hline $300 E+00$ & $800 \mathrm{E}-01$ \\
\hline $00 \mathrm{E}+00$ & $\partial 0 E-01$ \\
\hline 1.0892200E+00 & $100 E-01$ \\
\hline $5.5863700 \mathrm{E}-01$ & $100 E-01$ \\
\hline $2.7047700 \mathrm{E}-01$ & $7.8702000 \mathrm{E}-02$ \\
\hline $1.2389600 \mathrm{E}-01$ & $3.7630000 \mathrm{E}-03$ \\
\hline $5.5823000 \mathrm{E}-02$ & $1.2860000 \mathrm{E}-03$ \\
\hline $2.4842000 \mathrm{E}-02$ & $-2.1500000 E-04$ \\
\hline 111.000 & \\
\hline $1.7430700 \mathrm{E}+02$ & $000 E-05$ \\
\hline 1.1 & -02 \\
\hline $00 \mathrm{E}+00$ & $00 \mathrm{E}-02$ \\
\hline $3.5749800 \mathrm{E}+00$ & $000 \mathrm{E}-02$ \\
\hline $2.1307800 \mathrm{E}+00$ & $-1.0322300 \mathrm{E}-01$ \\
\hline 1.0892200E+00 & $-1.8111500 \mathrm{E}-01$ \\
\hline $5.5863700 \mathrm{E}-01$ & $-1.2438800 \mathrm{E}-01$ \\
\hline $2.7047700 \mathrm{E}-01$ & $1.1202800 \mathrm{E}-01$ \\
\hline $1.2389600 \mathrm{E}-01$ & $4.2251100 \mathrm{E}-01$ \\
\hline $5.5823000 \mathrm{E}-02$ & $4.7723100 \mathrm{E}-01$ \\
\hline $2.4842000 \mathrm{E}-02$ & $1.5682000 \mathrm{E}-01$ \\
\hline 111.000 & \\
\hline $700 E+02$ & $300 \mathrm{E}-05$ \\
\hline$E+01$ & $E-02$ \\
\hline $600 \mathrm{E}+00$ & $00 E-02$ \\
\hline $3.5749800 \mathrm{E}+00$ & $000 \mathrm{E}-02$ \\
\hline $2.1307800 \mathrm{E}+00$ & $-2.0474700 \mathrm{E}-01$ \\
\hline 1.0892200E+00 & $-3.7151100 E-01$ \\
\hline $5.5863700 \mathrm{E}-01$ & $-1.4363500 \mathrm{E}-01$ \\
\hline $2.7047700 \mathrm{E}-01$ & $4.6222100 \mathrm{E}-01$ \\
\hline $1.2389600 \mathrm{E}-01$ & $5.8558100 \mathrm{E}-01$ \\
\hline $5.5823000 \mathrm{E}-02$ & E-01 \\
\hline 2.4842000 & \\
\hline 111.000 & \\
\hline 1.7430700E+02 & -04 \\
\hline $1.1504000 \mathrm{E}+01$ & $000 \mathrm{E}-02$ \\
\hline $7.1917600 \mathrm{E}+00$ & $-1.5382000 \mathrm{E}-01$ \\
\hline $3.5749800 \mathrm{E}+00$ & $-1.7315200 \mathrm{E}-01$ \\
\hline $2.1307800 \mathrm{E}+00$ & $5.3097500 \mathrm{E}-01$ \\
\hline 1.0892200E+00 & $8.1762600 \mathrm{E}-01$ \\
\hline $5.5863700 \mathrm{E}-01$ & $-7.8673200 E-01$ \\
\hline & $\mathrm{E}+00$ \\
\hline
\end{tabular}




\begin{tabular}{|c|c|}
\hline $1.2389600 \mathrm{E}-01$ & $7.5490800 \mathrm{E}-01$ \\
\hline $5.5823000 \mathrm{E}-02$ & $4.6325100 \mathrm{E}-01$ \\
\hline $2.4842000 \mathrm{E}-02$ & $5.5220000 \mathrm{E}-03$ \\
\hline 111.000 & \\
\hline $1.7430700 \mathrm{E}+02$ & $-2.5000000 E-04$ \\
\hline $1.1504000 \mathrm{E}+01$ & $-6.2992000 \mathrm{E}-02$ \\
\hline $7.1917600 \mathrm{E}+00$ & $2100 E-01$ \\
\hline $3.5749800 E+00$ & $6.5282900 \mathrm{E}-01$ \\
\hline $2.1307800 \mathrm{E}+00$ & $-2.1533740 \mathrm{E}+00$ \\
\hline $1.0892200 \mathrm{E}+00$ & $4.0944200 E-01$ \\
\hline $5.5863700 E-01$ & $2.4570950 \mathrm{E}+00$ \\
\hline $2.7047700 \mathrm{E}-01$ & $-2.2868480 E+00$ \\
\hline $1.2389600 \mathrm{E}-01$ & $7.7769000 \mathrm{E}-02$ \\
\hline $5.5823000 E-02$ & $7.6560400 \mathrm{E}-01$ \\
\hline $2.4842000 \mathrm{E}-02$ & $-2.2730000 \mathrm{E}-03$ \\
\hline 1.000 & \\
\hline $2.4842000 \mathrm{E}-02$ & $1.0000000 \mathrm{E}+00$ \\
\hline D $\quad 10 \quad 1.000$ & \\
\hline $7.5375100 \mathrm{E}+01$ & $1.8000000 \mathrm{E}-04$ \\
\hline $1.9890100 \mathrm{E}+01$ & $2.0840000 \mathrm{E}-03$ \\
\hline $7.8792400 \mathrm{E}+00$ & $-1.8671000 E-02$ \\
\hline $2.9083400 \mathrm{E}+00$ & $7.8054000 \mathrm{E}-02$ \\
\hline $1.6388300 \mathrm{E}+00$ & $2.2632800 \mathrm{E}-01$ \\
\hline $8.7573000 \mathrm{E}-01$ & $3.1710000 \mathrm{E}-01$ \\
\hline $4.5048300 \mathrm{E}-01$ & $3.0503600 \mathrm{E}-01$ \\
\hline $2.2277000 \mathrm{E}-01$ & $2.1515000 \mathrm{E}-01$ \\
\hline $1.0534200 E-01$ & $58000 E-02$ \\
\hline $4.6455000 \mathrm{E}-02$ & $1.9451000 \mathrm{E}-02$ \\
\hline D $\quad 10 \quad 1.000$ & \\
\hline $7.5375100 \mathrm{E}+01$ & $-2.0300000 \mathrm{E}-04$ \\
\hline $1.9890100 \mathrm{E}+01$ & $-2.0860000 \mathrm{E}-03$ \\
\hline $7.8792400 \mathrm{E}+00$ & $1.9428000 \mathrm{E}-02$ \\
\hline $2.9083400 \mathrm{E}+00$ & $-8.7106000 \mathrm{E}-02$ \\
\hline $1.6388300 \mathrm{E}+00$ & $-2.8641300 \mathrm{E}-01$ \\
\hline $8.7573000 \mathrm{E}-01$ & $-3.3190800 \mathrm{E}-01$ \\
\hline $4.5048300 E-01$ & $-2.1210000 \mathrm{E}-03$ \\
\hline $2.2277000 \mathrm{E}-01$ & $500 E-01$ \\
\hline $1.0534200 \mathrm{E}-01$ & $200 E-01$ \\
\hline $4.6455000 \mathrm{E}-02$ & $1.6126800 \mathrm{E}-01$ \\
\hline 101.000 & \\
\hline $7.5375100 E+01$ & $-3.5500000 E-04$ \\
\hline $1.9890100 \mathrm{E}+01$ & $-3.1930000 \mathrm{E}-03$ \\
\hline $7.8792400 \mathrm{E}+00$ & $3.1956000 \mathrm{E}-02$ \\
\hline $2.9083400 \mathrm{E}+00$ & $-1.8559900 \mathrm{E}-01$ \\
\hline $1.6388300 \mathrm{E}+00$ & $-5.8318500 \mathrm{E}-01$ \\
\hline $8.7573000 \mathrm{E}-01$ & $-7.2766000 \mathrm{E}-02$ \\
\hline $4.5048300 \mathrm{E}-01$ & $7.9870700 \mathrm{E}-01$ \\
\hline $2.2277000 \mathrm{E}-01$ & $3600 E-01$ \\
\hline $1.0534200 E-01$ & $300 \mathrm{E}-01$ \\
\hline $4.6455000 \mathrm{E}-02$ & $000 \mathrm{E}-01$ \\
\hline 101.000 & \\
\hline $7.5375100 \mathrm{E}+01$ & $-1.8200000 \mathrm{E}-04$ \\
\hline $1.9890100 \mathrm{E}+01$ & $-7.2830000 \mathrm{E}-03$ \\
\hline $7.8792400 \mathrm{E}+00$ & $6.1663000 \mathrm{E}-02$ \\
\hline $2.9083400 \mathrm{E}+00$ & $-4.4416800 \mathrm{E}-01$ \\
\hline $1.6388300 \mathrm{E}+00$ & $-7.9983900 \mathrm{E}-01$ \\
\hline $8.7573000 E-01$ & $1.3061620 \mathrm{E}+00$ \\
\hline $4.5048300 E-01$ & $3.4708800 E-01$ \\
\hline $2.2277000 \mathrm{E}-01$ & $23020 E+00$ \\
\hline $1.0534200 \mathrm{E}-01$ & $1.7440000 \mathrm{E}-01$ \\
\hline $4.6455000 \mathrm{E}-02$ & $6.2628100 \mathrm{E}-01$ \\
\hline 1.000 & \\
\hline $4.6455000 E-02$ & $1.0000000 E+00$ \\
\hline 1.000 & \\
\hline $2.8980000 \mathrm{E}+00$ & $1.0000000 \mathrm{E}+00$ \\
\hline 11.000 & \\
\hline $9.5880000 \mathrm{E}-01$ & $1.0000000 \mathrm{E}+00$ \\
\hline $\begin{array}{lll}\mathrm{F} & 1 & 1.000\end{array}$ & \\
\hline $3.1720000 \mathrm{E}-e$ & 1.0000 \\
\hline
\end{tabular}




\begin{tabular}{|c|c|c|}
\hline \multicolumn{3}{|c|}{ G $\quad 1 \quad 1.000$} \\
\hline \multicolumn{3}{|c|}{$1.7100000 \mathrm{E}+00$} \\
\hline 1 & 1.000 & \\
\hline $5.778 e$ & $000 \mathrm{E}-01$ & $1.0000000 \mathrm{E}+00$ \\
\hline 1 & 1.000 & \\
\hline $1.164 \epsilon$ & $000 \mathrm{E}+00$ & $1.0000000 \mathrm{E}+00$ \\
\hline$* * * *$ & & \\
\hline $\mathrm{Cl}$ & $\theta$ & \\
\hline 13 & 1.000 & \\
\hline 8.3496 & $00000000 \mathrm{E}+0$ & $2.31688 \mathrm{E}-05$ \\
\hline 1.2506 & $00000000 \mathrm{E}+0$ & $1.80154 \mathrm{E}-04$ \\
\hline 2.8436 & $0000000 \mathrm{E}+04$ & $9.47782 \mathrm{E}-04$ \\
\hline 8.0336 & $000000 \mathrm{E}+03$ & $4.00139 E-03$ \\
\hline $2.608 e$ & $000000 \mathrm{E}+03$ & $1.44629 \mathrm{E}-02$ \\
\hline 9.3396 & $00000 \mathrm{E}+02$ & $4.56586 \mathrm{E}-02$ \\
\hline 3.6006 & $00000 \mathrm{E}+02$ & $1.23248 \mathrm{E}-01$ \\
\hline 1.4706 & $00000 E+02$ & $2.64369 E-01$ \\
\hline 6.2886 & $0000 \mathrm{E}+01$ & $3.82989 E-01$ \\
\hline 2.7606 & $0000 \mathrm{E}+01$ & $2.70934 \mathrm{E}-01$ \\
\hline 1.1086 & $0000 \mathrm{E}+01$ & $4.71404 \mathrm{E}-02$ \\
\hline $5.075 e$ & $000 \mathrm{E}+00$ & $-3.71766 \mathrm{E}-03$ \\
\hline $2.278 €$ & $000 \mathrm{E}+00$ & $2.19158 \mathrm{E}-03$ \\
\hline 13 & 1.000 & \\
\hline 8.3496 & $00000000 \mathrm{E}+0$ & $-6.49649 E-06$ \\
\hline 1.2506 & $00000000 \mathrm{E}+0$ & $-5.04895 E-05$ \\
\hline 2.8436 & $0000000 \mathrm{E}+04$ & $-2.66113 E-04$ \\
\hline 8.0336 & $000000 E+03$ & $-1.12499 E-03$ \\
\hline 2.6086 & $000000 \mathrm{E}+03$ & $-4.10497 E-03$ \\
\hline 9.3396 & $00000 \mathrm{E}+02$ & $-1.31987 E-02$ \\
\hline 3.6006 & $00000 E+02$ & $-3.75342 E-02$ \\
\hline 1.4706 & $00000 \mathrm{E}+02$ & $-8.97233 E-02$ \\
\hline 6.2886 & $0000 \mathrm{E}+01$ & $-1.67671 \mathrm{E}-01$ \\
\hline 2.7606 & $0000 \mathrm{E}+01$ & $-1.74763 \mathrm{E}-01$ \\
\hline $1.108 €$ & $0000 \mathrm{E}+01$ & $1.14909 \mathrm{E}-01$ \\
\hline 5.0756 & $000 \mathrm{E}+00$ & $5.63618 E-01$ \\
\hline $2.278 e$ & $000 \mathrm{E}+00$ & $4.41606 \mathrm{E}-01$ \\
\hline 13 & 1.000 & \\
\hline 8.3496 & $00000000 \mathrm{E}+0$ & $1.96645 \mathrm{E}-06$ \\
\hline 1.2506 & $00000000 \mathrm{E}+0$ & $1.52620 \mathrm{E}-05$ \\
\hline 2.8436 & $0000000 \mathrm{E}+04$ & $8.06086 E-05$ \\
\hline 8.0336 & $000000 \mathrm{E}+03$ & $3.39960 E-04$ \\
\hline 2.608 & $000000 \mathrm{E}+03$ & $1.24551 \mathrm{E}-03$ \\
\hline 9.3396 & $00000 \mathrm{E}+02$ & $3.99612 \mathrm{E}-03$ \\
\hline 3.6006 & $00000 \mathrm{E}+02$ & $1.14751 \mathrm{E}-02$ \\
\hline 1.4706 & $00000 E+02$ & $2.75504 \mathrm{E}-02$ \\
\hline 6.2886 & $0000 \mathrm{E}+01$ & $5.32917 \mathrm{E}-02$ \\
\hline 2.7606 & $0000 \mathrm{E}+01$ & $5.71246 E-02$ \\
\hline 1.1086 & $0000 \mathrm{E}+01$ & $-3.95201 E-02$ \\
\hline 5.0756 & $000 \mathrm{E}+00$ & $-2.64343 \mathrm{E}-01$ \\
\hline 2.2786 & $000 \mathrm{E}+00$ & $-3.49291 E-01$ \\
\hline 1 & 1.000 & \\
\hline 7.7756 & $\theta 0 \mathrm{E}-01$ & $1.0000000 \mathrm{E}+00$ \\
\hline $\mathrm{s}$ & 1.000 & \\
\hline $3.527 €$ & $00 \mathrm{E}-01$ & $1.0000000 \mathrm{E}+00$ \\
\hline $\mathrm{s}$ & 1.000 & \\
\hline $1.431 €$ & $00 \mathrm{E}-01$ & $1.0000000 \mathrm{E}+00$ \\
\hline $\mathrm{s}$ & 1.000 & \\
\hline 5.1906 & $0 \mathrm{E}-02$ & 1. $.0000000 \mathrm{E}+00$ \\
\hline 8 & 1.000 & \\
\hline 1.7036 & $000000 \mathrm{E}+03$ & $4.74039 E-04$ \\
\hline $4.036 e$ & $00000 \mathrm{E}+02$ & $4.06412 \mathrm{E}-03$ \\
\hline 1.3036 & $00000 \mathrm{E}+02$ & $2.13355 E-02$ \\
\hline $4.905 e$ & $0000 \mathrm{E}+01$ & $7.94611 \mathrm{E}-02$ \\
\hline $2.026 e$ & $0000 \mathrm{E}+01$ & $2.08927 \mathrm{E}-01$ \\
\hline $8.787 €$ & $000 E+00$ & $3.64945 E-01$ \\
\hline 3.9196 & $000 \mathrm{E}+00$ & $3.71725 E-01$ \\
\hline 1.7656 & $000 E+00$ & $1.46292 \mathrm{E}-01$ \\
\hline P 8 & 1.000 & \\
\hline 1.7036 & $000000 \mathrm{E}+$ & $-1.28266 \mathrm{E}-$ \\
\hline
\end{tabular}




\begin{tabular}{|c|c|}
\hline $4.036000000 \mathrm{E}+02$ & $-1.09356 E-0$ \\
\hline $1.303000000 \mathrm{E}+02$ & $-5.83429 E-03$ \\
\hline $4.90500000 \mathrm{E}+01$ & $-2.19258 \mathrm{E}-02$ \\
\hline $2.02600000 \mathrm{E}+01$ & $-6.01385 E-02$ \\
\hline $8.7870000 \mathrm{E}+00$ & $-1.06929 E-01$ \\
\hline $3.9190000 \mathrm{E}+00$ & $-1.22454 \mathrm{E}-01$ \\
\hline $1.7650000 \mathrm{E}+00$ & $3.83619 E-02$ \\
\hline \multirow{2}{*}{$\begin{array}{cr}1 & 1.000 \\
7.207000 \mathrm{E}-01\end{array}$} & \\
\hline & $1.0000000 \mathrm{E}+00$ \\
\hline $\begin{array}{lll}P & 1 & 1.000\end{array}$ & \\
\hline $2.839000 \mathrm{E}-01$ & $1.0000000 \mathrm{E}+00$ \\
\hline \multirow{2}{*}{$\begin{array}{rrr}P & 1 & 1.000 \\
1.060000 \mathrm{E}-01\end{array}$} & \\
\hline & $1.0000000 \mathrm{E}+00$ \\
\hline \multicolumn{2}{|l|}{$\begin{array}{lll}P & 1 & 1.000\end{array}$} \\
\hline $3.76000 \mathrm{E}-02$ & $1.0000000 \mathrm{E}+00$ \\
\hline 1.000 & \\
\hline $2.540000 \mathrm{E}-01$ & $1.0000000 \mathrm{E}+00$ \\
\hline 1.000 & \\
\hline $6.280000 \mathrm{E}-01$ & $1.0000000 \mathrm{E}+00$ \\
\hline 1.000 & \\
\hline $1.5510000 \mathrm{E}+00$ & $1.0000000 \mathrm{E}+00$ \\
\hline 1.000 & \\
\hline $9.52000 \mathrm{E}-02$ & $.0000000 \mathrm{E}+00$ \\
\hline 1.000 & \\
\hline $4.230000 \mathrm{E}-01$ & $1.0000000 \mathrm{E}+00$ \\
\hline 1.000 & \\
\hline $1.0890000 \mathrm{E}+00$ & $1.0000000 \mathrm{E}+00$ \\
\hline 1.000 & \\
\hline $2.170000 \mathrm{E}-01$ & $1.0000000 \mathrm{E}+00$ \\
\hline 1.000 & \\
\hline $8.270000 \mathrm{E}-01$ & $1.0000000 \mathrm{E}+00$ \\
\hline 1.000 & \\
\hline $3.780000 E-01$ & $1.0000000 \mathrm{E}+00$ \\
\hline$* * * *$ & \\
\hline 0 & \\
\hline 1.000 & \\
\hline $4.58400000000 \mathrm{E}+0$ & $9.20 \mathrm{E}-05$ \\
\hline 6.8680000000 & $7.170 E-04$ \\
\hline 1.5630000006 & $3.7490 \mathrm{E}-03$ \\
\hline $4.424000000 \mathrm{E}+02$ & $1.55320 \mathrm{E}-02$ \\
\hline $1.443000000 \mathrm{E}+02$ & $60 \mathrm{E}-02$ \\
\hline $5.21800000 E+01$ & $1.467870 \mathrm{E}-01$ \\
\hline $2.03400000 \mathrm{E}+01$ & $3.046630 E-01$ \\
\hline $8.3810000 \mathrm{E}+00$ & $3.976840 \mathrm{E}-01$ \\
\hline $3.5290000 \mathrm{E}+00$ & $2.176410 \mathrm{E}-01$ \\
\hline 1.000 & \\
\hline $4.58400000000 \mathrm{E}+02$ & $-2.00 E-05$ \\
\hline $6.8680000000 \mathrm{E}$ & $-1.590 E-04$ \\
\hline 1.5630000000 & $-8.240 E-04$ \\
\hline $4.424000000 E+02$ & E-03 \\
\hline 1.44300000 & $\theta \mathrm{E}-02$ \\
\hline 5.218 & $\mathrm{E}-02$ \\
\hline $2.03400000 \mathrm{E}+$ & $0 \mathrm{E}-02$ \\
\hline $8.3810000 E+00$ & $-1.467220 \mathrm{E}-01$ \\
\hline $3.5290000 \mathrm{E}+00$ & $-1.163600 \mathrm{E}-01$ \\
\hline 1.000 & \\
\hline $1.0540000 \mathrm{E}+00$ & $1.0000000 \mathrm{E}+00$ \\
\hline 1.000 & \\
\hline $4.118000 \mathrm{E}-01$ & $1.0000000 \mathrm{E}+00$ \\
\hline 1.000 & \\
\hline $1.552000 \mathrm{E}-01$ & $1.0000000 \mathrm{E}+00$ \\
\hline 1.000 & \\
\hline $5.46400 E-02$ & $1.0000000 \mathrm{E}+00$ \\
\hline 1.000 & \\
\hline $4.93300000 \mathrm{E}+\ell$ & $30 E-03$ \\
\hline $1.13700000 \mathrm{E}+01$ & $3.79620 \mathrm{E}-02$ \\
\hline $3.4350000 \mathrm{E}+00$ & $1.490280 \mathrm{E}-01$ \\
\hline $\begin{array}{lll}P & 1 & 1.000\end{array}$ & \\
\hline $1.1820000 \mathrm{E}$ & $00 \mathrm{E}+0$ \\
\hline
\end{tabular}




\begin{tabular}{|c|c|c|}
\hline \multicolumn{3}{|c|}{$\begin{array}{lll}P & 1 & 1.000\end{array}$} \\
\hline \multicolumn{2}{|c|}{$4.173000 \mathrm{E}-01$} & $1.0000000 \mathrm{E}+00$ \\
\hline 1 & 1.000 & \\
\hline \multicolumn{2}{|c|}{$1.428000 \mathrm{E}-01$} & $1.0000000 \mathrm{E}+00$ \\
\hline 1 & 1.000 & \\
\hline \multicolumn{2}{|c|}{$4.40200 \mathrm{E}-02$} & $1.0000000 \mathrm{E}+00$ \\
\hline 1 & 1.000 & \\
\hline \multicolumn{2}{|c|}{$2.8370000 \mathrm{E}+00$} & $1.0000000 \mathrm{E}+00$ \\
\hline 1 & 1.000 & \\
\hline \multicolumn{2}{|c|}{$9.680000 \mathrm{E}-01$} & $1.0000000 \mathrm{E}+00$ \\
\hline 1 & 1.000 & \\
\hline \multicolumn{2}{|c|}{$3.350000 \mathrm{E}-01$} & $1.0000000 \mathrm{E}+00$ \\
\hline 1 & 1.000 & \\
\hline \multicolumn{2}{|c|}{$1.110000 \mathrm{E}-01$} & $1.0000000 \mathrm{E}+00$ \\
\hline $\mathrm{F}$ & 1.000 & \\
\hline \multicolumn{2}{|c|}{$2.0270000 \mathrm{E}+00$} & $1.0000000 \mathrm{E}+00$ \\
\hline $\mathrm{F}$ & 1.000 & \\
\hline \multicolumn{2}{|c|}{$6.850000 \mathrm{E}-01$} & $1.0000000 \mathrm{E}+00$ \\
\hline 1 & 1.000 & \\
\hline \multicolumn{2}{|c|}{$2.450000 \mathrm{E}-01$} & $1.0000000 \mathrm{E}+00$ \\
\hline G & 1.000 & \\
\hline \multicolumn{2}{|c|}{$1.4270000 \mathrm{E}+00$} & $1.0000000 \mathrm{E}+00$ \\
\hline G & 1.000 & \\
\hline \multicolumn{2}{|c|}{$\begin{array}{l}5.590000 \mathrm{E}-01 \\
* * * *\end{array}$} & $1.0000000 \mathrm{E}+00$ \\
\hline \multicolumn{3}{|c|}{ C $\quad 0$} \\
\hline 9 & 1.000 & \\
\hline 3.39 & $0000000 E+04$ & $9.10 \mathrm{E}-05$ \\
\hline 5.08 & $000000 E+03$ & $7.040 E-04$ \\
\hline 1.15 & $000000 E+03$ & $3.6930 \mathrm{E}-03$ \\
\hline 3.26 & $00000 \mathrm{E}+02$ & $1.53600 \mathrm{E}-02$ \\
\hline 1.06 & $00000 E+02$ & $5.29290 E-02$ \\
\hline 3.81 & $0000 E+01$ & $1.470430 \mathrm{E}-01$ \\
\hline 1.47 & $0000 \mathrm{E}+01$ & $3.056310 \mathrm{E}-01$ \\
\hline 6.03 & $000 E+00$ & $3.993450 \mathrm{E}-01$ \\
\hline 2.53 & $000 E+00$ & $2.170510 \mathrm{E}-01$ \\
\hline $\mathrm{s}$ & 1.000 & \\
\hline 3.39 & $0000000 E+04$ & $-1.90 E-05$ \\
\hline 5.08 & $000000 E+03$ & $-1.510 \mathrm{E}-04$ \\
\hline 1.15 & $000000 \mathrm{E}+03$ & $-7.850 E-04$ \\
\hline 3.26 & $00000 \mathrm{E}+02$ & $-3.3240 E-03$ \\
\hline 1.06 & $00000 \mathrm{E}+02$ & $-1.15120 \mathrm{E}-02$ \\
\hline 3.81 & $0000 E+01$ & $-3.41600 \mathrm{E}-02$ \\
\hline 1.47 & $0000 \mathrm{E}+01$ & $-7.71730 \mathrm{E}-02$ \\
\hline 6.03 & $000 E+00$ & $-1.414930 \mathrm{E}-01$ \\
\hline 2.53 & $000 E+00$ & $-1.180190 \mathrm{E}-01$ \\
\hline $\mathrm{S}$ & 1.000 & \\
\hline 7.35 & $00 E-01$ & $1.0000000 \mathrm{E}+00$ \\
\hline $\mathrm{s}$ & 1.000 & \\
\hline 2.90 & $00 \mathrm{E}-01$ & $1.0000000 \mathrm{E}+00$ \\
\hline $\mathrm{s}$ & 1.000 & \\
\hline 1.11 & $00 E-01$ & $1.0000000 \mathrm{E}+00$ \\
\hline $\mathrm{P}$ & 1.000 & \\
\hline 3.45 & $0000 E+01$ & $5.3780 E-03$ \\
\hline 7.91 & $000 E+00$ & $3.61320 \mathrm{E}-02$ \\
\hline 2.36 & $000 E+00$ & $1.424930 \mathrm{E}-01$ \\
\hline $\mathrm{P}$ & 1.000 & \\
\hline 8.13 & $00 E-01$ & $1.0000000 \mathrm{E}+00$ \\
\hline $\mathrm{P}$ & 1.000 & \\
\hline 2.89 & $00 \mathrm{E}-01$ & $1.0000000 \mathrm{E}+00$ \\
\hline $\mathrm{P}$ & 1.000 & \\
\hline 1.00 & $00 E-01$ & $1.0000000 \mathrm{E}+00$ \\
\hline D & 1.000 & \\
\hline 1.84 & $000 E+00$ & $1.0000000 \mathrm{E}+00$ \\
\hline D & 1.000 & \\
\hline 6.49 & $00 \mathrm{E}-01$ & $1.0000000 \mathrm{E}+00$ \\
\hline D & 1.000 & \\
\hline 2.28 & $00 \mathrm{E}-01$ & $1.0000000 \mathrm{E}+0 \mathrm{E}$ \\
\hline a & 1.00 & \\
\hline
\end{tabular}




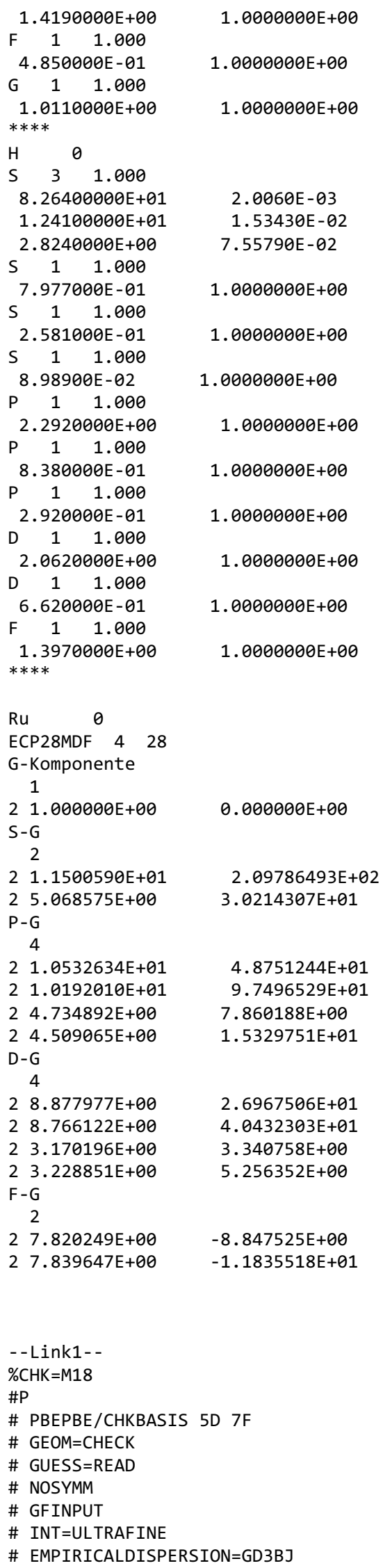




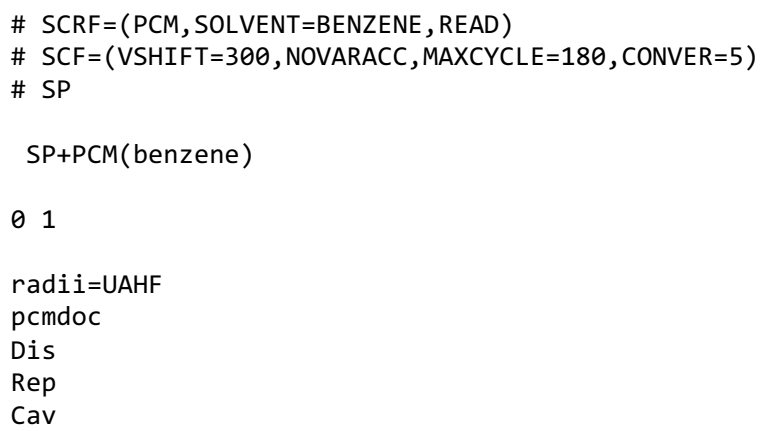

\section{References}

(1) Engel, J.; Smit, W.; Foscato, M.; Occhipinti, G.; Törnroos, K. W.; Jensen, V. R. Loss and Reformation of Ruthenium Alkylidene: Connecting Olefin Metathesis, Catalyst Deactivation, Regeneration, and Isomerization, J. Am. Chem. Soc. 2017, 139, 16609-19.

(2) Fulmer, G. R.; Miller, A. J. M.; Sherden, N. H.; Gottlieb, H. E.; Nudelman, A.; Stoltz, B. M.; Bercaw, J. E.; Goldberg, K. I. NMR Chemical Shifts of Trace Impurities: Common Laboratory Solvents, Organics, and Gases in Deuterated Solvents Relevant to the Organometallic Chemist, Organometallics 2010, 29, 2176-79.

(3) Dinger, M. B.; Mol, J. C. Degradation of the First-Generation Grubbs Metathesis Catalyst with Primary Alcohols, Water, and Oxygen. Formation and Catalytic Activity of Ruthenium(II) Monocarbonyl Species, Organometallics 2003, 22, 1089-95.

(4) Pauli, G. F.; Jaki, B. U.; Lankin, D. C. A Routine Experimental Protocol for qHNMR Illustrated with Taxol, J. Nat. Prod. 2007, 70, 589-95.

(5) Takeshi, S.; Sachiko, N.; Miyuki, K.; Toshihide, I.; Shinichi, K.; Akira, N.; Tsuneaki, M. Practical Guide for Accurate Quantitative Solution State NMR Analysis, Metrologia 2004, 41, 213-18.

(6) Scanlon, J. T.; Willis, D. E. Calculation of Flame Ionization Detector Relative Response Factors Using the Effective Carbon Number Concept, J. Chromatogr. Sci. 1985, 23, 333-40.

(7) Bailey, G. A.; Foscato, M.; Higman, C. S.; Day, C. S.; Jensen, V. R.; Fogg, D. E. Bimolecular Coupling as a Vector for Decomposition of Fast-Initiating Olefin Metathesis Catalysts, J. Am. Chem. Soc. 2018, 140, 6931-44.

(8) Janse van Rensburg, W.; Steynberg, P. J.; Meyer, W. H.; Kirk, M. M.; Forman, G. S. DFT Prediction and Experimental Observation of Substrate-Induced Catalyst Decomposition in Ruthenium-Catalyzed Olefin Metathesis, J. Am. Chem. Soc. 2004, 126, 14332-33.

(9) Kaithal, A.; Chatterjee, B.; Gunanathan, C. Ruthenium-Catalyzed Regioselective 1,4Hydroboration of Pyridines, Org. Lett. 2016, 18, 3402-05.

(10) Higgins, E. M.; Sherwood, J. A.; Lindsay, A. G.; Armstrong, J.; Massey, R. S.; Alder, R. W.; O'Donoghue, A. C. $\mathrm{pK}_{\mathrm{a}} \mathrm{s}$ of the Conjugate Acids of N-Heterocyclic Carbenes in Water, Chem. Commun. 2011, 47, 1559-61.

(11) Velleman, P. F. Definition and Comparison of Robust Nonlinear Data Smoothing Algorithms, J. Am. Stat. Assoc. 1980, 75, 609-15.

(12) Higman, C. S.; Lanterna, A. E.; Marin, M. L.; Scaiano, J. C.; Fogg, D. E. Catalyst Decomposition During Olefin Metathesis Yields Isomerization-Active Ruthenium Nanoparticles, ChemCatChem 2016, 8, 2424-24. 
(13) Turnovers at a specific reaction time are calculated as the molar yield of the product at that time divided by the initial molar number of 1 .

(14) Mol, J. C. Industrial Applications of Olefin Metathesis, J. Mol. Catal. A-Chem. 2004, 213, $39-45$.

(15) Hong, S. H.; Wenzel, A. G.; Salguero, T. T.; Day, M. W.; Grubbs, R. H. Decomposition of Ruthenium Olefin Metathesis Catalysts, J. Am. Chem. Soc. 2007, 129, 7961-68.

(16) Bailey, G. A.; Lummiss, J. A. M.; Foscato, M.; Occhipinti, G.; McDonald, R.; Jensen, V. R.; Fogg, D. E. Decomposition of Olefin Metathesis Catalysts by Brønsted Base: Metallacyclobutane Deprotonation as a Primary Deactivating Event, J. Am. Chem. Soc. 2017, 139, 16446-49.

(17) Precatalyst 1 contained an initial amount of 0.67 equiv. of free $p$-cymene, originating from its synthesis. This extra $p$-cymene stabilized 1 and was therefore not removed.

(18) Lummiss, J. A. M.; Beach, N. J.; Smith, J. C.; Fogg, D. E. Targeting an Achilles Heel in Olefin Metathesis: A Strategy for High-Yield Synthesis of Second-Generation Grubbs Methylidene Catalysts, Catal. Sci. Technol. 2012, 2, 1630-32.

(19) Demerseman, B.; Mbaye, M. D.; Sémeril, D.; Toupet, L.; Bruneau, C.; Dixneuf, P. H. Direct Preparation of $\left[\mathrm{Ru}\left(\eta^{2}-\mathrm{O}_{2} \mathrm{CO}\right)\left(\eta^{6}\right.\right.$-Arene $\left.)(\mathrm{L})\right]$ Carbonate Complexes $(\mathrm{L}=$ Phosphane, Carbene) and Their Use as Precursors of $\left[\mathrm{RuH}_{2}(\mathrm{p}\right.$-cymene $\left.)(\mathrm{PCy})\right]$ and $\left[\mathrm{Ru}\left(\eta^{6}-\mathrm{Arene}\right)(\mathrm{L})(\mathrm{MeCN})_{2}\right]\left[\mathrm{BF}_{4}\right]_{2}$ : $\mathrm{X}$-Ray Crystal Structure Determination of $\left[\mathrm{Ru}\left(\eta^{2}-\mathrm{O}_{2} \mathrm{CO}\right)(\mathrm{p}\right.$-cymene $\left.)\left(\mathrm{PCy}_{3}\right)\right] \cdot 1 / 2 \mathrm{CH}_{2} \mathrm{Cl}_{2}$ and $\left[\mathrm{Ru}\left(\eta^{2}-\mathrm{O}_{2} \mathrm{CO}\right)\left(\eta^{6}-\mathrm{C}_{6} \mathrm{Me}_{6}\right)\left(\mathrm{PMe}_{3}\right)\right] \cdot \mathrm{H}_{2} \mathrm{O}$, Eur. J. Inorg. Chem. 2006, 2006, 1174-81.

(20) Williams, J. E.; Harner, M. J.; Sponsler, M. B. Ruthenium Alkylidenes: Fast Initiators for Olefin Metathesis, Organometallics 2005, 24, 2013-15.

(21) Hong, S. H.; Day, M. W.; Grubbs, R. H. Decomposition of a Key Intermediate in Ruthenium-Catalyzed Olefin Metathesis Reactions, J. Am. Chem. Soc. 2004, 126, 7414-15. (22) This value is somewhat lower than those recorded in the previous experiments (70-82\%). The special NMR tubes used for these experiments are equipped with a glass plunger that separates the sample from the headspace, see Figure S1 for details. The plunger is not perfectly tight, and it does not completely prevent gas diffusion and exchange between the gases dissolved in the sample (mainly ethylene) and those present in the headspace (mainly argon). Ethylene and other gaseous products that have moved into the headspace are not recorded in the spectra, and the amount of ethylene missing from the mass balance may thus vary and depend on factors such as the tightness of the glass plunger (which, in general, is different for different NMR tubes and which may change with wear), the initial concentration of ethylene, the initial composition of the headspace gas (which is a mix of argon and ethylene), the reaction time, and the reaction temperature.

(23) Marvin 18.24.0, 2018, ChemAxon Ltd. (http://www.chemaxon.com),

(24) Pettersen, E. F.; Goddard, T. D.; Huang, C. C.; Couch, G. S.; Greenblatt, D. M.; Meng, E. C.; Ferrin, T. E. UCSF Chimera-A Visualization System for Exploratory Research and Analysis, J. Comput. Chem. 2004, 25, 1605-12.

(25) Schaftenaar, G.; Noordik, J. H. Molden: A Pre- and Post-Processing Program for Molecular and Electronic Structures, J. Comput. Aided Mol. Des. 2000, 14, 123-34.

(26) Spartan 16, Wavefunction, Inc., Irvine, CA, 2016

(27) Halgren, T. A. Merck Molecular Force Field .1. Basis, Form, Scope, Parameterization, and Performance of MMFF94, J. Comput. Chem. 1996, 17, 490-519.

(28) Stewart, J. J. P. Optimization of Parameters for Semiempirical Methods V: Modification of NDDO Approximations and Application to 70 Elements, J. Mol. Model. 2007, 13, 1173-213. (29) M. J. Frisch, G. W. T., H. B. Schlegel, G. E. Scuseria, M. A. Robb, J. R. Cheeseman, G. Scalmani, V. Barone, B. Mennucci, G. A. Petersson, H. Nakatsuji, M. Caricato, X. Li, H. P. 
Hratchian, A. F. Izmaylov, J. Bloino, G. Zheng, J. L. Sonnenberg, M. Hada, M. Ehara, K. Toyota, R. Fukuda, J. Hasegawa, M. Ishida, T. Nakajima, Y. Honda, O. Kitao, H. Nakai, T. Vreven, J. A. Montgomery, Jr., J. E. Peralta, F. Ogliaro, M. Bearpark, J. J. Heyd, E. Brothers, K. N. Kudin, V. N. Staroverov, R. Kobayashi, J. Normand, K. Raghavachari, A. Rendell, J. C. Burant, S. S. Iyengar, J. Tomasi, M. Cossi, N. Rega, J. M. Millam, M. Klene, J. E. Knox, J. B. Cross, V. Bakken, C. Adamo, J. Jaramillo, R. Gomperts, R. E. Stratmann, O. Yazyev, A. J. Austin, R. Cammi, C. Pomelli, J. W. Ochterski, R. L. Martin, K. Morokuma, V. G. Zakrzewski, G. A. Voth, P. Salvador, J. J. Dannenberg, S. Dapprich, A. D. Daniels, Ö. Farkas, J. B. Foresman, J. V. Ortiz, J. Cioslowski, and D. J. Fox Gaussian 09, Revision D.01, Wallingford CT, 2013

(30) Chai, J. D.; Head-Gordon, M. Long-Range Corrected Hybrid Density Functionals with Damped Atom-Atom Dispersion Corrections, Phys. Chem. Chem. Phys. 2008, 10, 6615-20.

(31) Minenkov, Y.; Singstad, A.; Occhipinti, G.; Jensen, V. R. The Accuracy of DFT-Optimized Geometries of Functional Transition Metal Compounds: A Validation Study of Catalysts for Olefin Metathesis and Other Reactions in the Homogeneous Phase, Dalton Trans. 2012, 41, 5526-41.

(32) Dunning, T. H. Gaussian-Basis Sets for Use in Correlated Molecular Calculations .1. The Atoms Boron through Neon and Hydrogen, J. Chem. Phys. 1989, 90, 1007-23.

(33) Woon, D. E.; Dunning, T. H. Gaussian-Basis Sets for Use in Correlated Molecular Calculations .3. The Atoms Aluminum through Argon, J. Chem. Phys. 1993, 98, 1358-71.

(34) Schuchardt, K. L.; Didier, B. T.; Elsethagen, T.; Sun, L. S.; Gurumoorthi, V.; Chase, J.; Li, J.; Windus, T. L. Basis Set Exchange: A Community Database for Computational Sciences, $J$. Chem. Inf. Model. 2007, 47, 1045-52.

(35) Feller, D. The Role of Databases in Support of Computational Chemistry Calculations, $J$. Comput. Chem. 1996, 17, 1571-86.

(36) Peterson, K. A.; Figgen, D.; Dolg, M.; Stoll, H. Energy-Consistent Relativistic

Pseudopotentials and Correlation Consistent Basis Sets for the 4d Elements Y-Pd, J. Chem. Phys 2007, 126, 124101.

(37) http://www.tc.uni-koeln.de/PP/clickpse.en.html (accessed 18-03-2020)

(38) Zhao, Y.; Truhlar, D. G. Computational Characterization and Modeling of Buckyball

Tweezers: Density Functional Study of Concave-Convex $\pi \cdots \pi$ Interactions, Phys. Chem. Chem. Phys. 2008, 10, 2813-18.

(39) Ribeiro, R. F.; Marenich, A. V.; Cramer, C. J.; Truhlar, D. G. Use of Solution-Phase Vibrational Frequencies in Continuum Models for the Free Energy of Solvation, J. Phys. Chem. $B$ 2011, 115, 14556-62.

(40) Harvey, J. N.; Aschi, M.; Schwarz, H.; Koch, W. The Singlet and Triplet States of Phenyl Cation. A Hybrid Approach for Locating Minimum Energy Crossing Points between NonInteracting Potential Energy Surfaces, Theor. Chem. Acct. 1998, 99, 95-99.

(41) Gannon, K. L.; Blitz, M. A.; Liang, C.-H.; Pilling, M. J.; Seakins, P. W.; Glowacki, D. R.; Harvey, J. N. An Experimental and Theoretical Investigation of the Competition between Chemical Reaction and Relaxation for the Reactions of ${ }^{1} \mathrm{CH}_{2}$ with Acetylene and Ethene: Implications for the Chemistry of the Giant Planets, Faraday Discuss. 2010, 147, 173-88. (42) Page, M.; McIver, J. W. On Evaluating the Reaction Path Hamiltonian, J. Chem. Phys 1988, $88,922-35$.

(43) Page, M.; Doubleday, C.; McIver, J. W. Following Steepest Descent Reaction Paths. The Use of Higher Energy Derivatives with Ab Initio Electronic Structure Methods, J. Chem. Phys 1990, 93, 5634-42. 
(44) Hratchian, H. P.; Schlegel, H. B. Using Hessian Updating to Increase the Efficiency of a Hessian Based Predictor-Corrector Reaction Path Following Method, J. Chem. Theor. Comput. 2005, 1, 61-69.

(45) Hratchian, H. P.; Schlegel, H. B. Accurate Reaction Paths Using a Hessian Based PredictorCorrector Integrator, J. Chem. Phys 2004, 120, 9918-24.

(46) Perdew, J. P.; Burke, K.; Ernzerhof, M. Generalized Gradient Approximation Made Simple, Phys. Rev. Lett. 1996, 77, 3865-68.

(47) Grimme, S.; Ehrlich, S.; Goerigk, L. Effect of the Damping Function in Dispersion Corrected Density Functional Theory, J. Comput. Chem. 2011, 32, 1456-65.

(48) Minenkov, Y.; Occhipinti, G.; Jensen, V. R. Complete Reaction Pathway of RutheniumCatalyzed Olefin Metathesis of Ethyl Vinyl Ether: Kinetics and Mechanistic Insight from DFT, Organometallics 2013, 32, 2099-111.

(49) Smith, D. G. A.; Burns, L. A.; Patkowski, K.; Sherrill, C. D. Revised Damping Parameters for the D3 Dispersion Correction to Density Functional Theory, J. Phys. Chem. Lett. 2016, 7, 2197-203.

(50) Cossi, M.; Scalmani, G.; Rega, N.; Barone, V. New Developments in the Polarizable Continuum Model for Quantum Mechanical and Classical Calculations on Molecules in Solution, J. Chem. Phys. 2002, 117, 43-54.

(51) Scalmani, G.; Frisch, M. J. Continuous Surface Charge Polarizable Continuum Models of Solvation. I. General Formalism, 2010, 132, 114110.

(52) Tomasi, J.; Mennucci, B.; Cammi, R. Quantum Mechanical Continuum Solvation Models, Chem. Rev. 2005, 105, 2999-3093.

(53) Zhao, Y.; Truhlar, D. G. A New Local Density Functional for Main-Group

Thermochemistry, Transition Metal Bonding, Thermochemical Kinetics, and Noncovalent Interactions, J. Chem. Phys. 2006, 125, 194101.

(54) Zhao, Y.; Truhlar, D. G. The M06 Suite of Density Functionals for Main Group Thermochemistry, Thermochemical Kinetics, Noncovalent Interactions, Excited States, and Transition Elements: Two New Functionals and Systematic Testing of Four M06-Class Functionals and 12 Other Functionals, Theor. Chem. Acct. 2008, 120, 215-41.

(55) Perdew, J. P.; Schmidt, K. Jacob's Ladder of Density Functional Approximations for the Exchange-Correlation Energy, AIP Conf. Proc. 2001, 577, 1-20.

(56) Zhao, Y.; Truhlar, D. G. Attractive Noncovalent Interactions in the Mechanism of Grubbs Second-Generation Ru Catalysts for Olefin Metathesis, Org. Lett. 2007, 9, 1967-70.

(57) Kendall, R. A.; Dunning, T. H.; Harrison, R. J. Electron Affinities of the First-Row Atoms Revisited. Systematic Basis Sets and Wave Functions, J. Chem. Phys 1992, 96, 6796-806.

(58) McMullin, C. L.; Jover, J.; Harvey, J. N.; Fey, N. Accurate Modelling of $\operatorname{Pd}(0)+\operatorname{PhX}$ Oxidative Addition Kinetics, Dalton Trans. 2010, 39, 10833-36.

(59) Harvey, J. N. Understanding the Kinetics of Spin-Forbidden Chemical Reactions, Phys. Chem. Chem. Phys. 2007, 9, 331-43.

(60) Schiwek, C.; Meiners, J.; Förster, M.; Würtele, C.; Diefenbach, M.; Holthausen, M. C.; Schneider, S. Oxygen Reduction with a Bifunctional Iridium Dihydride Complex, Angew. Chem. 2015, 127, 15486-90.

(61) Delaude, L.; Demonceau, A.; Noels, A. F. Visible Light Induced Ring-Opening Metathesis Polymerisation of Cyclooctene, Chem. Commun. 2001, 986-87.

(62) Webster, C. E. Computational Insights into Degenerate Ethylene Exchange with a GrubbsType Catalyst, J. Am. Chem. Soc. 2007, 129, 7490-91. 
(63) Romero, P. E.; Piers, W. E. Direct Observation of a 14-Electron Ruthenacyclobutane Relevant to Olefin Metathesis, J. Am. Chem. Soc. 2005, 127, 5032-33.

(64) Romero, P. E.; Piers, W. E. Mechanistic Studies on 14-Electron Ruthenacyclobutanes: Degenerate Exchange with Free Ethylene, J. Am. Chem. Soc. 2007, 129, 1698-704. 